$\because f 60(6)$

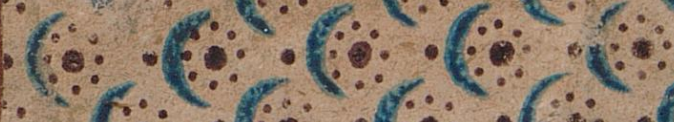

$\therefore C \cdot(\because) \cdot(\because)$ - ( $)(6)$ $\because \because 6)$ $6(\because \because \because)$ $6-60)$ $\%$ (6)

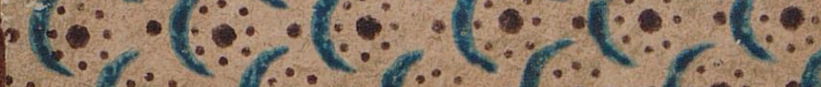

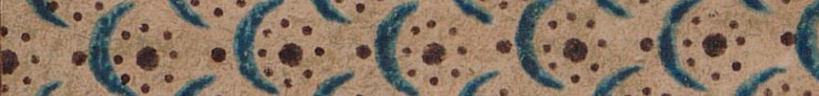

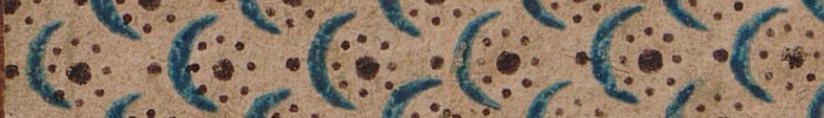
H.

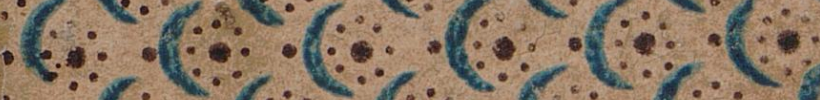

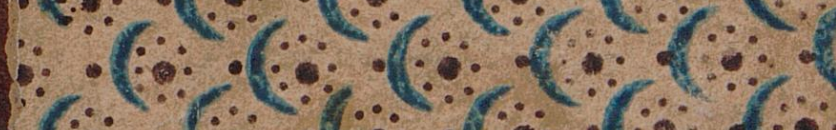

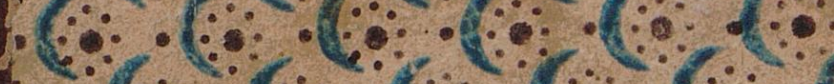
(1)

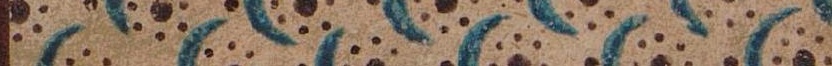

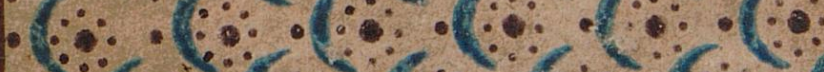

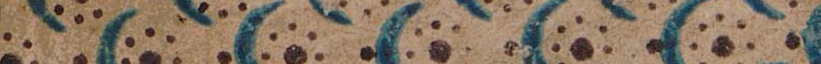
0
0

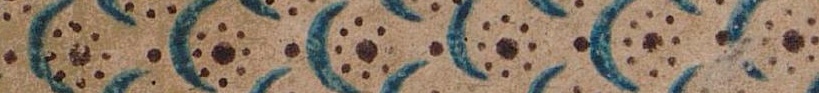

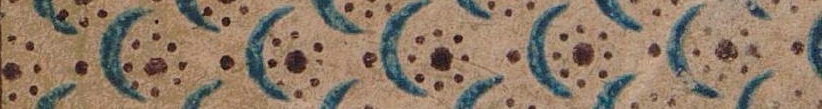

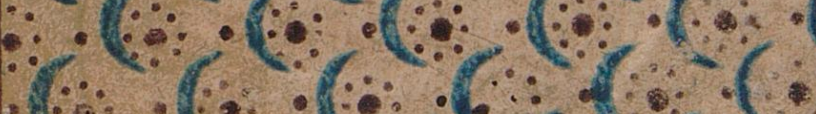

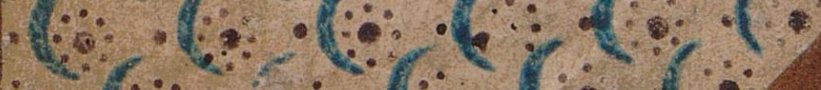
$\therefore(\therefore) \therefore(\therefore)$

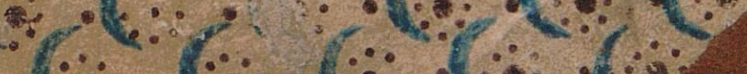

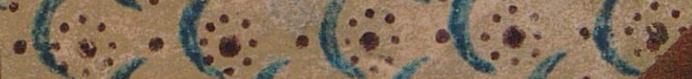







\title{
Det
}

\section{Deutide sinderfreund}

\author{
e in
}

\& $\in$ ( ค b U d

fits

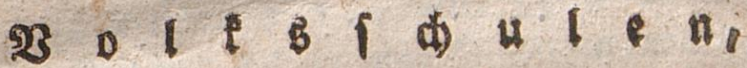

Dof

\section{3. शु. 2Bilmfen,}

Prediger an Der Narodial= STitwe Ju \$erlito :
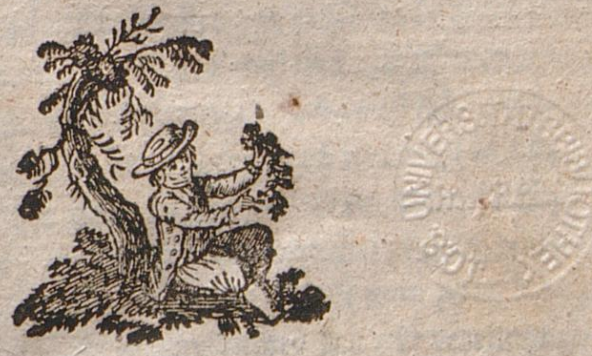

0000000000000000010000000000

3weite verbefferte mit Riebern un befängen vermebtte subgabe.

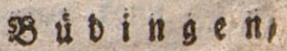

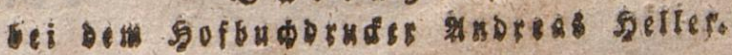




\section{t: 8 ?}

ditม

this o 8 is

(6.) $11.43: 3.9$

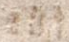

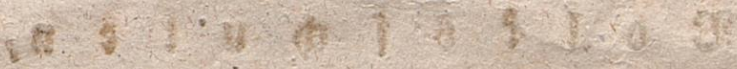

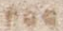

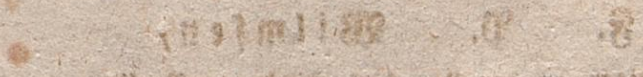

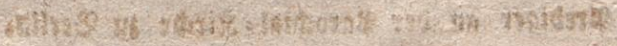

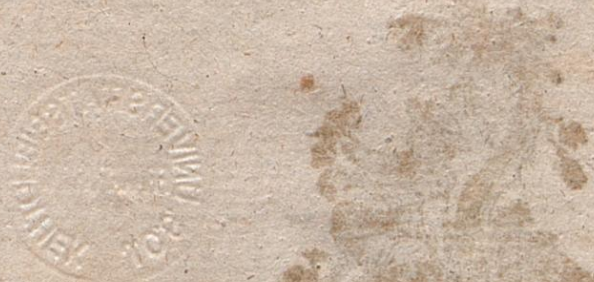

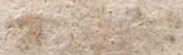
1963.

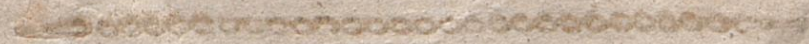
trobiang

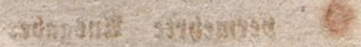

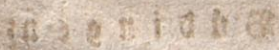

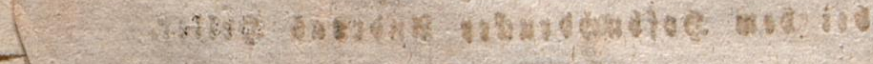




\section{2 ored}

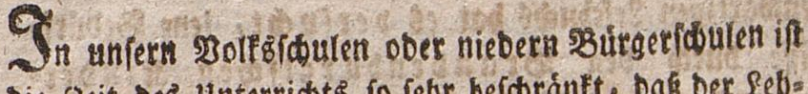

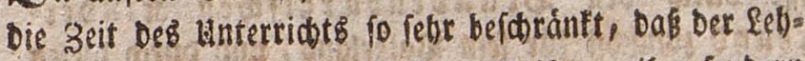
rer alles thut, loak man billiger $2 B e$ eife yon igm forbern fann, wenn er feine Edutiler babjin bringt, bas fie mit

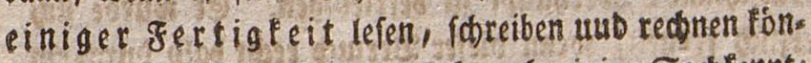
nen. Eollen fie auferberm aud now einige Sadfenuts

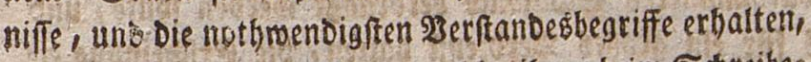

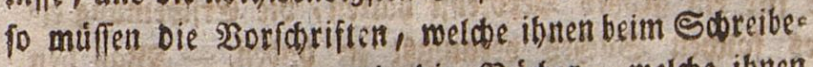
Unterriकt norgelest, unb bie Buider, welde ihnen bei ben Refe Hebungen in die Săñoe gcgeben merben, fo eingeriobtet fenn, Baf fie, indem fie fisceiben und Leferm, Durd Den Inthalt bis Befforictenen und Betefenen zum Denfen genobthigt, und mit ben nothmendigfen Sadfennt.

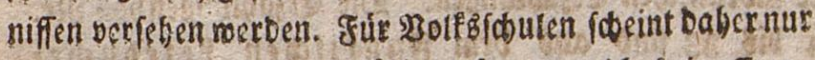

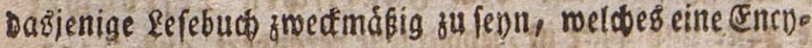

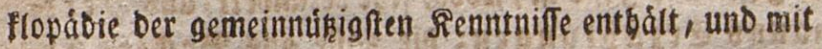
Der erforderfidell $\Re$ eidbaltigfeit aub Die móglidfre $\&$ a fri

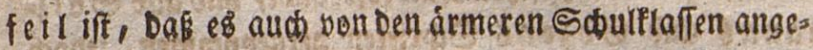

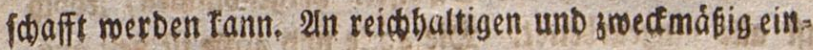

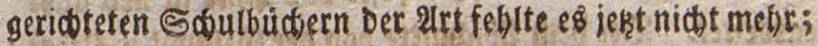
aber es feblte bisber nod immer an einem Esbulbudpe,

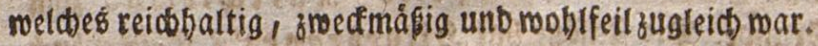

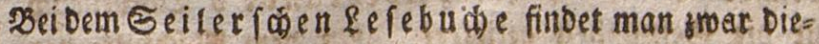

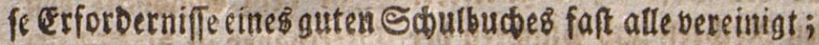

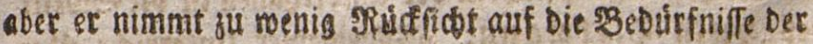
Retiber aus Den niebrigfent Boflestlafen, unb berfo noth: menbige Stufengang vom Reidtern zum Swheren mirb Barin faft ganz vermift; Daher es eigentich nur als eil

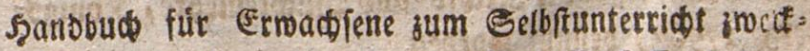
máfig gentunt werden fann. Der Berfaffer Dé̆ ge- 


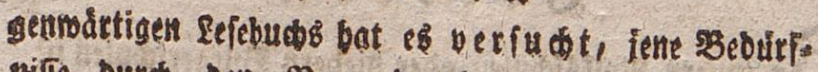
nife burd Den Brandenburgifen Rinder freund jundodft fúr fein Baterland, und burd biefen Deutfien Rinderfreund fur bie Bolligitulen

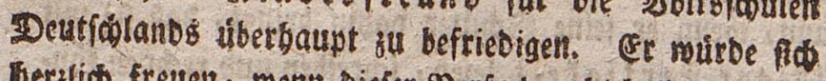

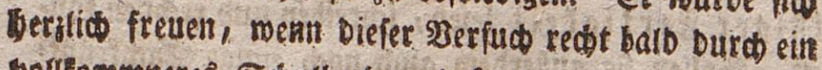
solltommneres Sajulbact) verbrångt merben follte, unb es fur eit grobes soebienf balten, Die erfobeinung eiz mes folden sBudes verantaft ju brben. - TBas Den Inthart Deefes S3ude's betrifft, io ift Darin mur weniges son Anbern entlebut, und altes entrehnte if fút ben lefonbern 3meat Des suches umgearbeitet. Die furzen Síne find neu auggearbeitet, ellen fo Der grósere Theil Der moralifoben Erzáblungen. Die Erzáblingen von ßr. 24 bis 33 fint aus bem sunfifoen fefebude; Die 34 fte uno 35 fte ift, jebod febr veraindert, aub Şer ro mants exidorungen; Die 3 Gfte aus secter ह ex-

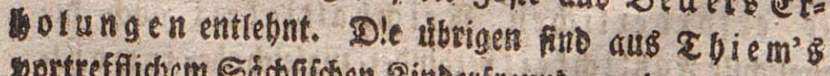

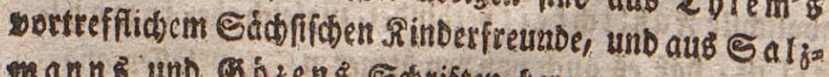

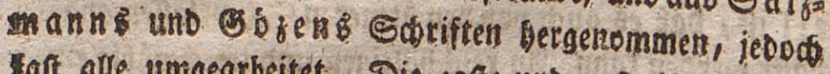
Faft alle ungearbeitet. Die 42 fte unb 49 Re if neu aus.

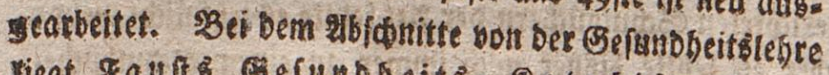

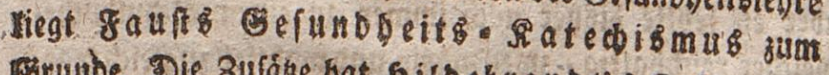

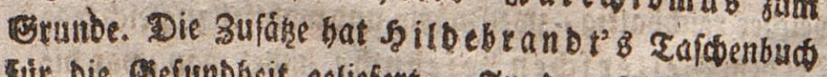
fut bie Oefundheit geliefett. In tem sbrobnitte bon Den SRedren unb \$flidten Der Unterthanen if Die Sdrift

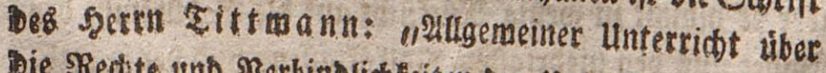
Die Retite unb ఇerbindridfeiten ber Untertbanen, Reip=

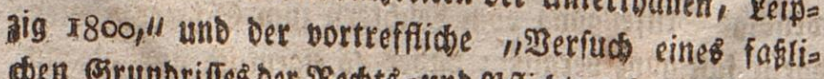

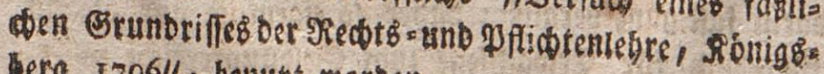
Leng I72611, benught morben. 


\section{9 a 1 t.}

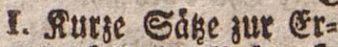
medung ber 2 lufmetf

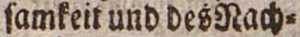
Denten:

II. Erådblungen zur \$be forderung guter Be $=$ finnungen uno sur Eđdurfung Deవె গูe fandes. . 36

1. Die ungegogenen Sin: Der

2. Die 3 erfudung:

3. Die úble Bemohnheit

4. Die fleinen Diebe

5. Der Tagebieb

6. Der fleine serfíchen: Der

7. Das mohtthảtige Sino

8. Das ordentlide Rino

9. Der eúgner.

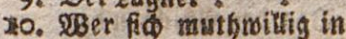
Gefahr begiebt, fonat Darin um

I1. Der ebritide Inabe 45

12. פBer nide Güren mill, mú fúblen

13. Der Freund in Der. groth

14. Der వanffü

15. Die muthwilligen RinDer

16. Der unafriebene

17. Det פsarmbergige

18. Der Eurdtfane.

19. Die gute Todter

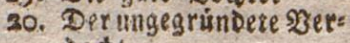

ax. Dab neugierige 2 Rn $^{\mathrm{D} D}=$ (1)
II
Seite

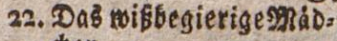

23. 5Renfobenfreundiade ${ }^{56}$ Oefinmungen

24. MBas beif: fomolles? 58

25. Befålligfeit 59

26. Die Eerláumberist 60

27. Die 23 abriagerin $6 x$

28. Der Biudéfpieler 62

29. 2uerglaube 63

30. Die folgen des fleifte und Der zaratbeir.

31. Ráføerei • 66

32. Der Bhierquáler: $\quad 67$

93. Settvestreib - 68

34. Hnupríbrigleir. 69

35. Die filatfoerin. $2 x$

36. Ein guter Dentiprud if ein freuni in ter roth

37. Berfübrung : 74

38. Der undanfbare डळळน= lev 39. Galfde Sdiars: 78 40. Dep unbefonnene Şрав 79 4I. Ebrri申 wábrt aut lángînen 80 42. Jugendide unbefon.

nenbeit 82 43. Haterfoied imítion Eparfamteit uno (8) eis 83 44. Der : Bienenfod". 84 45. Der ฮif́teid . 87 46. Diz Rnupenfeinde 9 47. Mit Soliefoerwebren foll man niфt foielen 96

48. Eine gute Sandlung aus fकledten serve= gungsgrimben 97

49. Jraurige Fofgen Der Biltbbeit 


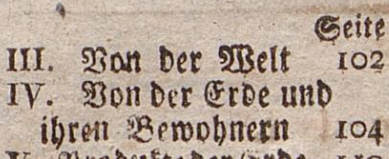
v. Produfte Der erde Iro

I. Dab Ebierreid $\mathrm{NH}_{3}$ Sáltgethiere soígel ? Intribitien 120 giriche : Tiretren. ổúrmer

2. D) ह अ A angenreid I 34 3. Da Es? inerafreiditi

VI. গon Dem STenjoden 144

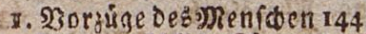

2. Der menfolide Sitruer 149 sinoden.

Mußfe!n . 150

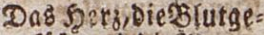
fábe und Die2d ern 15 t Son ben fungen tino Dem Litbembolen. 153 2zon bex פzerbauming Der Epeiren . 155

23on nemesebirn, Dem sricfenmare, und Den Merben. szon den Sinnen 160 23on der Saut, ben Sganren und DenशRa. getin $x 68$

7I. Sejunbbeit lę) 170

8. Befundbeit und Siranf= beit . 170

2. Szon Der fileidung I7I

3. 23on Der \&uft $\quad 172$

4. Du folle reinlid und oroentiti fenn : $\quad 174$

5. 23on den Speifen 176

6. Son Den Setránfen 180
7. Son ber 3etteguilg unid siutse . 184

8. 2rom EdInfen 185

9. Von den 28 ofnungen 18 ?

10. 23on Erbibungen und Crfáltungeí

II. $230 n$ Dererbaltung eins zelner sbeile des för Ders : 19I

12. 3an Der Sdonbeit und solfontmenbeit Deg förpers . 198

13. 20n Den sierbalten in Srantbeiten

VHI. SON Der Beitrech)=

nung u. Demsialenber 2 or IX. Merfroúroige Ma=

tur= erí)sinungen 208 X. Europr. 213 XI. Deurfalano 2 I9 XII. Bon Den Rediten und Pflicten Der Iln: tertbanen in wotleilla geriфteten Staaten 226

1. Bon ben Redten der Meniden 229

2. \$oi den Mflidten ge= gen Mitburger . 23I

3. S3on den Yflidten der Untertbanen gegen Die Dbrigfeit . 235

4. Son Dem 2Berbalten Der sjenicten bei Dem (Bebraude ibrer Re chte $23 \%$

5. Bon Den Stáriden in Der bürgerfiden Befell= foaft . 238

6. $30 n$ Den Serrfwaften und Dientiboten - 240

XIII. Lieber unb Cefánge. . $24 \mathrm{r}$ 


\section{I.}

\section{Siutse Sảke zur Ermectung oer 2tufmert:}

\section{famfeit und Deg ract)denfenz.}

ริ

(5) gebore su ben Rindern. Sinber miffen nod nidit viel, und Darum múfen fie unterrich tet merDen uno lernen. Daourct merben fie berftándig.

Ja) merbe in ber Soule unterrichtet, und Deriente ge, welober mir unterridt giebt, beibt ber $\mathcal{E} e$ b $\mathrm{r} \in \mathrm{E}$. Jid) bin meinem Sebter Danfbarfeit und Beboriam idouldig. So lange io unterriatet merbe, bin id ein Sduter. Cin guter Souter if aufmerffam; er bort nur auf Das, maś ber febrer lagt, nno Denft nui an Dab, mas er thun, oder begrelfen uno bebale tell folf.

Ein guter Edulfer fommt gern in bie Sdulep if fleibig, orbentlid), reinlid, firtfam uno friebfero tig. Er fommt nie zu foát in bie ed)ule, ift nidt sollo bei Dem fzerausgehen aus oer Sctule, uno rreibr (iid) nicht auf ber Strabe berum, (onbern gebt auf bem geraben 5 sege nac Saufe. So will ein guter Ed the ler leon.

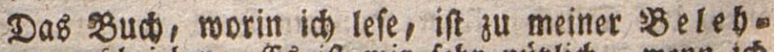
rung geidrieben. (EB if mir febr núblid), menn to

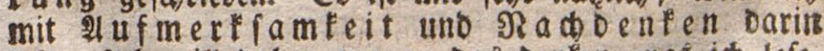
lefe. Job roll baber nur an Das Denten, was ich lefe, und mid bemuben, Das Eselejene zu ber ft eben. 2Benn id etmas nidbt verftebe, io will id meinen lehter bits ten, Dás et es mis sthlére. 


\section{Surfe Gåk̨e zur Ěrmectung Der Tufmexffamfeit}

2RBenn id aufmerliam und mit Rabbenten in Diez fem $B$ bude lefe, uno Daş, was ith gelefen habe, nidt vergefle, fo erlange it allerlei núblite Sienntnif= fe, und merde verfténdiger. SRidt alle finder er = langen Durch ben llnterricht nikliche Remtniffe. Man= d) bleiben unwiffent und unverfándig. WBelde?

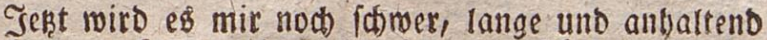
adtfam fu fepn; aber eb miro mir lunftig leidoter werben, wenn ic im entange die Múlibe nicht fiteue.

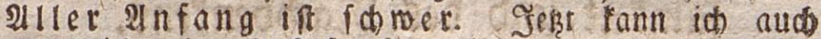
noth nich obne 2 infto lefen; aber menn ich mir Mribe gebe, fo werbe id es balo fennen, uno mich Dann freuelt, Dap ith eine fertigfeit im fefen erlengt babe.

- job tenne mein \&e(ebua), benn id meifi, mie ber Titel Deffetben beif́t. Er ftebt auf Der erftent

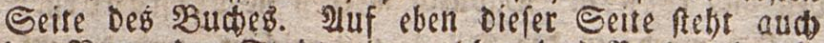

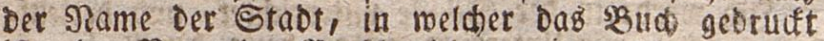
ift; Der Name ber s3udbandiung, in melther es bers

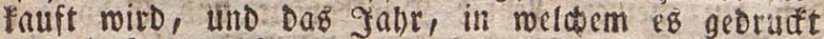
ift (oie Jabreszaf)!). - Id fenne mein fefebuad) nicit blóp an bem Eitel, fonbern aud an bem Einbalts De oder Dedel.

cine Intralt, in melder sgutaer georudt mendeti, beift eme $5 B$ u o oructerei. Ja fenne nod eimige

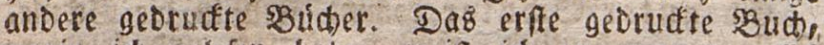
rovin id gelefen babe, meiß id) zu nennen. (ए8 beist

Der, weldber ein BBuch berfertigt ober berfafit, beibt Det Berfaffer ber S3udter. (Semóbntia) freht Det SRame Deś \$3erfaffer's auf Dem Sitel Des \$3udes. Erft miro vas 5 buch geidricben, Darn miro es georudt, uno Durd) Dấ Drutten tann man ein Bud) in furzer 3eit mebrere taufenomal bervielfáltigen. Diefe Sintt wirb

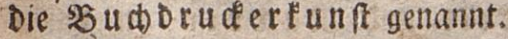

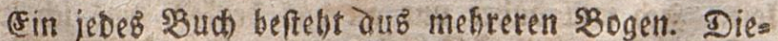
re SBogen merben uon Dem BZuchbinder zufammengehef= tet, bamit fie nicht berloren geben, Doer in Hnorbtums getathen fonnen. 
2Benn id einige Sibre sitter bin, unb nidft melor wadble, to gebore id) fu ben (Erroad) fenen. Dann bin id grofier, als jeßt, uno bann merbe ich audi) ver.

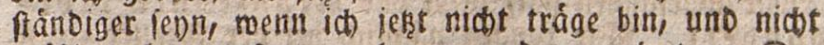
míbig gelge, anfatt zu letmen und zu arberten. Der

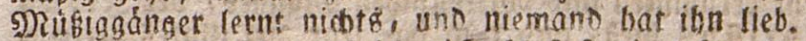

In Der Satule mus io niat blok fertig lefen, fons Dern auth Deutito foreiben, und mit zertigfeit rect)= nen lernen. 2 ser nidbt Befótriebenes lefen, unb nidt felbft foreiben fann, fommt in ber 2 Belt nicht fort; uno wer naksiednen nidbt berfteht, wiro oft betrogents uno meiß fich in vielen Jállen nicht gu belfen. Jँd mill mir redt biel Milke geben, fertig lefen, fareiben uno rectinen zu leriten.

Mancbe Stimer laffen fich gern belebren; e8 macht

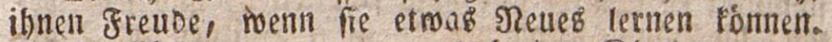
Soldhe Sinder nent man gelehrige Rinder.

याह id ein $\Im a b r$ alt roat, fonnte is noch nidt nod) ni(t) - unb nod untb. - Sm zmeiten Talyre

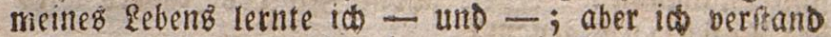

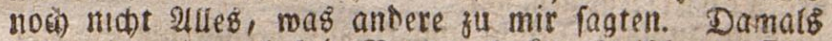
gab man mir nocb lein Broo uno fein fleifth fll effen; Denn ia batse nod nidht alle Bábne, uno tonnte alís feine fefte Spetie vertragen.

S(b) beise mit meinen פornamen -; mit meinen

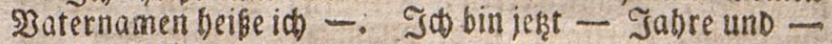
gronate alt.

Eieben Tage geboren zu einer 230 obe; vier $2 B D=$ wen, uno zree ober orei Sage, maden emen No nat auz. 3mólf 9lonate, ober 365 Eage gehoren 3 us einem Jabre. Seabs Nonate madten ein balbes Tabr, uno Drei Mronate ein Sicrteliabe aub. Die Nament Der zmolf Monate heifen nach ber Dronung alfo: Ta=

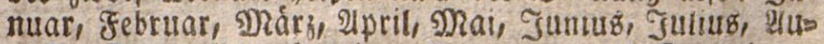
gut, September, Drtober, शrovember uno December.

SBent 52 W30 den, oder 12 gronate verfloffen find, to ift ein gable ju Enoe, und bam fángt fich ein

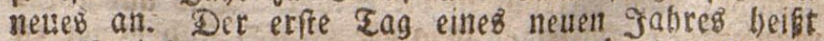

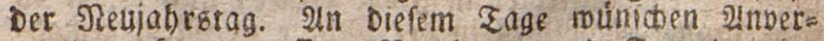
manbre, Sausgenoffen, Racharen und Freunde ein: ender Blúf und greube. 


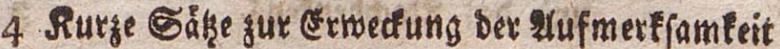

2Benn hunbert Sabte berfloffen find, fo ift ein Sable humbert zu Enbe. Das Sabibunbert, roldes fich un.

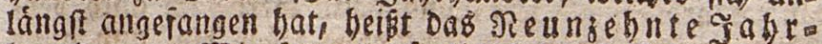
bundert. West fangen namlid bei Der Beburt eineB

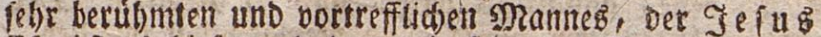

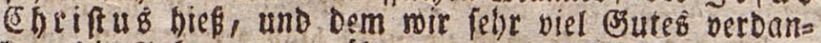
Fen, Die Sabre an zu fállen, uno nun fino idon 1812 Sabre berfloffen, feitbem er geboren mars.

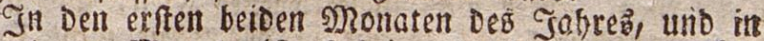
Dem leţten s)onate if es bei uns febe falt; es fált

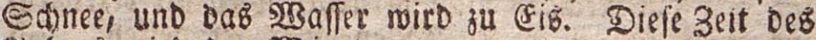
Gabees roito Der $2 B$ inter genaunt.

In ben brei gronaten, seld)e auf ben gebruar fols gen (wie beifen fie?) ift eb niat mebr fo falt; Das (eib fd)miljt; $e B$ fantneiet ntir nod) febr leiten, uno fefor we=

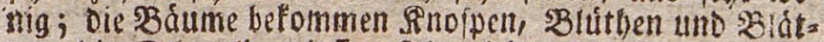
ter; bie Sombalben laffon fich wieber febell, uno einige SBlumen blúben, befonders Schmeglóftien, פeildien und Snaiblumen. Diefe fobone 3eit Deg Jabres roird Der zruftillg genantit.

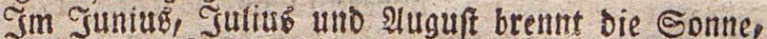
Die Euft wirb oft feb) beik, man flagt úber Şişe, unb

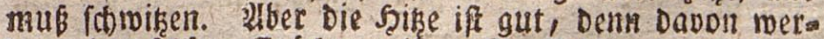

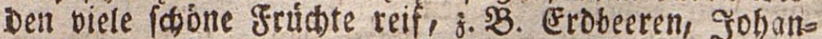
niäbeeren, Stabelbeeren, Riridien, Birnen, Hepfel, Wfirfichell, Aprifofen uno Pflamen. Diefe zeit Deb Sabres miro Der So m mer genannt.

Sm September, Detober uno Robember merbet alle Fridite in Den BSárten unb nuf Den Felbern einge= fammelt unb in Die शortathoffammern, auf Den 350 Den unt in Den Reller gebradt. Der Bauer hat foton im

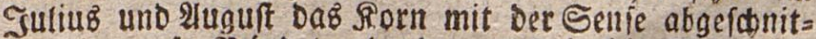
sen, in grofe 3 kundel gebumben, uno in bie Scheune gebracht, too es allşgeorolichen wirb. SRun grábt et. auch Die Rartoffeln uno Siuben aus; faneibet Die Difen Soblfopfe ab, unb bringt Dab́, mas er felbft nidt braudt, zum Berfauf in bie Etaot. Daš auşgeoro=

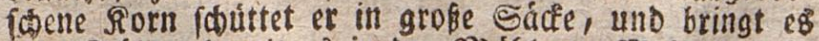

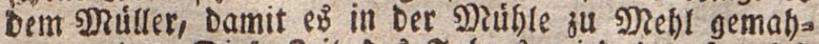

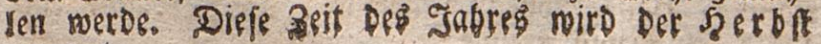
genannt. 


\section{unb beg Facbenteng.}

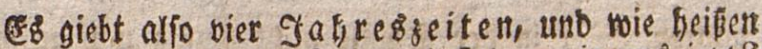
fie? in weldser 3eit Des Jabres befinden mir uns lest? \$ann ift Diefe Jabrezzeit ju Ende? Hno melche folgt

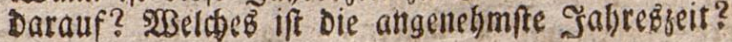

SBer hungrig ift, will effen; wer burftig iff, will trinten; wer mube if, will ausbuben; wer neugierig iff, will alles wiffen uno alles feben; wer mitleidig iff, will gern Dem unglúftichen belfen; wer eigenfinnig iff, will immer feinen wsillen haben; wer zánfifo iff? soill nidt in Frieben leben; mer frieofertig ift, mill nicht sanfen; wet frant ift, will gefund werben; wet gefallent ift, soill mieber auffteben; mer unbienffertig ift, toill झmbern Feinen Dicnft erjeigett.

2Bser nichts toeí, foll etwab lernen; mer frant iff, forl fich Des (Effens entbalten; mer nicht arbeiten mage foll aud niकt effen; mer feinen Eittern und fefrern niabt gebord)t, foll Strafe leiden; wer nidat boren will, soent man ihn ermabnt, foll fúblett.

WBer fich befomust bat, $m u \tilde{B}$ fich mafden; wer gefuno bieiben roll, muß másig effen uno trinfen; wer etmaß̉ lernen mill, 'muß́ fleíbig und aufmerffam fenn; wer gut follafen roill, muß an ange fleisig arbeiten, und weder :

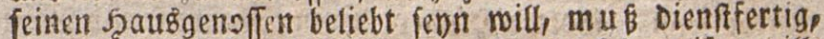
aufriq)ig uno freunolid fenn; wer etwab begreifen willp

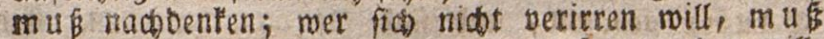
nach bem rechten 23 ege fragen; wer fatt werben mill, mü̈ effen; roer gelobt fevn will, mü fich anftándig uno berninftig betragen; wer feinen Eitern freude mas den will, muß in Der Sdule fleisig, zu Jaaufe fittram und geborfam, uno bet fremben Eeuten artig ledn; soer feine Rleiber lange baben mill, muह fie fobonen, uno reinici) balten; wer fonefl nad einem Sorte binfommen roill, $\mathrm{m} u \bar{B}$ eilen, uno nicht fáumen.

SBer viel Eselo einnimmt, fann aud biel Beib ausigeben, ooer er fann aud etwas aufiparen. Wer ein Scanowerf gelernt bat, fann fíd felbit ernóbren.

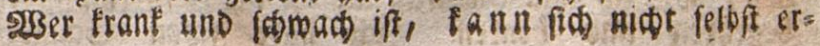




\section{Surge Cåke jur erwedung bet 2lufmerffamleit}

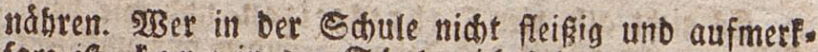
fan if, f a $n$ it in oer Ed)ule nidbis lermen.

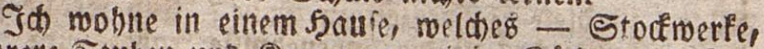
mehrere Sttben unb Rammern, einige Rititen uno Ret. ler, uno eiten Boben bat. In Diefem Jaanfe haben-

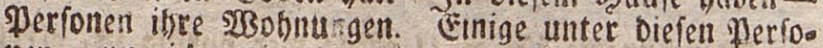
nen nenne ich meine - andere nentre ich meine -; Die úbrigen find meine jauggenoffen, weil fie mit mis

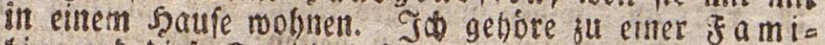
lie, unb biele Jamilie befteht aus meinen Eltern, mei= nen Beidbiffern und -

Daß Şaub, in melthem meine Eltern mobnen, ge= bort - Der, meldeem das Saaus gehórt, Weist ber

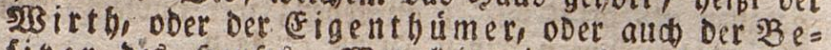

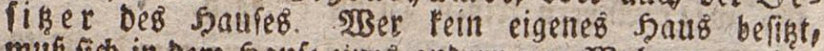

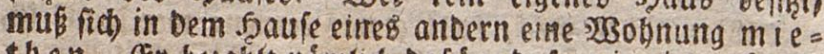
the $\mathrm{n}$. Er bezajitt námlich Dafúr, $\mathrm{oak}$ er in einem frems Den faute wohnen oarf, iábrtict) ein gemiffes Beld an

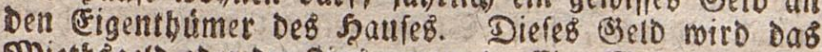

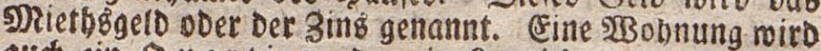
auch) ein Qu artier oder ein \&ogiz genant.

3u einer guten 2Boblinung geboren belle, gerảus mige uno trodene Stuben, Inftige uno geráumige Ram= mern, bequeme und belle steppen. Die Situde, Der Sieller uno ber sBoben múfen ebenfallß geráumig und luftig fenn. Solde Şáufer, beren Mautern uno æBám= De blok bon Stemen aufgefuihnt find, werden malfive Sf dufer genanut, und fint Die Dauerhafteften. Ein

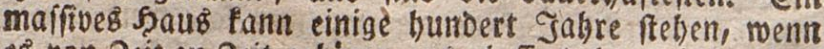
es bon seit zu 3eit gehorig auggebeffert (rcparit) wirb.

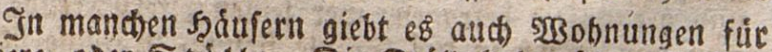
Zhiere, oder Srátle. Die estálle haben felten Fenfter, aber nirgento Defen. SBarum nict? In Den Ställen finbet man aud nicht Stúble, Eilahe, Epiegel, Epits Den ober Sarduffe. 2ber in manden Stallen frebt eim Bette. Sut wett?

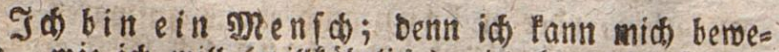

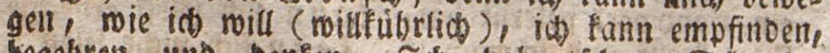
begebren uno benfen. Јa babe fobon Edmer? 


\section{und bez Nacbenfenz.}

Freube, Nitleiben, 2unaft unb gurdt empfunben. 2ud bie Zbiere fonmen 2 ergntigen uno Schmer $\mathrm{em}=$ pinden. Der şund freut fich, wenn er feinen Serrn fieht, et mimmert unt heult bor Sd)metz, metrn er bon einem andern şumbe gebiflen morden iff. Tht fañt meine Freube und meinen Sdimer; Durd, 230 rte for ets

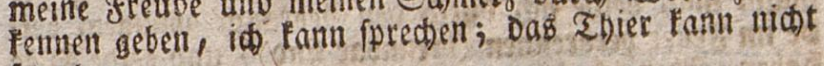
fpred)en.

Id gefle a ufredt, fann meinen Sopf in bie $S_{3}$ = he ridten, uno inn nad allen Seitert berumbreben. Die Tbiere geben zur Eroe gebuidt, unb fonnen Dent Jaimmel nidte anfeben. Tot fann fethen, libren, fublen; fomeden uno recten. Dies forntren die Shiere aud; fie taben, gleid Dem Menfan, funf Sinne. Man=

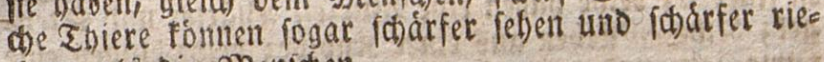
den, als Die Neniden.

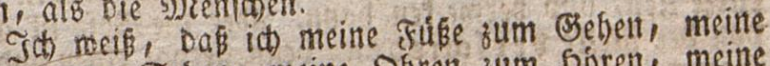
2ugen zum Eehen, meine Dhren zum Şbren, meine

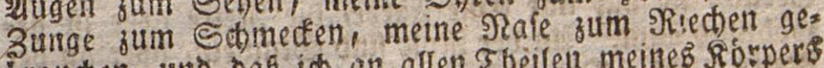
braudery, unt baß id an allen Theilen meines Ro:pers fúhlen fann; aber ein Thier weiß Dieß nicht.

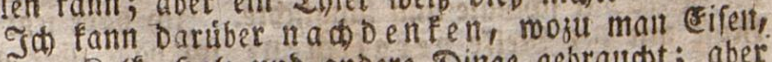
Steime, Ralf, Şolz uno andere Dinge gebrauctit; abet bie Egiere fonnen nidt nadbenten. Jo fann begret =

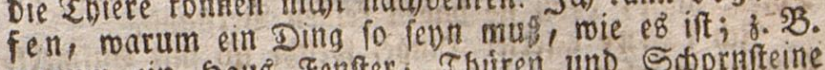
marum ein Şaus genfter, Thưten uno Sd)orafteine baben; marum der Dfen yon Egolierbe, und nidht von Jyolz gemacht merDen; warum man Die Pflanzen begles sen, uno Die Eroe umgraben mus. Go fann begreifen, warum ieoer sopf einen Sentel baben, und wate utm ein Meffer vori libarf, an Sitiden aber glatt unt ftuinpf fent muk. Iक meik, marum meitre Squble yon Leser, unb nidi bon Solz oder sgled, ober von इud gemant filib, unb warum ein 23 agen nidt melye

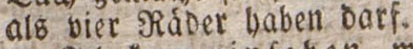

Jd) fann einfeben, marum id nicht immer thun Darf, was id will; ratum id thus folf, was meine Eltern und Rether wollen; marum ic folglam, fleifig und aufmerffam fann loll. - Tht weik, marum oie

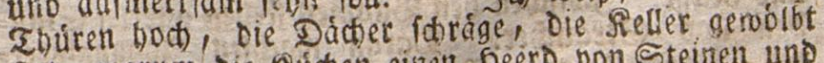

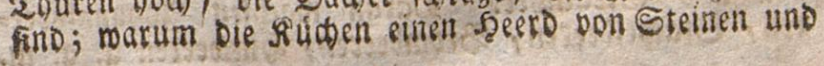




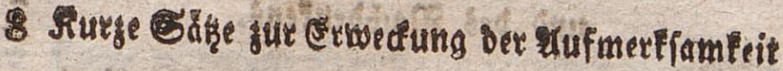

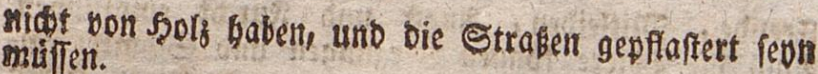

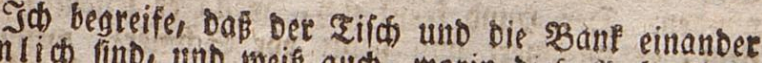

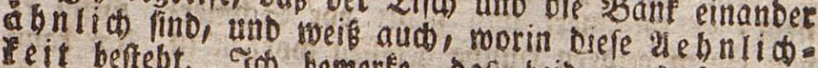

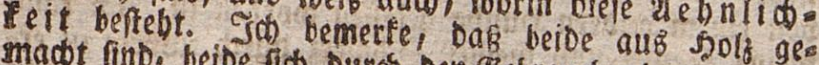
madt fint, beibe fid Durch Den Gebraud abmuken, beiDe im Jeuer verbrennen (brennbar find), uno beide Júbe haben. 2lber id fehe aud ein, Daf beibe eirtander is ins

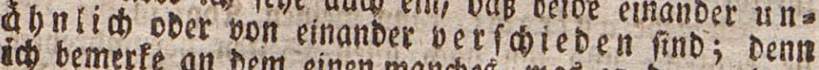
ić) bemerfe an bem einen manches, was an bem anbern sticht if , $: 58$. -

Die Siofe if Der Relfe dinnlict): Dern beibe fins

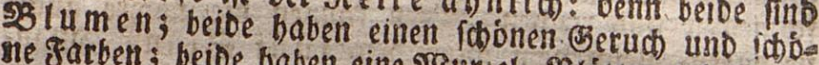

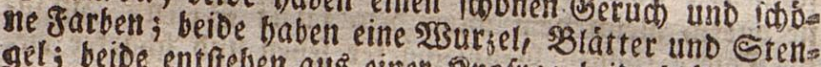

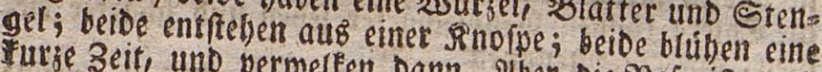
Furbe Beit, uno vermelfen Dann. 2lber Die Niofe iff aud son ber Selfe yerfdo ie Den; Denn fie bat einen anbern (seru(b), fie bat nur eime Farbe, Die Relfe aber if ges swóbntich bunt. 2in Der SRofe find Stadietn, aber an Dee

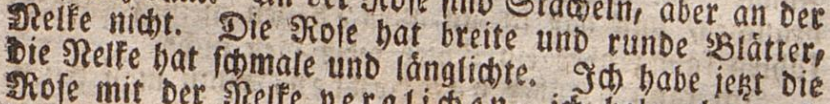
Siofe mit Der Selfe bergli is en, io babe aber auth beibe von einander unterfíle Den. Dieg fónten die sbiere nidbt, benn fie baben teinen serfant.

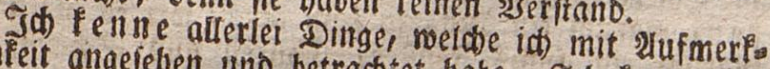
Jamkeit angejelben und betractetet babe. Job lenne sime SRenge Yflanzen, welde in Dem Barten wadien, 3. SB. Mohrtuben (Mobiten), Bobnen, Erbfen, Esurfen, WSein. frofoe, Fettige, Salatfráuter, alletlei Arten von Rob) ober Rraut, peterfitie, Emnittlaud, Salbei, Epara

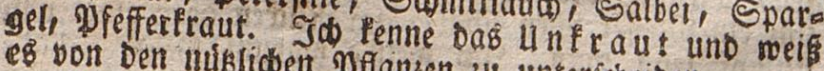
es von Den tuísliden Pflanzen zu untericheiben.

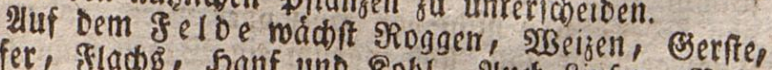

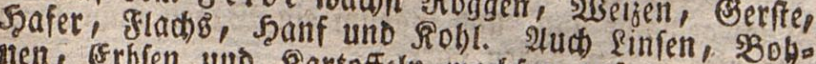

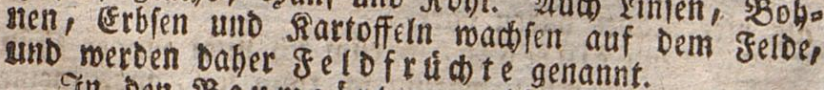

Jin ben soumgdrten wadien Birnen, 2epfel, Dhaumen (3metifsen), Ririden, 2ptifofen, Pfirfichen,

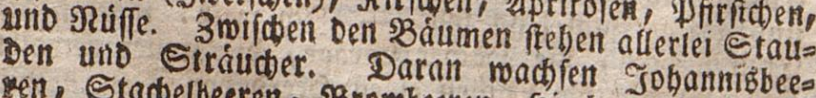
men, Stadelbeeren, sgrombeeren, Joimberren u. a. mo 
Ifre biele grtiots fann man effen, fie fitto efbar (und gefund), wenn fie reif find. Hnteife Sruidte find (imátidi.

Die $\mathfrak{B}$ åume berforgen unb nidt nur mit ibrelt faftigen Irúdten, fondern fie -erfreuen uns ald im Frubling Duref) ibre fanneermeifen und rofenrothen 3 lit= then, ersuiden uns an beikent Sommertagen Durch itho ren Schatten und wármen uns im sBinter burd ihe ŞOls.

Der 2 a u m frebt feft, weil er eine ftarfe $2 B$ urzel That, methe tief in bie Erbe binein gebt. 24uf ber 2rsur= jel ftebt Der Stamm, welder mit eitter feften $\Re$ ind mie mit einem Rleibe umgebelt iff. Llm bie Spige Deg Etammes berum fiken Die 2iefte, und an Den 2leften firen bie 3 weige, an ben zmeigen Die 3 lätter und

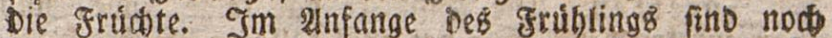

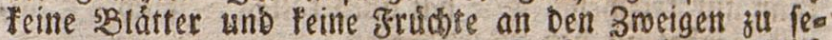
ben, fonbern nur sinofpen. Diefe brecten entido auf und Daraus entfethen Dank SBlútben uno Blâtter.

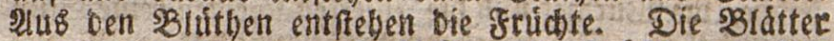

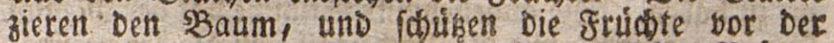
brennentit Solne. SBent ein Baum feint Blatter berliert, she Die Itridote reif fint, fo verbotren Doer עertrofinen Die grudite.

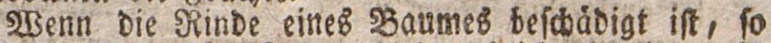
wirb ber sbaum franl, uno fritbet enblid) ab, ober gebt aus. Darum ift ez febr unrecht, uno verbient hatte

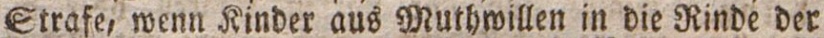

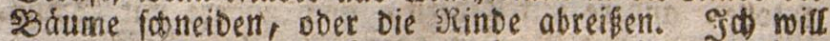
nie einen Baum befdadoigen; aber ich mill mid uber eimen gefunden und blubenden 23 aum freuen.

Dik I biere baben nidt einerlei Beftalt; es ît

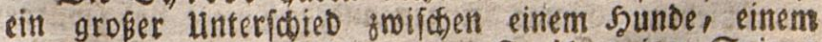
Eperling, einem Şect), einem frolo, einer Spinne und ciner Ednecte. Der Sunb bat bier gúfes, und gehort Daker ju Den vierfúkigen इbieren; bet Eperting hat nur zmei fúbe und zmei flúgel, er

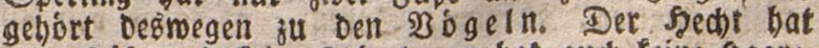

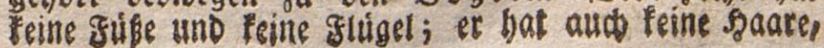




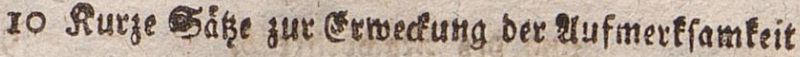

wie Der Sutub, und feine gebern, mie Der Sperting, fonbern Edaupen. Er farn niat geben, mie Der Shunb, und midt fliegen, wie ber Sperting, aber ee

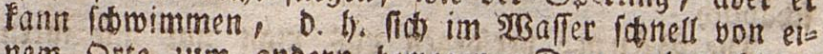
nem Drte zum anbern betwegen. Daju gebrautht ee Die gloffebern und Dent Schrmanz. Er genort zu ben Filich, uns leht im $2 B a f f e r$

Der grofid) hat jrour vier gtife, aber feine Sintero füfe frito lang, uno er gebrasudt fie weniger zum Be= ben, als fum Sdbmimmen. Er fann aud nid)t geben, ponbern nur Gupfen oder fpringen. Er lebt im शzaffer unb auf Dem fande, und getsort zu ben Umphibien.

Die Spinne (fianter) that 8 glife, uno fein rothes

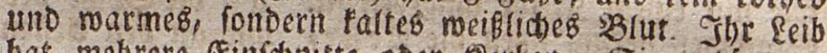
hat melhrete einfdnitte ober Rerben. Sie gethort 34 Den Jnieften. Die Edonefe bat reine gafe, und

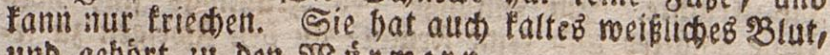
und gebout fu Den 28 úrmern.

gaft jebes Sthier bat eine befonbere stimme. Die Sifche, Die 2Búrmet und die Infetten icheinen feine Stim= me zu haben. Die angenelgmife Stimme hat Der menítb. Io babe gebort, wie bie Rachtigall uno oie ferche fingt, Der Stord flappert, ber sumb bellt uno fnuret, Die

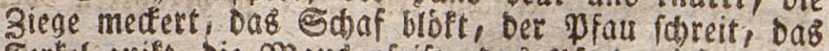

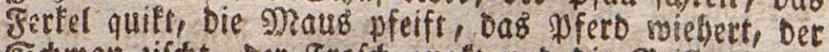

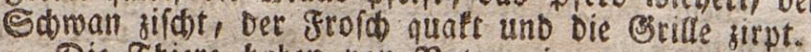

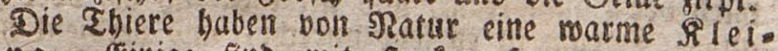
bung. Einige find mit frarfell şaaren ober mit 2Bolle, andere mit gebern, nod andere mit Saup= pert, eimige mit Borfen oder Stadern bebect.

Die wirben abiere, melabe in den 23aloern reben,

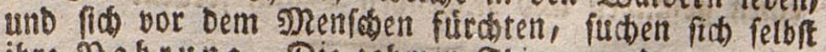
ihre Rabrung. Die zabment Ehiere merben von ben

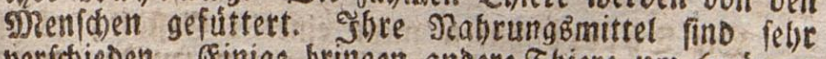
verfobieden. Einige brimben andere Thiere um (múrgen (ii), und freffen fie Dann auf. Diefe beiben $\Re_{a} a b=$ tb iere; anbere freffen toote shiete, welde fofon in

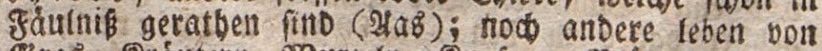

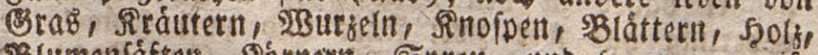
Bilumenffften, Sidinetit, Epreu, und fogat bon gif= tigen Pflanzen. 
Dofen, Silibe, Swafe, Pferbe unb siegent fref fell Brab uno Rituter: Szunbe und Raken frefen Fleifin); Suthner uno \&anfe Roun, befonders Berfe. Die sienen náben fỉd von Bglumenfáften, die meiften 2Butrmer von 2 Burjeln, die Siaupen bon Blastern.

Die áfieren \& lieder Det Thiere find fehe bets fáceben. Einige haben 2rme unb sBeine, námlid Die 2iffen; andere baben neber 2irme, noch SBeine, nod

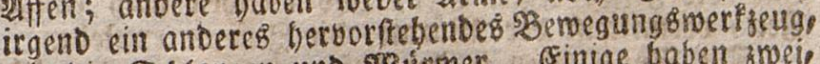
mie Die Edtangen uno 28 urmer. Einige baben zroeis andere vier, nod anbere fects ober act, ia einige for gar mebr alB g, unbert gúbe. Der Rellermurm, ein

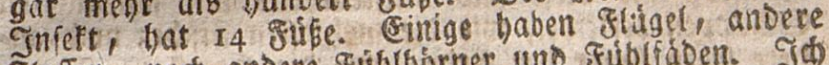

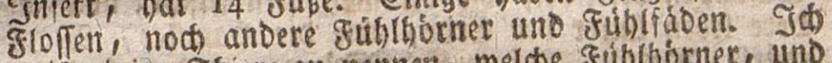
soeip einige Shiere zu nemen, melde gublborner, uno sinige, weldje gúblfabon baben.

Einige Ibiere babentuperorbentrich fá arfe Sin= ne. Die Raben und die Sande taben einen úberaus forarfen (seruch, uno ber sbler bat eis beroumberngs

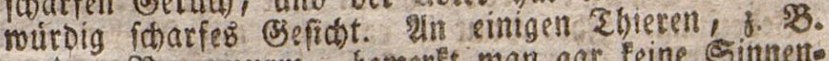
all bem Sicgenmurm, bemerft man gat feine Sinnentmerfizeuge, feine Dbren, feime 2ugen, und Peime Nafe.

Die shiere follafen, wenn fie ermitidet find, uno einige fotafen mit offenten 2ugen, $z$. 23. Die Sgafen; andere im Eteben, :. 3. Die Pferbe; manobe nut am

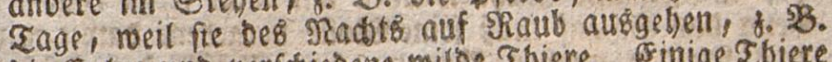
Die Eulen und verfótedene wiroe Ibiere. Einige Ithiere id lafen ben ganzen rssinter-binourch), unb maden nidbt eber auf, als bib Die enft warm roiro.

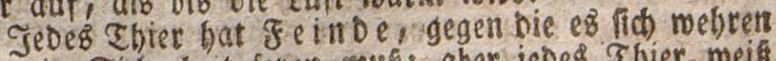
ober in Siderbeit feBen mub; aber jebeb Thier meiß̈

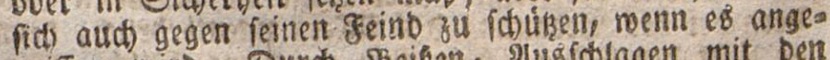
griffen miro. Durd Beiben, 2ubloblagen mit ben Simterfúgen, Groben, Sted)en, \&aufen uno Bertrie= den wiflen fie ibre Jeinbe abzumebrett, oder fich bor ithen in Sicherbeit fu fezent. Eiatige, die im Wafler leben, miflan bas yBaffer trube zu machen, wem fie verfolet rerben; andere vertreiben burd einen Beftant, ben fie oon fick geben, ibre feinde; now andere feflen

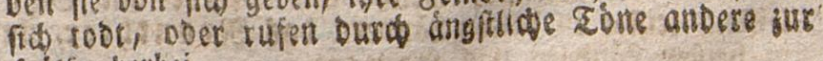
şúlfe betbsi. 


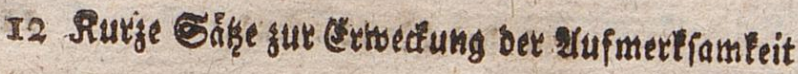

Mit groker Sorgfalt pflegen unb nåtren Die Iabiere ilire Jungen. (che fie nod geborens find, baben fie

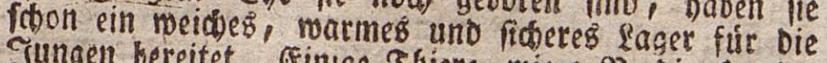
Tungen bereitet. Einige Zfiere, wie 3.3 . Die Sunbe, Saken, Pferbe, Rúbe und Siegent bringen lebendige

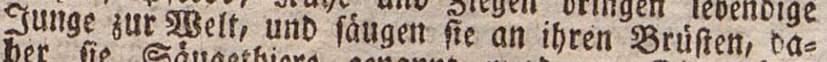
ber fie Săugetbiere genannt merden. Die 23ógel unt bie fifde legen (Eier, aus meldett nad einiget

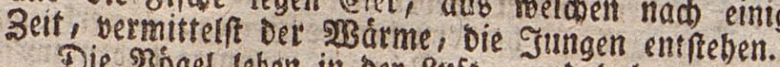

Die 230 gel leben in ber euft, und haben eine febre Jeidote Sefleidung yon SeDern; anbere Elbiere leben im YBaffer, uno diefe finto meiftentbeils mit ideleimigten Schuppen befleidet, mie bie melbeften fiiche; now ants Dere leben unter Der Erdo, mie Die feamiter, Mrauls

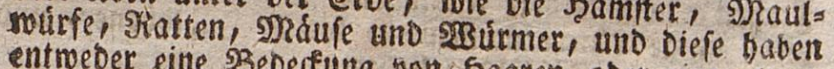
entweber eine 3 Beocefung yon Shaacen, ober eine glatte

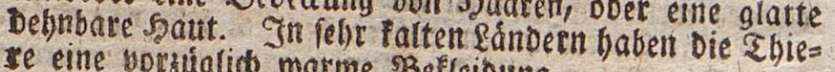
re eine vorzigliglid watme Befleibung.

Die Sa dug et biere haben eime febr veríbiebene

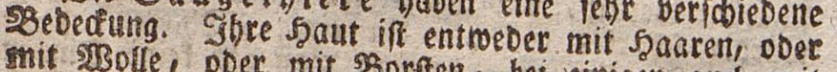
mit 2 Bolle, Doer mit Sorften, bei einigen auth mit Stacbeln, Sduppen ober Sabiliben bewadifen.

Der 5Rukn, meloben bie Sáugetbiete Den Fents

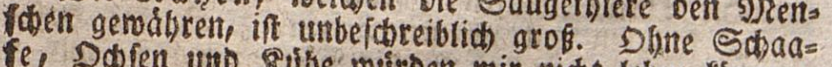
(e, Ddfen uno Ruthe vutroen wir nid)t leben fonnen: Denn das Sdaaf muß feine $58 B$ olle bergeben, Damit wir

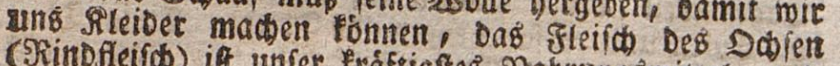

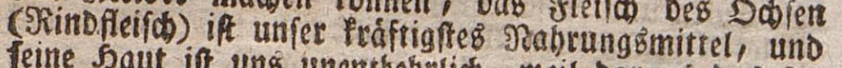
feine Saut ift uns unentbegrtich, meil Dataus Das \&es

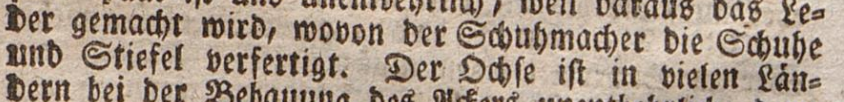
betn bei Der sebauning Deg शofers unentbehtlid, benn er fieht Den Pflug. Die Rub) giebt uns mild), wor

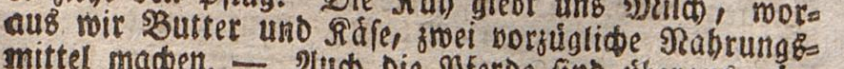
mittel maden. - Nud Die Pferde fino uberaus nüs= Iid) sum SReiten, Gabren unb Pfliggen, uno Die EFet fino in bergigten lánbern unentbelyrlid, weil fie to tharle uno unermudibete faftráger fitio.

Eben bie Dienfte, meldse uns Pferbe und Dafen

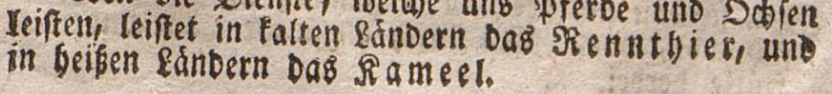


Die 23 gel erfreuen uns Durd ibren Befang, bie: nen ung zur Speife, uno finb ung nod auf manderlei

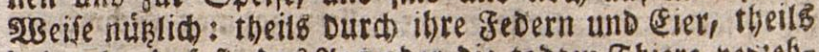
Daburd, Daß fie Das Has ober Die todten Thiere vet?et): ren, viele fódóliabe Sbiere vertilgen, uno befonders bie Jrófie, Echlangen unt Eibedfen, welche fid fo ferte vermegren, megfangen und vermindern. (5\& giebt $53048=$ yogel oDer búbnerartige Bügel, 23 a ffervógel Doer Sdmimmopgel, Sumpfoogel, Sillgoogel, 23 alobogel unb $R$ a ubobgel. Ja meis einen Şausvogel, einen 5Baffervogel, vier Eingógel, einet Eumpfoogel und einen Siaubvogel fun nenten.

Die zif

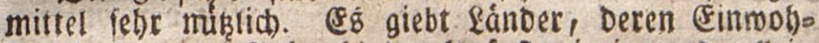
ner bab gainze Jabr binourd faft einig und allein von Silden leben. 2luch ber Thrân Der Sáringe und

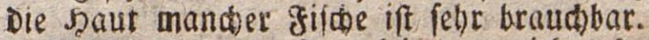

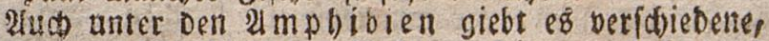
melche Dem Menf́chen zur গRabrung Dienen, befonders Die Scilibfríten und die gróliche.

Die Infeften werben unz borzúglid baburd fegr

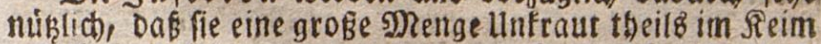

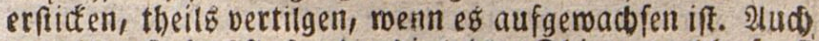

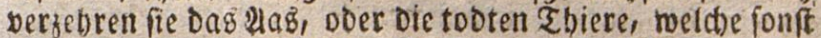

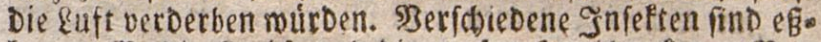
bar, 3. SB. Die Rerebje und die gropen Seulit)reffen. . 2on

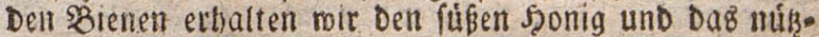

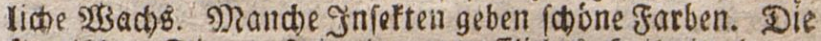

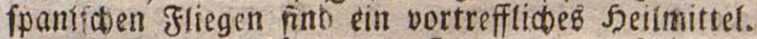

Inter ben 20 tirmern fino aud verfobiedente efs bar, z. $\mathfrak{B}$. Die 2luftern. Die giufcelfichaalen merben bon Den fiúnftern auf mancberlei 23 eife verarbeitet, befonder die Perlenmutter. Der lo núblide 2Babe

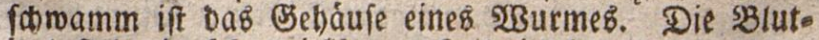
igel find ein febr mirfiames Seitmittel.

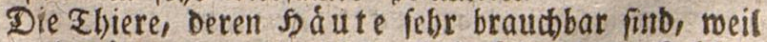

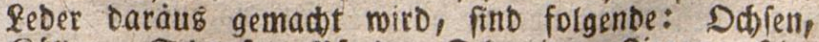
Rálter, Sđaafe, Dferde, Sd)meine, 3iegen, Shir.

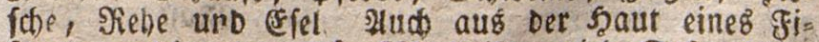

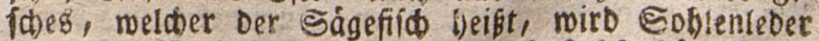
gemadt, Das̉ feber, weldes aus Dфienhâuten ge 


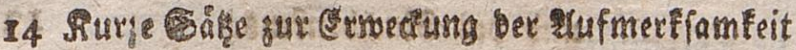

macit miro, heipt SRindleber, uno Daz, welches att8 Wferdetduten gemacht mird, beibt Robleder, meil man Die Pferbe auds Roffe nengt. Den zabmen Sdreinen wiro die fraut Rict abgezogen, fondern nur den wils Den. Id babe conon sbicher gerehen, melche in Schweinteder singebunden waren.

2uk Der effelshaut macht Der Đergamentmader

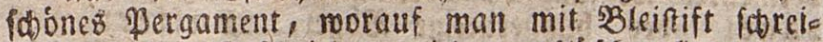
ben, ano Dab Bjefdriebene mieder ausilofichen fann.

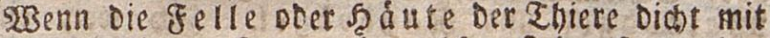
meidten molligten Sfaaren bewadsfen fino, fo merben

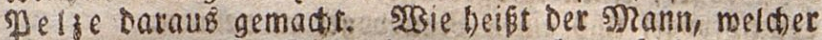

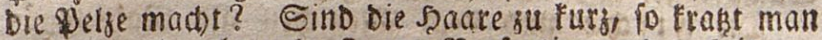

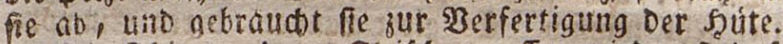

Die Ebiere, Deren gleild gegeffen wird, finb fols genoe: Id) fen, fálber, Sámmel, \&ammer, Sdbmeine,

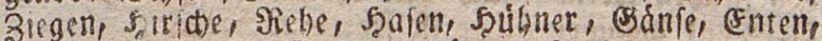
Surn, Saltben, Frammetöbógel, Serdien, Fafanen,

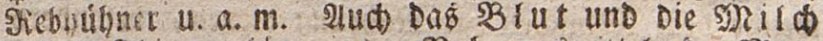

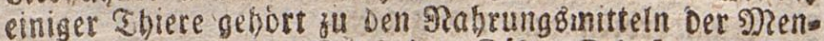
fthen, befonvers ole Milth Der Rúbe, Stafe und Biez gett. Fil mandien Eándern mirb aud Pf ferdernich getrun= ten. Die (efelómilct ift fehr gefund, und nuro fogat als ein Sherimittel bei manchen firanflbeiten gebrautst.

3ne Speife Dienen Dem Menchen Die Frúdte Der 5Báume und Stauben, und Die şBurbeln und SBlátter vielet Pflanzen und Siráuter, 3 . $\mathfrak{B}$. Die 28 utze In Der Deterfilie und Deg Sellerie, bie 3uctermurzein, die sBlátter ber Robirpftanzen, DeB Eauerampfer', Dez Epinats uno Der Salatpfanjen.

Der Menfa) foll mit Den Epeifen nict blok feinen sunger ftullen, fich fáttigen, fondern fie rolten itgm aud

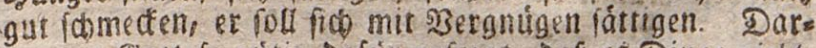
um hat (Bott fo gutig Dafur geforgt, Dak es Dinge glebt, womit man die Epeifen rou rzent, dab beibt, ibmen eis

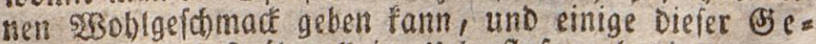
soúrze find taft tiberall im Heberflus vorbanden.

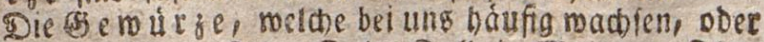
gefunden werben, fint: Salz, Ealbei, Whajorall, Thp: mian, Dill, Deterfitie, Zmiebein, Ritmmel uno Siorbel.

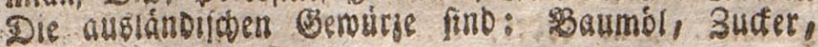


Pfefer, 3immt, Murcaten = Rúfe unb Muicaten = glú the, Radgeleir, Ingber unb Fardomom. थtuch ber Eifig getbort zu Den Eerwutrzen, Mian bat Bierelfig uno weimefig.

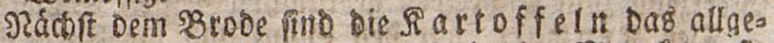
meinfte uno noblfeilfte Nabrungemittel. Man funn fie

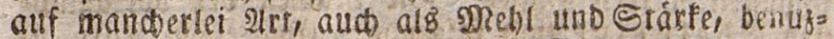
zen, und fie laffen fid Den, ganzer sBinter bindurd in

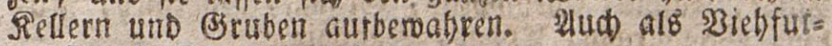
tef fino fie fehr brauchoar.

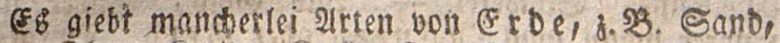
Setm, Jhon, Sireide, Ralt. Den Sand gebraution ter Maurer, um inn mir Ralf zu bermifon. Den feitren Eano freut mans auf Die nafle Schrift, Damit fie nict, verióche; audo beftreut man die Stuben Damir. - Dent

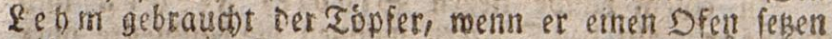
mill; ald jum 33 auten wird et gebraudt Die 38 auser. báuler baben oemóbntiá Rebmmánde. Die Escumeat und Stalle babert cineti gubboben bon feftgeftampftem Bebm. - Der Tbon miro von Dem Topfer ju Eoppen,

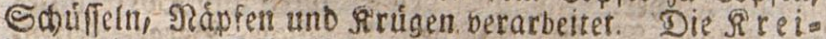
De mito fum Scbreiber und Malen, Der Ralf zum Bauen gebraudtr.

Die Steine merben auf mantiertei Beife benukt. Einige, mëldbe Sanofteine beifen, werzen zum Mablen

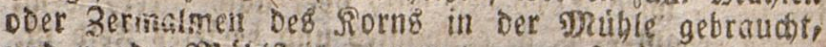
und merben mlíbiffeine genannt, wenn fie, bet,auen und

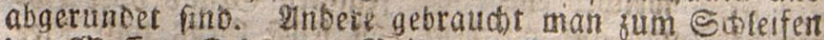
Det IRefer, Scheeren, SBeile unti Degen; not andere bum bुauen und giflaftern Der Straken, bejonderz̉ die Siaffereine, Sanbfeme unt Riefslfeime; autb Den Mar.

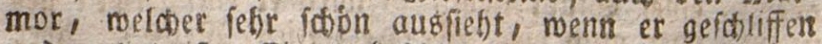
und polirt iff. Ceinige foftbare uno vorzuglic idjone Eteine Dienen Den Menichen zum Sdimud, unb diefe bets fen EDelfteitue. Der Diamant ift ein EDelftein unt zmar ein lebr nutslicber (EDelftein; Denn man tann (3) las Damit zerichneiden, und er if Dem Glajer unentbebrlid). 3u Den nuģbarfen Steinen gebout Der ge uerfe eit oder sereides giefel. Er mito befonders als gline 


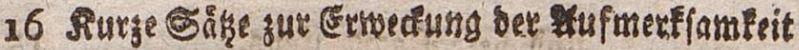

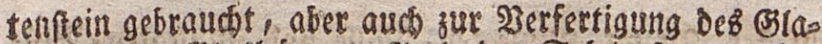
feB in Den Blasbitten. 2lud Der Ś liefer gehort zu Den braudbarften Eteinen. Er wird atm Dad =

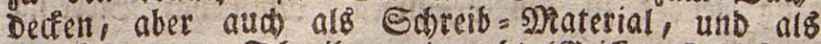
2Ssetfieug fum Sabreiben gebraucht (erriffel, Sd)iefer).

Det Rrvftall hat Den Elan; uns Die Farbe DeE

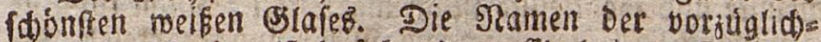
fen EOelfteine find folgende: Ehalcebon, Rarneol,

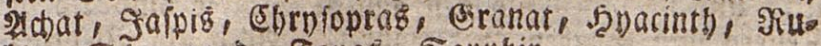
bill, Sdmarago, Eopaz, Eapphii.

In Der ErDe findet man Bsolb, Silber, Gupfer, eifen, 3inn, Brtei, Steintoblen, Eorf, Etrinfals, Coblfteime, Sdmefel. - 2a8 Bolb, Silber uno

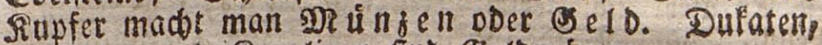
Souib b'or und Rarolinen find Boldominzen.

In sinem ranbe liegen Stábte, gre céen und Dírfer. In Den Dórfern robhnen bie Eandleute ober

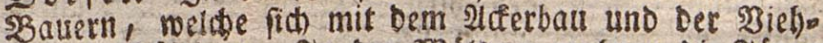

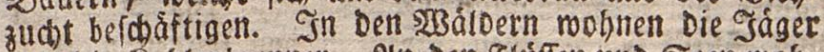
nno bie Sioblenbrenter. 2Un Den flúffen und Seen mohs

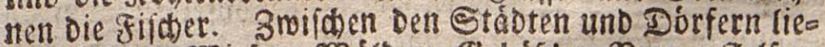

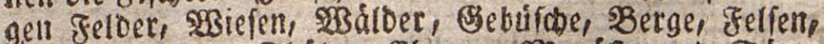
Súgel, Bebirge, Thåler, Ebenen, Mrorofte uno Sum \#fe, Scell, Zeidte, frifile, BBäbe und Duellen.

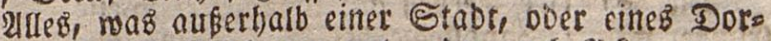
fé́ liegt, miro gelo genannt, oder audo 2dé, men

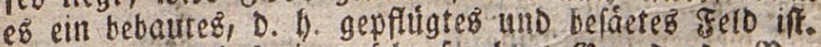
(Ein Feld, weidjeB cinen febr feudtent Grund oder 9BD= Den hat, und auf reichem yout felbit Brab und Rlee.

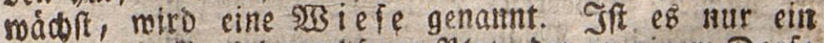
groker mit (5ras bewadfener \$lak, Der in einem Dorfe ober in eitter Staot liegt, 10 miro or ein 2nger ge

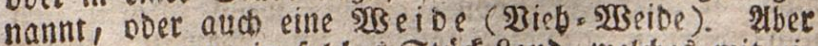
wie nennt man ein foldber Stúc Eand, weldhes mit eio tem 3aun ober einer Mrauer, ober einer Secte einges

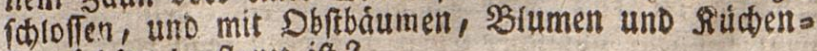
eserwádelen bepflanat ift?

Ein grobes geld, auf meldem farke und Dhe sóume in groper menge, aber niकt na申 Der 2 rits 
nung, bei einanber ftefgen, mirb ein $2 B$ alb genantut. 2Bie wirb aber ein foldeb Geld getrannt, auf melchem Feine Báume, fondern viele Etráucter bei einanber fie.

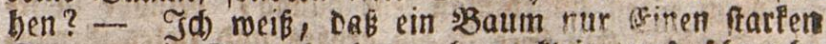
Stamm, ein Strauch aber metrere fleine und fobmade Stámme bat.

2uf vielen geldern giebt ef Saug al ober fleine Era

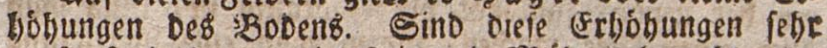
groß, fo Daß man viel zeit uno Nilibe gebraudt, um auf bie Splike zu fommen, fo merDen fie - genannt. Beftefte Der ganze Berg aus stein, fo mird et ein $z e l$. fell genannt. Zumeilen fiegt man allf Den getdern gant fleine errbobungen bon frilder Erde; Diefe rúbren bon

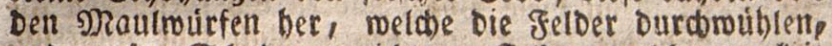
und groken Schaden anriditen. Feider, melde gar feie ne (Ertbobungen haben, werDen Eb benen genant.

Der ebene Raum, melaer yon zrei oder mefjecters

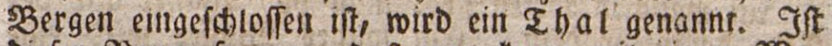
Diefer Siaum fo enge, Daßs man taum mit einem श्रुa. gell binourd fabren fanst, fo beift er eine Solludt oder ein Soblmeg. Bumeilen lino in den sergent Sertiefungen, Deer Sioblen, in meiten mehrere Ment= fit)en ober Thliere Plak finben fónnen; biefe werbent Berotten ober alló gelóflúfte genannt- - 2ber wie nenut man eine ganje Fieibe bon boben sgergens. meldhe roie aufgethuirmt neben einander und úber eina. ander liegen? Sanche SBerge find fo bocs und freil, Doer läbe, Daß man fie niobt an allen Eeiten erfteigen famn. Ein $23 e g$, Der tiber folde serge gebt, wirt ein $P$ a genannt.

2tuf ben gelbern tommt man fumeilen an folde Stúde Eand, weldbe voller sBaffer, und baber to roeith find, Daß̉ mant einfinft, menn man Darauf gea ben will; Diefe merden SR or a ff e genannt. 230 Der SBoben gani mit 23 affer bebecft iff, und zwar mit eio

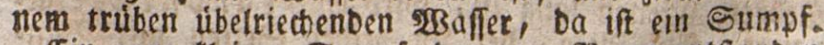

Ein gant fleiner Sumpf, Der vom Siegen entifandent ift, mirt eine $\mathcal{P}$ fúke, DDer aud ein Piuhl genannt. Da, mo Sumpfe find, batren fith perictiedene $28 \mathrm{ged}$ ouf, welche Daber Sumpfoigel genant merbent, befonpers Reibs, Stride, Rranide, Rabr= 
18 Rutge Coik̨e gut Ermedung bet 2ufmerffamteit

Dommern, Sकnepfen, Riebize unb эBa ffer bither.

dưd Seen finbet man febr báufig, beinabe in jee Dem fande. Ein Sce ît ein grob́es und tiefes Bemáfs fer, weldeb von allen Seiten mit fand umgeben iff,

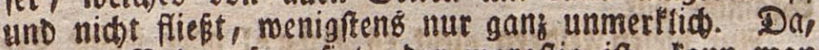
no Der 2300 en fumpfigt ober moraffig iff, fann man aud einen See madeen, inderm man cine Bertiefung

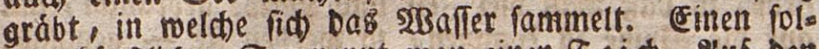

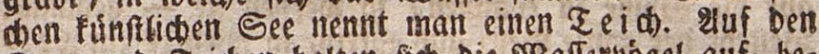
Eeen uno Eeiden balten fid bie $23 a f f e r v o g e l$ auf, be

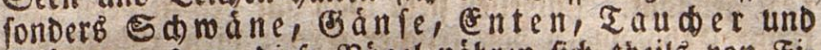
Mo ven; Denn diefe 230 get nábren fíd) theilz von Fio fden, uno Die Seen fino gemeiniglid febr fifdreids. In ben Seen mádît das Edilf, melder bon den Sorbmadtern gebraudbt wirb.

In manden Detten freft man bab 53 affer aub ber erbe berboriprtubein. Ein fordtes $23 a f f e r$ miro eine Suelle genanut. 2830 mebrere Queflen zufammen= fommen, entfeltit ein $\mathfrak{B}$ a d oder ein fleiner $\mathcal{F} 14 \mathrm{~B}$.

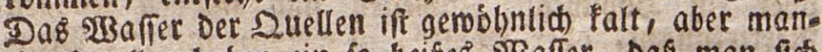

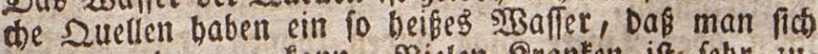

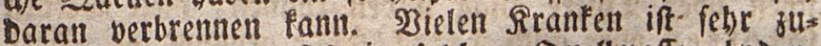
tráglid, went fie fich in foldhem ouellmaffer baden, nadbem eB fict ein menig abgefút, bat. Mande Quellen fließ̧en beftánbig, mande nur 34 gemiffen $3 \mathrm{ele}$ ten. $23 e n n$ eine Duelle nicht meht fliefst, fo fagt man won ibr: fie if verfiegt.

soenn man zwei Stunden lang rafah binter cinanber fort gehen muB́, um von einem Drte fum anbern zu fommen, ro liegen biefe beiben Derter eine IReile son einander. - Ein breiter Jahrmeg, ber zroilchen fele Dern, 203iefen uno SBergen, Doer auch tiber 3 serge uno Zháler hinmeg von einer Stabt jur andern fúbrt, unb

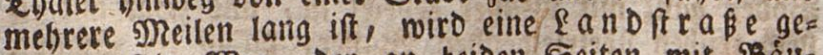

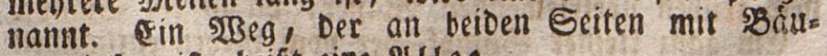
men befest if , beist eine 2lllee.

Man tann auf mancherlei $\mathscr{Y}$ rt von einem Drte gum andern Yommen, oDer reiren: zu fuffe, zu Dferbe, zu शुagen und zu Schiffe. Xm woblfeilfen uno fideeften reif man mit Der Poft. So nenat 
man einen 538 agen, welwer in jeoer 230 de an einem beftimmten Tage unb zu einer beftimmten Stunte van einem $\supset$ rte zum andern fábrt, und nidbt nur alferlé Eacten, SBriefe lino Beld, fondern aud Reifende mito nimmt. $2 B e r$ in einem foldten 2 Bagen reift, mirt ein Paffagier genannt. Der Mann, melder Den Wofte rogen fábrt, heifat der Poftillion, unt Derienige, velcter mitfábrt, um auf bie Eachen, melde auf bem Poftragen find, 2lat su geben, Damit niфts Davoll verloren gebe, beift Der Edirtmeifter oder S a aff ner.

$23 e r$ eitten sBrief mit ber joft megfoicfen mill, mas if) verfiegelii, uno Darauf den Namen uno Den 230 bn: ort Degienigen, meldoer Den \$rief erbalten fol, Deutlio

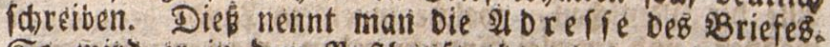
So wits er in bem Poftljatife abgegeben, und ein be.

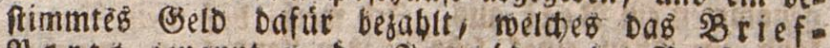
\$orto genannt roird. Ie nodbem ber szrief leidt Doet fonwer ift, miro menig oder diel oafúr bejabit.

Eitte Sieife, meldbe man in einem Eage vollenbers fann, wiro eine ₹agereife genannt. Die gróste Reife, welde eitt Menids mactien tann, if bie Sieife

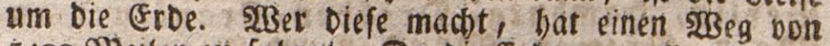
5400 Mzeilen ju fabrent. Da bie Erbe von allen Seiten

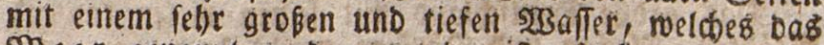
greer genannt roird, umgeben ift, to fann man am beften fu Sabife um die Erbe berum reifen. Dieß ha.

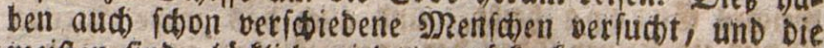
meiften find glidflito mieber zuridigefommen.

2tn Den fanbitrafien, in beli Etáoten, flecten unb Dorfern findet man Scaufer, in weld en jeber Reifende TBobmung, Nabrung (3ehrung) uno allerlei SBequem. lidfeiten fúr BelD baben fann. Diefe Scátuer beibet 2Bsithbुdufer, oder \&afth Dfe, ober Serber gen. Der, welchem sin folders Sgaub gebort, beist sin (5) a ft noirtb.

Da, no fwei sasege fid treusen ober zufammets Fonmen, if ein SDeidemeg. Man pilegt an Den Sdeiderwegen einen hólhernen sBegreifer aufzuftellen. Dies ift ein Pfabl, an welobem sroei oder brei bole ferne 2rme befeftigt find. 2uf jebem alrme frebt Des $\$ 2$ 


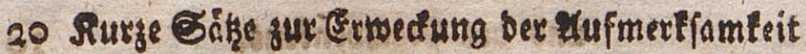

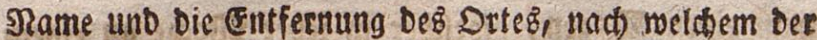

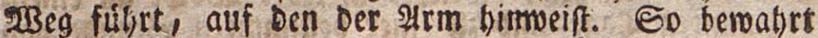
man Die Sieifenden por Dem Beritren! Denn mer Den recten $\mathfrak{B}_{\text {eg }}$ berfehlt, verirt fid.

2Ber oft auf Secien gemejen iff, bort und fiebt fefje vieleb, lernt allerlei sRenichen, Sbiere, Pflanjen, allerlei Stáde unb \&ánber fennen, befommt viele (Erf a b r un g. swer nod) nidtet viel gefeben uno gebort hat, ift un e $f$ ab ren. Rinoer baben now menig Erfabrung. SRarum?

Die Stad, in melder id moltie, beift: - Diefe Stabt liegt in einem \&ande, melctes - genann miro. Disß fano neme id mein saterlano, meil -? Spein gaterland if großs, es liegen viele Stäbte, Flecten und Dorfer Darin, in meldjer viele taufend metnj(hen wobnen.

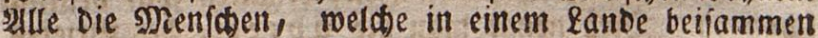
wohnet, uno einerlei Sprade reben, macien zufammells

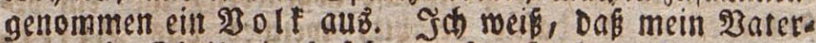
Jand ein Ibeil eines febr grofen Randes ift, melches Deutfat lant beist. Ta habe getort, bas 2300 Stíd. te in Deurfatano tiegen, und Daß́ Diele spillionen Mien. fenen Darin mobnen. Die grósten unter Den Deutitaen

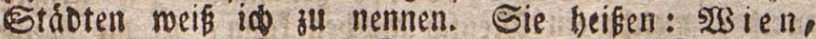
serlin, Samburg, Drebden, Branfurt am gRaill, Bremen, Munden und \&ugB̈burg.

Das grofe Deutíflano mit feinen viclen tauleno Etábten, Flecten und Dórfern madit bod) nur einen febr fleinen Theil bon einem viel grobern fande aubs? meldes Europa beist, uno aud Das grose Europa if nur ein fleiner Sheil Der unermé̈licten grofen Erbe: bie erbe if aber roieberum ein febr tleiner Sheil bee פrselt, 0. b. alles beflen, mas (sott gefichaffen bat. Denn zu Der $25 s e t t$ gebóren alle Die unzáblbaren Bes ftirte, Die wir in einer hellen s?adt am Simmel er= bliden, uno die Sonne allein if viel tausendmal gróser, als unfere ertoe.

2Benn mir aus unferem 2 aterlanbe togreiften, unb bis Dabin fámen, two es fiø endigt, und eit anderes Iand fich anfángt, fo máren wir an bie (S) ránfe unc

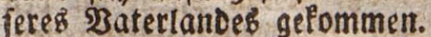

In gropen Etdoten mohnel Solbaten mit ibren Dfficisren (oirfe maden zulammengendamen die 
Barnifon ober baß smilitár, bie 3efaksung bet Stabt auB), Runfiler, Raufleute, Belefite, Sgand. werfer, Eagelógher und seftler ober sirme, ît foblechten, guten, fdobnen unb pródbtigen Szkufern. (Eine Stabt, in weld)er ein sionig ober ein furrft, Doer ein anderer regierenter Sgerr mohnt, wiro eine Re fibenzftad genamt. Srs ien ift bie Refibenz=

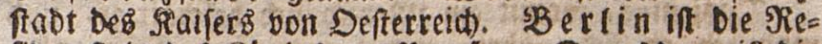
fibenjfabt Des Rónigs bon Preufen. Dres Den ift bie

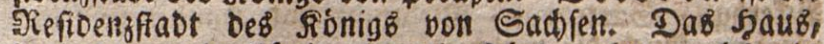
in weldsem ein Rínig ober ein furtft wofint, wirb eir Sd) lo B Deer ein Pa laft gentant.

sine Etaot, in relder lebr viele sauffeute mols = nen, uno wo alfo Saandel getrieben roird, nennt man eine Shanbelbftabt. Die Şandelëftábte liegen ge= wobntidy an grofen fliffen, weil man grofe \&aiten am beften zu Goiffe von einem Drt zum andern bringent fann. Diejenigen Dinge, mela)e ein Raufmann zum 23erfaut aurbietet, werben $2 B$ a aren genanm. Ein Şaub oser ein grofes 3immer, morin die $23 a a r e n$ in groper slenge aufberabrt merben, beift ein $2 \mathbb{B a}$. renlager.

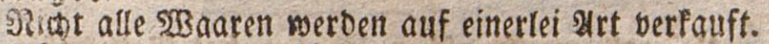
granche werden gemeffen, mandse geroogen, mant d)e gejabit. eimige merben fitudeife, nad ber BróBe oier sdjonbert vertauft. Das, womit man mist,

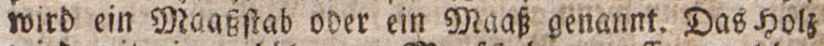
wito mit einem bólzernen gaangfiebe gemeffeli, melcher

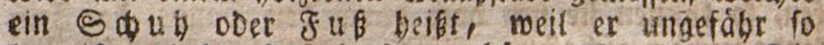

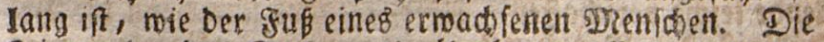
Seinmanb, $D a 8$ इ 4 , uno úberbaupt alle baummolles ne und fribene zeuge merben gemóbntio mit einer (E) He genteflet. Die Etle ift now einmal fo lang, als Der Suf, unb bat obngefábr die eånge eines थrmes.

Die fluifigen Dinge, 8. SB. Del, $23 \mathrm{cin}, 3$ Ber, EFe Fig, Sorup und Branntemein, werden nad $R$ annen Doer 2 uarten ober ma aben gemeffen. Man be Dient fich Daz̧u veríbiedener Getáfe von einer genau beftimmten Brópe. - Die Butter, Der Sutfer, Das SBrob unb viele andere 25 araren werden auf einet $\$ 3 a a=$ ge gemogen. Man bedicnt fia) Daju beridiodener (je 


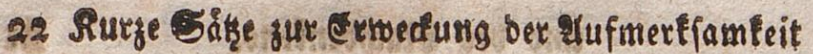

midb te, melde) Duenthen, sothe, Pfunbe und eents ner heiben.

Gríáte merben entweber gezáblt oder gemefo

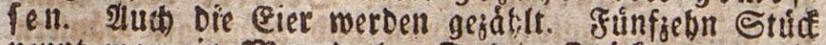

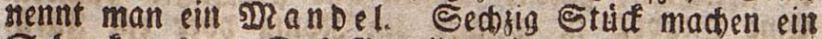

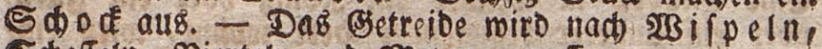
Edeffeln, Bierteln und Meken gemeften.

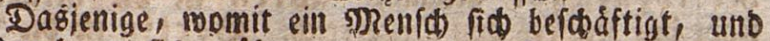
sooburd et fiob nábrt, nennt man fein Bs emerbe. 2loferbar, Bartenbau, Biebzudt uno Shanbel find Be: sorbe. 2ud bie seridiedenen şanbmerfe und Rinfte gehoren zu ben Bemerben. 2Ber fid mit Dem थdérbau

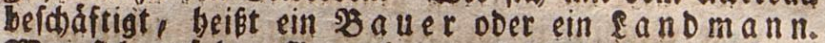
23er fid) auf Den (Eartenbau verftebt, beift ein (s) $a \mathrm{rt}$ = ner. - Ubimacher, Sildbauer, Maler, Rupferftes Qher fino Runftler. Zifchler, Drectsiler, Sdblofter,

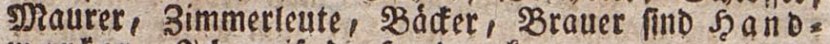

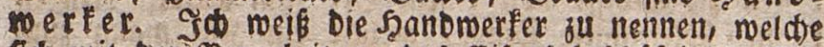

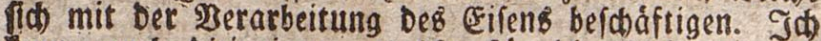
Rente aud bieienigen, meld)e fúr Die Sleibung und grafunng atbeiten.

Heberall, mo shenidien wolinelt, bat Bott bafúr gee forgt, baf fie, bei gleíß unib Sorgfalt, alles baberl, was fie zur sbefriedigung ibrer 23 e du r f niffe gebraus den. Denn menn gleid nidbt lebes \&and fo viet beroors bringt, al's feine sBewobner fu ithree Erthaltung bedit= fell, fo fonmen fie fich Dow Durch Den STanbel Das feh)

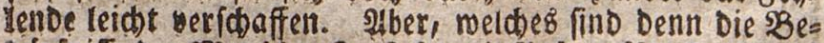

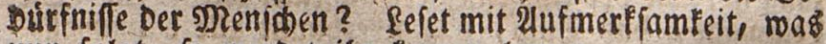
mun folgt, fo merdet ine fenten lerten.

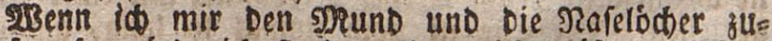
ffopfe, to murbe id ferben; Denn id múpte erfifeen.

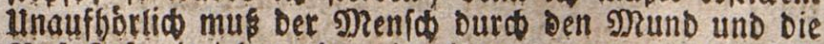
S?afe \&uft eimiehen ober einatbmen, wenn et leben foll.

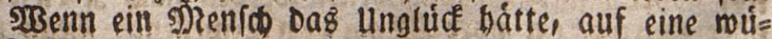
fie Snjel zu gerathell, mo er meder Speife, nod Irant, allo gar teine Sabrungsmittel fánbe, fo múfte er vor Shunger uno Durft fterben. - 2Ber im harten 20 inter weit wher Das felo geben muks und zulest nid)t mebs 


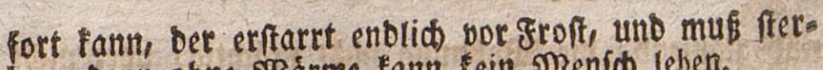
ben; Denn obne 93 árme faun tein Meníd leben.

2Bem man eit nethebornes Sind auf $b a b$ freie Feld hinlegte, und weder fúr feine Ernábrung, noch fúr feine Jieinigung, Ermármung unt sBefleibung forgs

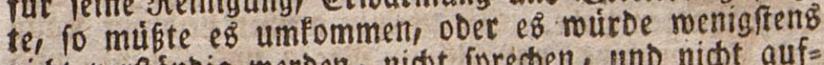
nicht verfánbig werben, niфht fprecten, und nicht auf= redit geben lernen; Denn bie sinder lernen vorjúglid, baourd gethen uno fpreden, Daf fie Den Bang uno Die Eprade Der Ermadienen nachabmen, und werben be fonbers burd die 2Anmeifungen und Belebrungen ber Erwadjenen verfándig. - 2llfo fuft, FBárme, গRal)= rung, Riteidung, $230 h$ hung und $\mathfrak{B}$ eifammenfenn mit feimes Bleiden ift Dem 9 Renfolen zur Erhaltung feines

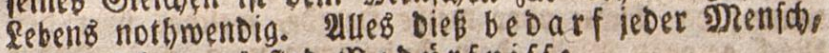
um zu leben; eb find $2 B$ ed úrfniffe.

26 er mir alle fonmen leben, ment wir aud Peinen 20ßein zu trinfen, feinen Sudben fu effen, unb feine feiDene Rleiber anzuzieben bátten. Diefe Dinge bedarf alfo ber गRenids nidt; fle find nidt nothmendig zu feis

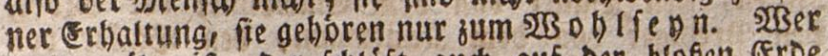
recht múbe iff, ber (कláft audh auf ber bloken eroe fanft und rubig; aber er idláft freilid lieber auf einem

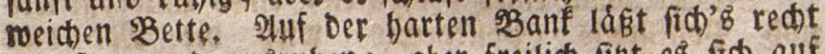
gut fizen und austuhen; aber freilich fint $e \xi$ fich auf Dem weid gepolferten Stuble bequemer unb angeneb= mer. Ein Rioff yon Dem grobften ₹ude thut redst gute

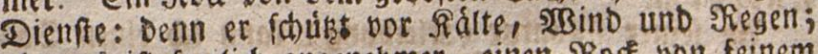
aber ${ }^{B} B$ ift freilich angenebmer, einen Siofé von feinem zud)e zu haben, Der mit fóonen sinópfen befekt iff. शilo reeide SBetten, gepolfterte Stúble, und SRodfe bon

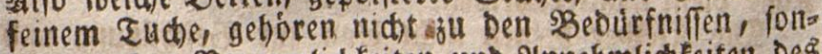

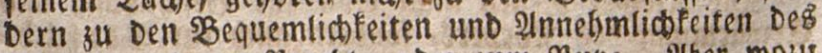

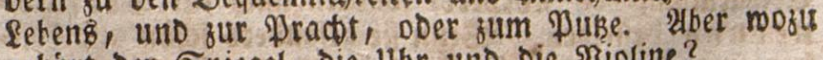
gelfort Der Spiegel, Die llbe und Die æridine?

Wenn wir binreiojenbe unt gefunbe Nabrung, braudbare Sileider und eine gute $53 s$ shnung haben, io mollen wir zufrieden fenn, wenn aud die Nablirung

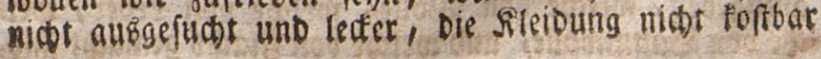




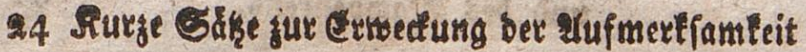

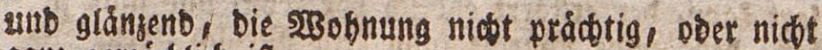
ganz gemádlid iff.

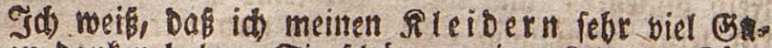

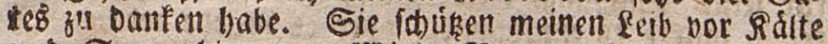
unb Sonnenhike, vor 2Bitto, Regen und Staub, uno bebeuten zugleid Diejenigen Theile meines Sorperş, mel= de fein gefitteter unb elirbarer פgenfa unbededt lafs fent barf.

Einige sleibung of ti ce finb aud manchen Szanbwerlern bei ibter 2irbeit niklid unb nothmenoig,

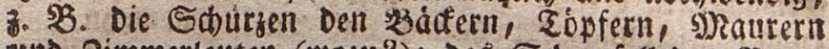

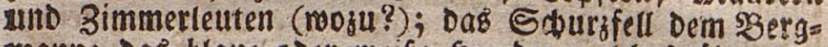
Mnann; DaB blaue doer weike Syembe, meld)es úber Den Rloff gezogen wiro, Dem fuhtrmann u. a. m. Daber fann man aud oft fonon an Der Riteibung merfen, maह̉ fúr ein Sewerbe jemant treibt, oder zu melthem Stande. er gehort. In feitter fiteibung fanti id Den Soldaten son bem Salnbeismanne, Den SBebienten von feinemi Sertn, Den Bauer von Dem Cinmobner Der Etadt, Den Prebiger von bem Saufmann, Den sBádter von Dem Shornffeinfeger unterficeiden.

Die Rleibung eines Soldaten, eines Unterofficiers unb Poftillions (Pofttnedt genannt. Die Fiteibung eineB 28 ebienten nemnt man, Die fivree, und bie Rleidung eineź Dfficiers Die uniform.

2n feiner shontur (Slleibung) Parn id) cinten $\Subset \mathfrak{a}=$ salleriften (einen Soldaten, Der zu Pferbe Dient, son einem Snfanteriften (einem Solbaten, Der zw gesue Dient) unter(कeiden.

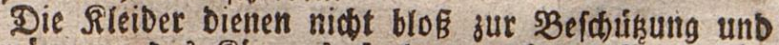

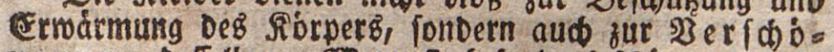
merung beffelber. S2Benn fie befonders imón und fofte bar find, to merden fie $\mathfrak{D}$ u iff eine reinlide Sileibung, weldie nett uno orbentlid fiket und ein gefumbeb, fróbliçeb Beficht.

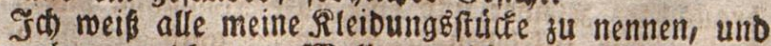
ansugeben, welde von $230 l l e$, weldie yon feder, melo de bort Baummotle, und melde bon Flados gemadt find. 


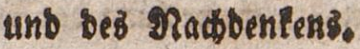

פreitueth Sut bat ber - bou - gemadit. Das (such meines $\Re$ Rotes bat Der - von - verfertigt. Die Reinmand, movon mein - gemaats ift, bat Der

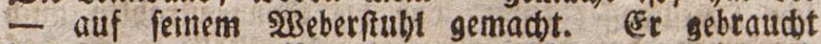

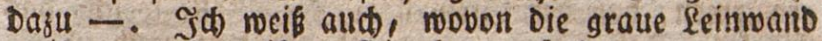
meif gemorden ift. Sie bat auf oer - gelegen. Neine Strúmpfe fint -. Die Stridterin braudite Dazu - Barn. Meine Etiefelt bat Det - von $R_{e}=$ Der gemadt. Das feder ift aub - von bem - ber fertigt norbent.

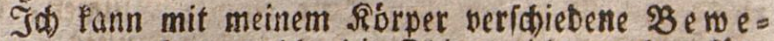
g ung en maden, welde bie Sbiere nidt madien fons. sen. Jab fann nictet nur gehen, laufeth, fpringen, fon= bern aud fotnell auffpringen, mich bidet, meinen giors per nad) alien Seiten menden, tanzen, rutiden, fnieen, mid) Hieberlegen, nieberferzen uno auffeben. So fann andern meine Eedanfen und meine Empfindungen burd Forte ju berfteben geben, ober (pre⿻)en; bie Thiere

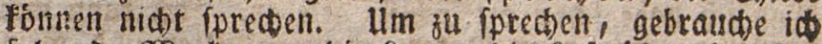
folgende 2Berfizetge: Die Sunge, Die fuftrobre, Der $3 a=$

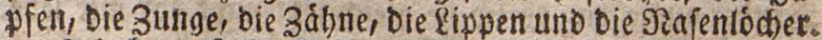

jo tann feben, nenn id habe smei gefunde 2 the gen. Tot fann boren, bern id babe jwei - -

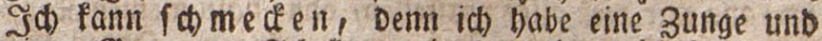

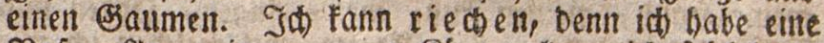
Rare. In meinen ganzen Rórper fan id fublen: eit befonders zartes Cefúbl babe id in Den singern.

Sक lebe ben grond, Die Eonne uno die Sterne

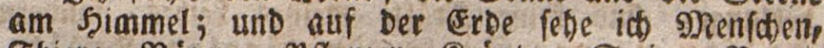
Shiere, Badume, Pfamen, Rráuter, Steine, Bergep

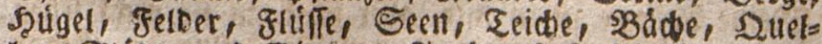

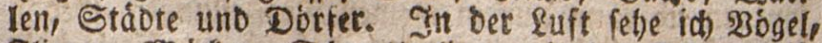
Jliegen, snúden, Edmettertinge; in Der erbe crblifo

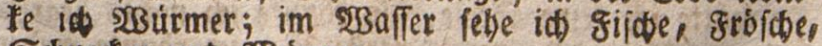
Sd)neden uno 2Butrmer.

Tat) hi re ben Befang ber szogel, bas Siollen beb Donners, Den Sकall Der slodten, das Sinallen eimer

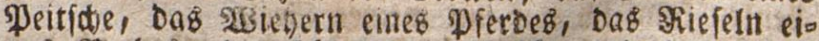

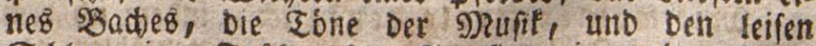

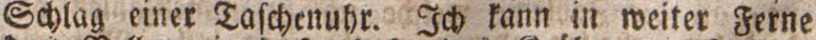

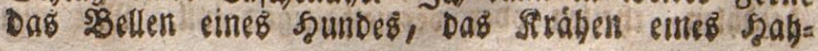


26 Rurge Sikge gur ertwedung bex Xufmerffamfeit

neż, Den Schall einer Bloce und bell sinall einer Flinte oder Ranone bóren.

Sa) fúble, $D a k$ Das geuer brennt, unto $D a b$ frí-

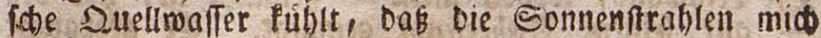
ermármen, Daß Der Stein barr, Die 230 lle meld), Daह̉ Eiś falt, Der Spiegel glatt uno Der fut rauh ift. -

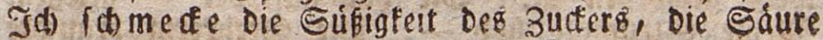

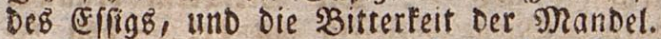

Ith rieche mit Boblgefallen Den Duft ber Rofep Des 2eildens, Der Szpacinthe und Der 2uritel. Iक rieche mit MiEfallen Den Duft einiger Blumen, uno

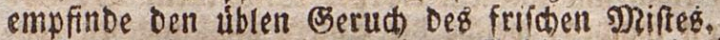

Tht erinnere mid einer Beldichte, die id vor einiger seit gebort; eineß Jremben, Den ich einmal ges Tehen; eineż Sdomerzez, Den th einmal empfunten; eitnes ßergnúgens, oas id vor langer 3eit genoffen; unb Deffen, waß id geftern in ber Sobule geternt habe. Iक foun mir vorfiellen, mie ein Sdbiff aubliebt; Denn idi) babe idjon oft Sdiffe gefehen. Th fann mir vorftellen, roie mein 2ater, meine Mutter und mein grruber ausfehen, ob id fie gleid jest nid)t vor mir febe.

Job fann min' an alles, was io gefelen, gebort, empfunden und gefúbit babe, Deutlich erimnern, Doee id fann mir biek alles vorftellen, obne Daju meinen Ropfo meine Şand, meinen fußB, meine 2lugen, Dbren und शRafe zu gebrauchen. Die Sirdfte, mit melchen ich mir etwaś vorftelle, mich an etroás erinnete, úber etwas nachfinne, etwaz empfinde oder etmas mill, oder etwas verlange, find teine Siráfte meines feibes, fonbern Sirafte meiner Seele ober Seelen= Rráfte. speine Seele if in mit, aber id fann fie nidbt feben; fonbern id fann nur in meinen Sorffellungen, Beban= teu und Empfinbungen merten, Dak id eine Secle babe. Szátte id Feine Seele, fo fonnte ia nictst begreio

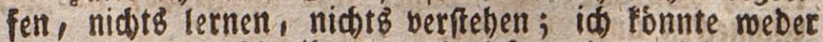
red)nen, noch fabreiben, nods lefen: Dent inbem id lefe, ober reds)e, muk id zugleid benfen, und Denfen fann id nur mit meiner Seele.

Nit meiner Seele benfe id, intern id red)= ne, an bie 3ablen, reldae ich zufammenzáblen ober besabłen, theilen oder vervielfáltigen foll. Dit meio 
wer Seele benfe id an Den פenenficen, yon meldiem ber

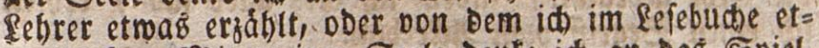
wab lefe. Mit meiner Seele benfe id an Daś Epiel, neeldes id) (pielen will, wenn bie Schulseit zu Ende iff. Mit meiner Seele Denfe id, indem id plaubern will, an bie Strafe, meldbe Der febrer auf Das Plall= Dern gefelet bat.

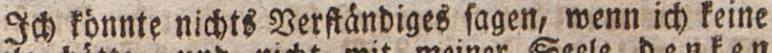
Scele bătte, uno nidbt mit meiner Seele De iffen

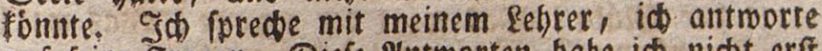
auf feine Fragen. Diefe Antworten habe id nicht erfit ausmentis gelernt, um fie bann berzufagen; ict babe úber Die Jragen meines \&ebrers nadgedacht, und Dann babe id geantmortet.

Th) módte meinen SRode nidt mit bem gertumpten

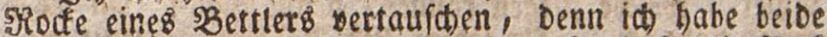

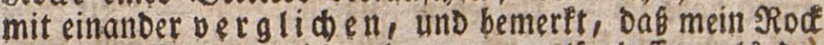
nicht zerriffen nab nidte abgetragen, alfo beffer, als Deet

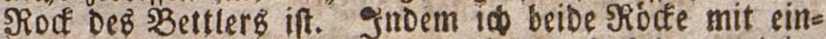
anter vergleiche, uno Dann urtbeile, Daß́ Der meinige beffer ift, gebraude id meine Seele ober meinen 2 er

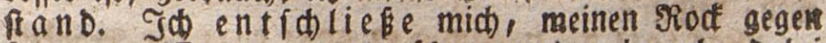
feinen zerriffenen zu bertaufden, uno gebraude Dabei meinen $2 B$ ille $n$ oDer meine $2 B$ illen है $\mathrm{r}$ aft.

İ babe gefeben, mie es Der sBauer madjt, mentr

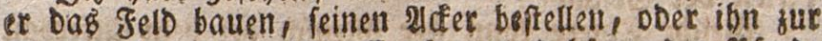
Saat zubereiten will. Er ipannt Dojien DDer Pferde vor ein fleines gabrzeug, meldeb Der Dflug heift, und vorn zmei Padiber, binten aber ein Beffell bat, an

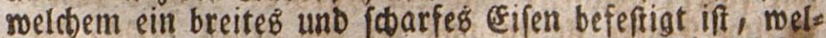

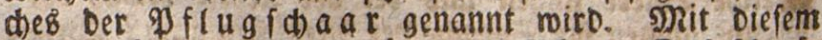

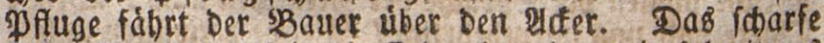
Eifen fanneidet thef in Die eroe ein, ein zmeites breiteres

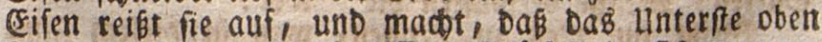
fommt, inbem bas aufgerifiene Stúc Erbe fio ummenDet. Dann wiro Der umgepflughte Plcter mit Samen von Rioggen DDer Bserfte, DDer safer Doer FBeizen beftreutet unb biefer Same mirb vermittelft eines bólzernen 2Betheugz, Das die Egge heist, und aus mebreren jufammengeferten Sarfen befteht, thinter 


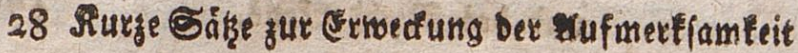

Die Erbe gebradt. Ginbem id biél alles fabe, erbielt i申 einen ३Begriff vom 2uterbau.

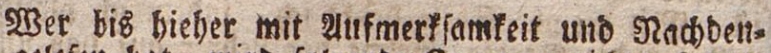
eets gelefen bat, mird folgende og ragen ridtig beants sootten fonner.

2Babี no ill ber Sungrige? 5 Wab will Der Durftige? 23as will Der Fleifige? $\mathfrak{B a b}$ will ber Faule? WBas

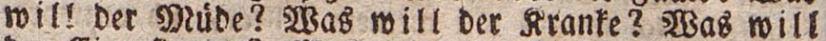
ber Eigenfinnige? $\mathfrak{B}_{B} B$ bo ill Der Dieb?

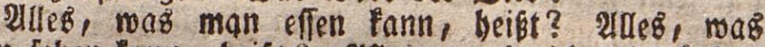

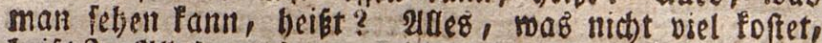
heist? ?lleś, maś man nidbt gebrauden fann, beist? alle Thiere, melobe ifre Iunge lásgen, beißen? alle Zbiere, weidbe fliegen fonnen, nennt man? In jeber Sano babe id - In jebem sǘc babe id - MRit mei

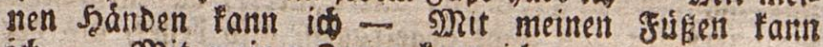
id) - Nit meinet 3unge fann id -

Die Rimber, weldhe ibren Eitern nicht gethordis? Seiben? Die Rinber, melide ibren Eltern freube mas den, beifen?

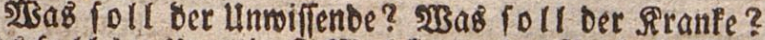
$223 a$ fo ll ber unartige? $23 a$ fa f a n faun Der Geidbidte? $23 a 8$ f ann ber Starfe? $23 a 8$ fann ber Eefunbe?

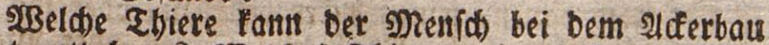

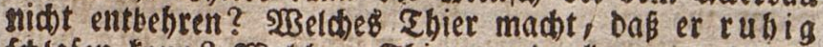

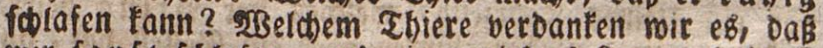
soir fanft foblafen, unb ein weiche \& fager baben? 23 elde Sbieke fingen unb bei Der थrbeit etwaB vor? 223 lide abmen bie Sprade ber Mreníben nach? Welde Dbiere fint uns unentbebrlich? sBselde bermuften unfere Bárten? Melde verwúften bie Jelber?

203eift bu alles fu nennen, was in biefem zims mer vom Saloffer berfertigt morden if? Mber auts alles, was bet sifder berfertigt bat? SBemerfit Du in biefem Simmer aud Dinge, weldbe Der Drect)Bler gemadt bat? Weift bu mir aud ein Ding fu nens neth, melocs von einem Rúnfter berfertigt, uno 3wat in biefer Stube befindid, aber niकt ficts 
bar if? alber mie beist bas Ding, meldhes in feiner

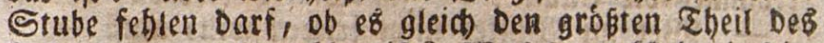
Sabreß ganj unbraudbar ift? $2 B a B$ bemerfft bu in bie fer Stube, uno befonders an Deinen Rileidungefftifen, bå̉ lonft an einem Thiere gefeffen bat? Tenme mir bie

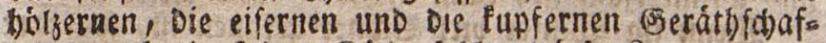
ten, welde in feiner suid)e feblen dirfen?

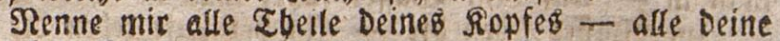
sgecurfniffe - allef, mas Du in Der Sकule gebraud) ft - alle Die Shicre, Deren Jieilíd ou gegeffen baft? -

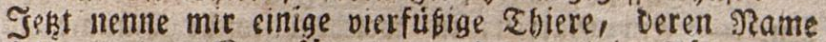
(iid) mit einem $\Omega$. anféngt - und nun einige 230 gel, De= rent Rame fioh mit eben biefem Buchffaben anfängt? Iक weik einige flide zu nennen, beren शame fich mit einem $\Omega^{2}$. anfäsgt. - Nenne mir ein Bemúrs, oeften शame fị́) mit einem $\mathfrak{P}$. anfángt. einige (e ro artell zu nennen? Ino einige Eberffeine?

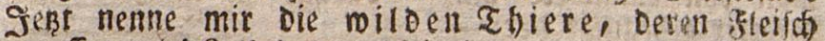
gegeffen mirb? und nun dicienigen, oeren Đeíz foftbar

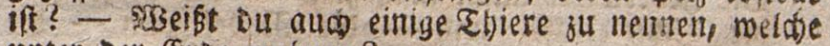
unter Der Eroe roobnen?

Nenne mir eintige Dinge, melohe von Natur cine gr tu ne Farbe haben - und einige, welde von ग?a=

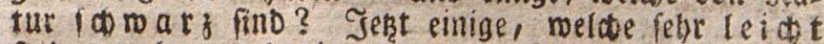
fino - aber auch einige, welde felor fid wer fino?

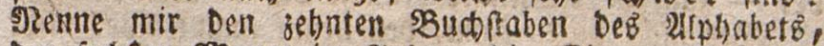
Den fediften Mronat im Gabre, bie 2 Binter = Monate 2 einen Jrubling $\dot{b}=$ Monat unb einen ferbft = Mronat? Run auct Den Mronat, in weltbem Du geboren bift?

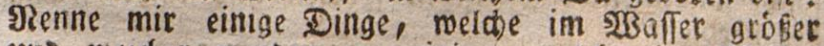
und meider werben - einige, weldhe in $23 a f e r$ faumelsen, uno einige, meldbe auf Dem 23 affer fómim= men? - शRenne mir etwab febr fúfes, etwas feft faurés uno etrasas bitteres?

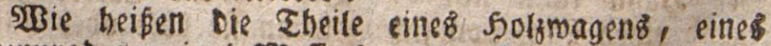
Epunnrades, eineś Me fters unb einer Ebure? Nenne mir einige gehler, wel= d)e Rinbern eigen finb - unb Die Zugenden eines gu: ten Sdúlets?

Im Serbfte ift Die \&uft - $9 \mathrm{~m}$ Sommer ift fie Im Serbfte merden Die Srifibte - Im Snublinge 


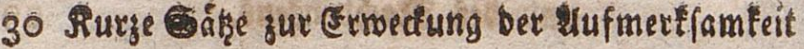

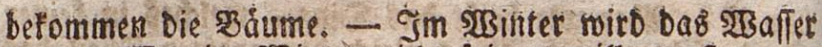
ju - $2 B e r$ im $2 B i n t e r$ nidbe frieren will, muß -

खer nid)t arbeiten mag, fonbern lieber músig gefit, beift ein - Meniat. 2Ber in ber Sdule nidt fleipig uno aufmetlfam ift, fann nichts - uno bleibt? -

$23 e n n$ in 23 inter viel Eddnee gefallen ift, fo fábrt

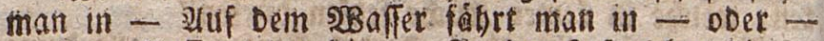
Jim $23 a$ affer lebent die - In Der \&uft leban die In Der erde robnen bie - In Sumpfe leben bie elle szátme, melche estare grúdote tragell, beis Ben. - Ein Garten, in welderm folde sBáume in Nenge madben, heist. - Die sBirte trágr teiue -; aber ihe Sfolz ift fehr - Die (Eid)en und Die siof = taftanien tragen zmar frutate, aber fie find nicht -

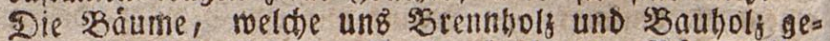
ben, fint folgende: - Strofe abgehatielte sadume fágt man vort eimander, unt - Daraus ju maden. Die 23retter gebraudt Det -

Die Jebern gebraucht mant fum - bie Dinfel zum - Die Edieferrafel zum - Die grader zum - Die gúbe zum - Die 2utgen zum - Die Dhren fum Die Neafe zum - Die Zunge fum - uno zun - Die gúfe zum - und - Die Såande zum - Die Zábne

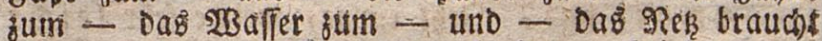
bet - zum - Die glinte braud)t ber - bei ben -

2Ber nidjt horen farn, Den nennt man? SBe: nidst felfen fann, Den nentut man? syser nid)t teben fann, ift - $2 B e r$ nicht geben tann, beift- $2 B e r$ úbee

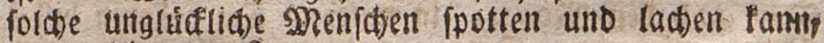
Der verbient - ?

Sebr gern hore id ben fofonen Befang einer und einer - 2lber Das háplidbe હef́chrei Des - mas id nidt gert bóren. Der Sperting fann nidt Der şabn fann nur - Die meiften Elgiere geben irgenb einetr faut bon fich, wenn fie bofe ober bungs rig, ober fróblich find. Das Sdmein - Der Şund - Das Ethaf - Dab YferD - Der Dafe - Die

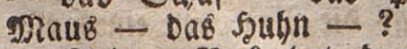

Meinen Roof bat ber - gemacht. Dab Brod bact Der - Den Robl pflangt Der - Die Echube bat Der - berfertigt. Das feber a den. Squhen 
madt Der - er nimmt bazu Die Felle ber - und Die Sfátute Der - Shite macht Der - Die Wfetbe be fallágt Der - Den $23 a g e n$ madt ber - Pelze verfertigt ber -

2Beithe sanomerfer beidáftigen fic mit Der 2̧erar=

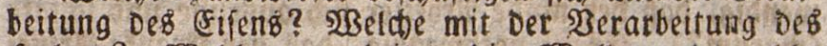

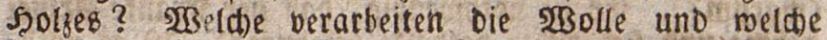

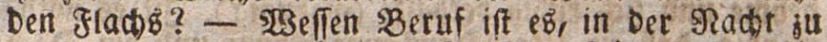
maden, Damit Die úbrigen Nenfden fidjer uno rubig foblafen fonnen? 2Beld)e Mrenicten múfen mitten in ber S?acht arheiten, Damit ibre Mitbúrger am 9Morgen effen fonnen? SBerufkgefdaften nid)t rein balten? Ino welde múffert bel il)rem (semerbe viel Beftanf ausftehen?

Sagt mir einige Şantomerfer, welche nur foldhe Dinge berfertigen, bie entreber fur SBequemlichfeit, Dder zum 23ergnugen, oder jur pradt bienen? Sienne mir Die Rizinter, welche Durch ibre Sunftwerfe unfer Sluge ergóben? Nentie mir die Siunft, reldbe unz be= queme und fatone 230 hanungen berictafft?

2Belche shenfchen múffen fnieend arbeiten? Weldse miffen bei ibrer arbeit flettern? $2 B e l d e$ máfen fries

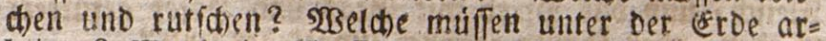

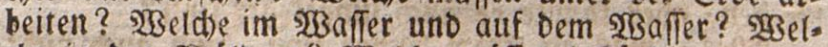

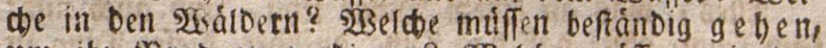
um ibr SBrod zu verdienen? 2Beldie miffen mit Dent

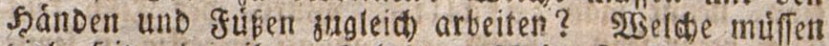

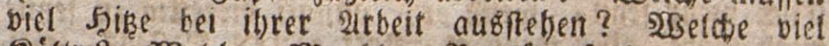
Rálte? Belcter Menfoten $B e r u f$ erfordert $e^{B}$, faft immer auf Reiren zu fenti? 2Beldhe múfen beftánoig 2Blut bergießen? $20 n$ meldien Sandwerfern fonnte man fagen: fie leben vom 2 kinde?

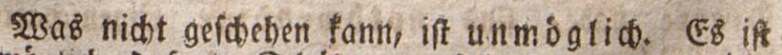
unmósalich, Dak sin Edhúler etmaź lerne, wenti er nidit aufmerffam unb fleifig iff. (EB if unmb́glid, Daß Der. ienige geiuno bleibe, melober unmábig ift unb trinft. Es ift unmóglid, Daß ein tobter Meeníd lebenbig roie. Der ericheine, unb bak ein tauber shenfé fich an ficio.

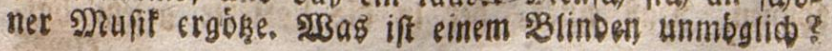




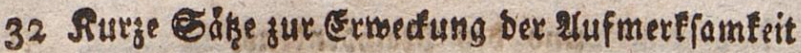

शुSas ift einem Rinbe von fect) Mronaten unmogglid? $\mathfrak{B a b}$ einem Rranfen?

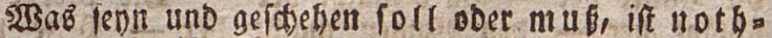

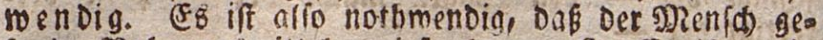
funde Slabrungsmittel genieß̧e (marum?). Es ift noth= mendig, Daß Der múbe Ilebeiter fich aubrulse und ficlafe

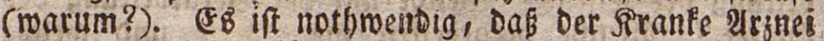
nethe und fid rubig verbalte (roarum?). Ift eह noth= wendig, dap ein leoes Jुaub eine Foure uno ein Dad babe? şsarum? \$llte Menitten muifen feerben; marum? 2llte Stlumen muifen vergeben; marum? 2थlle Sinder

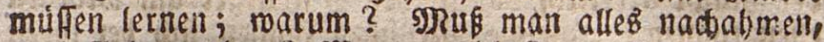

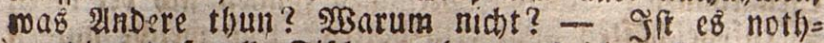
mendig, Daß́ alle Eirabe roth) angeftriden find, und

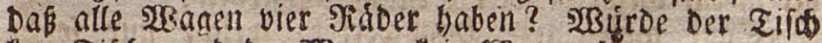

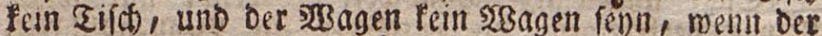
इif́ch meiß angeftriden wáre, und ber wagen zmei গid=

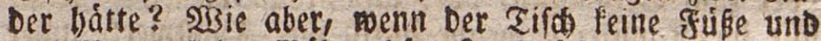

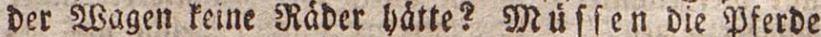
Dor Den $2 B a g e n$ gefpannt werben, um Den $2 B a g e n$ forfuzieben, ober fonnten fie eben fo gut aud binter Den sosagen gefpannt merben? Shus Der Şü mit

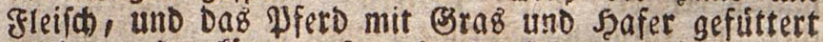
sorbell, ober fonnte e\& aud umgefehrt fenn?

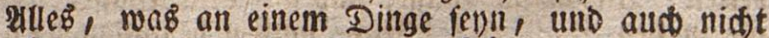

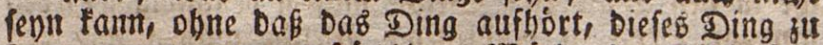

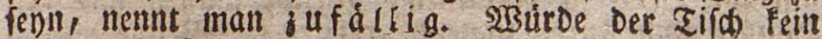
Tifd megr fend, wenn er, anftatt bierectig zu fenn, runb wáre, dDer menn er, ftatt eines SPaftens, jwet Saften hatte, Dder wenn ef nidbt blas, fonbern roth angeftriden, ober wenn er gar nidbt angeftridjen wáre? Daß alfo ein Eifod viercetig ift, etmen Raftent bat, und blau angeftrichen ift, Diek find lauter zufadlige (E)

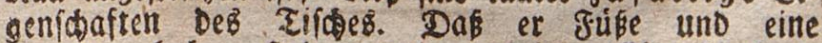
splatte babe, find notbroendige Eigenfarten. शुarum?

Dak vor einem Şaufe bez 2lbends ein Şunb heult, uno baß́ in Diefem Saufe bald nawher ein Mrenfich fritbt, if etwas zufálligế ; Denn ber suno bátte aud beulen, unb es batte fein greníd fiterben tonuen. Dás einer, Dar in Dí Sotterie gefertst hat, stmas 
ofwas seminnt, iff zuffálig ; or batte aud einfercen, und nidfts geroinuen fonnen.

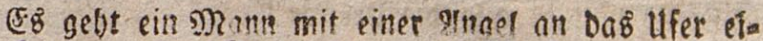

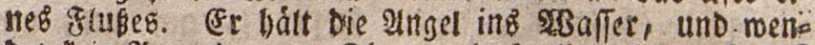
Dat fein 2luge bavon. Tbut er bab alles obne llifane? Stein, fondern er wifl Dadurd efroas erhangen, er will

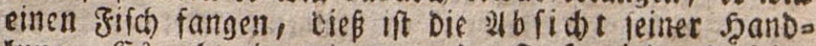
lung. (Ex geht ein anderer an ben Siufs, zicht alle faine

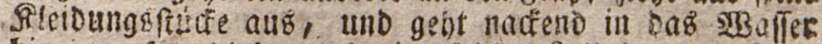
binein. Sat Diefer aud) eine 2lbfidt? Unt welde?

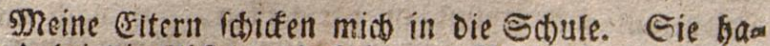

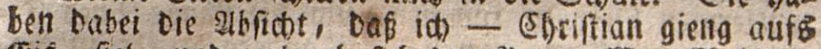
(eis, fiel, und zerbrad fich Den $21 \mathrm{rm}$. SWar Egriftan

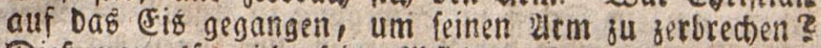
Diés war alfo nicht feine dibfít. Syatte er gar feine

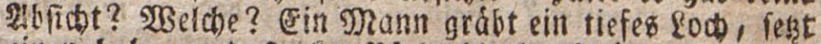

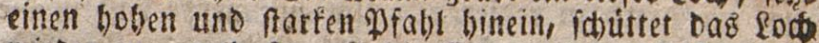
poieder fu, und fampft Die Eroe mit Den Júfen feft. Dann nimmt er now einen eben to grofen Pfathl, uno grábr ibs nidte weit von Dem erften enn. In Diefent beis

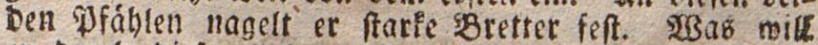
er Durd biek alleb zu Stande bringen? Eह if alfo feine elufidt, einen - zu maden. 2lber watum mill er ithn maden? Szat er Dabei aud eine 2ibficbt? 2Belde? ?

Seart mollte gern eine reife şirne bon einem bobes Baume berunter baben. Er foriftelte Den Baum, aber Die Birne fiel nidt herunter. Jekt berfudie er eb, Den sBaum 3u eiflettern, aber aud Dieß gelang ibm niक)t. Nutu bolte er eine lange Stange berbei und fotug bas mit to lange an ben sweig, moran die sBirne fab, bis fie berunter fiel. Rarl fuctete alfo auf Dreterlei थret feine 2ubficht zut erreicten. Dasjenige, moburds mais feime 2 bfidbt zu erreichen fudt, nennt man ein Mi t tet. 2Bie vieferlet Mittel batte Fart angewalid, um feine 2thfidt bu erreiden? wBie waren bie beiben erfen פRite tel? SBie mar DaẺ Dritte?

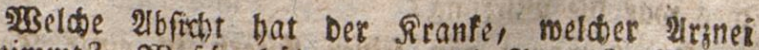

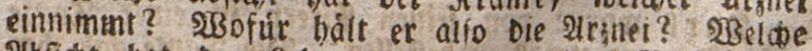

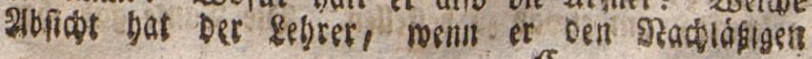
(5) 


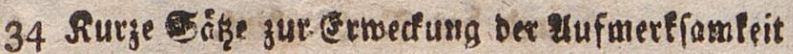

Echiler beffraft? शBaz folf afio bie Strafe lepn? פBelde 2hfict bat Des, melder fid im Sajreiben ubt? 230 fur bät er alfo die Ulebung?

Welder if bas befte Dittel, um fatt, um

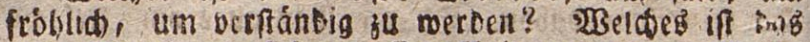
befte शitrel, um fich vor Rirantbit, vor langer 253 eile,

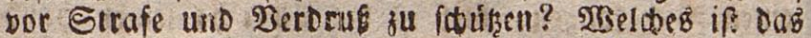
Miltel, um etwas ju finden, un unn 2mbern ctmab

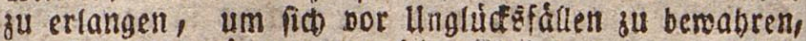
uin fict zu erroármen, um feine filetoer lange zu eribals ten, um fitb Eefluf zil verídaffen, um nach emem entfernten Drte fu formen, um Die fánge enzeg ₹io

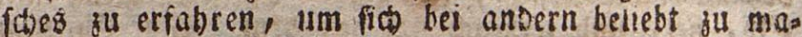
den, um ein Sdbif in sberwegung zu leken? - 2belo de פRittel metibet Der SBauer all, sm feinen 2icfer zut

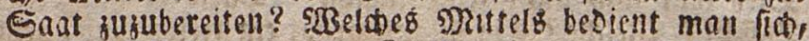
um ein Pferd fu regieren? Um eine grobe \&aft in Die Shbibe zu beben? Ilm bie Sthwere einer Sache ju erfab. ren? Un einen entfernten eine Nectridt ju geten?

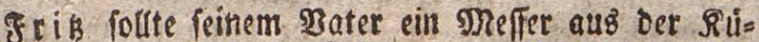

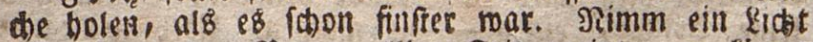
mit, fagte Der शater. 2iber Jrik meinte, er fónne Das Meffer aud in ginftern finden, und lief obne sidst fort. Es Dauerte feitren 2lugenbliof, fo borte inn Der 刃ater fallen Sdnell fam er mit Dem lidtre berbeis

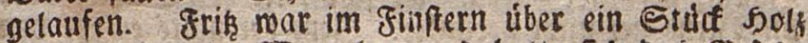

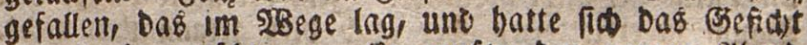
am Jeerbe zerídiagen. Eer mukte ben gamzen dibeno

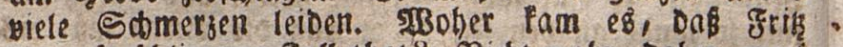
einen fo fdstimmen fall that? Sidit maise, baber, metl or bem Эatbe feines yaterö nicht folgen mollte, ober meil er eigenfinnig mar? Dasjentige, moraus etroas entfelbs, nennen mir die Urface, und DaEjentigen mas aü net lirfactie entifebt, nennen wir bie 203 ir. fung. Soseldes iff nun bier bie urfache? uno wets des nat Die 2Birfung?

fiart wurde von feinen eltern fu einer Eante geidiadt, um etros zu befiellen. Die Tante gab thm 


\section{uno bes Racbentens.}

ein grofes Stlice Ruden, unb einige 2lepfel, unb Rarl

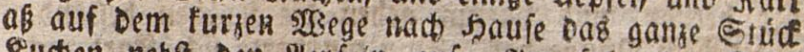
Siuten nebft Den Ilepfein auf. Im folgenten Zage batte er beftige feibfdmerzen. $25 a b$ mar die Liriade feiner firanflyeit, ober novon mar bie firanfbelt eine श्sirfung?

Bottlieb batte idone 2 lumen, aber an einem beifen Iage hatte er vergeffen, fie zu begießen. Da murs Den bie SBlumen welf, und neigten fid fur Erde. Was

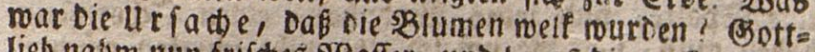
lieb nafim nun frifकes 23 affer, und begok bie welfen $9 B$ li: men. Eebr balo jeigten fich bie guten WB irfungen

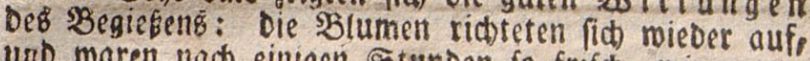
unb waren nach eimigen Stunien fo frifi, , rie zubor.

Der arbeiter ift múbe uno bungrig. WBas ift bie Urfad) bievon? Die Múbigfeit uib Der feunger fino alfo 2Birfungen; wobon? DeB Drorgen' ver= gebt die Suntelbeit, uno es mint bell $2 B e l d b e b$ ift oie lirfad e biebon? Sage mir bie 23 irfungen Deb

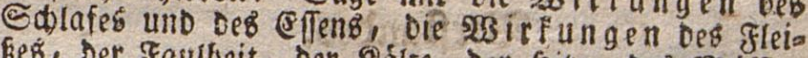

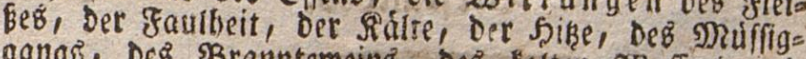

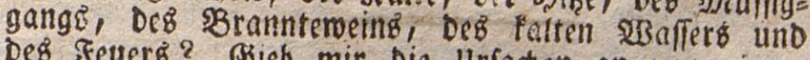
Deǵ Feuerb́? (sieb mir bie Ulrfacien an poil einem Shalle, elnem Stube unb einem ऊetore?

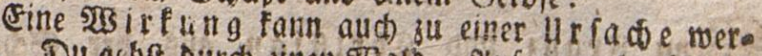
Den. Du gebft Durd) zinen walo. 2uf eimmal gerciebt

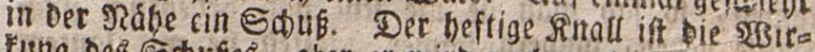

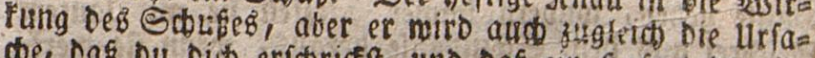

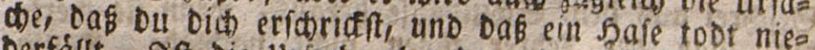
berfált. Tift oie Uriadje eber ba, Doer bie SBirfung? Dab 2Bort Urfache bedeutet aber nidit immer Dabienige, mober etmab entftebt; ober mober eimas fommt, fonbern oft aud): marum etmas to ift, wie es ift, ober marum etrabí gefohiebt.

Ra rI gieng mit feinem Szater uber ein Feld, und fahe, Daßs eine Menge Siráben eitrem sBatter, melater pflugte, auf Dem \&ube nacholgten. FBarum mogen bie Shiere das thun? fragte Rarf. Der \$ater fagte ihm, Daß bie firáben fia yon Dett watrmern nábren, melte in Der Frbe liegen, uno belonders von Den engertingen, moraus die Mailedfer entfeben. 


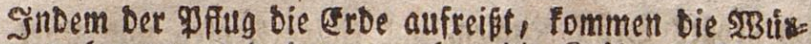
mer beroot, und Darum gehen die Rráben befzadndig binter Dem Pfluge her. Nun wunberte fít Rart nidt mebr úber Daś, was er fake, Detn er muste nun die

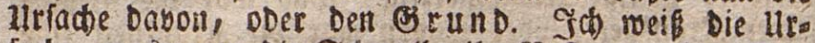
fache, mesmegen die Sdjmalbe ihr Neft unter Dem Das

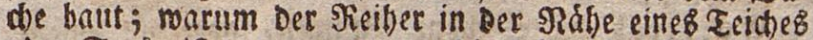
DDer Sees niffet; marum man die frtibner allf dem feos. fe, und nidt im Barten hat; marum iede Sdreibfeber

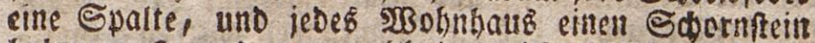

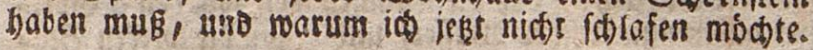

\section{II.}

Erzáblungen zur Feforoerung guter Ee= fimnungen uns zut Schárfung Dez Berftander.

\section{Die ungerogenen Sinber.}

2) Senn granz unb Egriftion aub Der Soule Famen, to fah man fie nie fill und ordentlid nad Shaufe geten, fondern immer fturzten fie mit lautem Beforei aus bem Equlfaule beraub, went fie merts

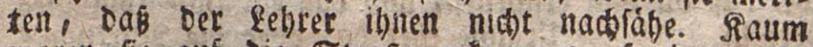
waren fie auf Die Etrake geformmen, fo jagten fie fito mild berum, und warfen einanber mit eroflios Gen, unb mobt gar mit Steinen. Szatte eb gerege

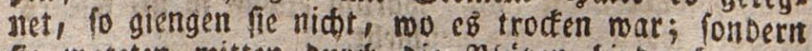
fie roateten mitten ourd Die Pfúzen binourch, und

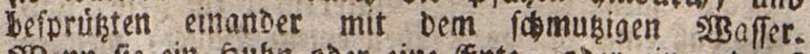
2Bemn fie eill Spubn ober eime Ente, oper -ein andere Thiet auf iffens S3ege antrafen, fo jagten fie $e^{3}$ bor fich ber, warfen es init Stemen, uno batten eine boso bafte Frende Datan, DaB arme Thier, to viel fie fonth

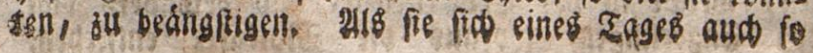


ungesogen auf Der Strafe betrugen, fam sit altet פRanis gegangen, und vermess ibnen ibre lingejogenteit. The

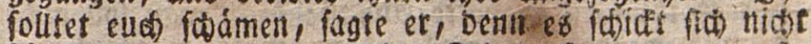
fir Rinder, welde aub ber Sd) fo biel sutes gebirte haben, mild uno ungezogen z" feyn.

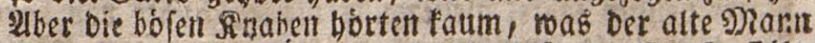
fagte, und liefen ladiend und tobent fort. Die sluffith =

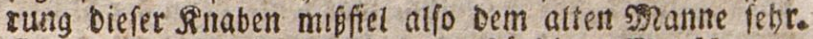

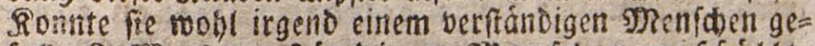

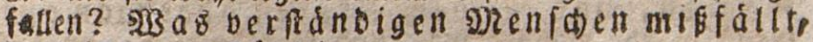
Daś if utlanfánoig.

ฐक) will mich immer fo betragen, bak verftánbige menfowen meit Betragen mit ssoblgefaffen bemerter toment.

Jrans fam immer mit bem sute auf bem Siopfe in Die Esulftube, obgleid ber fehter inm dieß iton oft berwiefen batte. 2iber ber moblgezogene sieg.

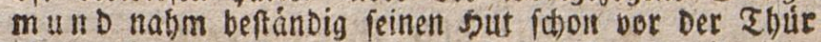
ab, madbre oie Squble rein, unt trat bann, mit elllet

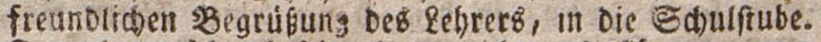

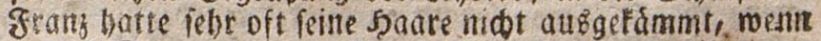
er beb Morgents in bie Sdyule fam, fie hiengen ifim ge= sobshlith in's esefitht, uno maren voll Jedern. Ift is anfánoig, fo in bie Sçule zu tommen? Wुsenn Ebrio filan erivab gefohenft befam, fo bedanfte er fich nie bas fir, fonbern brebte fich um, und gieng fort. Jif Dieß a

\section{Die Serfudung.}

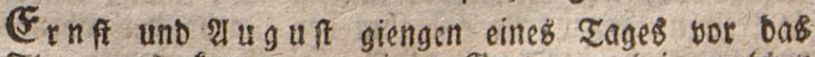
Sbor, uno famen vor einem Barten vorbei, meider offen ftano. Eie glengen aub Neugierpe binein, und fanden einige Qffaumentadume, weldse fo bolf bon reis

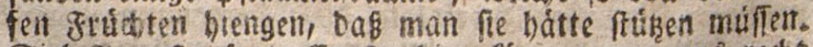
Eieb) 2luguf, fagte (Ernf, bier fonnes wir unb rect)t

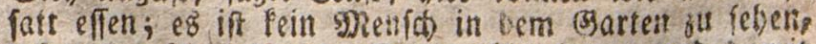
laß uns gefdraito emen 3weig abbredeen, und oamit fortlaufen. SRein, antmortete 2aguf, bas búrfen wie nidst thun, Denn bie pflaumen geboren uns ia nicht. (5i, was (s)abet Das? rief ernft; ber Nam, bem fie

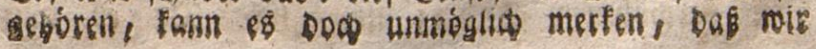


ein Daar genommen baberl, er bat fo biele, baß̧ man fie

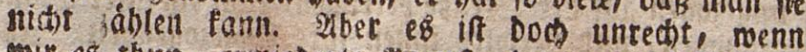
mir es thait, etribberte auguft: Denn man foll nidts hermtich regneb) mett, wab anbert gehort, menn es gleicí) mur eine siteinigfeet iff. stseift ou nidt meffr, mas Der

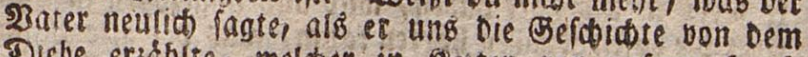
Diebe erjakhlte, melcher in Sieiten vor unferm Sgaufe

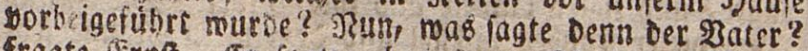
fragte Ernft. Er fagte: bet oem sieinen fángt man an, uno bei dem Brofen bort man auf.

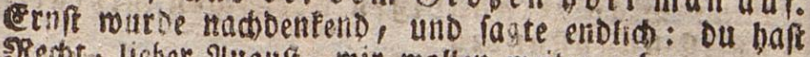
Feds, lieber 2luguft, wir mollen meiter geben.

Crnft mat in grofer $\mathfrak{B}$ erfudung gewefen, ef= maś Untechtes za thun, indem er Die sgegierde fúblte, Pflaumen zu effen, melche itm nidht geborten. SBie

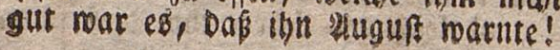

\section{Die ůble Csomobnbeit.}

So lange graitz in bem Souffe feinet Eltern mar, gieng er alle Eage, fobald es Dunfel murbe, zu Better oder er fólief fikent ein, und mit grober Muibe muste man ifn benn ermuntern, oft mubte ibn die Deatter fos gar mie ein fleines Pind ausjilehen uno fu Sette bringen, meil et fich gat nidt ermuntern founte. Dennod follief er fo lange, bis es ₹ag wurbe, und im Sommer fabe man ibn oft bei heflem sonnenichein nod im \$Bette lieo gen. Die Mutter ermabnte itn oft, fid Diefe ₹rćgbeit absugemoblhen; weil of $e^{B}$ fünftig nidt immer wurbe fo treiben tonnen; fie gab itsm Den Siath, Des 2abents in Der Stube umber zus gehen, fobalb er merfte, Daß̈ ibm ber Schlaf anfame, und Deb́ SPorgenb rafo aufs suntegen, fobald er gemedt wirbe, ober bon felbft ero madte. 2aber grans befolgte biefe ermabnungen nur refir wenig, und blieb bei feiner ublen Bemobnuteit.

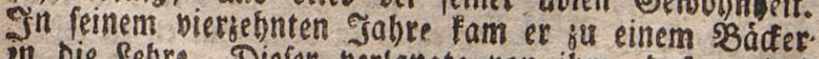
in bie fegre. Diefer verlangte von ibm, baß er Deß

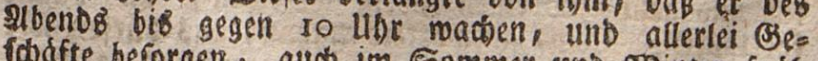
iddifte berorgen, aud im Sommer und $23 i n t e r$ frih um 5 uhe noieder auffetien follte. शber Dieß war Dem bermoghten granj unmogliq. Da ob nun 


\section{jux Befortbetung guter Befinnungen $2 c_{0} .39$}

nidit mebr frúb zu gette geben Durfte, fo foblief er be= fántoig bei Der 2irbeit, la zumeilen logar ftebeno ein. Einigemal fiel er um, uno fericilug lid Den siopf. Eein sehrters farafte ibn oft megen feimer Erégbett;

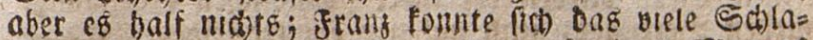

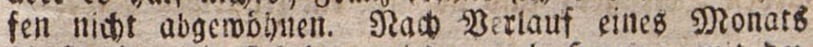
iabidete isa lein Rebrberr wieder nad) Saule, mit bet Berfiderung, caB er ith unmógtid bihalten fonnte, meil or gánzlich unbrautbor ley. Eranz murbe aud siemald ein thátiger uno gani braudbarer 21tbeiter. Eo faber if es, eine úble Eeroohngeit abzulegen!

\section{Die Eleinen Diebe.}

Rlaufens sinber hatten bemerit, Daß in Dem Batten

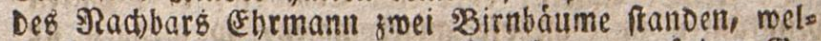
de berriche snúdte trugen. Sie famen auf Den Be. Danferi, úber ben 3aun zu feigen, uno fít einige sbito

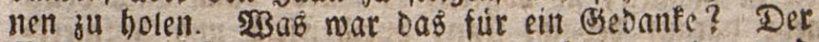
Radbar merlte endich, dak et beffoblen wutrde, und

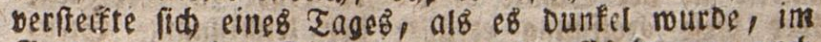
Barten, um ben Dieb zu ertappen. (58 Dauerre aud midte lange, to fabe er Rlauien B sinder uber Dell zaun fetigen. Sobeu und ángftich faben fie fich um, und alo fie feinen Menichen im (Sarten erblidten, lies fen fie eilig nad ben Birnbaumett bin. Eben rollteit fie mit ibrer Beute Davon gehen, alb Der faetr DeB Bartens berborfam, uno ithen in Den 2 Beg trat.

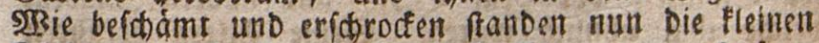
Diebe Da, mie flehend baten fie Ebrmannen, Daß er itgnen Dods biefe follect)te Sandiung vergeben, uni fie nidt bei ibrem \$ater. verfiagen módste. Ethrmann

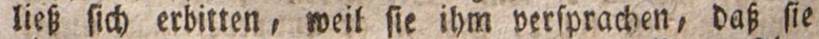
nimmermebr mieder etwab megnebmen wollten. 24ber Die böen Sinber bieften niकt 23ort; Denn nach eint=

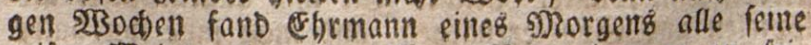
reifen \$Beintrauben abgeriffen. Sun gieng er zu fei= nem Nabbat, und bat ibn, leine Sinder megen ithree mieberboiten Diebereien j"! ftrafen. 2aber Diefe láug= neten hartnáatig, baß fie Doft geffoblen bátien, unb Der ミater glaubte ibnen. Ebormanin gieng feufteno forte 


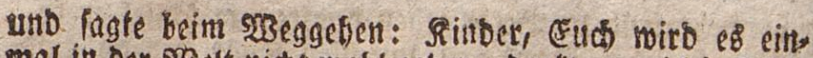
mal in ber $23 e l t$ nid)t wothl geben, Denft an mid)! Diefe Bort)erfagung gieng anto mirfind in Erfútlung. Die

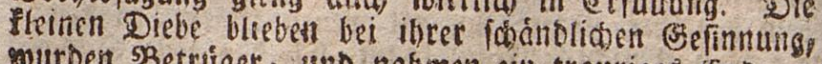
soutben SBetriget, und nabmen eill trauriges ende.

\section{Der ₹agedieb.}

Siegmund mat ber Sobn moblisabenoer EItert, uno baber fonnte er mandses Berginigen haben, mels des anbere Sinder entbebren mifien. Seine Eltern giengen oft mit ifta fpabieren, uno febrten Dann im= mer irgenomo ein, um allertei Erfriföungen zu genico

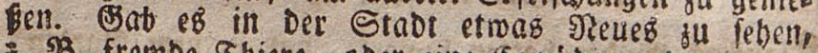
3. 2. frembe Ehicre, oder eine Eombive, ober waren Shuffeanten im (3iafthofe angefommen, to giengen fie

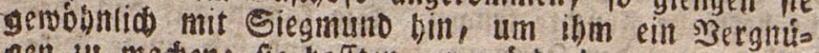
gen ful maden; fie boften, er mútibe bann aud befio

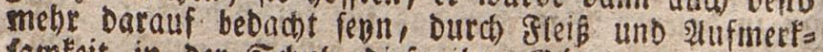

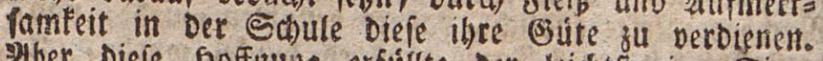
saber Diefe Sgofinuig erfúlte Der leiditinnige Eiego munb nictr. Ee befam einen tibermábigen Sjang zum

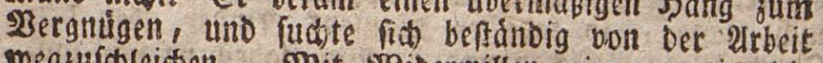
megulfaleidien. Mit rBiberwillen gieng er in bie

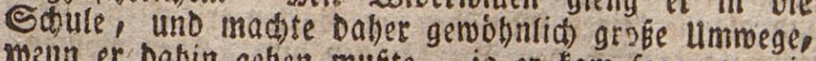
wean er babin geben muste, ja er fam fogar ztroei= Yen gar nidbt in bie Edjule, fonbern fpielte mábrend ber Edulzeit bor oem Thore mit anbern Rnaben? weldoe ibm ábnlid maren. Seine EItern erfubrer Dieß gmar, aber Siegmuno roufte Dann immer alier rei Entiकulbigungen vorzubringen, veriprach auch beo

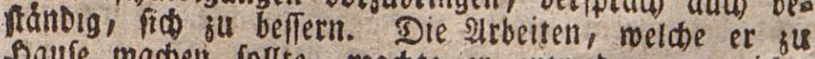
Sourfe madent foulte, madbte er entroeber gar nicht, ober to fluddtig, baßj Der fehrer unmogglic bamit ju=

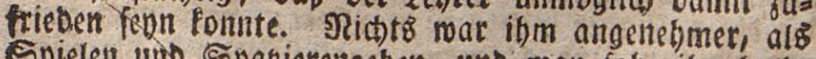
Spielen unt Epasierengeben, uno man fabe ibn balbe Eage auf Dem Felbe umber laufen, ftunbenlang zufe= ben, wemn die Soldaten exercierten, und wenn ${ }^{B} b$ int Der Stadt etwas zu fehen gat, fo fefilt Siegmuno nie. Sein gebrer nante ihn oft einen Zagedieb, weil of

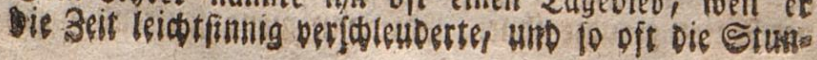




\section{3ur Beforberung guter Befinnungen $2 f_{0} \quad 4 I$}

Dett, weld)e ber grbeit unb bem fernen beftimmt warelt, zu reinem פergnúgen mifbrauchte; Denn baturd raubte er fin ja felbft bie loftbare Beit zum \&ertuen, welche nie soiecer fommt. Eligmund nabm nur an glter und an forperiicher Státle, aber niobt an Renntniffen unb Bjes

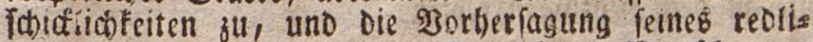

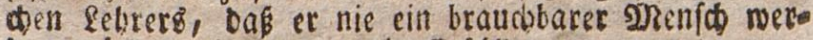
Den wúrbe, gieng genau in erfúllung.

\section{Der Eleine 2Berfotwender.}

Bater $r$ r $r$ a bieft feine Sinber frúh baz̆ an, Daß fie Durd 2lrbeit etwab̉ erwerben muftent. Seine Tódter

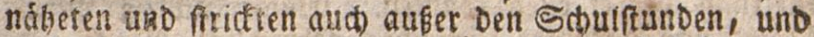
er faufte isnen Dann zuwoilen ibre flemen 2trbeiten ab. Seine Soblne orectiften, Doer madten alletlei Pappe 2itbeiten. Math biefe faufte ihmen (Etid) ab, menn fie fauber unb nett gemactst waren. Diefe Rinber batters alio iamer (seib in Sjánben, weldbes fie nad) ifferm 2Billen bervenden fonnten; aber ber $23 a t e r$ ermafinte fie oft, ef núblic onjubenden, uno Damit fararfam ume zugetien. SRarie unb Rarl, bie beiben jüngern Rins Der Erichbs, befolgten aud biefe ermabnungen, unb Feuften Aä) fúr ibr gefammelteśs seld allerlei Dinge melche fie nótbig batten, 8. 33. Mapier, gebern, Blei= ftifte, Metiler uno Steeren. SBie grok war immer ibre greube, menn fie einige Brofden Durch ibere 2 re beit ermotben hatten, und wie lieb mat ibnen alleg, mas fie für ibr eigenes seld getauft batten! 2ethee

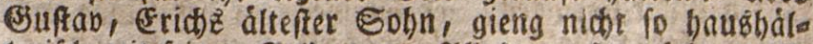
terif́) mit feinem (s)

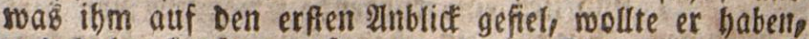
und Daber fauffe er oft ganz unnuke Dinge, ober auch foldie, die er lest getade nidbt beourfte. (er batte

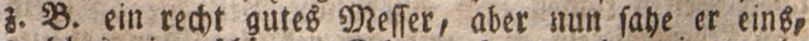
melders eine fobnere Schaale batte, oder ein wents gróber mar, gleich faufte er eq, uno gab bant, to Die feute forberten, baber er immer viel zt theuer eins

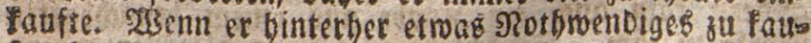
fen hatre, to febite es libm an (seld, uno er mollte

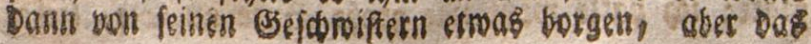




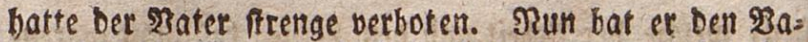

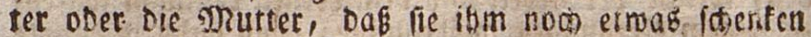
módten; abet er befan Dann immer jur sutmort: lerne mit Deinem Belde fparfam umgeten, faufe

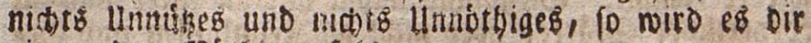
nie an Dem פiothigen feblen.

\section{\%. Das wohthátige Rino.}

Por einigen Sabren brannte nabe bei ber Etabt $\$ B$. ein ganzef Dorf ab, incem bei cinem beftigen Stur:

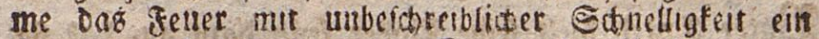
Saus nach bem andern ergeiff, she bie झ?achbarn jur Siettung berbei eilen fonntelt. Cinige actrig Sgren= foren, uno oarunter fobmadbe, gebrectiche sresfe uno Natronen, unmúndige siunder und atme Eageiobner, pertoren in einiger einjigen stunde ithre 233 blnungen, ib= re Sileioung, und alle ifre sabfeligfeiten. Bott, mas war $e^{8}$ für ein Jammer, diefe linglififlióen mit ib= ren arment, fum Sneil franfen Simberh, bon Siálte erftart (Denn es mat pad in Seerbit), leufzend uno seinens in Der Srre berumlaufen, und angftlid ein Dboach fucten fll fehen! Det recitf(d)affene Srediger

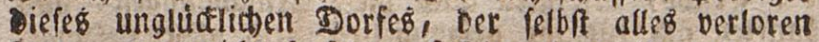
batte, wat nicht to lebr auf feine eigene siettung be= Dadot, als vielmelir Darauf, wie er Den Ungltidfioben, Die um ibn ber jammerten, fonnelle Sulte verichaffen fonnte. (Et gieng Daber auf Dent benabbarten Dórfern umber, unb ficte oie abgebrannten bet mitteidigen Seaten unterzubringen; er fammelte in Der গRábe und in Der Jetne Belo, Nahrungstmittel und Fleidungbs= ftude ein, unb liéb cine ribrende Erzódbung von oem fobrectionen SBrande in ben 3eitungen aboructen. Seine Bemúbungen waren aud niфt vergebenB. 23on alten Seiten tamell ibm anfebnliche Beitráge an \&elo uno Rebensinitteln ju, uno ber reblide Maann theilte alles mit eben fo grofer Freube, als Seniffenthaftigs Peit uno sorficos unter Die 2lbgebrannten aub. Lntee andern fam aud ein Sinabe aub einem benadbarten Dorfe fu ihm. Soúdtern trat er in bie Stube, und fagte: io batre mobl eine grofe sitte an Sile lieber 
zur Befortberung gutex Befinnungen $2 c_{0} \quad 43$

Sert prebiget, wenn Eie $\mathrm{eB}$ nidt úbet netmen molls ten. Sage mir nur, antmortete diefer freunolid), no. mut id dir belfen fann, id mill es red) gern tbun. 2ld) neit, belfen folfen Sie mir niche, ermieberte Der Sinabe : it bitte mur, Daß́ Sie Dieß̈ Beld und Diefent alten Siodf fúr bie armen Abgebranten annebmen mol= Ien: ef iff freilidg nur febr menig, aber id habe nidat mebr, uno idh módte Dod) fo gern fút unfere veruns glucten Radbarn etrob thun, Denn fie iammern mid fehtr. Meine Edweffer meinte zmar, mit einer fol den Silemigleit burfte id nidst fommen, Die fónte ja boch nur wenig oder gat niditz belfen; aber id fonnte es Dod) nisbt loffen, bieber zu gehen, und eb Thnen anzubieten. - Du haft ganz redot getban, lie= bes Rind, fagte Der Pretiger, und Ebránen Der SRutho rung fanden ibm babei in ben 2lugen. (Eine jeoe Bias be, Die aus gutem Szerzen gegeben miro, bat ibten Wsertb, uno alfo auch Die Deinige. SBleibe immer bei Diefer guten Gefinnung, uno fen reblich bemuibt, Daß Bute nad) beimen Iiráften ju befórdern, to wirf ou

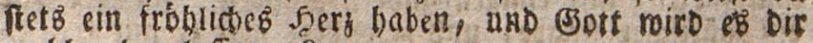
nobl geben laffen, fuc. $2 \mathrm{I}, \mathrm{I}-4$.

\section{Das oroentlid)e uno reinlidje Sino.}

थlibert batte arme, aber pehr redtidiaffene unb verftánbige Eftern. Sie robhten in einem engen Stúbdell, aber Dennocb fabe es immer orbentríd uns

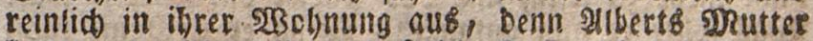
fonnte es nid)t leiben, Daß bie Sacten berum lagen, Doer Dá̉ Der Jublboben voll Eratb uno Sdbmuk mar.

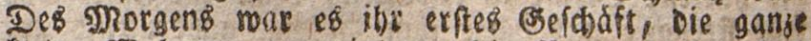
fleine 2Bobnung zu reinigen, Die Betten zu maeten und frifde suft in bie Stube du bringen. WBe batte moht Albert ein unorbentlicher Menich merben fonnens

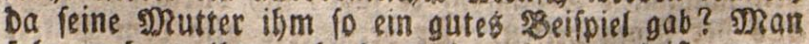
fabe aud an ibm recht beutlid, roie gut es ift, wenn Sinbet fid frúh an Dronuno und Reinlidfeit gemóh. tien. 2irbert bátte fich 3. 93. nimmerme'st entfoloffen, mit herumbångenden Saaren ober fobmugigen Sadnoen, mie mande unoroentlide Simber, in Die Sकute ju 
geben: es wåre ifm niobt móglid gevefen, Tage lang Den Samuk an leinen Etrefeln fiben zu laffen, Doer bie mit sinte befledten şande an feinet Sileidungsftuts fen abzumifasen, mie es fo biele unreinlide simber thun. SRie fabe man ifn anders, als mit atibyetámin= ten Soaren unb gewafdienen shamben in bie Ectule gebar, fein slod mat immer fargfáltig auggebúrftet, feine Stiefeln waren gefáubert, uno in reinen Schul= briquern nai Pein zlect unb fein Dlur zu finden. Eris men Sout warf et mie untet Den Sifक), uno mit bet Sinte gieng et insmer febr bebutian um: aud fehlte es ibm trie an einem Sajobentuche. Mlbert war bie greube feiner Eitern uno feiter Sehter.

\section{Der \&ígner.}

Seintid murbe yon feitren (Eltern nadi) bem $\mathfrak{P}$ oft: ba ale geididet, um einen srief abjuacben, an roels dem fehr viel gelegen war. Ruf Dem $23 e g e$ begegnete ibm Sranz mit einigen andern Sinaben. Branz war

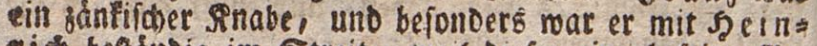
ria beftanoig im Etreit, weil Diefer eine beftige Ses

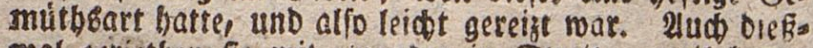
mal geriethetr fie mit eimander in Streit, meil femer Dem anbern aub Dem 233 ge gefien nollie. Tn Der Size

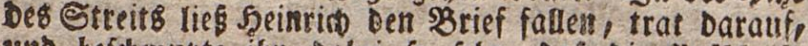

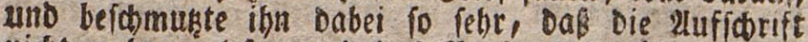
stidt mebr zu lefen, unb bas Papier Durdtódert wer. 2NBab follte er nun anfangen? 236 eni er zu Saufe fam, unb alles geftans, toas borgefallen war, fo batte er Die hátefte Etrafe fu ermarten, Denn fein vater mat felye frenge, unb batte ibm biefmal auB̈bruditich ge fagt: beftelle ia ben Brief redt orbentlic, benn $8 \mathcal{B}$ if mir febr viel Daran gelegen. Sointid) tam endlid) auf

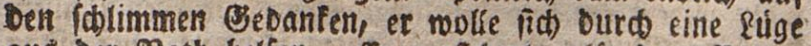
aus ber Noth belfs!. Er verfiderte alfo bem 2 ater, auf feine Jrage, mit grober Dreiftigleit, Daß́ er oent \$rief ridtig beftellt babe; ood foblug ibm bas Jers

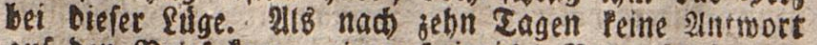
auf Dent Brief fam, gieng Speinxids 8 Bater leibft nat Dem \$onbaufe, um fí bu erfunoigen, ob aud Des 


\section{fut Befórderung guter Cefinnungen 26. 45}

Brief wirflich abgegangen måre. 23ie erftaunte uno

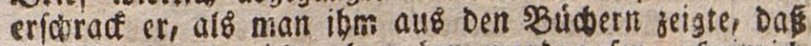
fein sBrief gar nidt abgegeben worden fer). Sheinriक follte nun geftehem, mab̉ er nit bem Briefe angefangen babe. Range leugnete or bartnátfig, baß or ibn nidte abgegeben babe; aber als ithm fein $23 a t e r$ veriprad, Daß́ er ibn allés vergeben wolle, wenn er geftúnder wab aus Dem sbriefe geworden (ev): fo geftano er ends lid alles. 2aber mie fehr mufte stemrido feine flige bereuen, als er bórte, Daß̧ er feinem $3 a t e r$ Durd ein

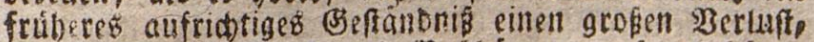
fich feibft grobe angft unb seefódmung erfpart batter

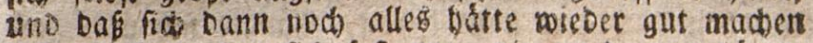
laffen. Er natim fich foft vor, nie mreber ju lúgents ano fieber eine veroiente Strafe zu leiben, als die uns malyrbeit zu fagen. 2atver es Dauerte lange, ehe er feineb ₹aferb 3utraten mieber geminnen fonnte, und Dię that ilym feht watje.

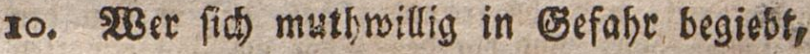

\section{fomm barin um.}

Enriftian Rakmann war Der Sobjn armer ET. tern. Seine ghutter ftarb, als er erft orei Jabre alt mar. Eein $23 a t e r$ roar ben ganzen ₹ag aúfer Dem Shaufe cuf 2urbeit, unt fonnte fich Daber menig uaß Den finaben betúmmerin. (fr muirbe alio gants obne

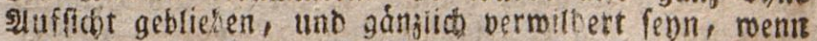

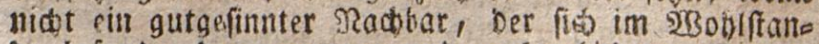
De befano, Den muttern uns noblgebilbeten Rnaber an Rendes Statt angenommen und erłogen fátte. Qther (Garifrian madte feinen Yfiege = eitern menig Frevoes Denn er thar wilb, ungenorfam und faul. Dft marn= ten und fraften fie ibn, aber er befferte fid immer nue auf furze Beit. Befonders miacte ibnen feine Berme=

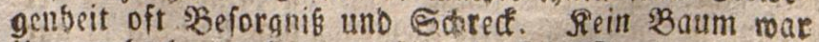
ibm 3u boch, er fletterte binauf; fein Eprung mar fo

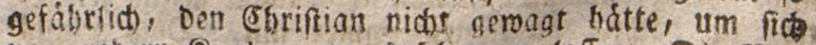
yor enbern Rnaben etwaḱ reten zu laffen. Die Ber-

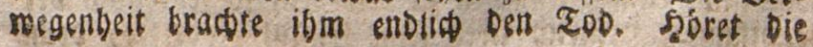




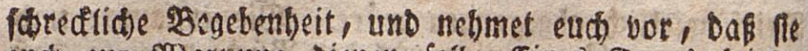

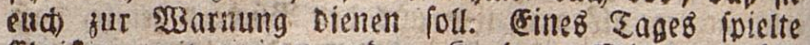

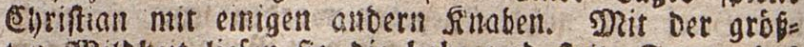
ten $583 i b$ beit liefen fie die hobe uno feile Frexpe beg Scaufer binauf uno binunter. Endith fam Estriftian

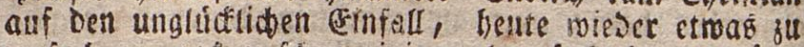

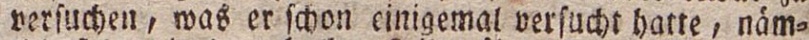
lich fich mit Dem balben leibe ther bas Bselánder Der ¿rcppe ju bángen, und fo von oben binab fu rutfiden.

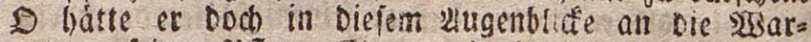

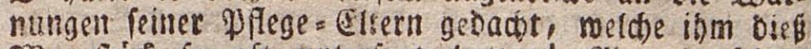
$223 a g e f(1)$ fo oft unterjagt hatten! 2hber in feiner

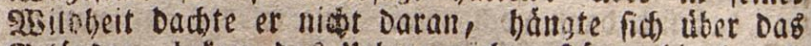
BetánDer, beiam bab Hebergenicht, ftárzte hinab und mar auf Der Stefle todt.

\section{Der cbeticto Snabe.}

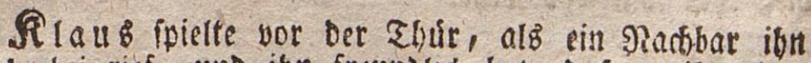
herbei rief, und its freundict hat, oak er itsm Den Biefallen thun, und vor Detn Fbor Die poft ermarten

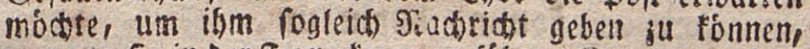
mèn er fie in oer gerne fommen fábe. Rtaus war fełse bercitmillig, biefen 2uffrag a volffibren, Denn er mar ein Dienffertiger Rnabe. Eilig lief er vor Daß̉ Thor,

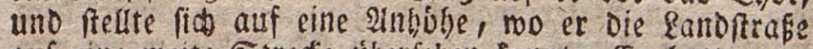
auf eitte meite Siredfe tuberfeben fonte. Er batte nun

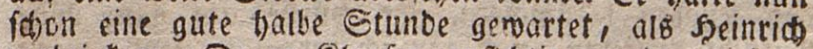
bortei fam. Da er fitatifen anfidtig murde, rief er ifsm fu: fomm mit mir, orúben auf Der 2rBiefe finb alle unire Sdulfammeraben, wir mollen jufammen sBall

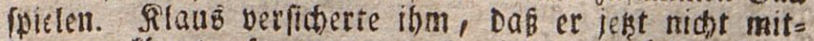
formmen follite, fo gern er auds mitipielen móchte, Demi er babe feinem Peachbar verfproden, bier auf Die poft of warten, und es iflm fu fagen, fobald er fie fom= ment fábe. 2hler wie lange willft bu Denn bier in ber Eonne felben? ervicterte Seinrid, bas baft ou ia gar micht notthig, uno ou beft nun ficon lange genus gemar= tet; ich Dód)te, ou fámeft immer mit. Dod Silaus war nitht jum weggehen fu bervegell, fo fehr aud ber beidtfinnige f̧einnid liber feine Einfalt ppottere, Denn 


\section{Jue Befsrderung guter Befinnungen $3 c_{0} \quad 4 ?$}

er hatte oft bon feinem Bater gebort: ein elrtider Mann bált fein 2rort 3war muste er nows eine volle balbe Stunde marien, ehe Die Doft fam, und barte Dabei biel Eonnenbike aubzufteben, aber mie grof war auch bani feine Freube, alo er enolid Den Poftwagen in Der Fers: ne erbictete, unb nun fettem seadbar die ermúnicte

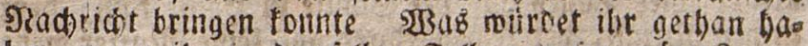
ben, wenn ibs in Demielben falle gewejen wáret?

\section{WBer nidjt boren will, mus fublen.}

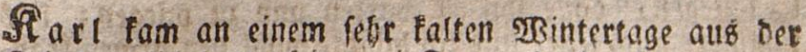

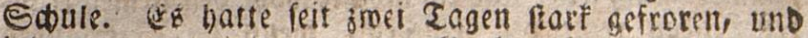
intem er mit eingen antern $\Omega$ aben uber cine sBriate gieng, fahe er, Daß oer fluß mit siz belegt war.

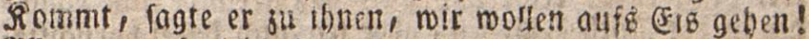
2alle waren fosleid) Daju bereit, und min flefen fie eime Ireppe binunter, Die naw Dem Jluge fúftre. Da fam eii ater gjann gegangen. Finber! rief er, wo wollt ibs b:n? Staut bem (sife nid)t, ef́ if nod) lange n:dbt frart genug, um elic zu fragen, ifr merbet einbrecten.

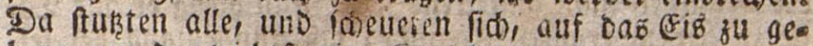
ben; nut Der leidtrininge Rarl fel)rte fich an Die sobbl= gementse 2Barnung nicht, fondern gieng ooth auf Das (Elá, er fpottete fogar ther Die endern, uno rief itgen fu: latámt euch, ibr babt tein Scerb; reer miro fich fúrciten! 2lber er mar faum einige Edjritte gegangen, Da tract er faton ein, und lag bió an Der Şals im शBaffer. 2ille liefen fotreient Debon, unb Rarl máre verloren gemefen, mern nidit Der alte Mann, meldjer quß gutberziger Beforgmí in Der Nábe geblieben mar, binzugelaufen máre, uno ibn gerettet bátre. Rarl jitte terte wie sin efpenlaub, mar todtenblas, unb fonnte

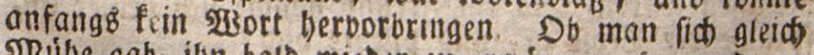
Múbe gab, ith bald mieber fu erroármen, fo murbe et Dod) redft frant, uno mufte einige ₹age im 3Bette lie= gen. Merfe Dir, lagte ber פater, als ei mieder ge= funb gemorden war, bie $23 a r n u n g:$ wer nidt boren will, mub fúblen.

2iber wenn nun alleb gut abgelaufen, und gart nidts eingebroden máre, bàtten Die anbern sinaben 


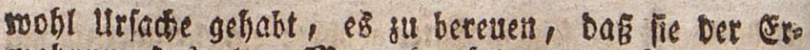
maksung Deb alten Mannes gefolgt raten?

\section{Det greuno in Det Roth.}

Sore, liebe ghutter, fagte Der fleine fartmann,

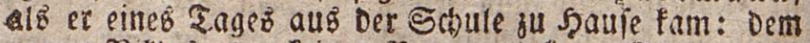
armen Diflab, Der feinen Bater und feine gutter mebe bat, geht es rect traurig; er if febr front gemorben, ano bie hofen leute, welche ibn fu fich genommen ba ben, laffen ith in einer abgelegenen Sammer gatiz als lein liegen, obne ihn gu warten uno zu plegen; oas janmert mid febs, und id móte mob! Den armen fran.

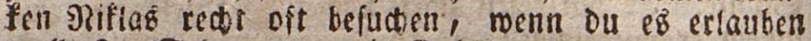
mollteft. Eefre gert, mein Eofin, antmoitete die Muts ter, bentt ez ift recit und gut, Defigreunde fid einall= Der in ber S?otb beifteben, aber fen aud Dabei borfichrige uno etfunoige biak jusor, ob Die Siantbeit Deines Freundes midt anfecfend, und fur bich alio teine (B)e fabr Dabci zu beforgen ift. Eogleid lief Sartmann bin, IIm: fich) zu erfundigen, uno bractite oie शRatricht, Daf Die firankbeit niobt anftedeno fen. Nun gieng er alle $\mathfrak{E} a=$ ge ji feinem franfen Ereunoe, fas ftunbenlang bei feis nem sBette, bolte allé berbei, mab er beourfte, unb bradte logar einige Etunten des s?achts bei itgm fu.

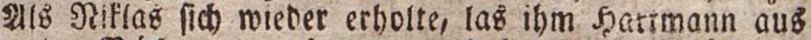
gutill Buchern etwaś vor, uno bradte lom ftárfende Eperfen, melche er fid von feiter guten gruter erbes ten batte. (Emer feimet פritichúler lagte einft ou bift bod ein redter Shor, caß ou ftundenlang bei Dem franten sillas fineft, is rotirde mict) Bafur bedan= fen. Wúrbe eś bir midt reble mobt gefallen, antmorteté Şartmann, wenn ou frant, unb bou allen 9genichen serlaffen wáreft, uno ein greuno nábme fich beiner an práa)e bir Eroft su, uno pflegte Dicts?

शitias murbe bald miecer gefunb, und bantle peinem freunbe Şartmann mit inniget squbsung fư feinen liebreiden SBeiftano. WBie roollie ict mid fretent, fagte er, went id bir aud mieder etmas gu Siebe thun fonnte, guter Şartmann, aber id vin arm, und meif oud nicht, momit id bir eine 


\section{zux Sefórberung guter Cefinnungen 2r. 49}

Jreube macten farn. Shach einiger Beit fam fartmann

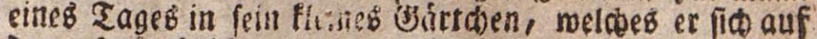
Dem Şofe fetbrt angelegt uno eingcrictet batte. WBie erftaunte er, als er alles Linfraut ausgerauft, bie flei=

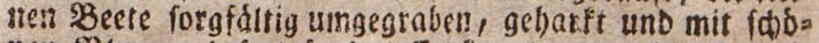

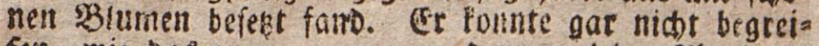
fen, wie Dab zugegangen mar, Denn now ben 26eno zแ-

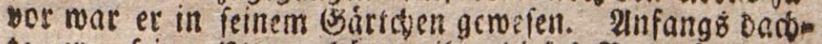

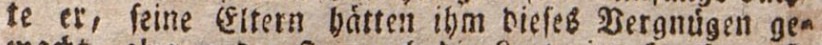
madt, aber weder fie, liod) Die felute im Şaufe mufs. ten etwas Eavon. Enotid erfubr soartmann von sinem

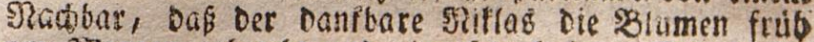
ant Mrorgen gebract: und eingeję̧t babc. Seit Diefer3eit leberen beste in ber berzlitoffen greundichaft, und b)dten moht ibe leben für cinanoer gelaffen, menn lie jemalö in diefen fall gefommen máren.

\section{Der Santfútitige.}

(8) oft licb Iebte mit feinen Gefómiftern unb maitfobisa lerin befadnbig in Etreit. SBenn feime fleine Edwefter

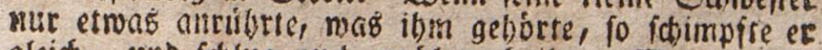
gleid), unb follug aud) wobl Bad) ibr. WBemn er fie madb Der Squie bradte, doer aus Der Edule abholtep fo hatte er beftindig mit ite zu zanfen; Denn bald giens

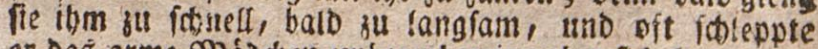

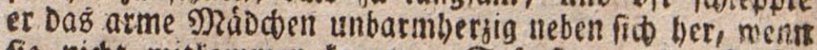

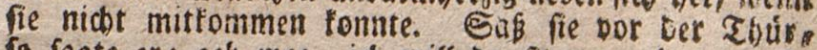
fo fagte er: gel) weg, id mill oa fiken, uno mern fie micht freimillig weggieng, fo ftié er fie mit (Semalt fort. (Eben fo madte er es in ter Schule, und Daber molfo te endid niemanto mebr neben dem zánfifiden Botto tieb fiken. Er fudte fogar eine ebre barin, jedem Frok

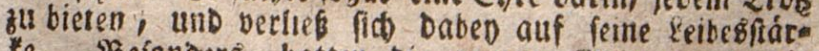
te. SBefonier batten Die armen Sileinen und Die Sdimachen, weldbe fid nidts mebren fornten, voc ithm fellie siube. Beftánbig fortete er tuber fie, uno feine गrefereien hatten fein ende. 2hut auf Dete Strabe fieng er sadnoet an, aber oa er bier oft einen Segner fano, Der ibm an Státte ober esemanotbeit

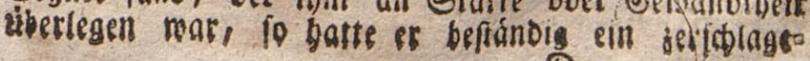


neß Brfidit, unb einft befam or hei einter Edhlogerei

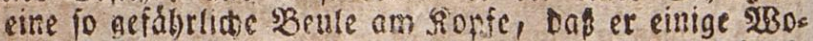
(t)en beftige Eodymerzen ausferten muste, uno lebents lang eine Narbe bavon bethielt. 2ther cud) Diek Uns glude befferte Den berwiberten tmo zornigen esortlieb nicist, Denn alß et ermadien mar, bradise er einft

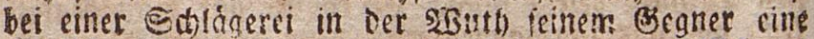

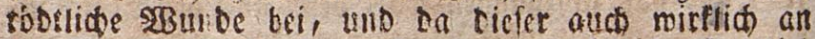
Der 2rsunde fiarb, fo mufte ser ungituclide esottlieb als ein Mórber feine ganje úbrige febensibet ins Sudts haufe subringen. Eo (chrectidid find oie folgen Det Bantfuct)t uno DeB Jathorne!

\section{Die mulbmilligen Rinoer.}

In einer Edule waren jrei firnben, welthe von ibret

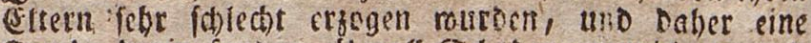

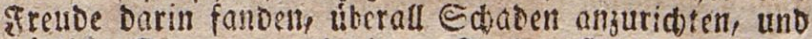
núslidbe Dinge zu verberben. In Der Ettule idmitten

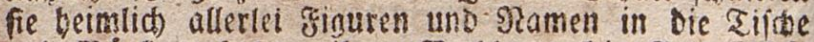

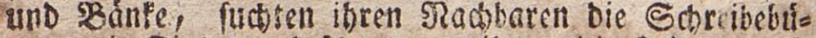
der mit Finte fu befomuken, ithen bie gevern alfs zufpalten, und ihre Saden zu verfteden. 2uf Der Strape madten fie és nicht beffer. Den Jruchthánbles riamen, welche euf sem Mtarte faken, watfen fie auf eine liftige $23 e i f e$ ihre gobrte um, ooer bemarfen fie aub itaeno cinem Ed)lupfrintel mit fioth und etci= sen. Bsiengen fie Des 2lbenos auf ber Strabee, fo folus= gen fie mit grofen Etoden an Die genfertacen, um bie feute ju erfdyrefen, Doer jogen an Den Sitingeln ber Jjâufer, uno liefen Dann fänell fort, ober verfecten

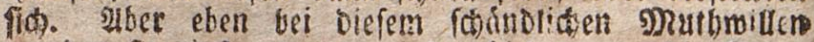
rourben fie einit ertappt, umb erbielten nun die Stras

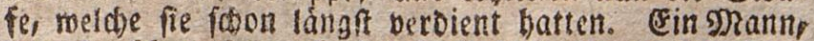

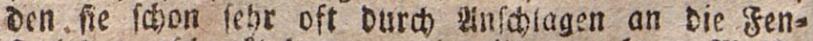

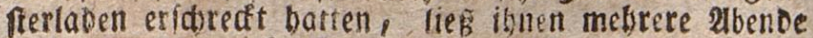
nach eirander aufpaffen, und esthlid) gelang es ibm

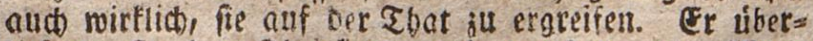
lieferte fie Der Dbriglett, unt wusten, zur $23 a r=$

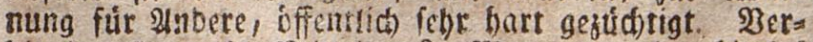
Dienten lie moht Mitleiden? fegr zu bedauten? 


\section{Det Unjufriebene.}

giloolph batte sohlbabenbe und febr guitige eltert? Da fie nur Den einzigen Sobn batten, 10 manden fie fehr viel an ibn, uno riodph batte baber alles, mas er fid) thur munfoben modite: gitte Rletoer, alle Eage gut fu effen, und manđeŝ. Şergnúgen. 2lber ebea Darum, meil eis ibm ju nobl gieng, mutbe er unges nigfan uno unzufrieben, Das beift: er freuete fich nies mals tiber Das, maś er batte, unto fand immer erwas

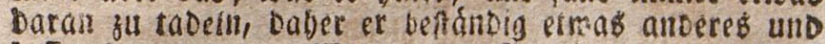

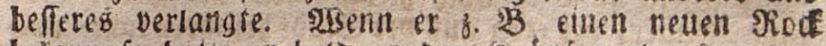
befam, fo batte er bald an Den finopfen etrab ausjlle

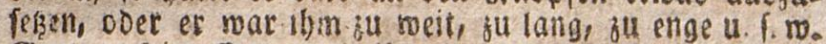
Bientigen feine esttern mit ithm ipapieren, fo flagie er bald

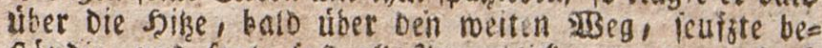
fándig, und fogte faft alle 2lugenblicte: menn mis lod mur ern Da máren! 23ar man enolich angeforrmen, fo geffel é̉ ibm wieber an biefem Dotte nidt, uno ec

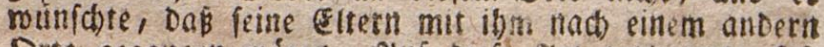
Drte gegangen wáren. 2üf Diefe sirt verbiterte fich Der unzufriedene glatph faft pebes sergnugen, uno mur

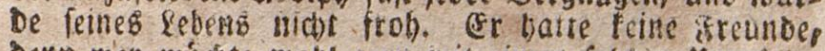
Dentr wer módste wobl gern mit sinem folden llnzufries Benent ungeben? Er batte aber aud faft niemalb ein frób)

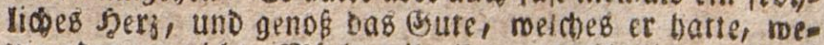
ung ober gar nicht. Modbtet ibt ibm wobl ábntid merben?

\section{1\%. Det Farmberbige.}

Runz unt $R I a U b$ gienget an pinemt febr falten

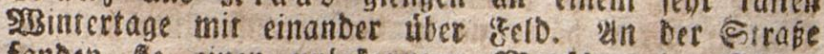
fanden he eimen unbefannten Meniton im Eanee liegen, welder feft zu loblafen fobien. Runz batte Mnitletben mit ibm, uno aus SBeforgtif, bak er er.

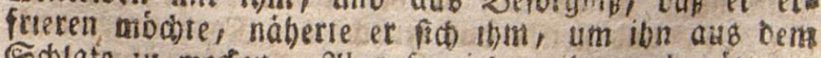

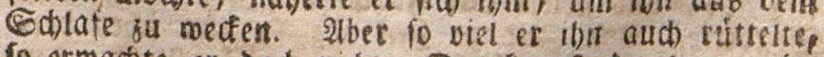
fo ermadite er Dod nicht. Dell fainft ou lange tute

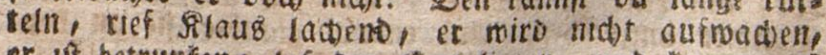
ex If betrunfen; lak Dell sierl liegen, unb fomm, is if talt, Rein, antwortele sunj, fo unbarmberig

$$
\text { D } 2
$$




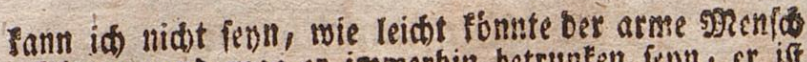
erfrieren, und mag er immerbin betrunten fel)n, ot iff

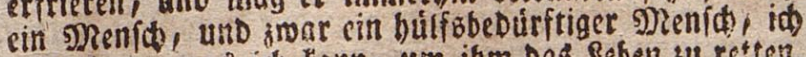

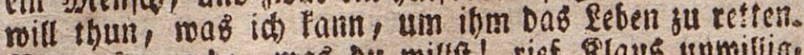

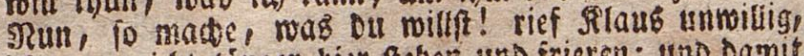
in mag nidst lánger biet feben und frieren; tno damit gieng er weiter. Rum beoedtte nut eifigft ben Sdlas

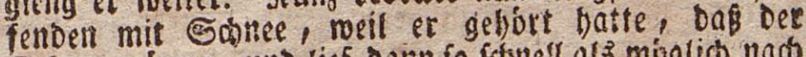

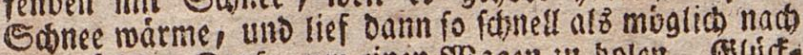

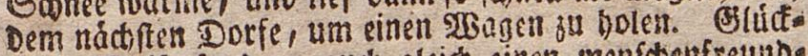

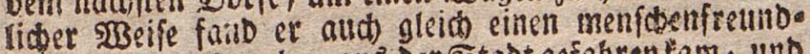
liden sauser, Der eben all ber Stabt gefahrentam, uno mit Deffen sclilfe er Den halbtodten Jremben fehr balo inz eeben bradite. Fróblich manberte er nun nacis Sqaufe, 2Bab urtheift ibr yom Sum? Uno wab ur= theilt ihe vom Ritaub ? SBeffell SBetragen mollet he zแแm 9Rufter net)men?

\section{Die Sutchtfome.}

23 ilbermille batte cine abergláubifơ e 2Bärterink,

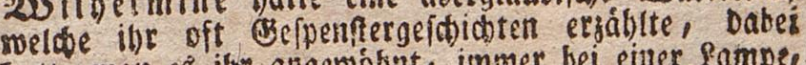

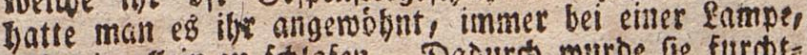
unb nie allein zu fallafen. Dasurat murbe fie furchs:-

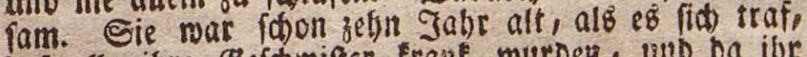

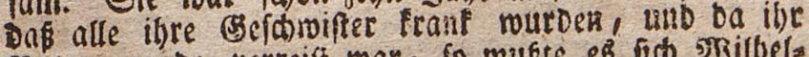
Sater getabe verteift mar, fo mufte es fich sobitbels mine zum erfienmal gefallen taffen, allein zu folafen. Daruber gerieth fic nun in grofe 2mgit, befonbers oa Die shutter feine fampe in itheer Rammer wollte brennen laffen, fonbern meinte: Dab grobe Mgäbders

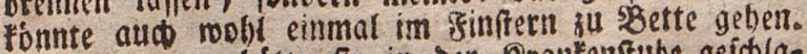

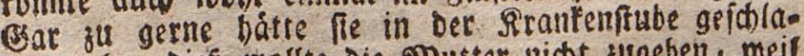
fen, aber Dieß modtre Die 2Diutter niकts jugeben, weit fie Daburd leidt bátte angeftedt merden fonnen Tseis nent gieng 2Bithetmine in ibre frammer, jog fid bas ftig aus, unb fecfte aus furdst Den Fopt unter bas

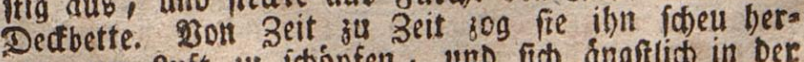

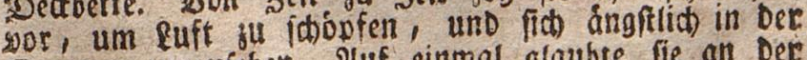
Fanmer unjurebetl. 2luf einmal glaubre fie an bee Rammertbuit eine lange meipe Befralt zu erblitent.

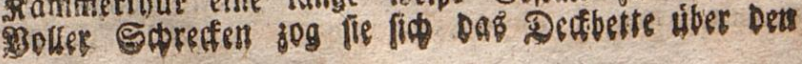




\section{3ut Beforberung sufek (Befinnungen 2c. 53}

Sopf, unb Der 2ingfifidmeiß̈ lief iht von ber Stirn. 2ange fonnte fie ef in Diefer Lage nicht aughjaiten; fie magte es endid allf einen 2urgenblide Den Ronf berbot=

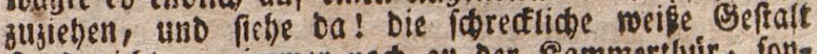
fiand nidft nur immer nods an Der Rammertluir, fons

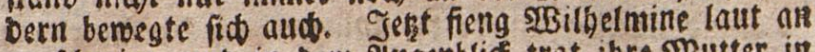
3u foresen, unb in Dem 2ิugenblid trat ibre Mutter in Die Rammer. Rber Sind, was ift Dir Denn! rief lle ibr ; ; tráumeft ou, oDer wadf Du? 2(d) Mzutter! פRut-

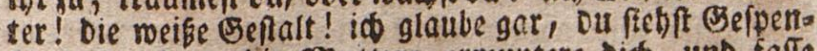
fter, erwiederte Die Dutter; ermuntere bido, unb fafe

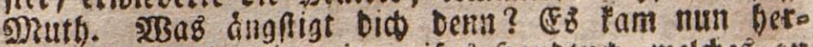

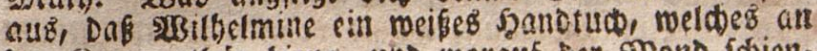
Der fiammetthúr bieng, und morauf ber פond fdien, fúr eine meise હeftalt gefjalten batte. Die s)utter batte an Der Raminerthuir geforcht, ob sBsifbelinine follief, unb

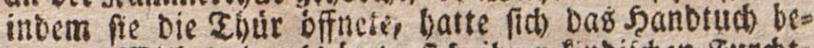

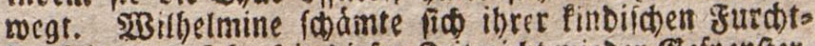
famfeit, und fabe feit Diefer zeit nidt mieder Eefpenifter.

\section{Die gute Todtet.}

SBitherm wat febr frant, und feine gute 5patter

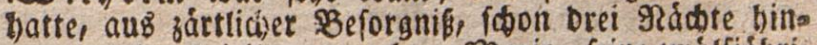
ter cinanter bei ibm gewadt. Maric, feine zmólfiebthi=

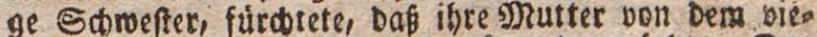

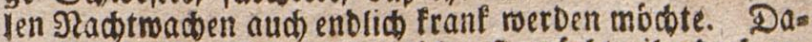
her bat fie ibre s)eutter berstid, fie módste ibr Dod ct= lauber, Die viette SRadt bei bem franten SBruber ou twas

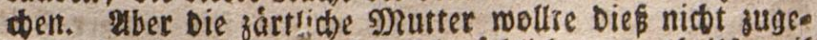

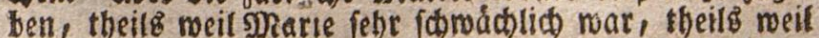

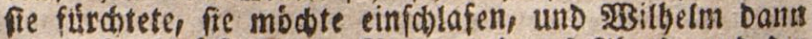
ganz obne suilfe feen. Neun wurbe eह̉ \&bent, und bie abgemattele Mitter mufte fic Dod endtid aufs פette tegen, weil its die dugen sufielen. Marie batte fim zmar aud auf sefebl ibrer Mutter zu Bette gelegt,

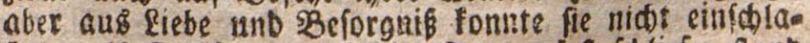
fen, als fie borte, Daß bie mutter feft folief, feanb

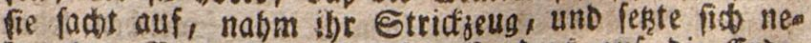

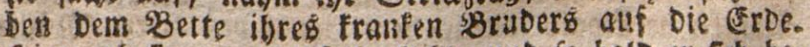

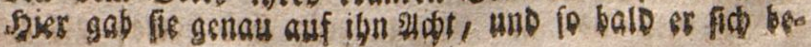


bewegte, thas fie fogleid bei ber Sants, um fid bu eta Punbigen, mas er verlange. So trieb lie $e \hat{b}$ bis an Den gnorgen, uno wie grob war nun ibre sreube, daf fie

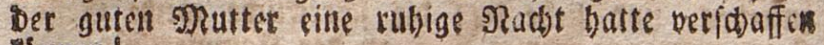
conmen!

Balb nadber wurbe bie snutter aud franl, erbolte (10) aber balo mieber; nut feblte es ibe an Siráften. Der 2lest hatt? int 5mariens Gegentsart gefagt: welin Die Sirante nur táglich cin wernig 23 ein trinten fónter to moiroe fie balo mieder fu Siráften fommell. elber wo follte bie arme frau baf berlo fum wein bernebmen? 2Bilbelmb firantbeit hatte gar ju viel gefoftet DRarie borte, dafi in dem fyafe, mo fie wolnte, jemano gefucts

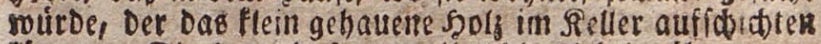
Fónnte. Sic, bat, baß man itir die 2libeit úbertragen módte, uno verfprad), redts emfig Dabei zu lepn. SRad bier fanern Stunten batte fte mirflid lo viel verbient,

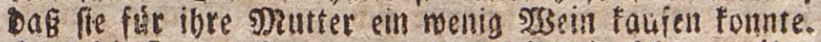
Dogieids fie vout ber ungenobinten atrbeit febr ermuber roar, fo lief fie boes fo foneth, als ob fie beute nod) gat

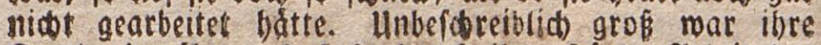
Sreatoe Dariber, Daß fie Durch ibre Sgande ertbeit Dọ guten Nutier biefe erquifung batte beridaffen fonmen. Die. Mutter mar to gerubrt utber Martens findiche

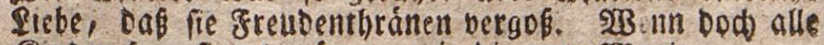
Simber fo gefintet róten, wie die gute SpRarie.

\section{Der ungegrundete 2 erbacht.}

Dem Saufmann spútler weren feit einiger seit

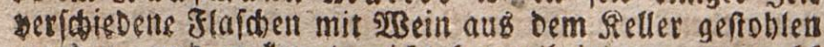
soorben, unt er fonnte nidht beraubbringen, mer mob)

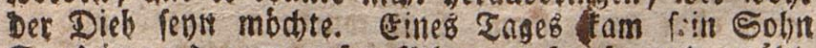

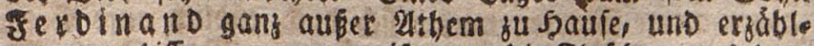

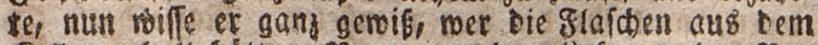
Rellet geholt bátte. Niun, wer benn? fragte ber $\mathfrak{B} a=$ ter begierig. Fein anderer, fagte Ferbinant, als Der theine ermalo, benn id babe ibn eben mit zmei \&la

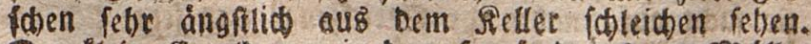

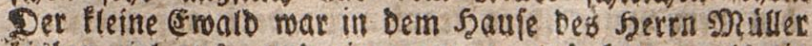
visber viet aug = und singegangen, unb batte, als ein 
jur Befơtbexung gutex Oefinnungen $2 c_{0} 55$

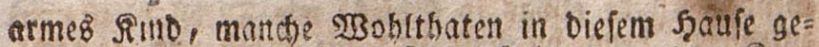
soffen. gotan breft viel auf ben fleimen muntern fina= betl, uno batte ithn bisher immer ben efrlidest

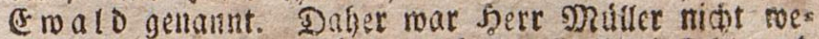
nig erfaunt, ats er borte, dab eroalo ityn befteble uno

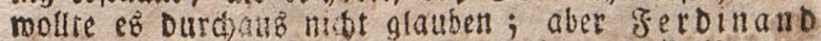

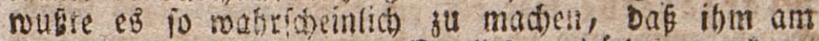

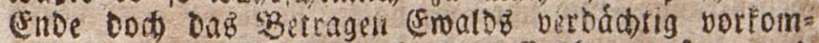
ment mukie. Er liés alio betr sinaben rufen, uno alb er eridoien, fabe er ifsn eite sBetle fibs ernefthaft an. Shât bu ein guteô Benviffen? fragt: er ihn bam. 23ei bicier grage fohien Eroato verlegen fu werden, uno erroblete. Bantworte eferich auf viefe frage, fubr

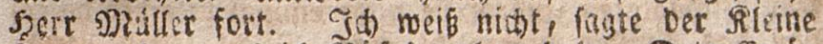
frammelnd, roas id şofés geiban habe. Dein Erro: then verráth Did), crmiederte Serr Muiller mit Unmeil lent uno fab tha dowei finfter uns broberto an. SBift ou haute in meinem Sieller geneien? Shaft ou zmei

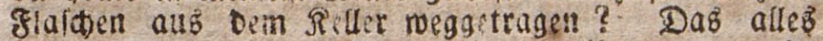
fonnte Eroald nidbt leugnen, aber alb ibm nun gerabe Sduuld gegeben mard, Dak er bie geffoblinen Jlafdert פBein ioegaenommen babe, verfiderte er obne Jurdot,

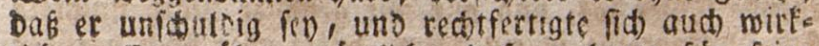

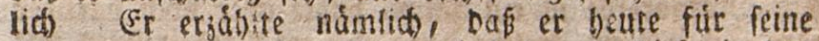

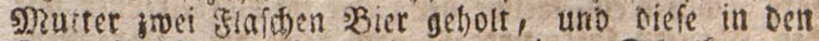
Ritler bei Selie gefest habe, um einem Sd)uffamera= Den, oer einen fiberen Rorb fll tragen batte, unb

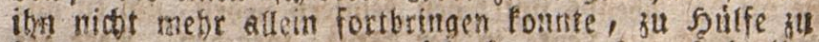
fommen; ais or wieber furtidyctommen fed, habe ifin ein groper Junge geneftet und vetfolgt, bis er Den Fiellet etreib)t habe. Bate or nun mieter beraubgefoinmen

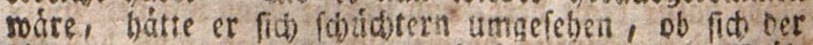
bofe junge niche etroa too verftecte babe. Saetr grillo ler ertunoigte fid bet erwatso ghutser, uno fand biefe llmpanose alle volfommen richtig. Ren that ef ibm felir leid, Daf er Den elirtichen und dienffertigen Ervald in einem fo bien żerdacít gebabr batte. Um ibn fúr

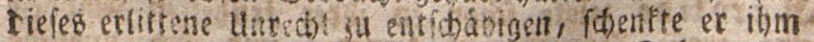

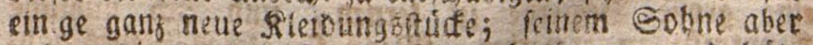
gab or Die Eegre: fed tunfeig beflutfamer, und buite

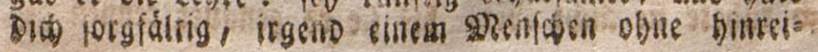




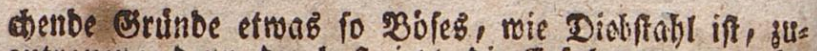
sutrauen; Detn bu baft ieşt Die Etfalbrung gemarbt,

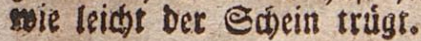

\section{Das neugierige Måd den.}

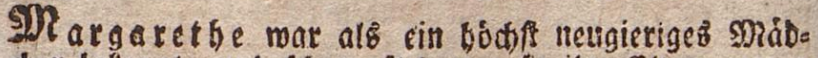
chen befannt, unt fiton oft hattert fie ibre eltern roegen itrer thoriditen Reugierbe beftraft So baid fie nur Dab getingfe Gerátifo nuf Der Strafe horte, lief fie an bas genfter, um zu felien, mas es gebe; uno eines Zages madte bie beftige sretsietde fie fo blino, Daf fie nit bem sopfe gegen die Jenferíceibe fubr, uno fich febr befacobigte, indem fie tiat eimmal bemerts battc, Daß Das genfter fugemacht wat. Nidbt felten verlor Sie auf Der Etrabe ijor Stricfieug, ober wab́ fie chen in

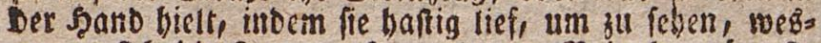
wegen fich bie Setute verfammelten. SBeinabe wäre fie einft Daruber um'B \&eben gefommen, tenn inbem fie in ibrer Unbeionnenigeit zufabe, roie ein Dafe, Der fid Yosgerifen batte, unb eben roieder gefangen worden

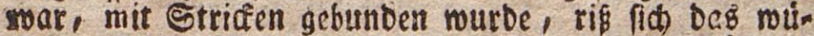
thenbe Ebier ID\&, unb nut mit genauer SRotb flistete (10) SRargatetbe in ein 5aug, buffte aber boch baruber

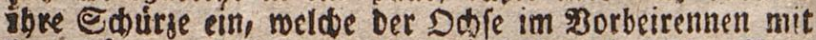
Den soormern fafte und ibr vom feibe rif́. Thre Reus sietbe verleitete fie aud fu bor ob en, und man fabe Je oft bes 2hbends unter Den fenfern fteben, um zu bos seti, mas Die Reute ill Det Stube fprád)en. 2tber bei Diefem Srorden lief fle einft fehr tubel an; benn cin Drann, Der fie Dabei ertanpte, zuditigte fie obne $4 \mathrm{~ms}$

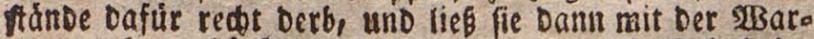
stung geben: finftig bordbe nidt wieber, fonft baft bus moh etros Edolimmeres zu ermarten!

\section{Das mifibegierige झRäbd)en.}

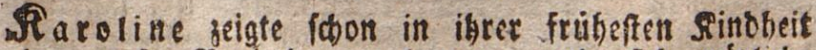
eine grofe segierde zu lernen, und fid míplicte

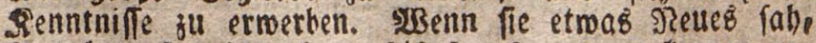
fo rubete fie nidt eher, biš fie so genauer fennen ges 
gut Beföcbetung guter Oefinnungen 26.52

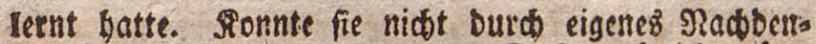

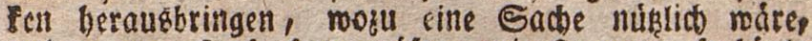
uno wartum fie fo feon miffe, wie fie wat; fo borte fie nid)t auf, ju frogen, bis ihre $283 i$ bbegierbe befries Digt war. Eegr gern gieng fie in bie Sabule, unb wena auch Dab 2 Better nod fo (क)lectit mar, Deanod f(t)eute fie nie ben weiten 2 Beg nad Der Schule. 2tuferorbents

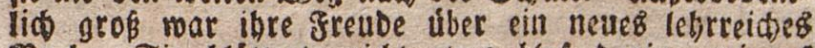

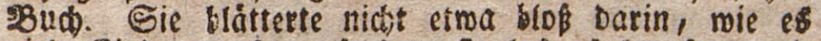
siele Sinder madell, fonbern fie lab̉ eहै langfam und mit grofer 2ufmerlfamiteit burd, uno Daber brieb fie auch nie bie 2tntwort fátuldig, wenn man fie fragte: was in Dem sBude enthalten feu? SBeinafie in alless

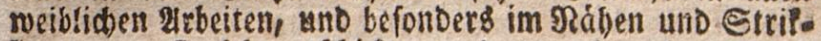
ten, war fie febr gefolitt, and um eहै nod) mebr zu metben, wurbe fie bie Begúlfiun siner frau, melde fie nar unter Der barten sedinguth unterriden molltep Daḱ fie ein ganzés Jaht bindurd, bom fứben MRorgen bis zum fpáten sabend, für fie arbeiten foulte, olgne $\mathfrak{B}_{e}$. jabilung Dafuir ju verlangen. 2iber als Dié faure Jabe endlid, uberftanden war, batte fie aud bie Freube, nicbt

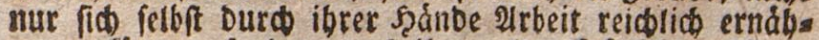

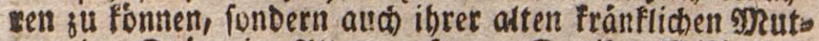

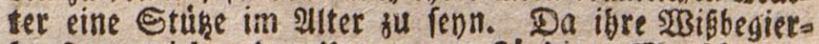
De fie antrieb, Den Ulmgang verftandiget פReniden fu fuchen, yon meleden fie lerten fonnte, fo blieb fie boe wielen Eborheiten unb Berfudungen bewabrt und ere freute fid Der श्ditung und fiebe allet guten Menfiden.

\section{IRenfकenfreunoric)e Eefinnungen.}

$\mathcal{E}_{n}$ ton mar ein úberaus gutherziget Snabe. Seine gróste greube war bie, 2unbern eine Freube 3u maden, und gern gab er etwas bin, wab ibm ferbfe lieb und werth war, menn er baburch snbre, unb befonbers feine Befdimifter, erfrewen ronnte. SBenn

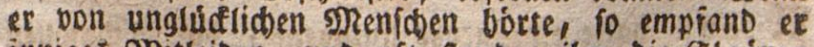
inniges 2Ritleiben, und oft franben ibm bie Thránen in ben 2ugen, menn fein \$ater uber Filobe von emem uninglúafúfalle erjablte, weldjer fiós ereignet batte.

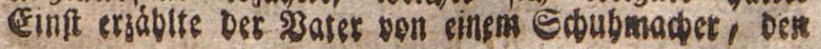




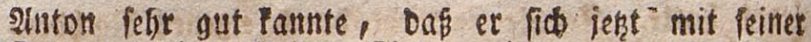
Frall uno brei fleinen Finbern in elner red)t trauti: gen Rage befénbe. Die armen Reute, fagte er, jam:

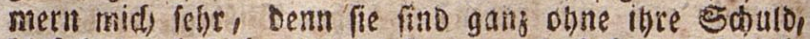
blof baburd fo berunter gefommell, baf fie von (d) lects: ten Mentifben, Denen fie Siedlichfeit zutrauten, um bes

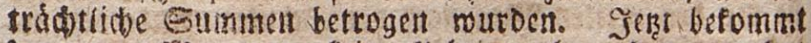
Der arme 2Rann gar feine surbeit mebr, Denn er bat

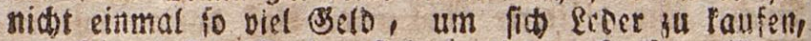
und feine beffen Sacten find bereits verfaiff. 2 Benti id) es nur cinigermaßen úbrig bátte, getn mollte id

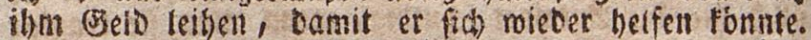
2lition hatte bick alls feller aufmertfan angebórt.

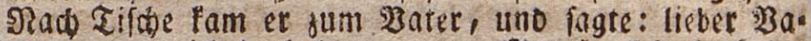
ter, wenn is Doch Dem armen s) Martin (10 blek Dek

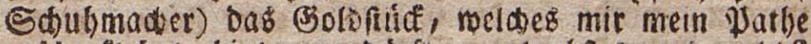
geidenentt lyat, bintragen burfte; erlaubft ou eह̉ mobl? Der Bater hatte anfanģ einigez Becenfen, Denn es war yoraubsufeten, Daßß Martin ausc) Diefe paar Fba: ser nie murde mieber bezahlen fónnen. Dow 2untoit

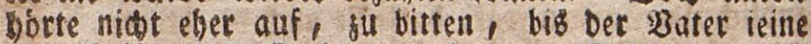
Crlaubmí gab. Frober mat oer gute enton noch nie gemeien, als in Dem 2lugenblife, als er fein Boldfute Dem armell Martin hintragent Durfte. Martin fontite nun cinett fleinen Bortath yon Reder einfaufen, 2uns

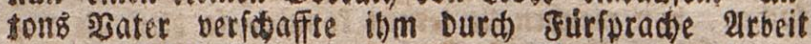
genug, uno balo mard bem armen פRasne fo meit ges bolfen, Dak et feine sBetten, welche et in Der SRoth batte verfegen múffer, roieber einloien fonnte, und von Nabutungefforgen frev war. gretlid bat nidet

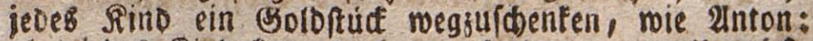
aber jebes Sind fann Dod etwas thun, un unglüf: liden zu belfen, uno fie ju erficusen.

\section{Wुas bifipt fómollen?}

Qf ug uft hatte eine grofe untugenb, bas Edmotlen ober maulen an fict; Denn menn er bon 'iemano be= leidigt zu fepn glaubte, to mar et viele ₹age lang unt

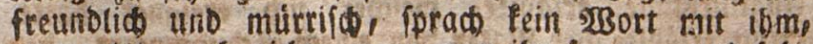
antmortete ardo nidot, merm man ibn fragte, suro fabe 


\section{gut Beffrberung gutet Sefinnumgen 26.59}

fo finfter aub, als ob or alle 2tugenblice um fich foilan gen wollte. Seach langer Beit war er enft wieter ge. fprádtig uno freunolid).

(Er betrug fid aber nidt nur fo unartig gegen feine Beffomifer und Epieffameraben, fondern fogat alit) gegen feitie (eltern, menn et yon ibment megen pitue B geblers beftraft worben war.

IIm fom nun viefe lliart abjugemóhnen, befabs Der 23ater 2lien im şanfe, menn 2luguft mis irgent

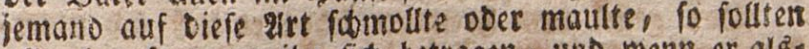
alle eben fo gegen ibn fich betragen, unb wenn er aldz: Dann auffictic, fo follten fie getabe nod) eimmal fo lan = ge mit ithm fctmoflen, als feine llnfreunblidfeteit ge=

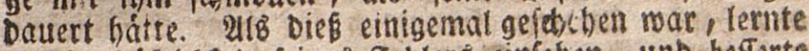

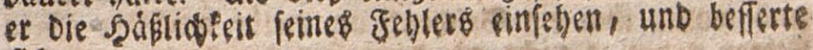
fiø.

\section{Befåtíg eit.}

Rlibert hatte in ber Edfule gelernt, Daß man eir nem ieben bie थdotung ermeifen múffe, die ibm fu= fommt, unt Das man fich Durch ein bófliches und ges

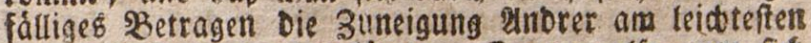
und fiderffen erwerben tome. Er war alfo, um fid 3u Diefer zugeno zu gewóbnen, immer aufmerffam auf fid felbft, uno eriaubte fich nie, aud menn ef als lein wat, irgend etroas llammíandiges.

In ber Eddule beobadtete er fetế eine anfiäntige Etellung Des feibes, aus 21btung gegen reinen fehter anb feine Miticufter. Er trai niemalö mit Dem Saure auf bem ropfe in bie Sdulftube, und vergás nie, fei= nem fecheer freuntlio eiren guten sporgen zu múnfdent. Sie fahe man ibn mit weit vorgeftredten fúpen uns zurúfofelefintem \&eibe fiken, ober mit Den \&rmen úber

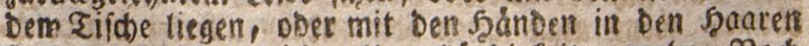
Praken, uno bergleiden unanftánbigfeiten melor. গRod

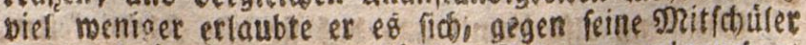

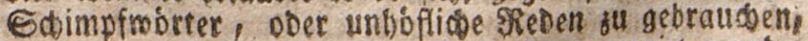
wenn er auch bismeilen von ibnen Daju geteiat murbe.

Finft gieng or mit einigen feiner $93 \mathrm{e}$ annten bor Dem Zopore (paksieren. Do begegnete ithen ein frember f̧ert) 
gu gsferbe, bet getabe auf fie juritt, um fie gu frageth in meldber Strábe ein geroiffer Eafthof láge, wo di einfebren moftte.

216 ber Srembe nabe fam, liefen bie antern Sena ben auś einer unartigen biósigfeit babon; 2libert aber brieb fielien, nabm feinen syut abe uno ants wortete auf bie frragen beó fremben bóflich uno bes

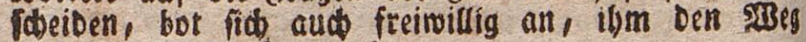
nad bem Earthofe ju zeigeth.

Das gefiel bem gremben febr: er lieb fid unters megs mit ibm in ein (Sefprád) ein, fragte nad) feitiel eltern, wie fie biefent, wo fie mobuten, und nad ant Dern Umfánoen. Bor Dem Bafthofe fiecg Der Zrembe

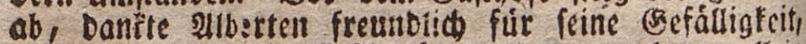
unb mollte ibm ein sefdenf att (Gelbe maden; allcin 2ilbert nabme eह nidbt all, Denn fein Dienfffertiger 2 atet batte itsm oft gejagt: man mus fich nitbt jiben ficineh Dienft, ben man andeen leiffet, bezahlen laffen. Jróbs

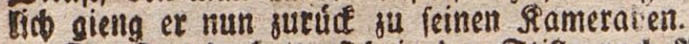

Der frembe batse fith in Der Stifle nad afbert eto sunbigt, unb als er erfubs, baf er nidt biof ein bófo lidber, fonbern audo ein ebeliớer und verfiántiger sinas be fev, unb febe arme Esttern babe, to ließ et ibn auf feine Soften nets fleiden, und nabm ifn nad entiget zeit in feime Dienfe, wo eb itym fehe mohl gieng.

\section{Die Dertåumoerinn.}

Senriette mollte fidi) sern bei ibren eittern uno sebrern beliebt madien, und meil is ibe ju (d)mer Dunfte, uno ju lange Dauserte, fid Durd) Fleis, Sitts famfeit uno ReDlidfeit biefe siebe zu erwerben; 10 legte fie fid aufB \$gerláumben: Denn fie batte bemerft,

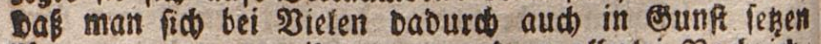
Ebrne, wenn man ibnen son anbern alletlei शRadsicts ten bringt.

Sie fieng aljo bamit an, baf́ fie alle Rleinigfeitellit jeben uniduubigen epaf, une jebe Unborfiditigfeit oder uebereilung ibter Gefobmifter bei ben Eltern heimlid

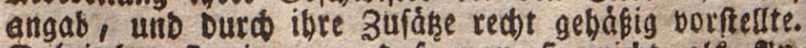
Dabei bat fie immer, Daf man fie nidt alo ants 


\section{gुน Befơrberung gutet Befinnungen $2 c_{0} \quad 6 x$}

geberinn verrathen módbte, meil fie fonft intem staffe ausgefeets fepn múrbe. Die Cittern, welde gegen ibre

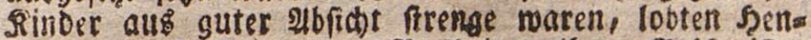

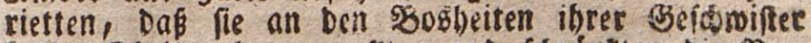

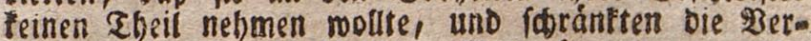
láumbeten in ibren unf(buldigen $\mathfrak{B e t g n t i g e n ~ i m m e r ~ m e b e ~}$ ein; aber Sgentiette rourbe uberall porgejogen und ju allen Euftharfeiten zugelaffen.

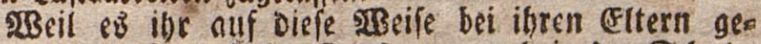

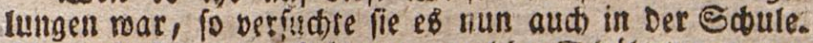

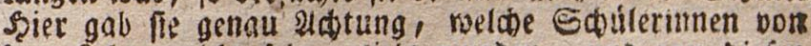
Dem febres nidbt fejer geliebt wurben, und von biefen

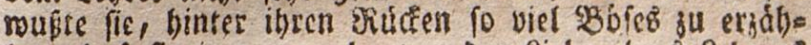
len, Daß fie immer mebr von Der qiebe ibreb Rebrers verlobren. Diefer aber bieft Sgentiettenz 2ubzage dar= um fúr wabr, mail er eben diefe Godurlerinnen fobon yon ciner idfledten Eeite fennen gelernt batte.

Jndeffent fam ibre boshafte Beriáumbung sod enbe

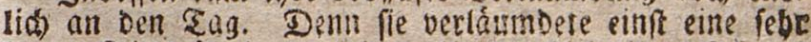
brave Mitriatúlecinn, meil fie ithr ole fiebe ibreb \&ehrers

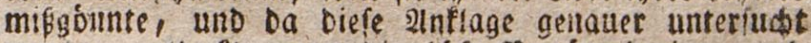
murde, entbefte man bie bófe Berláumberirn, uno beftrafte fie. Iut maffe niemand bon ber 3eit an mebr mit ibe ungefien, ober ibrell 2ssorten glaubert.

\section{2\%. Die $23 a b t f a g e r i n n$.}

Eine Sigeunerinn fam in ein Dorf, unb moffte ben Seuten für BelD mabrfagen. Einige maren auseit wirls lid to einfáltig uno abergláabig, Daß fie Den Reben Der liffigen zrau zuborten. Diefe fagte nun einem jeo

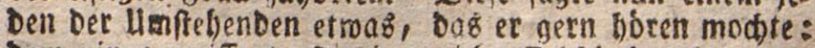
Dem einen meiffagte fie eine reiche (Erb/d)aft, Dem alle Dern eine glứtititbe Seirath), u. 1. w. Dafúr murbe pie dann aud reichlid beictenit.

Unterbeß batten bie Berichte non diefer Sandftreiderinn gehórt; unb meil folde Berrigereien frrenge veiboten find; io murbe fie unvermuthet alffgelyoben uno nach ber Etabt in פerroabrung gebracht. Satte

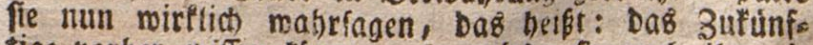

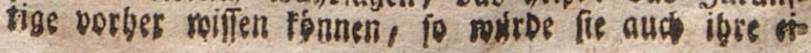


gene Eefangentrefinumg gemufit babell, unb berfelbel Durb bie ficucit entgangen fev.

Dennod aber gleubien die meiften das, mab bif

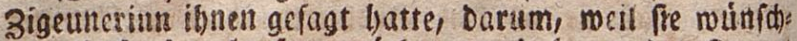

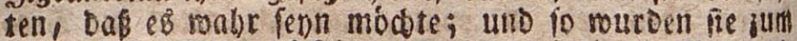
Theil Dabutd ungluffitiof. Denn Derienige \&: $\mathfrak{B}$, reels

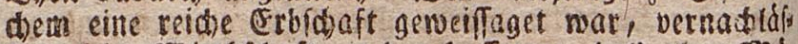

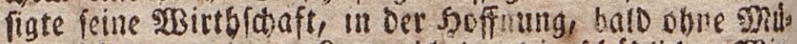
he reid zu metben. Qange blieben die fódoliden $2 B i$ fungen Diefes \$Betrugeb in Dem Dorfe noథ fídtbar.

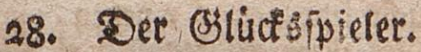

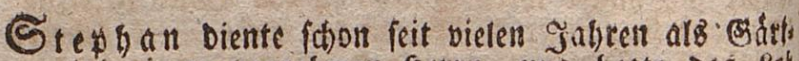
ner bet eintem vornet)men spetrn, uno baste das rob eines Pleifigen, geidiluten und treuen 2yrbeiters. (5t lebte babei aud febr zufticien, und múnichte meitet

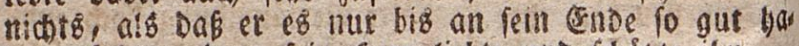

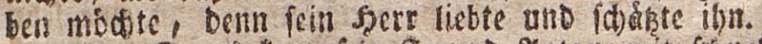

Eines zages fam fein greund 2untos mit fannetlet Edritten 34 ibm in Den Sarten, uno meltete ithm sole lat Fretude, Dak er 500 Shater ill Der forterie gemontent babe. Nun bin i申 auf einmal aus meiner शroth! tief er; fo termmerlid, wie bither, Darf ió) nun mein bisa den SBiod nitht mehr verdirnen. Ith gebe meinen Dientit bei Der Seerritoft auf (ex war Bectenter bei Demielbet

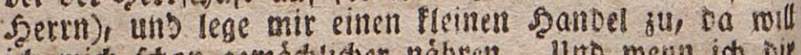

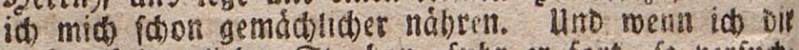
rathen foltte, lieber Stephati, fubr er fort, fo berfucts

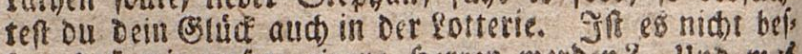
fer, Daßs roir unfere eigene Serren merben? Ulio wal

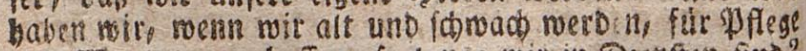
unt 23 artung $3 u$ hoffen, fo lange mir in Dienfen futo?

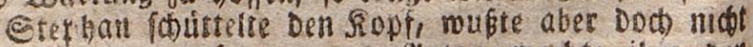
bief Dagiegen fu fagen, uno entron madite ibm bel Cervinn in oer gotrerie to mabricheintid, feltre itom auth Den 3uffant ser unabbángigleit to angenet)m vori

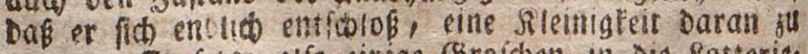

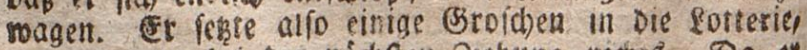
unt gemann bei bet nádffent 3tehutng michib. Da et mutblos werben mollis, munterte the shton auf, unt 


\section{fur Befórderung guter Oefinnungen sc. 63}

lagte: er follte ben Einfals nur eimmal verboxpells, und fortfabter, am Enbe múlfe fein Soob heraubs fommen; fo babe is feitre 500 ?haler gemonnen.

Etepian molte fein Beld nidjt gern yertoren babetr uns ferste alfo bon neuem ein. Das that er auch bie folgenoe Zett, aber fatt Der Ereffer murben immer Nieo

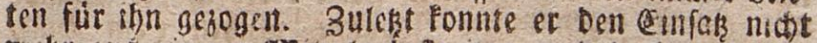
mel) auz eigenen Mnitieln beftreiten, und bod) wollte ee micts autboren, entisufeben, weil ef fich in Den fiopf gefelgt batte, er mitife cimmial geminnen. Uno mas

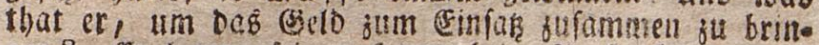
gen? Ee berrog feinen Sgerrn beim Berfauf der Bars

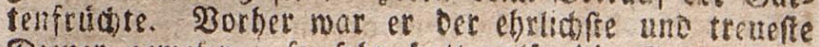

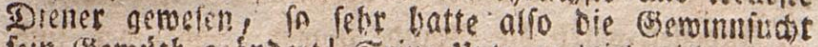
fein Esmúth geantert! Eeine untreue blieb niẹt lange verborgen, uno baher jagte ibn fein sperr fort.

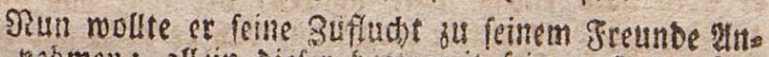
ton nebmen; allein biefer batte mit feinem ssetbe tibel gervirtbidaftet, uno nucb mieder in Die Rotterie geferste Dhne zu gerwinnen. Daburd war of in Edulden ges tatben, uno mujte lanofithtig reerben.

Es blitb alfo tem unglictichen Etephan nidtes úbrig, als chenfallह auß tern fanbe zu gehen, meil nies mano ifil als einen SBetruger ooer Dieb in feine Dienfte neslymen moute. Er fratb in grofer Dirftigfeit.

25et auf antere 2irt, aib burd) Atrbeit, Belb ete netben will, wiro am Ende immer unglúcfliti.

\section{24b rglaube.}

(B) fi a o wat to leichtgláubia, Dof er allez fúr mabe annabm, waź er bórte, ofine zu unterfucten, ob es auch mafie fenn fơnne. Diefe Eeid)tgiánkigfeit batte ibn aud zum 2berglauben gebracht; Denn wenn itm jemano fagre: in biefem ober jekem saule ipafe ein

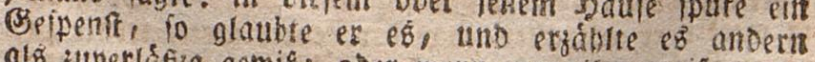

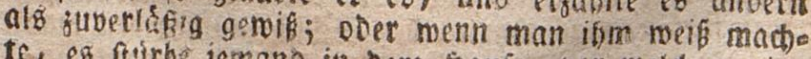
ts, eq fitirbe jemano in dem staufe, vor weldo m eine

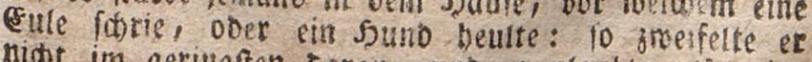
nid itn getiugften baren, who er glaubse allo eine 


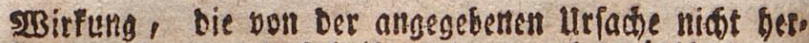
fommen fonnte, oas beist: or mar abergláubig.

Einfmal hefam er einen હd)abell, auß beiter Şaut, wie man zu fagen pilegt. 22nffatt Daß er nui eimen

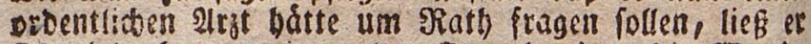
fith vieimefir von einer alten fran bereben, Die 23 mide mit cinem fogenannten Johannistofjiden (

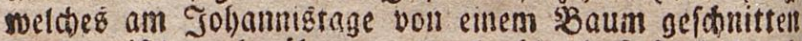

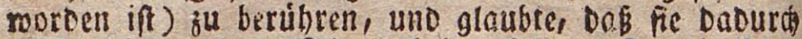
altein, Dime ansere Mittel, heilen follte. Da Die grall

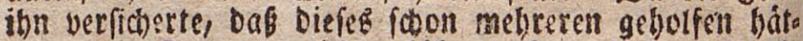
se, welde fie namentlich anfuibrte: fo vertieß er fich fo feft Darauf, $D a \beta$ er an feitre orbentlidje Rur basbte.

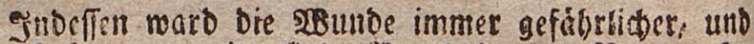

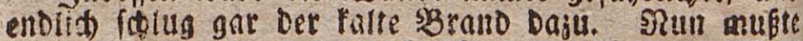

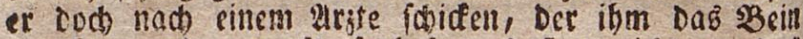

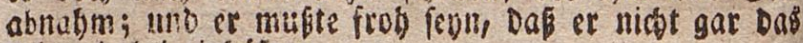
Seber babei cinbǘšte.

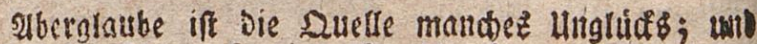

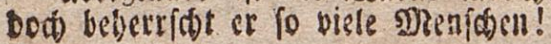

\section{0: Die Solgen Des flaífes uno Det Saulbeit.}

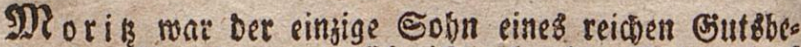
fibers. Mhit ith rar Ebriftoph, Der eofn eines

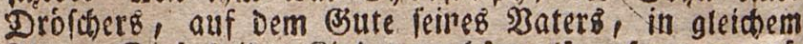
2itter. Diefe beiben Rinber wudien alfo zufammen auf, und (Ehrifoph murbe von Dem alten moriz fo berflich

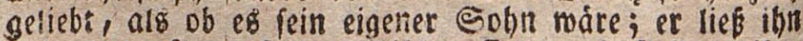
niobst mur oft an feinem zifóse effen; und fleidete ibm

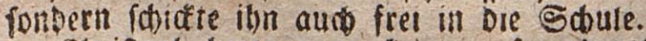

Ebrifopl hatte groar teine aukerorbentliche gádhigs Peiten, uno es roard ihm baber alles fehr (a)wer, was er lettuen follte; aber er gab fich viele Mnúbe. Sorgfák tig mettite er auf allež, wab́ ber lehrer lagte, letnte ju tzaule fleisig, roas $16 \mathrm{~m}$ in oer Eकule aufgegeben mar

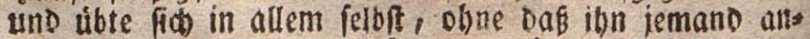
treiben Durfre. Durch Diefen unermúbeten \&ifer bradite er es balD Dabin, Dak er fernen Miti 


\section{3ur Deforberung guter Gefinnungen 26. OS $_{5}$}

auch Denett, melche beffere Ceiftergaben von Gott eme pfangen batten, als er. Gebermann liebte ith, unb

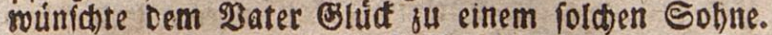

Morik aber war leidifinmig, und actrete nicbr auf Die guten fehren, Die er in Dar Edule birte. Epieo len, Sieiten, Sirichen, und Dergleitien Bergnigungest; maren ifm lieber, als fernen. Wenn er ermahnt wutDe, fleifig fu fenn, fo fagte er: id merke ein fanbo wirtb, und oer braudit nidit viel gu miffen; ment ios lefen, fobrriben uno rednen fann, fo bin iक gefolde genug, uno baju babe iot nod) immer 3eit.

So gieng ein Jabr nat Dem antern bin, unb reil er glaubte, immer nod zeit genug zu baben, io lernte er aud Das fefen, Sdbreiben uno গechnen nut febre mittelmáfig. Der Bater bárte eb freilia lieber gefem bell, ment fein Sobnt fleipiger getwefen máre; aber aroingen wollte or ibn nid)t, uno úberbies Dachte er ebenfallz, Daf́ fein Sobn in feinem tunftigen Stande

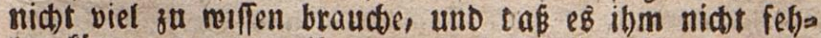
Jen fonne, menn er ibm nar Das (sutb mobl eingerido

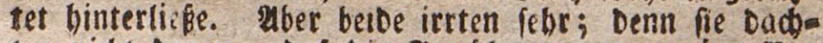

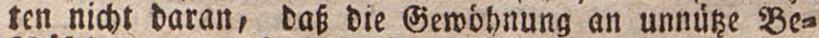
fideftigungen now meit follimmere Folgen babe, als

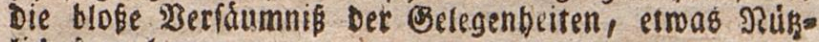
liđoế zน lernen.

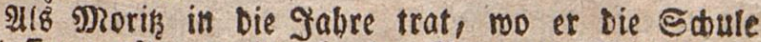
werlaffen muste, molite ifon ber Bater zur פrsirtbichaft anfúbren, uno trua ibm alfo balo biefe, balo jene (ares fodifte auf: aber פRorib gieng lieber feinen gemobnten Suftbarfeifen naw. Infratt auf Dem Gelbe zu (en), uno Die finedite zur grbeit anzutreiben, ritt er in Die Stad zu feinen Befannter, fpielte, unb ließ Die sined. te arbeiten, fo viel fie moliten.

Der Bater fobalt ibn zroar besimegen hart, aber half nidtz, umb er ftatb, wie man fagt, oor zetorus

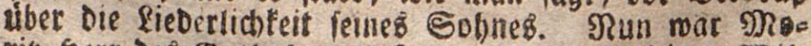
rish Sert Deb Gutbes, unb fornte gan nad feinem 2Bits

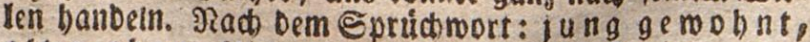
alt gethan, blieb et aud eben fo leidrifinnig, mie er sorber nat. Er lebte immer in Den ₹ag hinein, obne

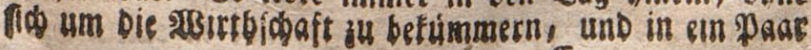


Gafren mar bas cut fo veríbulbet, baß es offentlict Derfauft merben mupte.

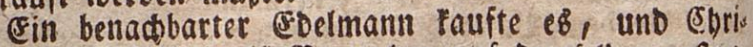

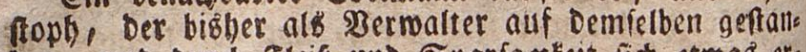
Den, und Durch fleiß und Sparfamteit fid) etwab ets norben batte, natym es in 叉acot.

DaB Eeto von Dem verfauffer sutbe reidte nidt? einmal zu, - Morizens Sabulden zu bezahlen, uno difo bátte er ein Eandiáfer merden múfent, mern fid Ebrifroph nidst, auts Dantbarteit und ysitteiden, feiner ans genommen, une ithm freie 20 obnung und freien Eili gegeben bátte.

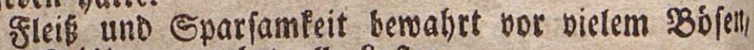
aber Drisiggang lebrt alle faffer.

\section{1. शR̃íchersi.}

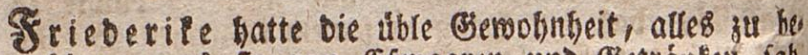
nafden, mas fie von Esmaaren uno Estrántent fab. Sie mar bebhalb oft von itren esttern beftraft morben!

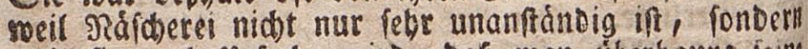
weil fie auch Ulrfache mirb, baf́ man uberbaupt leine \$Begierben nid)t máBigen uno unterbrúdén lernt.

Frieberife liés fin ourd feine Strafe abbaltem, ment ibr Die \&uft antam, zu nafdern. Die \&artentbuir mufitt um ifhrentwillen befándig veríchioffen fenn, fo langl

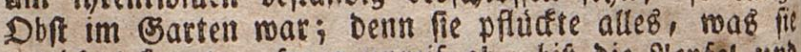
erreident fornte, fogar unteif $a b$, bis die 2lepfel unt sBimen an, uno warf fle roeg, menn fie nod) bart wae ren. Go verbarb fie faft ebent fo viel Dbft, wenn fir einmal in Detr Sartent fam, mie Dab Lungeglefer.

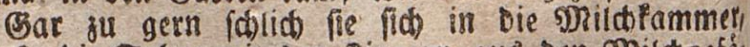
mo fie bie Sabne mit Den Bringern aus ben פRildseffá Gen nahm. 2Infangb glaubte man, Daß Die Rake bieft Ráiderinn todre, unto fobaftle fie ab; aber balo ento

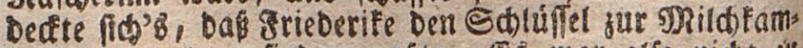
mer febr gut zu finben mufte. Es war alfo nidit zut werroundern, oaß die eftern gar fein 3utrauen mehr jill ibr batten, unb alleb vor ibe verfobloffen, wie vor eio nem Diebe. Einigemal war fie logar tiber Den syeein getaften, meløen Der Nater für sreunde in cinem ef? 


\section{Jut Beforberung guter Cefinnumgen 26.67}

fotranfe fteben batte, und war Davon beraufost und tootlich frant gemorden.

Eines Eages mar fie in Der Stube allein, uno pole de zeiten pflegte fie gern zu ibren গRáfchereien zu beruh: zen. Sie fabe fich um, ob irgent ein Eabrame offers fránbe, oder ob Saluifer ba wáren; endich bemertte

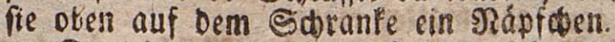

Sogleid madte fie 2unftalt zu pehen, ob etmas fúr fie zu naíten Darin ware Sie forkte eimen Stulft ar ben Ecorant, uno da biefer now nidt hods genug mars rtidte fie aud Den Eish bitan, ftieg ypm Etuble auf

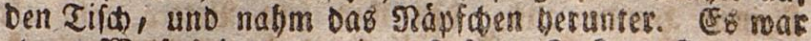
etroar 2setikes Darin, mie geftopener zuder, fie tunfte

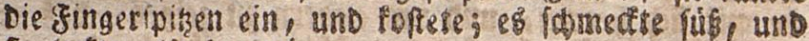
fie ledte alio begierig.

Dlóblic trat Die mutter zur shur bineis. griebes

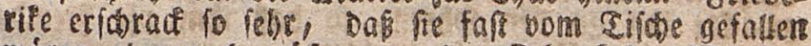
wáre; aber now gróber war ber Schrect Der Fiutter,

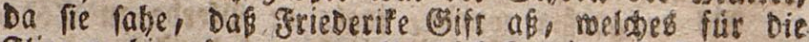
gliegen bingefent mar. Ungitudefino! rief fie, tras

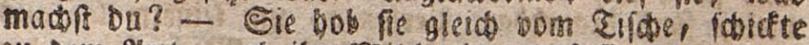

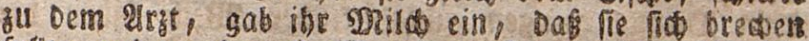
follie, uno randte alle mistel ane fic yon einem (domát)

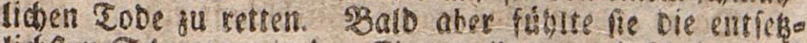
liaffer Somerzen in Den Eingeweiden, unb fotrie, baf man $e \mathcal{E}$ emige fodufer meit horen tonnte.

Der 2ras fom, und berotonete, Dofif fie immer now

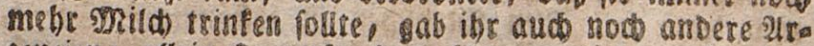
senteien; allein fie mugre bods fobon zu yiel genafost has ben; zroar blieb fie am feben, bebielt aber Dow entren

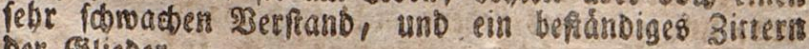
Det slieber.

2Ber feinen sBegietben unberminftig folgt, ben fule pen fie endici ing \$erberben.

\section{Der Shiercuåler.}

Der treime hortmann fant ein sergmian barant?

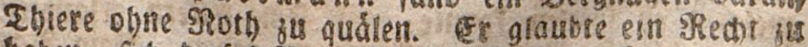
haben, fich brefes s3ergntigen zus madoen, fo oft er oie Eriegenipeit und Bewalt Dasu Gatte. Dhne zu bederta 


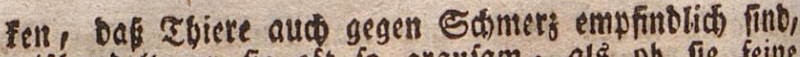
mifbandelte er fie oft to graulam, als ob fie feine

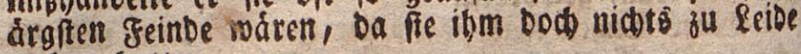
getbrin batten.

Er fieng Mraifaffer, banb fie mit einem gaben an einen Stod, und ließ̉ fie fo um Denfelben berumfliee gen, bis fie gans abgemattet water.

Die unichuldigen, unb in mandwer 2tbiatht núkliden

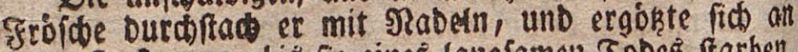

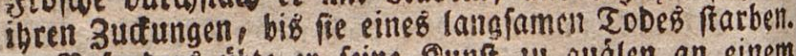

sefónderళ tubte er feine fiunft fu quálen an einem Fleinent Sounbe aus, ben ibm fein sBetter gefdenlt hatte. Den ganjen Eag fúbute er ibn all cinem Strils te mit fiob herum, uno um anbern zus zeigen, baj of

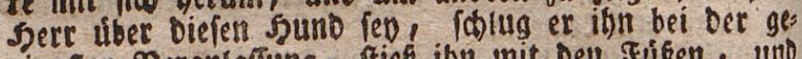

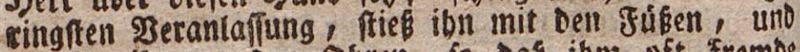
swadte ibn an ben Shren, to Das ithm oft frembe Lette Daruber 530 rowirfe maditen.

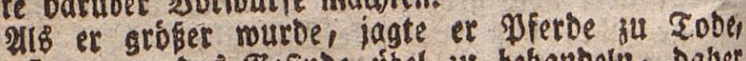
sino fieng an, Das Brefinde úbel zu behanbelin, Dabe: siele, fonft braudbare Derionen, um feinetwilten aub

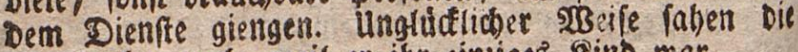
(Eltern thm nach, weil er ibr simbigeb Sino war.

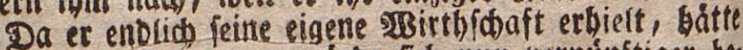
man meinen follen, er wurbe fich nun berninffiger be Iragen; allein er fegetre - nach Dem Sprúdwmort: jung gemobnt, alt getban - leine vorige 2luffubrung forth und lebte mait allen menfaten in befánbigem Streili

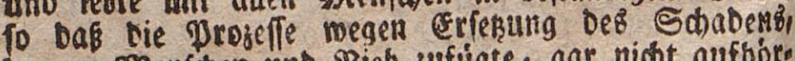

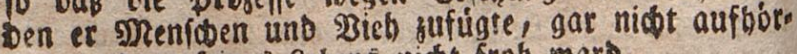
ten, und ex feines febens niobt froh warb.

Der eserecte erbarmet fid feines

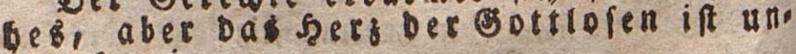
barmbersig.

\section{3eitbertreib.}

Seinrid flagte immer, Dak ithm bie 3eit fo lans

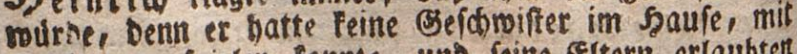
spelden et fpielen fonnte, und feine Eltern erlaubtel ibm niфt off, aub Dem Şaufe ju geben. Sein \$a sat sab ibm Dens satb, es foltte barauf fimmen, bes 
zur seforberung guter Oefinnungett $2 c_{0} 6 \mathrm{~g}$

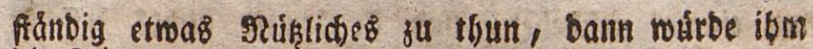
bie Beit nicht mejor lang merber.

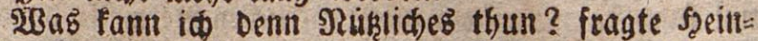
rid. Du fannft im Şaufe Deinen Eltern gur Şand ge: ben, uns ithten burch Deine Deenffertigfeit manche gnibe eriparen; ou famnft in ber Stube aufráumen, mand)e Beffellung maden, Deiner DJutter Sgot3, SBafler, und andere Frothwendigfeiten Gerbeiholen,

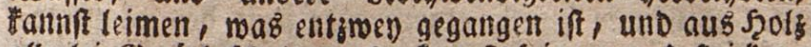

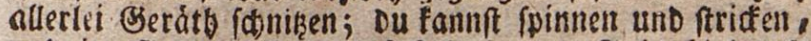
und im Barten giebt es faft Das ganje Jabe bindurd

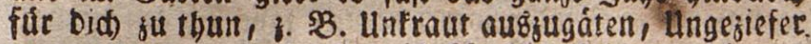

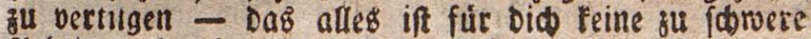
Ifrbeit und ein biel befferet Zeitsertreib, als immer fpielen, Denn Davon bat man teinen शuusen.

feintid) folgte Diefern Ratbe, uno befand fid redt nobil Dabey.

\section{Unvorfictigteit.}

Sgentiette wurbe bon allen, bie fie fannten, bie un worfidtige şentiette genannt. Bereidbte ibe biefer शrame zur Elbre? $2 B$ enn ibr Das folgenbe gelefen babt, to möget ine ferbof beurtbeilen, ob fie biefen Stamen sectiente.

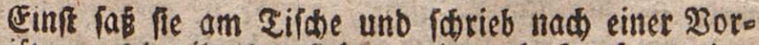
fdrift, welde ibr Der \&ebjer mit nad Saufe gegebent baste. 2uf einmal tórte fie eine Sutide tommen, mel=

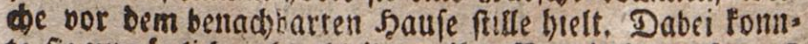
se fie unnóglid rubis bleiben, ibre Neugierbe muste erfit befriebigt feyn. Edonell fprang fie auf, uno in ber en warf fie bas Fintefób uno den Etuhl um. Die Sinte lief úber ben ganzen zifo binweg auf Den 230 Den. $2 s i e$ exfobrad foentiette, als fie fah, toab fie mit ibrer llit= sorlidotigfeir angeriobstet batte! roas follte fie mun thmut,

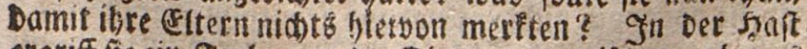

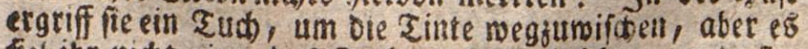

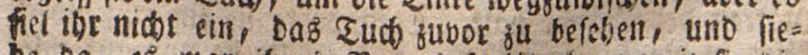

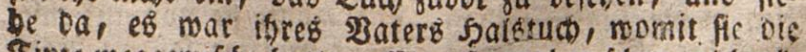

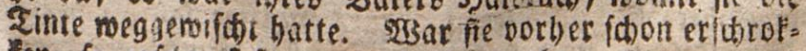

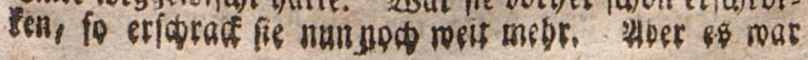


sun eimmal geidotiett, unb fie fonnte nid)tb beflers

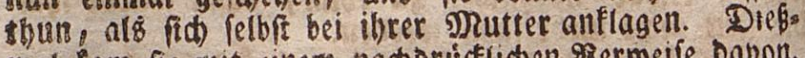
mal fam fie mit einem nactorúdtiden 3 serweife bavon. Sie nabin fid bor, funftig bebutiam fu fenn; aber foron am folgenten sage begieng fie ene abnlicte llnwor:

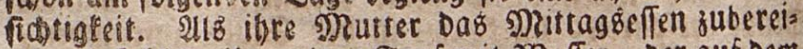
tete, befabl fie ibr, einen Sopf mit 23 affer, ter auf Dem Disn Ranb, aus? gett. Sgenriette giens ergriff aber ftatt DeB $23 a f f e r t D=$

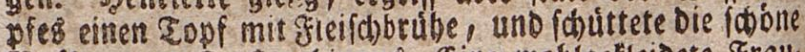

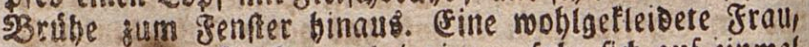
bie unter bem fenfter borbei gieng, fabe fic auf einmal lber und liber mit 58 rube begoffen. Thr ganjes Sileid soar verborben. Sie fam zu Senrietteng פRutrer, bes fdroette fids fehr uno verlangte, Daß fie ibr Dab Rleid bezablen folte. Diebmal blieb es nidbt bei einem nad):

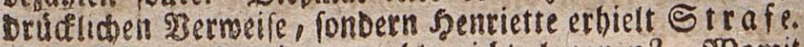

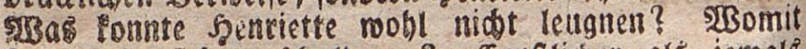

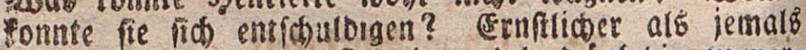

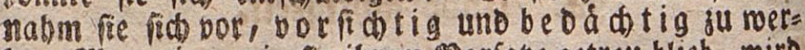
ben. अlber mie menig fie ibrem sorfase getreu blieb, wiro bie frolge fisizer. - Es roar ungefábr adit Tage nad)=

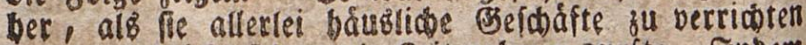
Gatte, mobei fie fio soenig Zeit nehmen Durfte. Snbem fie rafo aus Der Ríche in Die Etube geljen mill, be mertt fie Die syanne niot, roelcbe fie eben erft felbft

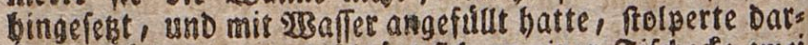

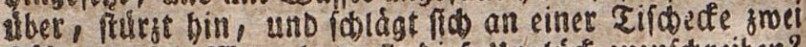

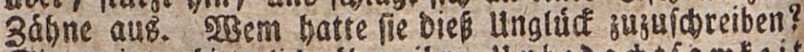
Eie meinte bitterlid uber iber II nbedactf amteit unb fonnte fit lange niobt gufrieben geben; Denn fie mat bura Den Berluft ibler sabme fegr entftelt. Sun bátte man Denten follen, Dab cin fo empfinotiches unglud fie

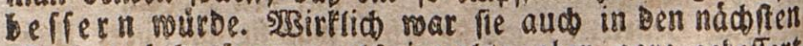
ISBoden behurfamer, als jemals; aber ganz gebeflett

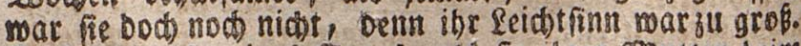
Dies zeighe fie eines $2 a g e s ?$, als fie ihrer gntutter beim

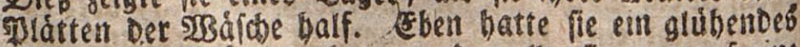
(Cifen in bie Dlátte getbon, unb mollte fie num auf eine

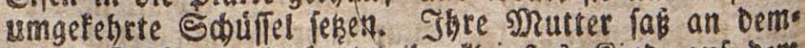
selben zirobe, und batte lor fleinftes Sind auf Dem 


\section{gur Seforberung guter Oefinnungen ze. ir}

Swooke. Dab Rino fpielte am silche, und hatte eben

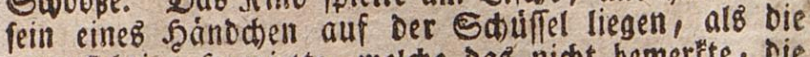

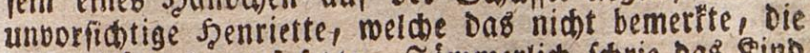

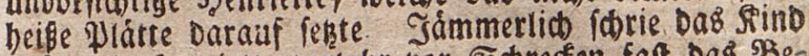

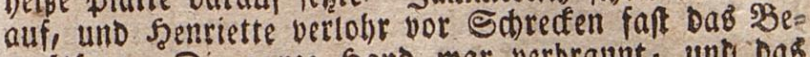
mustefenn. Die ganze Sand mar verbrannt, und bas arme Sino mufte Die beftigften Sdjmerzen ausftehen. fhenriefte meinte weniget úber Die \$ormürfe, uno bie

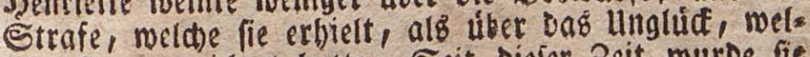
deb fie angeridstet hatte. Seit Diejer zeit murbe fie aufmerffamer aus fid felbft, und befonnener, und menn fie fich gleid Die unvorfictigfeit nid)t auf einmal gans abgenoblinen fonnte, fo legte fie bielen Feblet Dod ims

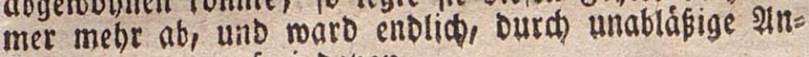
frengung, gans frei savon.

\section{Die silátfoberinn.}

Sophie máre ein rebt guteb̉ Mád den gemefen, wenn fie nue nidte einen groben Fehler an fiat gebabt

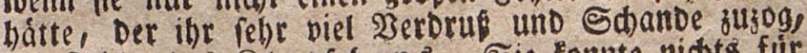

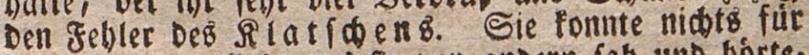
ficb bebalten; alles, was fie von andern fab und borte, ober erfubr, mufte fie mieder etzadblen; eB war ihe

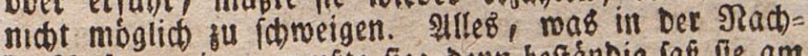
baríchaft vorgieng, mußjte fie; Denn beftándig faß̉ fie am

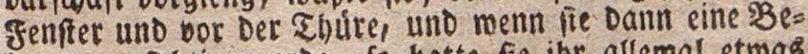
fannte anfictitig murbe, fo hatte fie ibr allemal etwas

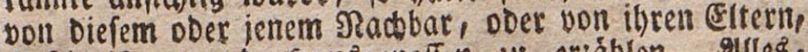

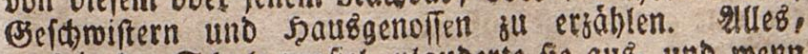
wab in ber Sd)ule vorfiel, plauberte fie aub, uno menn ein Rind Strafe erbalten batte, fo bredte fie es bald in bet balben stadt herum; Der:? jebem, Det itor be

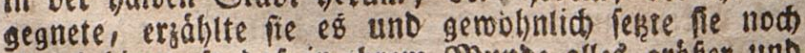
etroab binz̧, fo Daß in threm munbe alleb grófer unb fólimmer murbe, alB $e^{B}$ mirftich war. Durch biefe hápliche Neigung zum Silatichen fog fie fíd bei ibren

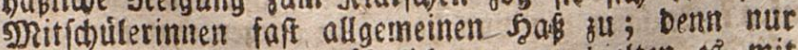
Diejenigen, welde ibe ábntid warer, bielten es mit ibr, alle úbrigen veradteten fie. $D a b$ that ihe freilid meb, aber fie war bod nidt Darauf bebacts, fid Die Plaucerbaftigleit abzugemóbnen. 


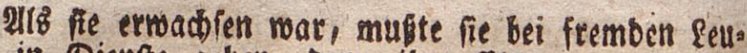

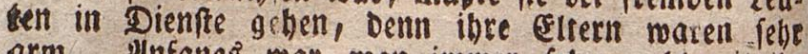
arm. 2unfangs roar man immer febr wohl mit ibe zufrieben, benn fie war reintid, orbentliob und millig;

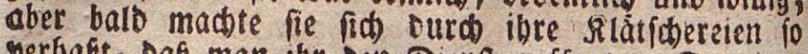
berhaft, Daß man ither bell Dienft auffagte. Es gieng ef bei jeber Szertidaft, unb endita mar fie in fo tibeli Suf gefommen, baß fie gar feine serríbaft mehr fint Den fonnte. Sie muste alfo ibre saterftad verlafferty suno ba fie es aud an fremsen Drien nicht beffer ma te, to batte fie tiberall baff:tbe Sthiffal, und fam ju

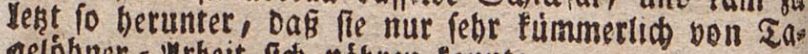
gelobner = atbeit fia nábren tonnte.

\section{Cin guter Dentfprud) iff ein greunt in Der Noth.}

(5ines zages, ba biele Sinber in ber Sdule zu smild. Geim ben aufgegebenen Denfiprud nidt ordentlicos auğ menbig musten, ergatblte Der febrer folgende lefrreide

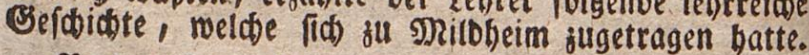

Balentin, ein unger Bauer, ber gute Sobn eis nes bófen $\mathfrak{B a t e r}^{3}$, batte now bei lebreiten Deffelbar ben áuBerff veríbuldeten uno vernachlásigten 2leferhof

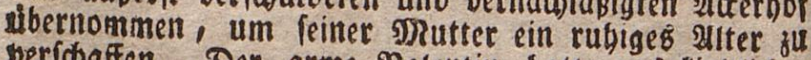
veríchaffen. Der arme Balentin batte aub findlider siebe eime grobe faft auf fit gelaben. Mit Rummer erroabte er am Norgen, mit Sorgen legte er fid शbenos zur SRube. Er batte nidet einmal fo viel Belo, um Rorn zur 2uвjaat ju taufen, ober Die sgeftellung feine 24 afers zu bezablen. 3war batte ein Nadbar aub NRitleiben fid erboten, ihm einen Ebeil feimes 2lfers bis zur Befăung zu befferlen; aber wo foulte Der

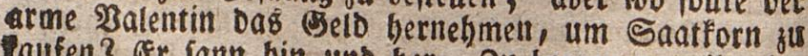
coufen? Er fann bin uns ber. 3u borgen war ibm bes Denflid, Denn wobon follte er wieber bezablen, oa oie Schuldentaft foboth fo grok mar? Bielleidst Dadtre er endiad, findeft ou शुorrath bei einem Samfer. (Ex fucte, und fand gludétid Die sorratbgtammer eines 


\section{gut Beffoberung guter Cefinnungen $2 c_{0} \quad 73$}

Şamftereี, und in berfelben fo biel 23 Baizen, wie er be Durfte. SRod warell bie Rórner unverfebrt, und fum Reimen gelchidt. 23on einer fotweren Sorge war nun Doch ber arme biftumerte Balentin freo. Freubig bera fundigte er feinen futio bem Nachbar, Der fogleicó bereit war, ibm bie Saat unter 3 ueggen. Jekt begab et fid auf feinen 2ffer, um Die Saat augzuffretten. Er that es untę Shráne!!, beln mie traurig mat nod

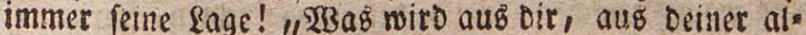
ten Mntter, Demen stribern und Ectreftern werden, Dache er bei fid felbft, renn bie Saat nicht gedeihen foutte? Biclleicht máre co beffer, ou dienteft bei gutent

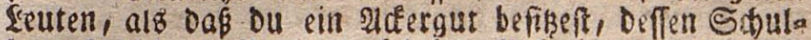

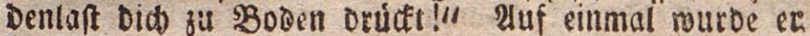
Deiter, und fafte Muth, Denn ibm fiel ein trofftidoer Denffyrud ein, ben er in Den Sinabeniabren gelernt batte. Diefer Eprad bießs: "Die mit Ê i) ránen fáen, werben mit greuden erndten, " oder mit

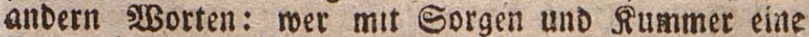
unternebmung anfángt, wiro greubenthránen meinen, menn fie gelingt. SBalentin fúblte fí getrofifet uno geftátit', inbern et bađite: aud meine Summer Thrás

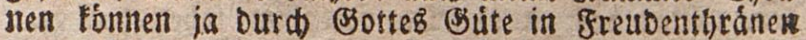
vermandelt merben, menn bic ernbte fommt: ith mill.

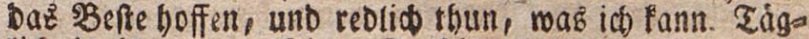
lid badite er an feinen Troftipruct, unb nun murbe

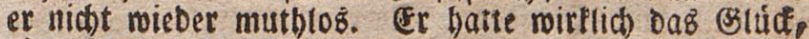
eine febr reiche Ernote fu madten, uno balb balf ee fid wieber fo meit, $\mathrm{Dak}$ er ein Yfere anid)affen fonnte. Damit bearbeitete er ben fleinen 2dfer, welder nod unberichuldet war, unb im 2Binter that er Damit gub)= ren fúr lobn. Dab eine Dferb bract)te ibm fo viel ein, Dafs et balo ein zmeiteB, uno endiid nod eit Drittes anidaffen, eine Schuld nach Der andern be= zahlen, und fich nac פserlauf einiger Jabre gan bon Stwuben fren maden fonnte. NRo शalentin in einem boben 2ltter, und im 2Boblftande, unt tile fpriat er bon feinem ebemaligen traurigent Edaidfal, obne bimzujufugen: "Do ic mit zbranen faen, merden mit grenden ernoten." 


\section{Derfürung.}

Stephan, ber Sohn eineb Tagelohnerbß, war fo ges

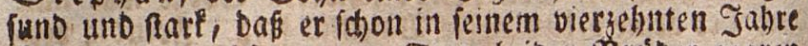
bóllig ansgerwadien war. Sente beioen soriber watel

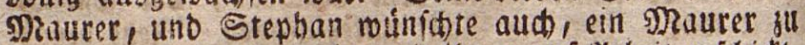
merben. Er murbe oaber mit ifhen auf 21 rbeit gefdict. fhier war er num faft unter lauter fimnlofen una bers

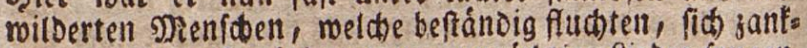
tell, uno menn fie einig maren, ungúbtige fieber fangen. Dabei uranfen fie beftánbig 3 tanntemein. Sebr bald forberten fie den jungen steplyan auf, mit innelt zu trin= fen. Diefer meigerte fich anfang8, weil er faton ein= mal cinent Edilud sBrantelecin getrunfen batte, und Davon ganz betáubt roorbent war. Aber nun fpotsetelt Die Befellen feiner, und einer fagte zu ibm: Tunge,

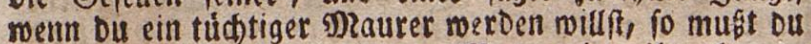
Branntemein trinten lernen. $23 \mathrm{ar}$ meinet ibs, hattent fie Fiedt? ?) Durd Daḱ, viele Sureben murde Stepfan endidi Dabin gebradbt, Daß́ er Dent sBranntervein ver= fubte; et (ibmedte ibm nidt tibel, und es Dauerte nicht

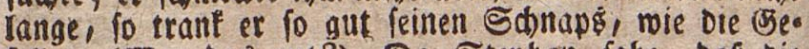

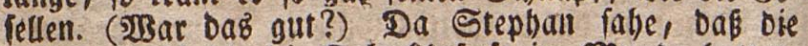
Esefellen beftánoig bie Tabackępfelfe im Munde batten,

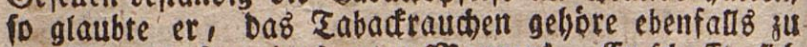

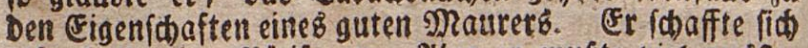
alfo balo eire pfeife an. 2lber er muste viel ausfese ben, ehe et ez babin bracte, mit Fertigleit fu rauchen. Dft wurte itm fo ubel unb weh, dab er Den Eabide

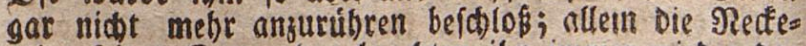
reien feitrer Rameraben bradten ibn immer mieber ba:

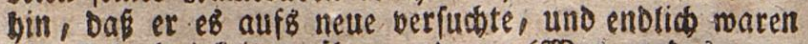
Die Sdmierigfeiten tibermunden. (Fुßat er Degrmegent gluteflid zu preifen?) Nan bielt fid Stephan im (ernfte fúr einen ganzen Nann, meil et alleśs mitmachen fonnte, mab bie andern madten. - 2lber nach einiger zeit fibien er niकbt mebr redit gefund zu fer)n. - Die fri= the rothe Befidetsfarbe, weld)e ex fonft gebabt batte, vertobr fíd ; er war blás uno mager, war immer trás ge und verdrofen, und batte feine fuft zum efiten, ia er fonnte fogar mande Speifen nidt melfr beroauen, die 
sur Befordertung guter Befinnungers 2c. 75

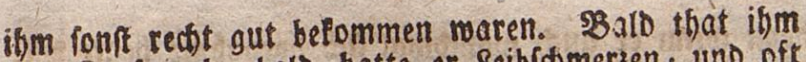
Det Fopf wel), bald batte et Eeibidimersen, und oft

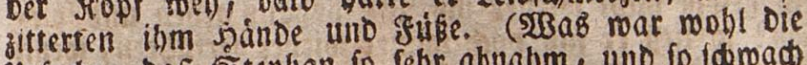

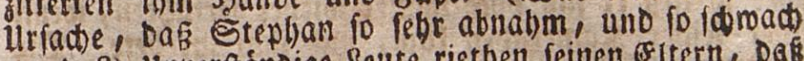
mutbe?) Unverffánoige feute riethen feinen EItern, Daf fie ibm Dod) zumeilen ein menig Brantitemein geten

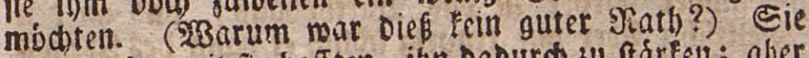
thaten $e \delta$, meil fie hofften, itgn Daburd su ftarfen; aber fie fondwatten itg nur nod mebr, und Stephan modbte nicbt geftelyen, waş für eine unorDentliche lebenzart er

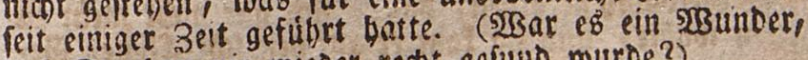
Das Etephan nie miecer recti -gefunb wurbe?)

Dab tor nod nict alles sBofe, wozul fich ber leidtfinnige Stept)an berfubren ließ. In eimem Sonn tage, alß er nict wufkte, momit er fich Die 3eit ber= treiben follte, fabe er cinige fiameraben in ein 2Bitthgs: haltz geten, wo Dpafif gemacht wurde. Da geht es luftig zu, Dadbte Stept)an, unb gieng bimein. Esinige reiner Rameraben fáken ba in einer niebrigen Etube,

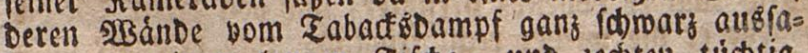
ben, an einem langen zifice, unt bedten tühtig. 23on ben vielen brennenden Tabacteppeifen mar die Etube to voll Dampf, Dak man nicht auf einen Ectritt weit um fid fehen fonnte. গadbdens man cine 2Beile bei eitander geferfent batte, that einer Den 200 fallag: ob man nid)t sarten (pielen mollte. 2Ille ma= ren $e B$ zufrieben, und Etephas murbe aud $\mathrm{Daz}_{\mathrm{z}}$ ein= geladen; aber er verfiand Das Epiel nidt. Dod bald fand fid einer, Der fid erbot, es ibm bul lebren, und ehe Der 2benb au Ende gieng, batte es Stenhan foton gelernt. 2/m nádiften Sonntage fand er fíd mieber ein, und nun follte or fobon um Belb fpielen. (Ex)

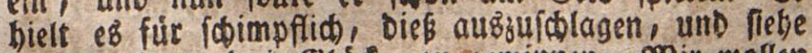
Da, er batte Daś Blifa, zul gewinnen. 2Bir mollen hóren, ob Dab ein fo grobés Slíá war. Stcphan bes Pam nun fehr viele Suft zum Spielen, aber et mat nid)t immer fo gludtlid, roie im 2unfange: oft berlobe er bie paar \&rojiden, meld)e er febr nótbig gebraudjte, um fid gribhfuide und 2lbenbbrod ju faufent, und Dant mufte er bungern. Das gefiet ibm freilid nicbt, aber Dennod formte er oon Dem Spielen nidot losfomment 
Denit watn et aud mandimal fich bornabim: beute mill

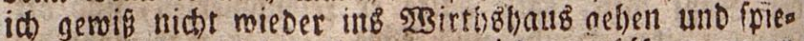
Ien! fo fieß er fid bod immer wieder verfúbren, meiti ciner feiner fomeraben fam, uno ibm zurebete. Dic Soffmung, Daś serlobrtie mieber zu gewinnen, rreb

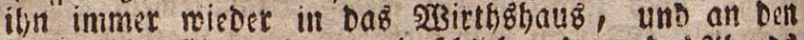

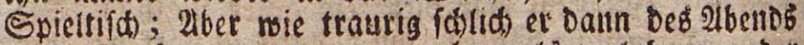
mach Scaufe, mens er nun abermalB̈ verlobren, obck ood nidis gerooniren batte! (Einft mar er saburch in fo grofe (Eelonoth geratben, bas er fiti) gar nicht mebr zh belfen wubte, uno ba fam or auf ben fátedtiatelr Bes Danfen, in einem saupe, mo et arbeitele, zu fteblen. Er nabm einen sRof, und einen filternen \&offel beg, nidt ofine grobe sungft und SBeflemmung. D, bátte et Dod lieber gefoungert, ober andere um eitre Babe ans gefprodien! 2al' ex ben foffel berfaufen wallte, warb er als verdeditig angebalten, fein Diebftabl fam ber

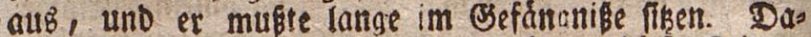
Durd fam er volfenos berunter, unb von biejer zeit an rourbe et nie wieber recht froblich, unb gelangte aud

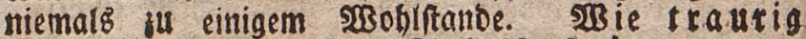
find bie golgen ber Spielfudt!

\section{Der undantbare Gduiler.}

Inton murbe yon peinet eltern zmar in bie Saule gebradit, aber nidjt bazu angebalten, Die edulule or: Dentlido zu befuden, Daber fam er oft su fpdt, uno mandie इage gar nidt in bie Sdule. Dann nach ibm fragte, fo biés es immer: 2unton babe fur leine Cltern meggeben múfen, oder et fen franf, Doet aud: er fonne beute nidbt fommen, meil er zu Saufe nothmenbig zu thun babe. Damit war ber \&eh)= ree freilich nicht fufriebet, benn wie war es̃ mobt móg. Iin, baf 2Aaton in Retintniffes meiter fam, wern er Die Sa)ule fo oft veriáumte? 2lber maz ben Rebrer bors

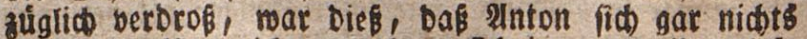
ous bem Intertidfte madte, fít immer treiben lief, und feinen ferneifer zeigte, befonbers nacboem ex ents lid to roeit gefommen mat, baf et ein wenig lefent unto foreiben fomnte; benn Diefer Sinabe war thoridot gis 


\section{zur Befơtberung guter Befinnungen $2 c_{0} \quad 77$}

nug, zu meinen, er thue nur bem Sebrer bamit einen Eefallen, menn er in Der Sobure feibig unb

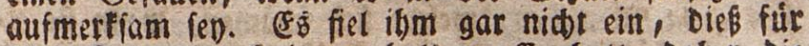

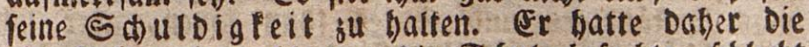
biet Jabre, in melden or Die Sd)ule befuthte, (d)ledit genug angewandt, unt wenig gelernt. Defto methe erftaunte Der \&ebrer, als 2hnton eines Tages in Die Sthule trat, uno inm anzeigte, bas er mun nidit melor in bie Sctule fommen mutroe. $23 i l l$ bid bein $23 a t e r$ in eine andere Saule bringen? fragte brr gebrer. sein, antroortete 2unton, ich foll nun gar nicht mebe

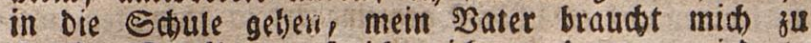
Şarfe. Daruber muß́ id mids munbern, erroiederte Der Sehrer, Denn ou gebft ia enft feit vier Jabren in Die Edule, und baft in biefer 3eit menigftens brei= mal in jeder 230 die gefehit, bift aud rie redbt fleis bis gemefen. - Mein Bater fagt, ich roúfte nun genisg, und er máre auch nur biz zum vierzelynter Sabre in Die Sd)ule gegangen, nun múfte er mid aufa Sjantomert thun, Danut itt) mir balo felbft mein \$3rob erroerben fónte. - 2ber meinft bu benn, fagte Der febter, Daģ Der Mneifter einen fehrling annebmen swird, Der weber fertiy lefen, nod fertig fodreiben uno rednen kann? Und mie millft ou fúnftig fertig me:Den, menn ou nun felbft Meifer gemorden bift, uno eine Fiedinung ficheriben, ober etwab aubreduten follet? - 2unton mukite bierauf weiter nichts zu ant= morten, als baß fein zater gefagt babe, er bátte aud

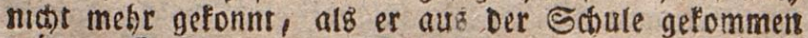
máre. Das mar nun freitic mahr, aber 2untons 2ater batte is auco Dafúr nie meit gebract) er lebre

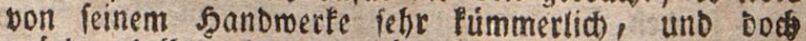
múrbe es ibn teidshlic ernágrt baben, wean er in Der Jugento mehr gelernt bátte. 2unton nabm alfo 216 . (h) Der, banfte aud feinem \&egrer nicht fúr ben Unters

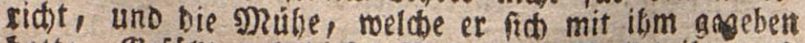
batte. Befált euch Diefes Betragen? 2 Bollet ibr aud

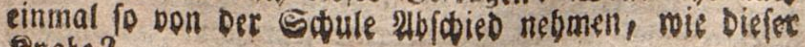
Sinabe? 


\section{Tralidue Edhaam.}

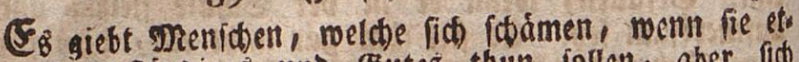
mas 2tnftandiges und Butes thun follen, aber fic

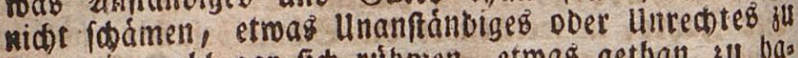
thun, la robl gar fid rubmen, etwas gethan zu bas ben, wab̉ unerlaubt unto fonánolich iff. Sold ein Menfos war pgillipp, Der Sogn eines senufmanues. er ichámte fich nidot, auf ber Strabe ungezoget unt mild zu fCDn, zu toben uno ful fármen, und fid mit feinen Befpiefen berum all balgen; ef fodsante fich nidti Diejenigen auf cine bodfift gemeine ent ju foimpfen, welo d)e ith alf irgeno eine 583 eife zu nabe tament; is et

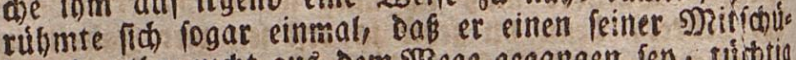
ler, Der ibm nidt auz Dem sBege gegangen fer, thimtig abgeprigelt, unb auf bie ercee semorfen babe. 2Baren

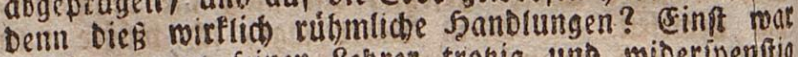
Pbilipp gegeit feinen lebrer trokig uno miberipenfitis gemefen. Gein sater erfubre $P B$, Denn er batte fid fers

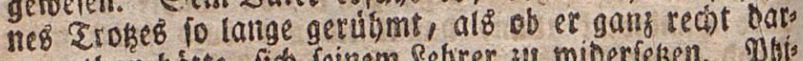
an gethan hadte, fiob leinem sebrer zu miberferen. Dhis

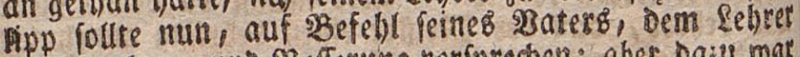
2abbifte thun, uno Befferung verfprecten: aber Daju rat

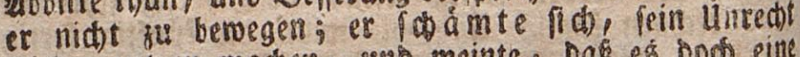
wieber gut zu machen, unb meinte, Daß́ eś boob eine gar zu grofe Echanbe fen, wenn es abbitten follte! et molite fid licber jeder andeen Strafe unterwerfon mein fie auch nod fo hart fers. Sisie gefált eud bies fes Betragen Dhilipps? Slaubet ibr mohl, Daß ef nad)abmungsmairbig fen? Philipp muste fich freilid julent gur 2ubbitte entrofliefers, aber et that es mit eis

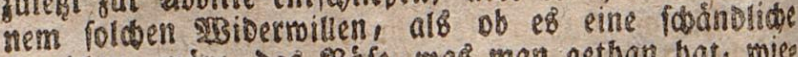
Szanblung wäre, das sBofe, was mant getban that, wies

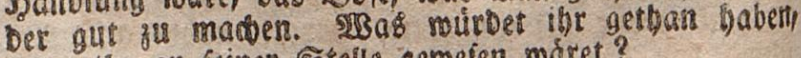
werni ibe an feiner Stelle genefen maret?

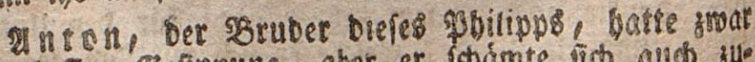
eirse beffere Cefimnung, aber or fodimte nict allob jue

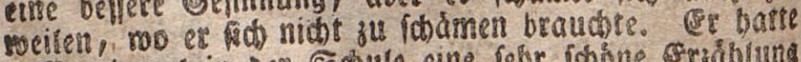

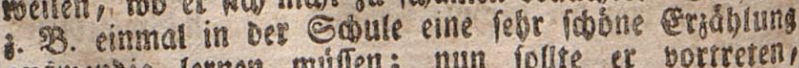
aašmentig letren múfen; nun follste ex bortreten!

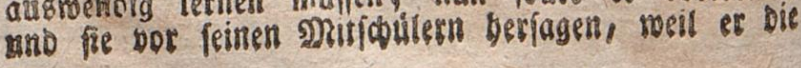




\section{jur Befordoerung guter Befinnungen 26.79}

(Befdictelid)feit batte, nidt mur febre beutlid, fonbern aud in Dem rediten Sone zu lefen. Plber er fóntmte fich, uno loollite anfangs Durdbaus nidbt bortreten, ob or gleid fonft feinem segrer febe gehoriam war. Erft nach vielem 3ureden, und als ber sehrer ihm ernftich Drobete, fagte er feine Erzáblung her, aber et that es mit nicbergefengtem fopfe, und iprad) Dabei fo teife und unbeutlid), Dak Der febrer unmogilits mit ibm fufried an fenn fomnte. Sibt, oa er feine Eache fo folredt gemedot batie, Durfte er lich nobl fodámen, aber zubor aud?

\section{Der unbefonnene Spaß́.}

2Renn gerbinanb Befpenfere Befdidten hatte eto zádblen boren, fo fonnte er oft bie ganje Nacht midit einfdhlafen, betm er roar unglaublich fur $\mathrm{d}$ t $f$ a $m$, und ob itgm gleid feine EItern und Sebrer oft genug gefagt

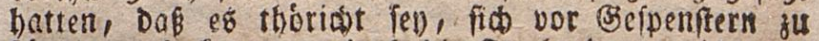
fúrdten, to fontte er bods Die gurdit Dabor nicht un=

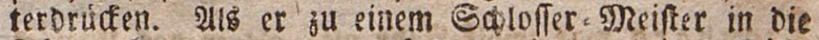
felice gefommen roar, muste er mit ben beicen éobo

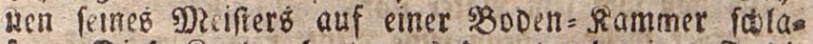
fell. Diefe Sinaben hatten eb Dem treuhersigen gerdi= nand bald angemerit, baß or fich bor Beipenfern fürdte, und befíloffen, fich eimnal mit $14 \mathrm{hm}$ einen Epá̈ zu maden. Der eine gab baber eines atbentos yor, Daß er fehe múbe róre, uno früh zu SBette gehen molite. Er batte aber mit feinem $B$ ruber verabrebet, Dak er fich unter gerdinands sgette legen, und menn Diefer im şette wáke, erft mit Retten reffeñ, bant plostich beroorfommen, und in ein reeifles seittuó grbilut an fein sette treten molle; Der Bruber follte Die Thitr ber Edtlaffammer verídief́én, Damit JerDinand nicht entmirchent fonne. $23 a s$ meint ibr ju Die

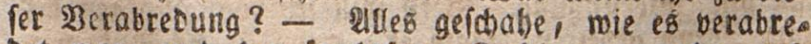
Det mar, unb ber furdtfarme gerbinano murbe auc mirflich burd baś Raffeln Der Sietten unter leinems

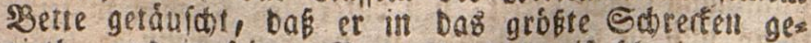

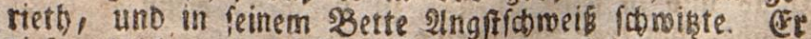
tief entich um Sxulfe, befam aber teine 2introort.

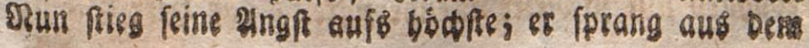




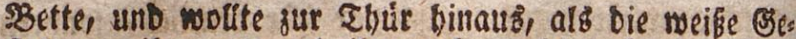

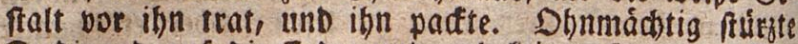
Ferbinano auf Die Erde, uno gab reinen laut von fi申. enolido merften die bofen suben, mas fie mit ifrem unbefonmetten Spabe angetidtet batten, unb wollten nun Den armen ferbinand aแ feinen Irth aher jetst war es su fpát. Geroinano lag leblos Da. 2ligftwoul ricfen fie ibre Eltern berbei, uno mit grö́ct g) Rúbe ward oer obumádstige fervinano wieder ins fes bell gebradt; aber er erbolte fid to bald nid)t mieber, Denn ein hiziges fieber war Die folge Der 2lngft, melde er aub̧geftanden batte. Sun bereueten die beiben Sinaben ibren Spaḱ, Dent fie batten fio tricht vorge frellt, Daß et to ubd ablaufen fonnte. Der \$ater ftrafte fie febr batt bafúr, und bemuibte fich, Jerbis nanden bon feiter thoridoten furdtiamteit nad uno nac su befreien.

\section{Clbelich wábrt am långften.}

Reonliard mar zmolf Gabe alt, alb er oaz linglué batte, Dafs ibm lein Bater farb Saus batre er fennen Berforger mebr, Denn feine Mutter mar fo Pránflid, Daß fie ibn unmóglich mit ibrer banbe glebeit ernahs reit founte. Reonbaro fapte Daber cen Enifolubi, felbft fein Unterfommen fu fuchen, um feiner sputter nicts zur faft zll fallen. Sann id ood fertig lefell, fchreiben und rechnen, Dabie er bei fich felbit; mie follte ia nicht ourd bie IRelt fommen, menn ito fleis

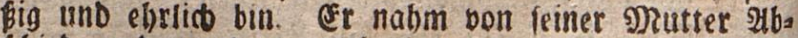
(chieb, uno manderte nach einer nabe gelegenen Stebt,

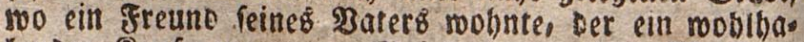
bender Raufmann war. Szei Diefem meloete fid Seon

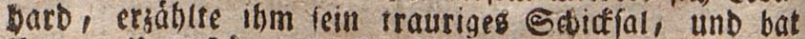
shn um llnterftúkung. (Sern mill iक vons Motgen biB zum 2 thenb arbelten, fagte er, menn fie fich nur meio

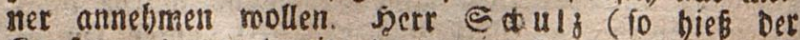
Saufmann) war bereit, Den vaterlofen stnaben in feit fous uno in feine Diente fu netymen, menn er bers foráde, thm treu uno chrlich fu bienen. Das veriprad

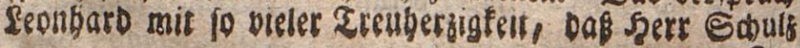


zut Beforbetung guter Oefinnungen 26.81

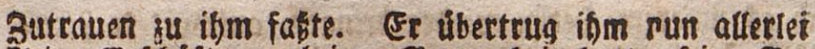
fleine Beimdfte, mobei et Belegenbeit batte, feine Bes Duld uno Sorgfalt fermen jul lernen, uno fand urrades mit ibm zufrieben zu feon. Befonders gefiel ifgm feine

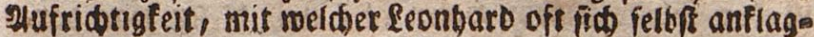
te, wenn er etruas niōt redt gemant, ober vergeffen hatte, unb bie Rernbegierbe, weldee er bei ieder Bselegen beit jeigte. SSald batte Der gute Rnabe fo febe bas 345

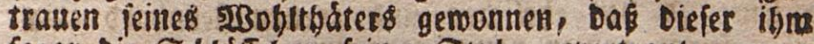
fogar bie Sdolúfel zu feiner Stube anvertraute, went

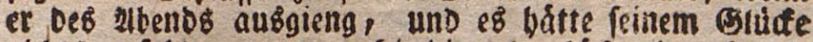
nidts gefeblt, menn nudt bie alte bóbartige şause

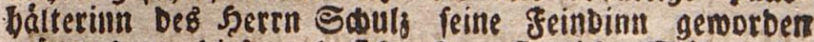
máre; Denn biefe gab fid alle etrinntide Múbe, ibn anjufctrodrien, und aus bem saufe zll bringen, meit fle an ibm einen láftigen Yuffeber batte, uno nun

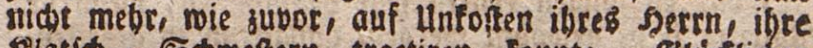

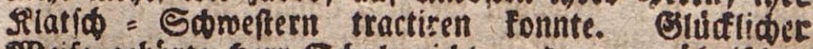

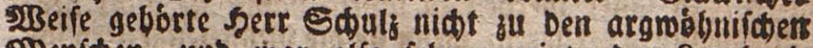
Mrenicten, unb toar alio jebr geneigt, Den Reonbarb fo lange fứr eines guten Rnaben zu balten, bis ex

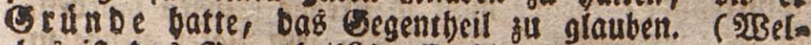
des ift Das Esegentbeil?) Er bieit Die sBefdurdoigungen ber alten Sgaugbaltterimn Daber für falid, beobad.

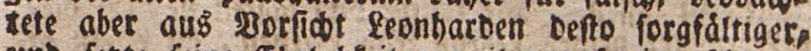
uno fente feine ceorlicteit jumeiten auf eine fommere Probe. Da er ibn abet nie auf einer lúge betraf, to traute er ibm aud feine setruigesel zu. Dft ichiffte er ibn aus, um etwas einufufaufen, unb gab ibm danks mebr Eelb mit, als er braudite, aber immer bradte Reonbaro bas Hebrige treulid wieber, unt nidet feftent batte er moblfeiler eingeffauft, als Seer Eduls gebactst

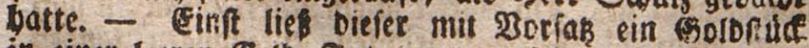
in einer leeren Eelb: Tats, um ju feben, ob Eeonbard soobl ebrtid genug lenn múrbe, eE nidt zu bebalien. Leonbaro fanb Das soldftúf, als getabe ein Diener

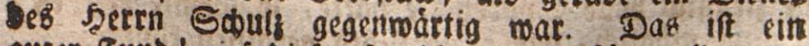
gurer Junb! vief biefer freubig atb, Dafur mollen wie unß̌ einen guten zag madben, lieber Reonbaro, benus

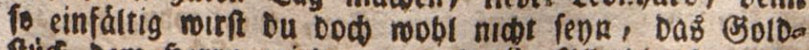
fluid Dem Sgertm nieber in geben? alterbings werbe 


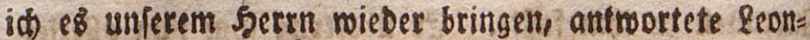
baro, benn it)m gebórt eb, uno nidht unb. Mit gutem Bemiffen fonnen mir es nicht belyalten, uno id mag mein gutes Bemiffen nidot verlieten. Er lieferte es auf Der Stelle feinem Şerrn ab, und biefer war bar=

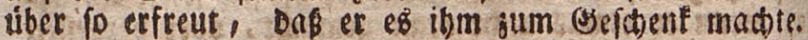
Eeit biefer zeit verior er niemalß̈ das Zutrauen fols

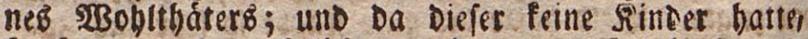
fo lekte et Den egrlicten uno treuen fconbaro jum Eeben feines ganzen शermógenb ein.

\section{Tugendidise Unbefonnenbeit.}

Serrmann batte einen redlidben, aber febe fitengent शater, Der ibn beftendig zur 2rbeit anbielt, und ibm nur felten ein 58 ergnúgen erlaubte, weil er ber Meinung

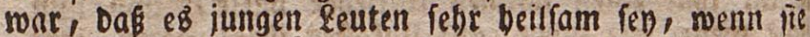
Frúbzeitig Daju gemobnt werden, ant)alteno ju arbeitell unib ef fich fauer merbent zu laffer, Damit fie nicht bernach Den Druth verfieren, menn fie bie Mrúfefeligfeiten itso

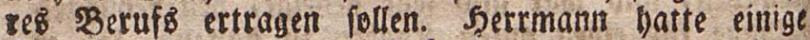
Sdulfreunbe, melche nidt to firenge crjogen nurben, uno Diefe fekten ihm in Den Ropf, fein sater gienge sil hart mit ibm um, und er babe nidt notbig, fid bab gefallen zu laffen. WBie fanue bus, fagten fie, bei bies fer febenzart Deines febeng froh metden, ou barfit ja

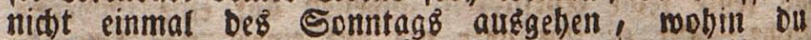
roillf! menn id mie ou máre, fagte einer unter ignem id) madie es, mie eb fajon viele gemadt baben, urto gienge in bie meite $23 e$ elt. Eo fat mand)er fein Bluff ges

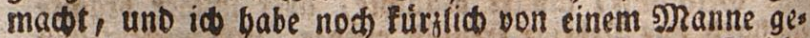

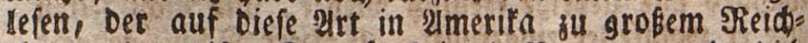
thum gelangt ift. Du máreft ia ein গRarr, menn bu bi lánger quélteff! Diefe thórichten 3uredungen fanbeh endlid bei Şerrnaann Eingang, und er gieng nun mirflich Damit um, feinen guten eltern zu entlaufen. Da of eine jiemliob volfe Sparbidife batte, fo meinte ot, of

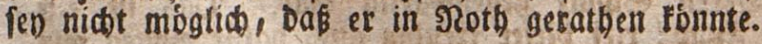

Eines sages matote er fich in aller gruble mit feis nem fleinen Sdake auf ben $23 e g$, niकt obne Serto flopfen; denn er fúblte bo bod, baf er fid an feinen 
gur Beffroerung guter Oefinnungen ic. 83

clteen febe verfunbigte. Inbef berubigte ex fid balo burd Die eitle SToffnung, Daß er fein Eltud maden, und Dann fege leidit Sergebung erbalten wurbe. Er wanderte cinige Eage nad cinander fort, uno eriditade.

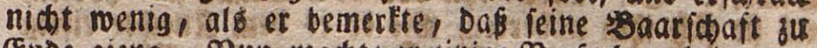
Ende gieng. Nun madte et einige ßerfudbe, bei guters Reuten untersufommen, allein uberall mies man ihn ab, theils weil er nidbt febr ordentlich ausfabe, theils meit er nod felde jung ano fobadablid war. Dennod ferte Serrmann feitre $203 a n b e r i c h a f t$ fort ; Denn ex fodante

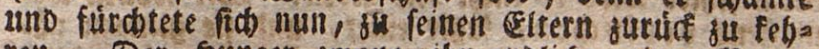
rell. Der sunger swang ibn endlid, einten sBatter zu bitten, Dak er ihn in leine Dienfe nebmen móbtes uno bet sauet war aud beteit daski; allein Serrmans folle nun allettei fobmere शlrbeiten thun, uno befam

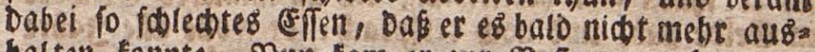
Walten founte. Seun fam er sur Befinnung, bereuete

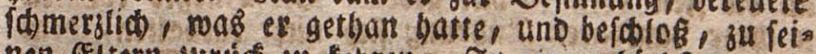

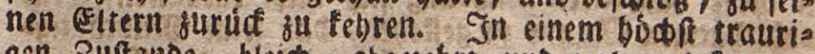
gen Suftarbe, bleich, abgegcber und berlumpt fam ex in feiner 23aterftaot mieder an, uno wartete Den 2lbend ab, um fid Dann in Dunteln nach Dem Salsfe feines Eltern binjuldicichen. Sein Bater ertannte ibn ante fangs nicht, und erlobract tiber den fléglitben 3uftano, in melderm et ibs vor fid fabe. Eebre ernfthaft aber Dod gutig empfieng et ithn! ferme פoutter weime sreute Dentbránen ibber igren verlorifen, uno nun mieber geo funbenent Soban. Reuevoll geftano er, Daß́ er nibs mebre merth fey, ihe Sobn ju betben, une demutbig unterwarf et fia bet betoienten Etrafe. Eie beftand

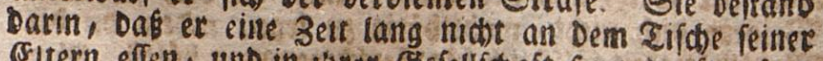

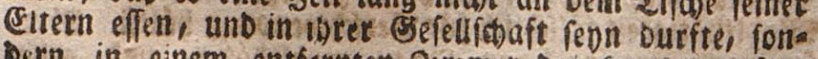
Dern in einem entfernten 3immer des sjaules emfam feine 3eit fubringen mubre. Setrmann wurbe yon biee fer beit an ein guter Eobir.

43. Unterfatied anifasen Eparfamteit und Cor:

In einet Hleinen Etabt murben von ber Dbrigleit

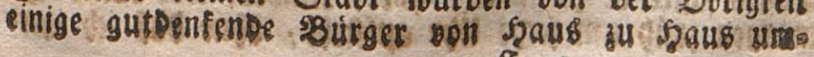
अ 2 


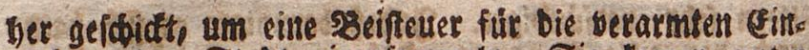
nobmer ber Etabt einzufammeln. Sie tamen unter anbern frutheorgens auf Den Sgof eines wohlbatenben 53auers. Sie fanben ibn vor bem Stalle unb biortent

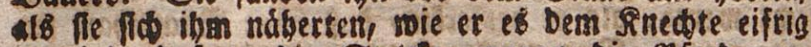
vermies, Daf er Die Errite, moran Die Pferbe ges ipanst gewejen waren, uber Rad)t im Nisgen an NBB= gen gelaffen, und nidst ins Erodene gebradt batte. "D meb! ber MRann if genau!" iprac ciner gum an=

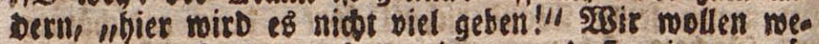
nigftens yerfuden, fagte einer, unt le giengen ud. ber. Der seere empfieng die gremoen febr freunblid und indef er mit ibners in fein saus gieng, bsadten fie

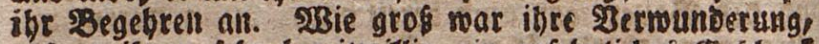
als er ibnen febr bereirmitlig sin anfebmitates Geldsent an Belbe gab, unb nod berfjrad): er molle alle gabre um Die zeit eben fo viel geben. Die SBurget tonnten in ibrer Danflbaren Filibrung fid niçt entbalten, Dem

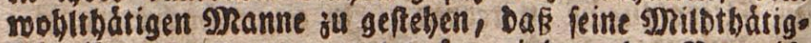
feit ibmen gans unerwartet fep, inbem Der Sertreis, Den er vorbin Dem Senedte wegen eiller fo unbebeutent Den Rleinigleit gegeben bátte, fie auf Den 2 rgmolgn ges bradt babe, Dais ef mobt fehr genau feon múfe.

"lieben frcunbe," mar feine 2nntroott, "eben bao "iburd, Daf id bas DReinige jeberieit ju SRatbe bielt,

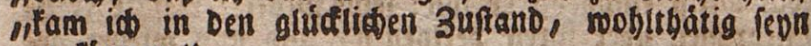
"13i tonnen."

Sødme Did nidbt Der Sparfamfeit, unb balte fie

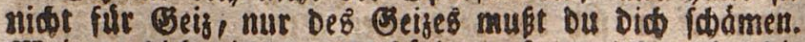

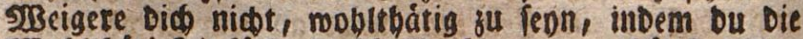

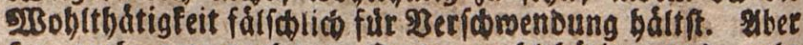

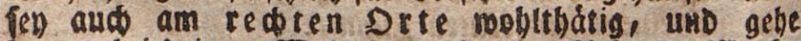

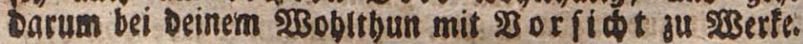

\section{Dex Sienenftoct.}

Bater 5 Biebermann batte vier Rinber; fie biefen: Rarl, Sernbarb, goite unb Szannden. Eines Zages fagte er zu thuen: bort, Sinber, wer yon cuc

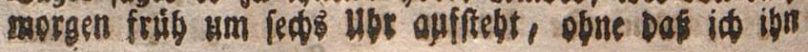


zux Beforberung gutet Cefinnungen ifo 85

wede, bem mill id eit redites geft madett. Die Sitto Der bordsten bod auf. 223 as benn fur ein geft, lieber Bater? fragle fotte. - Steb ou nur zu redoter Beit auf, obne baf ió bid mede, fo mirft bu erfabren, was CB fur ein feft ift, fagte Der \$ater. - $D$, id mill ge mis nod vor fects ubr aufíteben, obue bas bu mia medff, rief Lotte. - Sw aut! id aud! riefen alle.

Sest íflug Die Clode jebnt. SRun mar es 3eit, ju Bette zu gehen. Eie fagten alle bem $23 a t e r$ gute SRadt, und jeber Rinb fprad babei: Du follf? feben, פoter, baf id morgen frubs um feds ulgr aus bem ssette fev) mill. Nun legten fie fiof sa sette, unb jebes fagte fút fid), ebe es cinjolief: balb ledis ubr! balb redo ubr! SBernharb fótieb fo gar mit Sreibe uber fein sette: mor= gen um balb feds Lbr ftebt setnbarb auf! - Da fab man redt, Dás Der senio alles fant, mas er redot

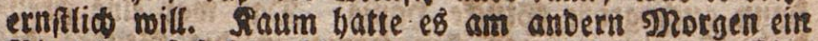
sBiettel auf ledsb gefolagen, fo maren fobon alle sin= Der munter. Jebeg ftano auf, jog fid an, und follid fid jur Rammer binaus, Denn jeDes glaubte Das etfe fu feph. Utber faft zus gleidjer 3eit famen fie alle in ber פBobnfube an. Eutren sporgen! riefen fie fretuoig eitus bem anbern ju. Nun, fagten fie, wollen mir bock feben, mab füt ein Jeft un' bet Safte maden mirb.

Sie giengen gum Nater. Ci! fpraá biefer, wehts Der \$ater verfpribt, Dett אimbern eit seft zu madeit, bann fonmen fie alle frub auffeben, Ners, it balte 23ort. 2tber erf tbut, Sinber, wab alle gute Sinber thun, fobalo fie aus Dem Bette Pommen. - Da fónms tent fie fit, mufoen fid bie Sámbe unb bas Befít,r, uno iputten ben פRuno mit frífem 23 afiet aแร. Sun famen fie wieber jum 3 ater, uno sannden fragte ungebuloig: madoft Du unB nun ein geft? - Da il' B! rief Der \$ater, unb warf jebem Pinbe cine Sappe úber

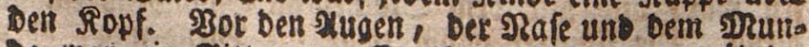
De war ein Eitter von Dratb, uno ber gamje úbrige Siopf war mit einem sude beocdt.

Merft ibr mas? fprad \$Bernbaro ju ben anbern Sinbern, Der Nater foneibet gemís Johity.

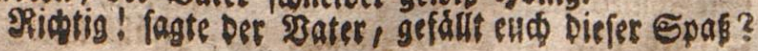


D ja! D ia! riefett alle yn folgten ben \$ater, bet Inu atio eine Rappe tiber Den Ropf nabm, und jebem Sinbe etwab zu tragen gab. SBetribaro trug eine Pfanme voll Soblen, die glúbeno waren. Sarl

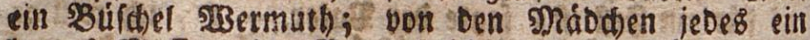
langeb פeffer; Der Sater felbft trug eine Bisblte, und bie g) Rutter folgte mit cinem Giebe uno cintem \$aat Sकuiffeln nach.

Jest fam bet gange Sug in Sarten an, uno nun gieng bas Jeft redt an. Der Bater madte baB Sau's auf, in bem bie sienen waren, uno trug jeden Stod Son feinem \$lahe neg: Dann nabm er ein sBúlcbel ISBermuth, bas et auf bie Pohlen gelegt hatte, und Jieß̉ Den SRaud Davon in Den Stod zieben. Da zo: gen fich Die SBienen zuntid, uno Der \$sater idnitt nun ent $2 B a d s$ heraus, weldies ar in Das Sieb legter Dann auch grope Stuide Jonig. Das mar eine Jrells De! Sun trug man Deth Sgonig in Die Stube; Die Simber forgten, uns Die glutter borte Semmeln, auf melche fie sonig fur bie Rinder freichen mollte. 2uch ber 5tater gieng fort, uno lagte: Sinber! nun madte id eud nod ein Jeft, id tafle fur sud Sponig

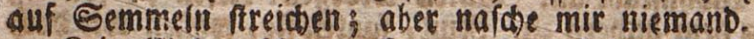

Rein Sind inathte auber - Samuden. Diefe mar

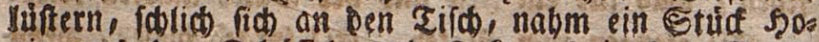
sig aus bet Sobuffel, uno ftefte $e B$ in ben s)?unt.

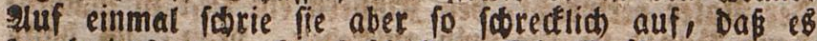
burch Das ganie Sgaus fotalte. Die Brtiber uno bie Ecomeftern traten ángfrich um fie, und fragten: was Pehlt Dir, fanmón? Bater uno gorutter liefen berbein suno fragter: mab feblt Dir? 2uber Szaunden biêt Den Druno auf uno fórie, ars wenn fie am Epiése ftácfe. Die Inutter fabe in ben snrunb, unb fiehe ba! ein soiell: den fás ibr auf Der sunge, meldbes im feonig gemes fen mate und mit Dem Stachel an sanndsens zunge bieng. Die Snutter nabm joar bie Bbiene veg, aber Die sunge fobrooll to ftarf auf, Dab Sanndien ben ganzen Sag Peinen SBiffen effen fonnte.

Die ubrigen Rinber asen ibre Semmeln mit Sonig. Sie famecten ignen feble gut, uno Rarl iprad): DaB̉ feff

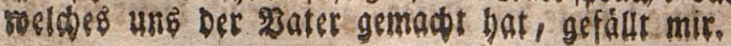




\section{jur Beforberung guter Gefinnungen 26. 87}

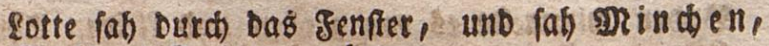
Des Racbars Fochter, gethen.

Dab arme Niniden! fprad fie; ibr \$ater bat Peine SBicnen, uno fann ibr Keinen sonig auf Semmetn ftrei=

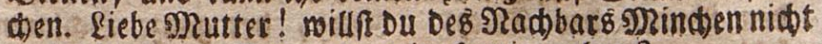
aud ein Daar Semmein mit Sonig geben? allect) gettr, mein Rind, iprach Die Drutter, gab ibr die Semmein mit şonig, und \&otte trug fie 34 MRind)en. 2Bas fúr eine greube Dab Mábden bafte! Wie fie fottchen bantte! Utno nun fomedfe lottwen i)e sonig nod einmal fo gut.

\section{Det gif cticich.}

Serr Sgerbft batte einen Zeid), in meldoem viele Siat= wfen und Sdjeien maten. SBenn er nim feimen Rin= Dern eine greude macten mollte, fo gieng er mit ibnen an

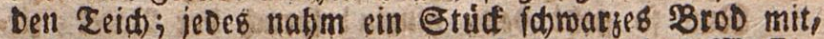

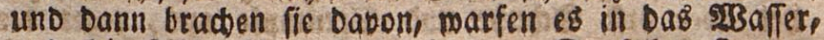
unt die fíche fónappten eह weg. Da fakent fie nunt oft cine Etunde lang, unb fahen wu, mie bie stíche aufs uno abjobmammen; Die Sáfer, Die im 2Baffer leben, bin uno ber fubren; bier und ba ein frofd ben Ropf aub Dem $23 a f f e r$ frecte, und - buf́ch! - mieder bithe unter war, went ibm ein Sind fu nabe fam.

7. Da múnicten bie Siniber nun oft: SBemn id nur einmal to ein Shier fangen, uno in ber शrábe fellen forinte! Sgerbft ließ es aber nie zu, Daß ein Rind Darnad) greffen burfte. $23 a r$ Diés mobl recht? Id glaube mohl. Ein gind ift fein Frofd und fein gild, bie im wBaffer leben. $23 e n n$ eins bon ibnen in Das Swaffer fiele, fo máre eहै auß mit ibm.

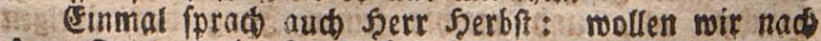
Dem Serdi)e gehen? Sa! ja! riefen alle und zogen froblia mit tbm fort. SBernbarb fprang boraub, uno fam zuerf bei Dem Zeide an. Saum war er Da, fo brebete er fid um, unb rief ben anbern Rints Dern ju: Rarı! Sanne! Lofte! Der \$ater madit einen Spaf! fommt gefdimino gerbei! Da tief alles, mas laufen fonnte Taufenb! maś mar ba! Das 2Baffer mar aus Dem zeite abgetaffen, unb ouf Dem 


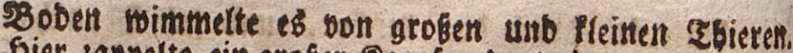
Sgier jappeltte ein grofer Rarpfe, Dort ein paar Sableien! Die fid in Dem Sdjlamme einjuwutblen fubten? Shmet. Ien, Sirebje, grejiche, Seáfer, garven u. F. Itw. bebecteen

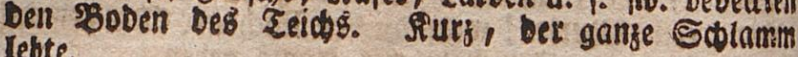
lebte.

Da båtte man die greube ber Fistoer feben follen!

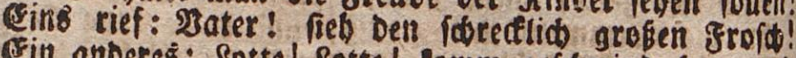
cin anbereś: Sotte! Sotte! Pomm gefómint ber, tail fieh Dett Sirebs! Sannden rief: o, wer bolt mir Dein Sáfer! Sieh, SBernbarb! Dort nidt weit vou bem Rar" Dfen! er bat einen gelben Saum um bic flúgel!

(Endlid) fiettg serenbaro an, unb fagte: ad, lieber Bater, wenn ous uns eine redote Suft madben willft, to Jak uns in Den zeid selgen! und alle fimmren bei: fiec

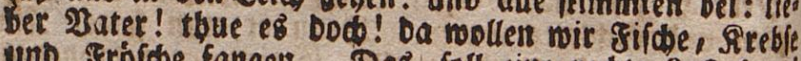
uno frófiche fangell. Das foll eine rechte Suft feon! SRun to gebt Denr bintein! ppred ber 2ater ; fire bet aber erft Ed dube und Strumpfe aus, leget bie obern Rleiber $a b$, in benen Die 2yrme fecfen, unt ftreift Die atermel auf, Damit ibe Die Sileider nidbt ju (comukig madbet. Dott feebers bie Sibrbe, in welde bie giiche follen fommen, biex ift ein Siorb für bie Sirebs

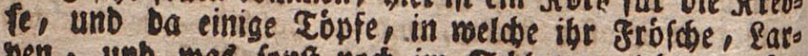
sen, unb was foutít now im Sollamme lebt, werfers

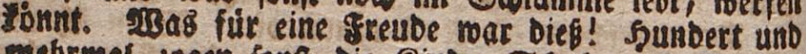
mebrmal rogen fonf Die Rinder Swube unb Strumb yfe aus, ments be ing ssette giengen; fo idnell muts Dent fie aber nie fertig, als Diefmal. Saum batte es Det isater gefagt, to mat aud idon alles fertig, unt fprang in ben zeión. Das war eine \&uft; to oft eir

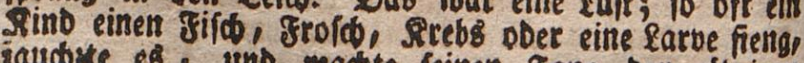
jaudse eb, und madte feinen fang ben ubrigen sunb.

3mei Stunben lang exlaubte thnen bet 23ates Diefe Suft; bann flatfote er in Die fanbe, unb ber fabl, bof fie nun aus bem Jeide fommen follten. So idonell gieng $e B$ aber nidbt beraus, als binein; jebes batte nod etrob ju fangen. (Ein' rief: nut Den SRebs now! bas̉ anbere: nur ben Stbmerl nod, tieber, guter \$ater! shet Der \$aser fagte: einb! 
gur Befóbexung guter Befinnunger 26,8

zoci! Drei! unb dief war bab 3eidell, Daf bie Sinder folgen mubten. Sie fprangen alfo betaus. stber wie fas ben fie aus! tuber unb thber waten fie mit Sdlamm bes Deft, und man fonnte fie weiter riechen als feben.

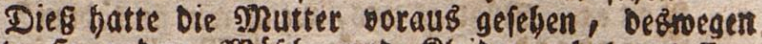
batte fie anbere $203 d$ fithe uno Sitcibung bolen laffen.

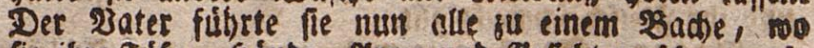

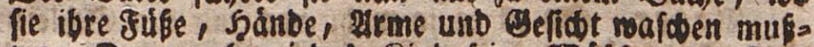

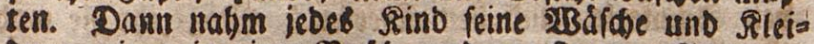
Dunge gieng in einen Bufd), uno sog fie an. Jekzt mas ren lie alle fertig.

SRu SPinder! fprad bet शater, ibr fiengt beute viele Tbiete, haben lie eud Sdaben getban?

刃ein! fagten alle.

23ater. Slaubt ibe benn, bas ibnen in Diefers Sobeben unb Eopfen wobs iff.

BBernbard. Das glaube i nicht.

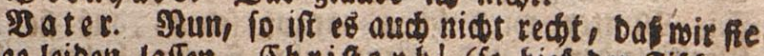

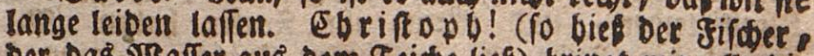

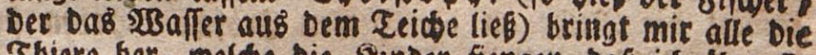

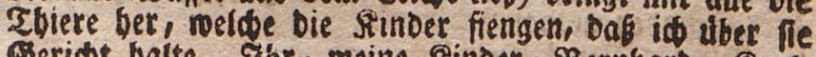
Beridet balte. Fate, meine Sinder, Sernbarb, Sart, cotte uno santre, unb Du, gute frau! fegt eud alle um mid, uno gebt 2Atung, of id redt ridte. SBernbard! menn iff es mir etlaubt, ein Ebjer fu tóbten?

פerntarb. TBenn es bir fidabet.

פa atet. Da Darf it alfo bie Rub nidt tobtets laffen; benn biefe lobadet mir is niwat.

28 ern barb. Ja, bie múfien wir tobsen laffert Damit wir ibr giteifo effen fónmen.

Bater. Mifo barf id ein Ibiet tobten, menn of mir fobabet, ober wenn iá lein Sleifi effen Paim. शiber warum tobtet man denn ben 233 allfifíc? Dee

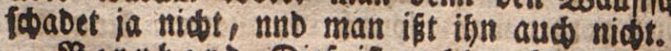

Bernbarb. Dié́ iff moll mahr; aber man nuage Dod fein Jitt.

Eot te. Linb bas girabeit.

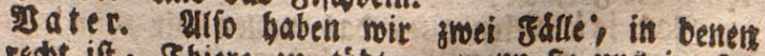

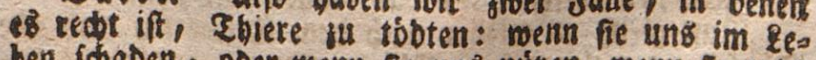
ben fopaden, oder menn fie uns nutizen, wenn fie toot

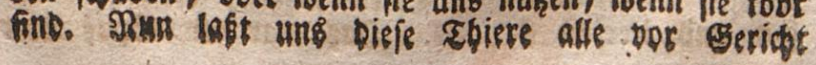


bringett, unb febrn, ob mir ein Feat baben, fie it tobren. Sier frelien exflich zroei fiorbe voll siarpfen. Jaben fie uns St)aoen gethan?

सlle. Nirin!

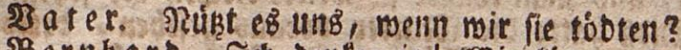

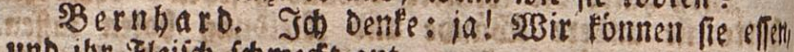
unb ibr Jleifid fomedt gut.

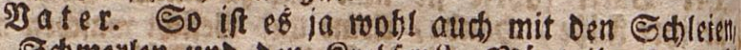
Den Sdomerlen und Den Rteblen? 23rogt it) fie moll eflen?

fotte. serfud eb nur, Nutter, unb fiebe mel de; bu fouft feben, baf fie uns redt gut fometen merden.

Bater. SRut, fo follent fie alle fterben! WBel wir fie aber ood tichs ufle auf cinmal effen fontmett fo forgt bafur, Ebriftewh, daf fie in Den Raften, Del

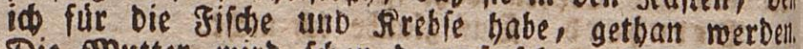

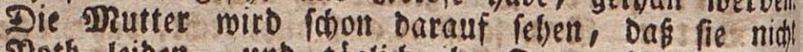
SRoth leiben, unb táglid ithr futter baben. SRut

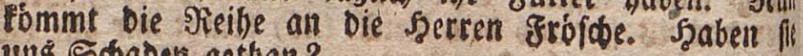
unB̉ Sdabek gethan?

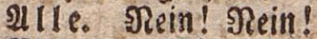

\$atec. Shüt $e^{B}$ ung, wenn wir fle tobten? Soll We eud bie matter vielleidft braten?

baunden. gi! it mag feimen groid effett. 2Esillf bu, lotte?

Eotte. Io will anbern bie sroblde laflen. Ta lobe mir bafur bie Sireble.

刃ater. Nun, fo mógen Denn bie Iróblare leben!

Eben to fprad man aud die Sarben uno die fiufet frei bom Iobe. Şert Sjerbft maim bant bon jeber art Diefer Egiere cins, zeigte eb Den Rimbern, uno fagte ibnen, mie eB̉ lebe, fid nábre, und mab̉ es núke.

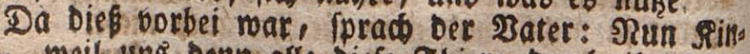
Der, weit un' benn alle biefe shiere burch ibr seben

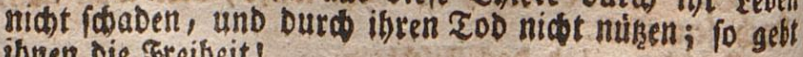
abren ois greibeit!

Ja! ia! viefent alle, Daś wollett wir thun!

PNun gieng eb mieber nach Dem ₹eid)e ju, unt alle Zopfe, in welden biefe Sbiere maren, trugen fie $b a$ bitt uno terten fie aub. Das war ein Spas uber 
gur Beforderung gutex Beffinnungen $2 c .91$

alle Epábe! Die Sinber freusten fid) alle, ba lie biefe Fhiere im ₹eide berumfriedben und bupfen faben.

Jekst foilte Der sug nach Spaure geben. (Ebe ber \$ater aber fortgieng, fragte or den SBetnlbarb: Waben

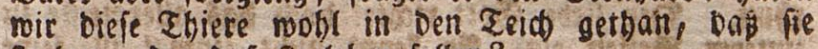
feerben, oder DaB fie leben follen?

Daß fie leben follen! mai sernbaros entumort. Nun, fagte ber 2 ater, to muifen roir aud Dafur lors gen, DaB fie Teben fonnen. Er liés Darauf DaB \&od

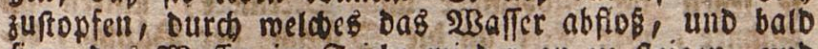
fieng Dab $23 a f f e r$ im Seicue mirber an fu freigen, unb alle Diefe abiere waren in Dem $23 a f f e r$ luftig.

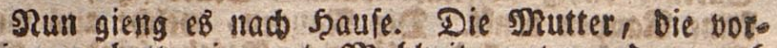
auช́ mar, batte eime gute Mablseit, unter andern aud cine Schutfet voll grebje gefodb. Diefe lobmedten Den stinbern berrlid, meil fie fid subor sin ऐaar Stun ben in freier euft bemegt hatten.

Da fie fatt maren, fagten fie alle: mir banfen Dit, Sater, Du baft uns beute ein redtes Jeft ges madt!

\section{Die Raupenfeinbe.}

Der 2mtmann De ull er batte cinen guten alten Båts

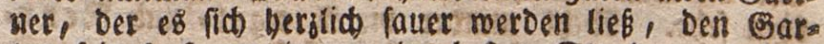
ten feines Sherrn immer im beften Stande su erbato ten. 2lber er foumte eben barum alld febr verbriếs lid merben, wenn alle feine Muíbe juweilen midbts

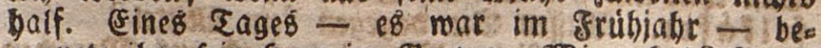
gegnete ibm fein Serr im Barten. $23 i e$ gelitb? fragte Der elmtmann. - 2ld, es geht leider fehr folectst, lieber Seert! antmortete Der Sátinter mit einem febe

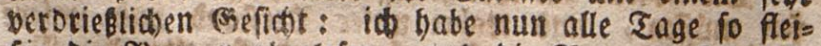
fig Die Raupen abgerefen, umb die Raupennefer berstilgt; und bod find faft in dillen solutben mieber

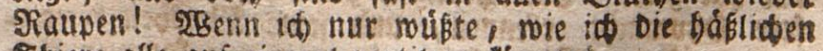
Shiere alle auf einmat vertitgen fonnte!

Der Siert. Eieber Dann, fed er nidt verzagt! mutre er nict!

Der of ártier. Benn i申 nur múpte, wo fie 
Derfämen, und wie id ibnen beilomment fontre! yt metnem fleife foulte es nidbt liegert.

Der fgerr. Der fleif vethútet manden Shas

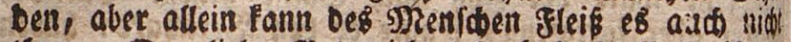
thun. Der liebe Cott ridotet Dow Das allermeifre ill unferm Seften ein. Er fernt nibt einmal alle fellt

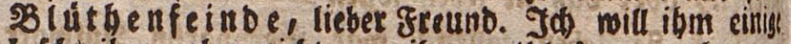
bef(d)eiben, aber nicot um ibn mutolos gu maden.

Sennt et benn Die frofitiometterlinge? Dir fe tegen in November uno December, mitten ifi Sthnee, ibre êier an Die Stámme ber sodume, vis

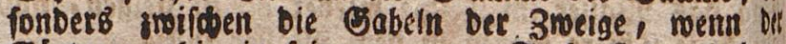
Bärtner ruhis in feiner warmen Stube fint, ins at feine SRaupen Dentt. Die Raupen, melde aus Diffen

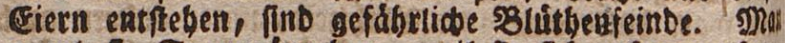
netnt fie Epannedupden : weil fie fich jufammenfpols nen, einen 2Budet maden, unb bann fortichretten.

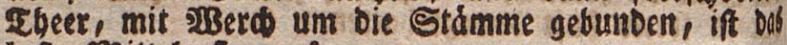
befte grittel, fie su fangers.

Der Edrtuer. Das folite man bod niót bell ren, mitten im Søntee Sdmettertuge! SBBie Ponnt fie bean bie sálte ausbalten?

Der Jgert. Sie balten fie aus. Die fob nell fubige is $\Re$ aupen, bie fo gefdmind laufen fonnet!

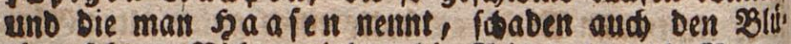

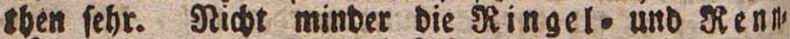
sdupden, wems fie nod flein finb.

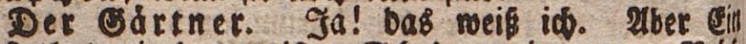
Caft tbut mir ben meiften Stbaben; ein grauer $\Re$ uff felf d fer. 2Biffen Sie bagegen tein פartitel ?

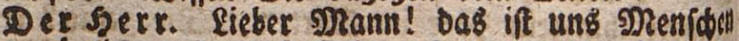
sidet moglid, alle biefe Hleinen feinbe zus vertilgen.

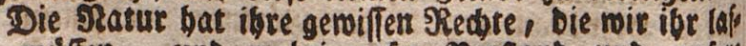
fen mulfen, und mobei unfer ssetftand uno unfert

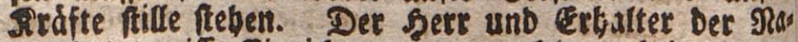
tur bat gewiffe Cintiobtungen gemadit, mobei wir ult fere Dbumadt effennen, uno feime 2 Beisbeit bensunt bern mufinen. SoBit geben, to weit wir fommen foil netn, und Danm baben wir unfere Pflidt gethan. Güt bas llebrige, was wir nidet fonnen, bat benn ber liebe Cott foron befier geforgt, als mir benten. 2sents 
Jut Defortekung gutex Cefinnungen 26, 93

mir ferbft alle sRaupen bettilgen foltten, was woltten wir bod nobl anfangen?

Die Eier bet Stammraupenfometterlinge, in ibeem Pelsmerf, Ponnen wir jur Stoth mobl vermins Dern, Denn fie riben uns vor ben 2tugen. 20sir fonmen fie bei Zaufenden zerfforen. SBei ben Iringetras. pen gebt Daß fdoon nicts an.

Der Bártner. Jab babe a meinem leben foton viel gevagt! aber die gingeteier firken gar 34 bod, und an Den bünnfen Reifern; mer mag ba binauf!

Det Stert. Eieht er mobl! 3ul Denen fóness wir fobon niфt lommen, mie mir mủnident.

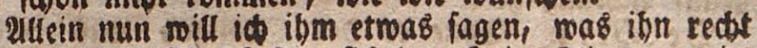
freuen wirb. Eine faft unfidtbare fleine fliege vertritt uniere Stelle. Diefe mird nad Sotteg roifer Eintid) tung unfer freunt, und ein gefdmotner feint Diefer raupen.

Sie bofert Durd Den feffen sitt burch, momit Diefe Cierden verleigit find, und legt in iebes ei ibr eige= nes Eicten. Rlein genug! rsenu bann Die fleine glies germabe augilonamt, fo frift fie bas auf, moraub bie tleme Paupe gemorden wáre. 21lfo fonnen Dann aus iefent Eiern feine भiaupen entfeben. Sie find von fleinen zeinden zerftort. (ex braudt alfo nidt in bie Godiffen Gipfel Der sgdume zal feigen, und megen bee Singetraupen fein Reben zu wagen. Dafúc fochidte Bott eine fleine grfiege bin, Die fie jerffort.

Der \&s riner. Nun, Das ift Dod munberbar?

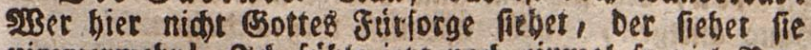

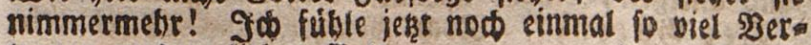
trauten fu Dem lieben Bott.

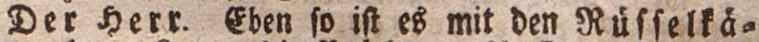
fern, beren Larben bie Blutben aušfrefîen. Da biefe

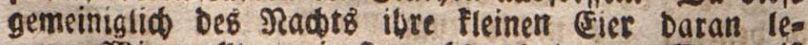
getr. SYsie mollten mir fie mobl abbalten? $23 e r$ mei aber, mab̈ Diefe roieber fúr geinbe baben, bie uns now umbefannt find ? In Der Natur ereignen fict lonft noch anbere umfánde, bie bet sbiutbenraue pen gútrtig finb, unb bie wir fobledterdings nict in unferer Bemalt baben. Git in Der sBlútbze it zu trod"-

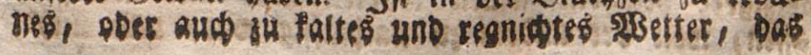


ad)t bis bietselin Tage anjdatt, fo mítb bas थ ufgu ben Der SBlutbfnofpen gegindert. Dann baben di Heinen sBiuthraupen Zeit, fid) einzuniften. 2Badfent aber Die solúthenofpen fabnell fort, fo tommen dit sBlutben den Raupen zuvor, tund fie múfen Davon ble: ben. SBet fann Das nun cintidten, uno nad feinem Eefallen maden? Rein Fónig tann mit feiner ganjent

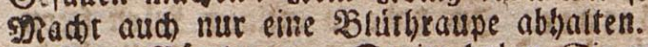

Der Sartiner. Darin baben Sie noby Redt।

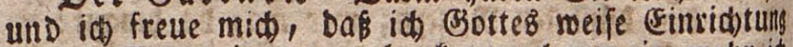
in bet seatur immer mehr fenten lerne, je mebs in barauf 2idtung gese.

De Serr. Ifoue er Dab Seinige, wab eitnt fteifigen Bártner jutommt. Júr bas Uebrige lafte of

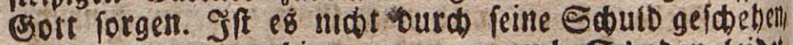
fo fer er ganz rubig, menn er aud Ecthaben leidet. Shat er Dod auf ber anbern Seite mieber Bortbeil, metth andere Eachen beffer getatben, als fonft. Der liekt Eott bat allezeit feite 2ubfidten, ment er in einem gabs re methr SRaupen entrfeben lábt, alö im andern, g)Ran. gonne thren, mab esott ibnen jutheilt. Ee meiß ibnet bod fis redfer zeit wieber (Einbalt fu thun.

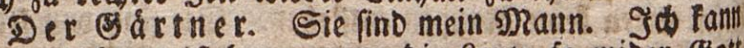
es gar niwt ausfeben, mein bie Reute fo miber Bott murren, wenn einmal mebr Siaupen, Doer Jelomáuil? Da find, alś fonft.

Der feerr. Semeiniglid murten aud nur bit gaulen, Die fid um nidts befúmmern, und teitle Şano antegen, ein Traupenneft wegzuichaffen.

Der es ârtuer. Rod meniger tann id eहै leis Den, wenn bie feute fagen: Das if cinmal mieber ele ne redite Strafe Gottes.

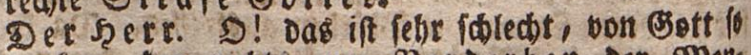
au Denfert, Der nidts zum serberben Der gRent

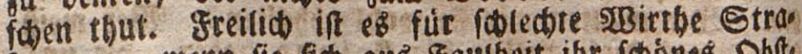
fe genug, went fie fí auz gautbeit ibr foónes Dbfit

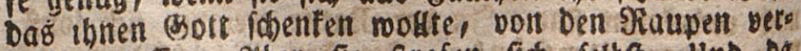

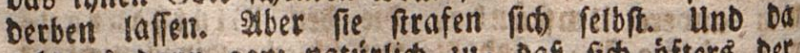
geht es Dant ganz natúrtid fu, baf fíd sfters bet fielíigere NRabbar feinen Earten mús mit abfreffer

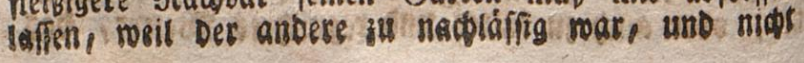




\section{zur Beforderung guter Cefinnungen 2e. 95}

gu redter seit abraupen liép. WBit wollen baz $4 \mathrm{nf}$. rige thun, lieber freund, und dann ben grosen Saufbater regieren laffer. Der meißs mebr SRittel ges gen Die Fiaupen, alz wir. Der hat feson geforgt, wie weit fie fommen follen, und bat ibnen mehr seinde jugeorbnet, als wir fennen.

Der esartner. D! fagen Sie Dow, mie es Das mit ift. Daś lobre in gar ju getn.

Der foerr. Da giebt es lo viele æobgel, Ráfer unt anoere Snieften, Die Den gefábrtidifen Siaupen, Den sluthraupen, gleid auf Dem fuke nadgeben,

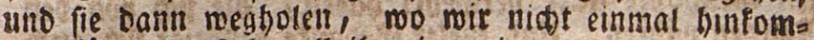

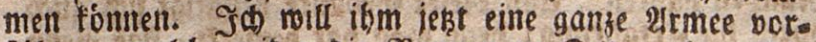

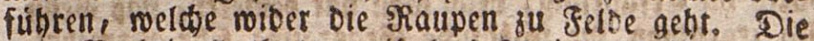

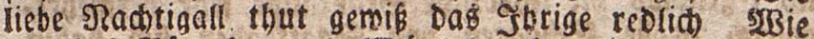

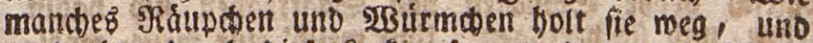
miro eben burd Diefe foctipeife yon Den unbanlbaren gnenichen gefangen.

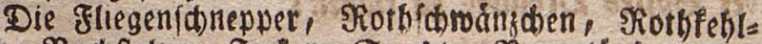

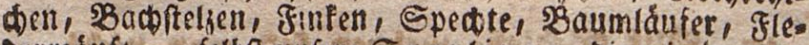
Dermáufe - felbft unfere Spertinge, die rir ja niabe

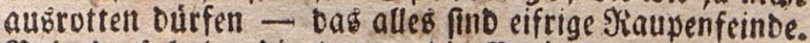

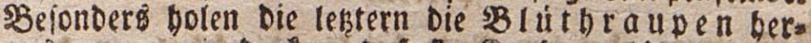
auB, wenn wir benfen, dafi fie Rnofpen abbredben.

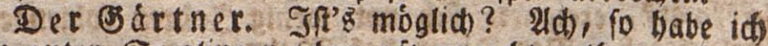
ben guten Epertingen icton oft unredit gethan; Denar wenn id fie fethe, fo bole it gleich bie glinte.

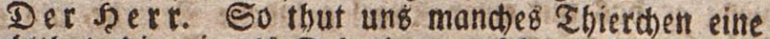

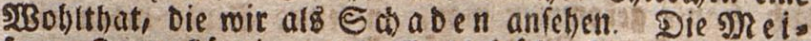
fen, 3 a unfónige uno (solo bábndien wiften die verborgenfren Exmetrerlinge, Die nir nimmermely fin. Den roúrben, cufjuipurren, uno piçen fie forgfáltig aus. 2lukerdem giebt es not fo viet grofe Daummanzer

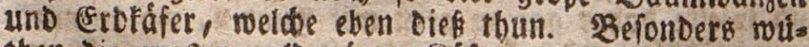
then die groken goltgrumen siafer unter Den Siatuen;

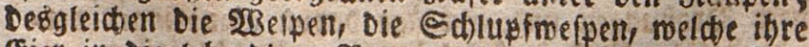
Eier in Die lebendigen भaupen legen, ba Dam die flei=

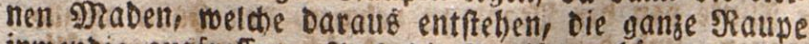
inwentig ausfreffen. 2uat) Die 2meifen gehoren zu Den Jeinben ber SRaupen: fie wúrgen unter itnen, wie bie

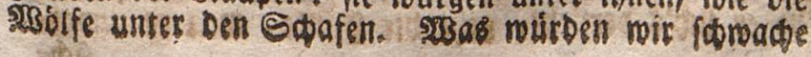


Drenichell gegen bab zabllope seer ber Fiaupen aus ridtels, menur Diefe Raupenfeinde uns nidt to februnterfturgten.

Der esdrttier. Eott fey bafúr gebanilt; ex bas alles nobl gemadt.

Der fpert. Yob bente immer fo von biefer Sto

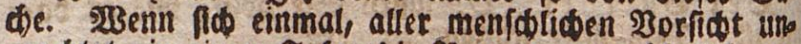
geadtet, in einem Jabre Die Raupen ungemóbntid veto mebren, fo roerden fid obne 3weifel, nad ber Drbmung,

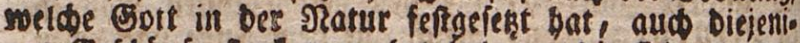
gen Gefísufe fare vermebrt baben, Die fíd von Dell Siaupen naduren. Lino Diefer Bebaute beffátigt fich aud Durct bie Erfabrung; benn man bat bemerl't, Daß ment

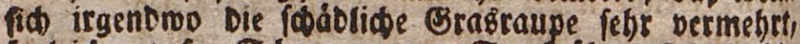

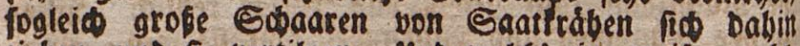
gieben, uno fie vertilgen. Und reeld' einte meife, moble thatige Eintidtuting if bae! Ift cs alfo nidt Sunber น́ber Eottes Dronung ju Elagen?

\section{Dnit Shiefgenefien foll man nid)t pizlen.}

2luf einem abeliden Gute bei Joadimstbal, in Det Ildermarf, ereignete fid oor mentg Jabren folgender

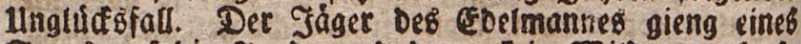

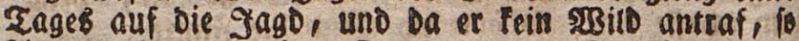
bieng er bab mit einer Rugel gelabene semebr, als et nach Shaufe gefommen war, in feiner Stube neben ejnet ungelabenen flinte auf. Ein junger Buride von 15 Jabren, ber mit Dem Jáger auf einer Stube wobnte, Pam bald nawher, un Stiefel zu plusen. Diefer \$2ut fide batte bon jeber Puft bezeigt, ein Ġáger zu werbent und fpiefte Daber gar ju gern mit flinten, wo er nut ibrer babhaft merten fonnte, to oft man ihn auds fitor Dură Drofiungen uno Sallage Davon abzubringen veto fudit batte. Fud biefmal fonnte ers nidst laffen, eia ne Jlinte ven ber $23 a n t$ ju nebmen, Den Sgabm aufjus fpannen, und bell 2tbzug aufzubritten, unb ungluatit

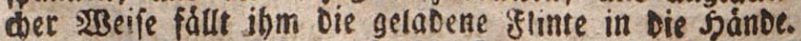
ebe or sid's verfiegt, gegt Die flinte, soeil ef unboso 


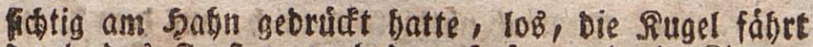
Durd Dab Jenffer, nad Dem Şofe, und ein Rinder máboden fintt, von ber siugel getroffen, zur Eroe, er= bebt fict zwar bald wieder, uno manit nod einige Sturitte édjent fort, muß aber bow mieber ginfins

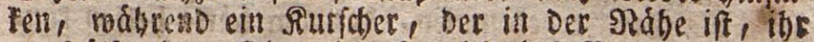

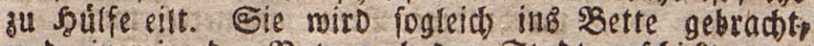
unb ein reirenber Bote nad Der Stabt geforidt, um cinen gryt z holen; allein fie firtbt nad) einer balben Etunde obne Sfulfe. Der SBuriche batte fich, von feinem bófen seroiffen geángffigt, aub Dem fenfree in Dent Barten binabgefturits, unb war entPommen; alfein foton am folgenden ₹age mard er entbect, und muste nun, zur Etrafe fúr feime Unnbefonnenfeit, Durch Die er fum Mórber geworben mas, lange im Befángnifife

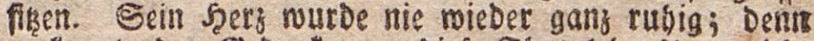
ex fomute Den Bebanten an Diefe Ebat lebendlang nidat aub feiner Seele titgen.

\section{Eine gute Sanolung aus follect)ten Ðe megungignunoen.}

Ein Solnfutidiser traf auf einer febr foblecten sando. Strabe einen Sradffiufrmann, Deffen $23 a g e n$ in RO $_{0}$ raff berfunfen war, und ber isn Dringent um sBei= ftand bat. Der \&obunfuticher marf ibme einige Droben= De Blidé 34, fpante aber obne ein 230 ort zu fagen, feine 》ferbe bor Dem verfuntenen sabagen, und fo wurDe er gludeftid wieber beraubgejogen. 2luf alle Danf= fagungen antwortete er nur burch finfere shlide, unb also ihm Der Fuhrmann Belo anbot, fprad er in eis nem zornigen Fone: id mag's nidb! (Ein Sieifender, melcher Dazu Pam, fragte Den Robnfuttcher: mie er bei

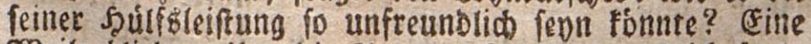

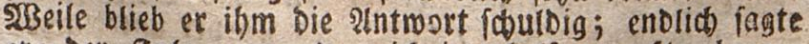
er: Dee futbemant, Dem id jekst belfen mufte, begeg= nete mir neutlich an einer Stelle, no id itsm augens

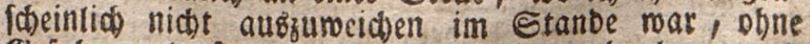
Befabr zu laufen, meinen शBagen zu jerbrechen, und no et mit feinem leeren $2 B a g e n$ jefor leidt ausmeis 
den fontute. Dennod nóthigte er mich, aus bem ssegt gu fabren, intem er auf Den Beiftano feiner Befáhrten trokte. Sgátre id thon heute, Da er allein mar, niat in grofer গ2oth angetroffen, er hátte ế ausbaden fole len! zber vielleicht mare ben gamzen ₹ag fein Juhto mann die Straṕe gefommen, Der ibm bátte belfen fón

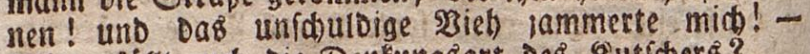

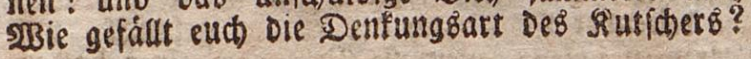

\section{Traurige Folgen ber 593 iltobeit.}

SFerbinand, Der Sobn einer armen ssittwe, wat won feiner frúbeffen Sintbeit an, ein milber unget)oro famer unb leidetfinmiger Rnabe. Sein $3 a$ set batte ith frenge gethalten, ftarb aber, als er erft 5 Jabre alt

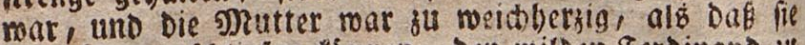

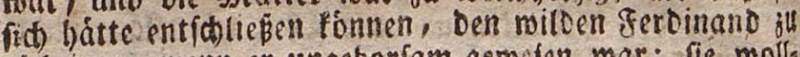
glid):igen, ment er ungctorfam gewefen war; fie mollo is ign lo gern blos ourd liebreide Ermabnungen und 2yarnungent bielen. 2lber Darauf adtete Der sBildfang nicht. Dft bat fie ignn fehr rúbrend, er modote Dow midt mehr fo gefabrlide Sprünge madsen, und fein feben nidst Durd Silettern in Befalje feken; aber froum war er ît auह Den 2lugen, fo fprang uno fletterte er, mie jubori

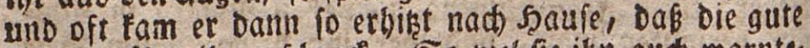
Shutter úber ibn erf(c)radf. So biel fie ithn aud marntes

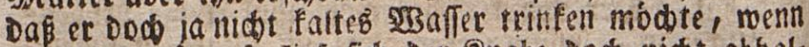

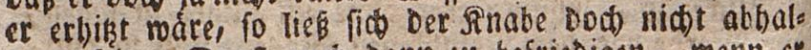
ten, feinen Durft auch Dann zu befriebigen, wenn at

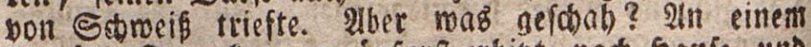

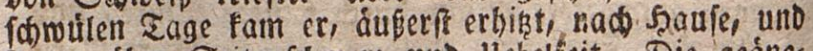
Elagte úber Seitenfommer uno Hebelfeit. Dic geángs

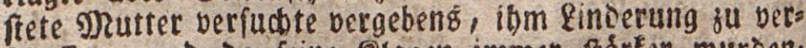
íbaffert, und oa feine Silagen immer fáster murben,

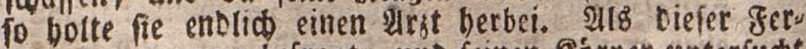
Dinanden genauer befragt, und feinen Siorpet unterfudt

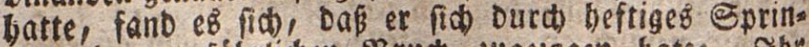
gen einen gefábrlicben szruch zugezogen batse. Thb fornt Denfen, lieben Rinber, weith einen Edrect bie arme Mutter biertiber batte, und fie wuirbe auferbem now Durch Die Untoffen getitten baben, welde ibr diefs 
zux Beforberung guter (Eeffinnungen zf. 99

Srantlyeit ithres milben Sobmes verutfadte, ment

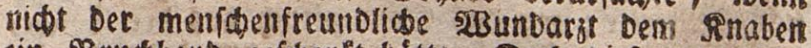

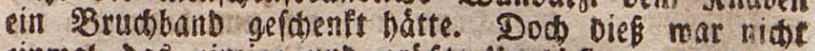

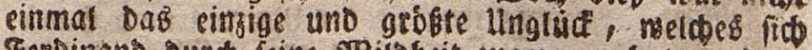
gerdinamb ourd feine WBilbbeit zugezogen hatte; Denn

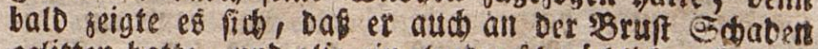

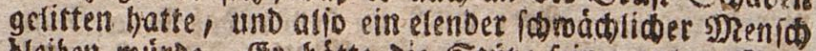
bleiben rourbe. Eet batte Die Stuke feiner guten Mut= fer in 2utter fenn fonnen, nun aber wurbe er Die llso facbe, Dá̧ ithe 2ulter fummervoll tno traurig nar. Sagt, was mollet ibr zu eurer $5 B a r n u n g$ aus biefer Bef

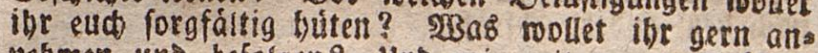
nebmen und befolgen? Und wo mit euren Rréftent baubbalten? SBarum mollet ibe Dieß tbun, und Dem wilden ferdinand nidt dentid merden?

\section{Das Raupenneft.}

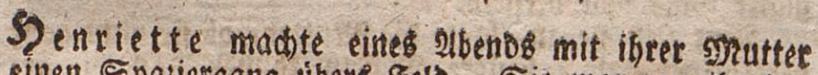
einen Epajiergang tibers felb. Sie war von ith $D a$. fu gewóbst, alles mit enufmerffamteit ju betrachten, mas un fie ber war. Dié that lie aud jest. थuf einmal blieb fic fteben, uno rief: Doutter! sDatter! fomm geforoind ber, unb fieh, was oa if ! Die Mut ser ram, uno fiel)! ba mar ein Nerfelbuich, Ber gans

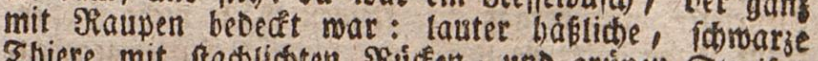

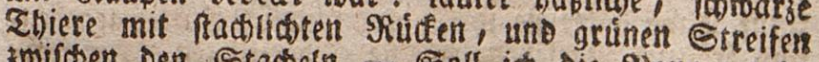

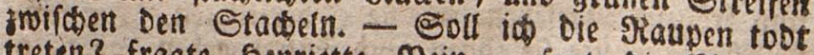
treten? fragte Senriette. NRein, - fagte Die DRutter; Denn wie Da fiebff, fo nábren fie fide von sieffelin, und

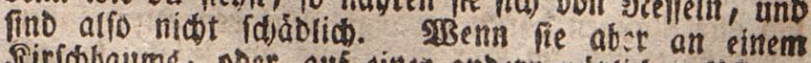
Nirfobbaume, ober auf citter anbern nusplichen Pfianze

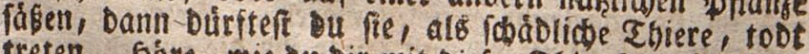
treten. Sobre, wie Du bir mit biefen Thierden eine recht groke greube maden farmit. Nimm lie mit nad sjaufe, uno futtere fie.

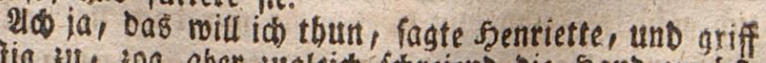

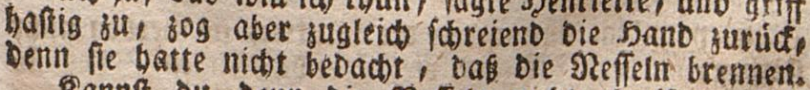
sanmft Du Denn Die Neffern nicht abreifen, obue (8) 2 


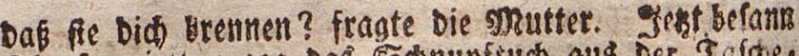

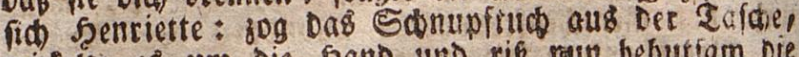
micfelte eb um Die frant und ris ran bebutiam Die gReffeln ab. Fretreig trug fie Die SRaupen nach Şaufe,

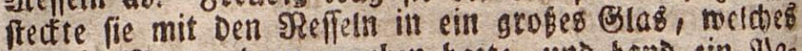
ibr bie Matter bazu gegeben hatte, uno bano ein Yas pier Daruber. - 2lber willft Du Denn, Daß́ beime Rau= pen erfticten follen? fragte die Rutter. Rein, Das will io nidist, antwortefe Senriette. - Sain fo muft Dis fleine fóder in Das \apier fredsen, Damit frifale \&uft in DaB BSlas fomms. Sief́ that Senrietre, und hatre ibre greube Daran, fu feben, noti die SRaupen ein sblatt nac) Dem anderi abfrafen.

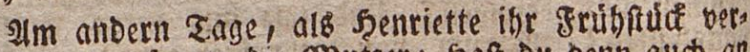
gefirt batte, fragte bie smetret: Saft ou nenn aud an Deine SRaupen geoadt, und thenen ibr Frubftud gegeben?

D! fagte frenriette, Die Faupen baben ned Das ganie Bেlar voli. Seffelti.

2lbet fiet fie an, fogte bie MRutter, ob fie nidht gains

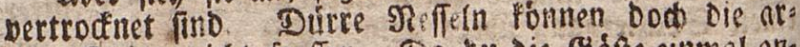
men Sbiere nidst freflen. Sa bu die Bafte cinmal an. genommen baft, fo if $e s$ aud beine Wflicht, ibnen alle כage frifobe Refleln zu bolen, uno fie. fo zu ernabren;

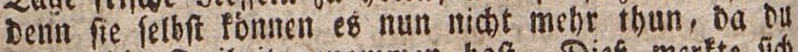
ibnen die Sreibeit genommen haft. Dief merfte lid Sentiette, und dergá́ ibre fleinen Báfte nidt roieter. Fün Tage batte fie ihnen nua reichlict Jutter genebent uno froblich fuge(ehen, wie fie es verzel)rten. 2im fects fiten sage woulte fie ibnell sud gutter geben, aber, 0 sBunter! Da fie Das Papier megnehmen molite, hatten (iia) alle Siaupen baran gebangt. Igrit Den Simterfifiell faken fie theils am Wapier, theils am Btafe, fo feft alb of fie angefeitat gemefen márent. Befatmind lief sentiette zur grutter, uno seigte ibr bie aufgebangten अaupen. SBeforglich fragte fie: aber mab felyt ibnent Denn? liebe Drutter, id babe fie Dod afle Iage fo reids lid gefúttert, und nun meroen fie mir Dod fferben!

Sen rubig, antroortete bie Mutter, fie merben nidt fterber, fondern bir now viel greube maden Laß fie nut ungeftort bangen. Daś that Sgentiette, und madte 
gur Beforbecung gutet Befinnungen $2 c_{\text {. }}$ IOI

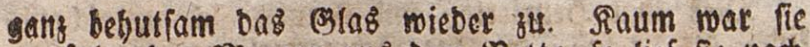
am folgenben morgen aus Dem szette, fo lief fie nad Dem Elafe, und fieh, ba gab eb fijoll mieber etrab Reues. Die Raupen maren verf(t)munden, und nun biengen lauter lánglidbt runde \$upphen $D a$, mit einet fleiten sitone auf Dem Ropfe. Sie lebten und beweg= ten fid bin und ber. Sanriette matote grope 2uget, folug die Şánoe zufammen, uno wuste niabt, was fie oaju fagen foltte. Einolia rief fie: Nutter! Mut= ter! fomm gefobroind ber, und fieb), was aแb metnet SRaupen gemoroen ift.

Sabb id es Dir nidit gefagt, antwortefe bie Natter,

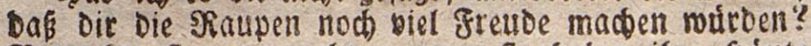
SBetrabte fie nun redit genau; fie baben ifore Staute abgeftreift, Die bu bier bángen fiehfi, uno baben fià berwanoelt in Dinge, Die man Dappen nennt. Laßj fie nur afle rubig bängen, unb fiet alle Eage nad bem Blafe. Bielleidit erbliteft du bald einmal mieder et was, was dir grofe Jretlde madt.

(EB traf riditig ein; nur mákrte es ber ungebul= bigen Senriette fulange, uno fedon batte fie faft afle Sgoffnung nufgegeben. alber nus waren faft einige \$23oden vergangen, als jenriette eimmal mieder nad it) = rem \&lafe lahe. Uno mas erblidte fie? Da mat alles Boll foboner bunter Edometterlinge in Dem Bjafe. Id fieh Dod, liebfte Snutter, rief fie, mab in meinem (s)afe ift! Eádeeno fam bie s)utter, uno als fie nun betbe gi= nauer jufaben, erbliceten fie ein neueś ş3under. (Fin Sdmetterling, Det in einer puppe fredte, orudte mit feinen zarfen Fúf beraus. Seine fltigel naren ganz flein, uno zufam = mengetollt, roie cin stúd \$apter. Er lief gefároino am Blafe binauf, uno bángte fid) an bab̉ Papier. Eci= ne flugel mudfen faft fitbtrich), unb nact einer sier. celftunbe biengen fé voufomamen ba. - So gieng eв́ nuล Den ganjen 20 ormittag. Gmmer ein Schmetterling nad́)

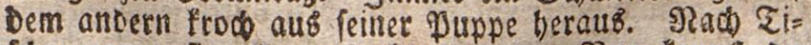
fore waren fie alle ausgefrodien - Nun fanmit ou bir nod) eine Jreube madjen, fagte bie פatter. NRimm Das હlab́, trag' und gieb ben Edwatrertingen Die Sreibeit. 


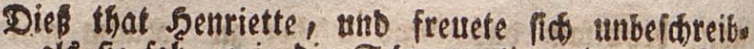
lid, als fie fab, wie bie Solmetterlinge beraus flats terten, uno bon einem Saume fum anDern flogen. 203enn fie hernad im Barten berungieng, unb einen braunen Sometterling mit fotwargen fledfen faht freuete fle fid allemal. Dot bift gemib aú) aub meie frem Slafe! Dachte fie.

\section{III. \\ 20 ○}

Det grobe siorper, auf weldem mir Menfoben wobs nen, bie Erof, if nue ein fefr fleiner $\Sigma$ beil bon Det $2 B e l t, 0.0$. von Dem, mas Bott geldaffen bat. ces giebt nod unzaltige Rorper ober ErDen, unter melóst biele unfern Eroforper an Brofe úbertreffen. Diele Rorper erbliden toir rum Sheil an Dem unermés. lioben Bemolbe Des Simmels in einer bellen Nactst. Sie foheinent uns megen ber Entfernung, in melcher

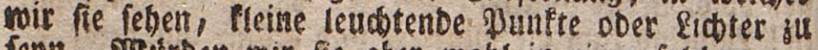

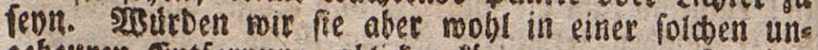
gebeuren entfermung ecbliden tónen, wenn fie nicbt rebre groß máren? Wit nennen fie Sterne. Det grofite unter Diefen Sternen fobeint Die Sonne zu fepn. Shre Strabien foiefen ourd Dis ungebeuren Ráume DeB Stimmels auf unfere Erde herab, erleud. fen uns etrórmen fie, and berbreiten úberall feben

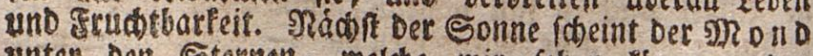
unter Den Sternen, meldbe wir leben fonmen, bee grofite. 2ua biefer Stern efleudstet ourd feine Strabse

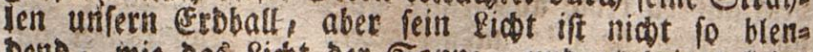
Deno, mie das fiát der Some, und es bringt teine 2Bárme berbor. Das grobe blaue Berwoblbe, weldes mit frimmel nennen, if ein suermeflider Raum 
in meldem bie Erbe, bie Sonne, ber Monb, unb uns záblige Sterne fammeber uno fich berogen. MIIe Diefe Sterne werbea 23 eltrórper gettannt, weil fie zu= fammengenommen bie $23 \mathrm{e}$ it ausmaden. $233 i e$ flein if alfo unfere erdoe, menn man fie mit ber sBelt vergleid)! SBlok Die Sonne if vierzethn bunberttan-

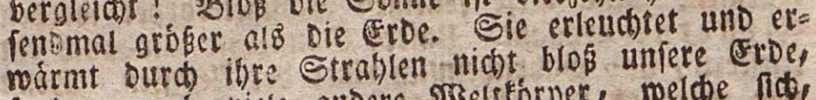
foubern nod biele andere SBelfforper, melde fits, gleid Der Erbe, um fie herum berwegen. Drei this

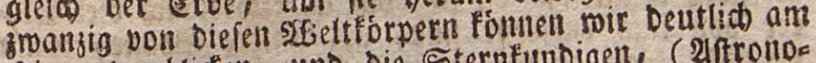
Simmel erbfideen, und Die Sternfunbigen, (2/itronde= men) baben fogar burd ibre linteriudungen und 2ubs=

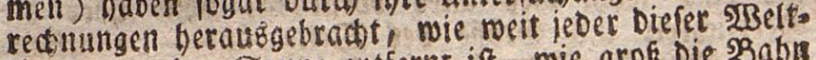
forper von Der Eomne entfernt ift, wie grób die sBabn iff, meldie er iu Durdhlaufen bat, uno mie viel zeit er Daru gebraudt. Desienige, relider Der Sonne am nád)fen fetht, (man bat ibn Me erfur genanut) Durd)= láuft in 88 Eagen feime SBahn um die Sonne, unio roll in eilier einzigen Eefunde 6 gneilen fort. Llnfere Erde bollendet erft in 365 Eagen 6 Stunden ibre Reife um die Eonue, und madt mábrend biefer zeit ben un=

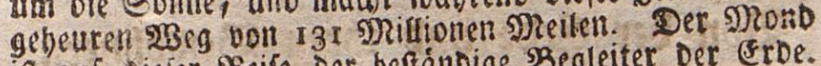

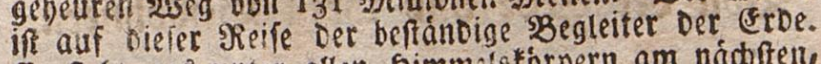

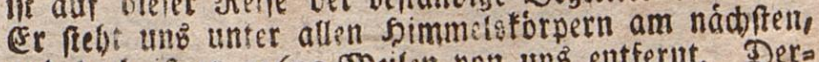

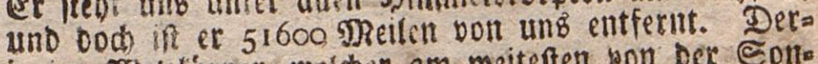
ienige 2Betiforper, meldber am meiteften yon Der Eolte ne enifernt ift (Der $u r a n u \xi$ ), legt feinen langen

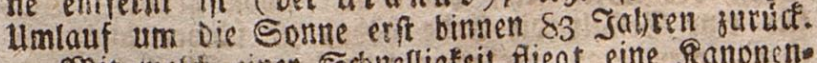
- Dit melit) einer Schnelligfeit fliegt eime Ranonenfuget! Demmot rourbe fic, bei aller biefer Schnellig= Feit, 25 Jabre filegen múfen, um von Der Sonne auf Die erde fut formen.

Die fgimmelstoryer, melde fid, gleid unferer (Es De, un die Sonne bemeger, und von ihr erlettiot werben, nennt man mit enem gemeini

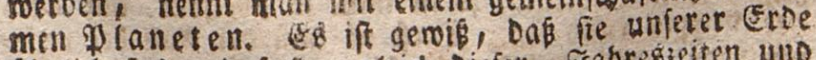
áfntich finto; Dafe fte, gleid Diefer, Jahregzeiten uno

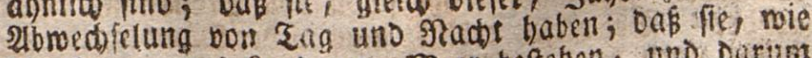
bie Eroe, aus Eand wiso meer beftehen, uno Durum

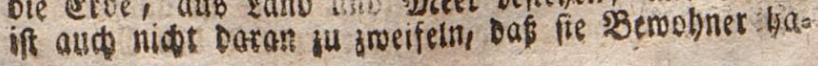




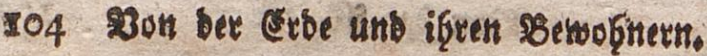

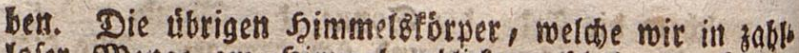
lofer Menge am Saimmel erblifen, icheinen gróbtent theils Sornnen fu feun, D. h. Foldhe Sorper, welde iber eigenes sidet baben, und mie unfere \&onne, Dunfle Spimmeletberper Dured ibre Strablen erleudten und efe mármen. 2Alle Diefe feurigent Simmeloblorper werdert gitefterne genamnt. Unfere Sonme ift alfo aud ein

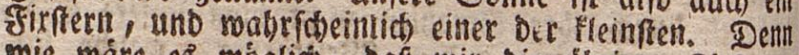
mie wáre $\mathfrak{C B}^{B}$ múgliâs, baß̉ wir die úbrigen Bixfferne

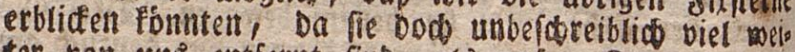
ter von unb entfernt finb, als unfere Sonne, wetht fie nicht Diefe an Erífe toeit úbertráfen? - Rónnet ibre enó mobl bieß alles borftetlen, obme tiber bie

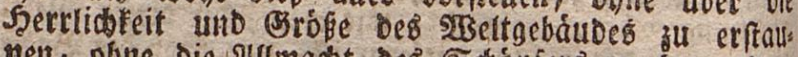

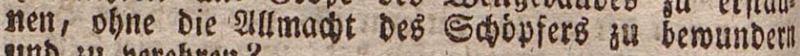
und gu veregren?

IV.

\section{Bon Det Crbe uno tbren Soemolnnern.}

B)

Tak bie e r be pehr grops, aber bod nur cin fleiner Theil ber $2 B e l t$ ley, baben wir idon gebort. $\$ \$ 3 a b$ fur eine Beftalt bie Erbe babe, ift fobreer auszzumas d)en, weit man nur einent febr fleimen Sheil Der Erbe auf einmal úberfeben fann, uno meil fie ung zu nabe

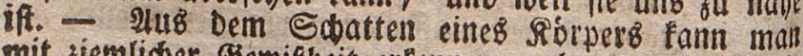
mit ziemlicher Bemifbleit exfenten, ob or rund, breit Doer edfig und fpisig fen ; und wenn ber Edjatten einez Rörpers bon allen Seiten allemal, fo oft er fin zeigt, runt erfdeint, fo ift nicht z" zoeifeln, baß aud Der siorper runo (e1). Dieß ift nun ber fall bei unfes rer erde. She babt mobt foton von Mronofinferniffent

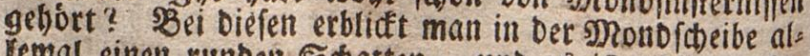
remal cinen runben Sdatten, unt ${ }^{B}$ if ausgemadi,

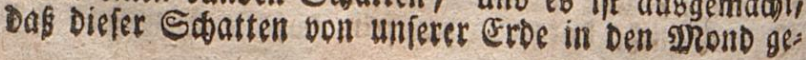




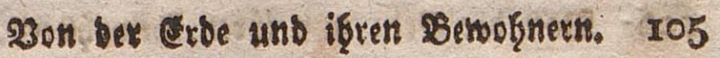

morfent wirb, fo Dap fie bei ihrem umanf am bie Sonne, in geraber sinie fwifhen ber Somne unb Dem Inonde

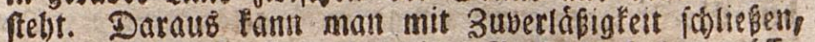
bak die erde einefugelartige Eseftalt baben múffe. - Eimen runber Sórper fann man ganj umgejert, fo

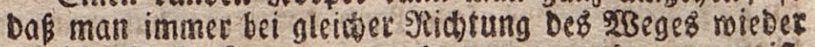
an Die Etelle fommt, yen Der man aǘgegangen ift. sBenr alfo Die Crobe eine lugelartige Beffalt bat, fo muf man fie sbenfalt umgeben, doer um fie berumreifen fönnell, und jwar auf Die 2irt, Dak, wetn man von feinem

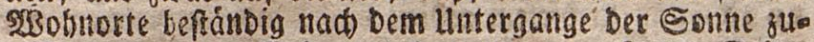
reifere, man am enthe von Der entrgegengerebten Seite, Doer vom 2uffange ber Somt ber, mieber nach Szaufe

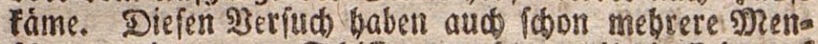
(d)ell, unb zwar zll Sdiffe gemocis, weif Die Erbe auf

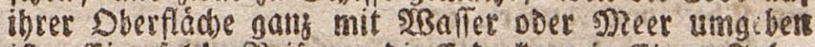
iff. Eine folde গieife um bie Erbe fann in Einem Jabre sollender merben, went man fic nisgenos lange aufo hált, unt $23 i n d$ und $23 e t t e r$ gúnffig find. 2Bit baben fhon getjert, $D a \beta$ Der gange $5 \$ 3 e g$ um Die Eroe eine Etrecfe von oingerábr 5400 Meeilen betrage.

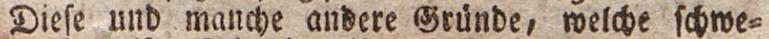
ree ju begreifen find, berweifen, Daß̈ bie erbe eime feht grofe Sitgel ift, aber eine unebene Rugel, megen Der vielen SBerge, weldse ouf Der Erbe find. Dod maden Diefe SBerge, fo hoch fie ard fum Eheil find, bei Der Erisfe Der erbe nidot mehr aus, als fleine Sanbtornet ouf ciner Reaellugel.

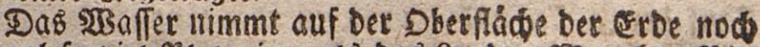

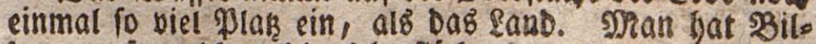
ber, auf melajen Die Sberfládte ber ganzen Erbe in Rieinen Dargeftelit ift; man nennt fre Eand arten. 2uf einer folden fandfarte fieht man jmei gtofe Sreife.

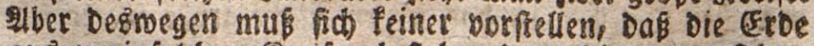
auz zwei folden Sireifen beftebe; benn Die ganje Dbera

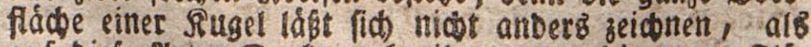
auf biefe 2rtt. Dentt euć, ibe roultet bie gante gld́= de eines 21pfets abbilben; mústet ibe ifn nidht als zoi erfabene runde Riteife Darfiellen? Betade fo muk es auch Derjentige maden, weldeer bie Erde abbito Den with, uno Daber fommen Die beiden grofen Sireife 


\section{3on bee croe unb iqren semob̨nert.}

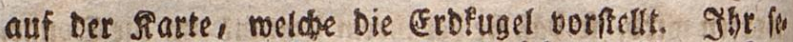
het, Daf auf Diefer Rarte einige Stutcte mit bunten gat: ben thberfitiden, uno einige reifs setafen find. Die

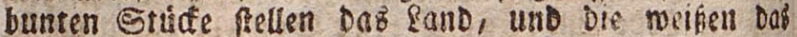
grobe 2Baffer vor, meloseb die ganze Erbe umglebt, und meer beiff. Das fefte sano Der Erve bat man in funf grobe Sheile getbeilt, weld)e Daher (E $\mathrm{r} D=$ S beile, Dber auco Melt. Eheile genasm merden. Gebet Eroftheil hat einen beionberen Namen. Der fleinfter

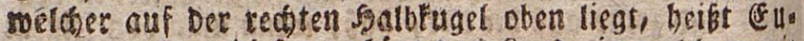
ropa, uno zu Dieferm gehort Dab̉ \&and, in melddem wit mohner. Datum pounen noir uns Europáer nenten. 2uf eben Diefer Sabblugel liegen now brei andere erto theile, melde 2 fien, Ufrifa uno Uuitraliett beisen. 2taf Der linten Şalbfuget liegt a merif a unt ein Ebeit bon 2ufratien.

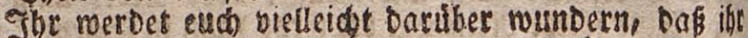
auf Der Eanotarte feine abbilbungen Der Stadote, SBerge?

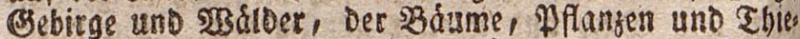
re, melthe auf Der Erbe find, fortberh lauter Namell findet. 2uber bedenft sur, wie ungebeuer grố eine Eandiarte merben míste, auf melcher bießs alles abge mast fenn collte. lind máre es mobi mógtia), cine for

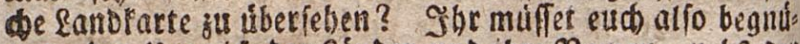

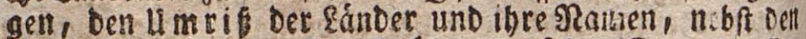
gramess Der vornebmften Erśbte, auf Der Rarte su fins Den. 2un oer gróften Sa)rift ertennet ibr bie शamen Der \&ander. Die fobmaizen frummen Enien zeigen Detr

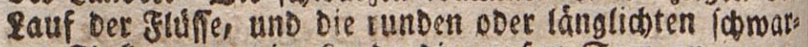
sen glede mittent im sanbe bie groken Seen an.

Dab 23 a f

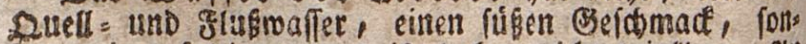
bern einen falzigen, uno if Daber nidst trintuar. 2An manden Deten wird Das unerméslide grofe speer febt. enge, Da nômlid), too ế von znet landern einges

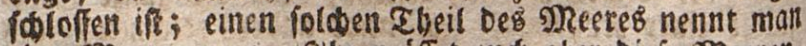
eine seerenge. She miffet eud aber Diefe Meeren gett nidts gar ju enge sorffetlen, fie fino menigftenz eine balbe oder ganbe Dieile, oft mebrere Neilen breit.

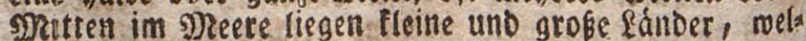
de oft gans you aneere umfloffen find; diefe merbell 


\section{Bon bet crbe und ifren soemofinern. :07}

Onfeln ober Eilande genannt. Sino fie nur zam Fheil vom פeere umgeber, to merben fie $\mathrm{Ha}$ alb Infeln genarnt. Der Fiand eimes Landeś am Mree re beift Die Rufte DDer Der Strand.

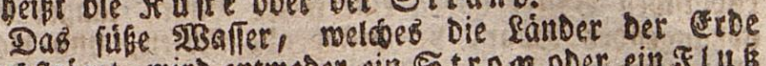

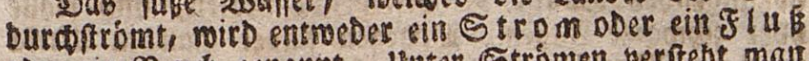
Dber ein $\mathfrak{B}_{\text {a }}$ क genannt. Ututer Stromen verffelst mant grofe fiefende esemáfler, melose fehe breit und rief

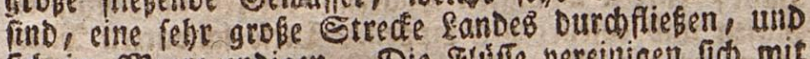
fí im Meere endigen. Die gुtuffe verenigen fich mit Den Etrómen, und find jum Theil aud febr breit uno

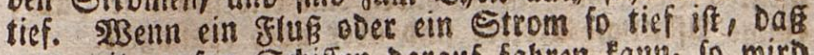
man mit groben Sdiffen Darauf fabren faum, to wird er foiffbat genant. Die Bertiefung, notm Das

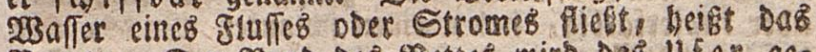
Bette. Der Rand Des SBetres wird Das ufer ges namut. In mandjen Tiúfent uno Strumen gieut es

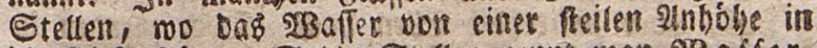

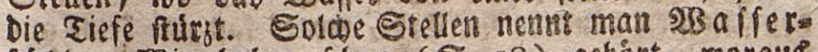
fálle. WBir baben fobon (S. 18) gebint, moraus Die gitife uno Etróme entfethen, unb weld be Bemálo fer man Seen ooer $\mathfrak{E} a n D=$ Seen nennt. Es giebt Eeen, melöre 20 und mebrete Dreiler lang und breit

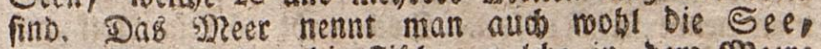
und baber werben bie fifohe, weldhe in bem smeere Ieben, Srefifore, und Die Echiffe, mit meldien mals auf bem Mreere fahnt, Seefobiffe genannt. Sagt man: Der Ses, fo if von einem Land= See bie Riede; fagt man: Die See, fo ift Dab̆ Mzeer gement.

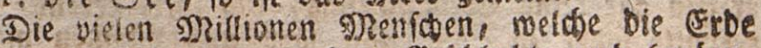

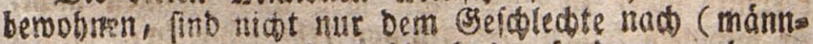

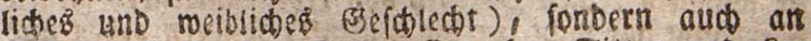
Eeftalt, Jarbe Der Şaut, Epradje, Eitten und Ses bentsart fehr verichieben. Dielentigen, melche in Einem sande beifammen mothen, uno einertei Beftalt, fato be, Eprad)e uno Eitten baben, maden zufammenge

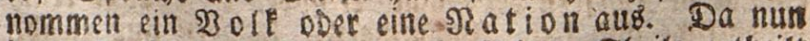
jeber Sheit ber erdoe wieder in fleinere. Sheile getheilt

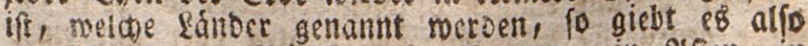

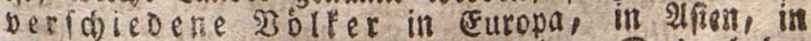
2firita, it stmerifa uno suftralien. Dod babes 
108 Bon ber Erbe und igren Benognetw.

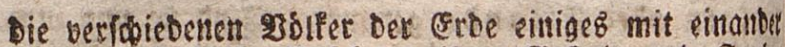

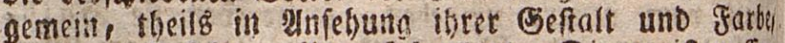
theils in 2infegung iftrer Rebensart. Die meiffen (Eu

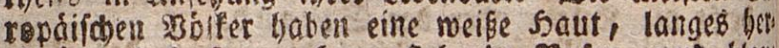

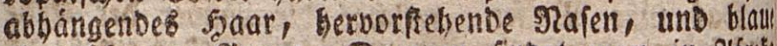
Doer yowarze 2ugen. Dagegen findet man in 2friti

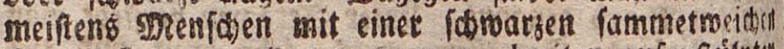
Sgaut, Purzen wouligien saaren, breiten aufgeftúlpten gajen uno rofentothen sippen. Diefe fobrarzen sgen" fdent werben Neger Doer Mrobren genamt. Dil meiffen SBerwohner affens habent eithe olibenfarbige Sauth

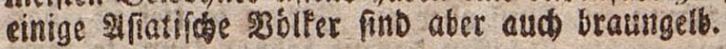

Die थmerif aner fino grofitentbeitb rotbbratl

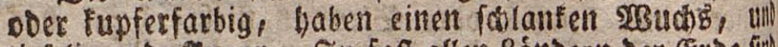

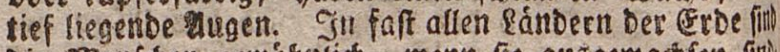

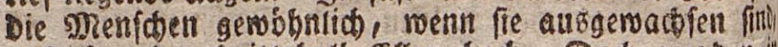
5 Euk, ober beittehalb Erfen boct. Dod werben in Den fálteften \&ánbern Der erobe, mo es faft gat Peine aty Dere Tabresseir, als Den $23 i n t e r$ giebt, bie sgenfats

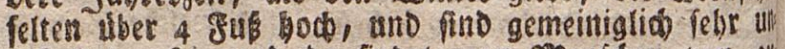
geffaltet. Shie uno Da finder man sientaien bon all

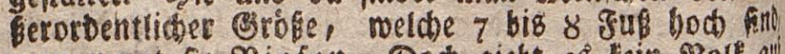

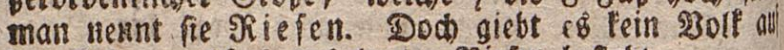
Der erbe, welches aus lauter Shiefent beffebt.

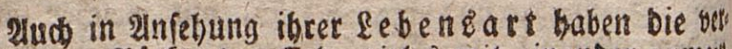
fobiebetren 20 blfer Der Eebe bieles mit einanber gemerl

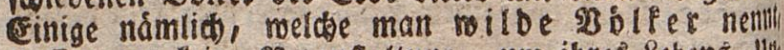

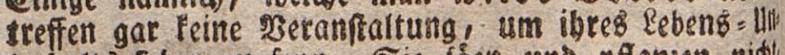
terbalts ficher zu fenn. Sie fáen unb pflanzen niabl|

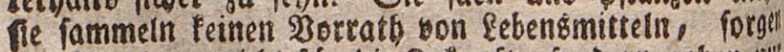

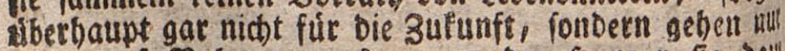
bann auf SRabrung auB, wenn ber Sunger fie Dall

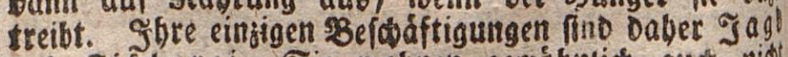

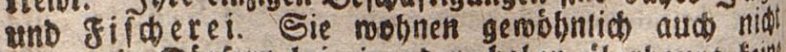
einmal in Dosfern bei einanber, haben tiberbaupt leith orbentlide und fefte sBobmungell, fonbern mar elente

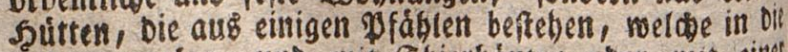
eroe gegraben, unb mit Ebiethauten ober mit einet

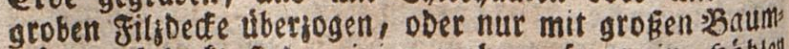

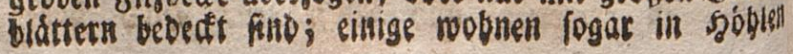




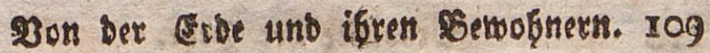

unter ber ertbe, unb gemofhntid fiehen bei biefen mits Den 20 bifern nur mentige Jamilion (Etámme) mit cin= anber in sertinoung, meiche aber feinen gemeinichafts lidben Dberberrn, feine Dbrigfeit, fondern bódffent im Siriegs, oder bei einer gropen Jagb enen 2 infulh = ter baben, Dem fie fo lange geborden, als Der serteg DDer Die JagD Dauert.

inbere golfer Der Erde, melde firtenbolfer Dber शromaden genant werben, haben jimat aud

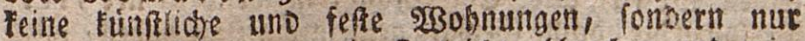
3elte oder Shitten, meid)e fie leidit abbrecten und roies Der aufiólagen fonnen, aber fie fint Docb viel verfánts Diger und gefitteter, als Die wiben $\mathfrak{B b} / \mathrm{fer}$, meil fie

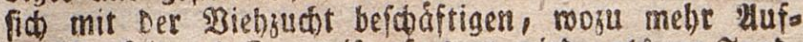
merffamleit unb Renntníf erforbert wird, als sur Sagd.

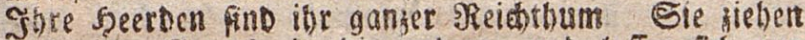
aub einer Begent in bie anbere, unb laffen fich nur Da nuf eine lángere zeit nieber, too fie gute $2 B 3 e i d e=$ Pläbe antreffett.

Nod andere ?olfer auf ber Eroc, meldis gefit. tete Bolfer genannt merben, befodéftigen fidd, auker Der Biebsuct, auds ned mit Dem 2l de epbaue, tind Dertichen allertei sinute und bुandrotfe. Sie

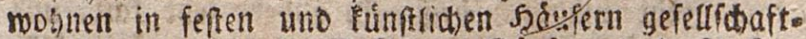
lid) bei einander in Státten, Dórfern uno gleden.

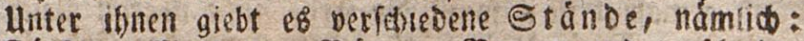

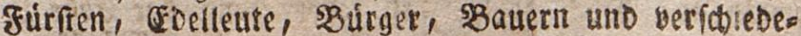
ne Berufzartell und Bewerbe, inbem einige ben

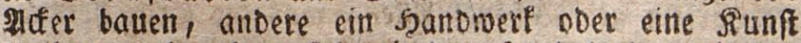
treiben, nod atbere fis mit Dem Santel ober mit Den

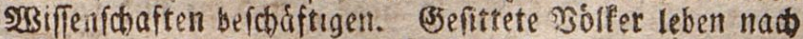

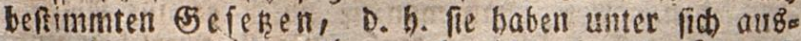
gemadit, mas̉ jeder thum und nicht thun barf, und mer unter ibnen mobnen will, muk veriprechen, fid Diefe Beferge gefallen ju laffen, und fie zu beforgen. Damit Diés von allen, auco bon Den unverfándigen uno SBosartigen, gefdeblen móge, fo máblen fie unter fith einige verfándige und reditichaffene Nånner, und geben ibnen Den 2uftrng, Darauf ju fetien, baf Jeber Den Befęen geforlam led, und Den Hngeborfamen ju 
freafen, went fie nicht auf Crimuctungen adten. Dil fe Berfonen merden bie sorigleit, uns eine fold grofe gefelliobaftliche 3erbinbung wirt eine burget

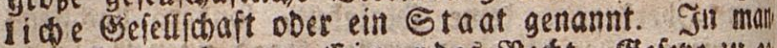

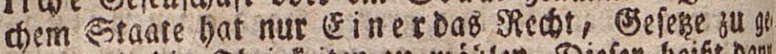
betr, uno Die Dorigfeiten zu máallon. Diefer beift oanl

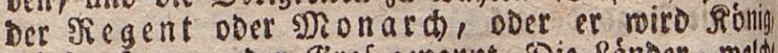
gurft, Jgerjog ooer Graf gerannt. Die Ranoer, meld

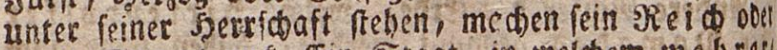
feiten Etaot aur. (Ein Etaat, in welchem mêtret Derfonen bie bódife (Serwalt gemeinfơaftlich babeit, with ein ein Eceiffaat ober eine se epublif genant.

\section{V. \\ 90 rodute Der erot.}

Da bie suft niat in allen Eegenbent ber Erbe bielel be $B$ ifichaffenlyeit hat, fondern in einigen Iambern od ganze Tabr hinburd beis, in anbern felye falt, unt wieberum in andern meder fu marm nod its falt, fot" Dern gemáfigt ift, to ift bie eroe niot tibernil gleid frubtbar. Dod bringt faft jebeb land ber croe is siel berbor, als feme sBemobner zu ibrer Eethaltum nothourftig gebraudent. शlles, mas bie erbe berbot" bringt, nennt man ibre Probufte ober Erzeug niffe. Thre $3 a b t$ ift fo grob, uno fie fino von fo berichiebener att, baf man fie unter gemiffe abthelo lungen ( von einanber unterfitheides zu fonment. Diefe gibtheis

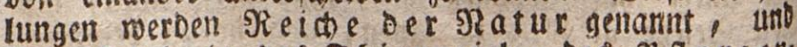
ibrer fino orei: Das g gierrei co, bas pflanjello rei a) uno bas sn in eralrei D. Diejenigen Lánden in nelöen gefitfete soblfer mohnen, baben mand) pros Dufte, befonders aus Dem Pflangenreicto, im Ulebers fiufle, meil ibre soerogher dab lano febr forgfartig 


\section{Probulte ber cerbe:}

bebautn. Sagegen febit es mandjen Lanberi getabe an Diefen \$rodutten, weil fie cinen unfrudtbaren $\$ 30=$ Den baben, oder fotrdat angebauet fino, aher fie bas bell mieberum andere probufte im lleberfluffe, melate Die gratur felloft berborbringt, f. M. Metalle, Sol? Doer Eals. Daburch find Die sgenforen auf ben Bes banten gefommen, Die uberfúfigen Produfte ibres fanbes nads fol(t)en fdubern binzubringen, wo $e^{B}$ an Diefen provuften febit, fie Da gu berfaufen, uno fic fúr Daś gelofie Beld Die ibnelt felglenden Protufte einzulaufan. So ift ber Shandel entfanden, mobei bie \$Baren entmeder zu Lanbe, vermittelft Der 5 Bagen unb faftbiere, oder auf Den gluffen uns auf Dem Meere, bermittelft Der Sthiffe, aus einem fande in bas andere gebradot mocroen.

\$Beife uno gutig bat eह Bott fo eingerichtet, bas jebes Eand, Doer menigftens jeder grope Erofrica), Das beist: leber betrádtlidte Zgeil Der Erde, gerabe diejes nigen Probufte hat, melde für die szeroobner beffelo ben, nach Maaßgabe Der Switterung (DeB Silima), Die nothnendigften uno mohtthárigften fint. So bringen 3. 23. Diejenigen fánber, meiche eine beife Suft uno teinen 23 inter baben, Die tráftigften, faftreiôften uno

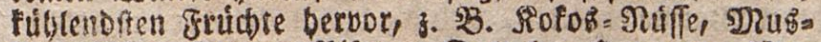
Fit = Salffe, Dliben, Difang, Datteln, Drangen, פRe=

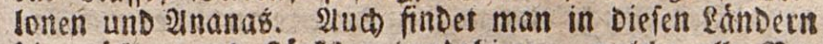

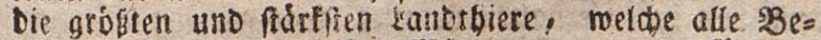
fobrerlidferten ber beifen witterung ertragen fonmen, obne Daburdi fll verderben, 3. S3. Die (E lephanten,

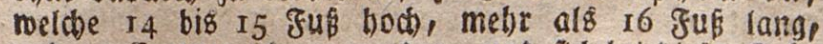
uno 50 (Entmer idwer merben, und fich bei biefer (Bri)= Be und Sctroere dennow fo leiot bemegen, Dấ fie tágs

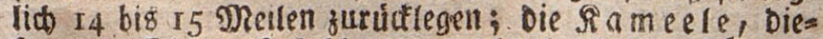
fe vortreffticoen Lafttbiere, welobe in heifen Lánbern unentbebrith finb, weil man Io bik 14 Tage mit ibnen Durd) brenmende uno mafferlofe Eanomuiften reifen fann, obne bas man notbig bat, fle fu tránten, uno sie mit

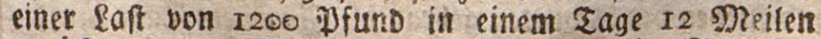

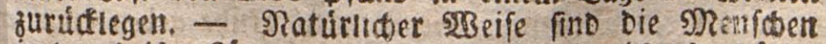
in oell beisen fánoern nicht fo farte uno triont fo thátige wie in Den gemápigten gimmelêftridpen, uns Darun 
Wat Bott ben $\$ 30 b e n$ in biefen Lånbern fo fructitbar ge mads, Daß er beinahe obine alle SBearbeitung bie fdónte feen fritidse in Dem groften Heberfluffe herborbringt.

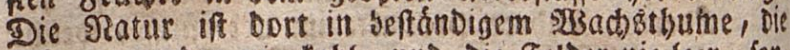

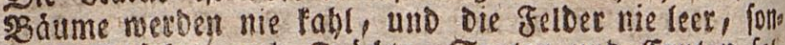
Detn 2Bluthen unb Sruichic, Saaten und Ernten fols getl ununterbroden auf einander. Da Die sgemofint

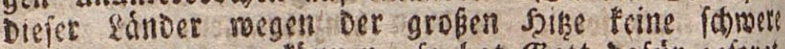
Sileibung etrragen tomen, fo hat Bott bafúr seforgth Dás Die EetDenraupe ithen Durw ibr feines esemelte Die leiditefte fleibung veridsafft.

(janz anbers find oagegen bie Đro Dufte der fals

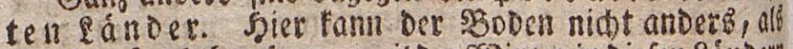
Go def unfruditbar fenn, weil ber sisinter in diefen Ránderth mur für menige 230 chen auffort, und bie in Den lath gett Epmmettagen unglaublich fobnefil emporgemact)ient

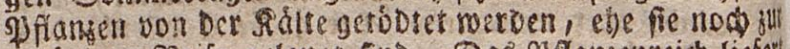
genórigen Sicife gelangl fino. Das Pfilanzenreid) liefert

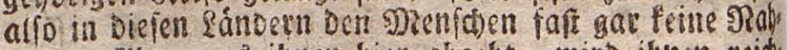

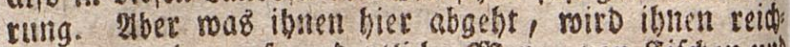

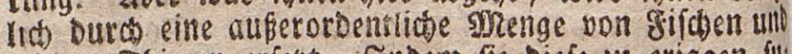
wilden Ibierela eríckt. Indem fie biefe fu erjagen fü

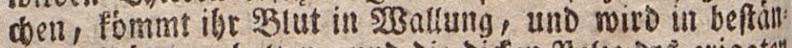

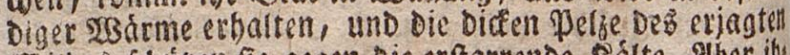

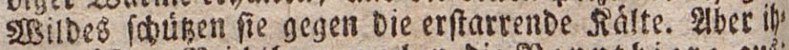
ren gróbten Sieidsthum macher die gienntbiere aüi Denn bon itgnen ethalten fie alleb, bos mit bon unferem Rindovieb, unferen Pferben und Sdbafen erbalten, unt

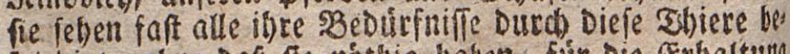
fricoigt, obme Daß́ fie nótbig baben, für Die erbaltunty Derfelben Die geringfte Eorge fu tragen. Die gants

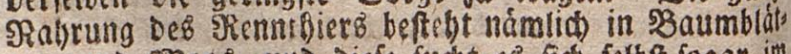

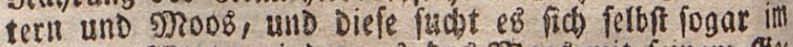

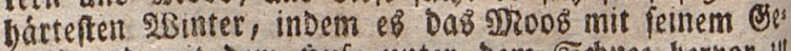
meih) und mit Dem șufe unter bem Schure berbor fll

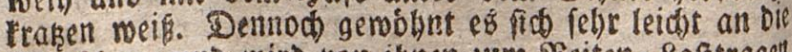
g) Renichen, und wiro yon ibnen jum SReiten, Eaftragen uno Biehen ber Schlitten gebraudbs. In einem Sage lauft

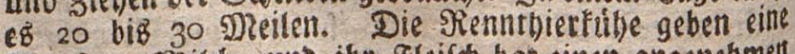

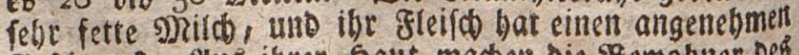

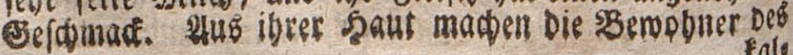


farten Eroftrids igre Rleiber, Shube, Satte, Bett= Defen uns anoere Dinge. 2üs ibren Siornern niffen fie allerlei Berátbe, aub ben sinocsen speffer, soffet und Sikdeltr, uno aus Den Dármen uns Eegnen Etrit:

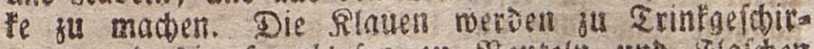

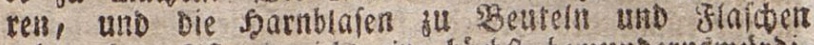

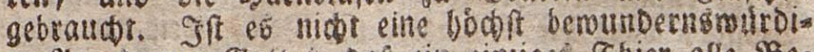
ge Anoronung (Sottes, oấ ein cinjigeb Thier alle SBea Durfniffe Deg Ineniकेen befriebigt?

Der erofrich, in meldsem mit mobnels, bat wea Der eine febr beibe, noos eine febe falte, fonbern einte gemábigte zistiterung, belde fico oft veránbert? unb eben Daburch zur (Erjetugung uno Ernábung ber

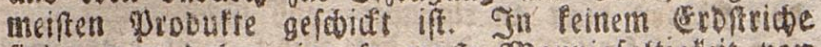
finbes man baber eine fo grobe פmannigfattigteit von

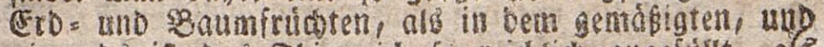
nirgents if bas Tbierscich fo reicblict angefullt, als in Diefem. Pleferbau und giebsuch find die beibent

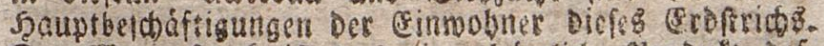

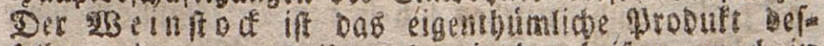
felbetr, bein er gedeiht meder in Den beipen, nod is Den Palten erofricher.

\section{Das Ebierreios.}

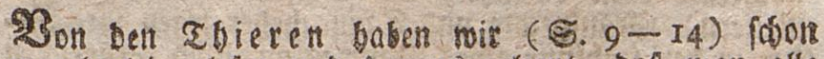
mancberlei gelefen und Daraus gelernt, Das man alle כoiere, welwe auf unt in Der eroe, im $2 B$ affer und is Der Euft leber, am beften non einandeb unterfósiben fann, menn man fie unter forgende 6 absbeilungen DDer Staffen bringt: S\&uggetbiere, शुogel, $21 \mathrm{~m}=$ phibier, gifoe, sufeften uno wirmer. MHe Eyiete baben bieß mit einanber gemein, dás fie einen Nund ( Mraul) baben, Durd nelden fie bem siorper feine Tabrung fufubren, und baf fie, bom folmger getrieben, milltibrtio ibre श2abrung gu fic nebmen. Dabei werben fie bon ibrem Raturtriebe ( Sn. finft) geleitet, unb bor alfem, rab ibnen foriofido ift, bewahrt. Diefe शRaturtriebe erfesent bei ifnen Den slangst Det Sernunft, uno find bei einigen Thieren

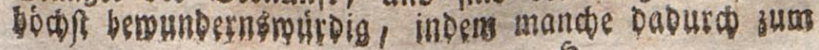
S? 


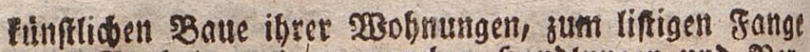

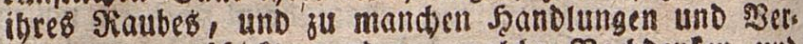
ridtungen gerdidt merben, meldse sadbenfen und IIrtbeilbfraft fu exforbern fobeiten. Sone vorberges gangene Anweifung uno Hefung madt Die junge Exill

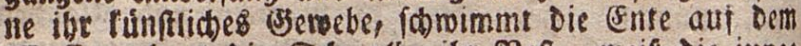

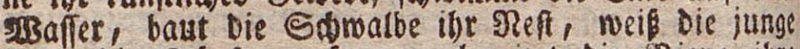
Rake bie smaufe fll fangetl, bereitet bie BBiene ibrefunftiden Zellen. Eben fo benunbernsmutbig if tis

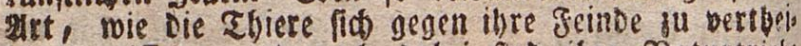
bigen miffen, und aud Dabsi find ifre Saturtriche wirffam. 2asenn bie Pferbe auf Der $2 B e i d e$ yoh cinem 2Bolf angegriffen werden, fo ftellen fie fich afle mit Den Sippen Dicht an einanter, und maden auf biele 2urt einen Sireis, in ben Der 2 Bolf nicht eintringen fann, weil alle mit den Şinterfúsen ausidblagen, und ifn Daburds zurtidtreiben. Die Dafen madoen of un: getebrt, uns vertbeibigen fich mit ven Sómernern. (Eis nige Thiere, welde im 235 fier leben, madien bas 23 a fer trube, unb entsieben fio fo Den Derfolgungen threr

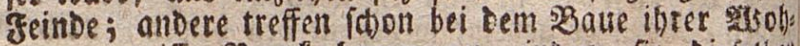
nung gersiffe 9 orfebrungen, inoem fie Diefelbell 1. B. in Didoten Dornfrtáudern, ober in ciner Felfen, ipalte verfecten. Die Elffer bebeft' ibr fúnflich ge

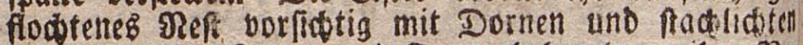
SReifern. Braupecte uno Eannenbeber legen ible S? fter in ber Sfoblung eines SBaumes all, und verfreichen oie uberflusige Deffnung mit (Ee)m.

In Unfebung Der fabigleit, ju empfinben, nimmt

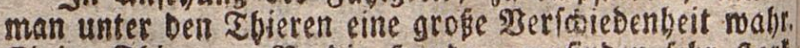
Eirige Tbiere, o. SS. Dis Şunde, empfinden lebr ftarl. sasie feht freuen fie fid, menn tie nad einiger seit ibeen Serrn wieberfoben; wie traurig finto fie, wettit fie ibren Sertn vetloren baben! Dagegen bemerl man bei vielen Ihieren, bejonders bei den Infeften und bei oen fificen, faft gar feine empfinoungsfábigs

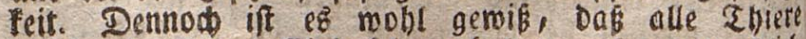
Durd Die Sinne Einorudfe erfalten, of man gleid an manden gar feine Sinnenmerfzeuge entbecten fann. So baben 3. 53. Die Edsmeiffliegen und andere Sin: felten offentar Den Sinn Des Genudb), ob man gleic 
leire seafe an innen bemertt, uno bie sithe boren

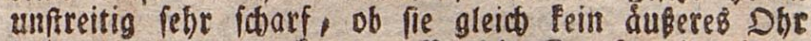
habell, Denn man fans 3. 35. Die Siarpfen in einem zeiwe baran gerobbnen, Dak fie auf Den Schall eimet Bilode fid verfammeln, um gefuttert zu merben. Eit. nige Eviere baben gan; auferorbentlich lobarfe Simne. 2on einer unermeßliden șbbe berab entbertt ber 210.

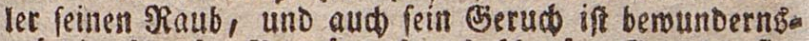

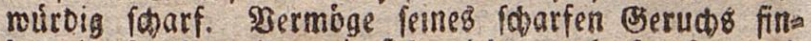
Det eill Sfund meilenweit fic mieber nad Saufe, und eben baburd entideft er unter vieten bundert Menrcoen

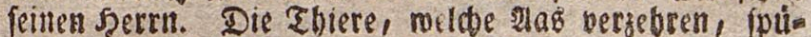
ren sin tobteb Shiet auf einter Strecte bon mebrevels

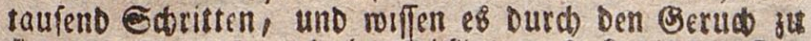
finoen, wenn es aunh im Didfigt verifectit liegt. Der

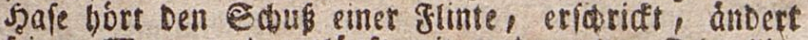
frimen

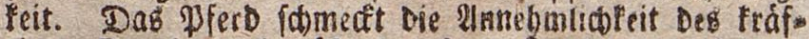

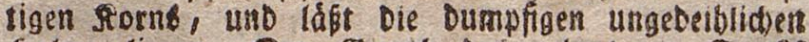
Salme liegen. Der Beruid Des getratenen Epects lofit bie sraub aus ibrem meit entfernten Schlupfinine

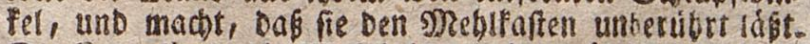

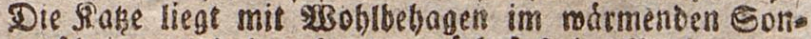

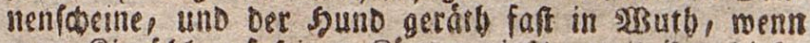
man Rienbbl auf feinen Robrper giefit, reil ibm Diefer

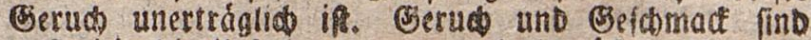
unftreitig Die Uriachen, warum Das grobfere STornviel

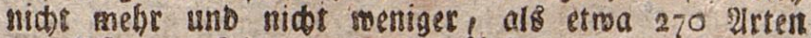
Don Pflamzen frige, unt alle ubrigen freben lákt, to fóson uno frdfrig fie aud fenn mógen. Daß̉ Pfero nábrt fich mit 262 pflanjen=2Urten, Die Sdineime frefo ferr beren nur 72 , uno beríhren feine andere, alb bies fe, wein fie aud nod to bungrig fint. leget einer Siaupe 30 yeríbiedene sarten von SBláttern vor; fie mirb vielleidt nur cine simbige est benagen, und alle ubrigen unberubst laffer.

2 Benn Die Ehiere enttráftet fino, und Dee Erbio Iung beburfen, fo fuden fie einen fittern unb beques mens Drt, und fallen in Den Schlaf, in weidsern mant de, 6. 23. Die Sgafen und Bemfen, Die 2lugen offen bebalten und aum mobrt tráusen; menigftens bemerfís 
man an ben Şumben, bafk fie oft ims Sdylafe bellen uno fnurren, wovots une ein Traum bie lirfacbe fenn fann. Einige Ifiere, befonders die SPaBen, de Eu= Ien, und verfobiebene SRaubttiere fotafen ber Iage, unb geben Des Prachts auf Raut aur. Pon Dem ges

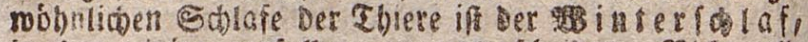
in Det einige yerfallem, fu untericbeiden. Sobt alle

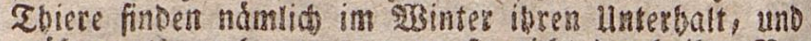

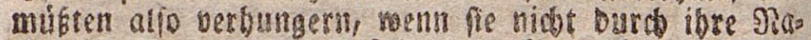
turtriebe vor Diefer Qiefabr gefóstimt moirben. Biele bereiten fich namtid im Serbfir mit benumbernzswite

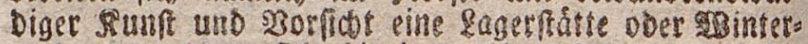
mobnung, legen fio binein, uno erftarren, bis bie SBarme Der Zrtublingbfonne fie wieber medt, uno in Det SRatur netse SRabrung füs fie bersiter ift. Diefe Erfarrung ift to frat, baf Die warmbtitigen Sbiere 3. 33. nie spurmeltbiere, mábreno Derfelben nur eine

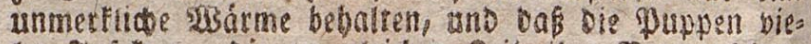

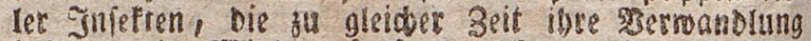
befteben, im sBinter oft fo ourctifroren find, Daß fie rie Eibzapfen, ober Blas flurgen, wenn man fie auf Die Eroe mirft. Dennoco abes lebr bas Darin follafen

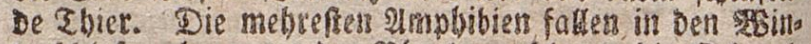
teríd)lof, aber unter Den 230 getn mogl nus die Sobral ben. PRande shiere erfearcen zwat nids im sointer, legen fid aber in sgerbfe tumfwolle und gut verwabre 2Borratbifammers an, unb tragess cine 9genge von פabrungs̈mittein barin fufammen,

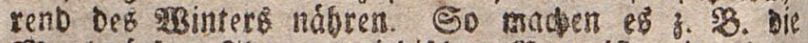

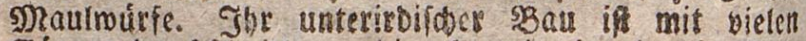

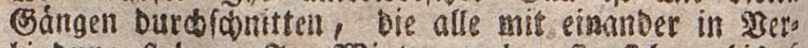
binbung fteben. Im sessinter grabein fie fid 5 bis 6 guk tief ein. Shre eigentlide 2830 bnung ift ein rebe

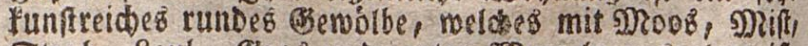

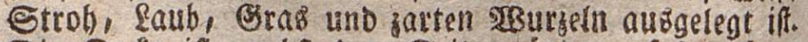
Die Dede ift, nebft ben Seitenmánben, feft zuams mengebruidet, und finftliob geslártet. Untes Dem Edonce. múbs len fito bie Naultourfe lange Bánge, unb graben

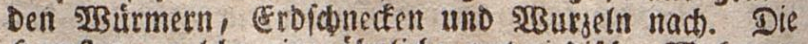

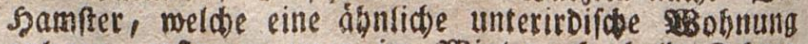
anlegen, erftarren zwar in 2 Binter, to bald Equef 


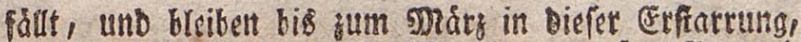
fammeln aber tod in Syerbfte einear groṕen sorrath yout fiorn, Den fie nichs eber angreifen, bis auf Dem Ferbe gar niouts melie gu finden ift. Won bieferm

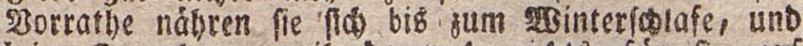

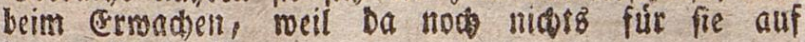
Dem Jelse ba ift.

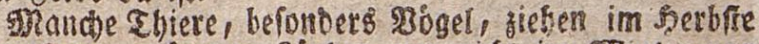
in eutfernte wámmete Rándes, um nitor im 2oimer vor Sålte uno Scunger umatommen, unt feloren im frubs

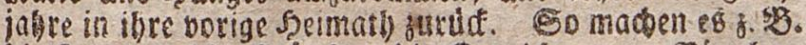
bie \&erden, bie Storoje, Die seraniche u. a. Bógel.

Erie inge Sungen forgen bie Thiere mir auberor: Dentitoer riebe. Edton bor ibrer \&eburt betriter fie ibnen cin meiched unb marmes Lager, uno zroar gerade an einem fold)en Drte, roo fid bindanglid)es Jutter fir fie finbet, unb wo fie bor lleberichmemanugen und andern Eefabren gefidert find. Einge Sbiere bringen lebenbige Junge fwr Trelt, und färgen fie an ibrett sbruiten mit sphilá) (Die Edugethiere); andere legen Eier, woraub Die Jumgen in Furber 3eit vermitrelft Det

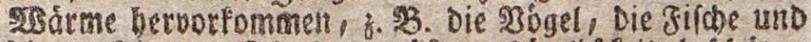

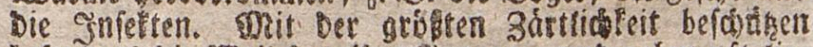
befonoers Die 25 eibden ibre Stsngen, unb geten oft lie= ber ibr eigenes \&eben bin, alb baf́ fie bie Jatyen oem ráuberifhen Zeinte uberraffet.

\section{(क) \& u e t bie ic.}

Die Sångetbier e fino grofitentheils bierfúpi= ge Ebiere, aber ef giobt aud sonter ifnen folthe, oie

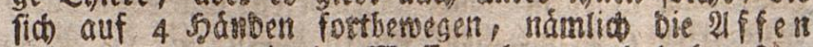
und andere, melche in 25 affer ieben, ento Daber fatt

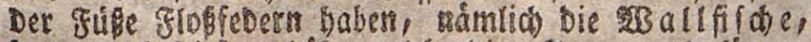
Denn aud Diefe gebóbren lebendige Sunge uno fánen fie, gehoren alio eigentrich nicht gu ben filden. - Der Rorper ber Saugetbiere if mit 5 a a e en boit febr ber= fobiebenter Státre, Range uno zarbe bebectt, bie aud bei einigen (\%. 23. bei Den Eobafen uno subein) wie 230tle getraufelt, ober als sborften fraff unb fruppigt find (3. 3. bei ben Sdmeinen) ober bie gar, wie beim sgel, fteife Stachein bilben. Bei manden fino Die 


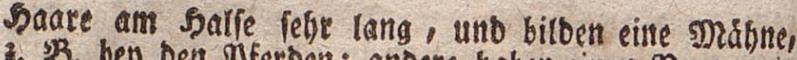
2. 3. ben Den Pferben; andere haben einen Bart, mie

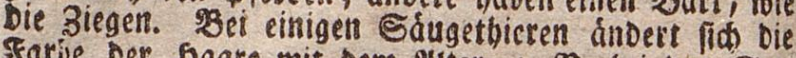

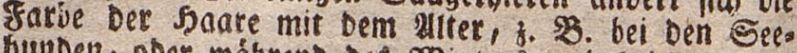

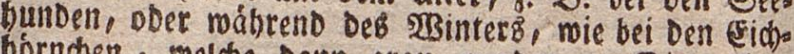
bornchen, weldhe bann grau reerben. - Die mebres ften Saugetbiere leben auf Der croe, unb mande, wie

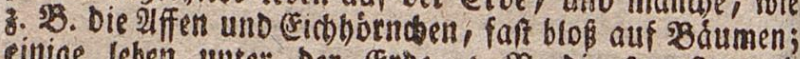
einige leben unter Der Croe, \&. SB. Die Sampter und Die Maulmuirfe; andere balo auf Dem fanoe, balo in 2rsaffer (Die SBiber unb bie Seebáren); nod anbere blog im $23 a f f e r$ (Die 2 ballfifice). Die Jinger und

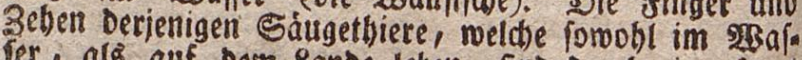
fer, alb auf bem Eanbe leben, fino ourd eine Şaut nerbunden, meldbe man bie Edwmimmbaut nennt, meil fie ibnen zum Sthimmen behurflict ift. Bei Den fles

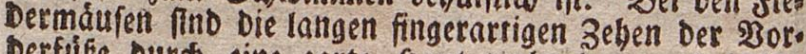
Detfúfe burd eine jarte Şaut berbunden, und Dabet fonnen fie ein menig. fliegen Doer flattern. Sie fino Die einzigen fiegenben Saugethiere. 2uf Der erbe fonnert fie nur fredien. - Cinige Săugetbiere baben borm artige Saufe, ndimlich Die Dferde uno bie Efal, viele baben gefpartene Silausen, 8. 20. Die Stbafe uno Die Dhfen. Die mebreften geben blö auf ben Beben, sur einige auf ber ganzen Subfohle. - Nuper Den §lauen unb Bábnten baben viele Sdugetbiere aud nod Sgirner erbaltent, um fid gegent ibre Feinde ou mehs ren. \$Bei ben fsirfiten fino bie f̧orner wie थefte cines Saumes geftaltet uno beifen (3) meibe. Eie rorben gemóbntio in jedem Sabce abgeworfen, uno Dann burd netue erfent, meldbe melar Enden baben, als̈ bie alter. Im februar und Nár: vestieren die Sirfhe ibr (seweib, uno fópn nad) 3 biz 4 NRonaten baben fie ein metres, weldtes anfangs febr meit ift. Die grofiten Semeibe baben felten mebr, alb̈ 24 Enden. - Einige

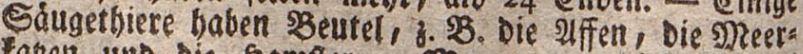
Faßzen uno Die Samiter. Man nennt Diefe \$Eeutel aud SBafentajden, weil fie an jeber Seite Der untern Sinnlabe aís badutige इafdent fiken, und von biefent

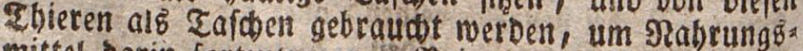
mittel barin fortz̧utragen. \$B. einigen \$eutelthieren 


\section{Probulte Det croe.}

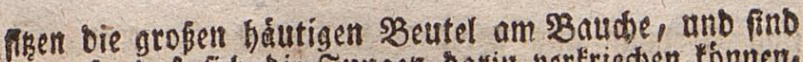
to groß, baß fich Die Jungen Datill Derfiried)en tonmen, menn fie faugen mollett. So ift $\mathrm{eg}^{8} \&$. SB. bei ber ssetle telratte. - Die $\mathfrak{Z}$ raudbarfe it o er Sdugetbies re ift auberorbentlid grof unb vielfact. Zum Reiten, Siebett, Raftragen unb fum Mderbaue bienen Dem פ?eth=

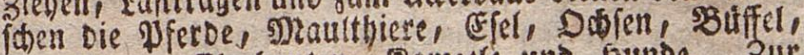
Rermtbiere, Elophanten, Sameere uno Junbe. Jut jago uns jun servact)en feines cigentbumb bebient

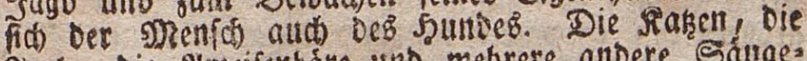
Jgel, Die Pincifentare unb mehrete anbere Săuges

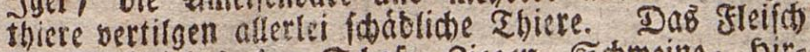
Deb sinboiebeb, ber Edjafe, Biegell, Stomeine, Jair = fobe, Sgafett Sanimbett, uno in einigen Ránbern auch

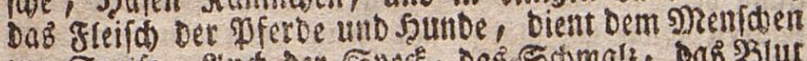
zur Epelie. 2ud Der Spece, Das Samaly, Dab 231 ur unb bie פritch Det Thiere Dient unz jur SRabrung. शu8̊

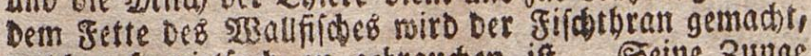
meldier fo vielfadi zu getraudsen ift. Seine 3unge

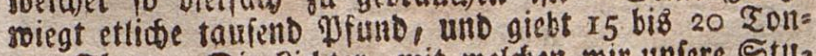
nen Tbran. Die Ridter, mit weld)en wir unfere Stu. ben crieuditen, find aus Dem Jette Der Defien wis

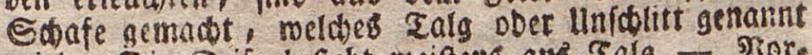
soirb. Dis Eeife befteht meiftens aub కals. - 230r. aiglio) grof und ausgebreitet ift Der SuBen, melwen Die fjaute uno felle Der Sängetbiere, iste Szaare, und befonderz ibre 280 lle ben פrenfonen gemábren. Man. de Felle werDen to zubereitet, Daf Die Sagare Daran bleiben, umb Dann beifen fie \$elzmert. CE ift Das

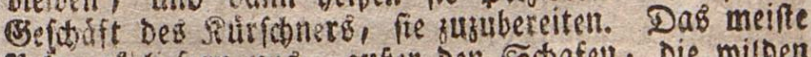
Delgmarf lisfern uns, aufer Den Echafell, Die milben Shiere, befonbers bie Zuchfe, zobel unb Sermelime. Die Jeáute ber milben Sdwoine unb Eeebunde mer. Den zam Befid)(agen Der Sioffer gebraud)t. 2 Benn Den Sakuten ber Shiete bie fgare genommen, und fie meich unb geidsmeivig gemadt roorben fint, fo merben fie Reoer genant. פRit Der 3ubereitung bes zeoers be=

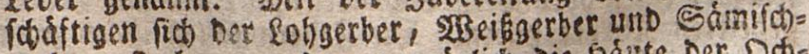
gerber; fie bemusen Dazu vorzúglio Die Jadute Der Da. fen, Ráber, Edjafe, Riebe uno ziegen. Eaffian, ein fónes glémendes leocr, mird aus Siegenfellat 


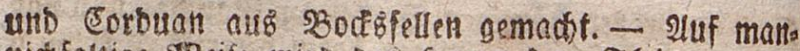

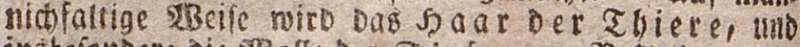

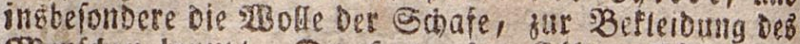
spenforell bemust. Die Saare Der fibe, Sidiber uno

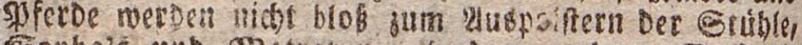
Sopha's unb Matrabell, lonbern auth fut \$erfertigung einer alrt bolt Danroffeln (Bárlatfósen) uno zur 33eteitung De8 bantucbes gebrouct), Defen feimere Inten sinigen smenfoen fur \$cileioutg, fo mie bie

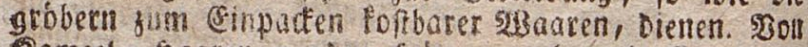

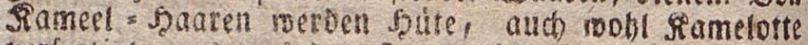
berfertigt, unb aus Den Şuaseit Der यngoriföen 3iege wiro Das fiameelgern gemadt, weld)es zur Berfertio

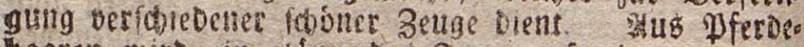
baaren with ein glángenber Settg verfertigt, mit meto den man Stuble tbetziebt. 2udt) fur Besiebung bet

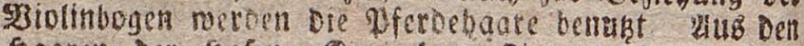
Saaren Der flafen, Senmuben, Bisgen, Spunbe umb SBiber berfertigt ber foumader grobe unb feine Sute. 2us Sd)afrodle madit man auf Dem isseberfuble fol:

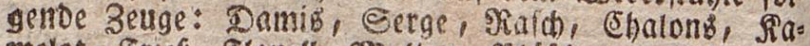
melot, Jtré, flattell, פrolton, Diuích uno Belbel.Die $\$ 8$ orft en Der Scheme gebraumt Der SBurftenbin= Der. Die ssemeibe, Die Sobrner, Die 3dbne (befontoers (Elepbartemábne oder (Eifenbein) uno oie Rnoben der Skugetbiere werben bon Dem Dreobier auf allerlei int

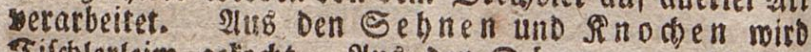

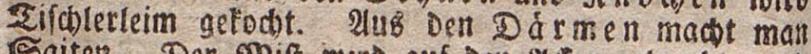
Saiten. Der gnift wiro auf Den Mater gebradbt, unt Dient fur Diungung ( Sruchtbarmactung) Deffelben. Ï hoifarmen Begenden bedient man fiot aud DeB̉ troofes nen Diftes fur feuerung.

\section{3: 8 i $i$.}

Die Bogel Fommen in 2trielung ifrer Bilbung

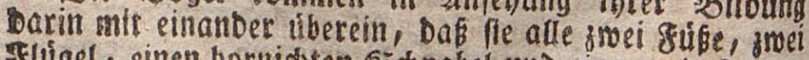
Flígel, einen bornid)ten Échnabel und einen mit Jebern

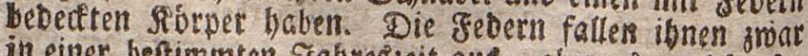
in einer beftimmten Jabrebseit aus, aber es madbfen fo=

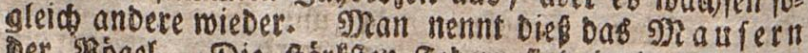
Der sogel. Die fárffen gebern fino in ben fittigen 


\section{Probufte bet erbe.}

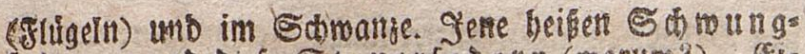
feoern, unb Diefe Steuerfedern (marum?). (Els

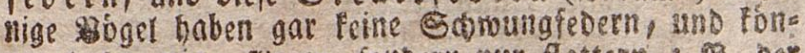
nen taber niwt fliegen, fonbern nur flatterm, 2. 3. Der Strarf, Der Refaar unb Die Pinnuine. Die mebres

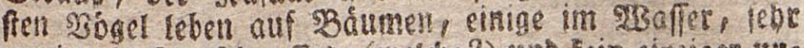

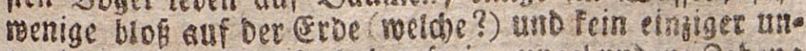
ter ber Erbe. Deande haben freie, ungebanterre Behen; bei anderr find Die Belen Durch' eire ectroimmbaut bers

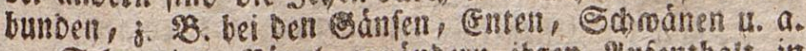

Eebre viele PQbgel beránbern itren 2ufentbait in

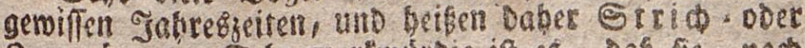
Sugoogel. Eebr mertnutroig iftes, Daf fie, nact einer fo langen 2ltwefentbeit, immase ibre alten seefeer mieberfinden. Die Droffeln uno Sirammetsobog gl zieben

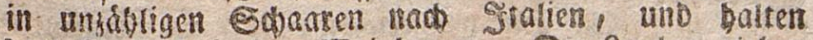

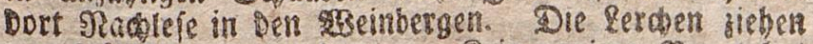
am fpatefer son tuts weg. Reen enziger Vogel hat Zátme, forbern diefe Thiere múferi ibre Sperie ento meber mit Dem Echnavel gerbeisen Dber ganz beríchluf"

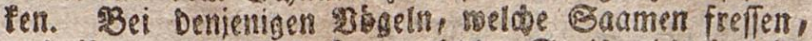

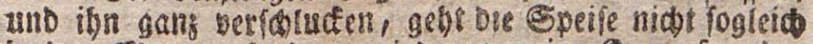
in Den nagers, fortern wird sabor im Siro pfe doer

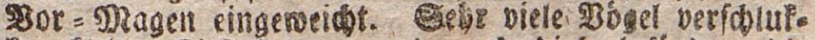
fen fleine Rieferffeine, uno and Diefe befórbern die

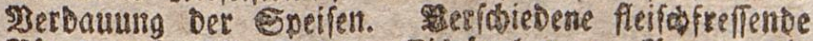

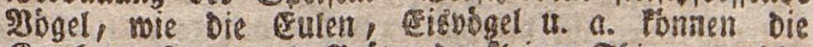
Sinochen, şaare und Britren Der Pleimen Thiere, melche fie veriebrt baken, nicht berbaten, fontern geben fie,

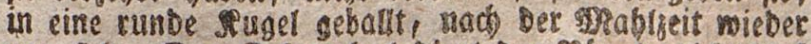

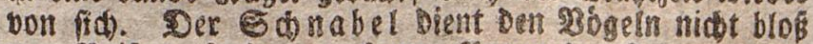

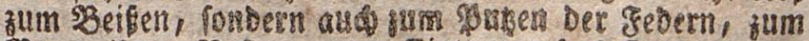
s3aue ibeer SReffer, zum eintragen Des gatters, jut

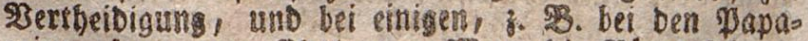

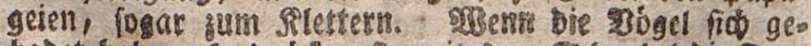
baøet baben, fo brdefen fie mit Dem Echnabel Die Eetts Druffen an Eseife, auß meldiem Daun ein feineb Del Dringt, und mun sieben fie bie geDern outc Den mit Def benenten Estinabel, ober befrteichen fie mit Dent

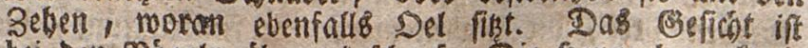

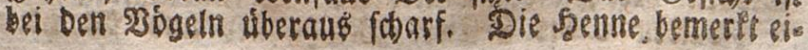


nett Spabiot in einet Entfertung, wo ifin fein menidilis

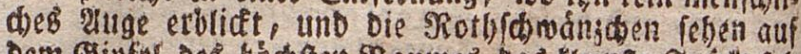

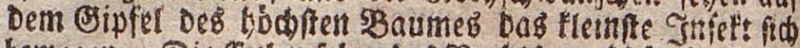

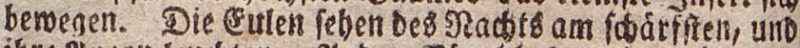
ibre Mugen leuctent. Anbere \$ogel baben eitien tiberaub

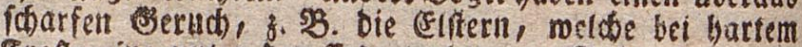
Srofte eine unter ber Eroe verborgente Mabe riechen. -

Die Borfidet und gelugbeit, mit melder bie 230 gel ibre Refter gerabe an foldben Drten anlegen, too fie am leidsteften igre \$Seburfutiffe befriebiget, uno

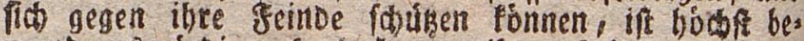
mumbernsmirbig, fo bak man ifnen faft meniobliobes शadbbenten unb verftánbige lleberiegung zutrauen mócs fe. Ebent fo borfiotig wákit lebe Eattung bie 38 a Materialien fu ibrem Nefte. Dicienigen Dogel, melde in beifen Sgimmetzftriden, ober an fobartigten Drten niften, nebmen iu inrem soute utut leidten uno

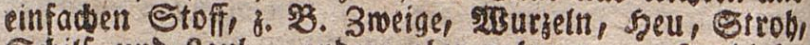
Edilf uns Eaub, anbere aber nebmert, auser Diefen

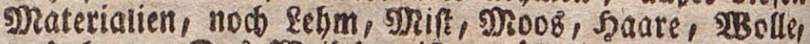

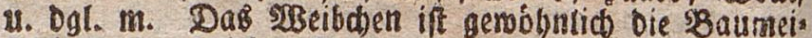
ferinn; nur bei ben Scjwartben berfteben fich beide (S): falediter auf Dab Nefterbauten. Die Beftalt Det Nes

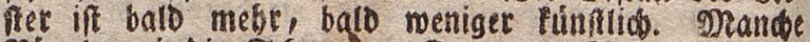
эsógel, mie die Samepten, Stappen, Ribise u. a. ma" den fió) bloß ein einfadies Eager bon Reistiol uno Strobbalmen auf ber platten erde; andere bereiten fić ein Funftofes SBette, in Den Ródern Der Mrauern, in bell

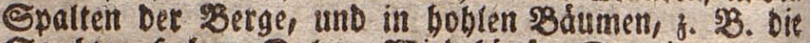
Spedte, Sgeber, Dolet, reiebehopfe, Spertinge u. a. Sebr viele, befonberb unter Den Siflneen, Tauben unb Singoigelti geben ibrem Refte Die Beftalt ciner Salbs tugel ober ciner Souffel; anoere, wie Der Zauntonig vie Beffalt eines sBadofens, nod anbere bie zorm ei nes sBeutels. - Essenn Das Neft gebauet if, fo $\mathrm{lgg}$ Die smatter ibee eier bintein. Die 3abl bet eier if bei Den berfaiedenen Battungen Det 30 bel fehr ver

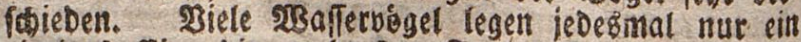
einzigez ei, bie meloreften Eauben legen znei, bie Mosen brei, bie Faben vier, bie ginfen fünf, bie Sqmalbent 6 bis 8 Eier. Rebbugner und 2 Badotetr 


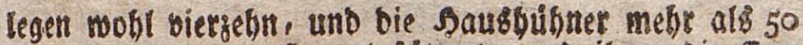
Eler, wenn man fie gut futttert, und ihmen oie हfier Had und nad wegnimmt. SRimmt man fie ifnen nidst weg, to bebrüten fie Die Eier, D. b. fie lesen fid Dar= auf, und bleiben fo lange Darauf flken, bis Die Rit-

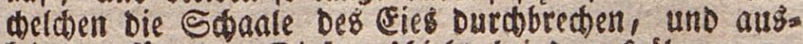

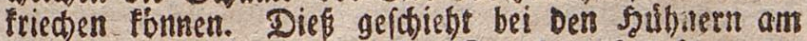

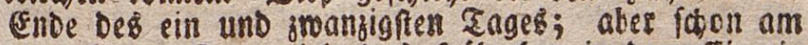

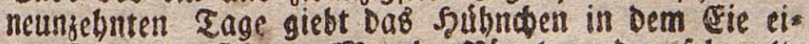

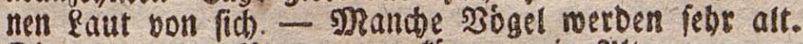
Die 2Dler une Yapagapen fómen ein झlter von roo Jabren erreiden, und oie Sdjwáne follen 200 bis 300 Jabre alt werben. BSanfe, ginfen, Stiegliģe und ₹ars ben nerben uber 20 Jabie alt.

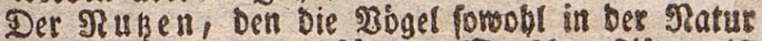

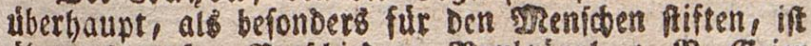
tiberauß

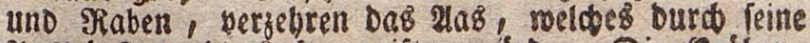
2lusbunfung Die suft vergiften wirtoe. Die Seraben, Die soúrger und anbere 230 gel freffen viele Jeldmáufe

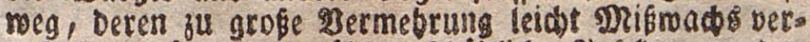

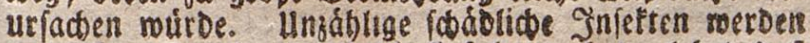
von 20 geln yertilgt, und Die erfabrung bat gelebrt, Daß

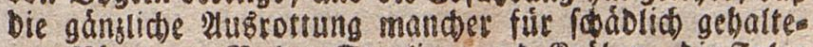
ner ₹ógel , 8. 23. Der Epertinge und Sráben, Die Jolge batte, baß Das Uungeriefer fiø unglaublich vermebrte, unb unerfestiden Sdaben ansidetete. Die Storde uno SReiber vermindern die grólabe, Edlangen und Cia Dedjen. Die Enten reinigen Die Gárten bon fobádliwen Edmeden ; Die Epertinge, Dreifen unt Sthwale ben verzebren eine grofe Menge der fódablichfen Raus

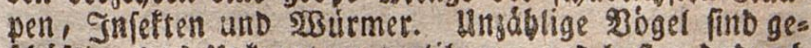
id)áftig, Das untraut ju vertitgen, uno lelfen baburch Dem mRenfiten einen febr grosen Dienft. 2hndere for gent für bie Bermebrung unto gortpflanjung míklidter

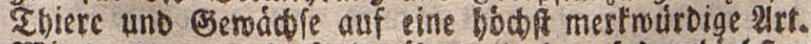

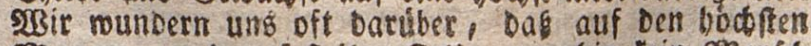

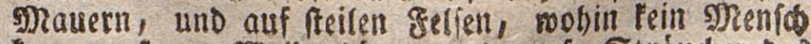
formen fann, 22seidenbaume und grofe Stráuder bes 2Jogelbserbaumes freben; bie Droffern haben fie Dabin

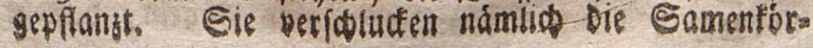


ner, geben fie unberbauef wieber von fiol, umb berpfiam

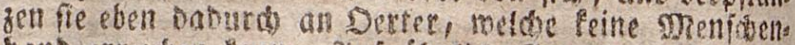

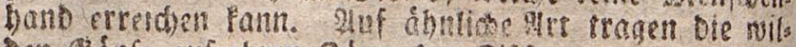
Den Soinfe auf uren 3ugen Den Biforrogaen in entfernte

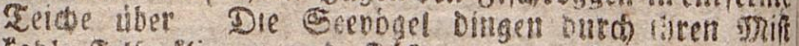
fable Belfentrippen und giften, soorauf nachber man"

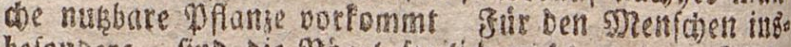
befondere, fino Die Bogel freilith mat is Dem Brave brauchbar, wie es oie Eduasthiere find, alicin fie ge

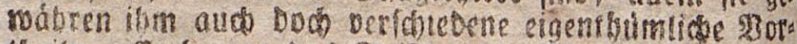
theile Er benus? Das ficifó, Die Eiet uno bas Reft son vielen, f. SB. non ben Gdnfen, Enter uno Scifhs nern, fu feiner Sahrang, unb gebraudt ibre gevern zum 2üusfopfen Det Betten, fum Bकresten, zu Binfer,

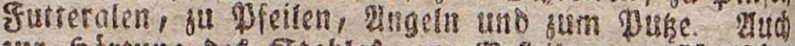

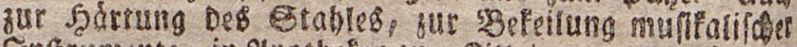
Snfrumente, in 2tpotbeten fum zitricen, uno anf mans de anbere 2urt find bie 230 gelfecern nutbar.

Der (ङ) aben, Den Die soogel anrichten, if unbes Deutenb, went man ihn mit Den sortieilen bergleichts melde fie uns veridnaffen. Cinige Fauboogel, 8. S3. Der contoor (Der grobte unter allen fliegenden 230. gelin), Der IEmmergeter us a fobten futlen, Sidber

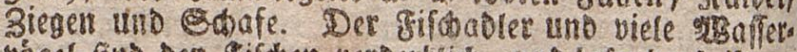
vogel fint ben gifchen verberticto, unb befonbers ibrem

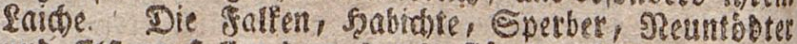
uno elfern ftellen Dem Spantgeftiget noob uno purgen

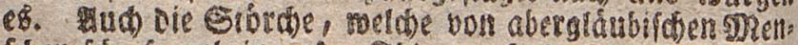
foten fur fegenbringende Eglere gebalten roerben, fint

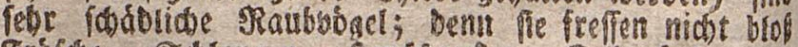
Irofose, Sdolangen, Sseufarectent, Gelomátufe uno 9) Rautrotirfe, fonbern aus iungeb Jeberbieh, Lerchen SBielien, gifobe uno gifálaid. Die Spertinge uno mandie Singobgel robaben ber Soat, Den sizeintrauben

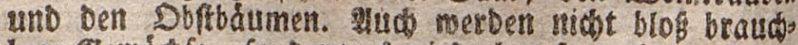

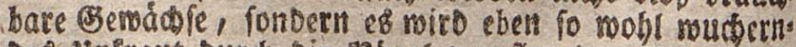
Der Uniraut Durch bie soger verpflanzt.

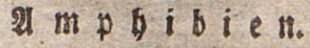

Die $2 \mathrm{mpl}$ bibien untericheiben fich botztiglid baburch ban ben E̊angetbieren und sBogeln, Daß̉ fie 


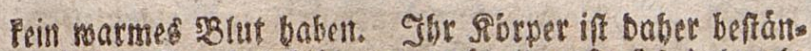
Dig falt. Bon ben fifonen untericosiden fie fict Daburch,

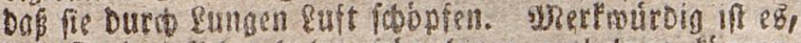
bak fie Das Wiberiblen lebr lange entbehren fómen, Daber z 23. Siroten in einers engen Baumlodos, uno foo gar mitten in Elumbibderi, wo fie role siagemater fits zen, graume 3ert leken. Ilud cin iebr boher erab von Sibe und Sialle tobtet fie nictet, besm man bat SBcia

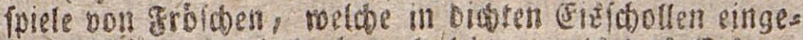
froren waren, uno tod) nod) febten, alb bab (Eib ge= fondrolen mar. Die meiften ampbibien gebell eme Ctimule boul fid, 3.53. Die Jrofde quafen; eimge aber 7. 23. Die grinen (Eiberen, fósinen ośntićs fumm zu fevn. Sie baben pine fehr verfobictone SBilbuna, Dena cinige fino vicrfífig, wie bie Sdildtroten, groflte und cidoren; andere habes eines langgefredten, robrenfor $=$ migen, Dumen Sorper, olnse Jufe unb ofne irgeno eit ábeteb 53enegunganertzeng, j. 53. Die Edblangen. Diefe fonness fid nut Daburds von einem Drte jum anbern bemegen, daí fie ibren Sorper fujammenzieben uno mieder ausirgecten. - Cinige simplibien boben cine fnowerse Sobale qu ibret Bebefung erbalten

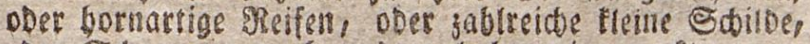
Doer Echuppen; now anbere baben eine nacte, nur mit Edoleim ubergogene saut, \$. 23. Die Eaubfroldoc. Die mehrefen bouten fid von 3eit ju 3eit. Mgerl=

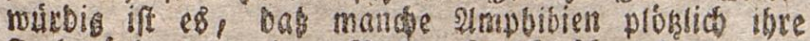

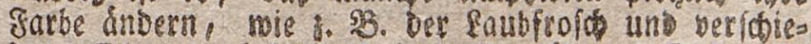
Dene Eiberetl, befonbers Das Ebamáledn. Daber ift es gefommen, bas man von einem veránbertidben $92 \mathrm{en}=$ faben fagt: er fer ein mabreb sbamáleon. Einige Ei= Deren unb Edolangen baben eine liberaug fónte bunts geflecte Saut. Die sRabrung Der 2mptibien, befonDerb Der Edilbfrotel uno oes Edulangen, if febe mannigfaltig. Mand ne nóben fich bloB bon einigen Battungen lebenber gnfeften. Faf alle Himpbibien fonnen bemunbernsmutroig lange faften. Cin Salo= manber lann mebrere monate lang obne Speife leben, und man bemerlt nidet einmal, $\mathrm{Dab}$ er Dabei betrácts lic) abzebrt. Son Scbildefoten reif man, bas fie ge=

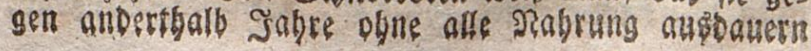


fonnen. - গow) bemumbernstwirbiger, als biefe 3\$ bigteit ibres sebens, ift Die Ecthnelligfeit, mit welder

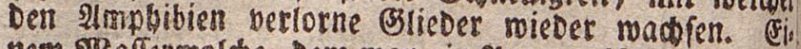

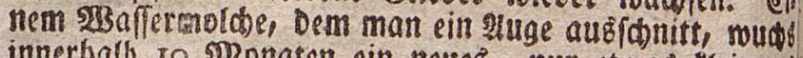
innerbalb to g2onaten sin neurs, nur etroab Heeineres 2uge mieber. - Einige 2imphibien, Dorzugflid cinige Sdltangert= 2urten, baben ein Sift bei fid, momit fie fith gegen ibre feinbe vertbeidigen. - \$Babrfibeintit

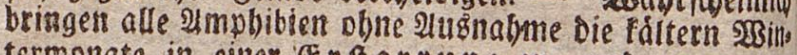
termonate in einet erftertung zu, unb zroar zum

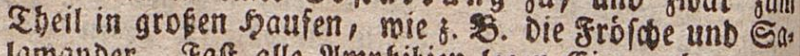
lamander. Saft alle 2tmpfibien legen Eier; aber malls ()e, befonders unter ben Eeslangen, geben Die Eier nicts elyer von fich), als bis das barim befindlidse. Sunth ge beinathe ferme bóllige 2luzbiloung exthalten bat. Sie wadfen febr langfem, leben aber aud zum Theil feft: lange. MRan bat Beifpiele, Daß Salidetroten uber 225 Jabre getebt babeln, uno roabricteinfich fónnen alfo ois Edtaligen uno sirofobille nod álter merben. Der Sero

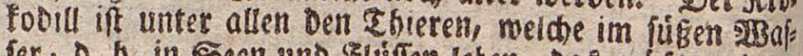
fer, D. b. in Eeen und filuifen leben, Das grobte; Dent

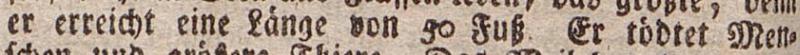
fajen unt gróbere Thberc. Das erseibcten legt gegent

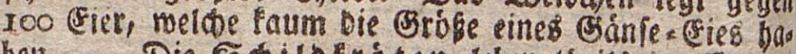
ben. - Dic Sollofróten leben theilö im Mreeren

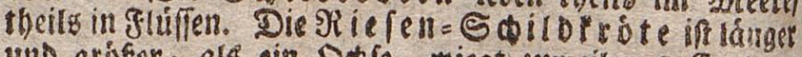
unt grófer, als ein Dafle, miegt zumeilen 8 Eentneit uno famn faffen von mebreren Eemtnern auf ibren fno's

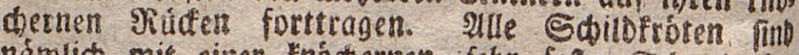
námlich mir einer tnód)ernen, febr feffen $S$ chaale be Dedit, Deren Dhertheil mit breiten hornigten Ex Eupper (Edil(Dpart) belegt iff. Diefe Schuppen fino bei mant den Batrungen fo frarl uno fotoufarbig, bas fie zu alo levlei sunftfacen, befonders gu Dofen unb ubrgebdusfent werarbeitet werbell. Die See = Sdbitofrote legt mebrere 100 eise, und hat ein febr icmacthaftes bleito. Die Sir te if nictet giftig. Die grumen 233 a fles frob. (a) e fino fiblau uno mutbig. Sie persebren פráufe, Sper. linge, unb reibft junge Enterl. Eogar úber grofe Sed) te werben fie Sperr. Sie find epbar. Die S di langen deben theirs in 5 saffer, theils auf ber eroen tbeils auf 


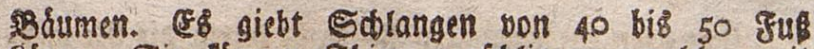
lánge. Sie fonnent Ebiere verfollingen, weldbe meit bicker alş fie felbft find, rocil ibre simnlaben fict febe weit ausbehnen, aber pauen fonmen fie nicts.

\section{\& if $\$$ ?}

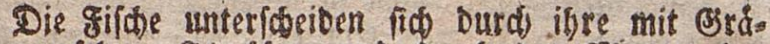
ten berfebene floffen uno burch Den Mrangel Der Sungen von allen úbrigen Ibieren. Statt Der \&ungen haben fie Riefern ober siemen erbalten. Die

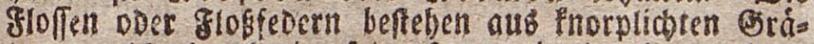
ten, melde Durd eine feine Syaut mir einanber yerbun=

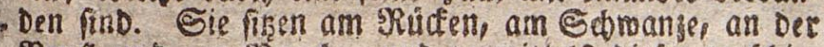
Şruft uno an şaude, und Dermittelf Diefer verídies Denen Jioffin Ponnen fid Die girobe lebr mannigaltig und idnell berwegen. Die Siement liegen an beiden Sei. ten bes Siopfs, und beftuers aus feimen Gafern, weldhe

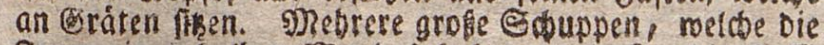
Siorm eines balben Mrondes baben, bebefen bie Deffo nungen ber sicmen, und beifen Daber Si i emen= Def:

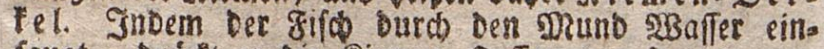
faugt, orútit et Die Riemen. Deffnungen fo lange zu,

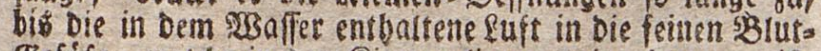
Geféser, melote in osn Riemen liegen, eingebrungen iff. Durd die Rirmen-Deffnurg geft fic Dann mieber fort. -

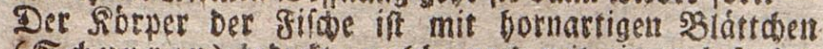

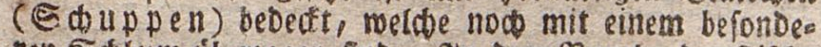
ten Edilem úberzogen find. In Dem Bauthe ber firatse befindet fias eime BSlafe, melde Daß Edumimmen erleich. tert, uno Daber Die S sifde balten fio nur in Seen, gluffen und Zeiden, andere nur in פeere auf. Die leBtern merben Sec.

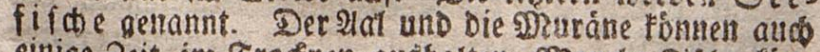

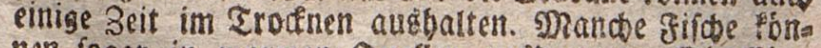
nen fogar in marmen Duellen ausDauren. Die Eier,

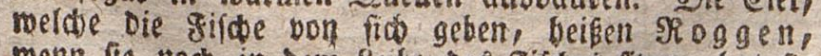

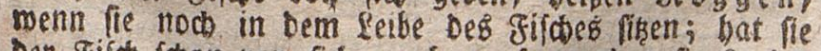

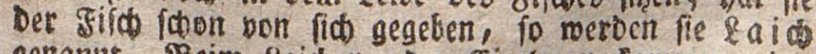
genanut. SBeim Eaiden ober Efierlegen Eommen viale silde an Das Hfer, um im Sdiffgrafe DDer an silipa 
vent ibre cier a legen, mo fie bann Durch Die Sonnen. mátme ausachruter merbeth. Die meifen filche leben

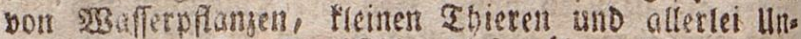

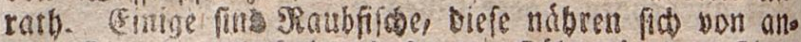
Dern gisichen, und baben Destwegen záhne in ben Rinte leber. Die Sadringe, unb eintge antere sifche maden zu beftimnten Gabrebzesten in unezmepticten Scharen

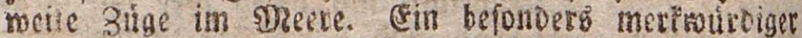

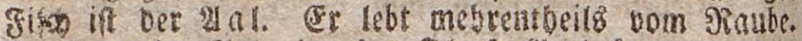

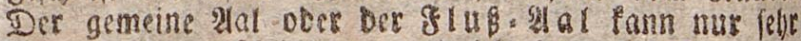
fleitle girche verfotingen, unb lebt Daber meifteng vort

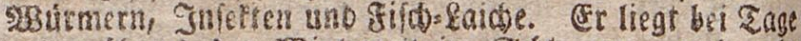
uno rodfteno ber xbinterzeti in Scolamme, uno geht

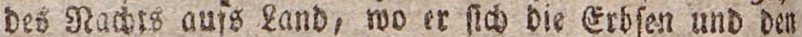

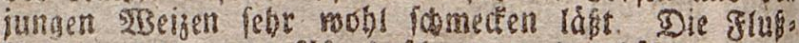
Iale merben an 30 yfunb fodmer, und gebdigren lebents Dige Junge. Der Sitter=24al, welder in unjeren gliffen midst gefunden wiro, bringt Demjenigen, wols

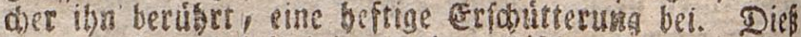
findet nuch nod bei eimigen anbern zifoten fratt. - Die

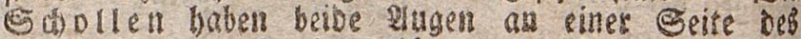
Sopf8. - Det \&ado gevort ju Den 3 ug = Fir de ell

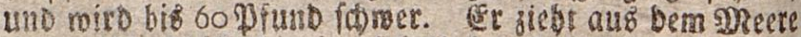
meit in bie gitufe binein, fobaíb Der Jrtibling gefonemen

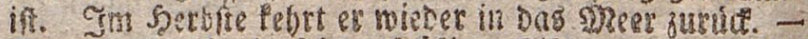

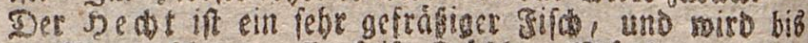

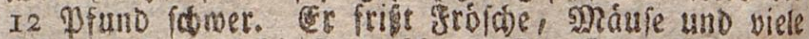

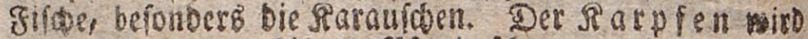

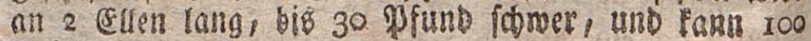
Jabre alt merders.

$$
\text { In f e f t en. }
$$

Die beiben Iegten flaffen ober athetheitungen bes

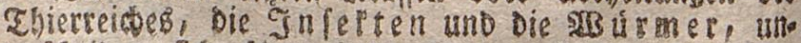
terfociben fich foron Daburdo son Den porbergetgents Den, Dap fie fein rotbes sblus, fonbern pate befen einet meifliosen Eaft in ibrem sibrper baben. Ihs ren গRamen baben die Snleften Daber, mell Soppli

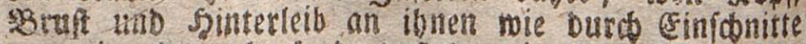
bon einander abgefonbert fino, ja bei Den mebrefres faft nut Durd einen gaben, mit singnber ver- 
bunben zแ feng foeint; Denn Tnieften bedeutet fo viel als Tbiere mit einfoniten. Ruberbem unter. fateiben fie fid nod burch Die zánen, weldge fie an ber Stiru tragen (fublborner), uno ourd die 3abl ib

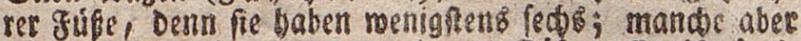
aud 12,20 , ja bis 100 unD 150 Juse. Es ift thrie geno ein grofer Unterfobico unter ben Sinfetren, fowon in Inebung oer SBrDettung ibres Rorpers. Sebe vielep 3. 5B. Die SRffer, find mit enee bormantigen Dede lifere zogen, unter welder ibre ftemen flugel liegen; andere fino mit femen szaren bevedt; bei Den Somettertime gen und einigen andern Snfelten fimo bie Jiugel mit fleis nen feocriben, ober vielmebr mit Stowpen berieben

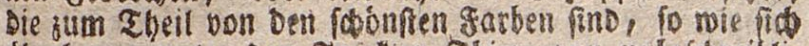
siberbaupt unter Den Sneften Ebiere pon unberctereiblim

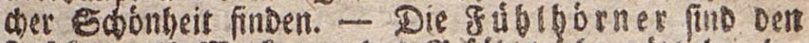

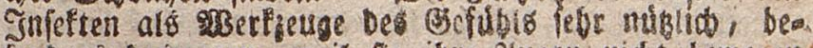

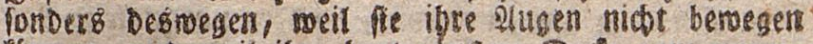
fonmen, uno weil iare baste dugers Decte ganz unem= pfindici ift. - Gaft auf allen Toieren find galeften anjutreffen, unb fogar unter ben Gnieften giebt $\mathrm{CA}^{3}$ ei nige, 3.23. Réfer uno Bienen, auf reldoen andere Sno

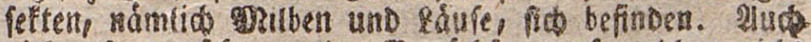

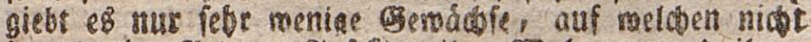
irgeno eine art von Snfeften ibre 298 obnumg uno ibren 2ufentbatt butte; is mange anter iłnen, i. 20. Die (sio ben, soerber voa mebr als 100 perferiebenen Battune

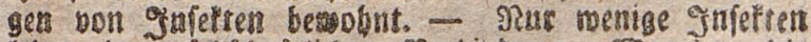
leben in gefellfobaftlicher Berbinbung. Mrancier die

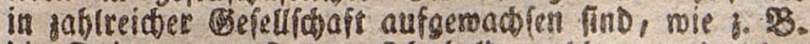
Die Epimneti, ferfreuet fich balo nabler, unb lehe cinfiederifo. Die mebreften Snieften banen fids úberats fuinftige Sobnungen obes Bebdule, ober fie

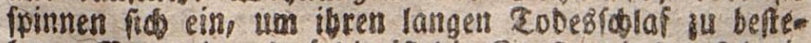
ben. Besunterndmuirbis ift Die Sunft, mit oer fic pis

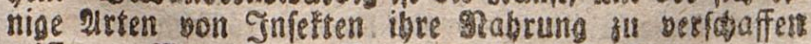
mifen. İser fann Epinngemebe betrabten, obne úber bie Sunft bes fteinen Zbieres is erfesunen? Eben to erftaunensmuirbig ift Die tridterformige fallorubes melobe ber simeifeniowe, ein Intett yon Der Brofofe eie ner gliege, in lofierem Eandboben gu madasn wris. 


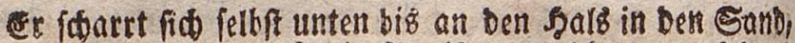
uno lauert nun auf bie elmeifen, nelcbe unberfebens an Den Fland feiner Brtibe Eommell, ums mit Dem lot=

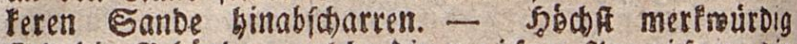
find die Gebaude, welde bie weifen It meifen, die in affrifa uno Amerilla gefunden merden, aus 3 gon und sebm auffúbren. Eie fino fegelformig, meift mit mel)s rern Episen befert, inmendig bod ausgerojibt, oft 10 bis I2 Juß foch, und furveilen io jabrreich bei einander

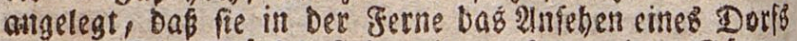
baben. Die SBánoe find mit grofern weiten Gángen Durdesogen, aber Dod fo feft gemolbs, Daks fie mebrete Menichen tragen. - Eben fo mertwuiroig iff Die $2 B$ ols nung ber sienen, ber sienenfoú mit feinen fernfti d)en zellen, Die feite smenfácuband po regelmábig rad

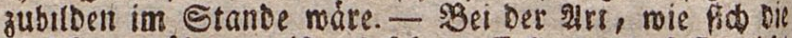

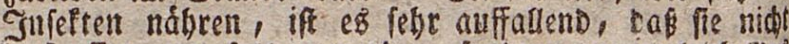
blos effen, um fatt zu werben, fonbern um zagleich plas

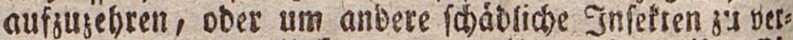

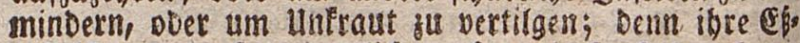
luft ift gan ausecorbentliob gros, uno pie fino rectis els gentlid gefrápig. Eine Raupe verzebrt in 24 Stunben Dreimal melix, alb fie wiegt. -

Die mebreften Infeften legen eier, melabe bie smut. ter naф einem bemunbernzburbigen Suftutt immet aufis genauefte an folde Drte legen, mo Dis finftige \$3rut am leidfeften und fioberfen ibre Nabcting finden fann. Mande legen \&. 38. ibre Eier nur in Den Sibts per lebendiger Snfeften anberer \&rs, in SRaupen, DDet in Duppen, ober gar in bie cier anberer Snffften. Dur renige Infeften gebibren lebenoige Junge. Die geftugelten Sinfelten neljmen mebrere Beffalten an, elle fie Dem Shiere ábntiós rerden, aus meldsem fie ents fanben find. Diele Beranderung oer Beftalt nent matt Die Bermandiung Der J̈nielten. Eigentlid. ift es feine sermanblung, fondern ez fommet babei nut Diesenigen Boeile fum şoridein, meldbe fo vers búlit find, Daf man fie zuoor nicht bemerfen fonnte.

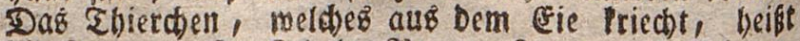
Die \& a rye. So find die Siaupen farven at: SGmets

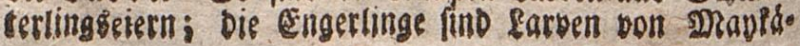


fer, biele 9graden fint bie farzen betwobenter Fliegen: (Eier. Diefe Larven thun niots, als freffen, uno freic fen einigemal ibre şaut ab (báuten ficb), morauf fie eine neue erbalten. Stad einiger 3 eit perfertigen fie fid eitte Sculle, in meldber fie gemobinticts fill unto rus big liegen, obne in freffen. In tiefer Beffalt beiken fie puppen oder R D m p gen. sabábrento ber Zeit,

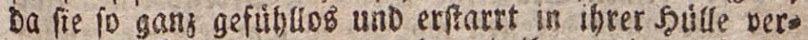
graben za fepor fobeinen, gebt mis ifnen oie grobe be

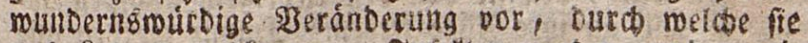
aus farben bolfommene Snieften roerben, und zu eis net beftimmten zeit bridot Das netre guneft oub feitter Szitle beroor. In biefen 3uftande madien fle nicht mebr, freffen wentig ober gar nicht, uns leben oft nue einige Stunben, nachbem fie zunor thre Beitummung

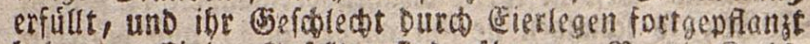
baben. - Cinige Snieften find efbur, z. 95 die Sireble uno Die groben Sheuichreden, welohe aber bei ung nid)t gefunden merben. Die Seidenraupe ift bem

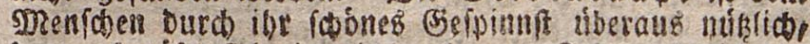

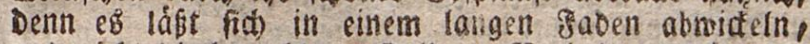
uno giebt bie braudabare Seibe. SRad jenter Sáutung wirt fie grober, uno einigs Tage nad, Der vietten Saku= tung ipinat fie fids ein. Das duserite Bemebe, mels d)es fie Den erfsen Zag fpinnt, iff febr unoroenrtlic; eB giebt Die Floretielde. $2 \mathrm{~m}$ zmosten Sage fipinnt fie

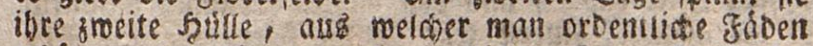

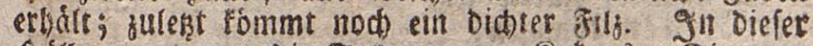
Stille neant man Die Eebibenraupen Rofons. Eie merDen in einem ${ }_{B}$ adfofer oder in beifem $2 B a f f e r$ getodtet, uno Dann roiro $D a b$ Bépinnf abgeb)afpeit. Einige fobtet man nidot, und aus biefen bridi, nadi etma 3230 )ett, eitt weiker Sकmetterfing herbor, melder (Eier legt und firbt. - Die sBienen gehoren cbenfalls zu Den Infetien, melabe Dein Denfíden unmittelbar núk= lict finb. Unfere Şaubbienen leben in SBienentorbent - Der sbienenfroceen, Dis wilden leben in boblen 9Baus

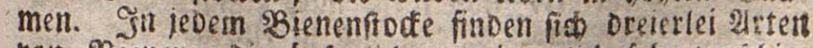

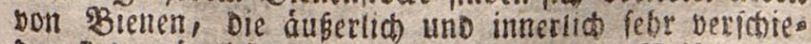

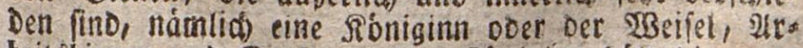
beitghienen uno Drohnen. Die Rónigint bált Die ganse is 2 
(S)efelfidaft zufammeth, unb erbålt Dronung unb รhåtigs feit in berielben. Sie allein legt eier, aus meidinen alle úbrige Bienen entfteben. Die qubeitz̈bienen find Hleis ner, alB bie Roniginn, uno ifgret fint 20 bis 60000 in jebem Stode. Die Drobnen fino mánnlidbe sBienen, uno unter allen bie gróbten. (Es fino liber 1600 , und fie baben Peinent Stadsel. Frsenn Die 2robeitbbienen eine

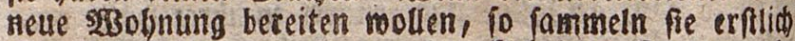
cine Art Sitt, Den fie bon ben flebrigen Sinopen abs nagen, uno all inte gufie fleben. Damit roerden aile SRisen uno gugen Des Stode's bis auf bie fluglocher

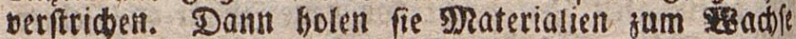
berbei. Dieß ift Der $B$ lumenfaub von ungdgligen $B$ lt men unb sBlutben. Gie benetzen ibn, uno verietgren itn Dam. Erft it ibren sRagen vermandelt er fich in

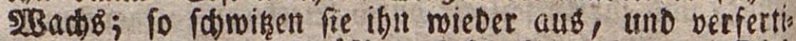
gen bavon bie regermáfigen fectsededigen zellen. Diefe Dienen theils fur \&ufberobhrung Des Jomig8, theils zu sleftern fur Die sBrut. Die gefülten zeflen vetfabliebent fie mit einer feimen 230 absecte, Damit Der

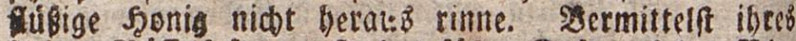
Fleinen SRuffels faugen fie Den fúfen Saft aus ben solu men ein, fálucten ibn binter, uno berarbeiten ibn in Jgonigmaget, Der wie eine tleine siare Gubfteht, unt morin ber Saft zu sonig mirb. Die Róniginn tegt in jebe Zelle ein ei, uno den ganjes Sommer bindurd 30 bis 40000. Suenf legt fie bie Ciet, moraus 2/t: beitsbienen Fommen, Dann die zus den Drobnell, unb ensliá) nod 10 Eier, moraus Sóniginnen werbell in befonberb Dazil gebauete 3ellen. In einigen Zaget

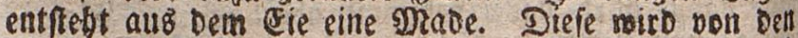
sBienen forgfáltig mit einem 23 rei gefúttert, bis fie fid nap etra 8 Tagen einfpinnt. Dana verfoliefen bie

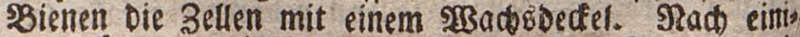

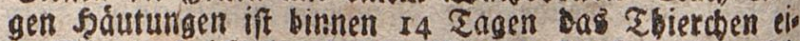
ne SBiene, bricht Durd Den $233 a$ sedectel bervor, mitt mit Seonig gefúttert, und fliegt naw einigen Stunbent mit ben ubrigen aus. İBenn fiot in einem Stocte bie sBienen zu febr bermebt baben, und befonders, wen mebrere junge fioniginnen oa fino, fo wiro ein ₹beil bas von ausgetrieben. Diefe nennt man sinen S do a rm. 


\section{Produlte ber Cride.}

Sie folgen ber Sioniginn, bangen fid ba, wo fie fid binfeght, in ciaem fegefformigen biot jufammengedráng= ten Spaufen an, werben fo in einem leeren Bienenforbe aufgefangen, unb fangen fogleict an, fich anzubauen. Sind mebrere Róniginnen in Dent neuen Stofe gefom: men, to finder man bie uberflúbigen am anbern ₹age getobtet vor bem Stode liegen; Denn nur eine fann

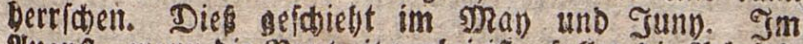

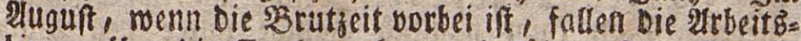
bienen úber Die Droblenth ber, und tobten fie. Sobald Die grofte im Spathetbfte eintreten, verfallen bie Bieo men, twie bie meiffen Infelten, in ben 2Binteridblaf,

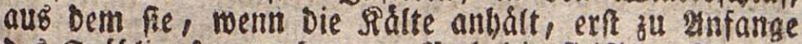

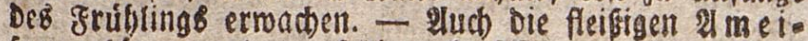
fen geboren zu Den gefelligen Infeften.

\section{3 มี $r \mathrm{~m} e \mathrm{r}$.}

Die æButrmer baben swar aud ein meíflideb falte

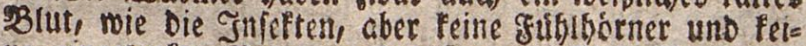

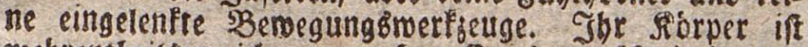
mebrentbeils meid, gant ofne finoden, faleimig uno

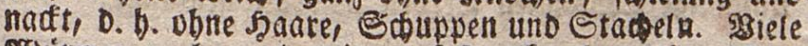

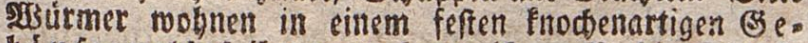

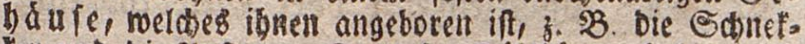
fen unb bie 2lufern. Stant ber Sublborner baben viete शुuirmer fogerannte git blfáde einigen yon betráduttider \&ánge finto. Beri vielen Sdonets

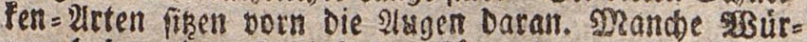
mer babes einen fo einfedien sórperbau, Daf man gar leine Briebmafien an ibnen unteritgeiben fann. In

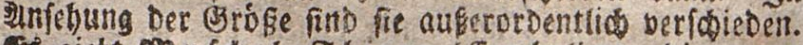
es gicht 52 u foel: Thiere (Sondulien), bie gegen - Eentner wiegen, unb bagegen wieber gaturmden,

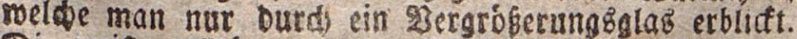
Die meiften 5stirmer batsen fich im $523 a$ aller auf, einige

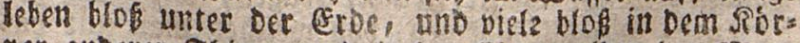

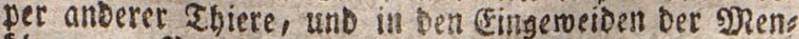
Idinen, 3. 23. Die Darmmurmes. SManthe 2arten Der 52surmer finder man zwar in grofien fsalsfen bei einans Der, wie 3. 23. Die 2uffern, aber fie leben Dod in Fel: 
ner gefelligen Verbinbung. Gatre Nagutung fuchen bie

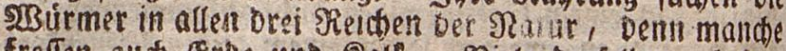
freffen aud Esbe und Rall. SBiele berfelben, befons Ders unter Den Sdmerfen, unb aud bie silutigel fón, nen auB̈nelbment lange faften. Ginige legen Eier, an=

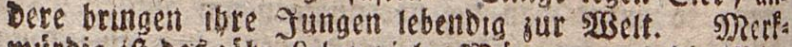

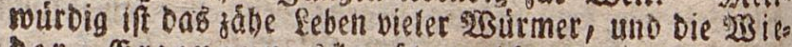
Der = erfeugungefraft, welde man an ibnen be

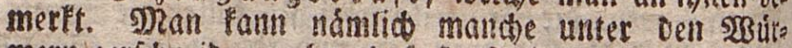
mern gerianeiden, ohne das fie ferben, unb nad fut zer Zeit nachfen bie abgeidnittenen Theile wieber, wie

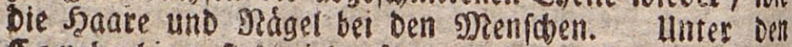

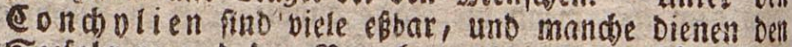

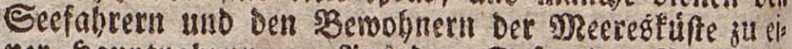
ner Sgauptnabrung. 2ubB Dem Safte Der sBladfiich? fann man Einte macten. Der sBaft Der Stectmurded

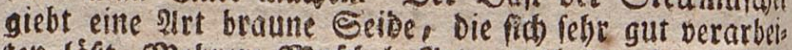

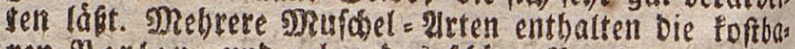
ren \$ertent, unb geben bas fosine Declen in uttell

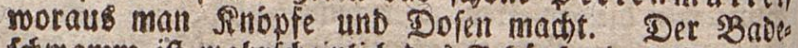

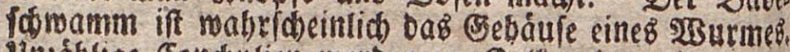
unżablige Condjplien metben zu Salf gebrannt.

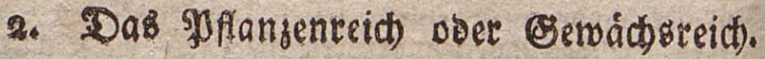

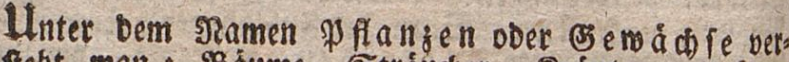
fregt man: sgáume, Stráucher, Práuter, उráfer, Sdiodamme unb Mroofe.

Die \$Báume baben einen Stamm, fratfe פBurzeln, 2lefte, 3meige, Inofpen, SBlúthen, SBlátter, uno Ertíbte.

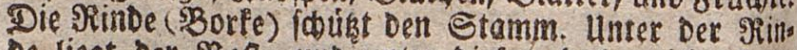

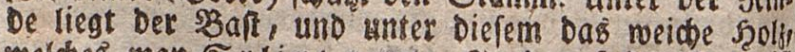
melches man Eplint nernt. Sn Dem feffen Sjolze ift

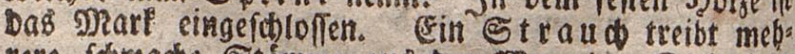

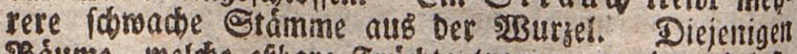
IBdume, welche efbare Frubite tragen, merben $D$ b ft: b dume, unb alle tibrigen, bon weldeen man nur $D a b$

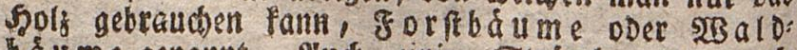

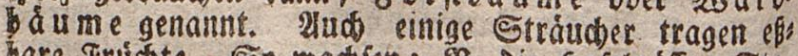

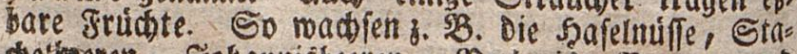

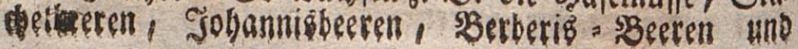




\section{Probulte det crbe.}

Simbecter, aud bie 2seintrauben an Etrådorm. Die 23aldbáume fino entweder \&aubbol zet ober

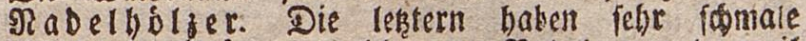

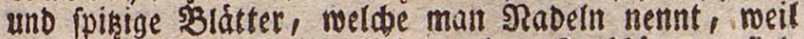

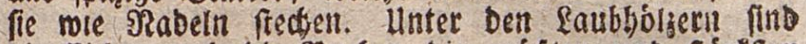
Die (Eichen und bie szudten Die gróftent und ftátfifen

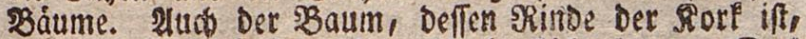
morats bir Pfroppfe maden, if ein (Eid)enbaum. Das -3udbenthols gebraucbt Der Eilabler lieber, alb ber 3im=

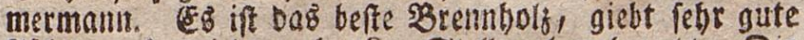
Niche, unt wiro aud vöm Stellmader benubat. Die sudectern Dienten zut Maff, unb geben ein gutes

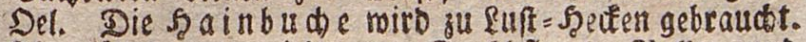

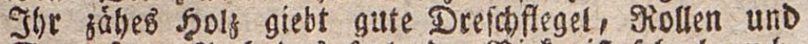

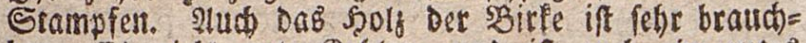
bar. E⿺ giebt sute sioblen, und if audi ein gutes

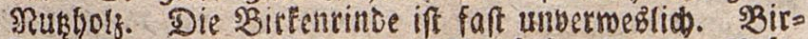

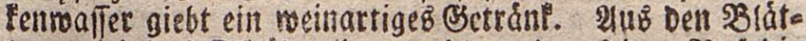

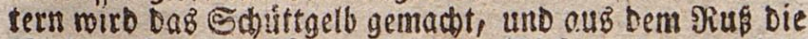

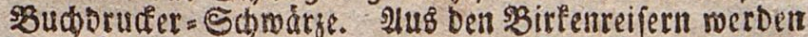
Die núblident Sefen gemad)t. - Die Erle (Ëlstp (Elfe), wóds) foch und gerabe, und am beftet in einem

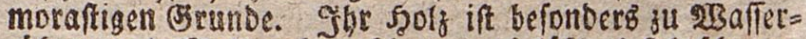

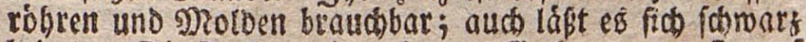

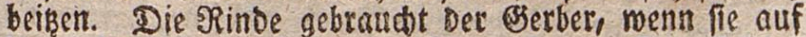

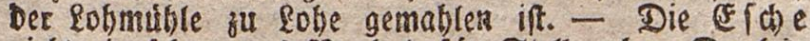

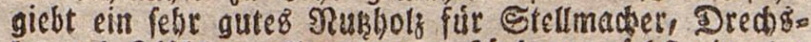

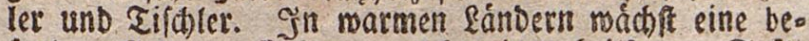

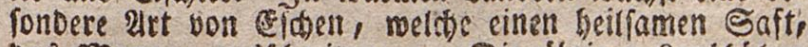
bas Manna, auśfbriken. - Die úbrigen \&aubhóbet

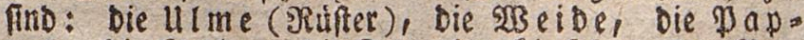

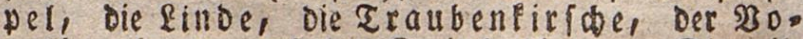
gelbeerbaum, und der Spindelbaum. Die mils De Raftanie ( Ro $\beta=$ Raftanie) unb Die 2 fazie fino frembe SBáume, roelche aber leß̧t bei uns báufig, jun Eheil aud (t)on als Fieme $2 B a l b u n g e n$ angeplanjt merben.

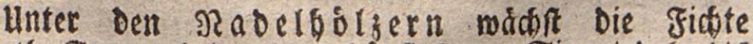
(भioth = Tanne) bei unB am bsufigftert. Sie mird 60 bis

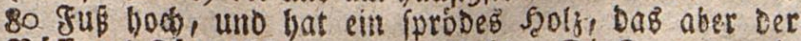

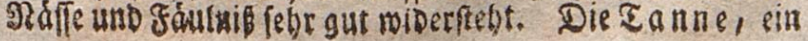




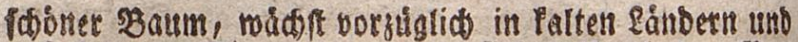

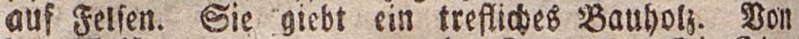
Der 23 eibtanne gerinint man ben Eerpentin. Die fi ie fer ober ber gienbaum (8sbure) roiro befonder\& ju

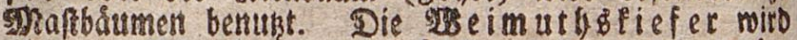

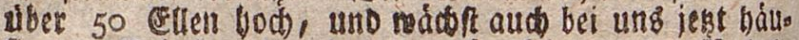
fig. - Dé \&erdenb a um trógt feine zarten Frabelin

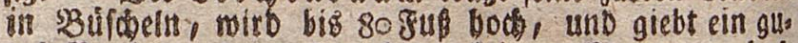

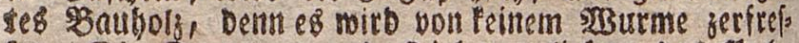
fent. Die Eanten uno giditen liefern Daś pect, einen bartigen Saft, ber in großen Sieffeln mit $233 a$, fer gefdomolsen, in Sadfe gethan und aużgeprefft

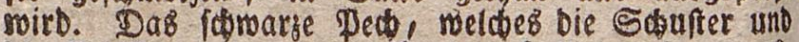
Die Sdaifer gebraudsen, if ein gefocoter und getrod: meter Ebeer. Der Theer wirb aus ben festen sebuts sein Des Fichtenbaums gebranit.

Unter ben a แห 1 å no if

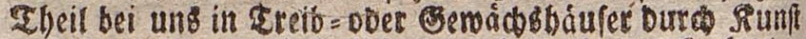
gezogett werben, find befonbers folgende mertivurbig: Ger sitronenbaum, Der pomeranzenbaum, Defien grúdiste audi Dranger genaunt merben, (Dafiet Dab SBort Drangerie); Der Se affecbaum, Deffen Jrtidite fleinen Sirrident ágnelln, unb Die SBotne entbals ten; Der Eb eebaum, Deffen geroffete slätter Ebee

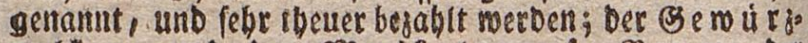
gelfen = uno Der M แ Rorbeerbaum unb Der 3 imm t ba um, Defen Fins

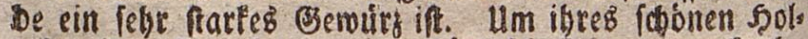

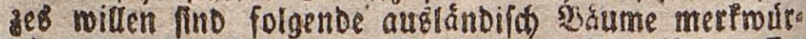

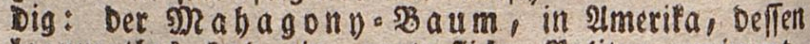

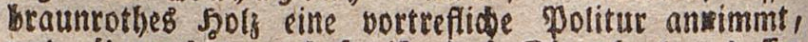
unb úberaus Dauerthaft ift; bae (Ebenbolz, Deffent

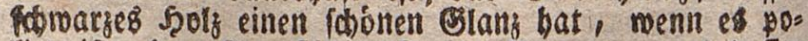
litt iff; Der Brafilienbolz= Baum, aus beffen

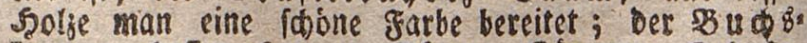

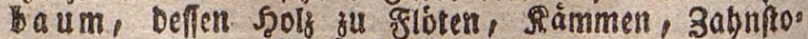
(d)ern und feinen Seratbrabafen verarbeitet mirb; Der Platanen= \$ a u m, welder aud bei unz jekt haufig angepfianzt mirb. - Eben io mertmúrbig fimo noch eis nige anbere aublämbifche Båame, meldbe befonberb̉ jur cernábrung ber 2Renfकen bienen, uno úberaub frudts 


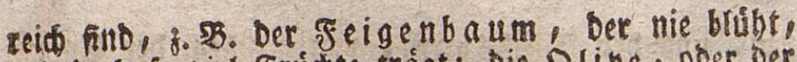
und bod fo viel Frúbte trógt; Bie Dlive, Doer ber Delbaum, Deffen fruidete borzuglich fur Bereituritg Deb

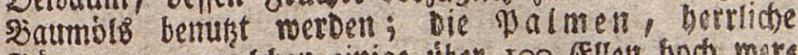
Baiume, volt weldten einige úber 100 Ellen boch mets Den, uno weber 2leffe nod) 3weige, fondern blob am

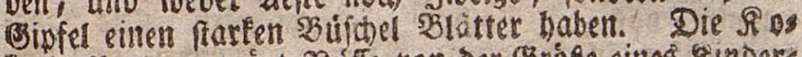
I o s, Palme trágt Ruffe von der stóse eines sinders Fepfó, in weldbem ein Szild faft enthalten ift, Der als ein erquidendeb Betránt genoflen wirb, und aud eir fajones Del giebt. Die Fafern, womit Die Ethale Der Seus umgeben iff, merben fu Striefen verarbertet; aub Den grofen szlattern diefer faumes madot man

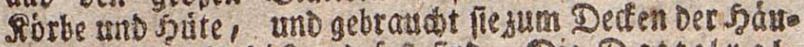
fer, weil fie febr dia und feft find. Die Dattelpal.

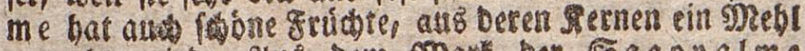
gemadt wirto. Itus bem Jart ber Sagopalme miro aud ein nabrhastes Mrebl gemadt. - Der 38 r o b b a m hat eire metorestormige gruct, bie ges rófee, mie $2 B$ eizentrob fidmedt. Diefe SBadume tragen beffántig fo reidolid) Frridste, Dof brei 38 áme einen sDeenfoten Daß̉ samje Jabr hinburá nábren.

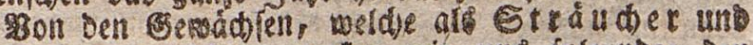
Stauben wadien, werten mir uns folente: Den Sreuzborn, bie Etedpalme ant rotben SBeeren, Den Edsueballenfraud, Dab (Ep p) \& (Eppid), sBintergrün), Daß̉ (Eeibblaft DDer SRaprifolium mit feiner moblriectenden sinme, Den Rellerbals, Deffen sRinde Den Seldetbaft giebr, Der slafen auf ber Saut fiebt; Den wirben Rofenfrauts ober bie Sagebutte. - Ein

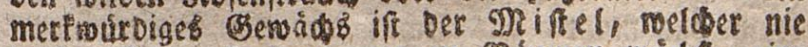
in ber erbe, lonbern nur an sadumen mádbift, eine Elle how mito, unb burdifótige sseeren trigt. 34

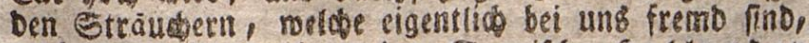
gebort Der $\Im$ a

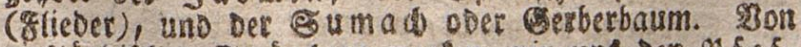

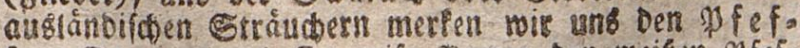
fer. Straud, beffen reife szeeren ben meifen Mfeffer, fo ric Die unreifen Den fórearzen geben; Den Spa=

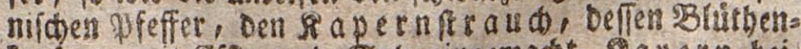
fnofpen, mit Effrg und Eals eingemant, siapern bei= 
Ben, unb ben Bbod Bbart, yon meldem ber Bummi Zragant fommt, Den bie zátber gebrauden.

Eine eigene Battung yon Wilanzen machen bie Garrenfeduter, Die Moofe, und Storodmme aus. Sul jenen gehort bas Siannentraut ober Edbah. telfalm, Dab zum politen gebraust wird. Die Mloos fe mader an sBámen, Eteinell, Rinoden und Jer. fen. Einige MRoosatten úbersichen bie SRoráfte, und

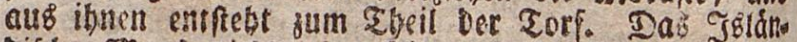
Dif(t)e शRoos giebt eine fehr gejunbe und nabibafte Speife. Unter ben Edowammen giebt es giftige und efbare. Sene baben Dunfle unb bunte Eactben, und eitren bohten Strel. Die Pillie find eire ent bon Edmocimmen. Die Drordein gehóren aud zu biefet

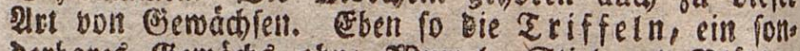

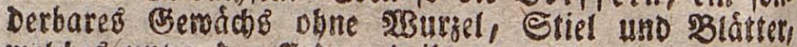
weldes unter bet ertbe gebeibt.

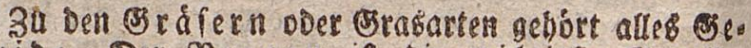
treibe. Der $\Re$ oggen if bie mimtigfte Betreiber art, meit er DaB ftaffrige SBroD, unfer baupteádblichs

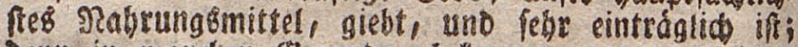
Denn in mandsen Eegenden betommt man von einem Sdyeffer Sioggen mohl zefn unto mehr Echeffel wieder. Die úbrigen Betreibearten fint: Der 5 Beizen, Det Epelz oder Dinfel, Die eserfe, Der Safer, Der

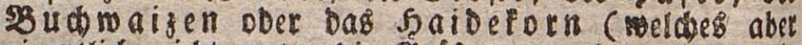
eigentrich nicht uneter oie (Bråfer zu redonen iff), Die

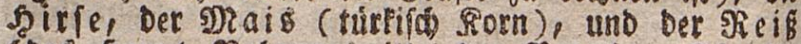

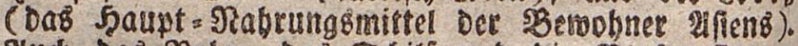

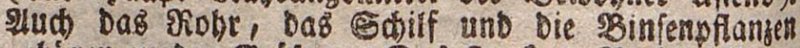

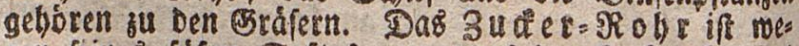
gen feines fuken Saftes, moraus ber zuserer bereiret

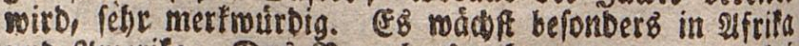
und Amerifa. Das 8 a mbuBr ohr, woven man bei unb Epazierfftode madt, miro ein ftarfer SBaum, und

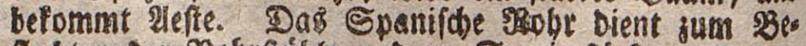
flewten der Siobrfuthle und zu Spazierfibofen.

3u Den Pflangen, melde cinen Theil beb gelbo

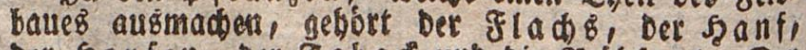
Det Sgopfen, Der zaba af unb die SRúblaat. Der 


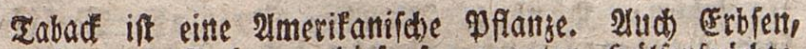
Sinfen und Bofmen, Diefe pogenantent Stúlienfrúchte, merden bdufig auf Dem zelbe gebauet. Det g?obn ge.

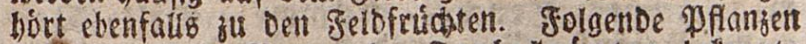
fint uner Dem Samen Der Farbefrá uter befannt;

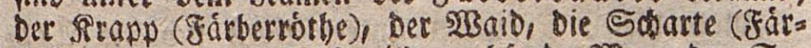
berbiftel), ber meiftenB roilb wachfenbe $2 B a 4$, Der Sas flor (cin Diftelgemdids), und Der Safran.

Unter Dem samen ber Bartengenádie Doer

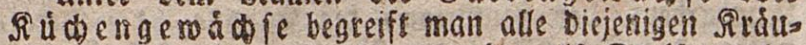
ter und Pfianzen, melche entweder als Epeifen zubes reitet, oDer als Bemuirze an bie Speifen getban merben. Dazu getyort ?. B3. Der sBlumentoble oer SBitfing, Doer

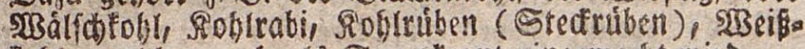
fohl, welther aud alB Sauetfratit singemaci)! mirb.

3u Den giben uno 23 urzelgenachien geboren

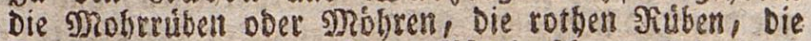
Fiuntelriben, welde Dft Io Pfunb former finb, unb aus beren Eaft mant einen guten Buder machen fann,

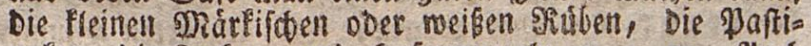

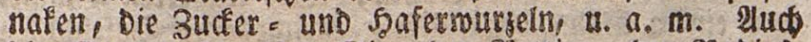

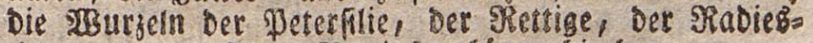
den uno Deß פreer = SRettigb geboren bierber.

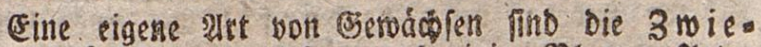
belgewad fe, zu reldem aud einige simmen= 2urten gebóren, t. 53. Die Syocintben, ₹ulpen, filien und sazettett. Folgenbe 3roiebelgewaidie fins efbar, uno rerben alBె Berourr an die Epeifen gethan: Die ges

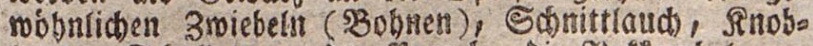
lauch), Schatlotten, Der Dorreb, Die Sioffambole. Die Rnollengend \&d fe find Den 23 urzel - Benédifen ábnlich. SRan rebnet Daju Dent Eellerie uno die $\Re a=$ puntifa (Siúbenrapunzel), Die Rartoffeln, Die Erópfer und bie erbntife ober Erbeichein.

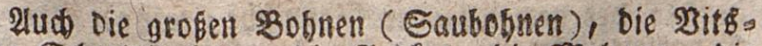
Doer Sdominfbobnen, Die Butfen, Die Inelonen, die Rurbifle, Der Ealat, bie Enbivien, die Preffe, Der

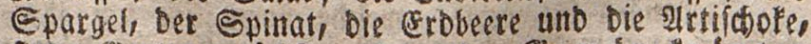

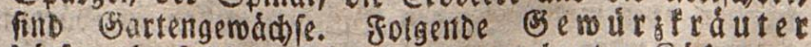
Durfen sbenfall in feinem gut angebauten Ritiengats 
ten feoten: Rorbel, Raute, Salmen, Melif. le, piefferfraut, portulad, Pimpinelle, Sauerampfer, éffelfraut, shaioran, Thy. mian, 2nis, Fendel, Filimel, Dill, Dea, gun, sgeifut, Senf und Soriander.

gson biefers Firdutern unterickeiben fic bie $2 \mathrm{r}$.

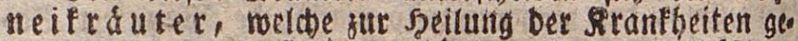

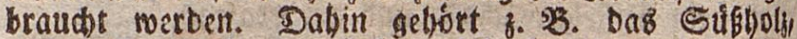
aus Deffen Saft Der Eaftikenfaft bersitet mirb, Det SRlbabarbet, Der SBalbriall, bie Simille, die Sdjal, garbe, Der Sauertlee, Der Sómentabn, Dab Jobannis. fraut bie Stiefmutterdien unb viele anbere.

So heilfam biefe firauter find, fo fisablid) find cinige anbere, meldbe baber giftige sérá uter genannt werben. 3 or biefen muß man fíd forgfáltig bứret!

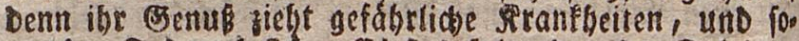
gar ben ioo nad fid. Es fino folgende: Der S do iel.

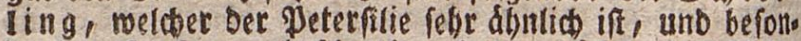

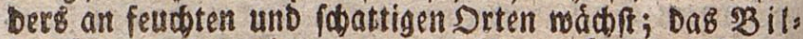
fenfraut mit einet grauen, blau geáberten şlume,

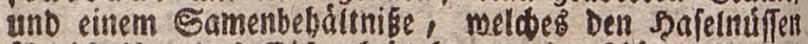
ábnlito ift: Das Eifengutichen, eine robone, blaue,

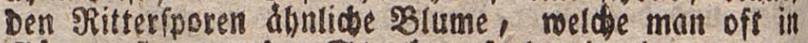
Bárten findet; Der St ed a p fel mit einer langen tridterformigen 58 lume, unb einer Samentaplel, wels

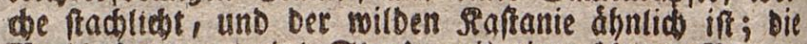
Bellabonna, eine Staube mit einer blauen sBlume,

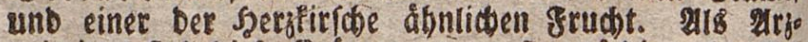
neimittel find biefe Sitátet, menn fie auf Die recble 2ut gebrausot merben, lebe beiljam.

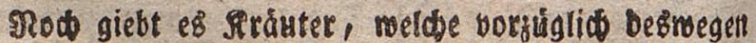
angebauet merben, weil fie ein gutes und nabrbaftes guttet für Die Sgausthiere geben. Sie meeben babet

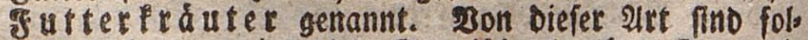
genbe: Der gemeine ober Spanifde (rothe) Silee, Die

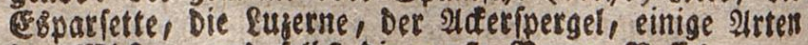
Der ssiden, uno lelbft bie groBe SBrenn - Neffet.

Diejenigen Geroddyfe, meldoe vorstuglid um ibrer fojónen ober mobltieddenben sBlútben millen in Bártten gezogen werben, beißen $\$ 31$ umen. Die meiften gebo 


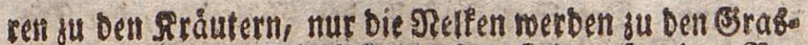

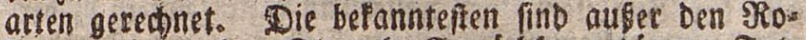
fen, weldhe ju Den Etraud = Bemád)fen seboren: Tule pen, Syuacinthen, Jonfitten, Eazetten, Narziffen, Li-

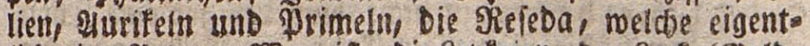

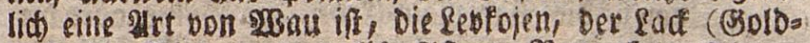

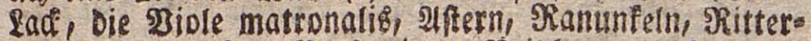
fporen, Tuberofen, Balfaminen, Seilden und Eupiten.

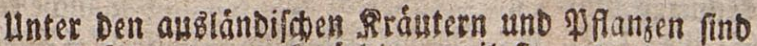
befonderb folgenbe mertioúrbig, weil fie entweber als Bemuirs ober duf andre 2irt fegr muslio frob. Dee gngmer, mit einem folifáfnlichen stengel, teflen

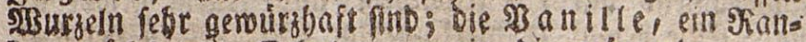
fengemádb mit Edoten, morin bie glänzenden uno fefr gemuirshaften Samenfotmer liegen, welde zur $58 \mathrm{e}=$ reitung Der Edofolabe gebrantht rerben; die alna

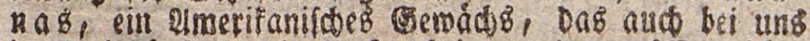
in Treibbáufern, bie ftarf geteigt merden múfen, baks fis gezogen wird, uno eime febr foffriote grudot bringt;

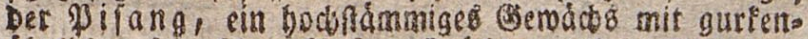

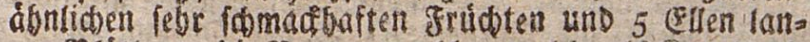

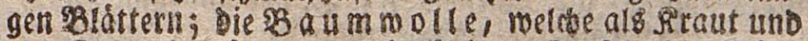

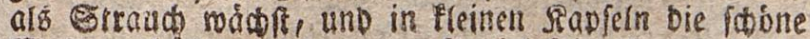
șolle enthelt, aus der man fo viel feine Zeuge (fiat=

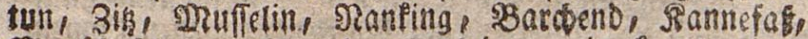

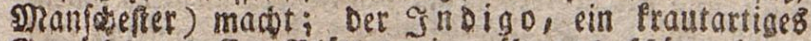

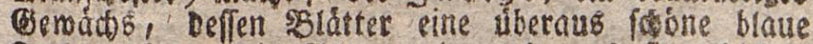
Sarbe geben; Die 21 loe mit mebr als fingerbiden, langen uno frablichten Sgláttern, meldue Daber aud in «metifa zum/Deten Der şáufer gebrakd merben. 23on einer 2urt bon 2loe erbalt man einen bittern Saft, bet sut 2urstrei gebraudt mirb.

\section{Das Mineralteict.}

Plle Drineralien lafien fid unter folgenbe viet Rlatfen bringen.

1) Erben unb Steithe. unter ben veríbies Detten Erben find megen ifrer Nukbarfeit yorzuglid mertwirdig: bie Riefel.erbe, welche Durd \$er-

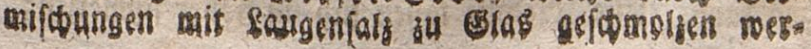


Den Fann. Der Bzergernftall, ber Eharcebon, Der Stipel, Der sBimśftein, Der Feuerftein ( Flintenfein), Det Jappis, Der Eafurftein bon trefflicher blawer gatbbe, Der Branat und biele anbere geboren fu ben Sicfeln. Die Ebonerde, wozu nidt blof ber gemeine Elyon

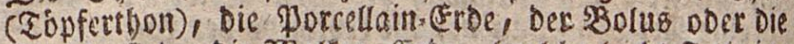
Eiegel = (erbe, Die Walfer = Erbe, (meläe Leid)t Fett ein: faligi), Der gliaunthon, Der Thon. Edtiefer, Eafel= Edjiefer und Dads = Schiefer, fonoern aud mainche cole Eteime, 3. 23. Der Saphir, Seubin, SmaragD, Fopaz unt ansere gerectret metoen, meit ihre seftand theile thonartig, unt auf eine fúr uns unbegreifliche ş3eife lo ausnebment bart, burchifotig uno feurig gemorden fitib. Eine befonbers merfmúrdige Stemart ift ber \&A yeffein oder sopffeill, welcher fo meis aus ber

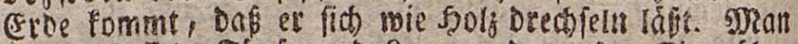
mađis Reffel, Tópfe uno Lampen Daraus. Eine ábn: ride Eigenfmaft bat oer Serdentinfe ein. Die Sial" Erde bat Das (Eigene, Daß fie fich mit spaffer erbint. Eie niro zum Sheil to hart, bag fie am Stabl zunfent

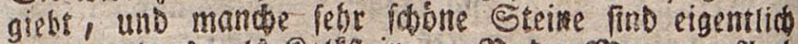

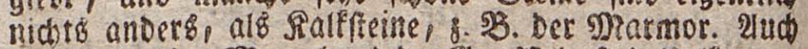
Die fireibe, Der Mergel uno Der Sinpbftein find salterten.

2.) Brentiche Mrineralien, welche mit els nem elgenen Berudse brenten, oder menigfteng gfims men, unt zut Unterbaltung Deß Beuerb bienen fonnen. Dabin gehort i. 3. Der Ectmefel, Der Bernifein, Daß̉ Ertól (Steind, Bergól), Das (Eropech) (Jubenped), 2k Ephait), Die Steinfohle, das Sieifblen (Bsraphit), nots aus sbleiftifte und Sathelgtieget gemactit werben, uno Daß̉ auch als Dfenidurárze gebraucbt wirb. Selft ber Demant (Diamant) get)ort fu Den brennbaren Mineras lien, of et gleids Der bárteffe unter allen befannten Siós pern ift, uno won feiner feile angegriffen miro.

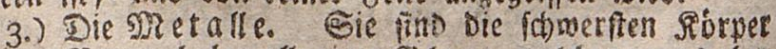
in Der Natur, baben alle einen \& lans, soldcen man oaber Den metallifoden Stanz genant hat, fimb bi eg i a m cbe

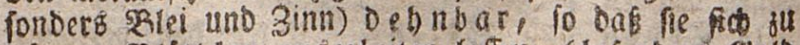
binnen sglätroben ausarbeiten faffen, (befonberg sold und Eilber) unb zábe, fo Dak man fie zu Dratb jieo ben fann. 24le Metalle laffen fich im feruer fobmelien; 


\section{Produtte det crole.}

Das Cifett uns Det Braunfein aber nur bei einem rebe farken Getrer. Man findet Die Mretalle in Der Eroe ent, meder geDiegen, D. \%. tein bon allen Bermicoungen, Doer verergt, 0 . 5. vermifdet mit andern DRueralien,

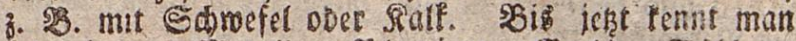
jolgenbe is gretalle: Pratina, (Solb, Silber, Quedefiber, siufer, éfien, slei, sinn, Sinf, 2Bismuth, Spiesiglab, Robalt, Sidel, Sraunftein, Solfram, Dolnbón, grfenif, Ulinaum und Sitanium.

Das mit anbers Rórpern vermengte Metall, Doer Dab Ex wiro in Den fogenanten squt te ll gereinigt,

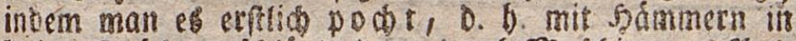

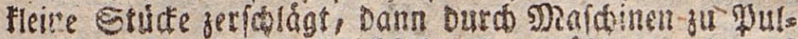
ber ftampft, biefes Durdflebt oder wádit, und auf

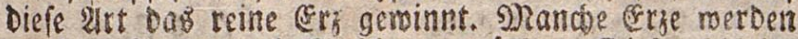

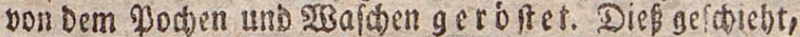

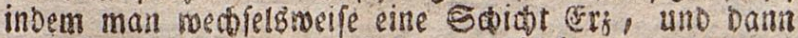

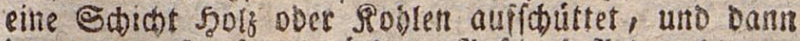
ben ganzen Saufer anzunbet. Muf Diefe 2urt macot matr Die Erge murbe und zam Sctmelaen gefdidt, uno rei nigt fie sugleid.

Dab Eifen miro jebr mannigfaltig benukt, indem man entweder Sfenplatten, fanonen unt Rugein Dars

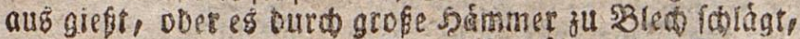
Doer eb ju Srath zieht. Die Cirenfábe, (Das Etabei fen) wiro ju gneffern, Edbeeren, Degen, Sábeln,

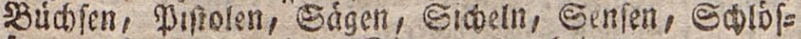
fern, und vielen andern Dingen verarbitet.

dus Dem Siupfer maden die Rupferformiede Ref. fel, Sópfe, Bedten, Dofen, Famen, \$f fanmetl, Dfens blajen und verfoptedente andere (Berátbe. Dures SDi. foungen matot man aub Dem Rupfor ₹ombate und Defifing.

DaB Sint und SBlei verarbeiret Der Simngiefer; man loblâgt es aber aud zu fehr Dúnen SBláttcoen, welcte Staniol Doer Folie beifen, und zur sbelegung

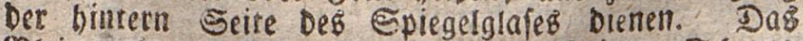
sglei roitb za Edrot uno Sagel (fleinem Sarot)

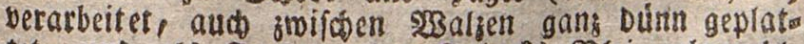

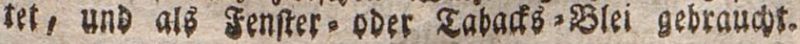




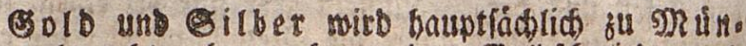

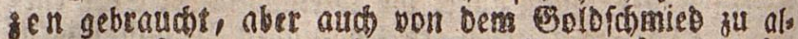

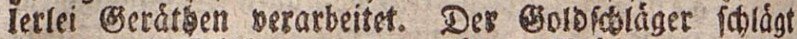

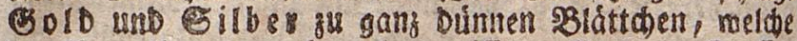
Die 2 ergolder su gebraucten mifer. \&uch fu Treffen roicb Das gors und Silber berarbeitet.

4) Die Salze. Sie unterfateiben farb borzuglio

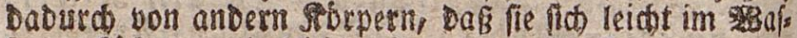
fer euflofer, uns teidst mit anbern Stoffen innig vers binten. Die sornebmiten Ealgarten fino forgente: Steinfalz, Ẽalmial, 2laun, פitriol, Salpeter uno Soba.

\section{VI. \\ S०n Dem Menfoen.}

I. Borzuge oes פenfchen.

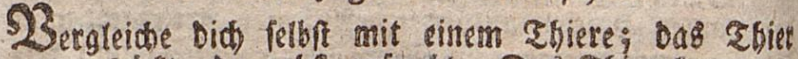

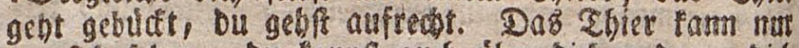
yor fie feben, bu fannft auds úber Dids und un bid́ fehen, fannfi Den Simmel nuit feinen Etertsen, tasunt Die Eonne und Den Mrono betracóten. Der faft Jgánder

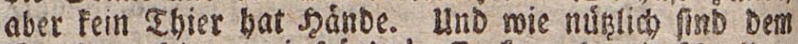

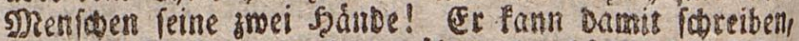
jeitonen, fonikere, maten, náben, oredifelin, Die Pfero De regieren, das sgrot bacéen, fáen, ernderes u. Dgl. m.

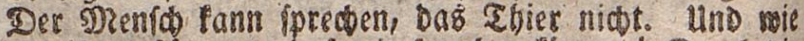

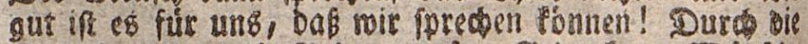

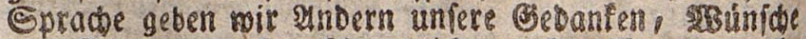
uno sBitten, unfere idsmerzlicten unb unfere angench. men empfindungen zu erfennen. PRonatep Du nidt

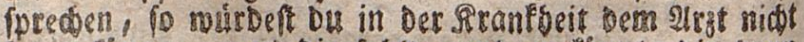

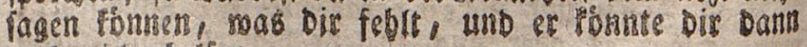
aud nidst belfen.

Du Pansft 60 , 70 und 80 , in 90 unb roo Jabse alt meroen. Die meiften Elisere merben nids halb in alt. Nur febre menige erreiden ein eben fo bobe 216

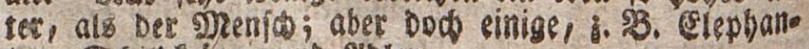

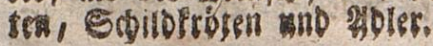


Det Menín hat mebr \&ebenzeraft, alb Die afiere.

Der Mrenid fann in allen Eanbern ber Erbe unb in jeber Suftart (Şimmelsfrich, Silima) ieben uno altebauern, in Der warmen, falten uno beisen; bas

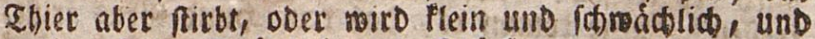
berfiert feine Sđóntheit uno Stárfe, menn ef aus feis nem Baterlande nad einem fremben \&anbe bingebradt wiro. Nur menige Tbiere fonnen in jebem Şimmelb. friche leben. 2luch bieran jeigt fich bie srofere febense fraft Deg s) renfeten.

Das Shier fann fid groar auch, wie Det Mrenid, willftifrlid (wis ef roill) von einem Drte zum an Dern beroegen; aber fo mannidfaltige uno fo funfts lia)e Berwegungen, wie ber Menfo, fann eв Dod mit

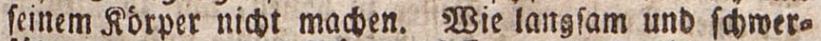
fállig bervegen fich SBaren, elffent uno sunbe, mentr man fie aud now fo funftion fum Sanjen abgerichtet bat; uno wie ungefdidt uno báfricb leben tie Dabet aus? Der sienít fann fogar, obne zu fprechen, blof Dura) Die Berwegungen feinez Siorperb́, befonoers Der Szánde, Des Ropfs uno der Magen, andern feine Bese Danten uno sisumfore fu verfetben geben, er tann die Bebebroenfprache reden. Scodfen fann ber sound Durcb firtimmen feine siorpers uno Friecten

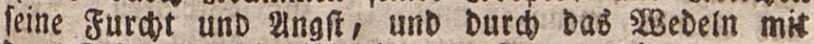
Dem Echmanze feine freube zu erlennen geben.

suab Dab Sbier lamn fúr lein Seben, leime Erbale fung uno Eiderbeit forgen, fann fich gegen (Sefaliren uno edngriffe iajuken; aber bei roeitem nidt auf fo mannidbfaltige exrt, wie Der IReníd. Diefer bat un-

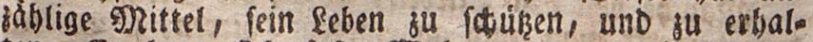
ten. Er baut fid fefte 2wobnungen, morin er yor Dem engriff Der roiben ahiere gefidert it, und fid

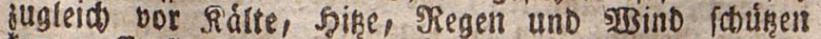
tann. Er Fennt to viele Sráuter uno \$flanzen, melá)e Die firaft baben, Rirantheiten zu beilen, Doer ifon oae bor ju bewalsren. Er tann fich ourd Dámme gegets

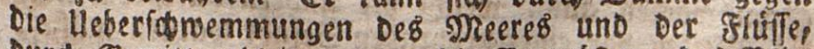
Durd Bemitierableiter gegen Dic \$sermúftung Deg SBlig. 


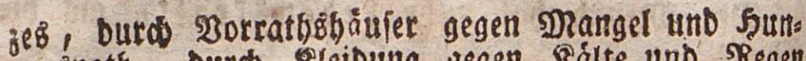
ge:Enoth, Durd Seleidung gegen Rálte und Regen id)uุen.

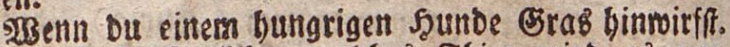

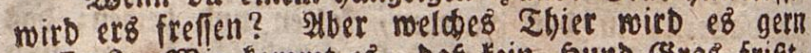

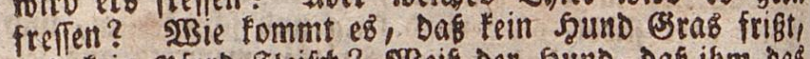

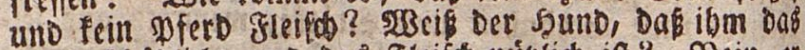

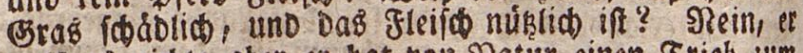

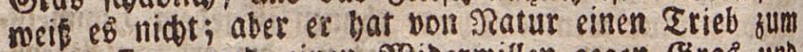
Silericteffen, unb eimen 2Biderwillen gegen \&rab uno

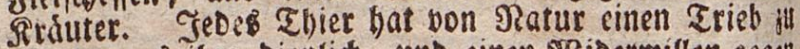
allem, mab ibm bientic), und einen $2 B$ iberwillen gegen

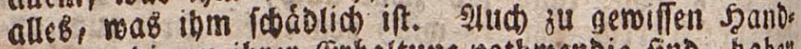
rungen, bie su ibrer exhaitung notbroendig find, baben alle Shiere you SRatur einen Erieb. Eo baben alle Bis $^{2}$ gel einen ₹rieb, fich Jefter zu bauen, unt zu fliegent;

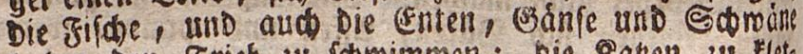

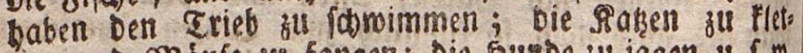
tern und Máufe zu fangen; Die șunde zu jagen u. f.t.

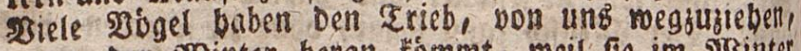
wenn Det 2 Binter heran tommt, weil fie im $233 i n t e t$

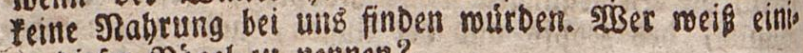
ge Diefer 200 gel zts nennen?

2tud Die Nenichen haben yon Natur Triebe. Deb

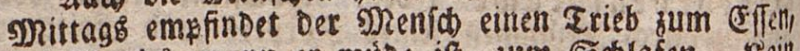

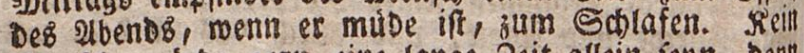
gRenín múrbe gern eime lange zeit allein fenn, bent

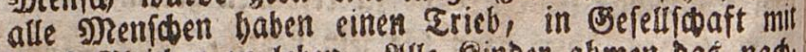

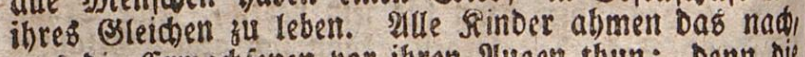
mas die errwadfenten vor ibren थugen thun; Denn bil MRentiden haben einen Na a ab mung strieb.

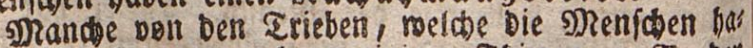
ben, bemertt man aud an einigen Sbieren, 3. SB. Den

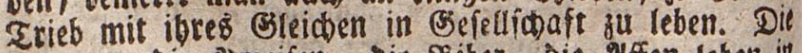
3Bienten, bie 2umeifen, bie siber, Die थlfen leben in großen Befellifaften bei einander. Die Bsemien, mel de mit unfern 3iegen viel 2lebnticteleit baben, gehent immer in Befellifbaft auf Rabrung aus, uno ffellen Sobibmaden auB, melche Die enbern burch ein ftats teb sperfen bor einer brobenden (Befabr marnen múffert.

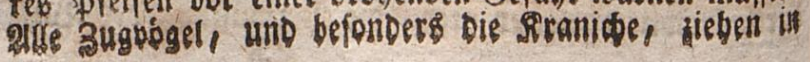


(Bsefellfibaft fort, unb geben fich in ber Gerne burd) ein raubeb Befchrei einander ju erfennen, ura niobt getrennt zu werbent. Sie fliegen in einer befimmaten Drbnung, und Diejerigen, wellde goran fliegen, merben nach einiz get seit von ben bimterfen abgetóft.

Die menichliden Befellfobaften fint aber Dod viel Drbentlióner einqeridtet, und bauern lánger, alB̈ Die Der Shiere. Die meníten leben bei einander in, Stäbten und Dörfern, um fich einanber bei itbren Iltbeiten uno

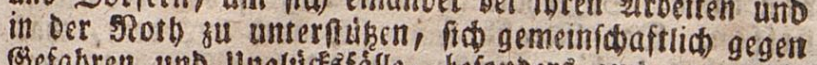
Befabren und unglúdéffálle, befonbers aud gegen die

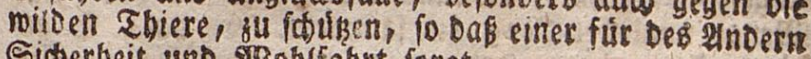
Siderbeit uno 530 oblfabrt forgt.

Die Berbinbungen ober sefellichaften, in melchens die Menichen leben, fino fermer (e) mannioffaltig.

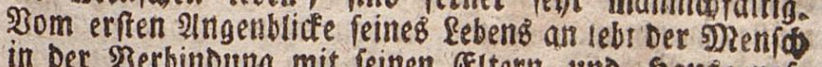
in Der Berbinoung mit feinen EIttern uno Sgauk̆genof fen. WBenn ex anfangt, feinen Berfand fu gebraudeeth to tritt ex mit \&ebrern und Miticatiern in פerbinbunge Dann aud mit guten freunben, Nacbbarn unb Mitburs

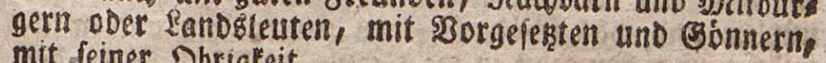
mit feiner Dbrigleit.

MRande Mreniden leben, megen Des હeidtaftes, Das

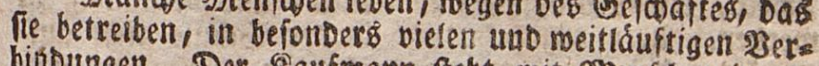

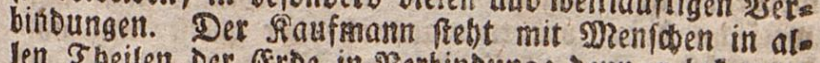
len Theilen Der Eroe in \$erbindung; Denn er betommt feine 5 Baaren aub berfobiedenen unb meit entferatent Rándern, is. S. Sitronen und pomeramgen aus Jtalien, \$2Bein aub Spanien und Irantreid, saffee aus 2Imea rifa unb enfien, oft mebrere taufents פReilen meit; Eis fen aub Edbmeben, 3inn auś England, sBolle aus

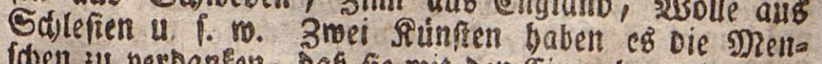
foen ju verbanien, Daf fie mir Den einmolinern der ent-

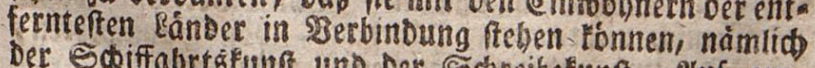

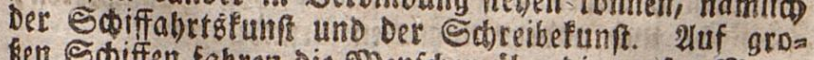

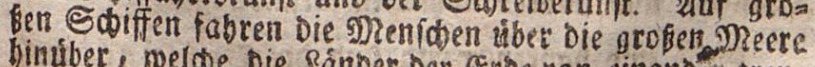
hinúber, welde die Ránber Der Erbe von einander trennen, unb ourch bie Sobreibefunft fonnen fie Denen, reelo de nod) fo weit von ibmen entfernt find, ibre ssedans fen uno sisuinfobe fo gut zu verfeben geben, als ob fie. fi由 mit ihnen unterredefen. 
Ein ieber mRenich fann un terficeiden, nas wable unb mas fallo iff. Eer tann fich unzáblige ridstige Begriffe machen; Denn er bat Daz 2̧ermégen, zu

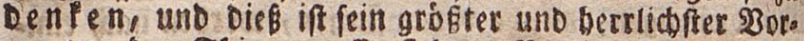

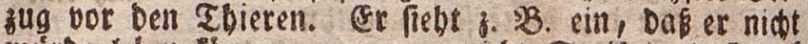
moirbe leben fonnen, wenn er nicht Speife uno Trant zu fich nábme, teine Sileibung und teine 280 binung hatte; Daf er alfo Diefe Drei Dinge ni det entbet.

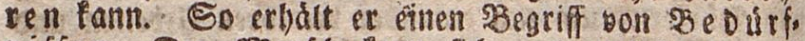
niffen. Der Mgenfat Paun fich aucd aus Dem, wab er gefelen, gethort, berftanden und begriffen bat, eint

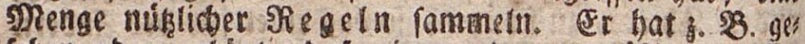
fehen ober gebort, baß̉ eimer, Der unmásig gegeffent

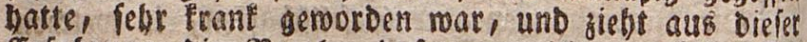
eErfabrung Die Siegel, Dẩ man niç)t unmápig effent múfe, mern man gefund bleiber molle. Doer or borth

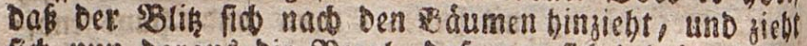

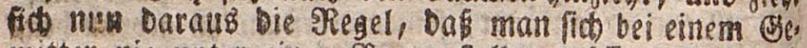
mitter nie unter eimen 5 aum fellen muife. 2uf bief?

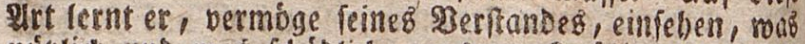

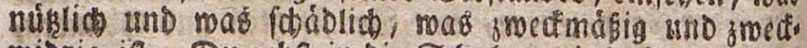
miorig ift. Du gebft in Die Sdule, unb baft Daben bett

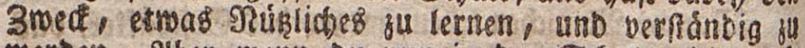
merben. 2lber wern ou nun in ber Sकule nidt aufs merffam bif, fondern plauderft oder fpiclif, uno um! ber gaffit, fo banbelft ou zreat mid rig; Denn auf Diefe Ant famnif bu beinen 3weaf, berfándiger ju merben, nicost erreichen. - Durd feinem 2 erftand

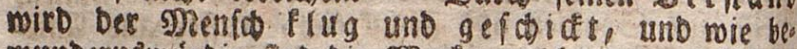
munbernbroirdig fino Die sBerte, melde bet menfablide \$Berfand bervorgebradot bat! Man betradte nur die

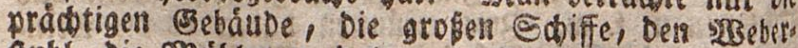

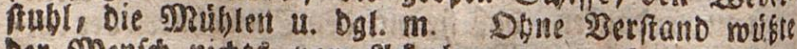

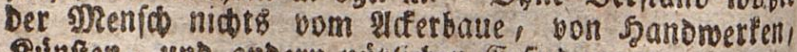
súnfen, uno andern nikgliden Eifinbungen.

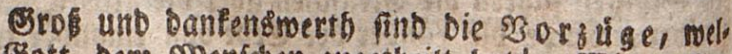
che Bott Dem Menfeben jugetbeilt has! sBir mollen uns Diefer \$orzuge freuen, und Boft Daburch Dafüt Danlen, baf mir fie meife unto gemifientaft gebraudent and lie รน erbalten fuchen. 


\section{Der menfotidbe Rórper.}

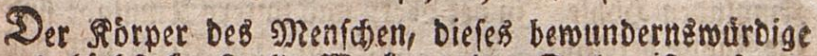

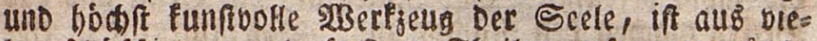
len fliffigen und feften abeilen jufammengefert, und alle biefe faft unzáhlbaren sheile bilden eine gia. (dime, Deren Bau wir nidt oft unb aufmerffam genug

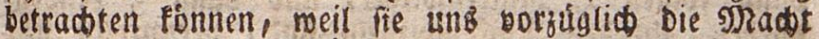

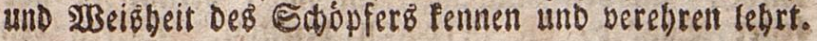

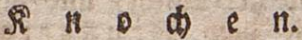

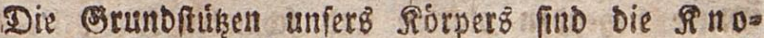
wen. Sie find ftarf, fefit unb bart gebilbet, bamit fie Das gltifich Dez Rópers unterfúken, und vor Dem

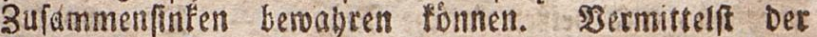
Belenfe fint fie alle feft untereinander verbun. bent. Die Selenfe aber find mit Rnorpeln verie. ben, Damit fias oie Rindwen nicht an einanber reiber

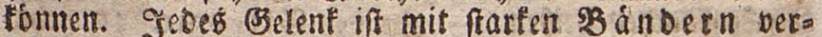
fehen, Damit es ni申bt auseinander geben fant, unb aus fleitten SBláżhen (Drúfen) bringt beftánbig eine Fettigfeit in bie Belenfe, Damit fie geidmeidig bleiber. 2Ille burd SBánber unb Rnorpel untereinanber verbun= Dene snoden, Deren man ungefábr 26 z záblt, madten Dab Serippe bes menichlicten fiotpers aus. Die Rinoden haben theils eine róbenformige, theils eine platse ober breite Beffalt, und viele fino inmendig ganz boht. Iud Die 32 3áf ne geboren zu Den Sinoden. Sie unteríbeiden fich nur Daburd yon Den tubrigen,

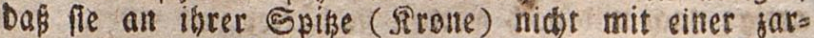
tell Şaut, ber Beinhaut, befleidet finb. 2ud die innere Sjoblung Der Rnochen, melose bas - mart ent= Dált, if mit fold) eiturn Sáuthen belegt. - Daß ganse Rinodsengebaude theilt man in ben Ropf, Deat Fi ump f und die B liedmaßen. Der Edabel des Ropfes if aus verfobiedenen Theilen zufammengeferst,

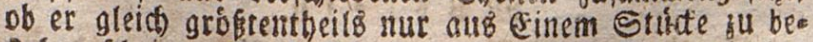

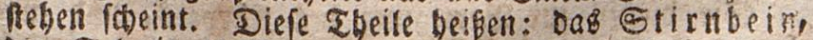
Die Edeitelbeine, Dab Sinterbauptbein, uno

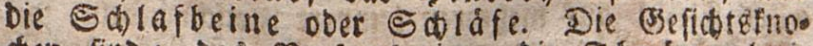
den find: Dab গRafenbein, die Thránenbei- 
ne, unb bie Sa a menbeine. Die lekteren bilben bie Sinitaben -Sjoble. Int Der obern und untern Rinnlabe finb

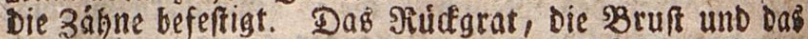

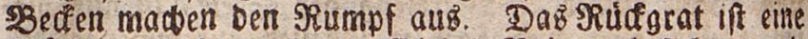
Eáule, welche ลuв 24 2Birbel= 2Beinen beffeht, und

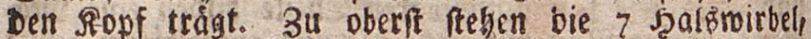
Dann folgen 12 Siliffermbitbel, unb Damn 5 Renbents

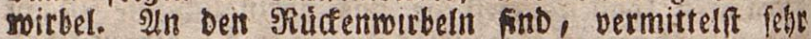

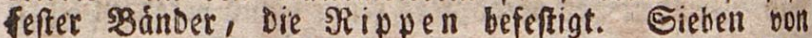
biefen $\Re$ Rippen fint gefrummt, unt bilden mebrere 930 s gets, Die fich mit bem 3rufibeine, einem in Det SRitte liegenden platten und fomalen Pnodben, vers

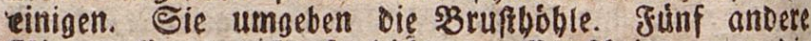
SRippent liegell midet fo Dicht am sBruftheitre, mie bie Dberften, uns heísen falfche Rippen. - Da, mo Dấ

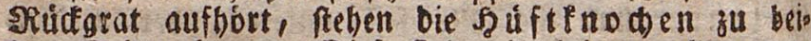
Den Seiten hervor. Diefe find mit einigen andern bers

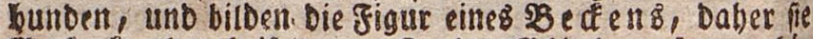

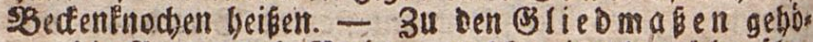
rent bie थr me und ge eine, melde einander lebr ábto Vid) finb, unb wieber aแz verfdiebenen einzelnen Theis Jen beffehen. 2in Den alsmen unteridheibet man bell Dberarm, Dent lluterarm, unb Die Shand ; Die Beine befteben aus Dem Dber = und linterichenfel, und Dem Sufue. Der Dberidentel fángt fich an Der Sulfte ant

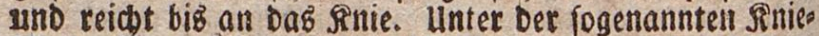
(í)eibe fängt fid Der llnteriobentel an, weldwer bis all Den ₹ur reicht.

2llle biefé Rnowen finb anfangs meiob unb fnorpele antig; nad unb nach merden fie bärter und feffer. SBei

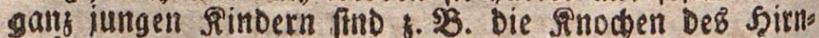
ídábels noch fehr neió), Daber man von ibnen zu lagets

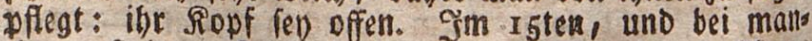

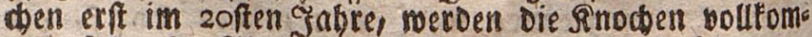
men feft; im alter merben fle leidter und brúdig.

s⿱⺈ 4 \& $\&$ \& I $\mathrm{n}$.

Sie bienen jur sbewegung bę Röriperb, unb mas

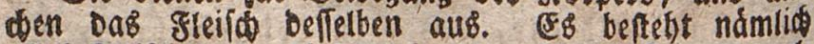

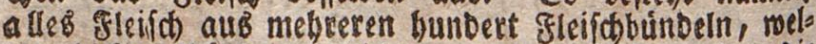

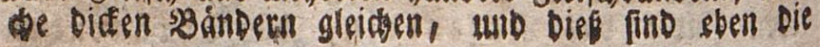




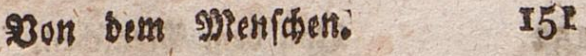

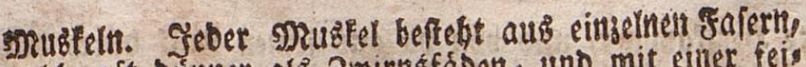
weldbe oft Düuner alş Zwirnsfáben, und mit einer feis neit Şaut, Dem fogenanaten 3 ell g eno e be, úberzogen

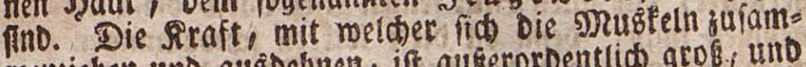
memsiegen und ausbebnen, if aukerorbentlich gros, und miro Die Re izbarfeit genannt. Diefe Rraft mirft theils mit unferm 5 sillen, wie \&. 23 . wetn wir unfere Atrme ober SBeine berwegen, menn wir geben, arbeiten, etwab ergreifen ober ferfbalten, theilb aber aud of he

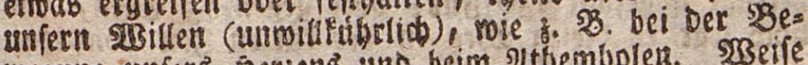

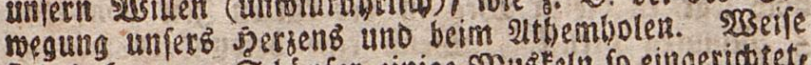
fino Daber vom Etbopfer einige misteln fo eingesidiet, bas fie nie mide uno follaf nerbert fonbern immer it \$etwegung feron ponnen, obne icmals fu erfoblaffer.

Dab Sgerz, Die solutgefápe uno Die 21Dern

Das Serz if ein hobler, aub ftarken gleirobuinDeln fufammengerountemer Rórper, Der unten in Der

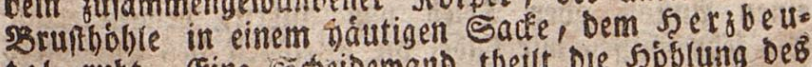

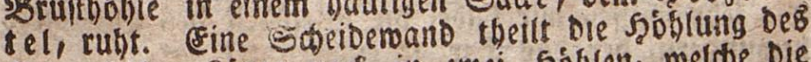
Shergens oer fange nad in zwei froblen, weldhe bie Serzf a m mern genanint werdent. SeDe Soerifammer if reber burd eine Sdeidemand in zmei Sjoblen ab: getheilt. פRit diefen veríticoenen Rammern fint bie

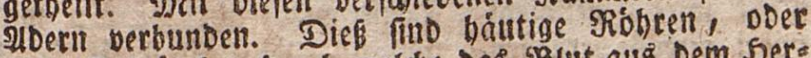

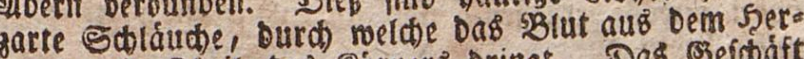
zen in alfe Ebeile bes Rörperb Dringt. Das Eeficáft DeB Serzenz iff, $D a b$ B Blut in die entfernteften theile

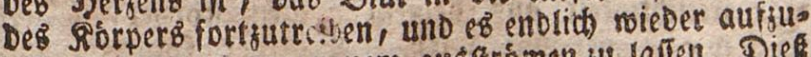
nebmen, un es yor neuem aub́ftrómen fu laffen. Dief nenmt man Den Rreiblauf DeB sB lateB. Er miro

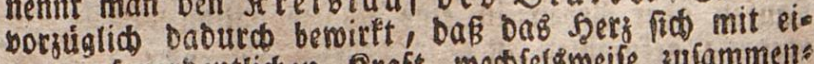
ner auferorbentlichen SRraft med fergmeife zufammens

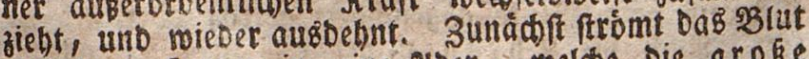
aus bem Sersen in eime 20er, welde bie grobe \$ulB̈aber genannt mirb. 2uś biefer etgieft in swei fleinere eloern, bann wieder in nod fleis nere, mit weldhen ber ganze fórper gleidfam burd)=

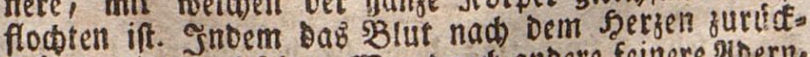
frobmt, nimmi eB feinen sBeg ourd andere feinere 210ern, melde SBlutabern heiben, uno flieft in bie rede 


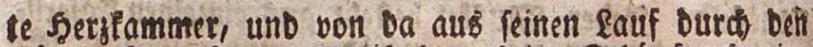

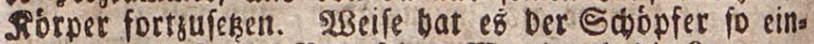
getictet, Daf alleş sglut feinen 203 eg Durd Die \& u n gen

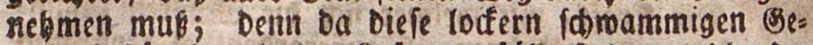
mebe beftánig mit Der \&uft angefúlt find, melàe Der gmenion einatbmet, to wirb bas islut bei feinem Durd: gange burd Die elunge abgefúblt uno erftifít. Det 52Beg, ben bas şlut bei feinem llmtaufe bu maden bat; betrágt ungefábr 150 Jußs, Doer gegen 75 Ellet?, und Dow legt es biefen 20 eg in Der furjen zeit bon etwa

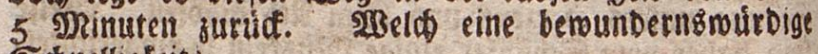
Ednelligfeit:

Das Ser eineb gefunben פRenfwen jieht fich in Eio ner Drinute fedjzig bis adjegigmal jufammen und alfo in einer Stunde orei taufent fechshundertmal; mie erfalls nenswuirdig if Diefe şemegungşfraft, befonbers, ment man bedentt, Daßs Das Szetz fid von felbft, obne irgend

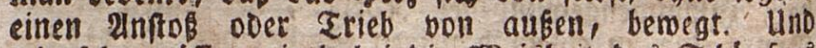
soie fehr múfen mir Dabei bie 2Beizheit Deß Echopferß bemundern, Der Dar Jjerz fo eingerichtet hat, Dáp feine geroegung ober zufammenziebung und 2lusbehnung

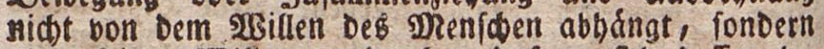

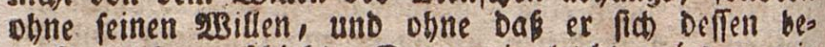
souft wird, géfiefht. Denn mie leidte witroen mir Dabei etrwas vergeffer, und augenblictiod hörte bann unfer seben auf.

2BBenn Du Did erbiagt baft, fo bringt eine wák sige Jeudtigfeit aub Deinem grmen fororper, welde (ड) weik genanit roird. Da Der Edjmeiß nut Danu aus Dem Riorper oringt, menn Dein sglut burd

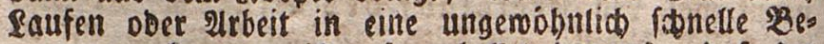
soeguns gefommen iff, to erbellet daraub, Daj Der Sdberis vom sBlute abgefondert mitb, ober fid abs ferzt. 2uch bie ₹bránen gebóren zu Den másrigent Seubtigleiten, meiche von Dem grlute abgefondert

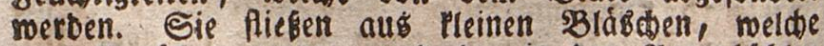
man Drufen nennt, und Die in Den 2lugenboblen

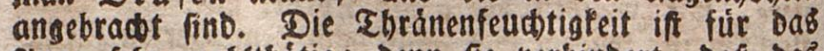

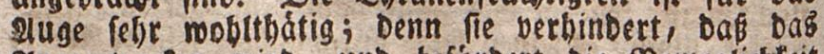
suge tiofen mirb, und beforbert oie semeglidfeet

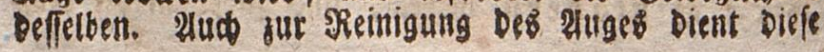


Feudtigleit, Denn fie fpúh)t gleidfam Denf Staub, unb alle anbere Unreinigfeiten, welche in Dab Pluge gea flogen find, aus Demfetben weg, uno Darum bat es

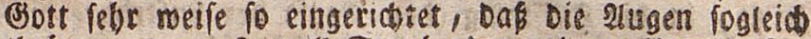
tbeänen, wean fie boll Staub ober anberer unreinigfett fint. - SBeftánbig Dringt aแઠ unferm Rórper ein ró́briger Dunft, aud Dann, wenn wir nictet fobmisen; Diefer Dunft witb ebenfalls von bem sglute abgefon-

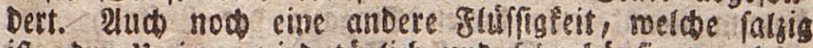

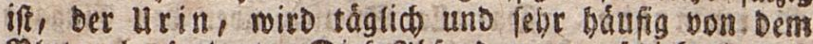
3ilute abgeionbert. Diefe 21bionderung geiobieht in Den Rieren, weldbe unten am Filitgrate liegen, uno Den Ul rin in Die 28 lafe leiten. Daber enthalten Die Neieren eine grofe פrenge sglutgefáfe, Durd) melche bas sBlut láuft, um fíh von ber wábrigen und falzigen Jeuditigo teit tu befriten.

SBon ben \&unget unb Dem थtbembolen.

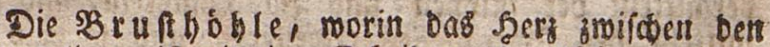
Rungen liegt, iff mit einer Sdeidemanb verfehen, welobe fie boll Dem Interleibe trents. Diefe Sweideraand be= fteht aus einer febuidbten פRusfel, melde gleid) einem Gelle auggefpannt ift. NRan nennt fie Das 3 merch= fell, und fie beffebl barum aus weichen muźleln, Damit bie sarten \&ungen, welche bis cuf bas swerdffelf berablaángen, nidbt gebruadt merben, ober fid reiben mógen.

2on Den smei fungen, weilde mir baben, liegt bie sime in ber recten, bie anbere in Der linfen Seite ber sBruftebble; Daber witb bie cine Die redote lunge und Die anbere die linfe genannt. Die \&ungen fint weidhe, fómammartige Sobrper, uno entbalten viele Suftgefáke ober suftbebaltter.

Fiber wie formmt benn bie Luft in bie Sungen? merbet ibr fragen. Diefs gefobiegt auf folgende art. 23orne am Şalje fáblet ibs Didet unter ber Şaut eine aus mehreren frorpelidoten Fringen zufammengeferzte Piobre; bieß ift bie \&uftróbre. Da lie bie \&uft in inei \&ungen leiten folf, to theilt fie fid, mabe vor ben Sungen, in inei 2lefte, Durd meldoe bie eningentfgente 
Suft in bie Rungen geht. Durd ben 9 Mnuth uab bit Tafe ziehert wir bie suft ein, melde Dann in Dem hin:

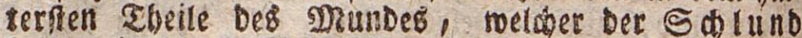
beist, in bie Deffnung (Den Fiopf) Der Ruftrobire bin eingebt. Diefe Deffmung ift nur fefer formal und engen gleidjan mie eime Epalte DDer' 'SRize. Sinbem die cint geatymete \&uft fith Durd biefe enge Epalte Deśngt, entifelit Der Zon, ben wir Stimme nennen, uno barum nennt man biefen Eingang ber fuftróbre tie Stimmrize. Da alle Speifen úber ben Eingang Det euftrobre finmeggleiten múfien, um in bie meje binterwákt liegende Epeiferibure zu fommen, fo if Die Etinumrige mit einem Decetet verfehen. Denn mie teicht fonnten fonft bie gerfauten Epeifen in Die

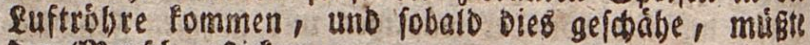
Der smentch exfficen.

Die Euft Dringt von felbit Durd Dis Suftrobse ith

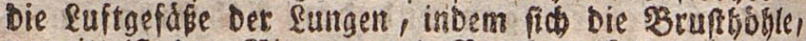

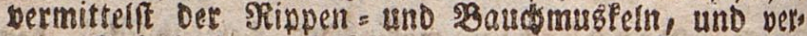
mittelfit bes 3merchferles erweitert, unb to bald fic Die SBruffhóble wieber zufammensiebt, wirb bie einges athmete \&uft aus ben cuftgefásen mieber beraubigetrier ben, und burds bie \&uftrobre meggefda afft, bamit bie nels eingearbmete fuft an inre Stelle einbringen fann.

İegt laffet unz einige 23ortheile bemerlen, welde Der sRenís oon bem थthembolen hat. Eerfftids mirt Daburd Das sblut in ben fungen, no fich Duta Dab 2ttbembolen inmer frifote Ruft befinbet, abgeffublt, unb zugleich bon feinen unteinen Theilent befreit, Demn Diefe

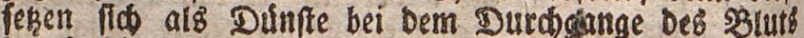
Durch) bie fungen $a b$, uab twerben nun nait ber auss

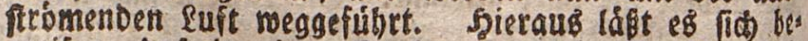
greifen, Daks in einer Etube, mo viele smenichent beis

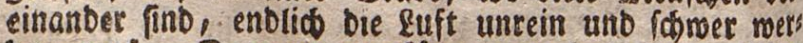

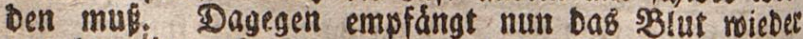
bon Der eingeathmeten suft Die befiten Theile unb with auf bie Art Durch Das stbembolen febr verbeffert. Indeffelt gefdicht biefe ßerbefferung Des $B$ lute\& nut Dann, wenn bie fungen frifकe, teine \&uft cingefogen baben; ift pe untrein und nict frifó, to miro Daf 
sliut nidit verbeffert, fonbern verfolimmert, unb bes Menich füblt Beángftigung und innere Sibe. Daro um fanu man fid in einer mit beifen Dánfers anges fullten stube unmoglids noll befinden.

Da Der शragen ein hăutiger Sack, nabe unter

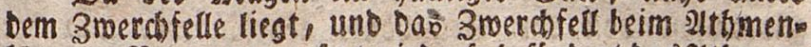
bolen in SBemeguting gefest wird, fo befórbert Das 2tbments bolen audi oie Berwegung Des Magen\$ uno Der úbris

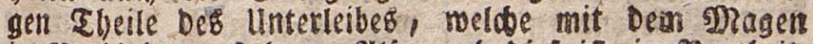
in Berbinbung feben. 2urpo auch Dies if ein פortbeil, Den wir bor ben attgmenbolen baben. Sjierzu formmt

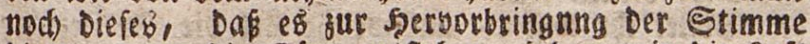
Dient; Demn bie इone entfteben, inbem mir die fuft

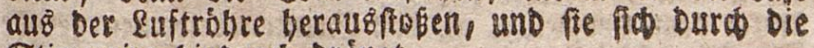
Stimmringe hitndurd Drángt.

\section{\$on Der Berbaung ber Speifet.}

פुBenn unfer Siorper etbalten metden foll, fo múf fen mir táglid Nabrung 8 mittel fu unB nebmen; Denn DaB ESlut leibet beftendig einen feber mertficien Bertuft, theilB burd Die 2lusbanfintung, theilB Durch bie Edfte, welde es Dent verítrebenen Eliebern Des Sibro perb zutbeilen muf, Damit fie befreben tobnen. Diefer sertuft muß wiener erfegt merben, und dié̈ ges

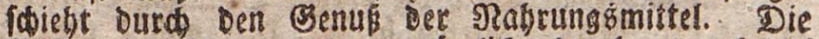
Rabrungśmittê merDen námlid Durd mancberfé פBerljetge verbauet, D. ho. in Saft unb 3 lut ver

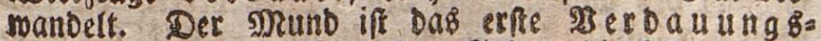
werfizag unfers sórpers. Intern bie feften Spei= fen, Dermittelf ber 3unge, melde aud ein spustel ift, in Dem Nunbe feftgebalten merben, find bie $3 \hat{b} b=$

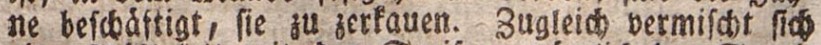
eine flúbigleit mit Den Epeifen, námlid ber Epei

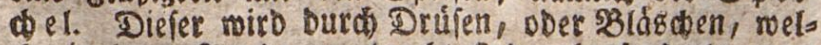
de in bern munte angebracti find, abgefondert; man nemet fie Epeideld rufen.

$23 i r$ miffen foton aus sem borigen, Dak binten im Edtunde, in ber Rachbaridaft Der Euftrobre, now eine andere SRohre ithre Deffnung hat, relche Die Epeiferónre genannt wiro. Die zentauten 
unb burd ben Epeidiel angefeudteten Speifen gelgen

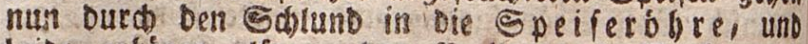

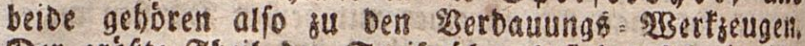
Der grófte Theil ber Epeiferobthe befindet fich in De? Sjoble bes unterleiber. Diefe Sibgle ift burd bas Biverchfell bon ber Soruntobble abgefonbert, und Der

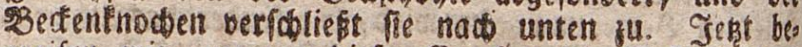
greifell mir, marum biefer Sinoden Die Beffalt eines 53cteng balommen hat; meil et námlid ben untert

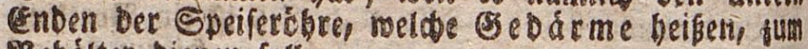
\$ebaltet bienen foll.

2Bollet ihe wifen, auf welde ort bie Speiferbibe

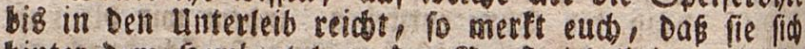
binter Dem Sperzbeutel an ben Beruftrirbelbeinen gerabe zum 3metchfell binab, und Durdis eine Deffnung Deffelo ben in ben unterleib gieht. Rurs nad ibrem Eingange in Denfelben nimmt fie fege an 2Beite zus, gleid sinem Regel.

Der Miagen, meldher mie ein queer lizgender $3 e t t$ tel aus mebreren Sacuten gebilbet ift, liegt zu pberfit mabe unter Dem 3werdffelle, bängt mit Der Epeiferóbre genau zufanmen, unb liegt mit feiner redten Seite an

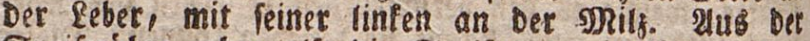
Epeiferóbre geben alfo bie Epeifen unmittelbar in Det Mnagen, unb swar burd bie oberffe Deffnung Deffelbell, melche ber magenmuno beift. Shier mifot fich eit fharfer Saft unter Die Speifen, Der fich Durw Die Sges megung Des Mragens aus Den Drúfen preft, , Die grto

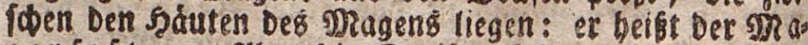
genfaft. - 2ber bie Epeifen folleri nicht beftandig in Mragen beiben, fondern aus Demiellen in bie Be Dérme gefúbret werben; barum bat ber magen aufer Dem snagenmunbe nod cine Deffnung erbalten, Durd meldere Die Speifen in Die Bebárme geleitet merden. Die BSed drme fino meide SRobren, weldee ats einet glatten, inmendig idoleimigten spaut beftelen. Eie madien nur einen eizzigen Darm $=\Re$ an al aus, wels

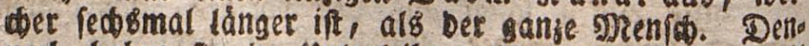
nod baben fie in unterleibe volitommen plak, meil fie eine gewunbene lage baben. Den oberfent Eheil Diefer Cédárme nennt man Den 3mólffingerdarm! 
Ber legte beift ber Maft= Darm. In bem 3molffins

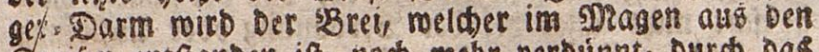
Epeifen entftanden ift, noch mebr verbúnnt, Durch bas sufammensieben ftarter MRub́felfafern zufammengetne. tet, uno mit Der \&alle, eines bittern und (d)leimicts: ten Seudrigfeit, bermifot. Durds Diefe Sermildung werden Die guten Fabrungatbeile aus Den Eperfen ab. gefondert. So geben fie Dann in bie ubrigen Darme?

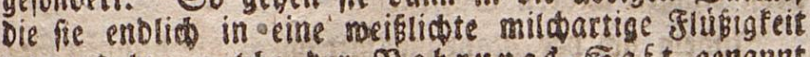
yerivandeln, weide bet $\Re a b$ rung $8=$ Saft genannt mirb.

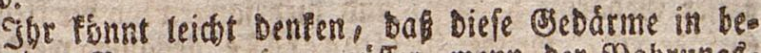

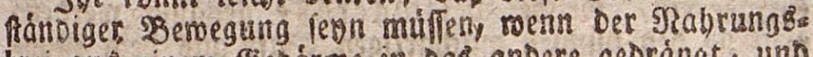
brei aus seinem sebárme in bas anbere gebrángt, uno

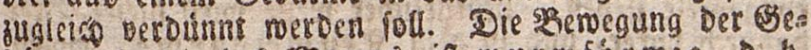
Dárme, fo wie Deह smageng, ift wo urmformig, D. \%. ungetabe eben fo, wie Die eimes solurmes, Der forts frieden will. 2aber Da bie Bedarme in ber sheble Des unterleibes Durd einanber gefolungen liegen, fo tonms ten fie fid bei Diefer beftánoigen soemegung leidt ans einanoer reiben, oDer in enanber wictern. Um Dieß zu verbuten, find fie Durd eine mit Fett berwachjene Spauts, Die man Das Eefróle nent, unter einaster verbuna

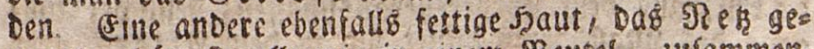
nannt, bait fie alle wie in eltem Seusel, jufamment? uno verbinoest, Daí fie nidt mit bem Baschfelle zhe farmmenwamfen. Durch einen beftigen Exrung oder Sall, ober eine übermáfige 2lnftrengung beim Şeben fitwerer Eaften fans dab SRek lódter befommen: bam tretent die Gedáme aus ibrer Sage und Der antenfod bes fomme eiren

Die \&eber, bas grófte Eingemeibe be\& Luterlei-

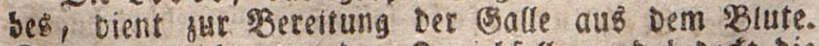
Eie liegt gleid unter bem 3merdfefl, uno bebeaft bie

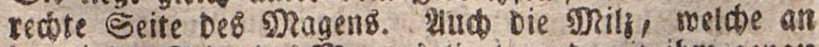
Der tinfen Seite Dex̉ 52lagens liegt, uno mit ibm genaus

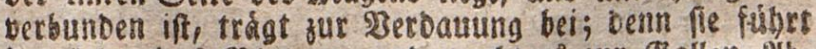
ber \&eber Das SBlut fu, und madit es jur csallen=2ab. fonbertung tauglich. Sie ift wie eine 3unge, námlict lángliderunb, geftaltet, uno auz vielen sBiutgefágen uno zellgemeben ufammengefert, Daber formammiकt. 


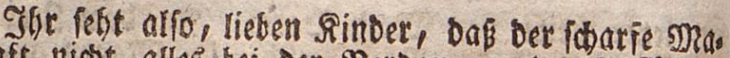
genfaft nidot alles bei der serdaunng oder 2unfofung Der Epeifen thuts, fonbern Dép auds Die rourmformige Bewegung Des MRagenB unb Der Gedárme, Die Daraus

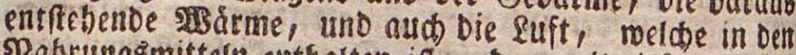
Nabrungemitteln entbalton ift, Dału mitwirfen. Silea zu fommt nun nocis, mie mit gefórt baben, die Balle nebfe einigen anbern Safften.

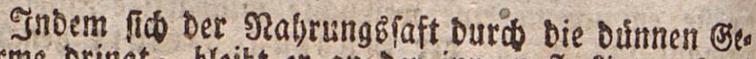
Dárme bringt, bleibt et an Der innern floceigten saut Diefer Bedárme bángen, unb bier fausgen ilbn bie fleinen

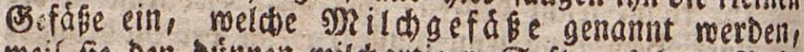
weif fie ben oínnen milchartigen Eaft auB bern Nabs rungébreic jief)en. Slaturtidjer meife mirb oiefer szrei Dasurch Dider, unb in Dem P rummbarme, mobjin er แun fommt, vertiert er reine glúfigleit faft gani,

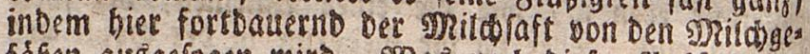

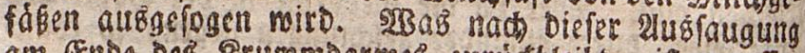
am Ende Des Rrummiarmes zurutatbleibt, if zur Ero

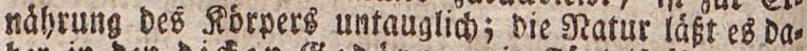
her in ben Di de en G edármen in gáumís úbergeben! und burd Diefe, befonbers Durch Den iogenannten gMa f fo

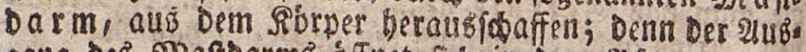
gang bes Mrafsbarmb bffnet fio in Dem elfter, ober

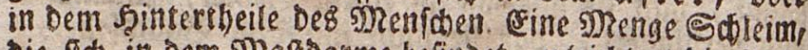
Die fid in Dem SRaftbarme befindet, erteidjert Die 2lus= leeruung bes barten untarbs, uno macht feime Sodarfe fúr Den Darm unfáádlia). SBei eimem gefunben Mens

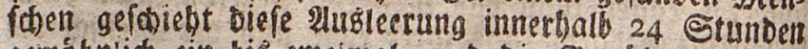

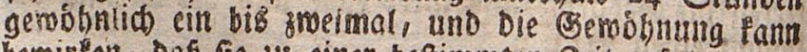
bemirten, Daf fie zus einer befimmien 3 eit exfolgt.

Der $\Re$ chrungsfaft foll, wie wir gebort baben, in sobrper almáblig in slut berwane elt werben, um

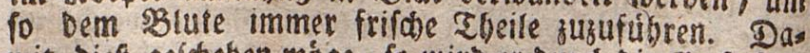
mit bieß gefdethen móge, fo miro er Durd bie \& e f r b g. Drúfen, in melchen eime mástidite Jeudjtigteit ents balten ift, berbunnt, unb bann Durch Die slutabern nad) Dem Seersen bingefúbert, wo er fich erft nad Bers lauf mefirerer Stunben in sglut vermandelt. Diefe

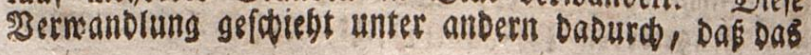




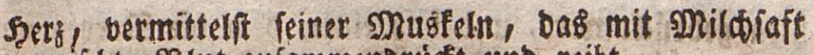
sermifdote sBlut fufammenbridt unb reibt.

פon Dem Bebirne, Dem Ir úce nmarfe und Den शerven.

Gar mifft fabon, lieben Rinder, Daf man Den linge lidtrutben (obalen) Srnochen, melower Den oberen und

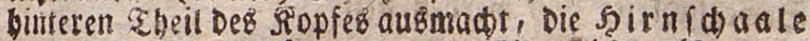
Dber ben Sirnfdido el nennt. Eigentli múpte man ihn Den Eetyrnióádel nemnen; Denn er bat von Dem

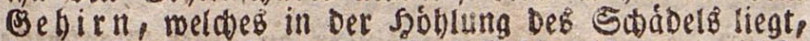
feinen Namen erbalten. Das Bethirn ift ber rocidjfe Thesil Des אopfes, uno ber widtigfte und jartefte Theil Deś ganjen meníblichen Siórpers; Denn bie geringfte

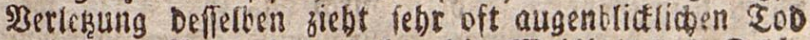

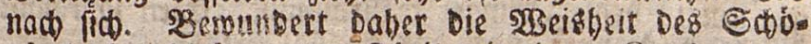
pferg, Der Diefen zarten Egeil mit einem Rnochen uma geben bat, meicher ithm gleidfam zu sitrem feften unt undurchoringtiden Sablibe Dient. SRerfet end bierbey,

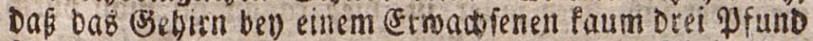

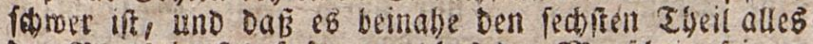

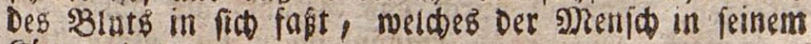
Siorper bat.

2us Dem Bebirne unb Sitúcfenmatie entfpringen vies Ie meise géden ober Samire yon veríntebener Diffe,

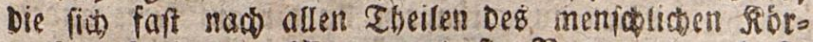
pers verbteitent - gran nent fie Rerven, und fie fino bánfig mit eimander verbunben ober berfíct)ten. Eie entiptingen alle paarweife. "2uz Dem Behim ent"

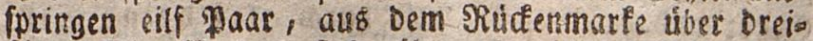
big Die stetven find uberaus michtige und notho mendige Theile unfers Sibrpers; Denn fie maden durt ifre Sieizbarfeit, Das wir empfinden. Daber

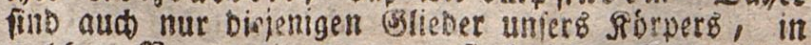
meldem Serben liegen, empfinblid. 2ule andere

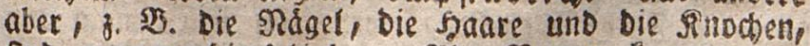
fint unempfinolic. 2lile Rerven fommen ins

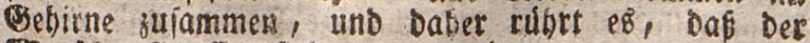
sIrenich alle Empfindungen verliert, menn fein Ces 
birn gebricet mirb, unb bafí eiter, Dem bie Nerben in 2atm zerfadnitten worden find, an Der sano feimen Sajmer mebre empfinder, weiln man all mit cinem Meffex hineinfounitte. - Die Rerben find aber niфt blok Die 220erfieuge ber esmpfindung, fondern aud Der $\mathfrak{B}$ enegung; Denn fobald ein Nerve jers fdnitten ober unterbunden mirb, berlieren alle Bslies Det, zu benen Der zerfotnittene Nerbe bingebet, ibre sBerveglicteif, uno merden freif.

\section{Bon oen Sinnen.}

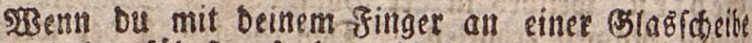
bin uno ber fábrit, fo bemertift ou, Dafs fle glaft iff

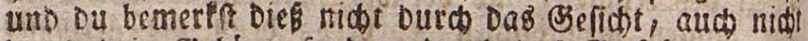

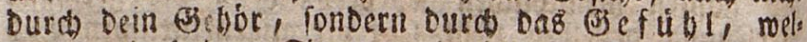

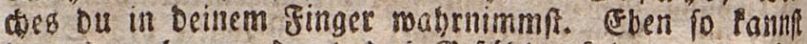

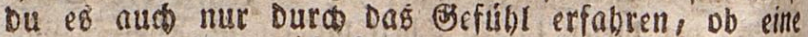
Sacbe bart ober weich, falt ober warm, naß DDer tropo fen, idwer ober leidt) ift. slber, ob ein Eetránt bits

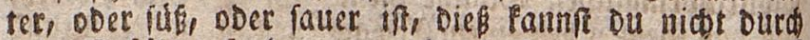
Dein Befubl, fonbern nur Durch Deinen Bsef $m$ a d erfabren. Somobl Durds Das sefúbl, als Durch Dets

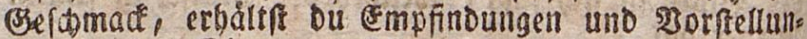
gen bon Den Dingen, weldbe a uber bir fino. Rofreft

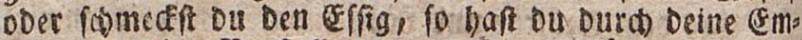
pfinoung eine Dorfellumg von feiner Eacure erbaltem, uno Diefe $90 r f t e l l u n g$ fónnteft Du Dir auf Feine andere

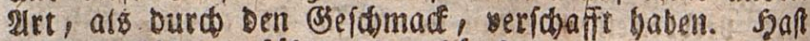

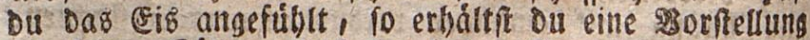
bon feiner Rálte. 2ứ Durch Den Sertud, Durch Dab̉ Behor uno Daz Befint; alfo Durch alle beine Sith the erháltft Du Empfindungen tsab פJorfetlungen yon

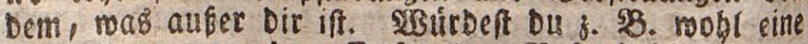
gorfellung von Dem Duft einer Siofe haben, went Du ibn niás Durct ben Beruch empfunben bátteft? Rómteff ba bir Delr Rinall einer Ranone und Die fobmarze garbe borfecllen, menn ou ienen now nie Behort une diefe nod nie gefeben hátteft? Befobreibe eimem sglinbgebornen die fatwarze garbe, und einem Taubgebornen eine fobone mufit, fo gut ou tannfe, 


\section{Don bem Emenfiten.}

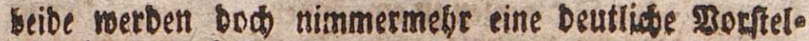
tung Davon erbaiten.

zuerf mollen mir úber Den Sinn bes Sefúbrs weiter nabbenfen. 23 ent wir füblen mollen, ob ete mas hart ober meich, falt ober marm, rauh ober glaft

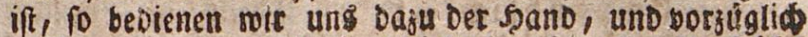

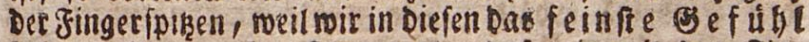
baben. 2aber nober fommt es, Daß noir mit Den finto gerpiken fo fein fúblen fonnen? WBeil Da Die Shaur am

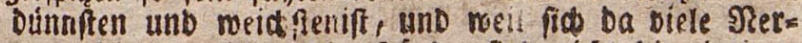
ven endigen. 2tber die Sánde find nidt die einzigent IBerfzeuge ber Empfindung, Der gamze Rórper if ibr sBertzeng. Deine Empfindungen find nids! alle bon eincrlei 2utr. Niddt wabr, eu Gafte eine unangenebne Empfindung, menu Du aus einer moljlgehsijten Stube

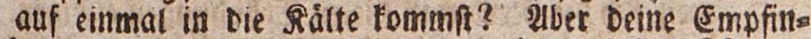
bungen finto felor angenehm, menn bu cus ber Rálle in eilte marme Stube tritt? ? Du fieb) bieraus, bas Deine empfintungen cben fo berícieben fimb, als Die Einbrü: Fe, welde bie duferen Begenftanbe auf bich maden. Sinb biefe esinbrufee nur folmad, to fino es aud Deine Empfintungen; ; find fie ftarf, fo baft bu aud fátr: fere empfindungen. 2Butroe nidot Deine empfinbung weit

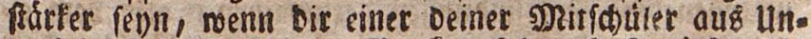

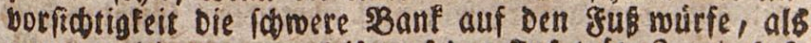
menn er bir nur ganz leife auf Den Gǘ tráte? wie befa tis ift bie Empfinoung, wenn man fidi Den ginger vorn am Nagel tiemmt, aber bei meitem niout fo beftig if fie, menur mar fíb Den me nicht ro empfinelid if, als am singer. Ein @tid in bie gubroblen rabmerst lange nicht to beftig, als ein Stid in Jie Sand ober in Den Urm, weil Der ontenf́ an Der Subifoble eine aberaus Diffe Dberhaut bat, unb

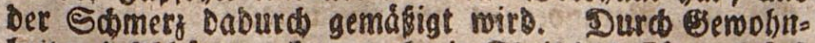

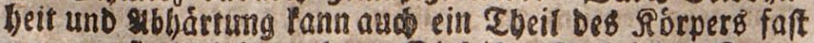
unempfindich merben. Dießs if 3. 28. bei ben getuer= arbeitern, bei Scomieben unb Schloffern Der ģall. Diefe feute befrändis mit bem feuter ungeben, uno Die

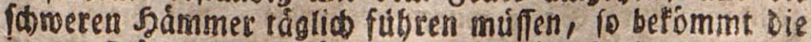

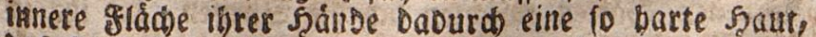

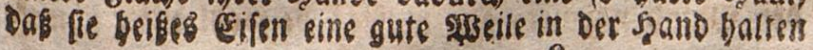


Fonnen, ofme Sdmerien zu empfinben. श2sorauf fomms es allo bei Dem Befuble an? Theils auf Die sBefwaf, fenbeit Des Einbrud's, theils auf Den Brad Det

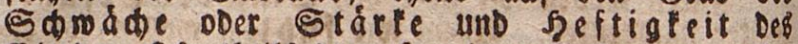
Eind ru d 8, theils Darauf, wie grob oder mie getino ge Die Empfindicteit bes Theil' ift, welder Den Einbruat exbált. Die grófte (Empfinotictecit bat Der פenít im 2uge, und Darum Derurfadt ibm aud

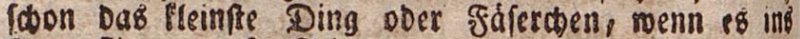
Iluge fliegt, grofe Sodmetzen.

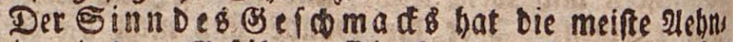
lidfeit mit Dem Bsfúble. Die zunge ift Das vorzúgs

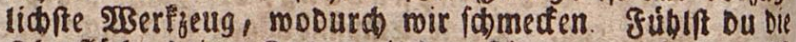
Dberflidbe Deiner 3unge mit Dem finger an, to bemerfl

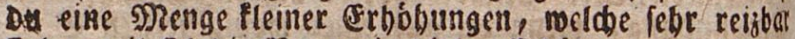
fino, weil fios bie geerben in diefen Ergobhungen endigen. Sie beifen Debrwegen Nerventórzchen. Diefen

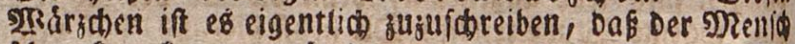
(domeden fans, und Darum nennt man iie aud Be.

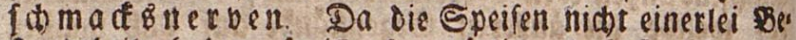

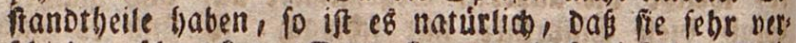

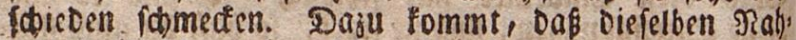
rungstmittel dem einen felbr angenebme, Dem andern fehts unangenelime eimpfintungen verturfacten, alfo Dem elo nen gut, Dem andern febr tibel ichmecten. Biebt es bod

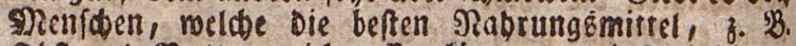
Dbft und SButter, nidbt efien lonnen, meil fie bei ibneth

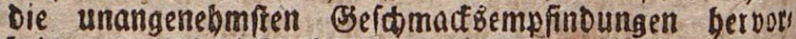
bringen. lano Dieß gekt fehr naturlico ju; Denn Die Epel" jên vermif́cen fíb im skunbe mit ben fobon vorbandes nen Sáften Deb Effenden, uno Diefe Sáfte fönuen Dow unmoglia bei allen Menichen bon cinerles 2rst fenth. פBie viel biefe Edffe, und mie biel befonderz Der Epcio

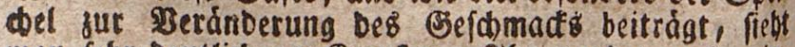
man febr Deutlid an Siranfen. She merdet eut erils nerti, lieben sinber, Daß eud alles bitter idomedte,

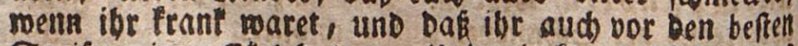

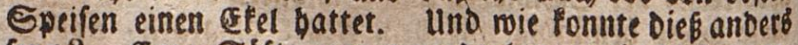
feon? Eure Edfte maren verborben, mit Diefen vers Dorberen Safften mifकten fíd Die Epeifen, bie ibr in ben anuno nafmet, alfo muften fie nobr bitter fommes 


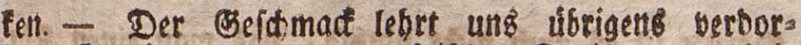
bene Epeifen von guten und frifonen Speifen untericheis Den, uno bemabrt uns alfo vor Dem Bentufie folder

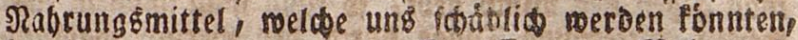

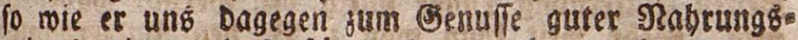
mittel reibt, und fie fưr uns angenéb matot.

Der Beformact hat einen rreuen (sefährten an bem Sinne bes Eerum 8 erbalten, und febr meife bat

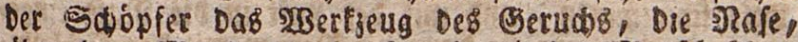

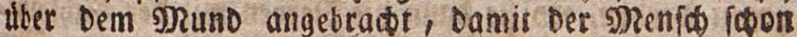
Durd Den Berud bon folden Dingen zuricfgehalten

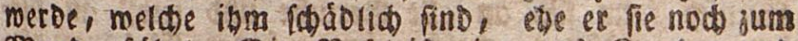
Munde fúbrt. Die Nafe if eine aus snoden und

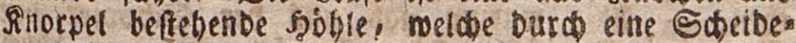
mand in jmei Theile getbeilt wirb, uno vorn unb himten geoffnet ift. Yhbre bintern Deffmungen erftrecfen fict

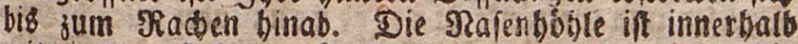
mit vielem Sdleim thertsogen, uno Darum heisht Die Spaut, mit welcher fee aubogefúttert ifs, Die Sd leim. hauf. Sie if voller Druffen uno Swleimbiáb̆en, meldo beftánbig, unb befonders bei einer Entzúnoung Der Sdileimbaut, Den Edieim abfonbern. Der Dide

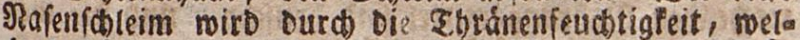

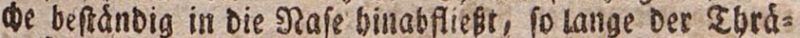

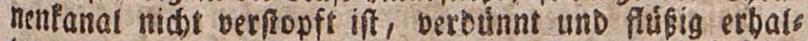
ten. Unter allen sinnen bringt ber Beruch bie beftig= ften efinbtude bervor, benn burd ubetricdenbe Dinge tonnen Dhmmadten entfeben, unb Durd) gute Sertu= de tanu man Die fidutiften Dhnmadhten bertreiben.

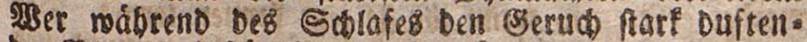
ber Blumen befrándig einjiebt, fann bom Schlage ges rubet werben uno fterben. - Daś, mas eigentlid ben Berud benirtt, ber Duft, ift etroas febr feines, unt

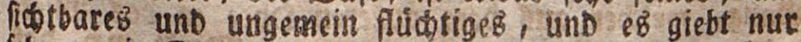
fegt menig Dinge, welwse feinen Duft bon fich gebere

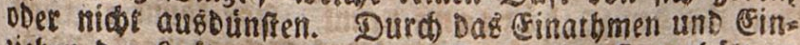
bieben Der euft wird Der feine Duft, Den fie enthalt su

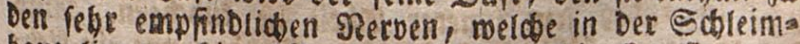
baut liegen, bingefubrt, wo er bann die Empinnourg, - ber der Sieis, welden mir Eerud nennen, bemirlit. 


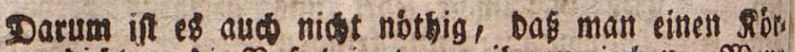
per Didit an bie grafe bringt, um ito zu rieden. WOent man gegen Den $23 i n d$ gebt, fo fant man ifon in eistst meiten Entfernung Dinge Durd Den (Seruch mabrnets?

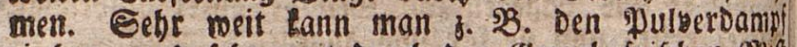
rieden, und febr meit burd Den seruch frif(hen Mifif

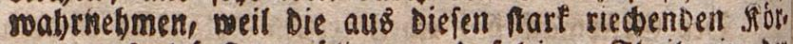
pern auśgedunfteten soligen und falzigen Ebcile in Det euft foprimmen, und mit Der \&uft in Die Siaje elagse aogen werben, no fie Daun die Nerven mit Der SAlleins baut reizen, umb fo Den Bertub veruriaden. - Dir

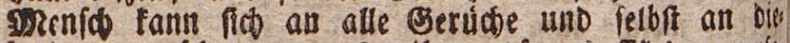

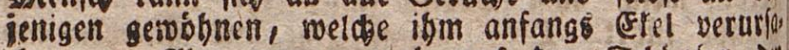
Wen. - 2her marum geb unf Der Schopfer ofl

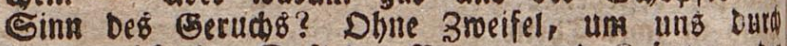
Den erquidenben Duft ber 28 lumen und Rráuter, Dit

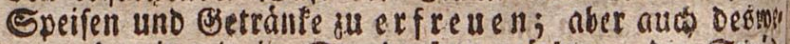
sen, bamit wir im Stanbe fenn mochten, Die Espat

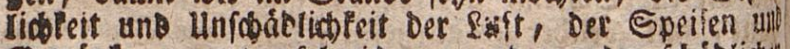

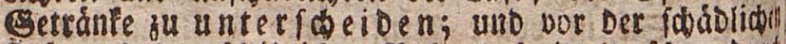
suft und ben fobablichen Nabrungemitteln faton burd Den Berad gewarnt sourden. Dean Die meiffen wobly siedenber Naburungetmittel fino beiffam, unb bie mes ften ubeltriedenden fino nadotheilig.

Saffet uns ther Den Sinn nadoenten, weldeen oi sinber, beionders beim linterridte, gebraudien folleth

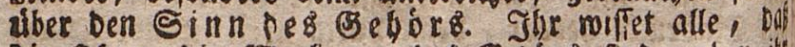

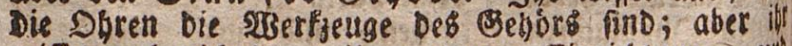
wiffet nod nidts von ibrer inneren eintiotung, utit? merbet erffaunen, menn ibe fie jept Pennen lernet. Dod du bete Dbr, id meine Den lánglidet gewunbernt Rnorpel, Det fo verfobiebene פertiefungen bat, if nut

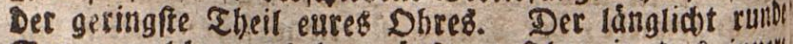

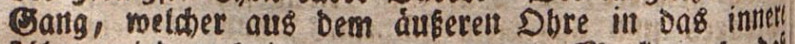
fubet, mirt nad inmen immer enger. Meetit esd, baf man ibn ben Beborgang nenut. Ghe tount es felof

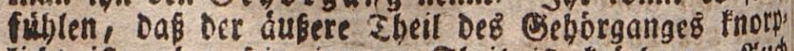

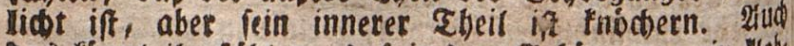
Dab fonnt ibr fúblen, Daß in Dem séeborgange ein Hleby siger Eaft befindlich ift, Der Das Innere oefferbeth

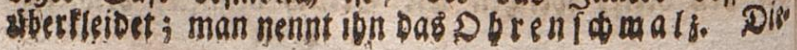


Pet Eaft if getbridt unb bitter, und foff bas Dor vor

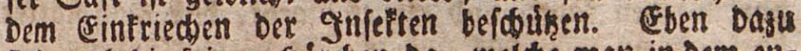
firb auds bie feinen Șárchen Da, welde man in Dem entgeten Ebeile Des Esthorganges finbet. 3ugleid bient

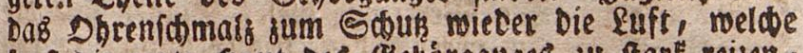
fouft Die zarte Saut Des Betborganges iu ftart reizen, und Daburd Sdmerzen bervorbringen mutrbe. Die ine nere enge Deffnung Dez Bellórganges if Durd eine lángs

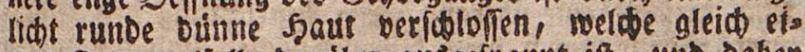
Hem ₹rommelfelle baruber ausgefsant ift, und Daber aud Dag Tro m melfe ll genannt wirb. Shinter biefer

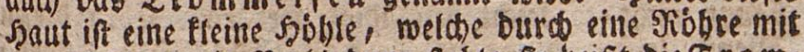

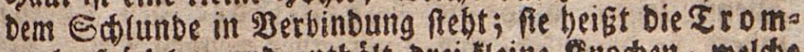
mel. Soblele, uno enthält brei fleine Sinoden, meldse man, ibrer befonbern sgilbung wegen, Sgammer,

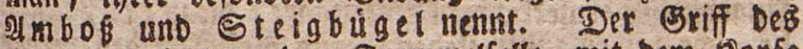
Stammers liegt aln Dem Trommelfelle, mit Dem siopre

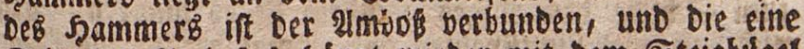
Eeite Des 2 tmbofes bangt wieber mit bem Steigbúgel

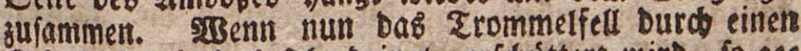
Schall, ber in Dab Dhr Dringt, erfátuttert mird, fo ges rathen aud biefe Drei Rinodern, Der seibe nad, in \$e:

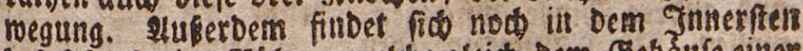

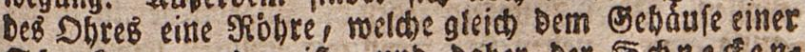

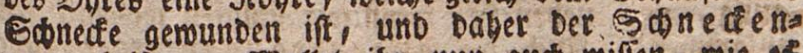
gang heibt. - 2Bollet ibr nun aled miflen, wie 16

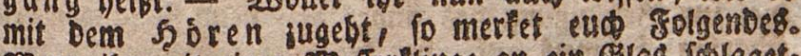

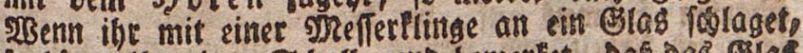
fo boret ige einen Sdall, unb bemertet, Daf DoB Chas gittert. In eben Diefe gifternbe Bervegung wirto mun

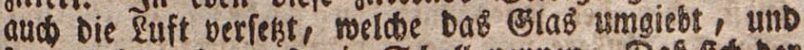

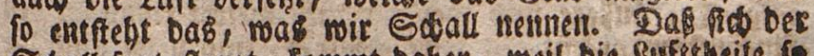
Sdaul fortpflanzt, tommt baber, weil bie furferbeite fo genau unter fid sufammenbângen, $233 i e$ bie $23 a$ ferertbeile.

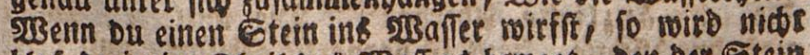

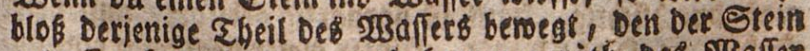
getroffen bat, fondern runb herum geráth Das $23 a$ affer

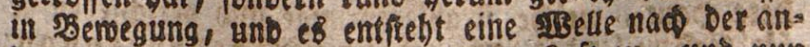

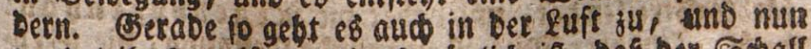

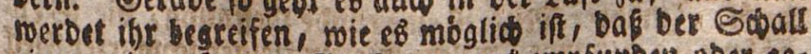

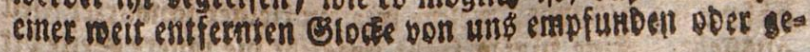


bort merben fant, und wie es sugebt, bas man fadtlet

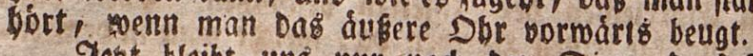
Jekst bleibt unE nur nod ber sinn Des है?

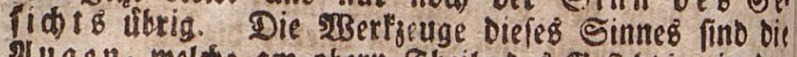
2ugen, welche am obern Ebeile bes Befidotib, in ben fogenannten थ u ge en b o bl en, befeftigt find. The ftumet "ś fúblen, Dás Diefe Sjobien aus sinoden gebildet find,

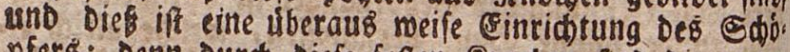
pfers; Denn Durch Dieie feffen Inoden find Die zarten 2lugen vor Stóben gefichert, und tonnen nun uber baupt nicht fo leidot beimadigt merben. Eben biefent Diemit leiften aud bie 2lagentieber ben शugen! indem fie Darunter, wie unter einer meiden Decte, ger fotikt liegen. $2 / \mathrm{m}$ sianbe bes obern und bes untern

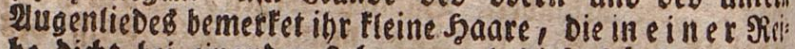
be bidt bei eimanber freben; aud Diefe Şärdent Dient"

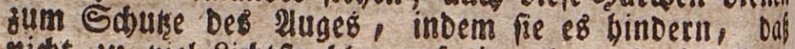
niobt is viel fidtfrtablen auf eimmal gerabe ins 2uge?

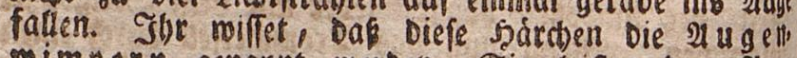
roimpern genant merber. Sie leiften bem 2luge aud nod eimen anbern Dienn, inbem fie Den Staul und anbere Dinge auffangen, meiche in's 2uge fliegen mollen. Broci onudteln feren Die 2lugentieber in sBemes gung, und fieben fie, neenn wir ficlafen wollen, fot zufammen. 2lucb bie 2ugenbraunen tragen fept

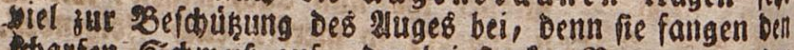
pharfen Schweib auf, ber bei ftarfer sBemegung ober idwrerer Irbeit bon unfeer Stirn flieft, uno Der bit

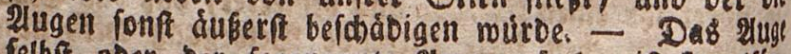
felbof ober Der fogenannte el ug a pfel, if fugelfors

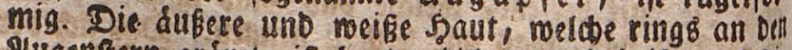
Aligenferen gringt, if bart gebildet, unto beift aud bie barte Şaut Shimere ibr, unb mit ibr verbunben, if

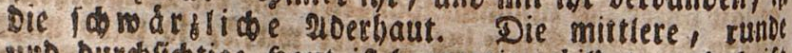

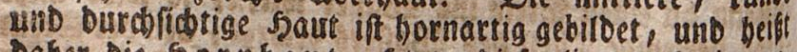
Daber bie Sornb a ut. Sinter Diefer liegt nod eine alls bere saut, meldoe firablenformige usio verfádieden gls fárbte Streifen haf, und onber die Sic s genbogenb aut

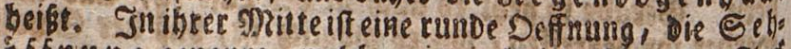
offnung genanns, welche roie ein fleiner fowmarjer \&left

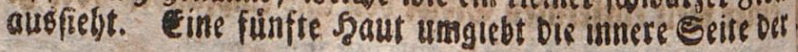




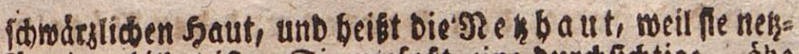
formig gebilbet ift. Sie umfaft eine Durdfichtige, dhe Jeudtigleit, welde bie gláferne genannt mirb, smeil fie Dem gefonmolsenen slafe abnlic iff. Morn in bicfer

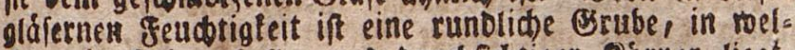
der ein fleiner beller und Durdfítriger Sörper liegt, Den man bie Sryftalt= \&infe nennt, weil sinftall fehr bell uno burdfirdtig iff, unb weil biefer sorper bie gorm eimer Rimle bat. Der SRaum, welder zwifthen Der

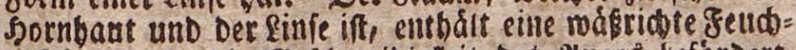

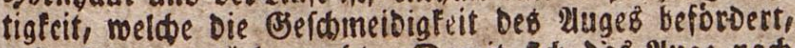
unib es beroeglid madit. Damit fid) Dab 2luge nact

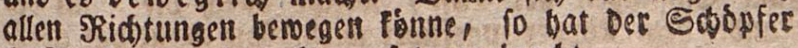
6 Nusfein an Dem nugapfel angebradst.

Ient, tieben Sinber, fentet ithe etfit bie zbeile, aus melwen cure 2ugen beftebell; aber ibr milfet nod nidt, mie eह zugebr, baf ibr felien, D. 5. mit euren 2usgen Die Dinge, melde um eud her find, Deutlion mabrieh= men fönuet. Dieß follet ibe jeķt lernen. In einer Dun= Peln Stube, oDer in einer finftern Rawt fonnet ithe

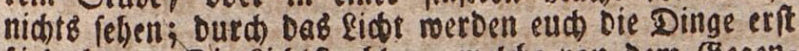
fidtbar. Die eidtifrablen, welde von Dem (segen= franbe ausgehen, Den ibe felben follet, oringen Durch bie

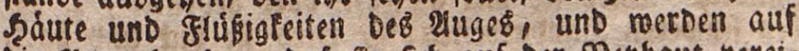

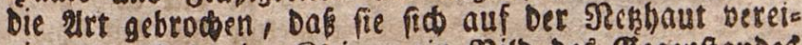
nigen, und Da im Rireinen ein sBitb Des Cegenftandes entrerfen, wie eb bet Spieget im Broben tbut. Sit Der Begenfiano unferen elugen zus nabe, fo fehen wit

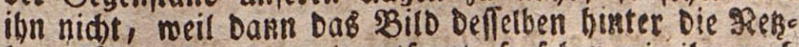
baut fálft; if er zu rocis entfernt, fo febers mir ibn oud

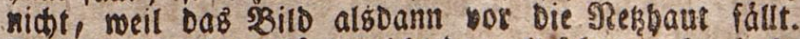
Daß wir die Eegenfánde niçt boppelt feben, ob mir fie gleio mit tmei 2usgen mabrnebmen, rúber baher, reeil bie Empfinbung in beiben 2lugen gleid ift. Sgzit jmei

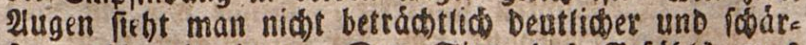
fer, als mir einem. Der Sinn Deš ceffúbrs mus

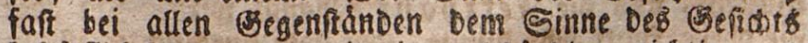

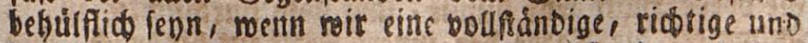
Deutlide $20 r f t e l l u n g$ von cinem Eegenftande erbalten follen. Nennet mir nur einige Dinge ober Eigenfobaf: ten Der Dinge, yon relden wir feine $230 t$ felliwntg bas ben wúrben, wenn wir fie niकt feben fontuten? 


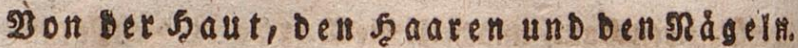

Unfer ganjer Roorper if in sine weide uno ftarle Dede eingebattt; mir nennen fie șaut. Sie ift cinet

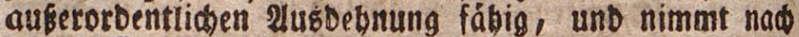
jebem Drude ibre yorige Seffalt roieber an, ober iff cla fif if. Sie bat eime menge SBlutgefábe, und Dar ber if fie an berfóbiebenen Stellen blåutich, ober aud

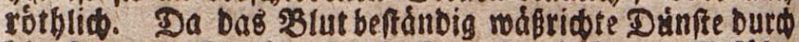
bie Shaut auşhaudot, fo ift fie auch mit folden Eefáfent

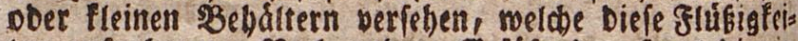

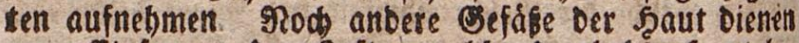
sum Einfaugen ber fuft, melde Durch bie Saut bes

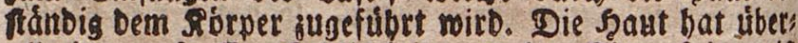
all eine grofe Emprindithteit; an ben gingerspitsen if

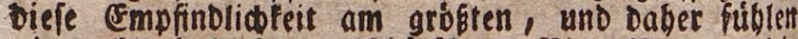

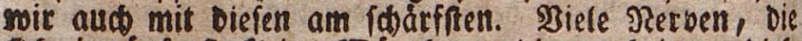
fide in duferft fleine s\$ärsden endigen, bringen biefe

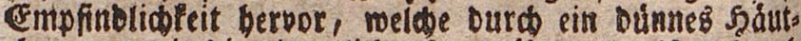
deti, momit Die eigentlide Sgaut úberzogen ift , Durd Die Dberbaut ein menig verminbert reiro. Diefe Dbethaut if unempfindlich; man farn fie mit einer $\Re a^{3}$ Del Durchifteden, Dbne Den getingften Sdmetz zu ems yfinben. 2rits fie viel getieben ober gedrudet , 8. 28 . len fdomeren Spanbarbeiten, fo mirb fie bid und hart.

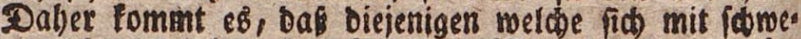

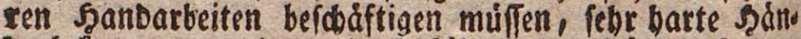
De belommen, uno menig Eefúb) in Den Sganben babett. Die Saárte unfrer gubfoblen bat eine ábnlide Urfacte.

Die garbe ber sgaut if bei allen mentoder

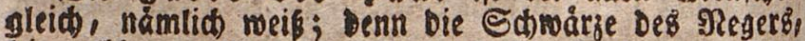
Die gelbbraune Farbe Des 2lrabers, Die fupferrotbe faro be Des 2merifaners unb Die meife Des Curopáers if nicht bie farbe ber eigentliden Sgaut, fonbern bie gato

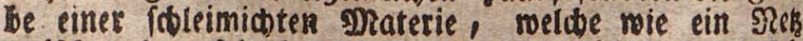
zwirden bet Oberbaut uno Der eigentliden Shaut fich bingiebt, und Die fettbaut genanat wirb. Da aber

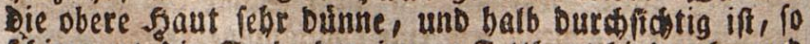
fómimmert bie garbe ber innern getthaut himbures, unb to fobeint es Denh; als ob bie Dberbaut Die garbe bättes melde eigentlid Der Jetthaut jugebort. 
Die dufece Esite Der Şaut ift grabtentbeils mit Syaaren bejek̨t, melde aber nur an menigen Stellen zablreido, lang und bid find, und an mandien Stellen gant fehlen, mie s. 3 . an Den Suffohlen, an Det innes rett fláde Der Sgano unb an Den Iugentiebern. Diefe

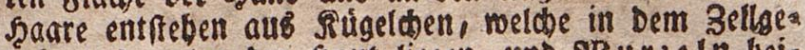
webe unt unter ber saut liegen, uno 23 urze in beiGen. Raum merbet ific ef glauberr, lieben Rinoer, bas jebes, auds bas feinfe foaat, eine boble, barte uno elaftiobe Robre, uno mit einem Safte angefúat ift, bei beffen ఇertrodenung Daß Saar abffirbt und auşfällt. Die ssourzeln fúbren Dem Şąre feine SRabrung zt, uno

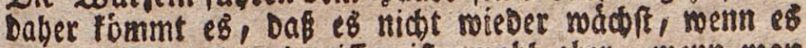
mit Der rourzel augetiffen if, wohl aber, welln mat es nut an Det $23 u r z e l$ abgeidnitten bat. 2bet mozt? merdet ihe fragen, migen bent bie vielett Saare bem

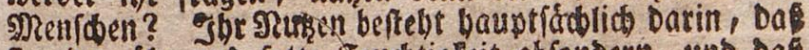
fie eime sabe unb fetre Seudrigleit abfonderti, uno bas fie Den unter ibum liegenten Theil bebeden, ermårmen unb bef́ctipen. Dieß erfabren Diejenigen, welde Die Ropfbaare grobtentheilb, ober gans verlobren baben: fie muffen, um fic yor ben Sømerjen unb Unannebms lidbeiten, welobe baraub entfeben, zu lobuhen, allers lei funftiole Sopfbebedungen gebraudien.

\$2Bir baben nun alle Sheile unferes funftio gehatse ten Rorperb, bis auf Die SR \& gel, fennen gelermt. g) Rerlt eud von biefen, Daß biele barten i glatten unb unempfinolioben platten an ben singern uno 3eben mit ibren 23 urzeln befeftigt finb, Daß́ fie Dielen Eliebern eine grobere Fefigleit geben, uno Daburd ben IRett foben Das Breifen, stnfafter, Beben und Ereten febe erleidtern. Sugleio bienen fie gum Sraken, uno aud Diefer Dient ift uns oft febt nilltommen unb fehr nosth= mendig. Pinber múfen fió iebocó forgfáltig buten, Diejen Dienft su oft yon ben sRagein zu begebren, Dem eb if unanftenbig. Feinlicke Rinber forgen all bas

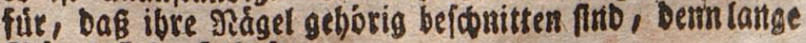
vidg el find elelbaft. 


\section{VII. (a) erunobeitslebre.}

I. CSefunobeit uno sranflyeit.

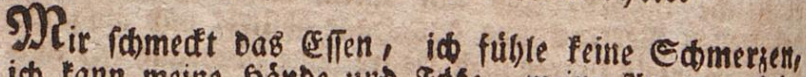

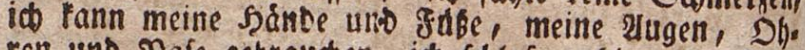
ren unb গafe gebrauden, id follafe rubig, uno fann

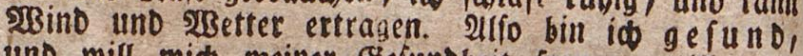
uno mill mid meiner Sefunbheit freuen, mill mid aber aud in Nots nebmen, Das id nidbt frant reerbe. Dern Dem Sianfen iff nidot mobl. Er ift imwad und matt, Das effen formedt ibm nidt, et fann nidit 23ind unb 23 etter ettragen, nidht arbeiter, et bat

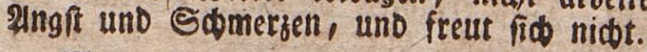

230 enn id einen gefunben feib behorten mill, to

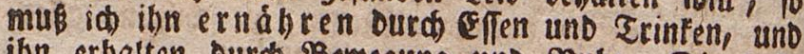
ibn erhatten burd Bemegung und Rube. Die befte 3Bemegung ift Die 2rbeit, und bie befte soube Der Sकlaf. Iक fann prant merben, wern id mid dits gere; menn id gornig, ober bofe, doer mild bin; ween id ju febr fpringe; wenn ít ju viel efle ober frinfe! Doer fu unredber 3eit unb sus oft effe; menn id mid Durd faufen und Epringent erbikge, unb Dann Pogleid trinfe; menn id su lange folafe, ober nidt ju red. ter Beit fu SBette gebe; menn its mid nidot waside uno nidst Pomme; wenn id mid nidft vor Dem fallen in 2ld) nebme, uno unvorfidtig einbergebe; menn iो mid) erfit erfibe, und Dann in ben zug fielle, ober mid bis aufo şembe ausziebe.

\section{Bon bet Sileioung.}

Die Pleibuig mus gerabe fo befouffen fevn, das man nidt unbebagliot Palt, aber aud nict unbelgagtid 
beis ift. Sie mus fid baber naw ber Sahrobseit und ber פBitterung riditen, aber aud noch befonders nad

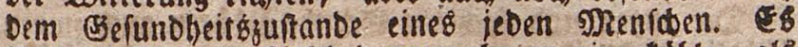
iff gut, fid von Sindbeit an melyr an cine fưble, als als eime febr marme Steidung zu gemóbnen, unb fid

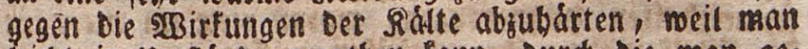
leidts in Umftánoe geratben fans, Durds die man ges nótbigt nirb, Der mármeren Rleibung zu entbehren. Edablid ift $e^{B}$, fiț tubermáfig marm fu fleiben, und cine \&aft von Doppelten Seemben, $23 \dot{m}$ fern, Dberróden und Pelien auf Dem \&eibe zu tragen; es bringt bei Der

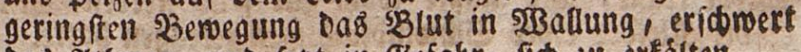
Das etţment, uno ferct in હefabr, fíd zu crfálten.

2n falten Tagen Pleibe man fid mitten im Şom mer, wie man fiot an einem 53 untertage fleiben wưrde. Uleberbaupt ift in unfern Segenben bie leidste Somo

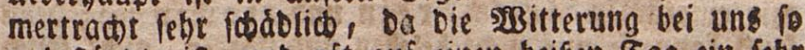
unbeftandig ift, unb oft auf eimen heifen ₹ag ein febr túbler, wobl gar talter slbend folgt.

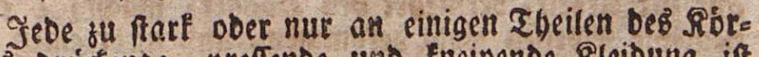
pers oruifence, perfende uno freipende Rleibung if

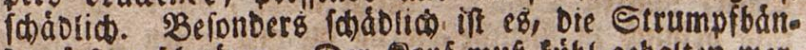
Der feft ful chruiren. Der Sopf muß fúbl gebalten wer= Den, uno jede fiopfbededung ift, fumal, roent man fratfez f̧ear bat, unnobtbig, ausgenommen fum ङकuh yor Der Eonne. Eelbft Die lteinften Sinder Darf man obne Esefahe mit blobem Siopfs in bie freie \&uft follte

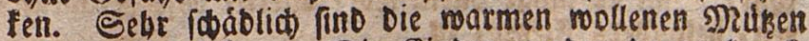
und Die \$elzmukzen. Die Seinder merden Davon frant, befommen llingeziefer unb estino, glúfe, Siopf = und Zabnichmerzen, und beforbers fकlimme 2ugen. 2/m menigften Dérfen fie bann einen Sout ober eine solübe

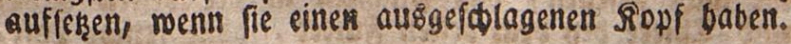

Dide Szalstuder, befonbers menn fie feft suge=

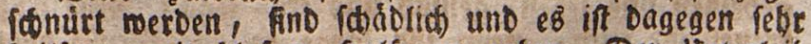
beilfam, mit blofere Şalfe ju gehen. Der Interleib

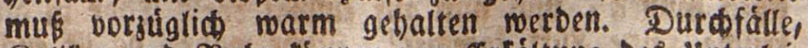
Roliten und SRubr fónnen von erfáltung Deb. Linterlei=

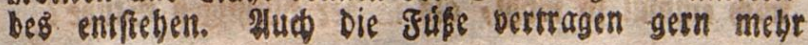




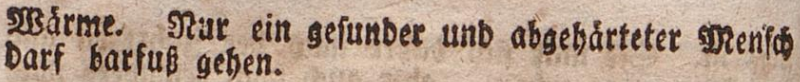

Die engen uno pipitigen Sdube gebieren aud gu bell

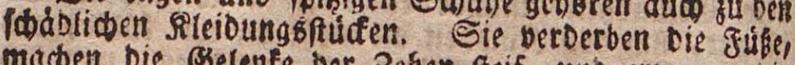
madben bie Belenfe ver Bchen feif, uno erjeugen die

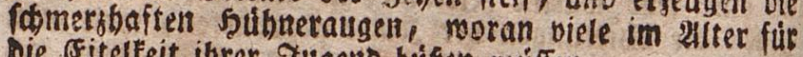
Die Eitelfeit ibrer Jugento buifers miffen.

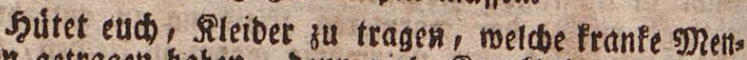
liden getragen baben, Dern viele franflbeiten find a th

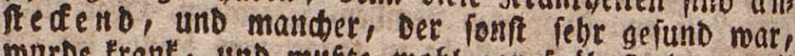
marbe frant, uab muste mobi gar frúb ferben, meit et bie seteiber eines Sdjwind fúdtigen getragen batte.

\section{Yoon bet \&uft.}

Cs fomme febe biel barauf an, bas bie suft, melde mir einathmen, frifa, rein uno tro of en fen; bens

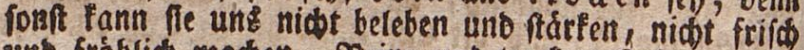
unb fróblids madben. Fieine unb trottene fuft muntert

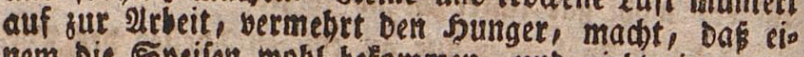
nem bie Eyeifen tobl befommen, und giebt cinen rus

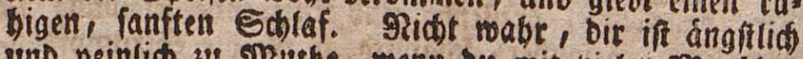
und peinliक zu פnurbe, wern ou mit viclen Mneniden in einet Heinen Stube lange begfammen feyn muft, unt weber Senfer nod Thusen géffnet werber? -

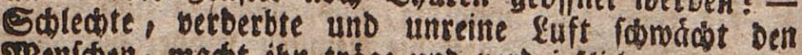

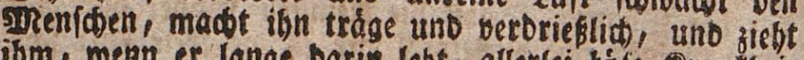
ibm, neran er lange batin lebt, alletlei befe srantbeisen, bejonbers gieber, gus.

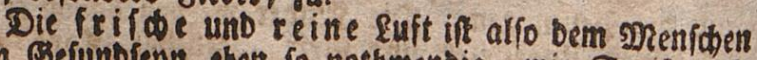
gum Eefunbienn eber fo nothwendig, roie Speife und Trant, unb wic bem sifche bas friche 233 affer. Szabt ibr nidot gefeben, baj Pfiamen in ber beften croes und Ebiere bey bem beften Jutter, ofine frifobe fuff, verberben? FBie Ponnte ber gRenías obne friche euft gebeiben und leben, geiuno uno frob lenn? WBie fege freuet ibe eud, merm ife lange in Der Stube babt fisen múfen, unt nun auf simmal bor's shor in bic fríde reine euft fommet? SRidt mabr, Da if eud tro sinmal to wobl, als in Der bunfigen Etube? 
soollet ifs miffer, mpourch Die euft verbirbt? Das folte ibe boren. 2lber merit es eud aud! SGrm in simet tleinen Stube viele smenfímen bei einander find, uno befonters darin bei einander follafent, fo berstibs Die \&uft. WBab iff nun Da zu thun? Man

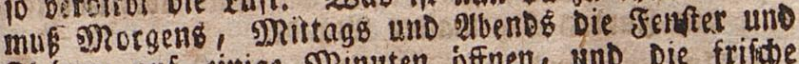
Thuiren auf einige MRimuten offnen, und Die frifice luft yon auken binemintefen. 2lber thun Das mol)s

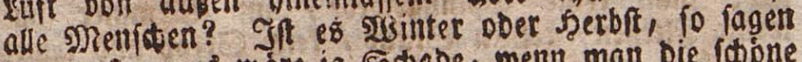
Die meiften: is wäre ja equade, menn man bie fobone 2sórme woute jum Genfer binaus geben laffen! llib in Sommer haben fie misber andere Einwendun. gen. Ilter if eś midt beffer, ein wenig frieren, unb Dabed gefund fepn, als marm fiken une baben fránfe lid, fouman uno verorieflic feen?

Now fohlimmer ift $e b^{2}$ menn in Der Stube, alts Ber ben wubbunftungen Der Meniden, aud now Der Dampf bott Dellampen, इalglidtern DDer Ridtidnu= pfeni oder vom súgeln uno vom piátten der szálćses

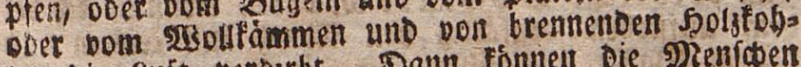
Ien die fuft verburbt. Dann fonnent die Mrenicten nict nur frant merben, fonbern fo gar erftiden. S2lan tann Die \& uft baburd verbeffern, Daß̈ man Efíg auf einen gluhendent Etein giest. Ein $23 i n d o f e n$ if ein gutter \&uftreiniger.

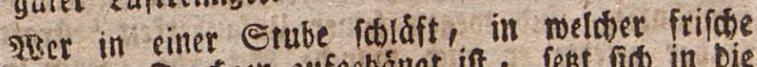

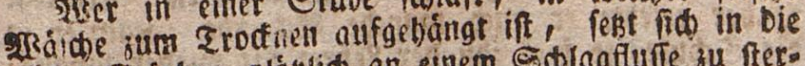
gróbte Befabr, plóblid an einem Sdragfluffe zu fter.

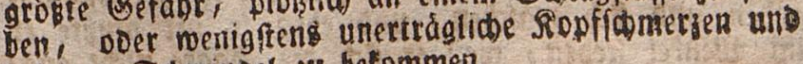
beftigen Sabinoel zu betommen.

Hört, wie es dem Gaftwirth M üll e r ging. Diefer hatte eine ganze Nacht nicht lchlafen tiönnen, und wollte gern am andern Tage ein wenig Mittagsruhe halten. Aber in der Gaftitube war unaufhörlich Ge. raulch. Seine Frau rieth ihm, oben auf eine abgelege. ne Stube zu geben, weil er da ganz ungeftöhrt fchla. fen hönne. Sie felbft führte ihn hinauf, und fchlofs die Thür ab, mit dem Verfprechen, ihn in einer guten Stunde zu wecken. In diefer Stube war eine grofse Meage frifche Wälche aufgehängt; das bedachte die 
Frau nicht. Als fie nach einer Stunde kam, um ihren Mann zu wecken, fand lie ihn vom Schlage gerühat, todt im Lehnftuhle.

Cben fo fobedolid find Dis Tusodinftungen ftart ried)ender slumen, uno frifo mit Ralf ubertund)tet, DDer mit farbers arigemalter 23 dusbe.

In einer orbentliden uno reinlíden 2robmfube fiebr man feire Epinngemebe, im Summer nut menig fliegen, feinen Staub, fein Stroh uno feinen Untarb, alfo 3. 23. Feine upfelftbalen, ober Sinos den. Die genfter lino bell uno tlar, uno man fputrt

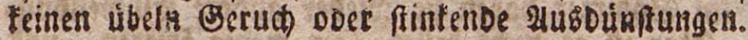

\section{Du folle rein uno oroentlich fern.}

ferbinano nabn fion oes Mrorgens nie die zeit

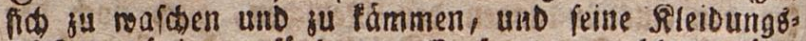
fucte geróng tu fáubern. Er fpottete mobl gat tiber feine reinlide So mefter grarie, wenn fie fid bei

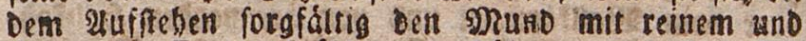

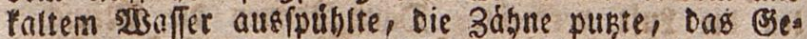
fitt, cen siopf und bie Dhren rould, uno cann ibt langes Sear mit vieter Inúbe atistómmte. Diefem gus tet seifpiele folgte ber unteinliabe gerdinand nict), fo oft ibn auch oer Dater und Die Mustar bazu ermabnten, uno negen feiner Unfauberfeit beftraften. Diber erlebte ef aud mande Sthanbe. Ram er in Die Schule, fo biek ibn Der fehrer oft mieber finaus. geber uno fid maficen, und eben fo oft muste er

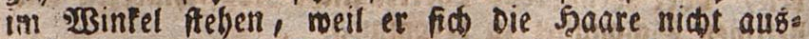
gețammi und ole Stiefeln nidt gereinigt batte. So et befam fogar enolico einen delbaften anftefenten शlugfolag an Den Şánden, Den man bie Rráke nennt, uno Durfte nun eine lange 3eit gar nidht mit andern Dreniden umgeben. Jeber beradotete ibn,

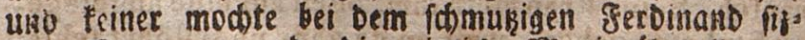
zent. Dagegen nurbe bie reinlicte s) tarie úberall gerts gereben, und bon aften פrenficen wegen ibrer Neins licbereit gefáåkst unb getiebt. Eie fabe immer gefunb uno frifo aแs, unb Durfte fíd nie fómen. 
Die Unceinlidefeit, lieben Rimber, ift bie Ur: fade von vielen Rrantheiten, uno befonbers von anfte? fenden Sirantbeiten uno bojen üusfolégen. WBer das

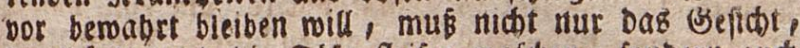

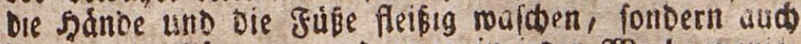
Den ganzen sórper, uno zroar in jecer 230 che menig* fens eimmal. Darum máre e\$ mohl zu wuinfach, Daf man an jebern Drte Aliffalten zum SB a Den bátte, uno Daß Daz SBaben cine allgemeine Sitte wutroe. Sar Dabura roiro bie Saut fo reill gebalten, baf lie fres aubounften fann.

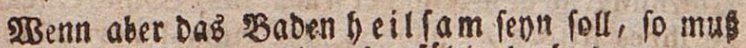
man folgende Siegeln vaben forgfáltig beobactien:

I) פRan mué fid vorfictig an folden Stellen bas Dell, no teine Befatir ift.

2) Dan mus gefuno uns nog) [enn.

3) Man barf nidt exbiß̨t fEpn, Doer fur borbet biel gegeffen baben.

4) शRan mus fid nidt langfam, fondern geformino mis Dem Ropfe und Dein ganzen Riorper, unter Dав̆ $23 a r f e r$ rauden.

5) NRan mus in sade nidht ftille fiken, fondern fic frart beroegen, ober ionmiminent. Und

6) SRab dèm sade inur man nidt ruben, fondern gemádic geben.

In B. hatten die mehreften jungè Leure Luft zum Baden. Sie gingen alle Tage, gegen Abend, in Gefellfchaft, nach einem Teiche. Einige konnten fchwimmen. Diefe wollten fich eines Tages, weil ein Fremder dabei war, als grol'se $S$ chwimmer zeigen, kleideten fich daher fchnell aus, obgieich fie noch vom Gehen erhitzt waren, fprangen ins Waffer, und durchfchwammen den Teich einigemal. Einen ron ihnen rührte der Schlag, als er noch fern vom Ufer war; dies war die Folge der zu fchnellen Abwechfelung der Hitze mit der Kălte. Die übrigen Schwimmer waren weit ron ihm entfernt, und als fie herbey kamen, theils gu furchtfam, theils au ermüdet, um ihn retten zu köm- 
nen. Gott! wer fchildert das Schmerz gefühl derjeni. gen, die gern gerettet hätten, aber nicht fchwimmen konnten! Nan lief in gröl'ster Eile, um vom nahen Dorfe einen Kahn und Stangen zu holen. Viele Men. fchen eilten zur Hulfe herbei. Man fand den Verungluckten bald; derzte hamen auch, aber vergebens war das Bemühen, ihn in's Leben zurück zu bringen. Sehrcoklich war die Lage derer, die den. Eltern des $\mathrm{Er}$ trunkenen die Todesnachricht bringen mulsten. Man denke fich den Gram gatex Eltern, welche die frohe Hoffnung, an dem fchon erwachfenen Sohne eine Stiz. ze ihres Alters zu haben, auf einmal zernichtet fahen. O vergeffet es doch nieht, lieben Kinder, dafs Vorfchtigkeit bei jedem Unternehmen nöthig ift, vorzügiich aber da, wo nahe Gefahr des Lebens droht!Sollten nicht endich fo viele Beifpiele durch ihre ei. gene Schuld Extrunkener, Yorficht und Behutfamkit lehren?

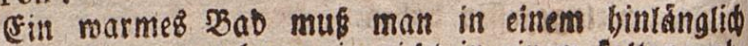
warmen 3immer nebmen, ia niot in einem falten; obs ne Diele 930 rficht mirb man fod burd pin roarmes $\$ 300$ mebr fhaden, als músen. Diefer gilt aud won ben fübáoern, nelbe bei Mnbáufung Deb Bluts im sopfe uno in ber Bruft febr beilfam fino.

Sidat blos leinell Sorper unb feine Rleioung foll man reinlich baltet, fonbern aus Das Saušgeráth, oie

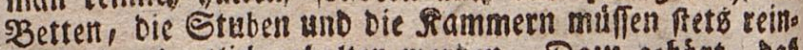
lid uno pibenslich gebalten werben. Dazus gebort, Das

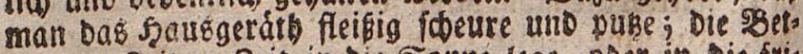
ten von Beit tu Beir in bie Eonne lege, Doer in oie fri-

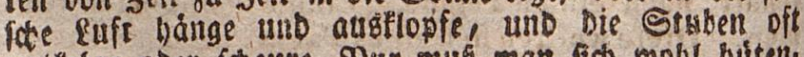
aldiflebre ober foceure. SRur muß man fich wohl buten, in einet gefdecuerten Slube, bie now nider mieber recth

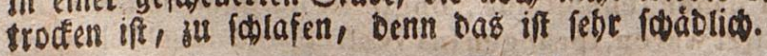

\section{Son den Epeifen.}

53 arum eflet unt trintet ibs? Nicht mafic, um ello ren Saunget fu fifles, um euren sorper ful erthaltent

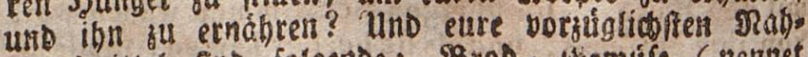
rungemittel find folgende: $2 B$ tod, Biemúfe (nertiret

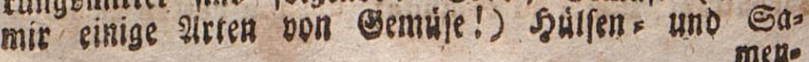




\section{Befunbbeitalefire.}

menfridote, ( wer fann einige nennen?) stuf, Driló)

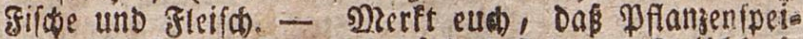

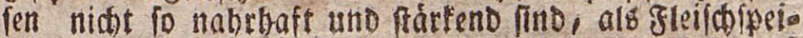
fen, uno bag gleídipeifen aud nabrbafter fino, als Epeifen bon firchen. Darum fouten unfere Mrablgeiten aub einem fleineren Iheile fifase uno cinem groperen Theile Bemúfe befteben. Bon blofen Bleidhipeifen ges

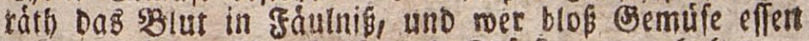
moilte, mutioe niwt Straft unb Stárfe genug baben.

Eoll Dir Das Effen immer redit nobl fomeden, fo

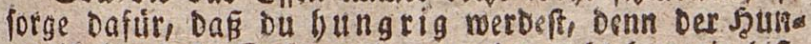
ger if ber befte foch), uno wenn ou red)t bungrig buft?

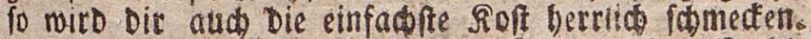

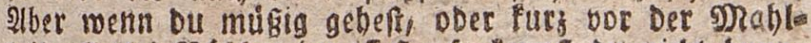
zeit allerfei siáchereien iffét, fo fannit bu nidot bunge tig werben. Du must fleifig arbeiten, uno bid in freier luft bewegen, Dann wirft ou genif bungrig jus Eifote fommen, und bann merden aud die Epeifen bei Dir gebeithert.

Qtber merle bir Dabei, toẩ bas Eprúdomort lagt: शrfuviel ift ungefunb. Denfe nicht: viel effent giebt viel গ2abrung, Denn wenn Du daś, maś bu gea geflen laft, nicht verbauen lannft, fo fidabet es bir.

Hüte dich, vielerlei Speifen darch einander zu effen, damit es dir nicht gehe, wie dem unverfändigen und gierigen Mo ritz. Diefer hatte einige Grofichen gefchenkt bekommen, und ging nun auf den Markt, um fich einmal, wie er fagte, etwas zu gute zu thun. Exft kaufte er Pfefferkuchen, und verzehr= te fie, dann Aepfel, dann gebackene Pflaumen, and nun noch fettige Kuchen, welche eine Frau feil bot, Das alles verzehrte Moritz in einer Stunde. Und wass gefchah? Er konnte diefe Speifen nicht verdanen, hlagte über Kopf - und Leibfchmerzen, ihm wurde ubel, und or quälte fich einige Stunden, ehe er fich übergeben konnte. Noch am andern Tage war er lehr krank, und mufste nun mehreie Tage faften.

Pflanzen muffen, fle man fie geniefit, forgfáls tig unterfudt merden, Damit frime Biftpfanzen poss 2n? 


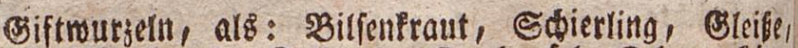

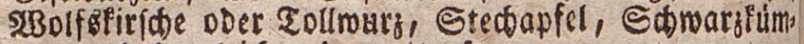
mel, und Dergleiden Darunter fey.

थlle ভ

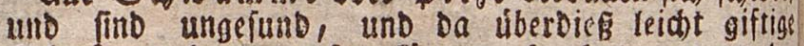
Edbodnme Darunter fenn fonmen, fo that man an be ften, menn man feime ist.

Reife Rartoffern find niát ungefund, menn fie won guter virt find, uno nidit úbermáfig genoffen mets

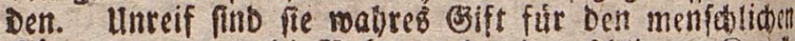

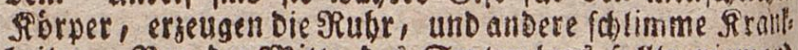
Weiten. Sor Der Mitte De\& Eeptemberb follte niemanto sartoffern effen. gette Speifen in menge zu genie Gen, if febr fabáblido. Der Surfen, an Dem beim 2H" fange DeB $2 B$ interb fo viele leiben, ift megr zu bem bdus

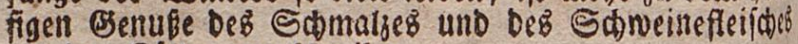
als Der Rálte zusufobreiben.

Sdarfe, gefalsene, uno gemitzte Speifen erbih

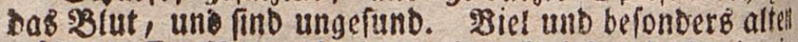
Siáfe zu effen if ungefunb, weil er Srriés und Steinte it

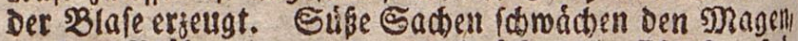

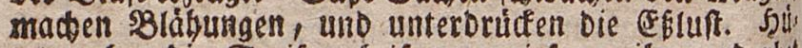

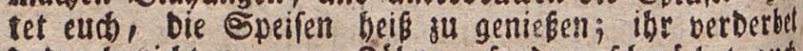

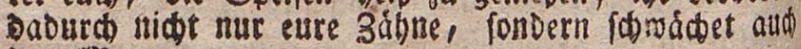
Den smagen.

supferne es efobire múfen gut diberjint fenn!

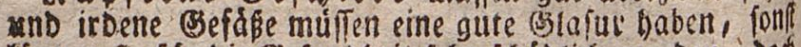

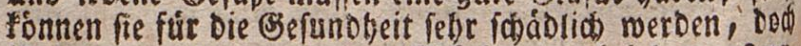
ift bei ben lestern weniger Gefabr, als bei ben erfern. 2ekfonbers mus man fich huten, faure Epeifen in fupfers

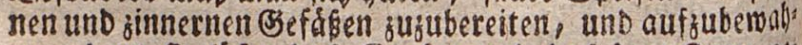

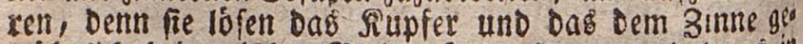
robibntic beigemichte Stet auf, unb vermandeln es it

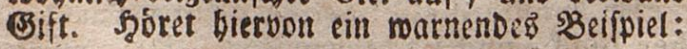

Der Schufter $B$ odenteich in H. Kochte Pflan menmufs. Nachdem feine Frau daffelbe in Töpfe gethan hatte, blieb am Rande des kupfernen Keffels, wie gewöhnlich etwas fitzen. Der Schufter, welcher aus unzeitiger Sparfamkeit nichts von dem fchönen Mafse wollte umkommen laffen, kratzte alles forgfäl. 


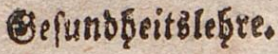

tig mit dem Löffel ab, was am Rande des Reffels iazzen geblieben war, und al's es begierig. Einige Stunden nachher empfand er heftige Leiblchmerzen. Er nahm einen Schluck Branntwein, aber die Schmerzen wurden nur ärger darnach, und ex brachte die Nacht unter fchrecklichen Qualen zu. Am Morgen war fein Leib aufgefchwollen, und es mul'ste ein Arzt zu Hülfe gerufen werden. Doch diefer kam leider zu fpät, denn fchon war der Unglückliche an den Mufse, welohes er fo unvorfichtig genoffen hatte, geftorben. Die Säure der Pflaumen hatte nämlich den Grünfpan aus dem Hupfer gezogen und fo dus Mufs vergiftet.

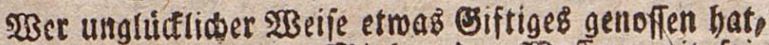

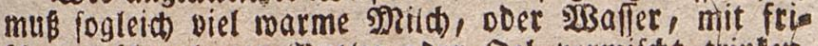
faber geidsmolzener 5 Butter Doer Del bermifid trinten. sBredimittel fino am soirfiamften, ment iemano Ediers ling, oDer 230 lfätirjकen u. ogl. gegeffen bat.

23eijenbrod, Suben ano Eemmel fatadent in gus groser s) Renge und warm genoffen, und find nidt fo

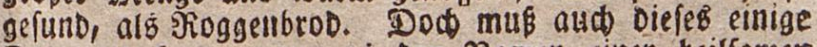
Tage alt feon, wenn eb́ Den samen einter beilfament Epeife verbiement foll. 3u Den vorzuglich fédelichen Epeifen geljort aud Dab ferte Bactroet ( fonder 8 Pafteten und Eorten, Die nur ein auberft ftarter Magen zu berbauen im Stande ift.

Mari e pflegte, wenn fie Brod im Yorrath backte, für ihre Finder lileine Salzkuchen zu backen, um ihnen ein Vergnügen zu machen. Eines Tages hatte fie diefs auch gethan, und liefs fich von den findern erbitten, ihnen die Kuchen fogleich zu geben, ehe fie noch halt geworden waren. Zwar hatten fie alle verfprochen, nicht eher davon zu effen, als bis fie lialt geworden wären: allein $\mathrm{Chrifi}$ ian, Mariens zweiter Sohn, konnte doch feine Begierde nicht mäf . figen, fondern verfchlang den ganzen heifsen Kuchen. So lief er aufs Eis, ward durftig, und trank das eiskalte Waffer. Auf einmal fühlte er Uebelkeit, und haum konnte er noch das Haus erreichen. Mit jeder Stuinde ward fein Zuftand fchlimmer, und nock. vor Abend war ex todt. Die Aerzte öffneten feinç श)? 3 
Leib, um die Urfache feines plötzlichen Todes zu er: fahren, und fanden den Kuchenteig in einem Kllumpen vereinigt, und noch ein Stück davon im Schlunde.

\section{Don ben Betrånten.}

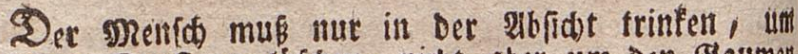
feinen Durft fu lofweth, nid) aber um Den Baumet zu finzeln.

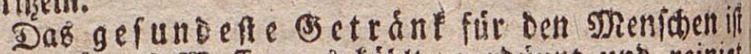
reines, flares 5 Baffer; es flablt, verbunnt tind reinigl Das Silut, erblist \$Ragen, Eingeweibe, Behirn uno Serben inj Dronung, uno madt ben spenidhen ruhig, bei

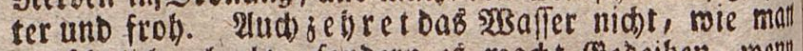

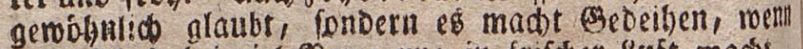
man fid babei vicl 3 crosegung in frifder suft macis.

Dic smen/doell trinfen gervobnlid biel za biel matl

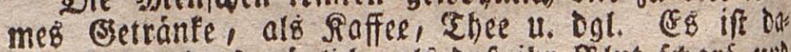

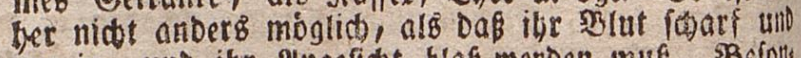
unrein, uno ibr e्यngefid t blas meroen mus. SBefolts

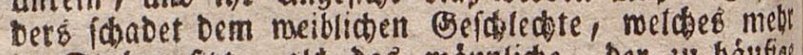

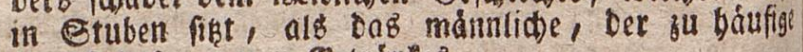
Cenußs DeB marmen Betrånlę̧.

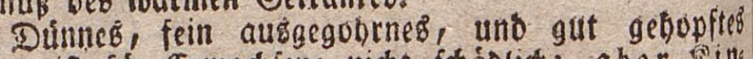

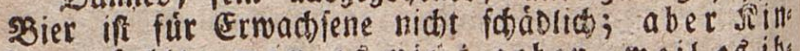
bern follte man é nirat geben, meil ebits: ne lertrinfen benimm $\mathrm{t}$.

Der 28 rannte wein ift unter allen Betranten bas

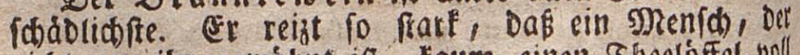
nidt an ibn gerobbnt ift, Faum einen ghceloffel ooll Davon bertragen fann. Er bindert bie \$erdauung! madt 2 Baflung uno Sik̨e, und wirkt auf Dab Sefjitn to farf, Das Derientige, weldber biel bavon genieft, alle Befinnung verliert, lino fo ichmerfállig uno frafts $10 B$ wird, Daß er nid)t mebr auf leimen Fúsen fetell

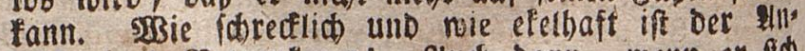
briat eimes sBetruntenen! 2udí) Dann, wenn er fid erfolt hat, bleibt er fobmach, und bies Befúbl Der Edmadoe verleitet ibn, aufb neue zu trinten. 24uf

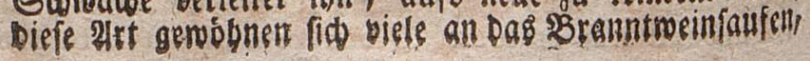




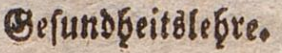

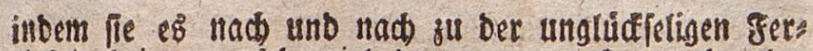
tigleit bringen, febr viel bavon zu trinten, obne bes raufidt zu merden. Die zolgen Diefer abfibeulichen Be= mobnheit find fáreditio. Die Sáufer fonnen die ge* noffenen Epeifen niơt mebr verbauen, unb vertieren Daber enbtio alle effluft. Bei einigen entfteht bon ber beftigen Reisung ein sB!utbuften unb Sungeniudt; Die gemobntiobe solge ift oie $2 \mathrm{Ba}$ (terfucht. Dabei merben

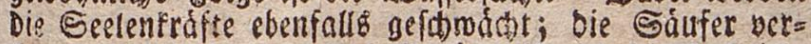

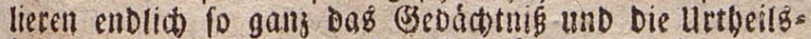
fraft, oab́ lie zu ben meiften Beidjafter gar nidt mebr

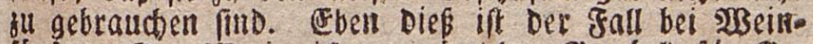
fáufern. Der 283 ein if zum táglioben Bsetránte für Be e

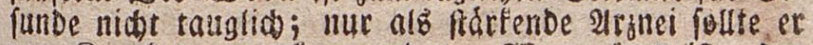
bon firanten gettunfen werben. 2Ber gefund ift, Des trinfe nur Dann 23 ein, wenn er Durat farfe Hrbeitel Doer langes (s)eben ermattet ift, Doer Dann, wetn er einmal mebs \&ebhafrigfeit und grobfinn als gemóbutiós

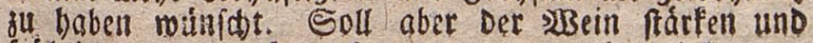
froblid macten, fo mus man man wenig trinten.

Heitmann in Br, erbte ron leinem redlichen Vater Haus und Hof im befien Stande. Schöne Pfer. de, Kühe und Schweine waren in den Ställen; die Gärten voll fchöner Obfibäume, und das Ackerland trugreichlich Korn und Hülfenfrüchte. Auch baares Geld erbte Heitmann von feinem Vater, denn diefer war arbeitfam und fparfam gewefen, fo lange er lebte. In den erften Jahren war auch Heitmann ein recht guter Wirth, und es ging ihm fehr wohl, Aber eben diefer Wohlfiand, in dem er lebte, verleitete ihn zum Müffiggange, und er gewöhnte fich, alle Nachmittage in ein Wirthshaus zu gehen, und da bis an den lpäten Abend zu bleiben. Dort fand er Säufer, mit welchen er f́pielte, und die ilhn nach. zund nach zum Branntweintrinken yerleiteten, um ihm. dann, wenn er berauleht war, defto leichter im Spiele Geld abzugewinnen. Bald genug fiel ex fo tief in das Lafter der Trunkenheit, dafs er oft, wenn er um Mitternacht taumelnd nach Haufe ham, feine Frau fchlug, und das Gefinde mil'shandelte. Fon diefem ward er endlich bei der Obrig- 
keit rerklagt, die ihm nun mit Gefängnifsftrafe droh. ten, wenn er fich noch einmal an den Seinigen ver griffe, Bei nüchternem Muthe verfprach er Befferung, aber am folgendon Tage war er fchon wieder betrunken. So verf'chwendote ex nach und nach all' f'ein baa res Geld, und fing nun an, feiner Frau heimlich Speck, Würfte, Linnen und Kileidungsftücke wegzunehmen, um fie an fohlechte Leute, die ihn in feinem Lafier be. ftärkten, zu verkaufen. Dadurch kam der unglücklis che Mann endlich fo weit, daf's er die Abgaben nicht mehr bezahlen konnte, und ein Stück Vieh nach dem andern um einen fehr niedrigen Preis verkaufen mufs. te, denn bei dem Saufen hatte er das Vieh verfäumt and fo war es in fehlechtem Stande. Eines Tages kam Heitmann fíät aus dem Ḱruge, als alle Einwoliner des Dorfs f'chon in tiefem Schlafo lagen. Halb betrunlien fchwärmte ex umher, und kam auf den unglücklichen Einfall, dem vor dem Dorfe wohnenden Müller einen Schreok einzujagen. Er taumelte hin, brüllıe vor der Mühle, war wieder fill, und fuchte ins Haus zu liommen. Durch das Gehell der Hunde erwachte der Müller, fah aus dem Fenfter, und rief: wer da? Heitmann fohwieg, und Tuchte dio Thür zu öffnen. Der Müller glaubte, es wären Diebe, welohe ihn überfallen wollten, holte leine Flinte, und rief: fagft du nicht, ob du Freund oder Feind bift, fo fchiefse ioh dich über den Haufen. Heitmann fchwieg immer noch. Der Müller rchofs zu, und traf ihn in's Bein. Er fiürzte hin. Man lief num mit Knüppeln hinaus, und fehlug fo unbarmher zig auf ihn los, dafs er einige Wunden am Kopfe, und einen ganz blauen Rüeken bekam. Als fich die Wuth des Müllers abgekühlt hatte, und fich in Mitleid verwandelte, holte man eine Laterne, unà fahe mit SchrekLen, dafs es Heitmann war, den man fo jämmerlich geprügelt hatte. Der Müller liefs ihn nun in's Haus tragen, und auf's befte verpflegen, denn er bereute feine Wuth und feine Uebereilung. Es kam zu einen gerichtlichen Unterfuchung, und der Müller war fehr bereitwillig die Heilungskoften zu tragen. Heitmann mufste für feinen dummen Spal's fehreoklich büfsen; aber such diefs befferte ikn nicht, und man was endlich 


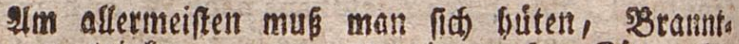
mein bu trinfen, menn man in grofer Rálte eine Feife thut; man fann fide feler leid)t Dadurd Den zod surielien.

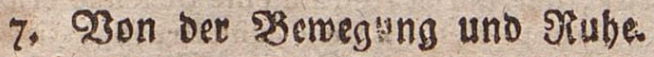

Die forpertide şerregung, befonberb in freier sufft, bat mannidbfaltigen SRußen fúr Den MRenfden : fie be soirlt Şunger und Durfit, bilft EfTen und Trinfen bers Dauen, und madht, Dấ $e^{8}$ gebeibt; fie reinigt Das

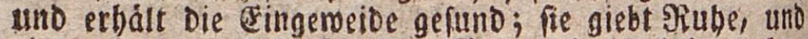
einen fanften Sclaf. Die forpertide Irbeit beiont

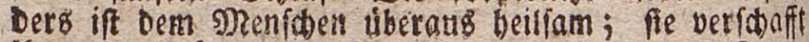
ibm eine brúbende Befundheit und ein langes Reben,

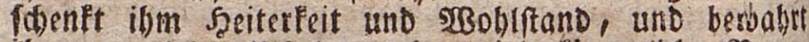
fon bor bielen llebern. Obne viele Pörperliche sgemes gung uno ofue 21tbeit faun Der Nenf(b) unmogglid ges funb lepn. 2lber man fann es bierin leid)t libertreiben.

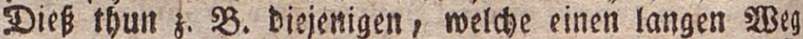
meden mollen, und glcid anfangs fo laufen, oap fie zulekt matt uno ltaftlob merben. Nie muß man io

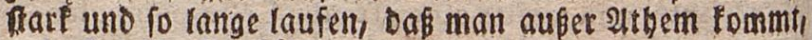
sitio Şerzllopfen fúbitt.

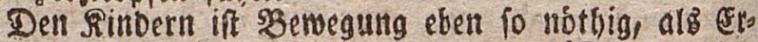
madjenten. Simber, werdie viel fisten mífen, werben ungefund. SBefonbers ift $e \mathfrak{B}$ für tleinere Pinder feht fidáblid, wenn man fie immerfort auf Dem 2rme trígh,

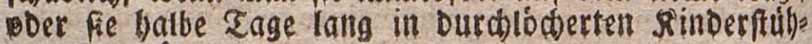
Ient fingen láfít.

Die sBemegung unb bie 2rrbeit fann nur bann fona Den, wenn Der MRenf(d) feine Rráfte úbermáfig anfrengt,

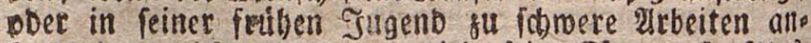
balteno yorridtet; Dann nito fein Siorper fraftlobs fumpf, unb vor ber 3eit alt. - FBer fid fo fart bes

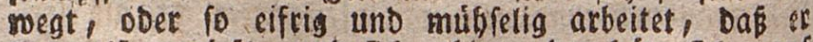

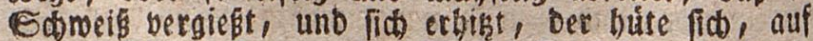
eimmal fitlle zu fiben, ober fid ber 3ugluft auşzufeßen, ober cinen falten Irune zu thun, Denn Durd alles bieß festr er feine Bejunbbeit unb fein Eeben in Befabr.

Grobmann ging gern auf Hochzeiten, und war inmez in folchen Wirthshäafern zu finden, wo es 
recht ausgelaffen und lärmend zuging, und wo die halbe Nacht hindurch wild getanzt wurde. Da tanzte er dann fo lange und fo heftig, dals ihm der Schweifs von der Stirn flofs, wie bei der fchwerften Arbeit. Dabei trank er befiandig. Bier und Branntwein, und gebehrdete fich, wie ein Unfinniger. An einem heifsen Sommertage wollte er fich auf diefe Art einen luftigen Tag machen, und als nun bei der heftigen Erhitzung nichts mehr feinen Durft lörchen wollte, fo rief er: gebt mir einen Eimer mit Waffer, das mufs helfen! Einige Anwefende, welche vernünftiger waren, erinnerten ihn, daf's er fich durch einen kalten Trunk auf die Erhitzung den 'Tod zuziehen könnte; aber Grobmann verachtete ihre wohlgemeinten Erinnerungen, und rief: meint ihr, dafs ich fo weichlich bin, wie ihr feyd? Ich kann alles vertragen, nur Wâffer her! Und damit taumelto er nach der Küche. Hier legte er fich neben einem yollen Eimer hin, und trank fich recht fatt. Das hat geholfen! rief er prahlend, Aber es dauerte nicht lange, fo fühlte Grobmann den heftigften Fieberfroft; man mufste ihn endlich nach Haufe und ins Bette bringen, aus, dem er nicht wieder auffiand; denn fehon ami lgenden Tage brach er Blut, und nachdem er fich mehrere Monate mit der Schwindfucht gequält hatte, fiarb er in der Blüthe feiner Jahre, als ein warnendes Beifpiel, wie unglüellich der Menfch durch Wildheit und Ausgelaffenheit werden kann.

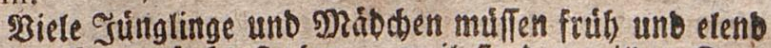
an Der Sungenfuht fterben, weil fie Den milden Tamz 3u febr liebten. sBer nidit beim इanz vollfommenen

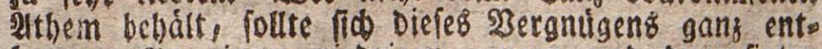
balten. In einem niebrigen, engen und Dumpfigten

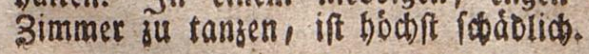

\section{Xom Sdblafen.}

2Ber rutbig fajlafen mill, mus fich nicht mit yollem magen nicoerlegen, nicbt h) is ig e Betrdute genoffen, fid) Den $\mathfrak{z} a g$ úber múbe gearbeitet, und ein gute $\mathcal{G}_{\ell=}$ wifen haben. - 2hn simem rubigen Edlafe ift fege 
viel gelegetr, Denn wer nibt rubig gefolafen bat, lants am SRorgen nitht munter und froh fenit, uno meDet Iraft, nod Suft jur arbeit baben.

Das Schlafgemach mus nicht marm unb niebrig, fondern falt, how uno getáumig lenn, uno to vial als

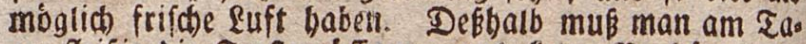
ge fieifig bie Jenfter offnen, unb feine Borbánge um bie Setten Gabeis. - 2luf unb unter SeDerbetten ju follafen, ift sidat gut; benn biefe shetten baben zu biel 2Barme, auds fammetn fict) bie bolell, unreinen uno oft franfen gusbunftungen Darin, und machen ben siós. per ungefuno. Befonderz verurfacisen fie glúfe, Ropf= Babn = Dhren = und Bidifdmerzen. Die befen Bets

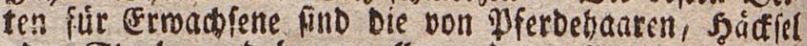
oder Stroh, uno baummollene oder wollene Durchnábte Defen. WBenn man fid aber einmal Daran gelvobut bat, atif ben geoerbetten zu íhlafen, io muffen fie im Soms mer alle abt, und im 23 inter alle 14 Sage an oie Ruft gebract), ausigeflopft, uno alle g) Jonate mit reinen lleberzigen verieben merben.

शlud) fúr Simber find SBetten von Pferdebaarefi, Strob und saloos am beften, mur múfen fie oft frifi aubgeftopft werden. Gederbetten fino Rindern now weit fकátider, alz Ermadfenen.

Man mur fio buten, in fremben 3 setten fu follas fen, roenu fie nidbt zuvor geluftet, uno mit reinenlle. berzugen verfeben find. Sit man baber auf ber sReife, und mus in eiren 2 Birtbsbaule ubernadoten, fo thut man mobl, wenn mant mit eitrem Stroblager vorlieb nimmt, ober fid unausigelleidet auf Das SBette legt.

Mann kann auch zu viel fohlafen, und das merkt euch wohl, lieben Kinder, damit ihr nicht Langfehläfer werdet, wie Ge org und He in rich waren. Diefe wollten, als Kinder, niemals gleich auffiehen, wenn die Mutter fie wockte, fondern liefsen fich wohl dreimal wecken, ehe fie die Augen öffneten, und Anftalt machten, fich anzukleiden. Darum kamen fie faft jeden Tag zu fpät in die Schule, und mufsten defshalb oft Strafe leiden. Die Mutter ermahnte fie yergebens, fie möchten fich doch endlich das Langfchlafen alagewöh= 


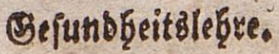

nen, denn künftig würden fie früh aufftehen mü ffen, und dann würde es ihnen fehr fchwer werden. So kam es denn auch, als fie zu einem Tifchler in die Lehre gebracht waren. Diefer war mit feinen Lehrlingem fehr ftrenge, und dahex muf'sten die beiden Langfchläfer viel Befehämung, und harte Strafen exdulden, che-fie fich daran gewöhnen konnten, früh und rafch aufzuftehen. Nun gedachten fie oft an die gütigen Exmahnungen ihrer Mutter, und beweinten zu fpät ihten Ungehorfam. Als fie fich endlich daran gewöhnt hatten, mit Aufgang der Sonne aufzuftehen, wurden fie noch einmal fo gefund und munter, als fie zuvor gewefen waren, und ihr Lehrmeifter hatte fie nun recht lieb, denn fie waren tüchtige Arbeiter.

Dine oringenbe ₹rotbmertbigteit follten niemalB fitis Der bei Ermadienen, ober mebrere finber in einem

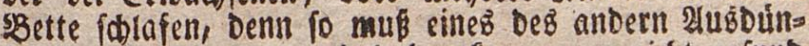
fungen einatbmen, unb Dabeb fanis man nidst gefuno fepu uno nid)t rubig fiblafen. Aber now fadelicter, und fểr gefábrliob ift es, ment ein gefunder s)ienid in einem SBette folloft, worin ein Franfer gelegen bat, obne Daß $e^{3}$ ruvor getúftet uno ausgetlopft worden ift. Ift die Sirantbeit febr bosattig gewefen, fo mus man Die s3etten verbrennen, oder tief vergraben.

\section{Son ben 528 baungen.}

SSBenn eine 2030 bnung gefund fenn folf, fo muifen bie Stuben und Rammern bell, geraumig uno fuftig fenn. In Dunfeln, Dumpfigten uno feudten 530 obungen mer: Den bie Meniden ungefund und (droad) $l$ gidtifich unb Fránfliđ), ia wohl Dumm, verbrieflid unb fobmermus= thig. Sinber gebeiben in folden s2Bobnungen nicht, fort a Dern werben blas, fdorellen, sebren aus uno frerbett.

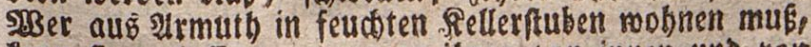
fann fie berbeffern, renn er ibnen yon innen uno vort aufen vieles \&idt, uno fo biel alB mogliab reine suft zu veriकaffen fudt, ben niebrigen gubboden erboblets

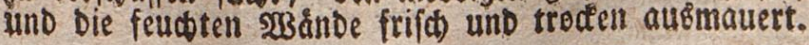

Stuben uno Rammern múfien alle sage gefebre uno gereinigt, uno wo moglion alle Jabre gemeiffe 


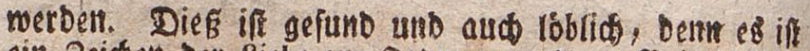

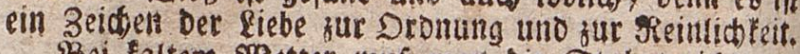
Bei faltem 23 etter muß man Die Stuben nicht un. mábig beizen, und fid nidbt an Den mermen Dfen feg: zeit oDer ftellen, am allerwenigften fid auf Die Dfens bani legen usho fotafen, Dena Das madis bent SRenf(t)en Dumm und franf. इotf uno Steinfóblen zu breninen:

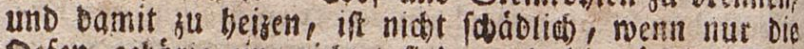
Defen geborig eingeriotet find, und bie nothige $230 t$ fid)t angemenoet wiro. (Eine folyr úble uno gefábrlioe

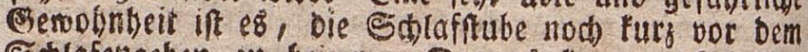
Sablafengehen zu beizen. Daraus fann grobes lut: glude entitehen, mie ibr aus folgender seidoidie lers nen tontist.

Fine wohlhabende Wittre, welche zwei erwach: fene Töchter hatte, mufste eine Nacht aus dem Haule bleiben, um bei ihrer hranken Schwefter zu wachen. Die beiden Mädchen dachten, fie wollten fich einnt al reht was zu gute thun, und heizten, weil der Abend fehr kalt war, die Schlaffube ungewöhnlich ftark. Nun gingen fie beide fröhlich zu Bette, nach dem fie zuy or den Ofen feft zugemacht hatten, Die Unglüchlichen! Sie ftanden nicht wieder auf; denn der Ofen bekam von der ftarken Hitze einen Rifs, und ein Stück Holz, welches nur halb verbrannt war, fing an zu fchwelen, und füllte bald die ganzö Stube mit Rauch an. Beide Mädchen mufsten ohne Rettung exficken. Zwar die eine rom Schlafe erwacht, aber vergebens hatte fie es verfucht, die Thür zu erreichen; man fand fie in fchrecklicher Geftalt auf dem Boden liegen; in der Todesangft hatte fie fich das ganze Geficht zerkratzt, und die Haare ausgerauft. Welch ein Anblick war es für die unglückliche Mittter, als. fie am Morgen ihre beiden geliebten Kinder, die Hoffnung und Stütze ihres Alters, nicht melrr am reben fand! O Iernet doch, fo lieb euch euer Leben if, mit Feuer und Licht behutfam umgehen, lieben Kinder, damit euch nicht ein ähnliches Unglück widerfahre!

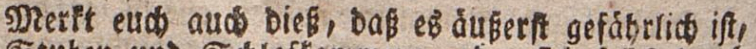

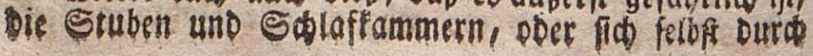




\section{Cejundb̨ritsteb̧re.}

Soljpoblett , melche in einem Fetretbecten ober itt eis nem Topfe find, zu ermármen, man wirb bavon slend, und fanu feblr leid)t erfticken.

\section{Don Exbiłungen uno Erkaltunget.}

23 enn man ourch beftige forpertide Sgerwegung, Durd

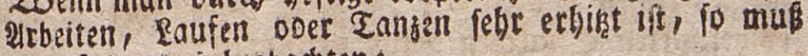
man folgendeś beobachten:

t) Nidot auf einnal rabig filsent ober liegen, fort bern in einter máfigen Bgerocguting bleiben.

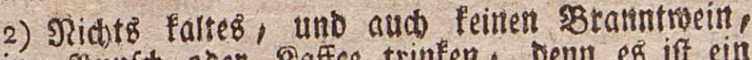
20Bein, Dunfd ober SRaffee trimten, Denn eहै ift ein

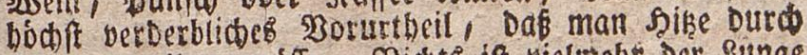

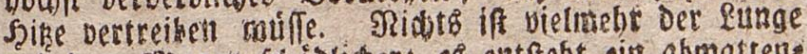
und Dem Mragen fodsoblider; ef entffeht eit abmattens

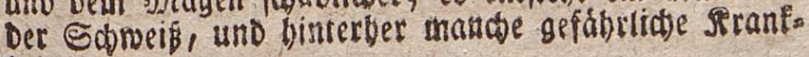
beit.

3) Nid)t Die Salut ober bell Robrper Der falten suft

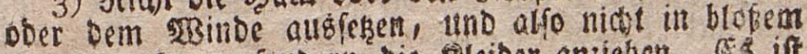
sembe gethen, fonbern die sileiber anzieben. Es ift freilich behaglicher, obne Rleiber fu geben, wean man

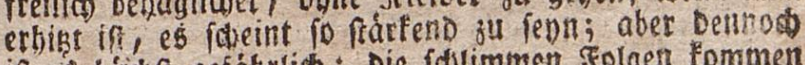

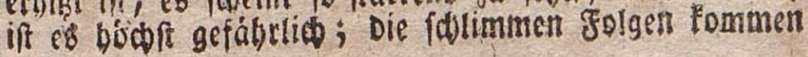
nact.

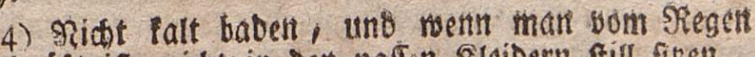

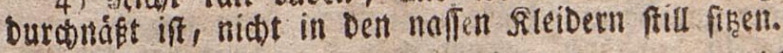

5) Sid niकt auf ben falten (Etbboben, auti tidat ins (Sras fosen ober legen, und am wenigften fo follas fen; Denn Davon tann man an allen Bliedern gelátumt merten, und Die Sdimindfudf oder Die Biajt betom: men.

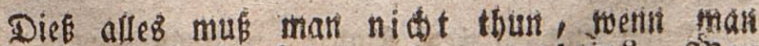
erfint if. Llno mab foll man bagegen thun? 2has foll fich bei einer gelinben sBenvegung fu extialfen, sinb

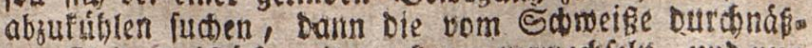
ten Rleidunggftudfe mit troderent verwedfelif, und nurs langfam feinen Durfit lo/hen. SBie aber, wenn Des 


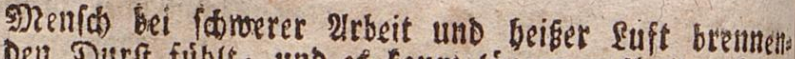
Den Durft fúblt, und ef faum lánger augbalten fantil foll er Dann nidbr auds trinien? Ja, er Darf eb zu! Srots mobl thun, aber nur nidt biel suf eimmal, auth Darf er Dabei nicts rufsen, fonderu er mus rafó fort arbeiten, ober fich beroegen, fonft ertéltet er fici), und wito Eranl. SRods beffer ift $e^{B}$, befonders auf der Sieife, feinent Durfe mit einem in sBer oder NBaffen eingetumiten studfden 58000 ju tofd)en.

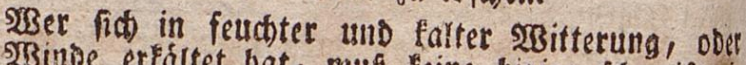

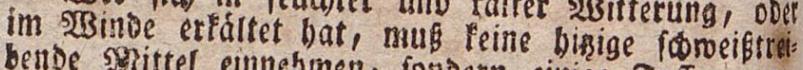
bende sglittel einnesmen, fondern einige Iaffen mat: mes swaffer, mit bem vierter Sbeil Effig vermilath trinfen, fich redst marm mit Rleidern bebecten, unt

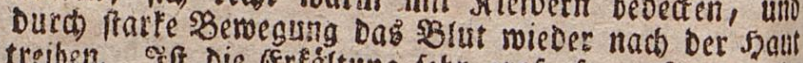
treiben. Soft die erféttung febr grob, fo muB man ein marmes subbab nehmen, Effig trinlen, uno fid ing

Tenn Der Riorper uno die gúpe naf unb falt ge morden find, fo muk man die naffer Rleider und Etrúm= pfe nidst anbebalten, fonoern Die Saut abtroutnen und warme troctene Rleider anzietien. Linterláft man Diefi

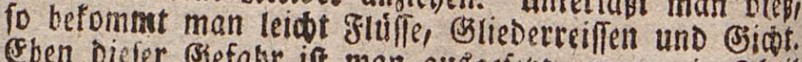
Eben biefer Bsefahr if man alisgefekt, menn ein sheil Dez siorpers ber 3ugluft ober Der Rálte ausgefebt iff itioef Der ganje úbrige Sórper warm liegt uno aubDút ftet; wemm man alfo, 8 . 3 . an einer feucten $2: 3 a n b$ fist, Doer gar Daran fóláft. - Man fann fíd yor ienen llebeln bemaíren, wenn man Die Sacut bon firmbeit

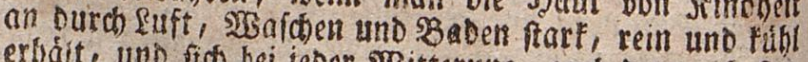

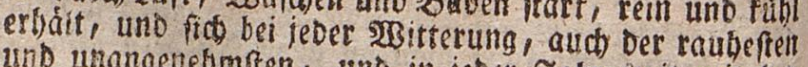
und unangenthmfen, uno in jeber Jabrebzeit mit bet gehorigen Botfict viel torperlide ssemegung in freier Puft madis.

Sgliret euch, licben Sinder, ju fruib Eommierfleiber anjuzlelien, oder eact gleid in Dent erften rars men Tagen Des frtiflings fo leitbt fu fleioen, alb ob ibr mitten im Sommer lebtet, Demn an fold)ent zagen fann man fid am allerleióteften uno gefábr lidifen erfálten, weil gemogntid bie Mbende smpfino- 
lidi falt fint. एक if bod mobl verminftiger unt

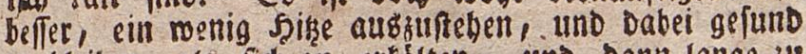
zu bleiben, alo fït, zu erfálten, und Dann lange zu leiben. Die Rålte ift ein Şauptfeind alleş Sebens, obs

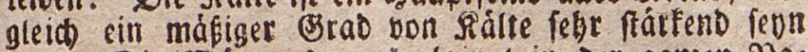

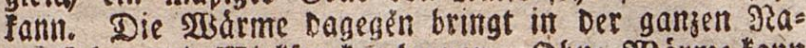

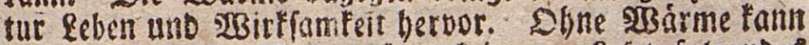
nidt einmal ein Eaamentorn feimen. Lefet folgendes auffallende SBeifpiel von Der auferordentlioden Rraft

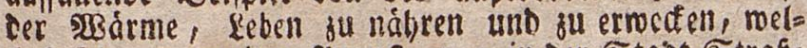
wes fict am zmeiten 2 uguf 1790 in ber Staot Stras= burg ereignete.

Hier fiürzte fich am gedachten Tage ein Soldat, Namens Petit, ganz nackend aus dem Fenfter des Krankenhaufes in den Flufs. Erst um drei Uhr Nachmittags vermifste man ihn, und er mochte über eine halbe Stunde im Waffer gelegen haben, als man ihn herauszog. Er war ganz todt. Man that weiter nichts, a's dal's man ihn in ein recht durchwärmtes Bett legte, den Kopf hoch, die Arme an den Leib, und die Beine nahe neben einander gelegt. Nan legte ihm dabei immerfort warme Tucher, befonders auf den Magen und auf die Beine, auch wurden in terfehiedenen Gegenden des Bettes heifse Steine, mit 'Tüchern umwickelt, gelegt. Schon nach 7 bis $8 \mathrm{Minu}$ ten nahm man an den obern Augenliedern eine klei, ne Bewegung wahr. Einige Zeit darauf offnete fich die obere, bis dahin feft gefchloffene, Kinnlade; es ham Schaum aus dem Munde, und Pelit lionnte einige Löffel Wein verfchlucken. Der Puls ham wieder, und eine Stunde darauf konnte er reden.

\section{Don Der Erhaltung cingeiner Şbeile Des sorpers.}

Uniere Sinnen = \$Berfouge múfen mir mit ber gróp. ten Eorgfalt geiuno zu erbalten fuchen, Denu un

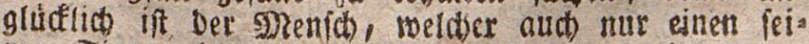
ner Sinne niकt gebraudien fann; er mus viele greu= ben und Innebmlichleiten entbefren, und visl Sei

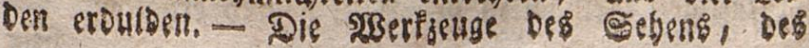


Sortents unb beb Certudib merben butch fleifige Les bung in facier reimer fuft gefund erbalten, gelodirft und geftertt. Diefen Drei Esimen iकabet mott to

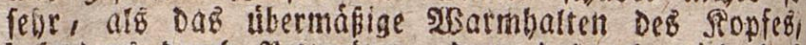
befónders Duras Pelzmitgen, Denn Dablirct roiro Das פiut im Ropfe angebçuft, eह entfteben glüfe unt Beftomitre, Deren Eiter oft zutudftritt, und Dant BSinbbeit ober ₹aubbeit verturfadt.

Den en is geft fáabet befonbers blententer, thit

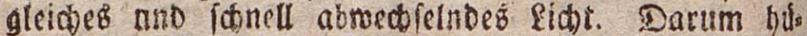
tet eud f. 23. bei Der 2rbeit, uns befonderb bei feinet

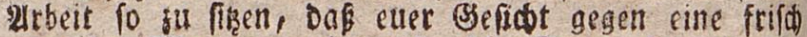
gerseifte mauer, auf melate oie Sonthe fdeint, ges

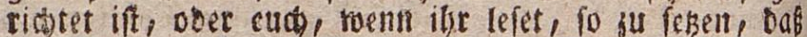

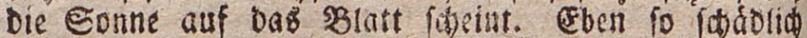

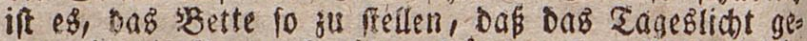
rabe in bie 2ithen frafilt. Soutet eud, alles zu febt in Der siálfe, ober fálef von oer Seife fu fehen, Doet elic) tange ill verborbener, mit Elaub, SRauch Dbet feutten Dunften angefullter fuft aufsubalten, ober in Den fangen 2 Binterabenden bei fotarfen Del = unb gidto Dampfeth, bet frarter Dfenthize, Doer in Der Dámmes

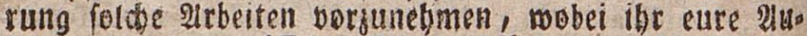
gen anfitengen múffet. Die Dadformigell \&iditifirmen

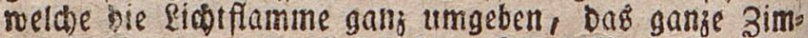

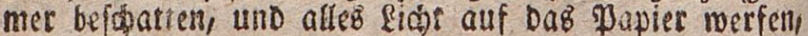
tauget niat. Heberbsupt follte man nur grobe 2arbeto ten bei ficte gerricterts.

Dem (Sebor fdradet jeder forarfe, ftatle unb uth ermartete Edall oder Sinall, serdorbelte \&uft, Feder. betten, Staub, vieler Stoleim in Der Nafe uno in Den Difren, und Daz Zufammenoriteden Der auferen Dfo ren Dura Múken unb Ropftinden.

Derm Bef

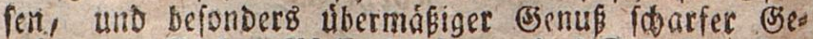

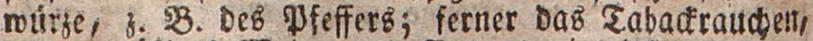

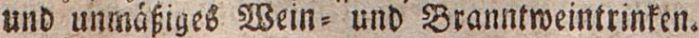

Dab (5) eflith I mirb befonbers ourd befanbige liebutis, burch ununterbrochene Ibátigfeit uno Durd forgfältiges Sieinbaltent Det şaut ge(und etbalten. 


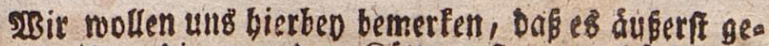
fábrlich ift, Die von Der Rálte erftartren Shänbe an beifent Dfen, ober gar am Jeuer ju ermármen: mas

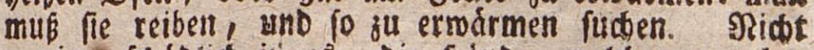

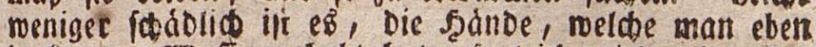
in faitem 230 flfer gebabt hat, fogleich mieder in wats

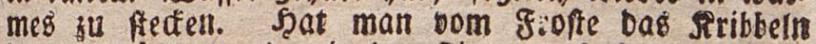
in Dein fadunden, ober in ben Jingern, fo feelt man Daš verlorne sefübl am befen Daburd her, bas man (iid) mit Ednee reibt.

Da unfere Eeele vermittelft ber Einbrúde, mele we Die Einue von Den áukeren હegenftánden betommen,

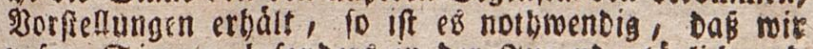
unfere Sinne, befonders in Der Эugent, tégliक und

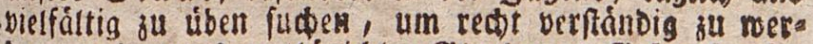
Den; uno vor Dem ithoricten BSlauben an Befpenfer beo mabrt zu bleiben. एक musbe meit weniger 2aberglauke

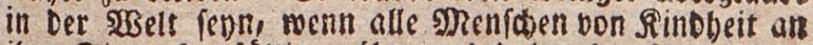
ibre Esinne forgfältig geribt, uno Dasurch gefuno erbate ten hătten. Sefonbers fonnen ung Brifict und Behorp wern fie nict setibt find, in Der Nacit betrígen, fo Dá bir furdthare Beffalten zu feben, uno fibredfidie Tóne ju boren glauben, wo dow meber etwas ju fehen, noch fu boren ift. Eind aber diefe Eime geborig geabt, uno gebt man beberzt auf bas, mas man fieft obee bórt, 10B, uno faft ${ }^{8 B}$ mit Den Şánben, fo miro mant finden, Dấ alle Befpenfter nut Setrug bófer Nens

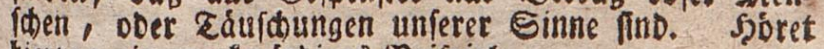
bietwon ein mertmutitbiges sBeifpiel.

In einem Magdeburgifchen Dorfe wohnte ein rechtfchaffener und verfiändiger Prediger, welcher feize gröfste Freude an der Erziehung leiner Kinder fand. Er hatte oft die Erfahrung gemacht, wie viel Unheil die thörichte Fuxcht vor Gefpenftern unter den Menfchen anrichtet, und liefs es fich daher bei der Erzie. hung feiner Kinder angelegen feyn, fie vor diefer Furcht zu bewahren. Sie mufsten, fchon in der erfien Kindheit, des Abends eine Zeitlang allein in Finftern bleiben, mufsten gewöhnlich ohne Licht zu Bette gehen, und zuweilen in Finfiern aus abgelegenen Gegenden des Haufes, wo fie genau Befcheid wulsten, 
-twas holen. Oft erzählte der Prediger Gefpenfter gefchichten, und zeigte dann immer, dals Betruf oder kindliche Fureht und Einbil dung dabei im Spie le gewefen wäre. Eines $\mathbf{A b e n d s , ~ a l s ~ e r ~ a u c h ~ d e r . ~}$ gleichen Gefchichten erzählt hatte, lagte er zu fei ner zwölfjährigen Tochter Marie: würdeft du dict wohl feheuen, ohne Licht auf den oberften Boden $z$ sehen, und die Garnwinde ron da herunter zu ho. len? Nein, gewil's nicht, lieber Vater, antwortet Maric. - Nun, wir wollen fehen; jetzt geh' ein mal, and hole fie; aber geh' bedächtig, und nimm dir Zeit! Marie ging, ohne fich zu bedenken, un fand auch bald, was fie holen follte. So lange wart ihr nicht die geringfte Fureht angeliommen. Abet indem fie die erfien Stufen der Treppe hinunter ge hen wollte, hörte fie etwas raffelnd hinter fich her hommen. Jetzt fing fie an, furchtlam zu werdeni doch hatte fie noch Muth genug, fich umzufehen. Abet freilioh erblickte fie in der finfternif's nichts, und indem fo nun weiter ging, hörte fie das raffelnd Ding wieder dicht hinter fich. Sie raffte allen it ren Wuth zufammen, und rief : wer da? bekan abrt leine Antwort. Es war ein Glücle, dafs fie noch lo tiel Muth behielt, denn fonft wäre fie gewifs die Treppe hinabgeftürzt, und hätte dann vielleicht Arm und Bein gebrochen. Indefren, als fie nun auf di zweite Treppe kam, und das rafelnde Ding nieli aufhörte, fie zu verfolgen, fchrie fie voll Angfl: Licht! Licht! und kam endlich ganz aufser Athem, doch mit der Garnwinde in der Hand, in das Wohr. simmer. Hier fahe fie fich wild um, und fiehe div ihr Verfolger war auf einmal verfchwunden. Sie er. zählte num zitternd, was ihr begegnet war, und kaur hatte man angefangen, die Sache zu unterfuchen, to entdeckte man fchon mit Lachen das raffelnde Gt fpenft. Es war nichts anders, als eire getrocknett Bohnenranke, mit einigen Schalen voll klappernder Bohnen, welche der guten Marie an der Rockliant hängen geblieben war; denn als fie fich diefe wie der anhing, und dantit fortging, war gleich das Ral: feln wisere da. 


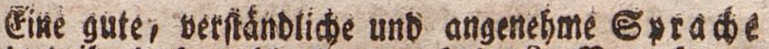
modiet ibr dod mobl alle gern baben? Nuni fo ge.

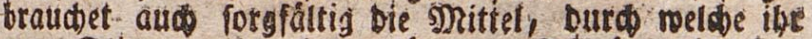
sure Stimme rein erbalten tonnet. Sdintret wiot ben Sals mit biefen ober fteifen Salbbinden irfammien. baltet die stafe rein, uns gebraudet alfo fleifig bas

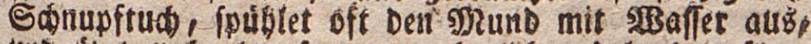
uno tibet elto, langfam, bernebmlio und laut zu fore=

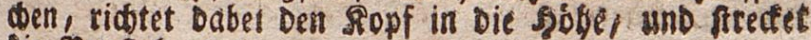
Die stuft bervor.

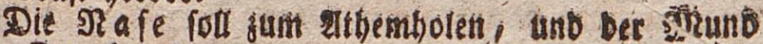

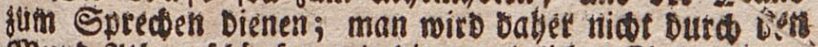
9) Muno Itbern fobpfen, roie die unreinliden sinber thusp toelde bie safe immer voll Salein baben, forbern burd bie Rafe, uno eben barum biefe rein balten, fid

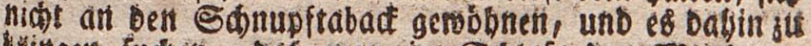

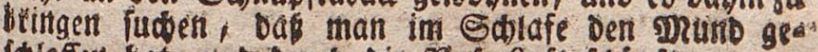

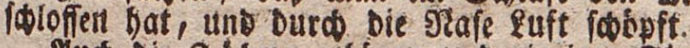

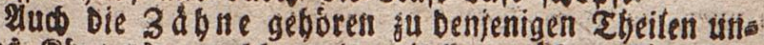

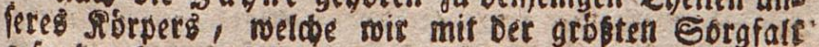
gefuno erbalten follen, bern fie find nidit blog zum. Epteden, fonbern aud fun Ramen Det Epeifen notb. senbig. Essenn Die Epeifen nidot gebórig getaut, uno baburd in einen SBeei verwanbelt merden, fo fann fie ber gragen niकit verbauten, uno bann ndbren fle aude

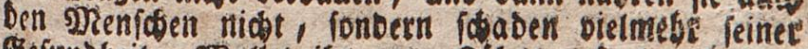
Gefunbheit. $23 s 0 l l e t$ ibe cure 3abre gefunb etbalten, to burtet eud vor allen Dingert, beike Speifen fu effeng uno viel rarme Betránfe tis gentieferi. Berbobnet crods nidt ant Den iddolidien Saffee und Shee, fotibernt trin. let lieber Paltes sasaffer, effet gelodie Speiren nidie thet, als bis fle lnutuatuin fino, reiniget Des sororgens

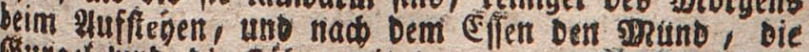
esurgel lunb bie 3dbne mit laumarmem sissaffer ( Denns

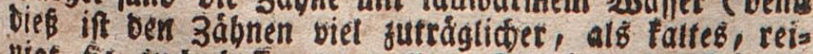
nigt fie aud beffer oon allem SDleim, bet fid ant= gefent bat, unb son ben jurúdgeblieberten Epeifen, )

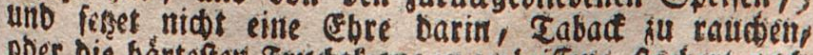
Doer bie bárteften frudotlerne zu jerbeiffen; ftodert aud

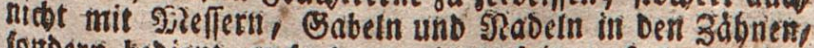

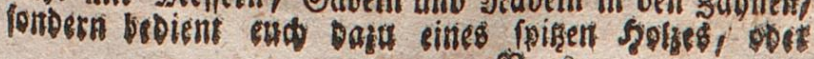
in 2 
einer geber. SBBer folledite zadine bat, foll fie nidt

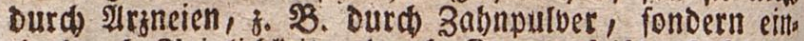

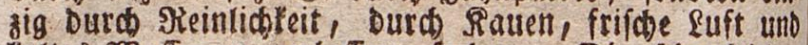

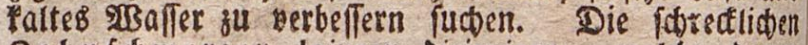
$3 a b n$ (d)merzen bringen Diejenigen, meldse Daran leiben múffen, oft zu Dem entrobluife, fids Die iranten zadhne austieben gu laflen. Es giebt gálle, in wel:

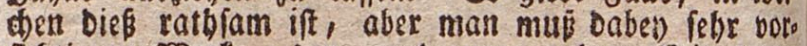
(iib)tig zu soerfe get)en, und jubor andere grittel bers futhen, efje man jum 2susgiehen fobreitet. Dft ift lles berlabiatg Des Magenz die urfache ber 3abnid)merzem,

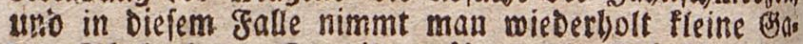
ben Sifhabarber. Suwbeilen rúbren Die Zafunfdemerjen yon Sollblútigfeit her, und Damn fotropte man im

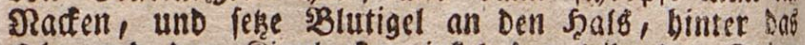
Dor uno Den Sinnbadenmintel der leibenden Eeite! bute fíd babei bor berifen Stuben unb vor Erbigung,

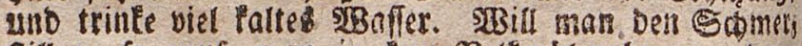

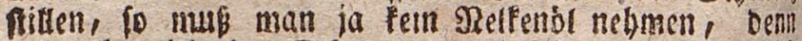
baburd mirb Der Echmerz mur befriger, unb endith

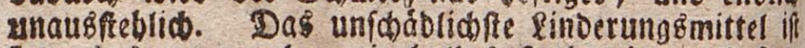
folgendes: man nethe ein balbes forb reine zerfobetil

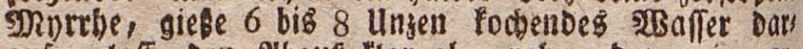
auf, laffe ben 2tbgus Hlar ab, nebme babos immets fort einen laumarmen Esiluat in ben פMund, unb ber balte ibs cinige MRinuten im Munbe, (Eben biefe $\mathfrak{W}_{\text {it }}$ Eung than 60 bis 80 Etopfen Moprtheneffens, in eite zaffe laumarmés grsalfer getrópfeit.

Da Der Epeidel zum Rauen unb serbauen oer Sepeifen fetgr nothmenbig if, fo mus man alles pero meiben, woburd viel Epeidel verlobren gebt, ;. 55.

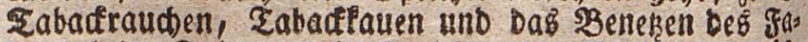

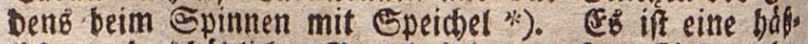
lide und iodadlicbe Bemobntheit manctier Rinder, bes

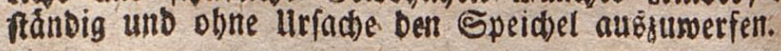

*) Beim Spinnen nuß man Den Gaben nit DSaffer, Das junur Durh 2 bier, Seife ober Stárfe tlebrig gemadt morben if , beneken, aub Den Jlads bon allen fafert uno Sdaben forgfáltig reinigen, fonfi fliegen Diefe beim 2atbembolen in bie Bruft, und veruriaden manderfi Uisel. 
Denm 23etmunft unb gute Befinmungen find bie cigen:

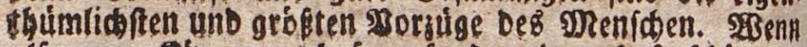
alíp euer Sobrper nod fo gefund und nod fo fabon iffi

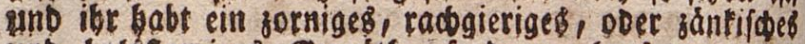
unb balśftartiges Semuth, fend ungethoriame, ober faule, ober leidfintige Sinber, fo mirb eld Pein bef:

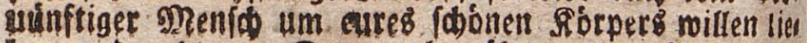
ben und adten. Darum bemúbet eut mit gleider Sorgfalt, Die Sefunbbeit unb Sdonbeit eurer Eefle

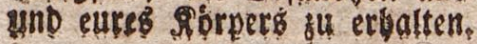

\section{3. $\$ 30 n$ Dem \$Bebbalten in STranEbeiten.}

Pinbet unb ermadiene leben nicht immer verninffie prbentrid uno máfig, und daber fino fie nidot immet gefiund, forbern fublen fich oft franf und fobmed. serbie forten fie fid bann verbalten? Dieß follet ibr list Yernett, lieben \$inber.

2Bes fid frant fültt, foll fid vor allen Dingen the

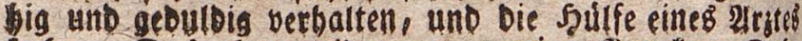
fucben. Das tbun leiber mur menige sirante. Sebl biele moilen in bet seranfbeif nictet rubig fenn, lonbern arbeiten, unb ibe Befodffte betreiben, uno Daburd maden fie bie Sranlbeit folimmer. Intubere mollen fid

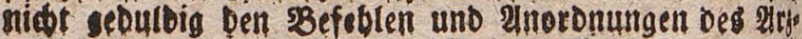
fes unterwerfen, fondern gefobmind gebeilt fenn, und nebmen Darum einen Ruaćfalber an, Der Dann freilión Die Rrantheit oft gefominb genug vertreibt, aber auf eine folde ent, Daf eine now gefábrtichere srantheit binterber fommt.

Ithe fraget, lieben sinber, mas suadfalber finb? Se nennt man bie niebrigen Betruiger, welde fid ribs

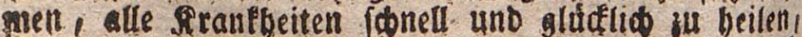
a fogar Die 2Bef́baffenbeit unb Den liefprung ber Rrant beit aub bem urine bes Sranken ficher beuntbeilen ju ionnen, und bie bod nidt bie allergetingfte Renntmib

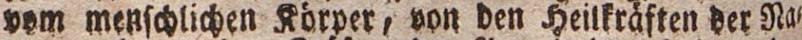
tur, uno you Den Siráften Der 2ranteimittel baben, Da

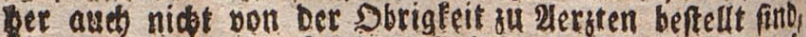

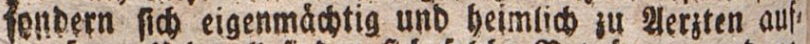

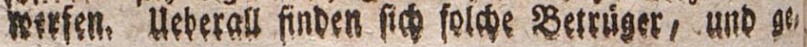


wibutidfint ef forde Sirten, ober Sdbarftiwter, ober verdorbene fonbmerfigleute. 3uweilen gelingt es ib neth, ourd ibre Arsneien einen firanfen wieber gefunto fu maden, aber baun bat allemal feine ftarle satur

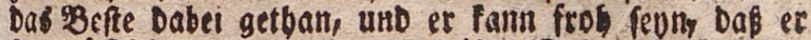
io gludtlid Davon gefommen ift. Seft oft Pommen aut ibre sgetrugereien an Den Tag, unb Dann werden fie ben ber Dorigfeit fo bart geftraft, wie fie es berdiea

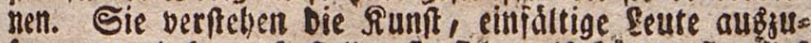
feagen, unb bermach fellen fie fid, alo batten fie alles ant Dem Urine sefeben, was ibnen biefe erft felbft in ibret Einfalt gef́agt baben. Einige ridten ihre 2BBeiber Dazu ab, Daś fie Die leute, melde Den llein Dez Rrant fen bringen, ausforíden, unb ibnen Dašjenige borber biuterbringell, mab fie nadber init grober Drableré auß̈ Dem urine prophejeien. Slndere borcten hinter ber Thuir, ober binter ener fpanifeben $23 a n d$, mas Die \&euten melide 2 risnei bolen wolleh, unter einanber reben. So babe id einft von einem berborbenen Shufer gehort, Der alb ein 533 unberboftor weit uno breit gerúbmt wurs De; Deffen Sdwager mar Swentwirth im Dorfe. 2Wenn mun ein Rranter fam ober fóidte, beffen umftánde ber

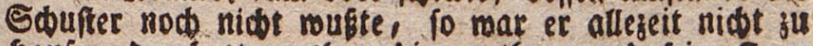
Sautfe, ober hatte nothmenbig zu thun, und feine frau befecllte Die Seute in einer ober zwei Stunben wieber. Gemóbntio lagte fie ibnen Dann: fie módeen nur unter Der zeit in ben Siras geben, und bas tbaten fie aud wob! don felbff. Det Edoenfmirth mar nun yon fei= nem Sdmmager, Dem 2Wunberboftor, Dazu angewiefen, wic er Die feute ausfragen follte. $23 a$ fie ibm fagtell

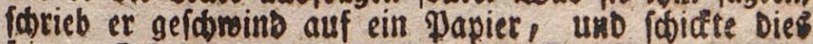
feinem Sdwager. Ramen nun bie Siranfen, ober ibre Boten mieber bin jum Squfter, fo trat er mit eiter

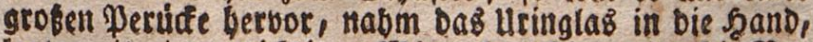
legte mit einer midtigen गRiene ben singer an Die SRas

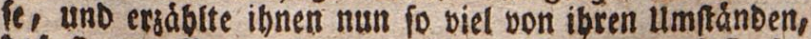

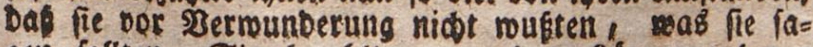
gen foflten. Sie bejabiten nun Dem eúgenpropteten eern, wab er yerlangte, and Diejer stheilte bann bas Eelo mit feimem Edwager. Die Đillen, Die er ben seuten gab, madte et aus blofer Semmelfrume, uno 
vergolbete Dber berfilberte fie, und jeine fieberpurber

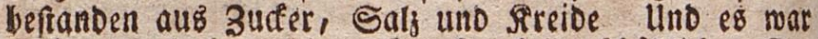
nod gut, Daß er feinen Sranfen feine fidsdiden Eas wen gab. Satimmer madie es ein suberer Duadials ber, ber bas falte zieber burd) Sropfen furirte, zu melo

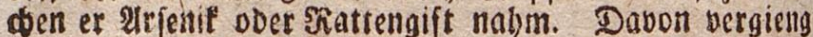
zhar bas gieber fóttell, aber bintertyer befamen die Qeure bon feinen Bifttropfen lablimmere zufálle, alb Dab Fieber, und blieben jeitrebeng ungefund.

Es ift aberglaube, Daf́s Stantheiten Durch seberen

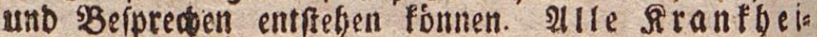
ten baben ibre naturlionen llrfaden.

In $H$. waren noeh viele einfältige Leute, welche an Hexen und Hexereien glaubten, fo oft fie auch rom Prediger und ron dem Schullehrer eines beffern helehrt worden waren. Michels Kind war rerfüttert, and wurde fehr elehd, Anftatt fich an einen vernünftigen Arat zu wenden, und das Kind mäl'sig und ordentlicn zu halten, gebrauchte man allerlei thörichte Mittel gegen die Hexerei, fo lange, bis das arme find zum Krüppel geworden war. - Ko n rads Find war von der ungefunden Milch feiner Mutter, die lehr ärgerlich war, krank und fehwach, bekam Lírämpfo und hatte heftige Verzuckungen, wobei es das Geficht fehrecklich verzerrte. Die abergläubifchen Eltern glaubten fteif und feft, ihr Kind fey behext, und begnügten fich daher, es zu bekreuzen und zu fegnen, ohne einen Arzt herbei zu rufen, und Arzneimittel zu gebrauchen. Es mufste elend fterben. - Hein emanns Kinder hatten beim Spielen im Garten den giftigen Stechapfel gegeffen; fie kamen fchreiend, unter heftigen Schmerzen, nach Haufe, und klagten den Eltern, ihre Noth. Bald bekamen fie fchreckliche Verzuckungen. Die Eltern, welche ihre Kinder noch furz zuvor fo munter und froh gefehen hatten, konn ten diefe plötzliche Veränderung nicht begreifen, und ohne erft nach der Urfache zu forfchen, waren fie gleich darin einig, daf's die armen Kinder behext feyn müfsten. Sie fchickten daher eiligft nach dem Kúbhirten in einem henachbarten Dorfe, der in diefer Gegend als ein VVundermann berühnat war. Diefer lam, 


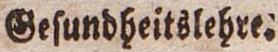

gab den Kindern einen Trank ein, wobei er mancherlei närrifche Gebehrden machte, um die vermeinte Hexerei zu bannen. Allein fchon in der folgenden Nacht ftarben zwei von den Kindern auf die kläglichfte Art, weil fie nicht zu rechter Zeit Hülfe bekommen hatten: auch das dritte mufste fterben, weil die Hülfe des Arztes zu fpät kam. Diefer öffnete nun die todten Körper, um die Urfache des Todes zu orforfchen, und fo fand es fich denn bald, dafs der giftige Saamen fie getödtet hatte. Jetzt maehten fich die abergläubifchen Leute bittere Vorwürfe, dafs fie fo thöricht gehandelt, und vom Aberglauben verführt, die ordentliche Hülfe eines gefchickten Arztes verfäumt hatten. Sie konnten fich nie hierüber zufrieden geben.

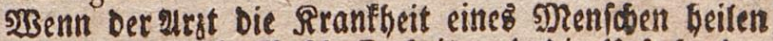
foll, fo mus et Die sefarfenbeit und Die lirfache Der

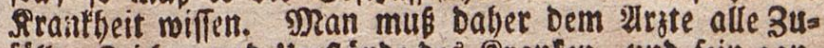

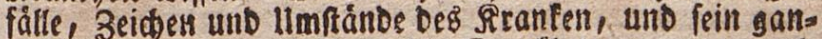
zes Befinden vom Anfange Der Rrantbeit an genau unb

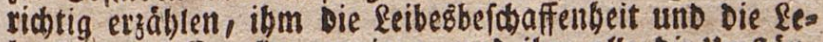
bensart Des Siranten anjeigen, und ibm alle bie llmftáns be fagen, melde Die Urjacbe Der Rranllyeit febn fonntell. एEs ift Daber gut, Dá́ Der 2lrzt Den Sranfent febe uns fprecbe, uno felbft die Natur und llrfadbe der Rrantbeit erforide. Der Sirante mus bann Den SRatb un' die sine

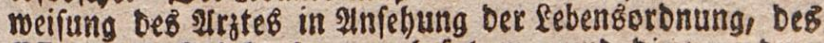
Effen' uno Irintenz genau befolgen, und bie berorones ten 2rzneien treulich unb zur redten 3eit gebraudett.

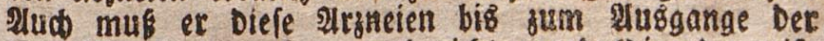
Rranlbeit fortbrauben, unb nicht ungebuldig ober mifo frauifo merben, menn Die Rrantbeit nidist gleid nach Der erften Mrznel vergebt, Denn Das ift eben fo ummogn lid, alB baß ein 98 aum auf ben erften Şieb falle.

Die Pfleger eines Reanten mulfen mit itm, als mit einem Ungludfioben, fanft und liebreich umgeben, ibn forgfältig warten unb pflegen, niobs biel nuit ibm reben, To lange bie Rranfleit now gefabrlió ift, uno bafúr for gen, baf́ eś frill und rubig um ihn ber fed, unb DoB ex immer frifote, reine und trodte luft bathe. 


\section{VIII.}

Bon ber వెeitrechnung und vom Stalenber.

Cigentlich beift Die 3eit vour 2ufgange ber Sonme bis zuns untergange berfelben ein $\mathcal{E}_{a g}$, und bieß wadre Det naturlide Tag. 2uber fo beredinen mir unjere Tage nidit, benn fonft múrben fie niemals aus vier unb swath tig Stunben, fonbert mitten im Sommer bódhifens aus (ed) $b$ z $b$ n, und mittent im 53 inter nur aus fieben biš adt Stunden befteben. Nach ber zeitrednumgs melche bei uns eingefübrt ift, nebmens mir $z$ ag uno Radot gujammen, uno netunen bieß einen Tag. Fsenn wit alfo von Jemant fogen: er ift auf adt Tage verteift, fo

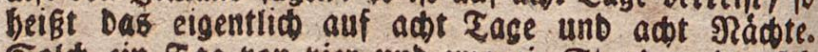
Sold ein ₹ag bon yier und zmanzig Stunben, Der fi巾 um פnitternacht anfángt, uns bis zur folgenoen snits ternadtst Dawert, beift ein burgertide er Fag. 2Bit gablen aber mur smólf Stunden, von פnitternacht bis mittag, unb Dann cben fo viele yon MRitrag bis SPlit sernabt. Die Ubren bienen Daju, um genau Die Etunden absumeffen. Man bat oreiertei Hbren. Die eine are if unberweglich, namblid Die Sonnenubs rett. Die Sonnenubren geigen bei Eonnenfकein bie Stunben mittelft eines 3eiger 8 an, Der in ber Intitte Der Ubr aufredot ftebt, und Deffen Eshatten immer auf Die zabt ber Stunde fált, meldie verfilfen ift. gadat alfo Det Sqantten bes zeigers jwirden vier unb funf, to iff es bals fünfe. Dove Sanduber fino jest faft

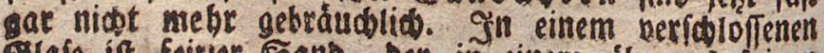
Elafe if feiter Eand, Det in einem úberaus feinen Straffl butd, eime uaren im Blafe angebradite Deffo nung in ein anberes Blaz láuft. Die Einnidtung if fo gemadbt, Daß et gerebe in ciner Stunde abläuft.

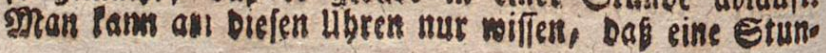




\section{Bon Det 3eitrednung und rom Ralenber, 203}

De bes Tages verfleffen if, aber nitht: bie wie vielfte Stunoe.

Die braudbarfen ubren ftnb alfo unftreitig bie siaberubren, benn man fann fie bei Sonmenichein uno in ber gimfernis gebrauden, une fie zeigen weit genauer bie 3eit an, alb̈ Sommenubren und Eanbuhren. Die Ráberubren soerben, menn fre gtof find, wie 3.98. Die 2 Banduhren unb Etubenubren, Durct Semictere in szerwegung gefert. Sind fie thein, mie bie ₹a fiden: ubren, lo feter man fie Durd eine buinne sufammenges tuimmte stablplatte, welde bie 8 eder beift, unb fic nad und nads ausbebnt, in sorvegung. Eine MRds Derubr seigt nidt nur die Stumben, (onbern aud die Dinuten, (Deren fectsig auf eine Stunbe geben, ia logar, menu fie Darnad cingetidtet iff, bie Sefunt: Den an. Eectig Eefunben maden eine gRinute aub. 2Bis viel Eefmben gehen alfo auf eine Etumbe?

Der Mn ond läuft in jebem gabre ungefäbr breiiefumal um bie cerbe, umb roiro eben fo mie unfere

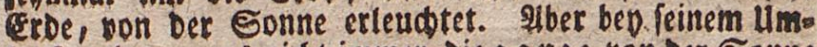
laufe zeigt er ung niकt immer die g a mze yon ber Sonke

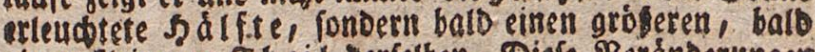
einen fleineren ₹b eil Derfelben. Bief́ Betánberungess feimer erleudsenben Edbeibe finden mir im Ralenber mit folgenden Tamen angejeigt: Ne umoud; erifes Biertel; Bollmond; lebtes Biettel. Den Reumbno feben wir gar nidt, meil uns ber monb albs Dann feime Dunfle Eeite jufebtt. 2lber einige ₹age nad

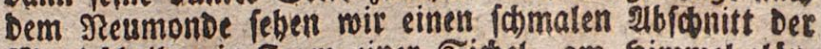
Mondiabeibe, in Jorm einer Siфel, am Simmel glans zen. Eieten כage nad bem Reumonde feben woir bie Monbrdeibe Galb etleudtet, und Dief mite bas erffe Fiertel genannt. Thun wirb Die grondicheibe an ies Dem शbent grófer uno runder, und fiethen zage nad Dem erfen פiettel if fie gan: runb, benn nun fefte bet Mond Der Eonne gegentiber, uno zeigt uns feine ganse erleuditete sadfte. Tun nermen mir ibn 20 oll monb. Der 20ullmont nimat alle 2tbenbe ab, unb hat fidi nad fieben Zagen wieder in Den balben snond bere mandelt, $D_{2}$. mir reten fesme Edeibe nur bark ere 
204 Bon bet 3eitredinung und voms Pulenbet.

leuctitet, uns jwar bes Mrorgens um 6 ubr. Donn fao gen wir: $C \mathscr{B}$ ift an rúät ber झrond ber Solne wieber náber, und neht

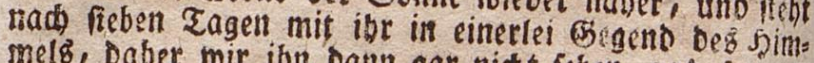
melb, Daber wir ihn bann gar nidt fekn, unb foiglid mieber $\Re$ e um ono baben.

Die Beit yon einem Reumonbe biß zum andern, mind

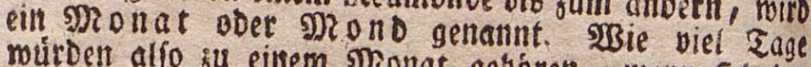
múrben alfo jus eirem Dronat gehoren, wetur fito ber

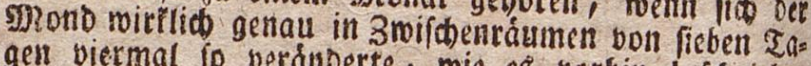
gent viermal to verdnberte, wie morben ift? - 2uber fo rechnen mir nidis, Delln wir múrben mebre als zwólf glonate im Jabre jáblen múf

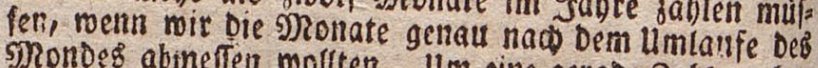

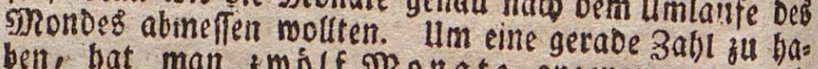
ben, hat man fwólf gonate angenommell, und jebem Monate einige Iage mebr gegeben, als et haben miffte, menn er genau nad den Umlaufe bes Nondeb abgemeffen merben follte. Der s) ond inein ift fur

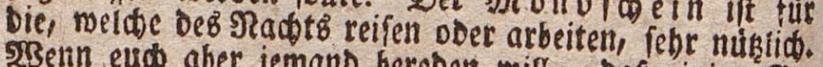

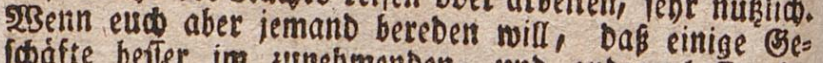
fodafte beffer in zunebmenden, unb anbere beffer im abmebmenden Mronde getatben, fo glaubet es nidbt.

2rbir lagen smat: Die Sonme geht auf unb gebt unter; aber eigentlio, geht unt berogt fic Die Sonne am scimmel niobt, fondern bleibt unberweglich an Dem: felben Drte fteben. श्रber unfere Erbe bewegt fiф um Die Eonne, uno sroar binmen einer Beit bon Drei Guss Dert finf uns feotig כagen utb fedbs Stunben, uno ins bem fie fich um die Sonne berum bewegt, Drebt fie fio saglei o brei bunbert funf uno fedzigmal um fad felbft,

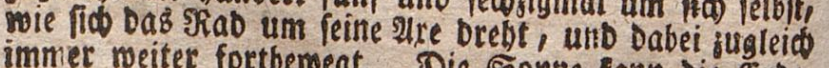
immer meiter fortberwegt. Die Sonne Fann bie Erben ba Diefe faft fo rund, toie cine Sugel ift, niobt auf cint mal $g$ an, befolinen, ober erleudfen, fonbern nur bies jelnige Jjálfte ber ranben eroe, melde ibr zugelebrt ift. Nimin eine Fugel, unb batte fie, menn os finfer iff, gegen ein fidt; nidst wabr, to nirb nur bie cime Şalf te ber Puger bon bem Ridte erleudotet, unb oie anbere

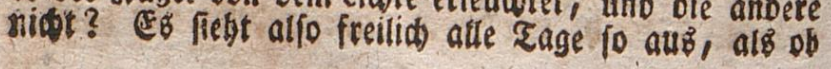


\$on Der 3eitvednung und vam Ralender. 205

sie Enne untergienge, meil wir bie allmáblige \$ene. gutig unferer Erde unmóglich mabenebmen fónnen, to

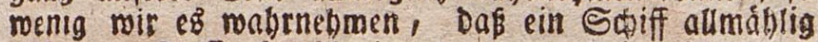
auf Dem Sluffe fortichnimmt, wenn mir sidtet etwa

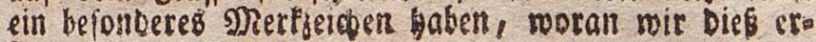
fenmen.

Dak man alfo bie 3eit in Iafre eintbeitte, und brei bunsert fúnf uno fediss Sage zu eittem Jabre rechnete, bießs fommit Daber, meil man alle Tage bie

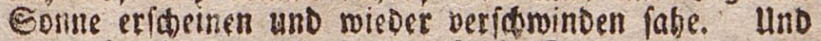
nun geft einmal rect 2ldt auf bie Sonne, to merdet

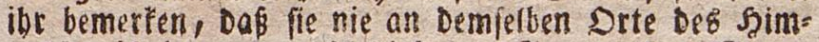

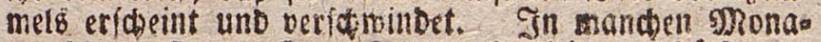
ten loseint fie nur furge 3eit, meil Diejenige Sálfte un=

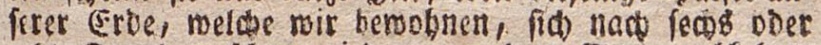
acot Etumben fonon mieder yon ber Eorne abfefirt;

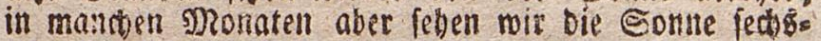
zelhn bis fichzebn Stunden am Simunel, roeil in Diejer aeit Diejenige Sadilfte unferer Cerbe, auf Der noir woh. nen, fici) fuir to lange 3eit ber Somne jukehrt. Es giebt alfo Monate im Jabre, no unfere ₹age furis

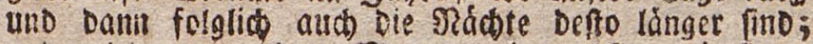
unb wiebcrum andere Mrotase, in melden wir furge Náçte und lange Tage haber. 3meimal im Jabre, námitión Den ein und jwanjigften gár uno Den orei und jwanzigfen September ift Der Eag bet ung getade eben fo lang, als bie radts, alfo beim 2infange Deb

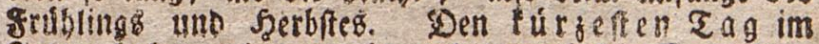
Jabre baben mir am ein und zmanzigfen December, und ben Iângften am ein uno smanigften sun!y.

Neun wiffen mir alle, mober bie 2 brestefelung

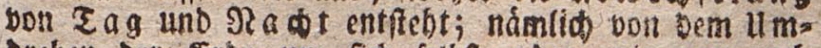
Dechen Der Erbe um fich felbft, ober, mis man auth fagt, um ibre 2tre. SBir miffen aud), Das am (ende

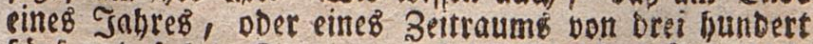

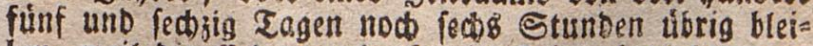
ben, meil die Eroe gerabe fo viel Beit gebrauct, um itgren fauf un bie sonne zu vollenden. Diefe uber = gabligen fects Etunben redonet man allemal im vier ten Jabre sufammen, unb madt cimen ₹ag barąuв, 
206 Son ber 3eifrednung und yom Salenber.

bert man ben Edoaltrag nennt, weil et bem jebefs. maligent vierten jabre eingef(t)altet mirb, unb swar im shonat Jsbruar, ber allo basn neun uno jmanjig Zage entbált. Daś Jabr, in meldbem biefe Cinidale tung geldiebe, beift ein S कoltiab?

sibenn bunbert Jabre verfolfen find, fo fogt mail ein Jabrbundest if ju ende. शुBir baben Das Enoe cimes Gabrhunberts erlebt, uno nennen das fu Enbe gegangene Jahithundert bas a dibebntel meil feit ber Esburt Jefu Ebrifti, DeB grb́bten

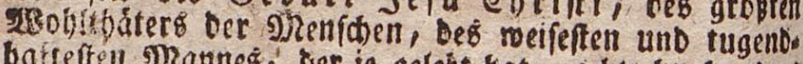
hafteften manneś, ber je gelebt bat, adbjebis bunbert Jabre verflofisen find.

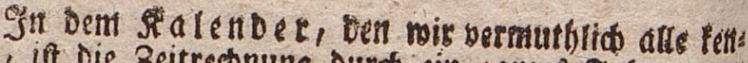
nen, ift Die Beitrectonung Durd ein ganges Jabs genaul

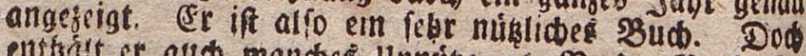

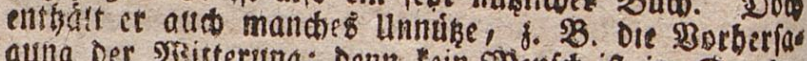
gung Der Fisitterung; benn tein solenich if in Etande atd mur einen $\mathfrak{z}_{a g}$, vicl meniger ein ganjes Jabr vots ber gu wiffen, mie bie wsitterung befdaffen fevn wer

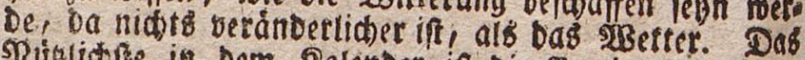

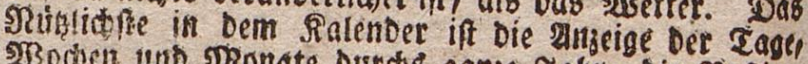
2Bowen und 5nonate Durds ganje Tebir, bie seftime mung ber geffrage, bie elbwect) fertungen oes Monbes, uni bie Injsige ber Eonten = und Diandfinfterniffe welaje in bem \&aufe beb Jabres eintreten.

Die SRamen ber wolf mo onate fino fege alt,

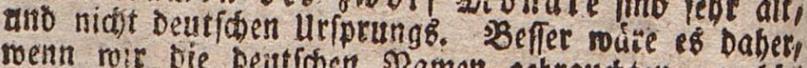
weenn wir bie beutfichen Namen gebraudien, welde fobon vor taufend Jabren ein Deurfiper Railet, Rarl Der Csobe, Den Monatert gab. Er nannte mit

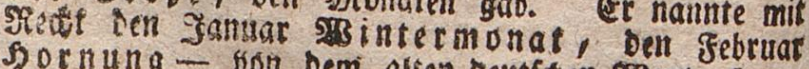
5o ornung - bon bem alien Deutfocen 280 orte 5 ori meldoes fioth bebeutet, weil es in Diefem 9 Drotiate ge.

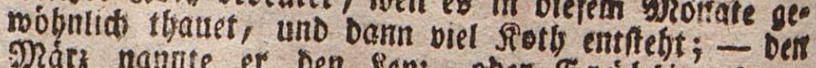
Márz nannte er ben feng = ober ze rubling g 8 moo nat; Den Stprit Dftermonat; Den Nai 230 nne monat, weil er sF3onze Doer. Freube bringt; Den Juni $\mathfrak{B}$ rachmonat, weit bant bie ssradader, $0 . \%$.

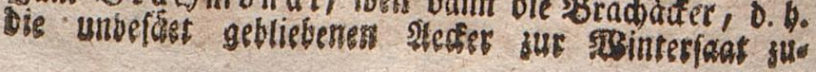




\section{Bon ber Zeitredinung un ven Salender. $20 \%$}

beteitet werben; - Den Juli Şeumonat; Den St: guft Erntemonat, Den September fferbft monat, ben Detober 23 einmonat, Den November 25 inters monat, uno Den December 厄) brift monat, meil Dab GeburtBfeft $\Im$ efu Elyrifi in oiefem Monate gefeiert mird, unb jwar, mie mir alle miffen, um SBeilgmacto ten. Diefes feft fált allemal auf Den fưnf uno zmanto zigften Derember.

Das Deterfeft fälit niobt immer auf benfelben Tag, uno nidht immer auf benfelben sRonat, fondern

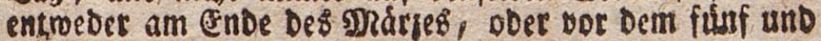
imanigften 2tpril.

Dab Pfingff feft fălt allemal fieben sBoden nad) Dftern eir.

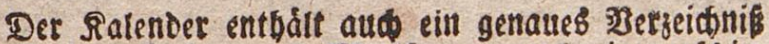

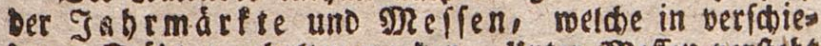
Denen Etábten gebalten werben. Inter s)effen berftht man grofe Jabrmartte, zu meliden fich fehr viele Seaufs leute aus fremben uno entfernten \&andern mit ibren शBaaren einfinden. In Deutídalano giebt es mebrere Drte, roo gReffen gebalten merden. Die befannteften fino bie Merfen, welite in Reipjig, in grante furt am Nain, Franffurt an Der DDer uno Braun fकre ig gebalten merben.

In unfern Ralentern frebt and ein stbidnitt von Eornen = unt Mondinfterniffen. Mit Dicien Sinfferniffen bat es folgende Beivanbniß. WBit miffen auś bem rorigen, baß́ bie Cirbe um bir Sonne, unb Det shonb um bie CrDe, aber auch zugieid mit bet

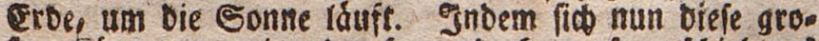
ben sorper um einander berumbreben, fo gefebiebt es zumeilen, Das einer Dem anbern Dab fidt Der Some regnimmt ober aufféngt, inbem er sroifcben itgn unb Die Gomne tritt. Da nun betbe, bie Erte unb Der Mromb, ibe fidt yon ber Sonne erbalten, und alfo finfter merben múffer, wenn isnen Dief́ fiabt entzogetr

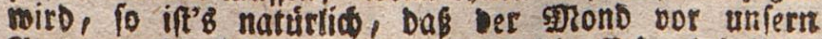

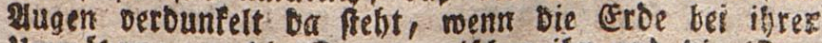

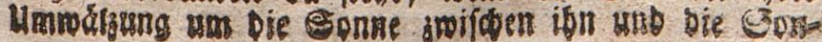




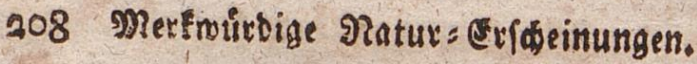

ne getreten ift, benn nun wirft bie cerbe iffent Swast

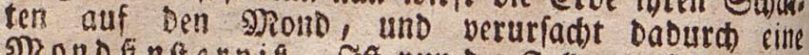
M on ofi nfterniß. Tft num ber fall umgefebrt, baß

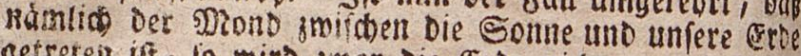
getreteit ifi, to wiro ziwar Die Erde nicht ganz Dabuld

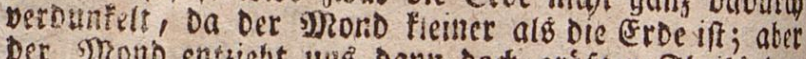
Der Mrono enzzieht unz Dann boch grósten Eheilis ben

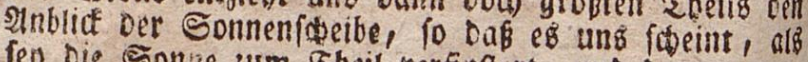
fer) Die Sonne fum Theil berfinftet, und Darum nells

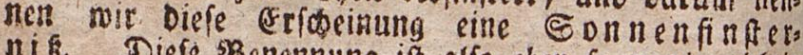
nik. Diefe Benemnung ift alfo eben fo menig ridtig unb paffend, als ber 2usbrud: Die Sonise geht auf uno gelte unter; Denn die Eonne wirb ja bei einer los genanntea Sonnenfinfrernis nist mirtliof oerfin.

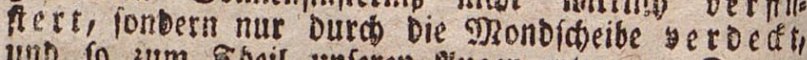
unb fo zum Ebeit unferen 2ugen entzogen. Da nu in biefen Balle ber siono ben gróbten Zleil Der Eon: meriftrablen auffángt, to mirb eg̉ bei Sonnenfinfter Miffen biënoeiter fo ounfer, bá Die Sterne am Sims

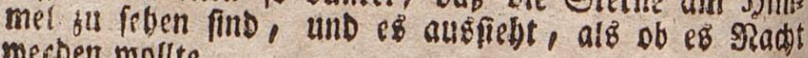
wecben wollte.

IX.

Merfwưtoige Natur= Erfatinungen.

Die Suft, meidie unfere Eroe bon allen Seiten ums giebt, jammt den Dúnften, welde fie enthditt, wirb die a: mofpháre ober Der \&uftereis geranm. bober man 3 . 23. anf boher Vergen, in biefem \&uft: freife binauffeigt, Deffo dunner wird bie \&uft, und befto reniger brúdit fie. Daber fommt eš, Daß bie \$Bergbemobner fácter uno fróblicher find, als die \$Bemobnes Der sbaler.

स्lle Duinfte unb Dámpfe, melde beftánbig von ver Groe und allen Dingen auf Des Cride auffeigen, 


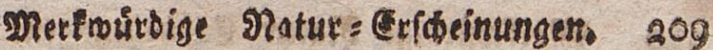

farmetir fic in Der etmofphidre, unb inbem fie fict gerbinben, entfebt Daraus Regen, Sdonee, SRebel,

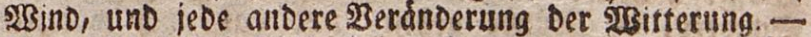

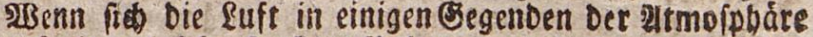
auf einmal regr ftart" ausbebnt, und Dagegen in andea

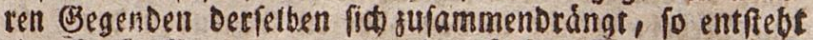
cine frarle sBerwegung unter den \&uftmallen, und biefe

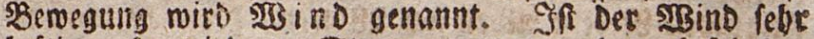
beftig, to nito er Sturm gemanni; Den beftigften Sturm nennt man einen Drtan. Ein Sturm zeta

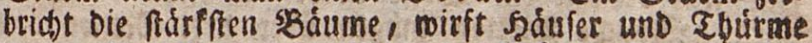

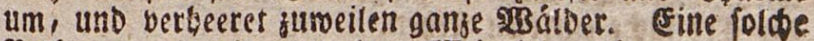

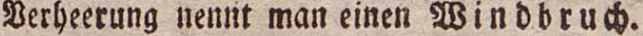

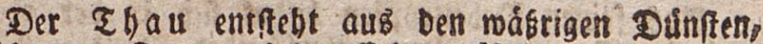
melche am Eage allö ber Ërbe auffeigen, Durch bie

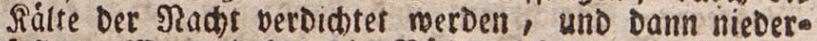
fallen. Nemn baher Die Nádote fehr roarm find, to fáut menig ober gar Pein Zhau. Befriert Der Ifou, to nennt man inn $\Re$ eif. 2Beenn eह getrift bat, fo find Die sáume uno stráfer fo meik, als ob fle gepubert

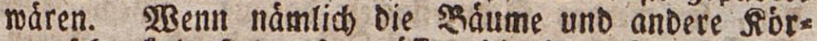
per felyr falt find, fo miaffen die Darauf gefaltenen Dúnite notgmendis al cisistbeildben merden. sgei gros Ber Rålte gefrieren logar bie suédinftungen, Die aus unferens פRunbe geben, unb DaB Şaat mirb Davon, mie mit einem Reife, úberzogen.

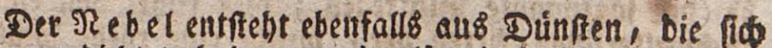
f(bon verbidstet baben, unb alfo eigentliक nictst mebr Dúnfte fint. Da Das şaffer, auś melidem er beffegt in febr feine Sheile zerthellt iff, fo formimint er in Der Ruft. SBentn fich biefe feinen Egeilen verbinden, lo bil= Den fie fegr feine Tropfen, melche albann nieberfallen. Dann fagt man: Det Re bel fállt. Edeint Die Sonne unnittelbar auf Den গebel, fo mirb bieß fobmims

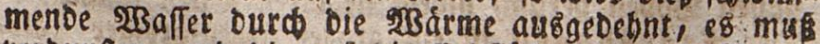
verbunften, unb bie auf bie Erofládbe gelagette Nebets Maffe mul lid beben. MRan fagt Daun: Der Nebef

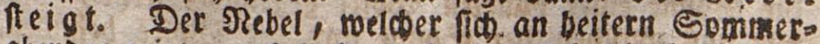
abenten feigt, entfet)t Daraus, Daß fich Die Ruft abge. túblt bat. glúfey melde niकt jugefroren limo, ratse 


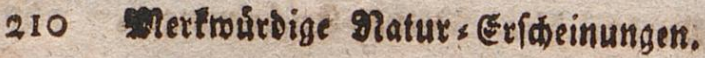

Gen im sisinter, menn es" ftart frierct, weil bie obert 2Bafferiobidten, wegen iber gréferen Didtigfeit unt

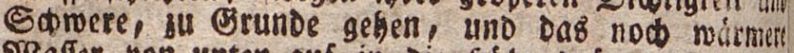
23affer von unten auf in bie Sjobe orangen, melaikes zroar verounftet, aber fogleid wieder tropfbar miro. -

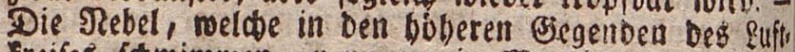

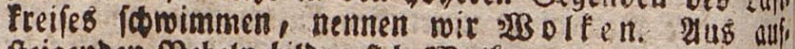

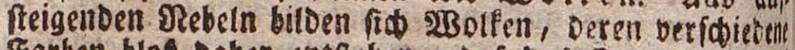
Zarben blof Daber entfében, Daß Dar Eonnentidit aut

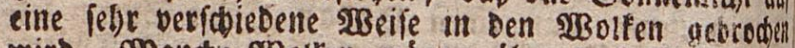
wiro. Mandie solfen móget úber Drei Meiten poi ber eith iláde entfernt fetben.

शนв feinen onebeltroyfen bilbet fíd in ber obera Suft ber 5 R egen. SMan untericheibet Etaubregen unt plakgregen, Etridbregen unb Ranbregen. So a gel il

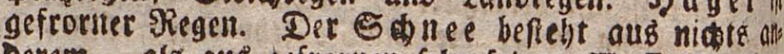
Derem, als aus gefrornen febr feitien sहaffertbrildery melche bei friller \&uft in Befralt fectigiadiger Eterth

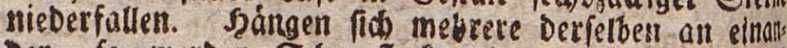
Der, to merben Squeefloden baraus. SBenn allie

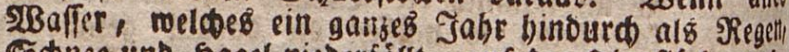

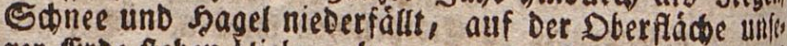
rer Erde fieben bliebe, obne zu verbunften, to mirte is ungefabr 30 3oll boch tiber Dem ganzen flacben Rante freben. - Zumeilen bat ber Regen eine róthicose fatts be (Der fogenannte slutregen); Dief rufget von einigeth Sdimetterling 8 = 2urten ber, welche eine totbe MRateril

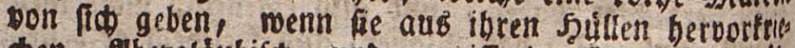

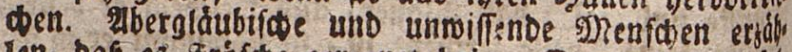

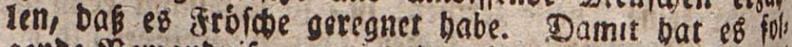
gende soemanoniß: wenin es nad langer Dúrre cinmal

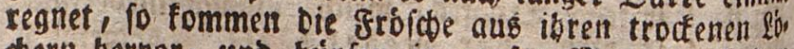
dern bervor, und biipfen in grober Menge auf Derl nafien, SBoden berum, mbem fie ibren Durft loiden, Der 3egenbogen if ein grober fiebenfarbiger seallo firfel, melcher in ben Miegentropfen fictbrbar mito, wetith Die Sonne ein.t Dunlelr 23olfe gegentiber fetht, unt mir ung smischen ber Sonne uno Der 2 offe befintert. Die Sonneniftrablen werben námlice in ben berabfal

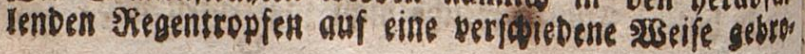


Mertwuำige शatur = Crfateinungen. 2II

dien. Die oberfte Farbe Des SRegenbogenz ift bis rotber und bie unterffe Die violette.

Es: giebt in ber Natur cinen Etoff, von befien 28 es ifhaffenbeit uns nur Dief betannt iff, baf er einem Riorper Die Siraft giebt, andere siórper bald enzlojie ben und bald abjutrofen, mobei fich faft immer ein litbtfunle ober eine flamme mit einem gróferen ober geringeren Bsráuffé zeigt, unb berienige Róbrper, Der Davon getroffen iff, eritouttert mird. Diefes Natur : Ereignig wiro Elefricitá genannt. Reibt man 3. 2B eine trine und trofite siabroffre mit einem mols. lenen gappen, uno bălt fie úber fleine Etudte Papier, to merden biefe sinigemal angezogen und zurude ges ftofen; rommt man Diejer siobre mit oem finger na= he, to frebt man im Dunfeln einen Sunter, bort ein siniffern, unb fúblt ein Stechen im \&inger. Shält man bie Sibbre, naduem fie lange uno fart gerieben iff,

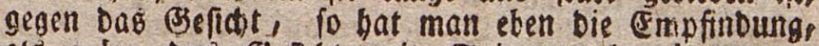
als wáre bas Esefiat mit Epinnenmebe überjogen. Eben dié̈ bemerft man, menn man sBernftein, SAbe: fel, Porzellán oder Siegellaf reibt. Man bat eine eigene Mgafóine erfunben, burw weide man bie Ele? tricitát fehe leidt etregen fann, Die Eleftrifirma

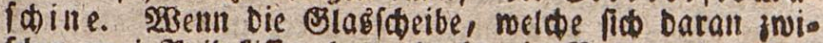

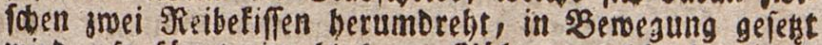
mird, to fangt eine blecbertie Sióbre, weldbe bamit in

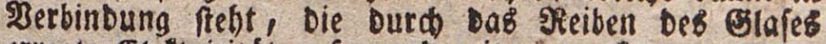
erregte Eleftricitát auf, uno mit einem trummen, an jebem Ende mit einem blanfen sinopfe beriebenen Drabte, Der einen gláfernen sanogriff bat, lect man Die Eefeltricitát aus Der SRóbre; Denn indem man fie mit bem Sinopfe Deß Drabteś berubrt, fábrt Der elefo trifose funte heraus. Man fann Diefen gunfen to frart maden, baf פRetalle Daburd in einem 2lugenblidée geidmolzen, und zoiere getbotet werben fónnen. Diefe eleftrifide Shaterie befintet fich nun aud in Den श्रolfen, melabe man Bemitterwolfen nennt, uno wenn fie in Befralt eines zadtigten Eeuerftrabls ausfábrt, 10 fagen mir: es blikt ober wetterlelidtet. Der sinad, welder genobntid auf ben sblis oper

$$
\text { D } 2
$$


Metterftrabl folgt, nennen mir ben Donner folat

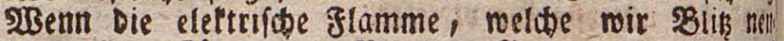

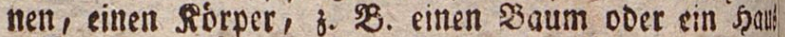
triff, fo serftort fie ibn, inberm fie ibn entweber is

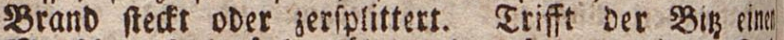
פRenfỏen, fo betáubi, lábmat oder tootef er iba. Dou

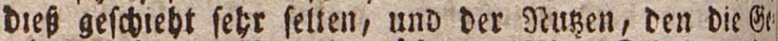
witter brimgen, ift meit groffer, alB Der Sdaben, on fie anrichten. Sie fúblen Die luft ab, uno remigen fn

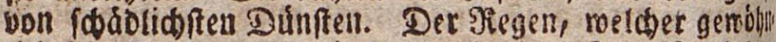
lich bie Esemitter. begleitet, macht bas rand frudthat

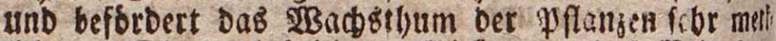

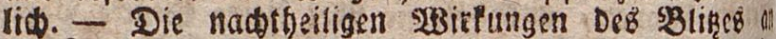
Gebảuben verbutet man am ficherfent Durd nohs ili getiolete SB lizable it er. Diś fino slicrme Stul gen, weldbe am obert ente mit einet rowarfen Entit berielen fend muffen. Um fie bor bem sroff zu bemob ren, ubergieht man die mit Sinn ober Sirnif, und on golbet bie Espise. Szerte, genall an einatrour befefight Siupferbledbe thun eben biefe Dienfte. Der Bzlikabieith

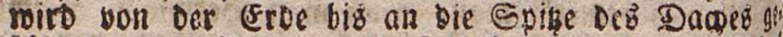
fubrt, unb bidt an Saure befeftigt, bow 10, id er bis in bie feudite croe hinabreidit. 2in cinem fol den Slizableiter fäbrt ole eleitrifo Materie, obmt Das Szaus zu befoddoigen, berunter in Aie Eroe. Eili

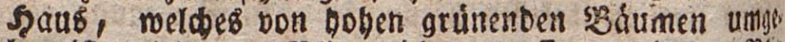
ben iff, soirb vom stike nidjt gettoffen, weil ber Blih Tios naco ben sáument binziebt. (Eben Darum mul

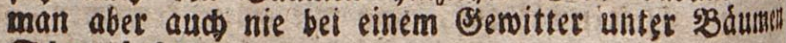
Sduk juњen.

She babt wobl fobon oft yon greliditern oor Sermifden gebort? Dieß find fleine Flammen obr Eidret, welde fito an fumpfigten Dertern feben laf fen, unb eine bupfende semegang baben. Sie ents fteben aus brembaren Dunften, welche fict) entzunben! uno fo lange leudten, alo fie bremtnen. SMan bat Diefe leuchtenden Dumfte Darum Jrtlictrer genannt! meir bizrweilen reirende, meldbe ifnen nachgiengen, Das burd bon ibrem $23 e g e a b$, und mebrentheils in sumt pfe sefúbrt nutoen. Eine ábntide ssemandtuiß bat 
es mit ben fogenanten Sternfornuppen unb Jeuerfugein. Trsenn bieie leuditenden Siorper aub Der obern Euft herabiobiebeth, fo fiebt eb gerabe fo aus, als of ein Stern vom stimmel fiele. Sie fotiefen mit Der grosten Beftomindigleit brennend fort, uno laffen fumeilen einen rorblionen Strid in ber \&uft furuid, ber fió) altmáblig verliert. Zumeilen bórt man ein Bejifíbe, momit fie fich beroegels, unb oft gerfpringen fie mit sinem sinall. The giot iff blendend bell.

zumellen fieht man an Der mitternádotliden ober Norb - Seite bes Jjimmels einen bellen Shein, aus meldem biomeilen Strablen berboríhiéren, uno ber

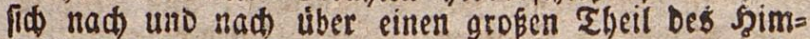
mel'b berbesitet. Det Scimmel fiebt banu suleşt gan rotb uno feurig aแB, uno gemábrt einen úberaub $100=$

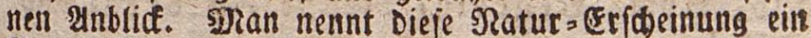
Rorolid,t, unb fie ift unftrcitig, fo wie bas 2 Better =

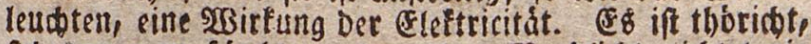
fid Devor 3 u furd)ten, Denn DaB̉ Rorblid)t ridbtet tie Edaden all, uno bat aud nidhts 930 feह zu bebeuten.

\section{X. \\ (5) 4 i}

SSir wiflen auB Dem vorigett, Daß berienige Zbeil unferse cerbe, in melwem wir mobnen, Europa beift; und Das Die 5 grofent Theile Der Erde roieder in Flei= nere Theile, ober in $\mathfrak{L} a$ noer uno Staaten einges theilt finb, melde auf Der Eandarte Durd Die veríbies denen farben bezeidnet werben. Die Ranoer, in melo be Eurropa setbeilt ift, baben folgende SRamen:

I) Deutfolanb, unfer Baterland, weldes mits ten in Europa liegt ( $\left.f_{0} \Xi_{0} .219.\right)$

2) Die Sobrocin ober Serverien, ein Pleines bergigtes Ranb, beflen Einmobner Sobmeiker ges

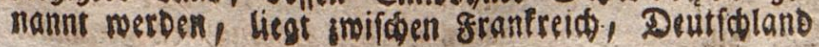




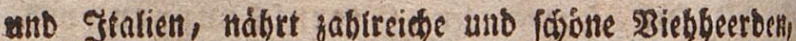

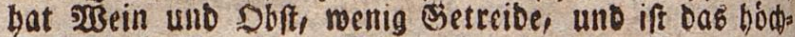
fite fant in Europa. SBit molien uns Die Ramen von zwei anfebnlidien Stabten merfen, meiche in biefem Sande liegell. Sie beiken: 3 úrich und sern.

3) It a lien, ein grofez, unb febe frudtibares \&anto, mercbes man baher ben Esarten von Europa genant bat. (EB if teid) an SReis, 2B?in, Del, Eitronen, Dos meramsen, Jeigen, 21 pelfinen, unb faboner Seite, und bat einen Heberflus ant 2 Beb. Sit Italien findet mant unter anbern die ftarten 'Buffeln, viele SRaultbiere unt

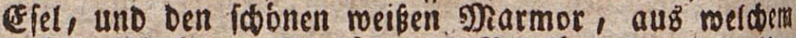
unfere Bildobauer Statuen und Bitbfaulen maden Utha

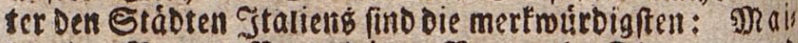
ranb, Rom, Beneoig, Seapel, glocen: utto (e) entua.

4) Eranfreid, ein groşes, frudtbares \&anto, meldees reid an 2Bein, Betreide, Del, Dbft und cBlet

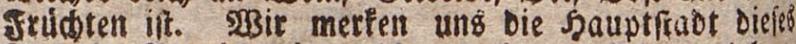
Ianbes: Paris, in melder megt aló fiebenmal bun Dertaufend Mrenfáen mobnen, unb bie Etábte: Toultotı sorbeaur, marfeille unb Strabburg. Die Einmobnet grantzeid है metben granzofen genannt.

5) Spanien, ebenfalls cin grober, unb zum Theil febr frudtbare\& \&ant, in meldiem bie feinfte Sdafo soolle, gute Seibe, fegr viel 2rsein(beronberš mallaga) und Del (Dlivenot), Baummolle, Budfer, sRanna smo Tabnd gewonnen mito. Die Spanifhen Mferde! Eiel uno Mraulefel fino vortrefflico. Die Szauptfatabt

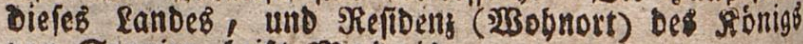
yon Spanien beift Madrid.

6) Portus al, ein fleineb, meiftentbeilgs frudtba

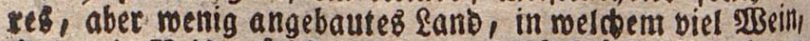

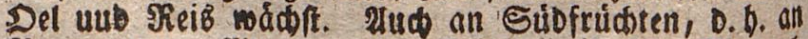
Dometanzen, Eitronen und geigen, if fein manget. ERan finbet in Portugal meit megr Efel uno Mraulefel, alb Pferbe. WBie man bei unz auf Pferben reiset, 10 reitet man in Dortugall auf Maulefetn. Die Sauptifad

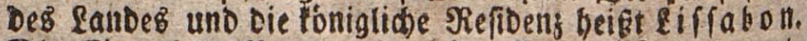
Die Eimothner Portugals nerben Portugiefen genannt. 
7) Solland ober bie Rieberlande ift cin leives moraftiges, bon vielen sianálen burdofonits

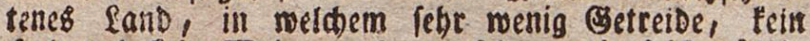

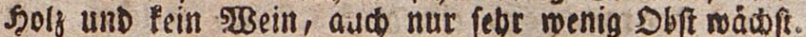
Die Szollander berfteben fich aber fehr gut auf Die Sieba

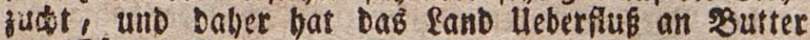
und ráfe. Da eb am smrere liegt, fo fehlt eb aud nidt an Eeefíden uno Seefals. Die grofite Etaet in biefem lande beift $4 \mathrm{mfter}$ am.

8) Brobbrittanien, Dber: englanb, Sdott: land uno Irland, beftelit aub jroei geoben Sinfelir. In Englano baut man bortreffliobe Berfte, uno oaber ift ardo Das engliface sBier Das befte. Die Biebjuct of in Diefem Rande febr bots getriebell, bzfonders Die

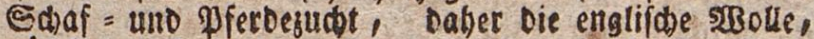
nádoft Der Epanifoben, bie befte ift, unb Die Englifoun Pferbe fur bie ldonften in europa gebalten werben. Das englifole feDer ift berúthmt. Sehr reid if Eng= lano an Steinfoblen, und Dab̉ beffe Binn if bab cangs lifobe. - Sdottland ift an erifen uno gifonen, bos fonders an Scáringen uno Stouffíden febr reid, bat trefflide Shafzudt, uno lleberfub an Eteinfobten. 2ud Irland bat treffliche 230 lle, Betreide uno gri fobe, befonbers Eadfe, in lleberfus. Der fionig von England wobnt in \&onDo ns, einet der grósten Etáte

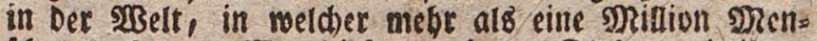
foben mobnen. $25 e r$ Diele ungebeare Stabt nad ibrer ganzen fánge burdonandert, bat einen $233 e g$ oon bei= nabe anbertbalb 5neiten zu maden. Sie elitbált 8000 grofe uno fleine Strafen, 34 S)Rarttpláke, uno noch 7 r andere Pláke, uno beinabe 500 Sirdsen und Rapellen. ßeftánbig tomment auf bem filuffe, an welobem fonton liegt, auf Der Ibemfe, Sdiffe aus allen Ibeilen Der erbe alt, unb man reduet, Dafí jábrlid mebr alo Ize00 Sdiffe aus = uno cinlaufen.

9) Dd dnemarf, ein Ileines ebenes Land, welotes

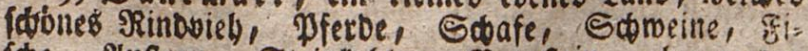
(íbe, Auftern, Steintolyen, Bernftein, aber weDer

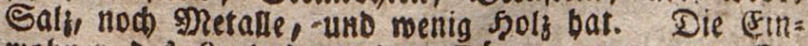
nofuer Des Eandé merben Dánen genant. Die 
Sgauptftabt Geift Sopengagen. Shiet nabut ber Sib gig von Dadnemart.

10) Normegen, ein grof́es \&anb, meldeb aut Dem fionige son Dánemarf gethort. E\$ ift boll hober und rauber SBerge, Deren Bipfel zum Eleit beftánoig mit Sdbitee bebedtt finb. Diefe פgerge entbalten Den भieidy shum Des \&andes: benn in ibrem Smnern findet fia's Sile ber, Siupfer und elifen in פRenge. 2deerbau und פiebs

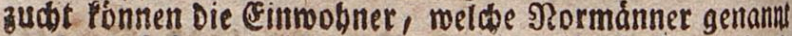
zoerben, faft gar niobt treiben, Deun ibr felfigtes sand bringt weder Betreibe nod) Erab bervor. Defto meht beídaftigen fie lith mit ber Sagb unb Sifherei. In Den

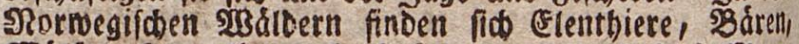
2rsolfe, Jeermeline und shajen genug, und Das Meeri an weldiem NRormegen liegt, veriorgt Die গ2ormánner mit facbien, Sadringen, Stodfifoben unb 2uffern fo reido

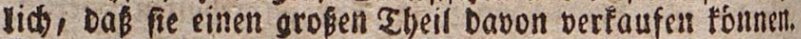

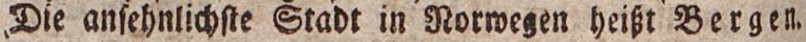

II) S中roeden, eines Der grofften lánber in Cu rolla, aber bennod einz Der drmften, Daber es aud nur wenig einmohner that. SRur an (Eifen ift Sdmmeben

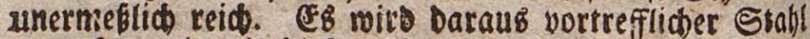

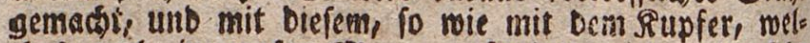
des aud in grofer glenge gefunden wiro, ein fehe

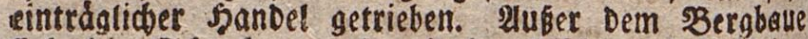
finb Die Sdaneben aud mit ber fiŕcberei befóáftigt. Sthr Eanto if yoll grofer firdoreider Seen, uno liegt solt einer Eeite am speere. Dab giennthier if it

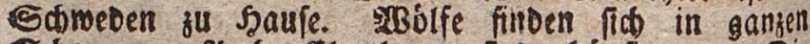
Sd)aaren. 2uá) Elentbitre fino băufig. - Die

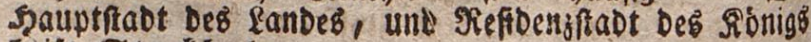
Geift St o dibolm.

12) $R u \in$ lano if $D a b$ gróbte Ranb in Curopar

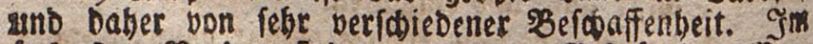
duferften slorben findet man nur Beftráude, sBes reh, Marienglab, Detzthiere in grofer Menge, gie

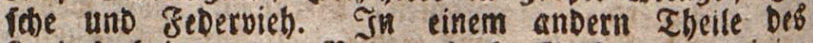
gantes bringt Der $\$$ BoDen Doch Bjerfte $4 m$ b einige

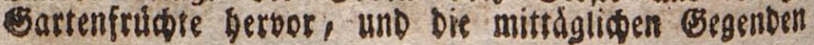


Daben Iderbals, Dbft unb gute Biebzudbt, wilde Pfer-

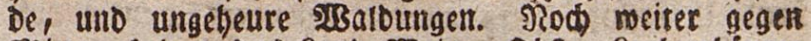
Mittag bringt Dab \&and $23 \mathrm{sein}$, Dbft, Lorbeerbáume und Setreide, morunter aut Der Reis ift, in Mnenge berbor. In biefen Begenden find Die Efel und die Res meete die gemóbntiden safthiere, die sBuffelocolen jie ben ben pfits, uno bie Pferbe meeben erlegt uttb gegef fen. Sziele sBewobner Diefes frudtbaren \&anoftrids 8 mif

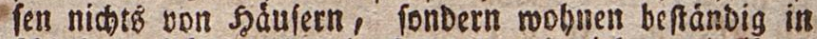
follediren Sgutten ober in Selten, und jieben mit ibren Sjeerden ats einer cegend in bie anbere. Biele fotlas gen in gelfen, şoblen oder Erbgutter ibre 2 Bsobnung. auf. Diefe sermobner Fiuglando beifen sataren.

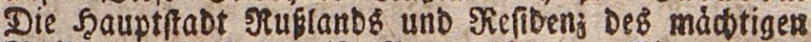
Sirfíchen Raifers beist petersburg. Eine anbere febl grofe Stadt diefes fandes beift No o s f a u.

13) Die Tưfei if ein febr frudtbareb unb mate

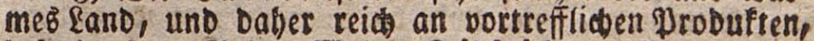

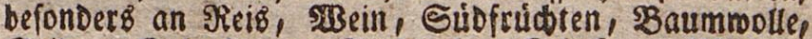
Seide, Sabadt; an Sinobieb, Sdafen, Pferben; Cleln uno Raulthieren; an Drarmor, Mlaun, Etwe: fel, Eifen und Salpeter. Dab tuirtifóe Barn ift be. rúbmt. 2uz Der Seide macten Die Túrten práøtige

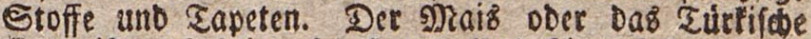
Sorn ift aud bei unB befonat. 2us Biegenfellen madt man in Det Surfei Den fóonften Sorouan uno Saffian. Die Szauptftabt beś Lanbeb beift R on fi a ntino pel.

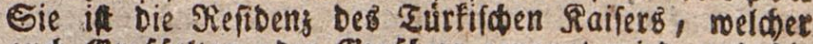
alid Sroffultan Doer Brobbert genannt mirb, uno bie grifite Stabt in Europa, aber nidt bie fóntete: Dent fie bat faft lauter bolserne ş̧นfer, uno frumme fobmuhio ge Strafen.

14) Ungar\# if zum Theil ein febr gebirgiges unb malbiges Sand, fum Sheil aber aud auskerft fruchtbar, uno befonbers febr reid an Esolb uno 23 ein, in cinigen Begenben aud an Betreibe, MRaib, FRiह̋, Saftan, Sonig, Mrandelt, Dbft, fibonem Rinoviebe uno f men Pferben, an Steit = uno Duettalis, uno faft allen mirreralien. In Dem Eanbe einiger llngarifoen fluffe retben Botoforner gefunder. Aud bier bedient man 
fich bes 25tiffers beim शderbaute. 2In Siften bat bas

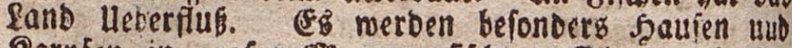
Rarplet in grofer spenge gefifot. Die vornebmitent

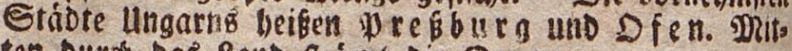
ten burd Dab fant ftromt Die Donati.

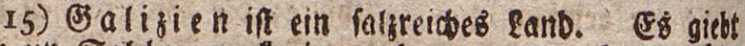
bier els Salbergmetle, in reithem man ungeheure siobs

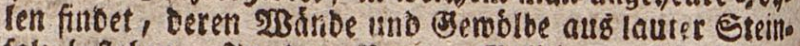

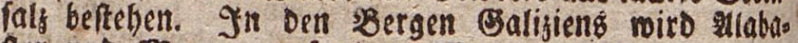

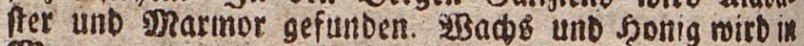

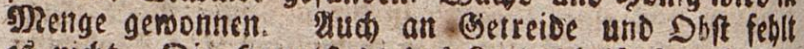
ez nich). Die Satiptfant bes \&andeg beifft \& c mberg.

16) Preuben if gróbtentbeils ein ebenes und fructbaces Eant, voll fofoner $2 B i e f e n$ unb $23 i c h$ weibert.

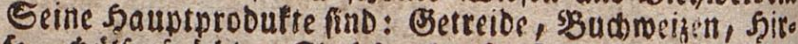

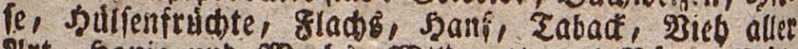

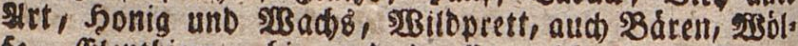
fe, Elentbiere, bie und ba eluerodín und sBiber;

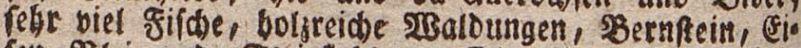
fent Blei uno Steintoblen. Die $\mathbb{B}_{\text {eid }}$ fel ftrómt burd Das Eanb. In einem gheile Dreuseng giebt ef vortrefflide Pferbe uno Daffen. Die gróften etabte

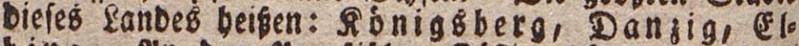

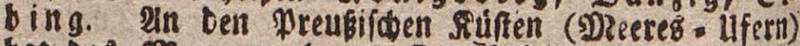
bat Das meer ungebeure EanDfelder uno Eanoberge (Duinen) aufgetbutrmt, meldbe man físon jum ₹heil urbar gemadt, 0 . 6. bebaut uno mit scumen beflants

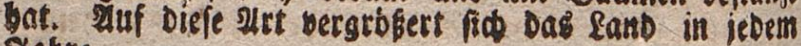
Sabre.

17) $\$ 30$ b men, ein geburgiges unb malbigeb, abee Doch in gansen febr frubtbares Eand. $24 m$ reidper ift eB an Eetreide, J2onis, 2BadbB, Dbft, EDerfteinen, Sols, Eifell uno Binir. Die Boobmen fint febr betriabs fam, unb gefoldtt, befonbers im Seinweben unb Spiken. rloppeln, in ber Berfertigung Des Elafes , im sergs baus uns in ber Druffi. Die Elbe Durchifrónt Dieß Sanb. Das sriefengebirge ttennt es bon Dem benadbar= ten Sdlefien. Die Sauptifabt sobbmens beift $\mathfrak{D} r a \mathrm{~g}$. 18) gndbren if auf allen Eeiten yon Bebtirgen

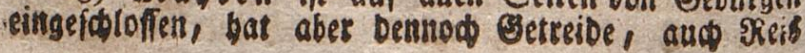




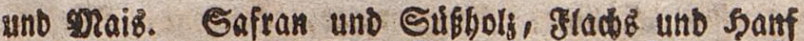
soirt in Menge gemonnen. Die fablteiden. \$Eergwerte

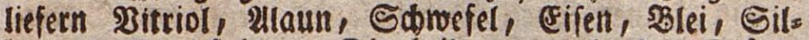
ber unb Steinfohlen. Die beibell vornebmften Stábte beifen 3 r únn und DUtmuts.

19) Sकlefien, ein zum Ebeil bergigteb fanb, bat febr fleifige und gcidiate Cimpohner. D:e Ed les

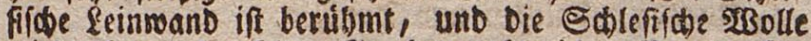
wirb theuer bezablt. In ben zablreiden sergmetfen Des fandeb miro Silber, Siupfer, Eifen und slei ges funben; Steintoblen finb in ber grbfeten Mrenge vorigan= ben. Die Doer Durdiftemt Dieß \&ano. 2yn ibrem

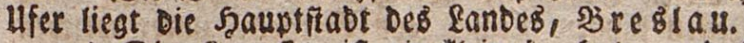

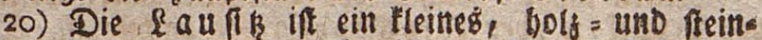

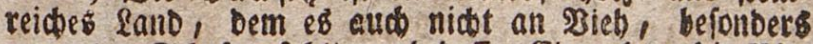
an guten Shafen feglt, uno beffen Einmognter bie SBies nengudt, Den Dbft = uno Cartenbau, uno zum Ibeil

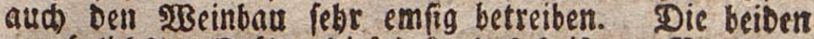

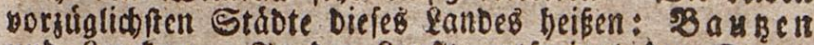
und \&udfau. In ber faufich entipringt ber Eprees Siub, an meldoem Bertin tiegt.

Die eánoer sobmen, gnduren, Gqlefien

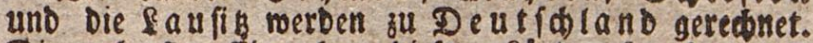
Die mebreften Einwobner biefer 4 länber fpreden aud bie beutíce Eprade als ibre ginutterfprade. Dod) bas ben bie szofsmen eine eigene Eptade, aúfer nelder fie aber aud uod bie beutide ipredert.

\section{XI.

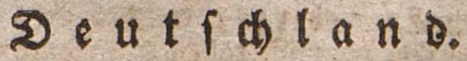

Deutichlanb if unter berr 20 europdifoben Làmbern, son melasen wir eben etwab geléen yaben, einz ber

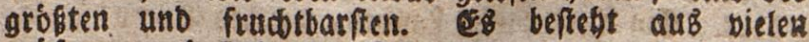
gróberen uno fleineren Staaten, unter beren $B_{e}$

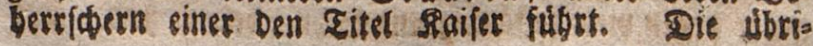



gen fint Sibnige, Grobfergoge, Jerzoge, fritten

Ein ficinet ₹heil Deutrílands liegt am Meere, námtió an Der Dftee unt Sorofee, uno an bem

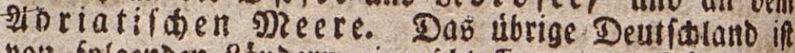

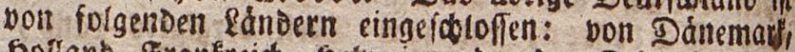

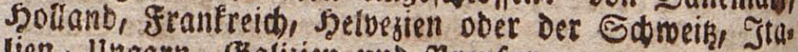
lien, Ungarn, Baligien unb preuken.

Deutfalanb entbált viele serge und esebirge. Einige barunter fint io bod, bak ibre sipfel faft Dab ganje Sabe binburds mit edaree bebedt find. Cing

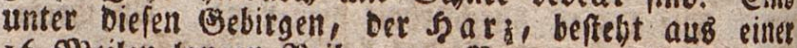
16 Micilen langen Feiben won SBergen, unter rolden Der B todell oder 23 lodés berg ber botifte iff. Det Ed)warzmald, Der Efuringer-20BalD, Dab ergabitge und bas fibtelgebirge fint ebells falls lebr grobe sBergletten.

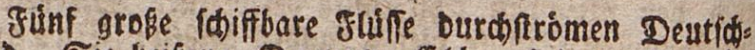
yano. Sie beifen: Donau, Elbe, Doer, SRbeil und $283 \mathrm{e}$ er. 2ufuerdem giebt $\mathrm{eB}$ in Deutidland now fehe siel tleinere flúffe, Die aber aud fobiffbar fint, uno eitre grofe menge Seen, unter meltben Der SBD:

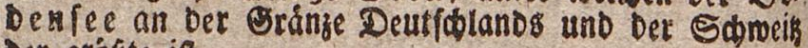
Der gróste iff.

SRur febr fleine Eanbftridte in Deutidland find utte angebaut, unb biefe beifen Sacident. Die meifent Deutínell Lánber finb nobl alsgebaut und fructboar, und Daber (ind Die \$robufte Deutichlanto úberaus

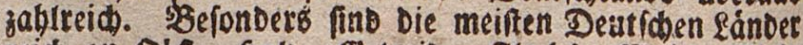

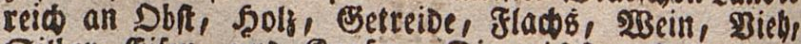
Eilber, Eifen, uno Supfer. Die reidften Silberbergs merle find im Erjgebirge, weldes eben von feinem Reicthum an Ert ober MRetallen ben Namen bat, unb

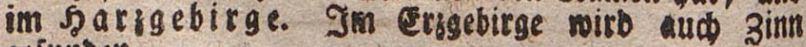
gefunden.

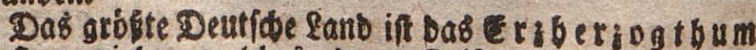
Defterreid, meldoes Dena Railer von Defterreí gebort. Ein Theil Diefes Eanbes, welides (E) D rol beift, if voll boher soerge, bie biö in bie $230 l f e n$ reichen und unter Deren viele befténbig mit Squre bedeofr find. 2uf 
diefen Bergen wohnen Bemien, (wilbe, unjern Biegen

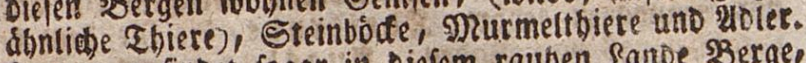
Sa, man findet fogar in tiefem rauben fande $\$ 3$ ergen meiche aus lauter Eis befteben. Sie werden es le t: fi er genannt. Die Svroler manbern in gans Deutfons lano alo Sganbelsleute umber. - Das Ersberjogtbum Defterreic bat fehr reid)e Sergwerfe, melde bortreffli: des Eifen und Zuedfiber liefern. - Die Sauptitadt Des Eandes beift SBBien. Eis liegt an ber Donau, ift Die Fefibenz Deß Seaiferz von Deftercei uno volfreichte Etadt Deutítiand:

Dab́ Sínigrei an Der Donau, unt if ein frudtbares Eand. (5) gethort bem Rionige von Pfalz=5gaiern. Die Sauptfadt

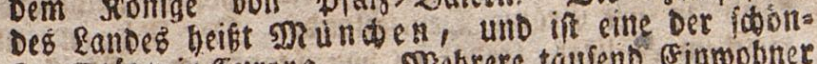
ften Stáote in Europa. - Mebrere taufeno Einmobnet

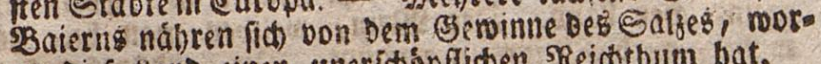

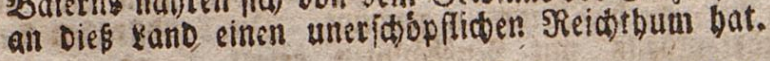

Franfen Doer Der gránfifde Reis liegt in

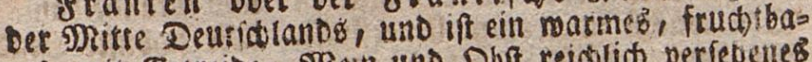

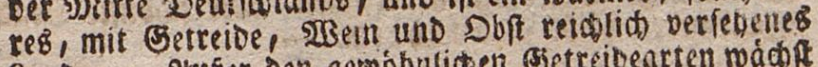

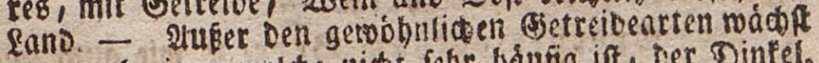
bier now eine, melobe nicht febr bánig ift, ber Dintel.

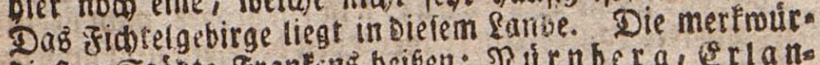

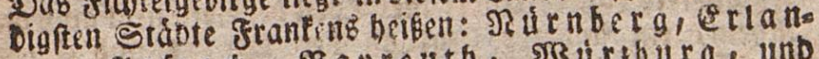
gen, थifpat, SBapreuth, 2Butrburg, unb

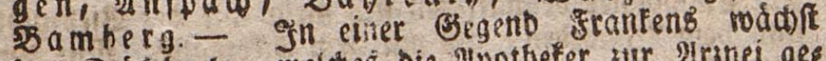

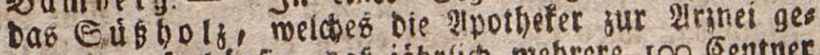
braumen, fo báufig, baf jábrriç mebrete 100 Eentries babon vertauft merden.

(5) maben Doer ber Sdmábifate Rreis ift ein auBerordentlich frudjbares uno marmes lanb. $D a=$ ber bringt CB Setreibe, W3e!n und Doft im Leberfuffe ber vor Dod giebt es aud boe, unirudbibare Segenben in Somaben, Denn zroei bohe une raube Bebirge, Det

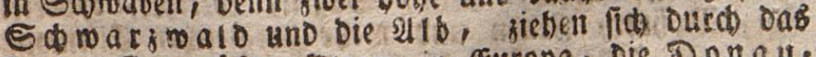
lano. Der grófte Etrom in Europa, Die Donau, bat bier feitten uriprung Die mertmurbigften Sdmas. bifhen Stádte find lolgende: Stutt gia LD, Die SRefis 


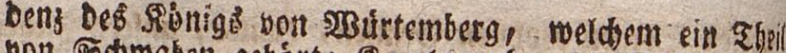

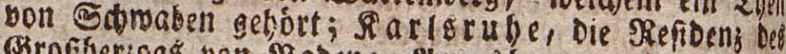

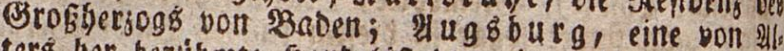

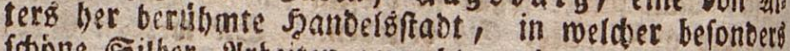

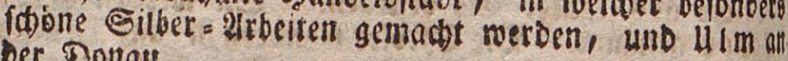
ber Dothats.

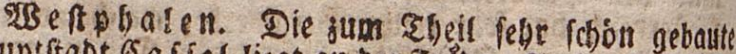
Sauptftabt E a fiel liegt an ber \&ulba. In ber গiadbars fobaft Diefes Eanbeb liegt granlf furt am Miain

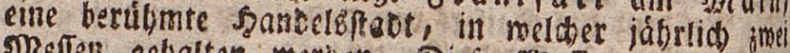
Meflen gehalten werben. Diefe Sheffen werden vou

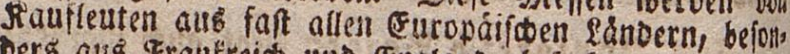
Ders aus zrantreich und England, beiucht.

Die $\mathfrak{P f a l}_{3}$ am $\Re$ bein in ein fobones frubtbates sano all Der Braimze Deuriolands. Der Sebe in, all melchem 68 liegr, trennt efs von grankreid. Eह if

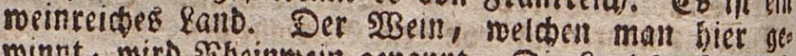
soinnt, miro Sibeinwein genannt. Die Eandfrafen fint grobtentbeils mit Stufbaumen befebt, und in Dell Theinbergen fieben datbte Raftanien = und Mrandeibáume. Doft widoft in grofem Hiberfuffe. Die fajboffe Stadt Des \&andeś beift פrannbeim. -

Das Brofberjogthum Berg, fo wie aut fum

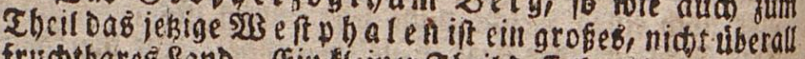

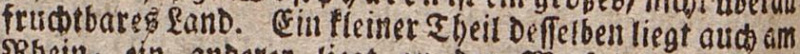
SRbein, ein anberer liegt an Der $2 B$ fer und E $m$ ?

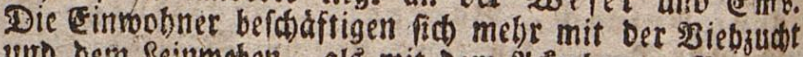

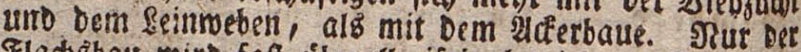
gladisbau mird faft tiberall eifrig betrieben. Die $\mathfrak{B} i t$ refel er Reinmant wird thetier bejablt. In Dera gebirgis

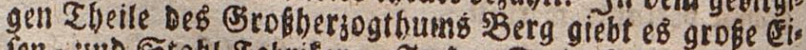
ren = tund Stable Sabrifen. In Der Stadot Jferion mets

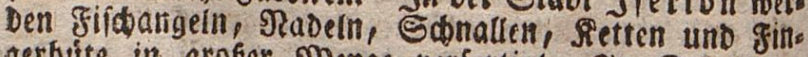
gerbute in grofer Nenge verfsttigt. In SDbelm

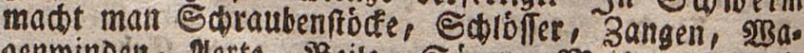

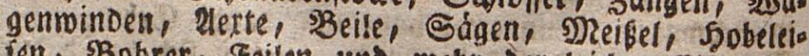
ren, SBobrer, feilen uno mebr Dergleidenen Eifenmano ren. 3u Den mertmúrbigften Sergichen Stabten ges boren folgenbe: 2Befef, Mitnfter, bie sanbelke

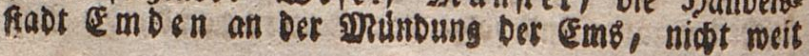




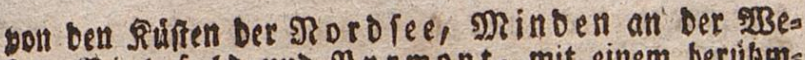
fer, sielefelo uno Purmont, mit einem berúhm= ten Sefundbrumen.

Nieberfadten ober ber शieberfádfifde Steis. Die Elbe turdffiémt Biefes \&and, in twet. dem es grofe unbebaute Stridte oder Sanibell giebt. Ein Theil von Mieberfacten if gebirgig: er entbált bas hobe, raube, aber filberreide Sebirge, welches Der Szarz genant rirb. (Einige Begenbelt Niedet. fabieng baben leberfuff an çetrelde, unt in ben láns

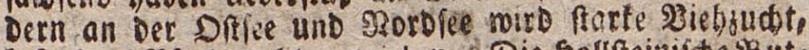

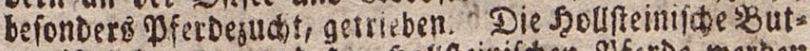

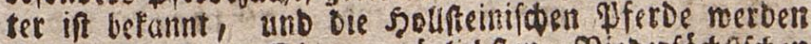

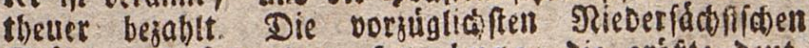
Etábte finto forgende: Şamburg, Die gróste deuts

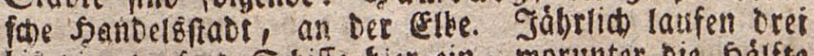
biz vier taufeno Eabiffe bier ein, morunter bie Sálffe Eeeidifife fimb In Dem geráumigen szafen Dicier Stant liegen befánbig Ediffe aus̉ Englanto, Dảnemart, Eđmeden, Rápland, 2merifa und 2ffen Dor 2nter, belasen mit Den yrobuffent aller ertotheile, befonders

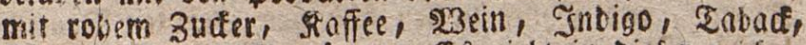
5Baummolle und Bemúrien. (CB giebt in Diefer reiden Etabt methr als 300 zuderfiebereien, und fo biel Rat=

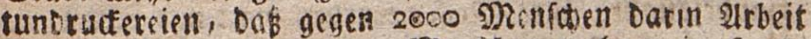
finden. Methe als 100,000 \$reniden mobnen in STam= burg, uno bie meifen ndixen fid vom Shantel. -

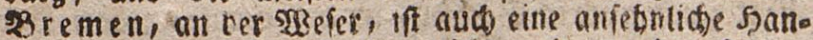
Beliftabt. - Eube de liegt nidot meit bon Der Dpfee, une treibt ebenfalls anfebntiden Şandel. - Sa annoder

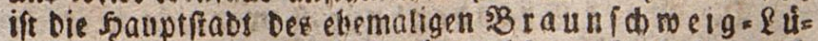
neburgf(den ober stannoberiden fanbeb, uno liegt an Der \&eine. - SBraunfoh weig war fonft Die Sauptfadt eineb Serzogtbum है sB ra un (d) weig, und bat zroei betúbmte Mrefen. - Mr a ge Deburg ift eine febr ftarte feftung an Der elbe, und eine bebeutende sanbelgftabt. - Salberitadt mat

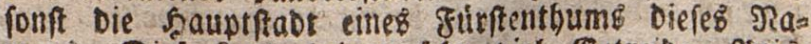
mens. Dief fant bringt fehs biel Getreibe, 2tivig,

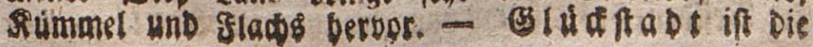


Sgauptiabt bes serjogth um \& Sglifecin, eines Dannichen Eander.

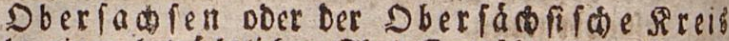

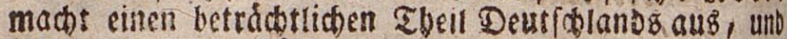
befteht aus crei sándern, melche Sachlest, sBralts Denburg und g o mmern beifert. In Dem mittagglis wen Thenle siefes Rreifes liegt bab E $\mathrm{r}$ z ge birge, aus

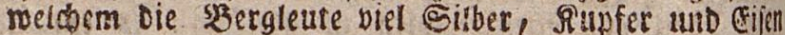
herborbolen. Der novertide Sheil hat enen niebrigeth

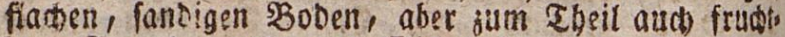

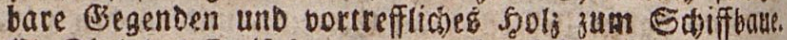

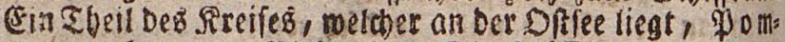

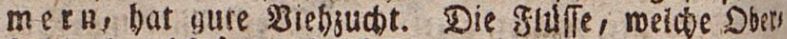
facten Duróftrómen, find folgente: Die ef l be, Die Doen Die , ay el, Die Eaqle, Die 23 arthe und die Epre?

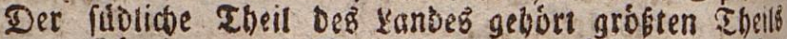
Dem Rónige von Sachlell, der nórdliche gebót beinabe gan; bems fiónige oo in preusen, ber aud Siturf ut fit von sprantenturg height. - Sa aden if

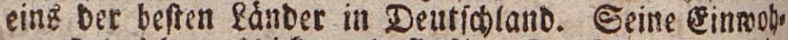

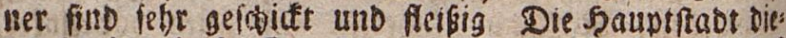

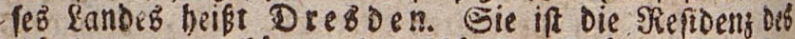

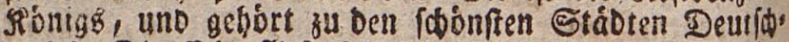
lands. Die Ertbe fliest Durch Diefe Stabt. Zutch \& eips

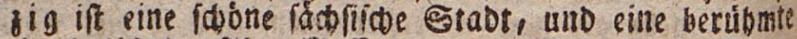

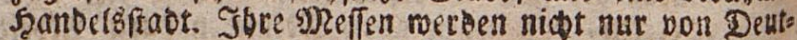

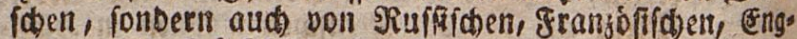

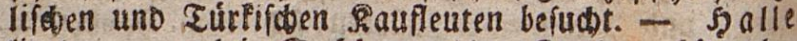
liegt stwar aud in Sadien, an Der Eaale gebort abet

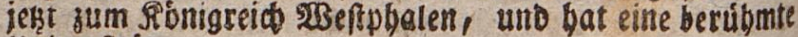
univerfitát.

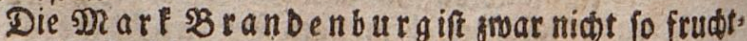
bar, als Sacjien, aber febe forgfalrin angebaut. Sil miro voll ber ober, Elibe, Jabel, Spree und $23 a r i b e$

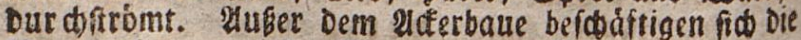

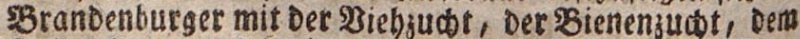
Eeibenbaue, und in einigen Eegenben aud mit bem s\$eins baue. In ben Stábten giebt es anfebnliche शु a $n$ u falo tu $\mathrm{r}$ en unb $\mathrm{g}$ abr ifen, befonder' SBaummollen $=580 \%$ $\mathrm{ken}=$ und Eeibeufabriten. Die Şauptrfabt Des \&anbeš und

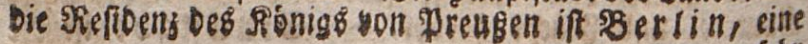


Febs grobe und gróptentheits fóbin geboute Stabt,

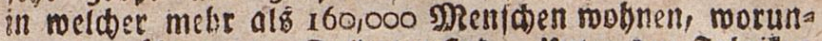
ter ungefábr 25000 Soldaten fino. Lnter Den gabriter

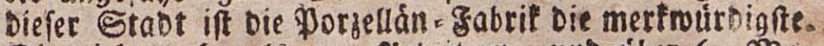
Sie giebt mebr alB 300 21rheitern, unb úber 60 Maa fern sefđáftigung. In ener anbern Fabrif fint einigé

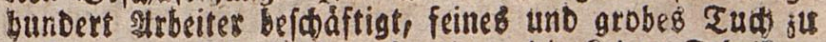
yerfertigen. Die Zuferm: Deteien, bie \&eber Jabritem, Die SBrantereinbrentrereien, oie Seiben = und Faummols Ien = Fabrifen uns die squd)brkafereien geben mefreren

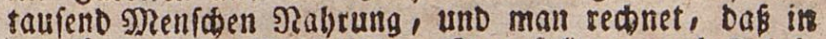
Den fámmtliden serlinifoben manufäturen uno gabria

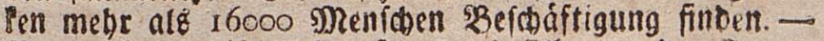
SBerlin liegt jmifden Oer Doer und Elbe, an Der Epree, rinem máfigen sluffe, Der aber $D D C$ grobe belatene Siábne trágt, uno fít jmei sneilen von şertin mit Dee Shavel veceinigt. Da Die shavel in sie Elbe fálit, fo

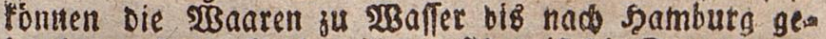
bract merben. Stut mit Der Doer ift Die Spree ourd einen Sianal verbunden, unb Die $523 a a r e n$ *ónnen Dahere auf Der Epree und Doer bió in Die Dffee gebtadt wet: Dell. MotBDam, eine láne Etatt mit prádtigert Swibfern und Euftgárten, liegt an Der Jgabel, vore f́tónen 3 ergen ungeben, unter melden viele sBeinberge inb. - Frantfurt an ber Dber, eine Sandelg. ftad, in roelder meffen gebalten merden, liegt in eitner

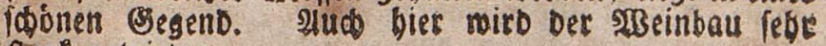
frarl getrieben.

Pommer it if ungleió frudtbares, alo bie Marte Szranbenburg, und bat Daber lleverflús an EsetteiDe. Die Ddfen, Ruthe, Pferbe, Shafe unb Eqweine finb in biefent fande vorbliglion grop und ftart. Die Jlúfe Ponmerns find fímreich. Die DDer fróme

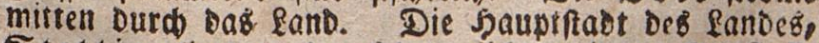
Stet tin, liegt an Der Doer, nidt weit vom 2lusfluffe

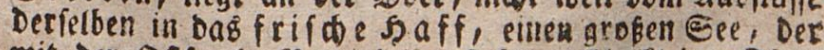
mit Der Diffee in Berbindung febt. - Ein fleiner Sheil bon \$ommern gebórt Dem Ronig vori SdmeDen, unb heift baber $S d$ webif $\phi=$ Dommern. Die Szaupte ftabt Deflelben beift Stralfund. 


\section{XII.}

30n Den פiecteten und gुflichten Der Iths tertbanen in wobleingeriojteten Staaten.

Rimber fimb, fo lange fie in bem şaufe ibrer eftettl leben, Diefen Geborfam fobulDig, D. b. fie Dirffen nidt thun, was ibnen gut bunft, ober in Den Simn formt fonderm lie múfifth tbun, was' ithre Eltern mollen, allo

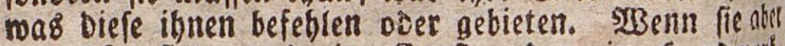
etrodefen fimo, uno eime Siunt, oder ein fanbmert?

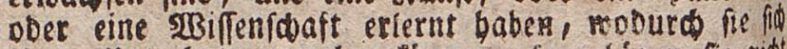
ibren Unterbalt erwerben fónne日, to gethóren fie siobt

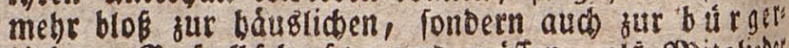
li

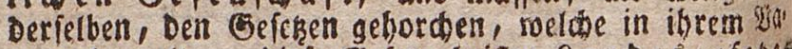
terlanbe getten; Diefe હeferse heifen \&and es gefeles

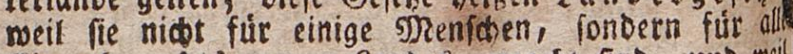
(Einmobner des ganten \&anbe\& gemadis find, und mell fer yon ibnen allen befolgt merben follen, Damit Dorb

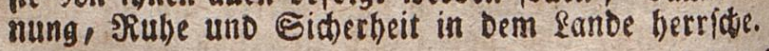

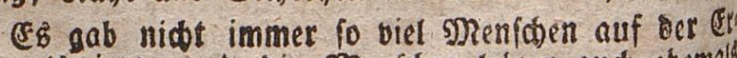
De, als jekt, unt bie menicten lebren aut ebemalls niobr in Etabten, Dórfern und eanbern gefellifiaftitio bei einander, fondern molnten jerftreut, in foblectsten

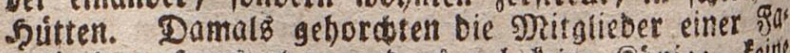
milie bem Şausbater, uno $\mathrm{e}^{\hat{f}} \mathrm{gab}$ feine Rónige, feint Jürften uno Dbrigfeiten unter Den Menfohen: Feinet war mekr als. Der anbere, Denn alle bauten bas gell

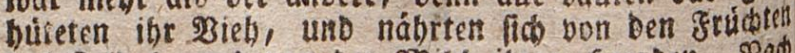

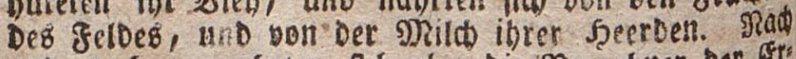
und na申 vermegeten fic aber Die şerwobner ber Et 
be, unb nun mufien fie nothwendig nobler bei cinathe Der wohnen. Da es nun yon leber gute und bófe Meen. fiten gab, fo entfano febr balo Erreit unter ibmen, als fie náber zufammen mobuten. $533 e n n$ Dann einec fíd nárter fúblte, oder mebr gerfteno batie, alb fein SRatbbar , fo nabm er Diefem mit Gerwalt fein Eigent

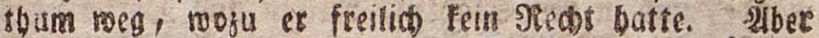
man nannte bieß Dernoch Dar siechr Des Stá rffee ren, Dber aud, weil eb́ Dabei nuf bie Etárte Der

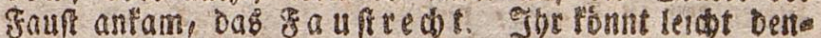
Fen, lieben Pinder, Daß die Sanachen, Die Sanftmetbigen, Die griedfertigen, und bejonders bie sweiber uno Simber febte ungltưfich maren, fo lange biefes fo gee nannte Reát galt; Denn feines woor Damals feines Eis getribumb und felbft fesmes febens fidber, Feiner fonnte in Fithe und friede geniegen, wab er fid mit פRibe. erworbett batte ; Etreit und Rrieg nabm fein EnDBs roeil liber Beleibigte fico, to baib er lonate, an reinem

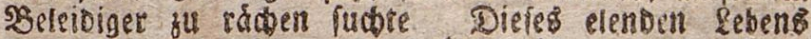

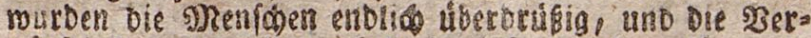
nunftigfen unter ibnen beiprbers fonsen barauf, wie Diefam unfug abrubelfen fenn mócter De famen fie

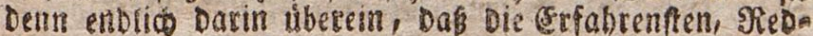
lid)iften uno sBbeifeften untes ibnen aušmaden follten, was jeber fu thun and fu laffen habe, unb alle filtige

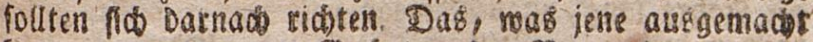
batten, nannte man Beferp oder gzeroronungen. Stun wurbe Der Zuftend Der Meníden febr glúctlid ges

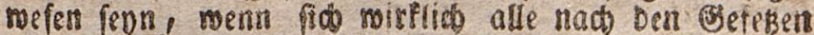
getioutet bátten; allein Die llnberfändigen, Die 3ornigen

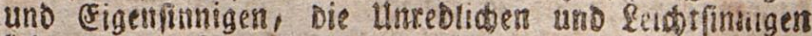
tehrten fich oftmats niaist an Die (sefere, fonsern thas

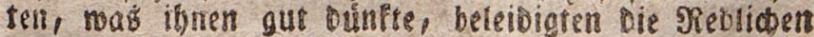

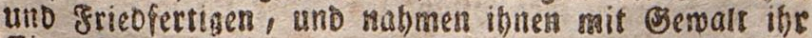
Eigenthutur. Da murben Diefé mit einanter einig, ge: miffe Perfonen unter fith zu wáblen, melte darauf ange

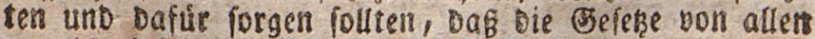
treulich befolgt weitben. Diefe Serfonen nannte man Jufammen genommen oie Db ig f cit, und alle úbrige Eimmobner bes fandes murden ll n te $\mathrm{r}$ i $\mathrm{b}$ a nen genannt, well fie jenten untertban, D. b. geboriam fepn folters.

$$
\text { क्र } 3
$$




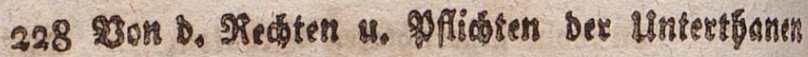

Eben fo, mie Die Rebrer in Des S\$ule, Die celtern in ibrer Familie, Die sluffeber in siner Fabti?, unb bie

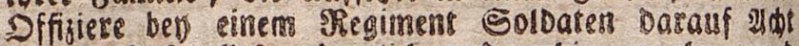
haben, DQF afles ordenrtion und rubig zugehe, und Feiner ben andern beleidige ober beranbe: Pben fo has

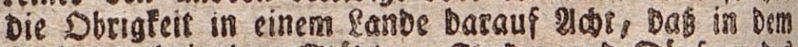

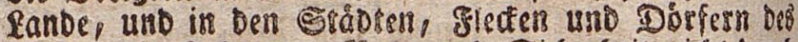

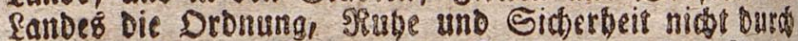

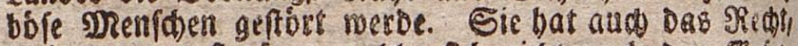

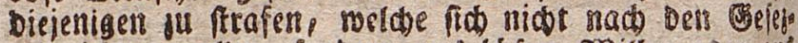
jell ridgten mollen, fonberll aus bófem $23 i l l e n$ oder aul geidtfinn, llntube und llnorbnung antident?

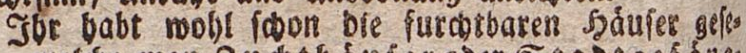

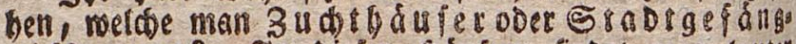
niffe nenmt? In biejen sadufern finder man tauter

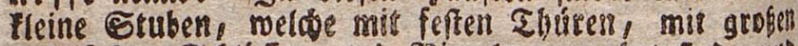
uno feften Edblóflern und SRiegeln bermabrt find, unlo

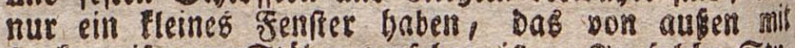
ftarten eifernen Etáben berfehen ift. In foldos stus

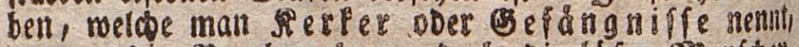

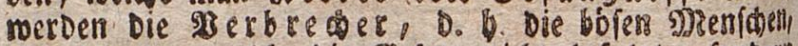
eingefpertst, meide Die Esereke sicht befolgt, fonbern

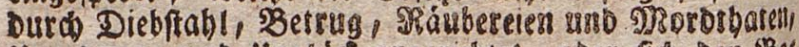

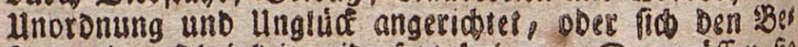
feblen Der Dbrigtrit roiberferst babert. Da múffen fir oft Jabre lang, mande logat jeitlebenz fúr ibre 23:5

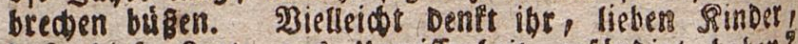
Daß Diefe feute aus unmoiffenbeit gejünoigt baben? Rein, fie habelt allerbings gerougt, mas fie thun unto

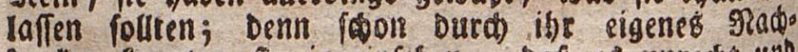

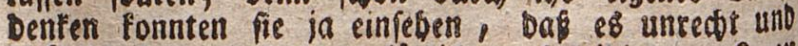

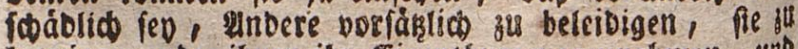
betrugen, ober ibnen ibr eigentbum megtunehmen, unt Dafe es Dagegen ibre \$flidtr fev, Der Dbrigfeit zu ges borchen. und obnebin fonnen alle Einmogner eines Sanbes genau erfabren, roas von ber Dbrigteit gebon ten ober verboten ift, weil oie obrigtentliden seferes und Berordmungen offentlid befanut gemadt werbert. Dieß gefdiebt theils in einer Sd)rift, meldoe in jebet 930de gedrudt beraus tommt, und fúr wernig BelD ju baben ift, (fie beift bie zeifung), theile Dadur\$! 


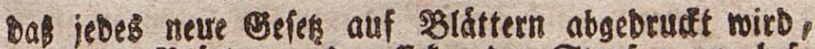
uno biefe sBlärter an Den (Eofent Der Strajent angehef: tet werben, fo bas fie jeder lefen fann. Falio fants feir unterban fíb mit feiner unmiffenbeit entídulbisert, menn es die Sefȩ́e úbertreten bat.

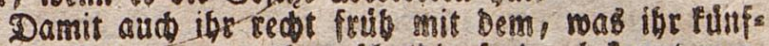
tig alo lunterthan zus thun fouldig feno, befannt werDet, und nicts aub llumbiffentbeit Dagegen fúnoigen mó. get, fo lefet Das Jolgende mit grofer 2lufmerffamfeit, and yráget es eurem Eedádtniffe tief cirr.

\section{SBon Den Siecteren der erten/d)ets.}

I. YUle blciben mus, und ifm roeber gehoten, nod perboten soerber Derf, wird fein fie de genannt. So bat \&. 2Z. jeoer MRenfक Daś gied) bat: fein Eelb, fein Saus, feine Rlciber unb Eaben nad feinem Soetieben zu gebrasden, wenn ef nur bamit esmbern feinen Sabaen aufugt.

2. 23enn alfo iemano meils giedat semaltfam anjl: greifen unb tu yerleken fudjte, mir \&. 20. mein Beld megnebmen, unb es fu feinem sathen gebraudsen wollte, to ourfte it ibn burd 3wank ober Esersalt bavolr $a b=$ balten, ober mid gegen ibm vertheibigen, unb ibm baz Genonmente mieber abnegmest.

3. Jeber Drenfó Darf feime Inlagen, Såbjigfeiten and Sirdfte fret und ungebinbert auBbitben; D i e $\beta$ ift Dab erfte uater bent alloemeinell menfosen gRedten. EEB Darf alfo feines burd graufame unt

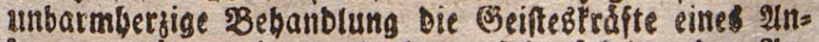
beren gertutten ober verdeeben; EB Darf feiner ben IImDetn binbern, Diefe Firáfte us uben, unb Durd Hebung ju erboben, und ju vervolliommen. 2Bent alfo of. $\mathfrak{B}$.

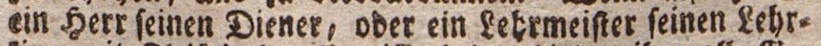
ling mit fleis in bes lumbiffenbeit erbielte, ibm alle Be legenbeit uno Mittel nábme, um etwå gu lernen, ober ibn gar in Jrrtbuimer fublbrte, um Dann mit ibm madten

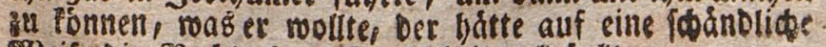

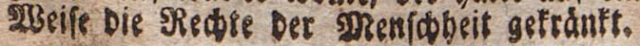




\section{Don D. SRedten u. Pfichten bet Untetthaten}

4. Teber Mrenis Darf nide nut lein Seben erfaltent es befouthen und vertbeidigen, fondern aud tiberbaupt

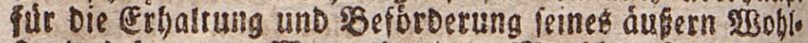
Rander forgen. SBer alfo einen Renfaber fo graufam suno gemalttbartig bebandelt, Dab fein seben ober feine

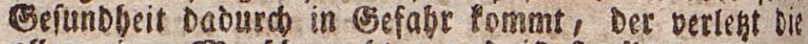

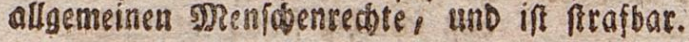

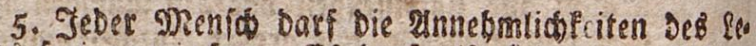
bens frei genieben. ef barf alfo feiner ben Indern

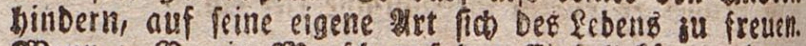

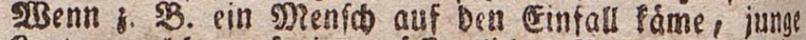
Seute, welde auf einem iffentlisten plake mit Epils Ien foc vergnigen, gewaltfam Datan zu bimbern, und fle reg gu striben, obgleido ibre Spiele gan uniduls big fino, fo todre Dief eine frafbare sanolung, Denti

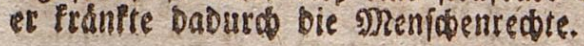

6. Seber smenín Darf alle Die Sacben erwarben! befizen unt getroubsen, relde fur Erbaltung feimb Rebens, unb gut 2 seforberung feiner Bludfeligfeit bies

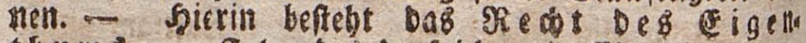
15) u ms. - Folgendes Darf id mein Eigentsum neth

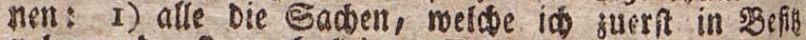
nelime, ba fie nod feinem \$nbern zugehorten, Detit

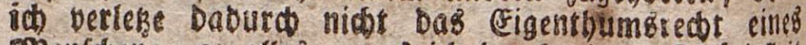

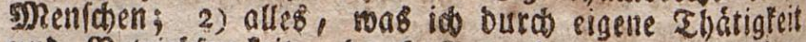
und Betriebfamteit, burch sinmenoung meiner sget

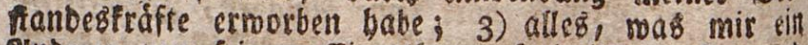
2nberer bon feinem Eigentbume freimittig auf irgento

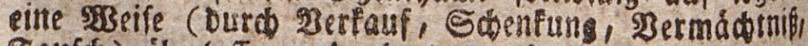
Sauí) Liberloflen unb abgetreten bat.

Cinige unter Diefen geenfotenreaten fino verdus

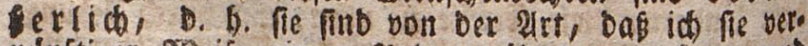
munftiget शrseife cinem 2nbern tibertragen tann unt

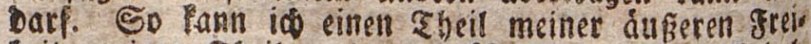
beit, einen Ebeil meiner siddfe uns meiner Erbals tungsmittel, ia felbft einen zbeil meiner Bstucteflig*

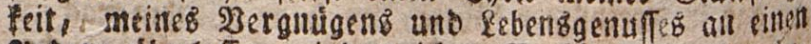
Indern uberlaffen, inorm iob \%. \$B. fur Jemanden bes

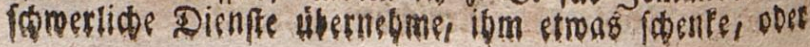


ein \$ergntigen nufgebe, um einen SInbern bei feiner

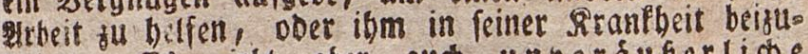

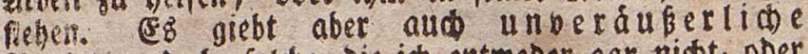

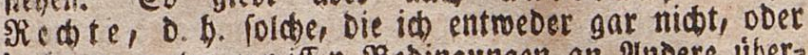
Doch nur unter geroiffen sebingungen an 2nndere libers

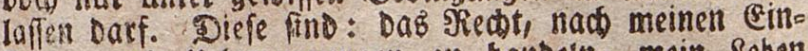

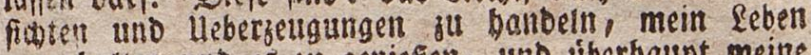
zu citbatten uno es zu geniefen, unb úberhaupt meine Glúfeligleit ju befordern und bu vergrófern.

Mrein (E) igenth ums te of fann id einem Inbern abtreten, entweber ofine alle sebingung, burd $\subseteq$ d) $e n=$ fusg, oder mit gerwifen 3 Bedingungen, weide der

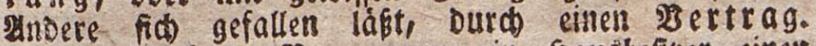

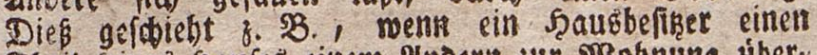

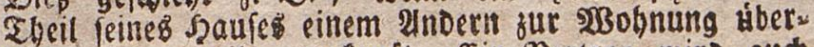

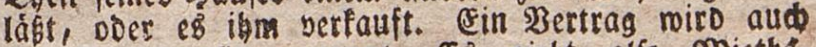
sin Rontraft genannt. ESB giebt allo Mietbs Pad $t=$ Rauf = Routrafte u. a. $\mathrm{m}$.

Die Dbrigteit idúkt ieben sgúrget beß Staates bei Dem Esenuffe feiner Siedite, und fidbert itjm Diefen senus.

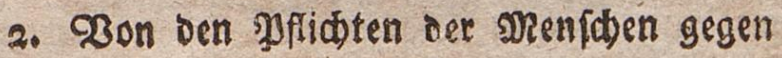
ibre soitburget.

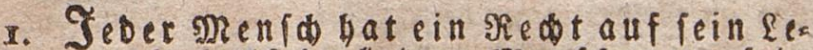
ben; alfo follf bu feinen menfoten um fein Seben bringen. - 2Ber einem spenfouen vorfánglid

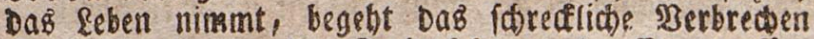
eimes Mordes ober Todrfólages. Ein borfátz: rieter Mobrber wird won Der Dbrigleit am \&eben ges ftraft (bingericotet), meil es ein biodit gefófirlider

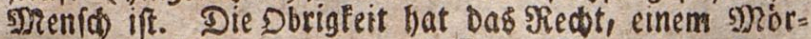
Det Das seben nefjmen fu laffest. 23 er einen feiner Berwanbten tóbter, mirb badrter gefreaft, als ein ens berer Mórber; aud Derienige, meldser Jemanden mit befonderet Braufamleit tóbtet.

2Bser in ber saise, ats tlebereitung ober aus Un. sorfictigleit einen sjorb begeht, fommt auf meitrere Jabre ins 3udthaus. Eben fo wiro Derienige geftraft, 


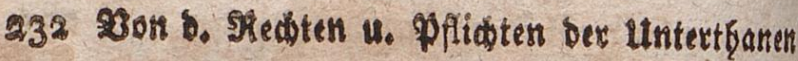

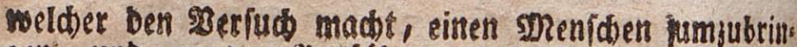
gen, und an Der subfúbrung Des gDorbers gehinbert

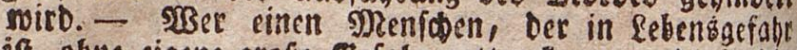

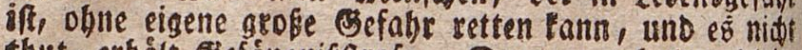
thut, erthe̊lt Eefángnibifrafe. Dagegen erbalten dieje nigen soelohnung, welowe fo edermúthig find, mit eiges

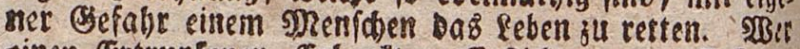
einen Erttunfenen, Erebenften, Erftidten, ober Erfrot:

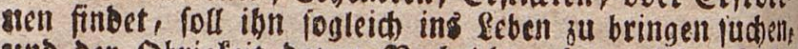
unb Der Obrigfeit Davon Nadbridt geben. PBer folde Pertungluatte rettet, erbalt sine Belohnung an Belbe;

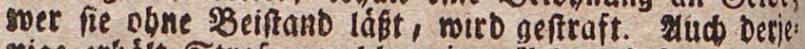
nige erbailt Strafe, melder einen anndern oefwegen ver" fpostet, weil at einen errbenlten lo sgeichnitten bat.

2. Jeber Meníd at ein Redt auf feine Befundheit; Daber follf ou feimem gren

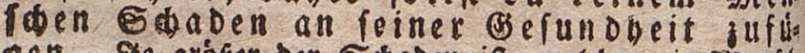
gell. Ie gróber ber Śbaben ift, welden ein grenfh Dem थnoern an feiner Befundbeit zufugt, befito gróbet

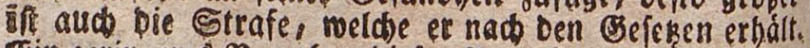

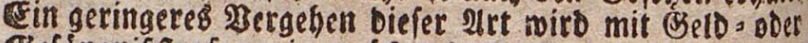

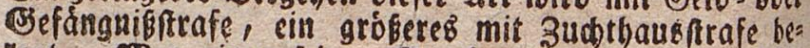
legt. 2Ber eiment feiner Doitburtger verrounbet, erbält

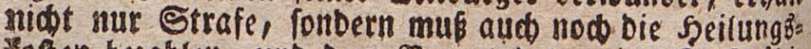

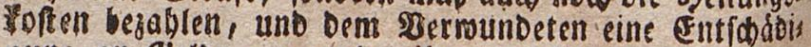
gung an Belbe, megen Der ihm verurfacten S由merzent und Der Stiorung in feinem sperufe geben. SBer eineti

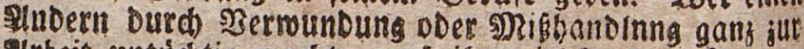
urbeit untudotig madt, mus ihn zeitlebens crnábren.

3. Jeber Menid bat ein $R$ eds auf reine gecibeit; baber forlft ou Peinem meniden reine greibeit nebmen. 2 Ber einen mentalen serbindert, Das ju thum, nas er reditmd́ffiger $2 B$ eis

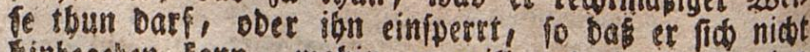
Einbegeven fann, nobin er mill uno barf, Der bat

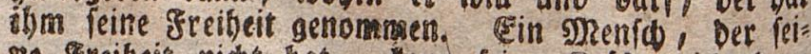
gie greibeis nicht bat, tann feime grráfte niat Daju

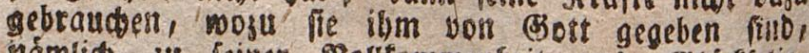

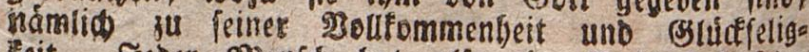

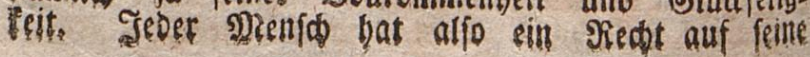


in mob̨leingeriateten Gtaaten: 233

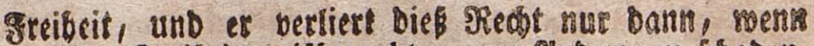
er feine greibsit misbraud)t, mm smbern ju fobaben.

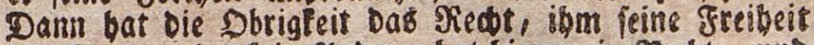

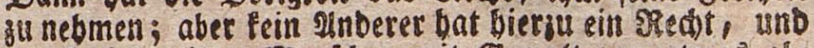
wer baber einen snenidben mit Eerwalt yon etwas abs hált, wors et thun Datf, ober ilgn gat einfyert, mirb geftraft. 2rser Sinder iben eittern ober Erjiebern Durç

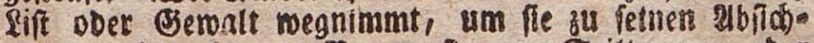
ten fu gebrauden, i. 33. um fie zum Seiltanzen, ober

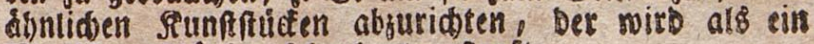
Menideert = MRtuber febr bart geftraft.

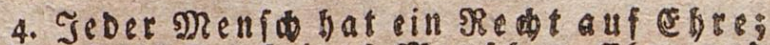

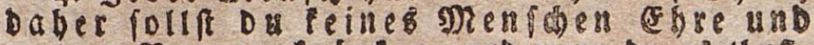
guten samen franfen, uno roenn ou eg thusfe

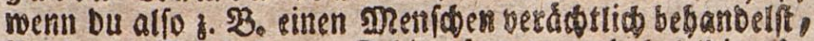
ion Durd Bebelseden, Edimpfworte uns beleibigende

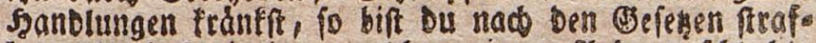

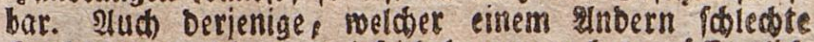
Szandlungen, Die er mirflid begangen bat, iffentlich borwirff, mirb von ber Dbrigleit als ein ebrenjobán. Der beftraft; Denn nur bie fórmtide Inßlage, aber nidt bffentlide Sefdimpfung, if erlaubt. SBBer andere is einet Sdrift, ober curch ein Eemárbe, auf meldjem fie in einer ldobetlicben ober veráchtliden Gefftalt bar=

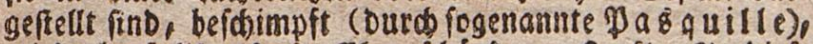
witd ebenfalls als eill ehremideanber geftraft. In Diefe

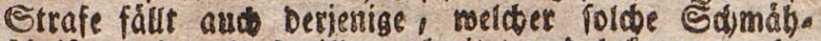
ideriften ober pasquille verbeeitet uno befaunt madt. 2uber ber Etrafe mus Derjerige, roelcher amberce bes

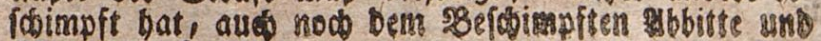
ebren . Ertiánumg tbur.

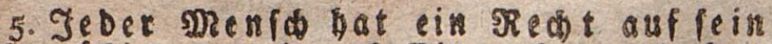
rectmdetg erworbelles eigenthum; Daber follf ou nide fieblen, und feinem spenchen in

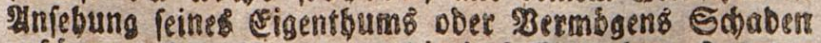
zufúgen, befonbers menn oir bas eigentbun anderer sut SBewaberng anyertraut worden ift. - WBer bab,

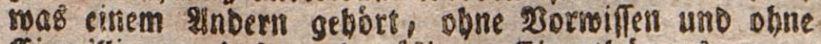
einmilligung Des recostmákigen eigentbúmers meg=

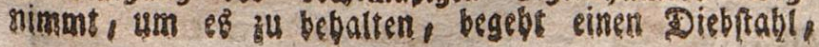


234. Oon ס. Feciten u. \$pfititen ber Untertbanen

unb wird von ber Dbrigleit bart geftraft, wenn ber Diebrabl entbedt mirb. 2lud cerienige mito a!b ein Dieb befraft, Der etwab fintet, uno es weber Demiento

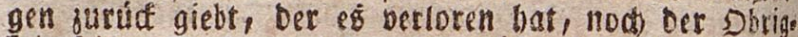
feit feinen funo anzeigt. Te grófer ber şerth eines geffoblemen Suteś ift, Defto grózer if audo Die Strafe Dež Dieber. - W3er bei einem Diebfable Thúren unto Edifloffer erbridbt, oder tóbrtide Imfrumente hei fin fubrt, DDer gar Dem Eigenthimes Bemalt anthut, mili bárter befrraft, als ein gerwibntictser Dieb. Eben bief

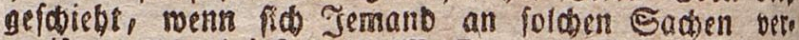

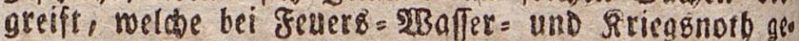
retter morden find. 2Ber foldes Sacien niebit, meide

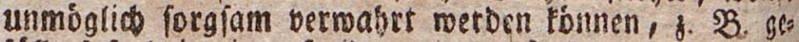

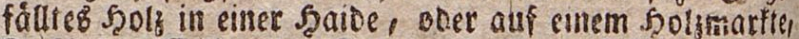

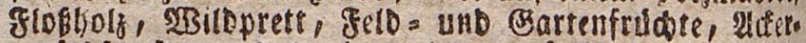

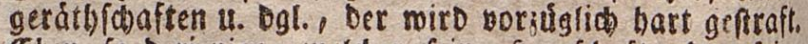
Eben fo Derienige, welder feime Serrfolt ober feine baukgenoffen beftieblt. - $2 B e r$ einen andern gemalto fam liberfallt, um ibm feir Eigenthum zu nefhmell,

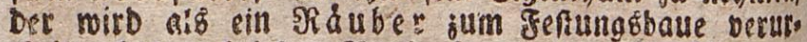

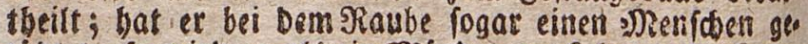
tóotet, fo mirb er alb ein Mordoer am Rebel: gefirtaft. -

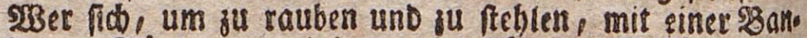

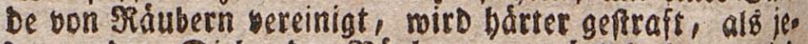
Der anbere Dieb ober Récuber; vorsúglico bart miro Der 2uf úber einer folden Saube geftraft.

Die Strafen bes Diebftabls und Raubes treffel niof blof Diejenigen, melde Die Şauptperionen Dabei gevefen finb ; fonbern auds Diejentigen, welche auf its

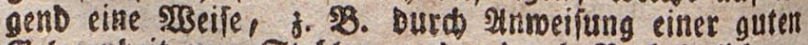
Beiegenheit zum Steblen, Dber Durch Nerbeimlioung Dez Diebfrabls, als Diebes=sebler, an Demiclo ben 2intheil genommen haben.

2ud biejenigen werben von Det Dbrigfeit geftrafth melche aus FRad), \$BoBbeit, SRurbmitlen oDer unachto famleit frembes Eigentbum bef d do igen, \&. SB. Das

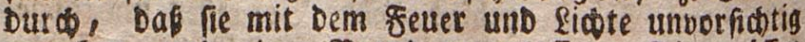

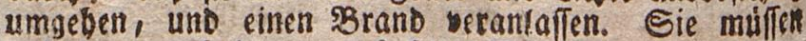
auserdem now Den berurfadten Edbaden erienen. 
in mofleingetidteten Staaten. 235

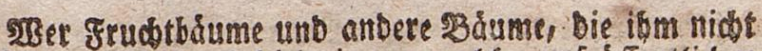
gehoren, befonbers biejenigen, welche ouf offenttictent Pláben und an Den Sandfrafent freben, umbant, Der

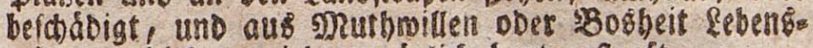
mittel beroirbt, niro vorighlio hart geftraft.

Irser bobhaft genug iff, Geuer antulegen, wirb alb ein 5) orobrenner bon Der Dbrigfeit am Leben ges

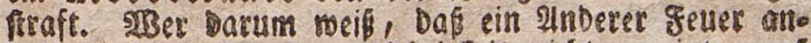
legen mill, uno ef Det Dbrigleit nicht, anzeigt, mus Gefángniß = oder 3udbtbaurftrofe leiben. Dagegen bes fommt Detienige eine onfegntiche sgelohaun an Beloes

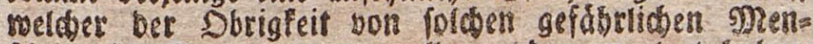
iden, Die Seust antegen moller, ober angelegt baben, fidere Rastiost giebt.

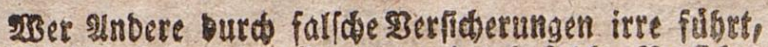
unb bintergeht, Doer ifneth gat Durch folcoe Desficole rungen eimen Sobon an ibrem sermogen zufuigs, Der

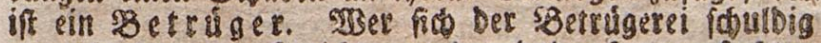

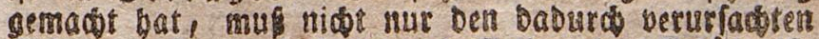

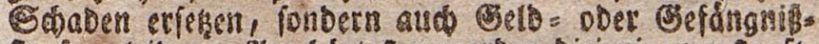
frafe etleiber. \$m batteften metben biejerigen geftraft,

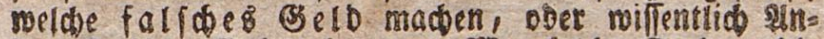

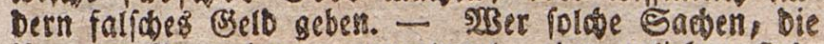
ibm gut \$ermahrung anvertrcut, Doer gefiebem frob, ableugnef, uno unterfd ldgh, miro bårter geftraft, alB

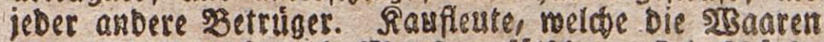

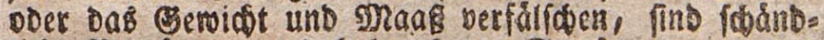
lide şetruger, unb babeft barte Etrafe gu ermarten, menn bie Dbrigfeit ibe Betrugerei entbedt.

3. 2 on ben $\$$ flichten bet untertbanen gegen ben Eandeshertn und gegen die Dorigleit.

Det Ranbeảbert bat ein SRe⿻̋ts, von feimen Uttertbas nen ju fordern, oaf́ ein ieder berfelben bie burgerlichen Befere millig befolge; dena menn nidit alle 3 turger eines Staats fid Den Belezen unterwerfen, unb ibren Bebroriam leiften, fo fann ber

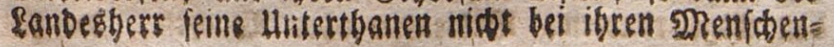




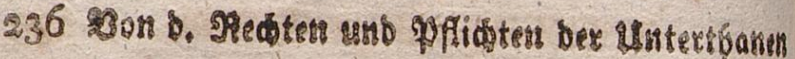

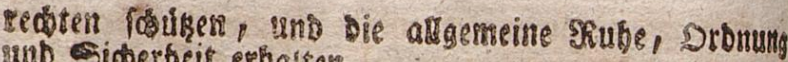
and Siderbeit ertholker.

Da bie Dbrigfeit Die Stelle bes Eandegheren bettrits , fo Darf fic bie Untertbanen Dazus anbalten, Dof ein jeber Derfelben zum allgemeiners 5 Beffen alleż bas thue und leife, was die Esfege von ibm forbern. $2 \mathbb{B}$ re filos alío weigett, die gefermásigen 2ubgaben uno zollt

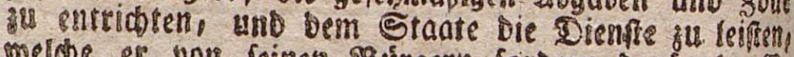

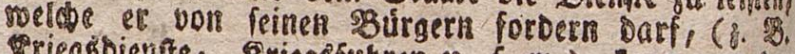
seriegsbienfte, Siriegsfubren u. f. w.) Fann var Der Dbrigleir Daju gejnorngen werber.

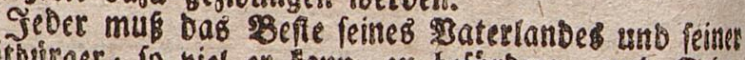
smitburger, to viel et tam, zus beforberm, und Edhas

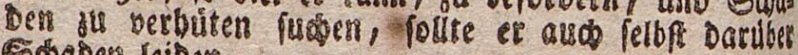
Swaben riber.

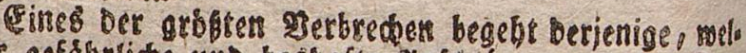

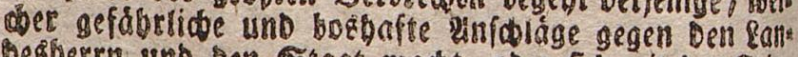
Degbertu unb Den Staat madt, ober fid mir Den Seith

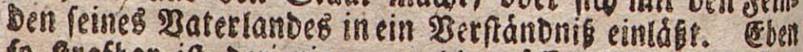

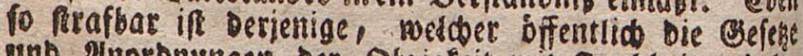

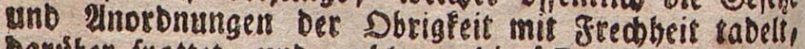
Daraber fsottet, und woht gar bie offentlid angebeffes ten SEefanntmaobunger Der Dbrigfeit abreíst ooer befitto Delt. - 22Ber 2nnbere berebet, $D a \xi$ fie fios mit ibm $g^{85}$ waltram Den sefeblen Der Dbrigleit miberfeken, ober mit lingeftum etrwas fordetn follen, Daś gegert Die હ̈e

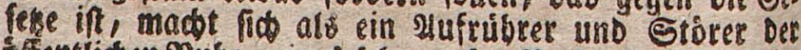
iffentliden Shube, eines febr großen Nerbredenz fobulbigs uno wito mit lebenźldnglioer einfwertung beftraft.

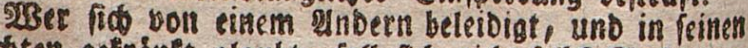

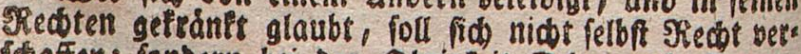
Ifaffen; fonbern bei Der Dbrigfeit Sauk uno esenug. cbuung fudben. Derangreifende Skeil aber foll fide obnt 28iderrebe Den sofehten Der Dbrigfeit unterwerfen, und Die Strafe leiben, welche auf fein Berbrect)ers geferst iff. Seber Untertban foil ber Dbrigteit mit Der gebubs: renben 2htsung unb Eghretbietung begegnen, uno bier Pelbe audt Durab duskerlidpe zeiden an ben ₹ag legen. Daber fint Edmábiforiften, welde gegen Die Sbrigo teit geriditet fino, Doppelt freafbar. SPeiner foll fid

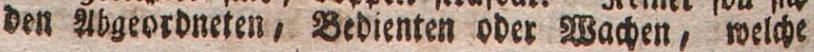




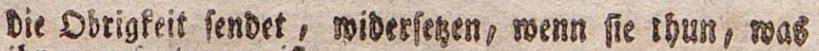
ibnen aufgetragen ift.

4. Bon rem obechalten Der פRenfohen bai Dem (3)ebrauctios iser Siectic.

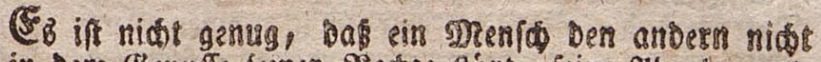
in Dem Benuffe fernes rechre ftert, peitse Mbgaben ent=

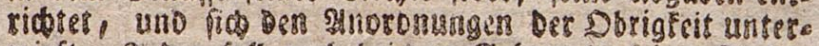

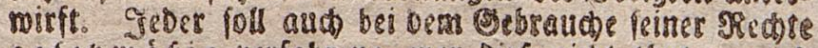

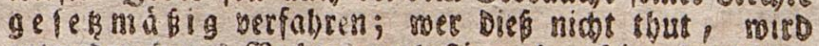
entroboer fenes SRectes vertuftig, oder feine Sgandiung miro fúr ungústig ertlárs.

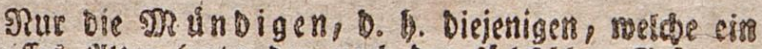

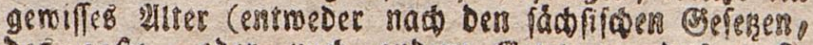
Das 2 Ifte, oder nach andern Eelesen, Das 25 ffe

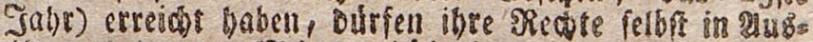

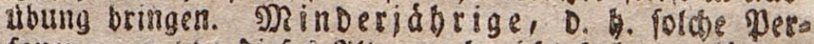

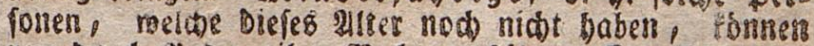

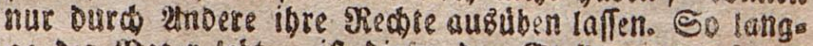

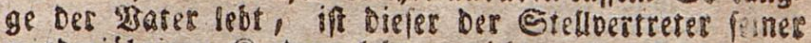

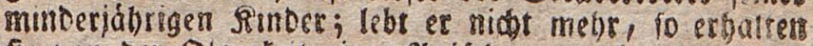

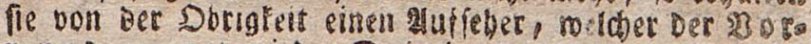
mund genannt wiro Ditienisen, welde unter sineres 23ormulide fteben, rerien Mi lindel genannt. Sie Dirfen, obne Cinwilligung ibres .\$ormundeb̆, niçts

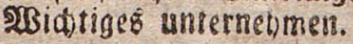

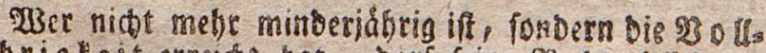

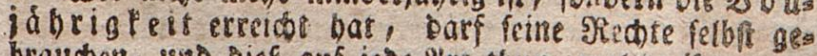
brauden, unb biek auf jede art thun, reeloe ibm dors theilbaft zle feon theint, fo lange er Dabei Die Recose 2inberer nict fránft. Eer oarf alio \%. 98 . Merträge

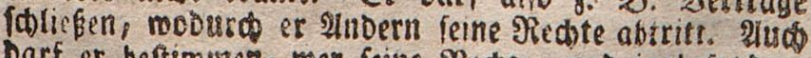
Darf er beftimmen, mer feine sRedte, und inabeiondere fein Eigenibum nach feinem zooe baben foll; er barf ein Eeftament maden.

Reiner Darf úber uneriaubte sanolungen

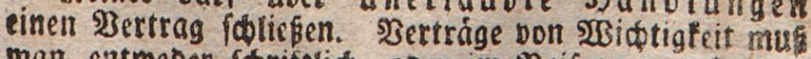
man entweber foriftlid, doer im Beifenon pon 3eucen abjobliefen, oDer von Der Obrigteit beftátigen laffen. 
238 Bon o. Redten u. Pflidften Der Untentfanen

Bum Beicber, baß man einen settrag oser Fiontrafi mit Jemenden abgefaloffen babe, plegt man (seid obr

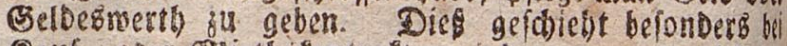
Rass $f=$ ooer ngietbsfontraften, indens man ermab Dar

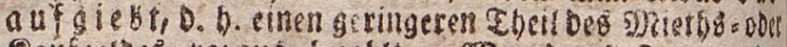
Raufgeloes boraus bejalit. Figer Durch 3mang obir sgetrug dabin gebrabst morden iff, emen \$ertrag ill

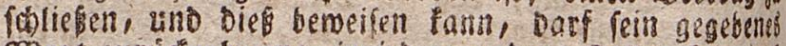
2Bort zurúatnelment; in jedam anbern falle aber mul er fein gegebenes sebort baltent. Die Dbrigfeit forgt bafúr, bas jeber feil gegrenes sobort baite, und Den

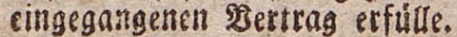

\section{Qen ben Ctånoen in der burgetict)en (B) ifllichaft:}

Da bie Sbrigfeiter mebr. Serwalt baben múfert, all Die ulbrigen Mienfden, fo mubten verfós is bent

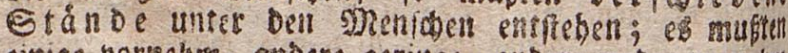
einige bornehm, entbere geringe, andere weber vormetrim now geringe levn, ober zum mittelfi an be geboren.

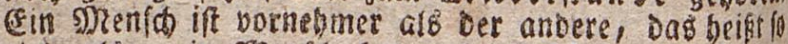

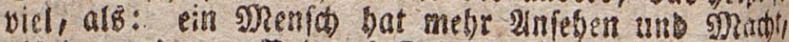

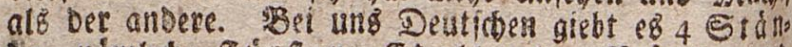

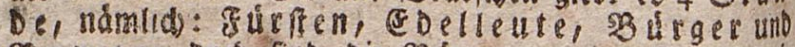

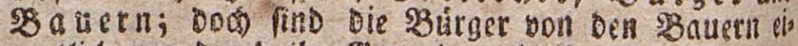
gentlid nur burts ibr (Semerbe und igre Rebengart uns terf́bieden, uno nidit Durd Den Stand. Diefe Stánbe fint erblin, D. D). Die Sernder erben den Stand Deb

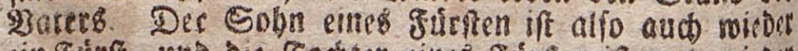
cia flutf, und bie Eochter eines fúrften ift auces witber sine Jurrfin. Shan mennt ienen Prinz, uno diefe Deingeffin. Die Eobme und Sobner eines Ebelmanns firib auch mieder abelich, oder geboren jum \& belo ftande; Die Rinder eines birgerb fino von Esburt

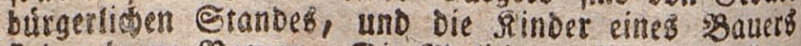

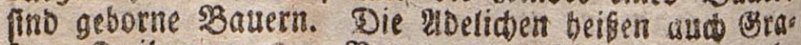
fen, greiberten Doer SBarone, und Dieienigen, melde to beisen, geboten zum bojen goel.

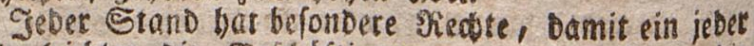

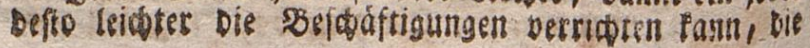


feirem Stanbe fufommen, und Pein Stanb Dem anbern binbertich merde. Die Rectete Deb भidelfandes be=

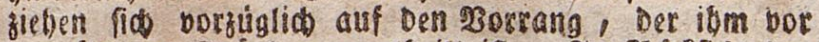

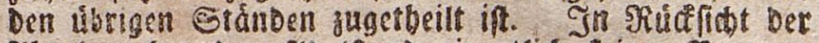

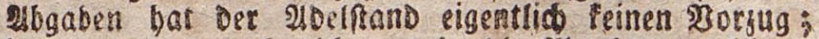
Dern wenn er aud nidit getade Die 24bgaben entriátet, melde bie úbrigen Stánice entriøten muifen, to bat er Dafúr mieber andere sBefgreerben ju ertragen, welose

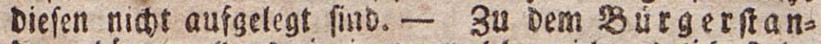
De geboren afle bejenigen, melose nich abelito find: unb auch nidot Bauern genannt werben fonnen, meil fie buirgerficte Eemerbe treiber. Diefe Serwerbe find: Die Shandlung, Die Rinfte, Die Shandowerle, Die SBier:

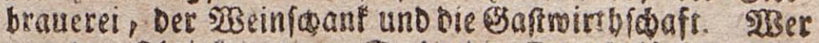
von Der Dbrigfett einer Stadi Die Eritaubnig bat, ficos in Der Stadt nieberzulaffen, uno ein búrgerlides Bes merbe fu treiben, bat Dab $2 B$ uirgerre 1 erlangt.

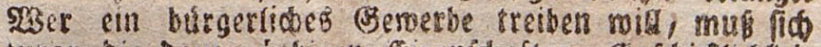

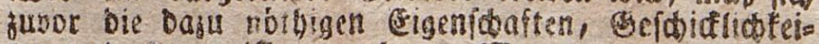

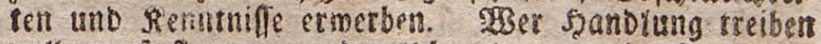

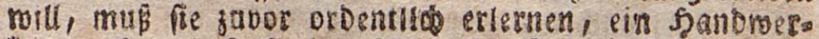

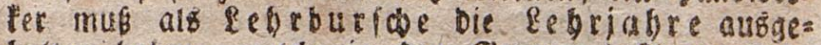
haiten thaten, welcte in ben Belenent beftimmt finto.

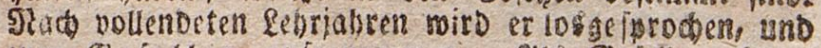

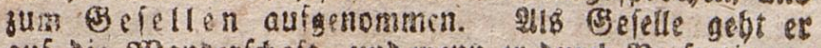
auf bic SBanderfobaft, unt menu er burch Derfertigung

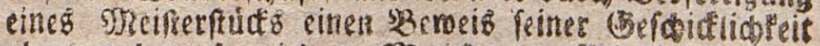
abgetegt has, fo miro er Mreifter. MIs greifter hat

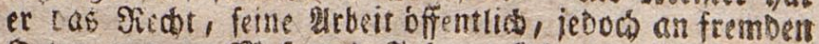

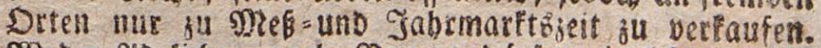

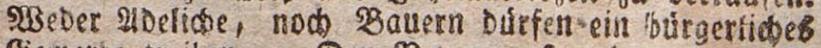
Berverbe treiben. - Den $\mathfrak{B}$ auernfta and madten alle

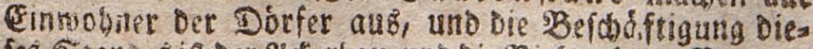

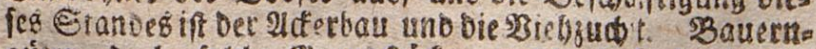
gúrer, 0 . h. folde Brrunoftide, meld)e be fonbers zum Ilferbau und zut 2iebzucht Deffimmt find, Duirfen in

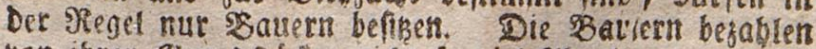

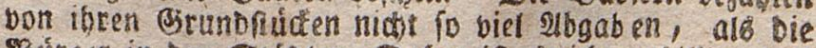

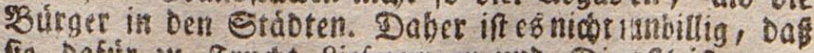
fie Dafưr zu gructst= Rieferungen uno Dien?teifungen 4. 5. za Rrieggfubren, verbunden find. 


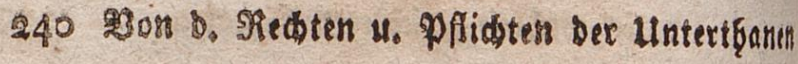

6. D30n Dex Dienftboten uso Serrfarafteh,

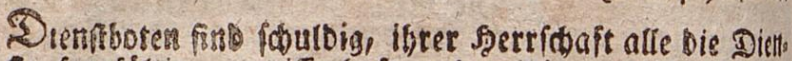
fe lorgfátrige gersilfenthaft und witlig ful leiften, mojt fie van Derferleen angenommen worben find. Sie lollen ben Befeblen ibrer fsertidaft gehorfam feryn, nuEgge nommen in Dem galle, menn Diefe itjnen ermas unter

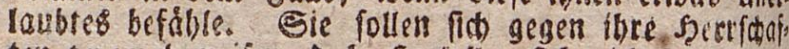

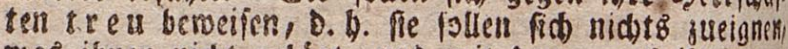

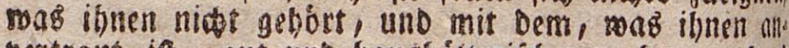
bertraut iff, gut uno bausbaditerifoch umgethen, und of

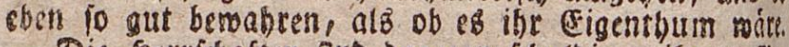
- Die Sgertichaffen find Dagegen fatulbig, ifrem Ese finde Dero arsbeoungenen foln fur beftimintent 3eit tll

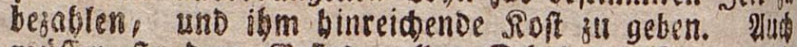

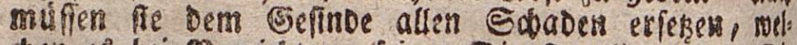
de en es bei Berriabtung feiner Dienfte erlitsen bat. Die

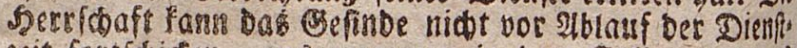

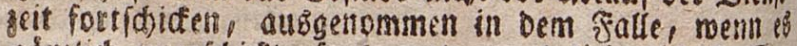

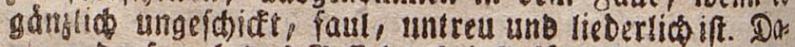

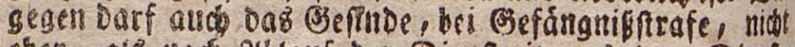

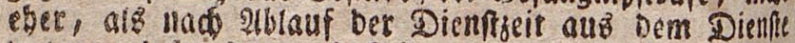

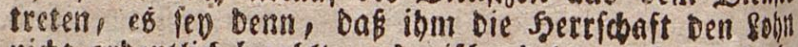
nicte orbentlic begablte, es mifhandelte, unb unertaubo te Sgandiunger von ihm verlangte. - Szerríhaittr fino serbunden, Den abziebender Dienfitboten ein Zeus"

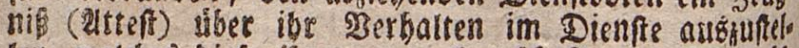
leil, welobes Diefe ibrer neuen szerritioft vorzeigen mulf fer, meil fie obne ein folctes 3eugnie nidt gemietbet merden Durfen. - Ylus Dem Dienffe getretenes eseint De muß fich fogleid mieber vermiethent; ober menn ef Dagu Feime Belegenbeit finber follte, fí beskbalb fogleid

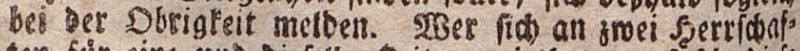
ten für eine uno Diefelbe seit vermietbet, mué für Diefe

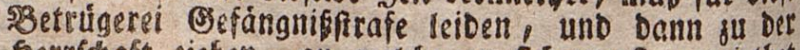

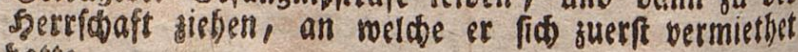
betce. 


\section{XIII.}

\section{Lieber und Gefánge.}

ז. M2orgentieb.

M und Dant; ethebe Brott, o Beele! Der Gerr bort meinen lobgefang, lobfing' itm, mei= ne Seele!

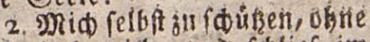
smadt lng id, tund follief itm grieden, wer cabeft die Si= deribeit Der Radit, und Rube fur die Múden?

3. Dubifteहै, Evot und Şery bet welf, uno bein if uniet Sebeti. Du biftes, ber esung erbáit, unto mir's iekizt nea ge= geben.

2. SRorgengebet.

SRe1. פon Bott roill id nidt lafieri.

2l Immádtiger! id bebe nein Slug' empor zu Dir. \$reie dir, Durd den id lebe, und netter Dane Dafur ! Serr, Deine Şulo ift GrOB, uno niemals hat $D a \cdot 3$ Qallen Der Unfoute dir mi Bfal= Ien, Das aus Dem Sierzen flós. 2. DaB nidbt in tiefen Бक’́ummer Des Sebené Siфt berlifdt, und bak midb, frei bon fiummer, ein fanfter S $_{\text {d laf }}$ erfricht, Dieß́b Danf id Deiner Mađt, u. Deiner Bater-Sreue ; Durd fie bir id auf's neue mit beiteru SRath erwa巾t.

\section{Eottes Bite.}

$23_{i e}$ grofift des भilmádt'get (súte! If Der ein Mení⿴囗十 ben fie ridt rúbrt? Der mit verhártesem(semutbe den Dane erfatioft, Der inm gebuhrt? Rein, feine Siebe ju ermeffen. fer) eroig meine grófte pficit: Der soer hat inein nod nie vergeffen, vergib mein 5ुers audo feiner nicbt?

2. Wer bat mido munberbare bereitet? Der (sott, ber mei= ner nidt betarf! sBer hat mit Earigmuth mic geleitet? Er. Deffen SRath it oft verwarf? פुber fárft Den fricoen im (s) = miffen? WBer giebt Dem (Beiffe netse firaft? Wer lákt mith fo viel But's aenieben? Sein 2Arm ift's, welфer alles fdofft!

$$
\text { 4. Morget = B̈efang. }
$$

Me I. Nundanfet alle (3) tit ic.

Dab (sravin der Natt ents flok, oie Sonne febret mieder. und mas auf extDen lebt, fingh Dir deE Danfes Lieder; Dir, Der Dut Berg und That mit Deinem Thau erquidf in und aud

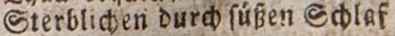
erquidêft.

2. Beffảrtt eilt unfer Beift mun wieder zurn \&efdáfte, Dä Du fir un erfabit, wed bu (2) 
Quft und Sirdifte, und Dein (3)= Deiben gabft. Stel' un ferner bei, Dafis unjer Eifer uns uno Anderk núblich (ev!

\section{Indentert an Bott.} Mel. Sobt Bott, ibr.

$\Re$ Cod reben wir, und haben Brod, und ein gefundeb $b$ fut

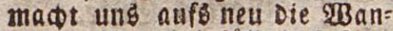
gen roth, und fulltt Dab sers mit s) (utb

2. Ev lange diefes Serj nod fdrägt, Die 2ugen offen ftetyn, foll biefes Sher;, von Dane be= toegt, auf Bott, Den Beber, febrit.

\section{Beim Solube ber balb= jäbrigen fection.}

mel. खas Bott thut, Dab ift.

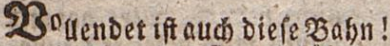
grets Dir, Der fie uns füforte, Der Brobes auch an ung $g i=$ than, Deß 23 eisheit une re= gierte. Du gabft uns firait Der şiffenfobaft uno unferer Pfitot zu leben, und weiter fort zu ftreben.

2. Bohl ung, wenn Deह \& $=$ willens SRut in unfern sersen mobnet: fie firomt ung fúfe grettien ou, ihe fanft (3efúb!

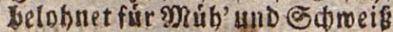
Den treuen §leî́, fie låŝt ung mit Sertrauen Die bellfte Bus funft foauen.

3. 2Bergeib, wenn faumrend unfer Juf nidt raftbe Edritte waate, Der SRuno, voll Mis = troun und פुerbruß́, oft über Laften flagte. Sinfort roll nie Der 2urbeit Mủh ung fdreffen,

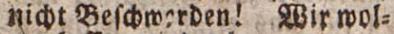
ren beffer watben!
7. Rad geendigter 2Irbeit. Mel. Lobt (sott, ithr Chriften.

Boubradut ift unfer Tagemert? शun laft unz fróhlic fern! Die Sreude giekt jur 2rbeit Stárt', uno unfern Seib (se: Deihn.

2. $30 n$ unjerm jugendfiфen Meibn fev Sárm und Muthitit fert, vernanftig foll oke frelt" De fevin, und angenehrn Dens netri.

3. Wie gut if unfer Boott, wie gut! Er giebt gefundern leib, ein frobes soers uno iri foen Nuth, und foriel seits vertreib.

4. Labt uns ifm Danfla! renn! Die Suft beim froben Jugentrwief erfic de nis in unis

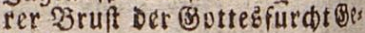
füb!.

5. 59: it (3) nabe blicft er Danls berab, fieft urfire frendern gern, die zreuden, die er relbit unz gab; O stinter, lobt beft 5ृerrn!

8. Dantlied itad ber 5) 5hals seit.

Danft Dem Sterrn! Mit fro" ben Gaben frutet er Dả gant? SanD; alles, allez, wa' mit Gaben, fỏmmt aus feinet $20:$ terbano.

2. Dantt Dem Serrn! fft giebt une feben, giebt und Segen und Eredeifn; foâfit ung Brod und Eaft der See ben, uns bu fátren, ou et" freun.

3. Donft Dem bern! Bet" giḱ, D Eeele, beines gutert

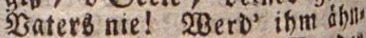
lid, und erááble feine 23 untor foút uno frub. 


\section{lieber uns gefänge.}

9. Ermunterung jur Reb= liobteir.

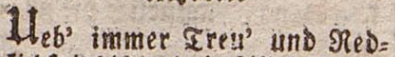
liøfeit bis an dein fillez \& $\mathrm{rab}$, und meiфe feinen finger breit yon Butres reaen au.

2. Donn miret ou, mie auf grúnen $2 u^{\prime n}$ Durळ's \$ilgerle= ben gebn; Dasn Eannft Du, fonder furdit uno (Grau'n, Dem Tod entgegen rebn.

3. Dem 238́femict)t toird alles fomer, er thue, maser thu'; Das Safter treibt ifon bin und ber, und lást ibm feine Ruh.

4. Der foóne Frúbling Iaot if m nidbt, ifm ladt Eein 2 el) = renfelo, er if auf sift uns Srug erpidt, und wanfot fid niकt: als (B)

5. Der WBind im Sain, das Laub am Baum faur't ibment= feBen fu; er findet, nad Des Lebehs sraum, in srabe fei= ne গiuh.

6. Drum ůbe Treu' uno Fed: (ic)teit big an Dein fiffeg (3rab, und meide feinen finger breit voll Gottes Wegetl ab.

7. Dann fegnen enfel beine Gruft, uno meinen Shránen Drauf, und Sommerblumen, Dull von Duft, blúb'n aus Den SEfránen auf.

\section{ro. Frube Gottegfurdit.}

श्सीel. Iु grámetr.

Sabe beine Suft, o gugend, immer gern on Den Serrn, und Dem Reiz Der Tugend! Was dein frommes Serz be= gebret, Dein 2 erfanb nicot er = fano, miro dir Dann gemábret.

2. Sđonell laß́s nidbt voruber: eaufden Diefe Suft! Deine

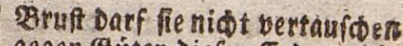
gegen (3) wer Diefer Erden, Die Dein Serz voller Exmerz balo vergiften metden.

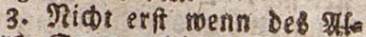
ters Sorgen did zerftreu'n; Jugerto, neis! Gळon am frúa hen SRorgen, in Der Blutbe Deines febens dien' itm gerno Denn Dem Serri dient Dis nidt vergebens.

4. Stäof, Befundbeit, lans ges Peben miro Dafá, nákbt egs Dir, Dein Erbalter geben. Weigbeit nur fen Dein Begebs ren! Danis wird er Dir fojor mebr, ift's Dir gut, gewábren.

5. Sudin Den gefunben $5 Q_{3}$ gen lá niobt ab is Das Brab cinen פolidf gu ragen! Du baft Dant, frey vom Serberben, Epelenruh': Dann fannft Dup Desa ou lebseft, ferben.

\section{Sed gut, fo biff Du gludidion!}

Mer. Woblatif, mein sery verlab vie melt.

23

(i) ant (अ)tes Der Butes liebt umo im thut, mie mobl if ihm im Seerjen, Den ganten Tag $p$ Die ganze Ramt! Ptiकts if? Das ifin unglucflid mact, felbit Seiben niळt, niфt Gomer:en.

2. Rein! wer bem lieben Gott feft glaubt, fid fein OBort feitle Shat erlaubt, die Jefus ibn beift meiben; mer alles fiebt, was Jeius fiebt, fiф gani uno gar an ifin eraiebt: bem fehlt es nie an greuben. 3. Irote frob fieht ber bent Simmel an, Det Bstes thut, fo viel er Eann, Der feine Múbe fdeuet, ber alle, nie fid felber, liebr, Den śndres 
Craurigfeit betrủbt, Den In= Drer Jreude frewe!!

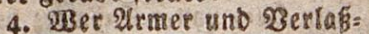
ner fich, wie รefub (Ebriftud, brúderlid und outfreid fonell erbarmet, wer \&utes gonnt, und Butes foenft, aud Dem, Der ibn aus Boabeit frántt, mer Geinde felfot umarmet ;

5. פoer, fern von fever ar: gen lift, ein Strenud der fie: 2Babrbeit ifi, ein feind von fal= form secen: wefi ja it jas soeß Bein if nein, wie lieb mire Der bell spenfwen febn! nie lieb oft auch den 250 fen?

6. wer gern mit aller feirer sraft, in geter \$bfít) (sutes rbafft, erfpart fict bier viel Seroen, geniest aud einft in jener Wett, Die unaufbortid' Blúf vosbált, unenolid fel'ge Jreudett.

7. Fob firrote nicd vor fei= ner groth, i i furdis feloft nido meinen $50 D$, wern id bas (sute liebe; menn idi, mie Jefuß ung ermabnt, mit Secl' und Eeib, mit DRund und Sand, nas redot uno gut if, ube.

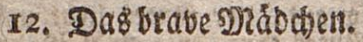

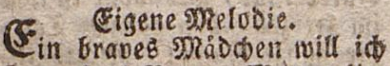
(enn, Retb Bater, Nutter lie= bent, ibr saddeln nar foll mico erfreu'n, ifor Düftrer 8 lic be= trúben!

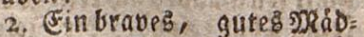
den if Die feindin alles $B^{\circ}=$ fen, bast affe freinofouft, affe Lifit und olles milde $2 B$ ejen.

3. Ein brave? 2Rádgen pran= set nidat im bunten fileid, wie 5) fauen; nidt oft - nie lang miro fein (Befictit im Spiegel eb bef(t)auen.

4. Gin braver Nä́bchen finnt nidtlang, fid mobifo aubुt fieren; grat?, ungetionftelt if fein Gang, iaturlic Die Mas tieren.

5. Cin braves Sind ift nidt su viel, ift Dantbar, if befceic Den; unfadálid, lebrreiф if fein Spiel, unfouloig feint Sreuden.

6. Still wirs ein braved Meád den fenn, wern attore ter Den-fomeigen; es plaudet! nidet in इag birein, nur per then $2 B 3 i k$ ju jeigen.

7. Ein braves Mádcien hat Dert nie, uno rauft fid nidi mit findern, madt andern $\&$ : ten feure 'grúb', uno fudt fir nidt zu hinvern.

8. 23065 - fagt ein bravel gntibiben - mir, wenn id mein Spinntad trete? Mot beffer iff mir beim (Elavier, aith beften, roerni id bete.

9. Des 9) Mangene will id früb auffes'n, aum \&eien uno jum Gitggen. SPrit) folt mall

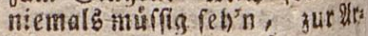
beit niemalezmingen!

10. (S) tht toill io allen sertis foeniebn, woniedemliebreid Denfen. Der PBruder $2 B$ ohl foll miderfreu'n, Der Sकweftern sumatter fránfen.

II. JMit mernem máb'att 2toenbbrob will its gern antil laben; fonmer if Die simuth) - grof die Nothy, fein 23rodjl eflen batien.

12. UnD follt' its mohl eith gåd den feb'n, halb nadt im Winter beben, Das fef'n,uno nidt ium Scbranfe geh'n und fein \$semand ifm geben?

13. 23:e nofs ift mir is tre ner $\mathfrak{~ r u f t , ~ m e n n ~ i d ~ n u r ~ \& a t e ? ~}$ thue! Sin ios nidotb 30 fee mit bemugt, tile feblt's mir dans an SRtbe. 


\section{Riebre und Gefånge:}

74. D'rum, Mảbden, madt és alle fo, fene brav mo fromm Darneben; Dann finnt ibr aud, mie id, fo froh bie foinfien Sabre leben!

13. Der redtidauffene Juing= ling.

Mel.2ffo bitost bie Weit gel. Seil, Seil bem Güngling, Der fí frǘt zum 3iel die roeisheit mäbit, mit Muth uno S3orfiøt Iáuft, uno nie des redten Big's verfelltt!

2. Der, twenn fid die şegier empirt, mit Nacborud fie bes fámpft, fie ber 3 ernunft ge: horden lebat, ibr feuer zeitig Dómpft;

3. Seil bett, Der niebern (Seiz uno Neid, unedre mis= gunf fliebt: fic úber 2indrer (s) ${ }^{2} e^{2}$ erfreut, uno am ibr 250b) kamát; ;

4. Gern giebt, menn er zu geben hat, und menn er nidis befibt, jum wentigften mis 3 sath und That Der Firmen Tugento หútz̧: ;

5. Der fid im Blúd nidt überhebt, Der nie in 230 lfen fleugt; im unglúd nie veria= geno bebt, fiф nie dem Safter beugt;

6. Den freinden milts, Den greunden treu, nie eine pfliot ver'ezt, und to er sugend findet, frei yon 30 rurtbeil fie fodutzt:

7. Er wirb Deß פzaters Ehr und Sobn, Der Dsutter freude renn; und if er alid fein $\AA_{0}=$ nigaforn, fo if er'e werth zu
14. Dúent Botte\&.

mel. Alfein (Bott in Der 5ूob) revebr.

Tob bin - unb nod niфt fang bin id, nict lange nod mein $23 a t e r$, mober ifter? mo= ber bill id? nober Der erfte 23ater? Ent mar eils jeber (Grofe ffein, fein mzenfos tar, meil er moltie fenn, - wie find wir denn gemorden?

2. Wer gab mir 24a' uno Dhr und Sano ? IRund, 24t5em, Blut und leben? MBer Sinne, TBiren und शserftand, Dem (S) ten midjuftreben? Sat rebe mid mis sreuben an, meil id gefin, reden, handeln fann, und mas id mill, fanm denten.

3. Die Sonne hat Eein \$Renfक gemadt, fie ift ung viel zu ferne; firn s) Ceiner PRact, und feiner 920nd unb Eterne; fie maren, ef) mir maren, $D a$; fein menídid slug', nidt eines (ah, fab) Mrond und Sorne werden:

4. $23 e r$ madte Denn Die $\phi^{\circ} 0^{\circ}=$ ne iseít, Das rabe und Das Ferne? BBer hat am frimmel bingeftellt die Sonne, SMond und Eterne? 230 her Der $T$ hies re grofe 3abl? oser madte Felder, ß̌erg' und Thal, uno Báume, frủdte, Blumen?

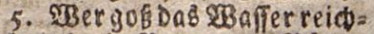
Iiđđ aub, in Brunnen, Både, Eeen? 2308 freut im 23 inter Fiofen aub? 203er beist Die FBitloe melsen? ober giést ben Regen auf oie 2u? भुer fúbrt Die grbolfen, trópfelt Thau auf 2 Biefen, Jelber, Reben?

6. Dab alles, alles (Brof und Siein ift Dein खBerf, Unfidtba= rer! Du bift Der ErDe, Du af= rein ibr Squpfer unb bermat). 
Per; Durb beine Maabt, auf bein (sebot, binido; ift, allers befter Bstt, Durd dich iff alles worden.

7. 23on (3) ott if afles?, mas ith Gab', Dhn' ibn Eann nidts gefibeben! Der sott, Der Als Jen susgen gab, mus alle?, al= Ies feben! Sein it oie 2rselt, aud id bin feirs; nidts ift st gros und nidits ju flein, Daf ex bafur niot forge.

8. UBie frob bin id, Du radteft mith jum seugen Dei= ger (Gite. I $\phi$ Danfe oir, uno sent' an bid mit freudigem

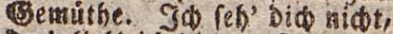
Dod lieb ido Dico; Bott, Du

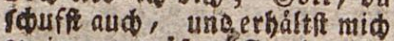
รu immer gróperti รreuben.

\section{x. Contt ît allmádstig.} 2Ret. 24tes ift an Bottes Segen. Seb

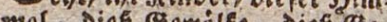

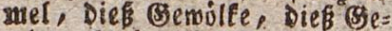
wimmel, iene suellen goldnen Qidtb, Diefer 5 aum uno feine solutthe fufteren Die bódfe $\mathrm{Cu}=$ se nur ein wort, uno weiter nidtz.

2. Wecbe! bat fie nue ge= orodben, uno es if beroorge = broden jeoes Stáubden, jedes Saar; fo viel Sonmen, fo viel

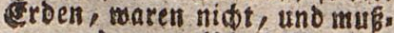
aen merben, als bas 5Bori ge: iproden mat.

3. Rinder, Diefe bơtefte (3) to, Die Den saum uno feine Elit the, und aud eud erích af:

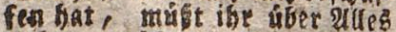
Iieben, múbtibr alle nie berris= ben, nie mit einer bojen ghat! 4. Iक ifr toúrbet es be= reven; Dens ifs Eonntet eud nidt freuen, beffen weider al= les fiebt: alles mas wir thun uno laffen, alfes lieber, affer saffen, aud yor fein Geridt einft giebt.

5. D wie madt es grofie Ereube, Darf man boffen, situ? genweide Deb 2llmådtigen it feyn! Safit unb inn Dann ber: liad fieben, nie Durd bofe shat betrúben, alle, alle, grob und Slein!

\section{IIlgegemwart Gotte?}

Mel. Mir naф, foridt Egriftu?

(B) ott! alleg, mas mir talt und ferm, uno grof uno flein bier feheth, Der Wurm in Stalls be, und Der Stern, Dort an Deb Simmels Sibler, ros levt uno nebt, ift Durch Diab Dar uno lebret uns:- Du bif unb nabs

2. 3mar faffet beine Ráge nidt Der blóde (j) eift bienieden: Dod) fảrft ę mádtig un’ gut Pfliф), føúks? unfers lebenls Erieben, menn obne filugelin finolidonir, Did fef'n in beis nen $23 a r f e n$ bier.

3. Itie, Szater, bift ou voi uns fern, nidt fers aud vors unz Sileinen; D'rum trauth wir dir unferm Seern, menti mis Derlaffen foeinen, menti Edwrabheit, Siranebeit odit too bera ungemiffen leben brobt.

4. Ou bift ung nab, wit

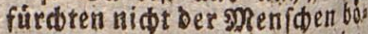
fen SBiffen, benn wir nur in: mer unfre \$fidot getrell und gern erfitlen; uni foredt nid" wenn Du um uns bift, Der ment (Dett Uebermuth unb lift.

5. Du bift uns nab', D'tutt foll uns nie yor Nadt tind Dunfel arauen, aud nidt vot unfrer srbeit SResh', getrult 
find wir, wir tratsen anf oid; wetn Deine Sand uns foúbt, shun mir mit \&ult, was frowmt und nußs.

6. + 2úgegenwartiger, ou Jifr un ttab' an atten Drten! Diф bintergef'n mir nie Durn Sift, und nie mit frommen 23orten, Du bift unz immer nab', un's du fiebft Den ver= borg'nen Ifaten zu!

7. Silf, Dof nir mit bem guten S\$фein uns nimmermebr betrugen, Die $9 j a b r b e i t ~ I a ß s$ un beilig feon, nie unfre Iba= tenlugen, auф von deráltern eluffict frei, fed unfer serz Dem Buten treu?

8. Du bif bei unż, auch un= gefeb'n, uno fern von unfern Sieben, famn obne did uns nicbts gefdefy'ts, nichtb unfer Seben trúben, ins unbefannte, fremde sand, fúbriz ou aud uns an Deiner Santo.

9. So fev es immer surfer Elud: por Dir, o Bott, zu mandein, und inmer mit dem frohen Blid auf Did, nur rect ju bandeln, ju denfen inmer: nah' bift oup Du fiebeft unferr Ibaten su.

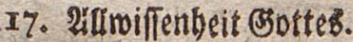

Mel.2Alfo hat (Sott Die şelt gel. Puळ unferm 2isandel fiebif ou ga, fenaft alles unfer ghoun, aud unfer Sgerz erforideft Dus mir wirfen oder rub'n.

2. D'rum fey aud Der (ese= Danferein, DBott, Dufiefft ibnja, unoniefes, mastwir thun, nur Sdein, ou jift uns immer nab"!

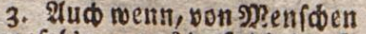
ungefeh'n, unz decitoie dunE= Is Radt, wenn ohne zeugen now fo fobin bas laftes Iodte und lacot:

4. 2ud bann foll bir nure beilig feun die fille cinfamfeit Damit soir uns in Unfobuld freu'n, Der föinen Jugenszeit?

18. (3)ottes unerméliche Bute.

IRel. 2Bie foún leucitet Dar Morgentern.

(3) ott, beffen ftarte Sand dic welt eriduaffen bat uno nod erfólt, id fकaue Deine Búte! शBohin id (eb)', anjedem Drt, Guf ErDen bier, am Simmel Dort; und wenn id mein Ses: mutbe auf bid, Sकojper! frelloiglenfe, und did Dente,

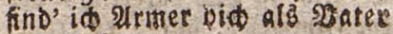
Hno erbarmer.

2. 'Sकau' id fo weit mein 2use reidot, gen foinmel, fo entbec' ic feidt Die Spluren Deiner Gute. Der Gimmels. forper Lauf und 9racot, Das Gternenteer, Das Du genadst? erwedenmein (Semuthe, gro= Ber Stbopfer, Did zu fingell. Dit ju bringen Chr' und Stárs Ee; grofi find deiner sánde werfe?

3. In ungemittern, Gturm und थีBind, Die Deines Efro= nes Dienter fand, entbed' id Deine Búte. Das Jestér, wie Der भुaffergruno, madt beine gropeliebe fund. gats fróbli= dem Eemúthe mill id táglid Daraufadten, sno betradten beine werte, Bott Der Wabs: beit und Der Stárfe!

4. Dein Eroreiф, अot\$, Das jebermann giebt Gott unb 'zreuben, reh' id ane unb ímede Deine Báte. J中 rif: 


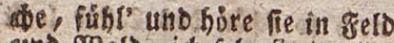
and Wald, id febe fie in aller forsen giutbe. Sen dir jeu= -gen alfe Dinge, fo geringe fie mir foeinen, Bott in Broken snd in fileinen.

5. D! zellg mids über felo ano 3eit, ats Dir, o Serr Der Serrlidfeit, Durch Deine grofie Siebe! Ruf bic Iá mich in श्रा: Jem feb'n, fár jedes (Bute dids erbib'n! Belebe meine Iriebe, (1) meit $25 a t e r !$ bir in Allem ou gefallen, fer mein eirziges 5 ifreben!

79. Berectigfeif Bsotte8. Mper. Made Did mein Beiz bereit,

(O) int in simmel mofnet, ex erbars met affer fich, feine fangmutb fdonet unfrer bier, wenn aud wair bofe sBege mandelt, und oft unredo handelis.

2. Mber aucb Beredorigelét gibt die meife liebe! Daß das Safter ungefdeut, niфt fein Sattyt erbúbe; DáB unb nidt Redt und N1fict unbedeutend bliebe, frraft aud feine Stebe. 3. Dá Dor Lafterbafte nod Gier nad 5 efrung frebe, aus Der Súnbe bartem Jod mieber fic erbebe, feidet (s)ott inande Noth, mancbe bittre seiden in ber Sunve Jreuden.

4. आ $\phi$, Der Geele füse Ruh, und des \&eben frieden, fommt Dem Lafter nimmer zu, ift ifm midt befaleben, ad, es oroht jebe Roth foredliber ifm im: mer, frieden bat er nimmer.

5. थक, fo foin if Die शRa= sur, Docb Dem Lafter nimmer, uno fo freunolid grunt die Siur, dod fús ibn nicht im= thet; Troft uns Sidt, giebt ibm nidt, Bottes milde Eoms ne, Segen nid: uns 230min.

6. Laf ung Deine freundlid: feit, Bott, nie forglos mas den, und in unfrer beffen zert, forgfam fenn und madben, dab̈ mir, Bott, niemniE Epott mit Der Gunde treiven, treu den Buten bleiben!

\section{Die liebe Bottes.}

5Nel. Bott Des Simmels und Der Cride.

$R$.

Riebe, die ou miф zum Billo Deiner (Sottheit baft gemadi die mit mebr als 2 atermilde Der Berlorenen gedadt; Sieber Dir ergeb' id mid, Dein it bleiben eniglid.

2. Siebe, die ou mid etro= gen, Durci) Der sputter = Siebe Sinn; mid) zu spenfwen bin: gezogen, ia ourda alleb, mas id) bin; Clebe, Dir ergeb id mico, cein a bleiben emiolid.

3. Siebe baf du mir verfun: Det, mie nod nie gefiebet mar; liebe in mir angegundet, Die mio aubfült ganz uns yar; Liebe, Dir ergeb' iक mi $\phi$, dein ou bleiben eniglitb.

4. Liebe, die burci liebe rei: nigt, wo nidyt' anders rein's gen fann; mit $\mathrm{i} \in \mathrm{m}$ reinften miф vereinigt, mie ez nur die Ciebe fann; Siebe, Dir ergeb" id mid, bein zu bleiven enigs lid.

5. Liebe, die für alle 23 efent mir ein Serz yoll siebe gab; mid) vor alten auseriefen, fort= suliebeli uber'm (9rab'; liebe, Dir ergeb' id mid, Dein 04 bieiben emiglica.

6. Rein! id fann nicht tot: derteben; fann niकt molfen 


\section{Liebet uns Gefånge.}

i申 bin bein; Dafúr merd' id id aud feben, Durch Dab Eeben felig reun. Siebe, oir ergeb' id mid, Dein au biei= ben erotglia!

7. Du fannf aud nicbt toi= Derfeben, bif ourd liebe etwig mein; nirft mids gludflid bet Dir feben, buras segluden fe= lig rebn. Siebe, mir ergiebrt Dis Did, mein zu bleiben eroig: Jiф!

21. Die Tor etgung.

Met. 3il bes sebens sreuben, $\mathfrak{B}_{\text {is }}$ it id filafen merbe unterm fíblet Gand, fübrt Der Serr Der Eroe mida an feines Shand ! Dructen mid auth Su? gen, bleib is ibm nur treu. to mird ieben Morgen feine (Búte neu.

2. Sommen tribe Eage, to ver 'ag' id nict; bemme ieose filage, Denir Die Bibel fprict: fann er Dein vergeffen, Der Dem 2loler siaub fatrfam zuge. meffen, und Dem fáfer \&aub?

3. Duldram und befdeiden geb) id meine Bakn; mest foll id nidt teiden, als is tragen Eann. Eetnten wird Dort Freuben, wer hier Ehránen fa't; relig, mer auc SetDen ftill mit Danf empfábt!

22. Treue \$orforge \&otte\&. met. 2uferfechn, ja guferfehn.

Ya fúrmabr! ung führt mit Fanfter Sand ein Sirt Durdse gilgerfano Der Dunfeln Erbe! ung, reine fleine sgeerbe. Szal= leluiah!

2. Wenn im DHnfer aHd fein Şåffein irtt, er madt, Der treue Sirt, und lást den Seinen ein freund fein fobeinen. Sallefujah!

3. Sider leitet auz des To= bes Srau'n er ung auf grimen 9u'n, auB Sturm und $23 e l l e n$ fur sublung leifer Dueflen. Snllelujab!

4. Freund fict blid't fein $2 u g^{\prime}$ auf un? berab! Scin ianfter Sirtenftab bringt Troft uno friebe. Er madet fid niфt múbe. Salle buiab!

5. Ja, firtrabr, er if ge= trea uno gut! suct unfer SBobnort rubt in peinen $2 \mathrm{r}=$ men! Sein Rame ift Erbar: inten. Sgallefuiab!

\section{Der gute Pater im Simmel.}

Mel. Iob finge Dir mit Sgers uns शुนกD.

Dev şater, ber im simmel mobnt, bat diefe weite $2301 t$ fúr unz gebaut, und Sonn'uno Mond gar ferrlic aufgeftellt.

2. Sat weite FelDer angeregt, und gutig bingeja't, toag Dbft und fobone Blumett trágt, und mar die Senfe mábt.

3. Er fiebt berunter in ber gadt auf reine Sinder alpe, uab roant and liebt im Don= nerfdlag trie in ber sadti: gall.

4. Er fouf ourd feinen sies bezhaud, nad feirem erben= bild, auf Eroen gute शुater aud, mit Sgerzen fromm und mille.

5. Die 2 áter bautn insontte Saus fid ibre Sulten an, und fomtuden fie ben Sindern au, ro gut ein ieber fann.

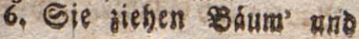


Blumen auf, umgåunen grŭ: neg Jelo, uno tragen mander. lei fu Sgauf fúr ibre fleine Welt.

7. Denn nimmer in ber $\mathfrak{B a}=$ ter Bruft von treuen Sorgen Ieer; Die Sorafame eit ift innen fuft, und fommt vou oben ber.

8. Bott, Defien Euft auf Meer uno lano su unfrer greute fdeint, Dee bat Durdb

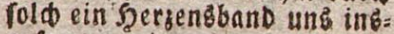
gefamt vereint.

9. Er gaf Den Baternamen Iiळ, Daß́ unz von feiner Treu', Der Ranse Sdónfter emiglid. oin beilig seugniß fen.

24. Preis bes 23 eltettScbópfers.

solel. Sobet Den Sherrn, Den wádtigen.

Sodpfer bar viselten, boll gottlid = exbabener Stárfe! IBBer ift im Stande, Ju dáblen Die splenge Der 20Bere, Die Du gemacit! D, mie entziot mid Die Nradt, Die id an ibnent bemerfe!

2. SBlide, 0 Seele, in iene exhabene zerne! $23 e t$ f Dort oben Die Gerrliden, zaff?: Iofen Gterne? Eomme und stond, Eroe, und wab fie be= wohnt, seaget vom Édopfer - id lerne! -

3. Serne von Gturmen uno Wo!fen und Sergen und Mee: ren: grob und unendio ift Bott, Dem fie alle genoren! Serne bom siobt, uno reenn im Donner er fpridt, ihn, Den 2ulmádtigen, efren.

4. $23 a \xi$ er gerdafien in flet= nen uno gropen sievieren: ร̧écuse und planzen und Stei= ne, und giråte an shietlit! prebigen faut: máøtig ift, De!

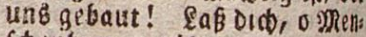
f申enberb, rúbren

5. Dhne ifon wárbe dein St ben uno 230 oblergeb'n wanfeni Dab, rase ict rebe und thut Denfleinften Eedanfen, jeglir des Gut, Deine gropfen, meil Blut, Gab' it Dem Edobyfer zll danf in.

6. Dunermeslide Dactieber Iitáfte uno feben, Die du din Pflanjen und sobieren und Mrenithent gegeben; gros if Dem Sffun! Ervig erbeb id Did nun! Dir foll Eein MRenl miderfteben!

\section{Die finone Esotte ErDe.}

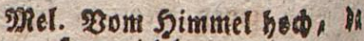
fomm iøber.

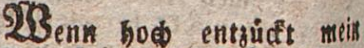
2uge fieft, miefiban, Botht Deine eroe blúht, und talls fend $w_{B}$ efen, grof und fleint fich inres fidonen 230 innorib freu'n;

2. Uni-fie voff holber freunb fidefeit, fí iedez ibrer findo" freut, fo smitterfico fie alle liebt, uns milde Rabsung ik Dem giebt ;

3. Tric biet Det Baum in reiner pradt, und doet dic Blumenniefe ladt; und talls fent Stimmen ouf Der flut Dein lob erbob'ts, Shert Der Ratur!

4. Der grab uno galo D'tauf madfen ließs, von Strơ men fie burdwáffern bief; yol Dem aud aller Gegen quilth Der ibren reiden Edoof ets fítit: 


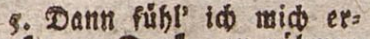
wedt oum Danf, ju rubmen Sen mit Mreisgefang, Def

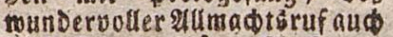
diefe ero fo fán erfauf.

6. Ituf, o mein Beiff, erbe= be laut Den, Der Die 23 lt fo fdoin gebaut; erfreu', fo lang es ifm gefélit, Did immer Dies fer foojiten Belt!

\section{DaB Steid ber Eeifter. Morige Melodie.}

SBSer zafflt, unendider! bie Sieib'n Der Edaaren, Dis fid Deiner freu'n, binauf ou bir

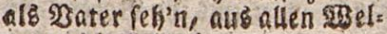
ten ju bir feb'n!

2. Hinábloar ift der Geelen Sdaar, bie einft an Etaub gefeffelt mar, mun lobgeroun= Den, unfre gelí nicht mebs in ibren Sdranfen bólt.

3. Linźblbar iff Der Eeelen Schar, Die unbegreiflid mun: Derbar, ou nod jur Erde fens. Den wirft, uno Dusch Dea SOO zum leben fübrft.

4. 24uf Diefen Enobar nidt altein find 2rsefen, Diefid Dei= ner freu'n, binauf ju bir, als 23ater feb'n, dir jatsdien, be= sens dich erbób't1.

5. Cin umgéblbares Beifter= heer rieff Du, Du 2 thbefeliger, ing Reben; unermétlio grof

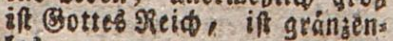
los.

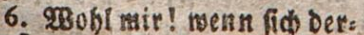
cinft mein Beift aud reinen Seffeln fic entreift, oring' id in eure fel'gen Reib'n miq, 9Ditanbeter \$ottes, cin.

7. Hnd Dann vernimme mein fáarfrer's Dhe Das bóf're Lied in eurem (ङhor, und meine Secle frebt und ringt eud nad, menn fie bem $93 a t e$ fingt.

8. Dem Szater, deffen 24 madtsruf aud fie mit eud verfonsiftert fouf, - nur meia ner bufle bier ein Brab, linz Rerblidfeit Dem Beifte gab!

\section{Das (s) gu feds.}

Mer. Mein erft Befüht (e) Preis uno Dant.

Dmeldein (3rúcf: cin Mení zu fenn? SBer folle Bott nid) preifen? wer des SBeruf'

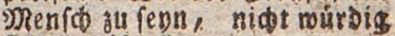
fid erweifen?

2. Th bin eis 233 under Deis ner sypact, uno Deiner meifent (3)ite. D Mení, , nimim Dei=

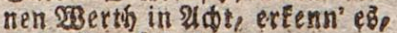
mein Bemuthe!

3. Den Leib, Dot! bera Danf' iab bir, Du gabfe mir Dies fe Seele, शुerfano, Szernunft. Befúbl mit ifs, Durd die id Deste un๖ máble.

4. Der MRenfe ift's nupe Der sedentann, fonft tein Bses

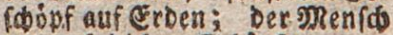
nur rufi diф, Sobipfer, an fanx Anorer debree werden.

5. Inn foufin bis zur Befels figfeit, gabfi ibm ein Ser vols Triebe, gunt soritgefubl, jue

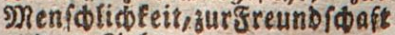
und zur liebe.

6. HnD Diefer Baben grulle idofft ibm umáblbare frels Des: giebt ifm fur Sugento Meth und Sraft, unD शib) 4at Troft in Seiden.

7. Dia, Diф erfenn' idp meinen Bott, erfenne Deine Eserte, id, id erfenne dein Gebot, uno 23 eibheit, (3) uno Sthrte. 
8. D weld) ein (Bluf, ein Meniक) fufeon! Did, Bater, soill ith preifen, und Des $53 e=$ ruf'b, etn mpenfó zu fevil, mith marbig bier erweifen.

28. Der Sథuk Der Engel.

Mel. Serr Gefu Ehrift, mein's Lebent lidot.

Sieh, פater, Dein beglide tes Sind freut Deiner (3iste fid. Da engel meine freun= defino, miegrof́, miegrobi bin id)!

2. Uin meine 2 slege fanden fie, als id ein Saugling roar. Durd fie traf meine Sind heit nie Die orobende Befabr.

3. 218 is am fanften leit= ban's gieng, fab'n fie voll swid auf midd. Utno wenn Die SPrut= ser mid umfieng, da fegneten fie mid.

4. 9uf ifren Sånden trugen lie mids Durd (sefabr und פoth. mein junges Seben foúzten fie vor franfbeit und vor ToD.

5. Dft war id betend inre Ruft, und bei bem Saitenfpiel, Da flósten fie in meine 3 ruft Der Bottesfurdt Befüht.

6. D modet id einft, mie jebt alb sino, Der Engel fret= De fevin! So mitleiosudi, fo gut gefisunt, fo zártlid uno fo sein.

7. Nie mein mein Sdus= ceiff uber mid, und flief' bon meinem Pad'; er jaudze, lleb' uno fegne mid bei jeber gu= ten Zhat!
29. 23 eifmactstied. Mel. Ebrifub, Der ift inein Seb. $^{-}$ Sereint eud, Eकomettern, Errícer, in andadtbotle Reif'n; Der foonfte \as fommt mieber, er foll une beilig fern!

2. S)it innigem Bntguten lob: Jefum, (Gottes Sohn! Er fam, une 子u begludien, berah vom Simmelsthron.

3. 2u mitleissoultem Triebe mard er ein Menfó, mie mir, und mandelte voll liebe allf Diefer EcDe bier.

4. Er lehrte Batt ung fell: nen, und toas (Sott moblges fólts; biefis uns ibn 23 ater nell: non, den grofen serrn der Welt.

5. इBßos ung ! Durd feine Sebre fest man nun meit und breit, Des menfoben gróbte Ehe re in פrenfdenfreund lidfeit,

6. Und in ein frommes Stre ben, Don alfem linreat ferli, zum 230 obl Der $\$ 3$ elt zu leben: o Enriften lobs Den Serrn!

30. Iretbe = Gejang an Seburtsfefte gefu.

cigene spelodie.

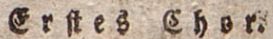

Sreut euक, Sdweftern! freut euch, Soruber! Eingt Dett foern geweifte Eieber! 2uf die Sreuden feiner sinder fieft ber szater freumolicó nieder.

2. Singet, Daf́ponutertit eallen, alle Simmel miederbal: len! Unfre froben Đreiaggefáns ge, 刃ater, lab fie Dir gefallen! Z weites (C) or.

3. gBenn vereint, in froben choren, Sinder ibren $23 a t e$ 
efren, Derfie alle zórtlid lie: bet, follt' et Das niकt gerne Giren?

\section{भा 11.}

4. Sa, bu wirft eह gerne Goren, menn vereint, in fro= ben Egioren, Jinder, Die Du gártlio liebeft, did, Den guten Bater, ehren!

Erftes Ehor.

5. Sieh, wie unfre Sjersen brennen, toeil mir did jeßz Sonter nennen, und ais, obne Jurdit und Sitterm, findid fu Dit anfen fonner.

6. (Sott! wer lebrte uns hich tennen, babmir olab jeßzt $23 a=$ ter nernen, und baß Einolid unfre șergen, gegen oi bor Lieb' entbremnen.

\section{Sweiteb हhor.}

7. Ward er heute nide ge. boren, ber zum Seliand auser= fobren, uns - Den sbater Een=

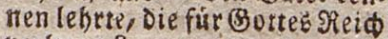
verloren?

\section{af $11 \mathrm{e}$.}

8. Ung, firr (5)ottes Reid verforen, maro eir Seiland quéerfohren, Der uns fl Dem Bater führte! Sceute mard er uns geboren.

9. D, fo freut elsd, Edwe: ftern, sruber! Eingt Dem Serrn gerweibte Sieder! Emig fiebt nuf feine Jinder, unfer Sater freundido nieder!

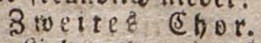

10. Piebe gab ung unfer $\mathcal{C e}$ ben; bat den Sjeifand ung ge: geben! Eroig wird un \& (Sottes: liebe, mie oie \&uft bie 233 elt, unfomeben!

$$
\text { \& } 1 \mathrm{c} \text {. }
$$

II. Siebe anb unz unfer $\sum_{e}$ ben; het den Seilane uns ge= geben! Emig wiro uns \&ottes: liebe, wie oie Suft Die $2 B e l t$, aufobueben! 3r. Die Rinber = Riebe Sefu.

\section{Eigene भRelodie.}

Riebifer Jefus! voll Erbarmen trugit Du Sinder auf Den 2ir men ; freuteft vicd, fie angublif: fen uno an Deme כruft zu orícfen!

2. Dit melden fanften Mienen fonenteft ou den $S_{e}=$ gentibnen, dafife, frei von Ju:zend = sưnoen, Ruft an ala lemşuten finden!

3. 25 en! wir gleid Dich hier nicht reben, frewnd Der fins Der, vor unêtehen: baft Dus Denrind uns niकt minder lieb, alo jene fleinen fimber.

- 4. Jekrt nas fiesft Du mit Sergnügen, afle finder in Den פBiegej, alle zóditer, alle fina= ben, bie von Seerjen lieb Dich

5. Durd Did gab unß Bott Das Seven! Du baft alles ung gegeben! Du millft, Dak mir gut auf Erden, und im Soins mel glúaflid merben!

6. Jefub! ja der fiefin mit greaben, wenn mir fromen das Bófe meiben, menu mir ofne

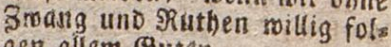
gen allem Buten.

7. Menn mir ffets Dir nehr,

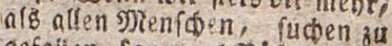
gefallem, fann uns Bófes nids ts begegnen; Bu, bu millit uns enia fegnen!

8. Jofus! Du madif, wenn wir follafert, wie ein Sirt be feinen Enafen; ou bift bei uns in Gefaifrell, fomnt und millft ung gern berabren.

9. Greudig breiben unfre Serjen allo im Ungluce, aud in Sकnterjets, menn toir glaus big ju vir flefen, roie renn wie oich yor unह fábert. 
10. Wenn wit unfre efterm lieben, nie mit গJorfab fie be= truben, allen Sindern Butes gónnen, (o)uteต̉ thun, fo viel wir fintuen.

It. Wentr mir gern uto ffei= biglernen, แท vom Figenfinn entfernen, alien, Die ung (3) ters febren, willig folgen und fie eliren.

12. Went mir gerne vor did Ireten, Jefub! uno mit 21 = Dadot beten, unछ von dir er= zablen laffen, und dein orsort su Srergen fofien.

13. 2B०ดी แกร! ment mir Deine Lebren gern, mit Ernft uno einfalt, boren; wenn wir Deine (Gut' ermeffen, Deine? Thaten nie bctgeffen!

14. $\mathfrak{B} a B$ wir núbzfides be= gebren, millft ou millig uns gemábren! Du, Der alle Saare gáflet, meifin, masjedem 5 Men: foben fehlet.

15. Dein ift Bottes Madt uno Stårfe! 3ablloz, Serr, Iino Deine rberfe; mas fann uns die Rube rauben, ment wir findlich an oid glauben!

16. Safs uns effrig Dabin freben, oich zulieben, Dir zu leben, ftets mit Euft an oíb An Denlen, Der nur \&utes uns will fiderien!

17. Módt' did unfer Sers umfoffen, und, mie Du, die Suntee baffen! Muditen twir idi nie betruben, uns oia úber alfes fieben!

18. Nein! o Jefu, feine Siebe, feine aleidet deiner Sie= be! D Dis freuth ber Men= cisenfeele it, Gaft (5eduld, nods sonn wir feblen!

19. Эa, ment uns die รeh= let retren, millft bu gnăbig uns veraeiben; millft Den S\$a=
Den getn verguteh, uno vor Sunden una befuten.

20. Soers Des Simimels und Der E:Den! Silf unz ńbnlider Dir werden, dab wir einft, thit aflen Frommen , Greund Det finoer, su dir Eommen!

32. Das Cebet tez Szetth. Melr 30 simmel bod, dh fomm' iф ber.

2 Siater, Dem fein andret gleidt, fo reit bas jeer bet $230 l$ en reiфt; verebre, met did denfen fann, uno bete vid als Yater an!

2. Dein Reid, ;s Dem nur Der gebort, ber díb von galls gem Sergen efort, vermebre fid uno jeber fes dir, und beinets 33 illen, treu!

3. 2Bab Du, o 23ater, willfit) ift gut; Seil Dem, Der Deinen IBiken thut! Sfud wi: , aud mir, Serr, roolten ibn, Dell bubetn (Beiftern gleid, voll: gieb'n.

4. Dul bift es, bet bie welt esnágrt: felbet Thieren unter" balt getwábrt; D'rum bittelt wir yon Dir, Dem Serrn, mas wir beburfen; Du gibft's geth.

5. Dubift die liebe, bifnoll Suld! DIa $\bar{\beta}$ uns, beim (Be: fubl ber Sould, Seerr, beine Bute nod erfreu'n, und uns fein Srudern aud verseib'n!

c. SnB un' Den SReiz fut Sunde flieh'n, une immer el: friger bemuif'n, nur $D a \varepsilon$ is thun, mas Dir gefáltt, unD un ein ruhig Serz erbált.

7. Errlofe unह, erlô' แกร (3)ot, aus jedem Jitumer, jes Der Nots! ! \&aś ung nod ftets beno, auf oids let'n, im nod dein Seil erfieh'H! 


\section{Sieber und Befänge.}

8. In Deine Stmmela STei= Jigibum, nuth dort erficalle, Gott, Dein Ruhm! Du bift Der Seery Der Serrlidfeit von Errigfeit วัน Emigleit!

\section{Die fimblite Sorgen: lofigfeit eities remen Şerzenz.}

Eigene Melobie.

2 शrenic, Dernimm des Edopfers Ruf! Er, Der Den Sireis der crde fouf, Deßs ci: genthum die frimmel find, ruft, dir in feiner Sabopfung zu: 0 Eterblicter, mag forgeft ou? Sh bin bern शater, ou mein Sini!

2. Der Did gemadt, forgt auch fur Didb; nidt auf oie Eroe fotránfit fid Der grofie NIan des Simmes ein. Dießs Qeben ift ein 2ugenblide, ein Frúflingetraum Dab idoinfte Blúd: ou folft, o minim, unfterlich feun!

3. Beonafe Ber unfterblicts= Eeit, oer úber Ervoe, 2belt und Beit ein Şer;, bas edel benft, erbebt! Empore dia in meis

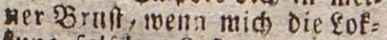
fung falfoter \&uff vom Buten abjufübret frtebt?

4. Die Rafen um der snfter Satupt berblúken, ef ifr Eflav eE glaubt; ibr fodandicher (3es= nuf entefrt. So bin sin 》ul= grin in oer Beit; nur \&reuden einer Emiafeit find meiner gan: bea Sorge werth

5. (3ieb mix, o Du, Der aer: ne giebt, ein Sgerz, Dab tur Das Bute liebi, oas tugend: Saft und heific in! Mrad an: Dere groß snügt, und meiner Pfidid ge: treu, ein mabrer meifer uno ein Ebrift!

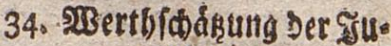 genojeit.}

Mel. Mir nad, pridt efris Rus unfer geld.

2 Jugendzeit, mie flúgels idnell eiff ou vor une vor uber! ROD Iadt une alles ftoin und hell; einft wiro uns alles trüber. $D$ golone Sage, trie viel (s) flob'n, febrt nie zurúcf!

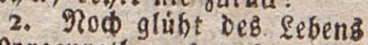
Morgenroth auf unferin fri. fiten $\mathfrak{B}^{3}$ angen; liod fome of uns unfer táglió Brod, to nut, fo fúb; wir bangen ant

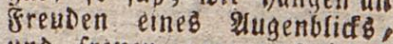
unt freuen gand uns iebes (3)

3. Sein Bram, fein (sift Des Summers foleidt in $\Xi$ draf Durcí unfre Bsfieder; und ob ein Samerz unz nieberbengt, mie bald entilieht er miener? Wie balo fommt, unerfámpft; Die SRuf' tund fübrt nns neuen greuden zu!

4. Tod liegt dez Sebens lángre $\mathfrak{B a h n}$ vor unferm Bfict; mir beben fie taum erft u Durdalatifet an! $23 a$ Iást fíd nod erftreben, twens, treu ber Pfidt, mir eifrig thus, was Tie gebeut, 3 u fruh nidot rub'n;

5. Noch if, - Seil ung? - Die Beit der Caat; mie mirb bie Érnbt'entzúden, wenn guter 2eerf' und eder That, mir viel un une erbliden: mo, mer bier fárglión ausggefireut, su fpát, toas er ento bebrt-berellt!

6. SRerr! Der ung fotif, Gaike 
Gugenderaft belebetunf're(Gie: Der; Den Beift mit edler 2BBif: feniळaft ernábrt: nimm unire Sieder, die mir dir bringen, gnádig an, du, ben fein lied erreiden fann!

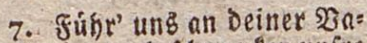
terband: nod fomanten unfte Curifte; mebr' in un? Tugeno und Serfand, lebr' un gewif fe Iritte; uno frraudeln toir in unferm sauf, fo giff ou váterfiф un: auf!

\section{23adfamfect ưter sun felbft.}

Nel. Sat finge bir mit şers Imi mono.

(3)tt! unfre Gugeno, wie fo fotmat if fie in (Buteri now), wie leidt gielt fie Dem géferi nat), und tragt bes saftery S०d! !

2. WBie balo mirtet atif bas meithe Ser' bes erfen ein: sruff's firaft; mie leictit er: watht bei euft und Sobers die erfte seibenfotaft!

3. Serbergen roolfen toir an nicbt: wie viel \$efabr unk Droht; mie leidt vergeffen iff, mas Mfficht uno mas uns gott gebot!

4. D' rum laffet Sitten แก im firaft bet Dem, Der afleg giebt, Der Dab 2zollbringen in uns fकafft, Der un als Sater liebt!

5. Docb nidt nur beten, maden atid) und forgen molien mir, baf wir nod treller in Gefrand oer firaftelebentier;

6. Das mir nie jagbaft fitle ftef'n, in (S)uten immer trág, und imtner mutbig meitergeh'n Der Zugeno ffmalen 23 ?
36. Fientheit ber Seele witi Deb \&eiber.

mel. (Bott fev Daut in allet Welt.

(3) ituflids: ift, ner eint als grann, ofne frige fagen fann: Serzeng Reinfeit if mein Fisfm, fieurdheit bfieb mein ह: 3enthum!

2. Der wird ftare und wird boll smath, und befidt gelun: bes slut, Der vor Eafiern fio beroabrt uno Die Siraft Derglt gento mafrt.

3. Froben Nutbes farin et ferin, Darf fich feines Butter freu'n, neil fein szatek uno Eein freund ưber feine thite" meint.

4. Seine Uniáulo bált tt merth, meil er Bott uno Ehri: ffus efret, Deffen sluge nidis entfitiebt, Der aud ing so"s borgne fieft.

5. $530 \mathrm{~m}$ Bemiffen niфt er: ftrecft und bon Lafiern unbe: flect , jeoem frei ins Antili feb'ti; o! Das ift wahrbaftig foon!

6. Darunt mill iós immet fort seib uno Seel an jedent Srt halten unbefle d t und rein: Dann, Dann merd' it gludfid revn?

\section{Dab Bemiffet.}

গ⿰冫广orige melodie.

Geder if in feines sruft eit nes Richters fid beruft, Det beileber sandlung foridt: of fie recht fell oder nid)?

2. Sedegute trbat, die ibt alfo thut, die freut eud biet; eater Sidbter billigt fie: biefet Sogn entgeht eut nie! 
3. Esenn ise abor unredt thut, o fo meidt der frobe Muth; bindet cudb Die 2ugen 3u, ish habi Dennow fetne Siuh'.

4. Súb if DeE Gewifieng Sobn, it Des Simmels Sor

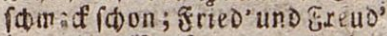
in eigner sforuft - 0 mas gleis фet oiefer \&uft!

5. \&ber bitter if Die pein, fels? datlagerficts tu fepn; $e^{2}$ of fublen, manfen foledt, bandie niळt naф \$ficht uno SRe $\phi t$.

6. Sdbellet bes Bemiffents siadot, lieben finder, Tag uno Radit! (3ott ift's, Der eb̉ in e:id iduf; ebretebals feinen Ruif!

7. Nảber fónint' unb (35ott niøt fenn; ncber feitnen 23 inf verleif'n, Derunblebre, was un' nußzt, und bor den Şers Derben loútet!

8. Das Bervigen lif deer Stab, Dee unb bom 23erderben ab auf Den $2 B$-3 zum (slucfe fubbr, unb an (j) ttes fant regier.

9. Denn, mot tåurఏen un aud leidt, und reil dab \$es wiffen foneigt, to falten mir Die shat nidt foledt; und die Shat ift bod nidt redo!

v. Aber Dann iff nidst be= Dadt, Dá̉ unb 巨कomadbeit feliffam incat. Das (serviffea, finder, liegt im \$erftand, und Diefer trugt.

xr. Deshaíb, Sinder, iftes faion, um Erleudtung Bott ju fieb'n, mie einft Salomo es that; - und Sott gab if)m, mas er bat.

38. Det sBerth Des RebenB.

Befannte פrelodie.

Reben if Des Simntels gróbte L Babe; ift des tiefften, nórm=

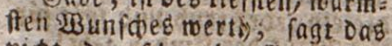
aidt Der fdomacte \$reís am
Stabe, der dencroo mit 3ise iern bort?

2. Sagt Das nidt ber Såuga ling in Der rbiege, nenn dee falte (S) auer ifon befalts, uns

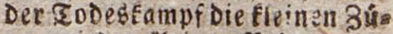
ge, jeden fleinen Reiz an ibrs entftellt?

3 East das nidt mit bats gem (3irs'n die Taube, menrs Des Beters Mordfudt fie be. broht? Sagt das nidot des fletnle SBurm in Staube? 2id : fict mindend leidet er Dent Too.

4. Eelfit Dab Dajenn - nue Dés Sebens Sdaatten, ift bens firaifen immer now ein (3) felbft Die Bráferbalme nuf Detr 5) ?atten ferben traurig unter Sonneiglut.

5. Uro aub (Eráberit, jmio fobetr oicitem Moore, orangen Irublingelumden fith bervor: gmifden Dotnein bebt oie junge Sibfe frob ibi glánzendes (s)es ficht empor.

6. Siror ung 9irmen brofient taufend plagen, bun der $2 B$ ie: je bis zum fruben (8rab; nber taufend, taufend freuden fa. get, baß eit guter Bott Das seben gab.

7. lino gerúbrt im mútterli= den Sperzen, fikt am wege dic öludiefigfeit; irautert, nenn ibir mablen, bittre (5dmerjen, fatt Des Segensi Den fie bolo uns beut;

8. Seut Deti Sirten bintet reiner Speerde; mie Den Gure flen, feine jrenten dar, liebs nocis immer ibre fleine erob, Die if $r$ Sikg in golonen Beiten mar.

9. Ta, Daz \&eben il Dos Siat: inelg (sabe nerts, Das Dane in unfern 20ern foblágt! Fủblt Das nidt, aud bei ber flein. ftell solbe, wer ein reines spen ini \$urea trágt? o 
39. Der mabrbaft greie. Eigene sprelodie.

STzer ift ein freier Mnann? Der Das Eefen verehret, nicts thut, was es vermebret; nidts, wenn er es aud fann: Der ift ein freie: Mann.

2. Mer if ein freier Mann? soem feinen fromunes Biauben cein freder Epótter rauben, fein Letbtfinn saeiftern fam, Der if ein freier mant.

3. פser if ein freier झूann? Sen nicht Beburt nod. Sitel, nidot Sammtroff ober fittel Ses greniden bergen Eann; Det if ein freier 57Rann.

4. OBser ift ein freiers) Der treu it feinem Stande; cud felbet vom 23aterlande den subant DutDent lann; Det if cin freier grann.

5. $28 \mathrm{Ber}$ ift ein freier Der, mus er Out uno Seben gleiф fúr die ₹ugeno geben, bo d nibts verlieren Eann; oer sit ein freier mann.

6. 2Ber ift ein freier spann? Der bei Des Todes Rufe froh auf Des Brabe Stufe nod

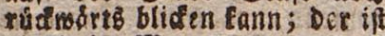
sin freier sptani.

40. Joфjळáh̨ung Der Elttern.

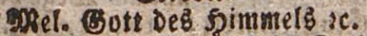
Pinber, bie ihr nod im sireife eurer guten Ëttern meilt, uno um Sileid und Erant uno Epeife forglos nur zu ifnen eilt, und Durd fie in jeber gifidot \&ebr' empfang't uno uns. terridot ;

2. Sinder! o bebenfet frubs, mab fie thun fúr eller Glía! Denft die namenlofe Soúbe, oud vom erften 2lugenblid eu. ses 2ufotuht'ns biह gur Beit eurer Reife ftets gemeiht!

2. Citerntists if obne
Sdranten. Stinder, nie be lobnt ibr fie; Danten aber fónat ifor, Danfen fút Der Eltern ?ieb" und Múh', fúr fo mande lano ge Ract, f folummerlos fúr elid Durctoract.

4. "Sie aแb reinem serien lieben, ihnen treu und folghan fevil, nie mit Sorias fie betris ben, jedes lareat forell bo retu'n, obme Silugein ibner trau'n, und auf ibre gointe forau'n;

5. " Riemale troßzend wide: ipreden, ithre Wusfor fellt verfteh'n, fill, gelafin, ible St)wádien, - fie firo Ment foben! - viberieb'n, uno ie chren, bis bie Bruft, in bif Todes 2rem fie ruft;"

6. "rRingen, Dấ Det geno pflege nidt an eud vers

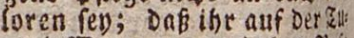
gento צrBege, euren isoben stu? tres, big ift an bem sill ftebt, jebems eafter trohelii, gebt;" "

7. "Eure Siraft Den Elten leiben, menn fie fraftlos fitio uno folvado, fie mit Irof und Süff' erfretien, bei Des alter' ungema DanE und Wflidt; Rinder! Die" vergefiet nidet ?

\section{Die Strírfe eltertidit: Siebe.}

Mel. sulle ift an Botteb Seget. geine Riebe gleidot Der Siebu N Die in Giternberien mallt; iede fiebe fonft mirt falt, aber! 0 ! Die flammentriebe guter eitern far ibr find bleiber enig, mas fie fint.

2. $D$ ! mie forglict in bet sflege fino nicht estern fur iht find Und die raubften $2 B$ ge find if nen immer Blamenteg? die ein jedes frúb uno ipát fát Dดร 23 phl Der Sinder geht! 


\section{Rieber uno Cefânge.}

3. Threr finber wegen $n d b=$ ren fí oft Eittern Eúmmerlit, und verfagen sreuden fid, wel= de Eeine offidoten mebren; Da Der finder $230 h$ l getweibt, fie fein Dpfer ie gereut.

4. 5301 er 3 ater, um aus glutben reine Rimber zu be: frei'n, fárote fid nidt felbft Gitiein? BIuten murd' Die Mont= ter, bluten, ribe Sltut ibr fing aus शoth; 2Rutterliebe trop? Dem ToD.

5. Mustertretre, Saterliebe,

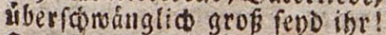
D, mie elent waren mir obne oiefe Simmelatriebe, die an Suld unerblid grob, Bott in eitern Sgergen gok.

6. Elternliebe, (s)t und (8) $n=$ ben, Sersenztugend, funft, Befóide, @eifebbitoung, un= fer Gltúf, nllez, was wir ist= mer baben, alles \&use ftromt Quż Dir; Bott Der Siebe, Dane Dafur!

7. Sinder eurer ettern pfle= gen, rev, bis einft ibr श्age bridt, immer cure fiebfessficos! Seil Dem Sind, und Eortes Ge: gen, Das ber eltern 2 es er freut, bis an's (5rab mit 23!u= men ftreut!

\section{Die Pflidut DeB Ese: borfamb.}

Sefannte Mefobic.

ern will id geborfamients, Jolgfametit if Sindern Sierde, meine eltern ou er= freu'n; fey mir innige sogier: De! D mie fonnt' id bie se= Irúben! Die mix (8)ott zu füh)= sern gab; fie su ebren, fie su lieben, rey mir sreube bis ins Brab!

2. Şab' iक meinen EItern midt aules, altes zu verbanfen? Emig folldie heil'ge wftiat meiHer Dantbarfeit nidtwanten!
Shre beilfanth Befekle will id treu und folgfon thun; Segen wiro auf meiner Seele Durd mein ganzes feben rub'n?

3. Reine Wiederfpenfigfeit múffe meitie bruft entehren; Eitern mact fie Serzeleid uno vergeblid ibre sebren! צ3eb mir, menn id die betrubte? Die nad Bott mir alles find! æBár iø's sserth, Daß man miф

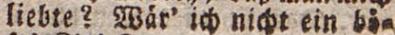
fes Sind?

4. Folgfamteit yereble midp mad' mid meinen eltern were ther; Seele, fonft Durdbrins gen Did ibre Seufzer einft wie Sdwerter! Sebendlangfev meis Seffreben, fie Durd liebe zu er= freu'n, gand für Bgott und fie zuleben, uno im IUD' in $x$ হrote ơ feyn!

43. - Batertanos = Sico. Eigene melobie.

Zut Simmel erbebe bio frets: Dengefang! Sow tone aus fróblidera Setgen der DanE! Einieder, vous 往aterfandelies be Durcoglúbt, fing freubia Dette Sódopen ein feierlid fies?

2. Er lást anż betuohzendies gládlide fand ; befobith uno beglidet tins mit feguender Sand; rein Segent ergieft fid auf alle berab, er wendet Ge. fabren fo váterfio ab!

3. Die suen une frigel fefy' luftig umber; bie Solme von gotonen frúdten fo fower; Die Serge nitpraugenden 3 áumen befézt, Die Triften von wáfferne Den sáden benesât.

4. Tód lieblider, als Dort Die Sonne, ibr 2 ill frablt Religion fo beglüa end, fo mild, erleltobtet uns Erbebemobneris

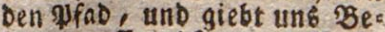
lefrang, Crquideung und $\Re$ atb. 5. D wofl Dem, Der's fubles, $\Re 2$ 
ste olúclid et ift, im Sande? no sriede und siebe fid fuft; im Sande, wo redot und $B e=$ rcdtigfeit mofnt, uno no fid Die gruthe Des Siedliden lobnt!

6. Sor Blúfliden múnfoet

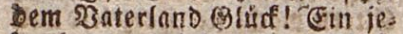
berbezetuge mit freudigemzslia:

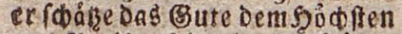

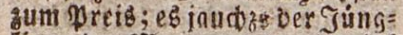
Iing, Det Mrann uns der (Grete! 7. Dnz Chriftentfum bleibe an' theuer und werth; (Bott

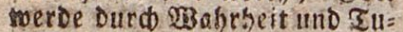
gend verebrt, (Berectigteit zie: re Den furftliden Ibron, Den Foben aum Sdbreten, Den frommen zum Sohn!

8. CB blúben Beraerbe und Rafrung im Land, unD Segen WHD 230 blfabit it iegfiden Stand; ein jeder befordore, fo viel er nur fann, des gater= fants Be Befte, u. freue fid o ann! 9. (Gott! fev un ftets freuno: lide uno fordere Du DrB SBere vinferer sánde in Sriede und Siib; fo Ieben mir glúdito, ino trallen von bier jum beffe: sen 3aterland, oroben au Dir! 44. Rieb am (Beburtỏtage. SR. Irmu. flein if meine Shutte. Gobner fas, did feh id wie: Der. $D$ fen feftido mir ge grúbt! Der bu touroig meiner lieder, murbig meiner fretiden bift; beit'rer indt mirfeutder Spergen, fabiner focint bie Sonne mir. Sक bin frob und bine Surgen. SDoner Iag, Das banf ic oir!

2. Dem id meine sieder fins Be, bem mein beiker sanf ge. bubrt, Serr und SDovfer aI= ler Dinge; o bu haft micis treu gefübrt. FloB nidt feit Dem erften Snge, der mir einft mein Eeben gab, Deine Suld mebr, Qlb id fage, fegenzyolf auf wiक berab?
3. Da it ungetwiB not manfte, ie der Scbritt nod frau: delno mar; Da fein Eallen dir nod banfte, Da raon nabmit ou meiner mafir; fougteft foot

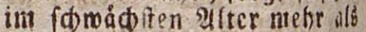
váterlid für mids. Welder Dant, o mein Erbaiter! is twobl arofi genug für did?

4. Eider baft Du mia gelis tet, bielteft mic mit flatit Sand; hatten mir meil Blúf bereitet, eb" mein Sgers dip nod verftand, liefeft aud mit treuen Sorgen für mid nit: malb, niemalb ab; forgett hel: te, forgeft morgen, forglt fit mid) bis an mein Grab.

5. Briebfo du mir ein langel Leben, oder einen fruben $\$ 0 D$ : willf bu mir vief greudengr: ben, oder gicbit Dis Sorg' unt Poth; mie Du willf: von deis nen Sånden nebu' ic alles Danfbar all. Du mirit allebi alles menden, beffer, als in Denfen Eann.

6. Nimm Denn, श3ater, thith gserfpreden eine beinen 26 all: Dels an! s) Rine Jreae nie fl breden, das gelob id ernflid an. Dir mein ganjes Sertil geben, dir mein ganzes sers meibn: S3ater, ons foll thill Beftreben, meine bo bite sren: de fenn.

\section{Snorgentied. \\ Eigene 2relodie.} 5) Cuf! Bruder, auf! Det Tas
bridt an; Die bobe Gomme fabset herant. Suf , nuntre Sameftern (Boridoer), fauttet nicht, uns minft, unह wintt ifir freunolid lidot.

2. Sa! wie fie bort in gib nigipradot herab atif grúnel Fluren ladt! D, feht fle Ge aes uno Gedeibn auf Den er madten Erdball ftreun!

3. Sie ládpelt miloigliф utte 


\section{lieber und Befểnge.}

umber, als fie eine Miutter már', uno fprácb: „ifis Sieben folummert nidit, freutelid an meinent goldnen Sidt!"/

4. llito iprad : moerlaft Die

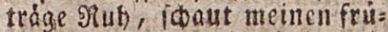
ben werfen;u, und lel't in mei= nem Angefict des 2 bohithuns Sreuben, folummert nidt!"

5. Bott, beffen Slllmadt fie

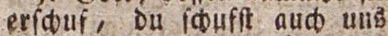
mit Dem Seruf, gu shun fum Dienfte beiner mselt, was un= fern firáften mojglic fálit.

6. Bror fam fúr ung nod) nidt die Beit, zu thun - o mår' fie nidot mefor meit! Dod (S)t'हु julernen, niemals rubn: Dief́, Bruber (Edme= fern), (et) jebt unfer (5hun! 7. Diebt! o ieft! fie ndhert fit), uns alleg, alles frewet fid, und afles bante, und alle bingt, Dem Gotte, Der fie miever = bringt.

8. 2uf, Bráber! auf, jum Lobgelang deb Sdopferb ton' aud unier Danf! DanE feD Dem Seerrn, Der fie gemador, aud yon ung Sindern ießzt gebracts? 46. S3zim Ermadien.

Mel. T3arum follt id mic Denn grámen.

Gefus medtbid nieber, blict Jid fegnend an; Deine stue genlieder hat er nufgethan.

2. Reue Lebendf rafte bat er bir verlief)n; o vor sulem bef= te beinen slite auf ihn!

3. In Der Drorgenftitle, Sees re, harre fein; fabop? aus peiner sulle feligez Bedeifn!

4. Sadau getroft und beiter, omein Beif, umber! Jefub, Dein Begleiter! mas bedarfit Du mehs \&

47. Die siorgenfunbe. Eigene Dreluoie.

5) iemorgenftunde bar(sold im 2) Runde ; ein meifer Spruњ voll Sraft und Eaft! Da füglt man miever urd alle (s)lieder jur 2rbeit Suft, zur 9rbeit Siraft.

2. Det Mefte Cäuf́ln, Der Serde Siróafeln, und allergio gel $2 B$ ald Beicorei; Die muntre Seerde, vie rafoen of prove, dieb alles ruft: berbei! berboi!

\section{2hendico.}

Mel. In allen meinen S baten. (3)er grono if nufgegangen . Die goidnen Sterne vran: gen an Simmel bell und flar. Det $2 b a i d$ feht foswarz und fotweiget, und aus den 933 ie= fen fieiget Der weise reber nunberbar.

2. 2Bie if Die 2Belt fortlle, uno in Der Dámmrung Sưtte fo traulico una fo holo; afs eine fitle fiammer, no ihx des इaseb Jammer verfotafen uno vergeffen follt.

3. Seft ibr Den Mond Dort fteben? Er if nut balb au fe= ben, uni ift buas rano tito folon! So fino wohl tiarade Sacden, Die mir getroft bela= ben, reilunfre Shaen fienidt febn.

4. Bott. Iấ Jein Seir uns [đauen, auf niकt tร vergạnglidos trauen, nidot Citelleit un freun! Laß̧ uns cinfältig mer= Den, und vor bir bier aufert= ben, mie finder, froma uno froblict feun!

5. NBofle endid fonder Brá= men aus Diefer ขBselt uns neh= men Durd einen fanften Tob! und wenn bu un' genommen, la ou unfer Sperrund unfer bott:

49 Beim Sdlafengeben. 9R. 2uf, Bruder, auf, ber Eag. 5)en fúben Sol laferbitten mit Du, befter Nater, Hns yom I 3 
Dir! Sieb Deinen múben firin: Dern rub; indem fie foinm. mern, madje ou!

2. Du maltef mit Barmber: figfeit fets úber unfre rebeng = zeit; voll lieb und शBeigheit theilf Du fie in Iog und शadt,

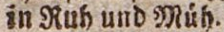

3. Veas gut ift, 2 sater, formt gon bir! Des guten viel em: Dfangen mir. Nimm unfer Dan= Eend Sallen an, für bas, wä Du an unz sethan!

4. 203ir wollen beine sinder peon und bleibin, und unz hod erfreun, safi ou Gort uafer $53 a=$ ter bite, Durob unfern sceilano gefum Ehrift!

5. IrE $D$ er efr meníd auf erden mar, da bradoten fie ism

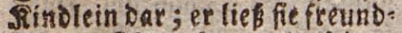
(idi) zu fid nabn, und reines Segenstus empfabr?.

6. Er bleibt und fegnet uns aud beut! Cin Sery voll reiner Stinblidbeir gieb ung, eill Serg ๖on Leidtfinn rein, und lấ une fromme Sinber fenn!

7. gisir fammeln uns , mit frobem 2ruth su rub'n in Dei= ner Baterfut; of $n^{\prime}$ Deit $B e=$ Geis, Du, Serr ber welt, Eein Saar von unferm Saupte fált.

8. N3ir folunmern ein; Du reif'fi bie frift, renn's unfer Iekter Solummer ift ; ad! follt es die le Nadtgefdebn: fo las uns bort Dein Pnttis lehn!

\section{Selbftprtifung au gbeno.}

Snel. Serr, meiner Seele gro= fen wertf.

cin Tag ift mieberum voll= bradt; foun nabet fidis oie Sunfle stacit, mit Sclaf uns zu begluden. Du lieber פater, habe Dane! Dir tonet unfer sobgefang mit freusigem Ent: intifer.
2. Dis foenfteft beut unb mandes solud, uno hieltet Sd)mer von uns surúd, mit freuen ung des febens. \&ф! fonnten roir am 28bond nun ung fretboig Das Befrannnis thun: wir lebten nidt vergebens!

3. Erfülten mir aud unfre \$ficot, uno frónfien unfre (ैi: tern niat, nods unire treten Eebrer? lind raren wir beitit Unterriát, nads gut gefinntet finder patidi, aud aufmerts fame Sorer?

4. Sino wir aud willig und bereit, das Gute fieto mit Solg famleit uno eifrig auţ" úben? Sino bir genteigt, aut ein sgergehn bei anbern gern zu úberfésn? als Büder fir zu lieben?

5. Uno DenEent wir: in Dis fer Radst if umfer Eauf vielfeidt pollbradt! - SBermag Dann ofne beben fid unfre Sell Dir zu nafi'n, um ibren sobn Dort zu empfab'u, nad eineti frommen leben?

6. DBott! Du meis'传, wit fino nod formad; fieb ung mit 23aterlangmutb nads! WBir mol: len beffer merden. Steh ung nit Deiner Súlfe bei, Daß unt fer Seerz gemeibet feg, Dir, 20 ter, idon auf Eroen!

\section{5r. Sifobgefans.}

cigene retelobie. Cineis uno zrane, frohe Sang Dient, Dab Seritt laben. Brober Beift! fel ge preift fúr die edlen Bsaben!

2. Retien bunt, frife, ges funo, fiken wir zur Etelle. Danet DemSerri froh und ger" fúr die fretibenquelle!

3. Frobflid fenn, giebt osec Deibit aud sum fleinfen spah: le; niфt fo jü fómedt's ge? wis in Dem Surten = Saale. 
4. $58 \mathrm{etm}$ ibe eft, fo vergebt nie Dabsi Des 2lensen! 2ho, ibr Slebn, wer tani's febn , und fiф niфt erbarmen!

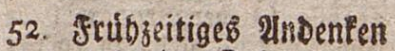
an Den SOD.

Mel. In bab mein Sad frot? heimgeftellt.

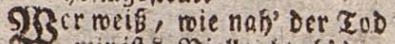
mir if ? Bielleibt eh' bie: per zag verfieft, bin id ver: meltt. wie Dürę Laub, beร Jodes giaub, und mein Sebein bebect toann Staub.

2. 20 ie manches Sind, 10 rofentuth, fobold, wis id, fant in ben sod; nod fab it reine wangen glübn, id labes blithn: bod ad, mie foneti war e'b Dahin!

3. Uळ 23ater! meine Lebens= keir fliefot eilig hin jur Errigs Eeit! כoalo if fie náber, uno Der Sderz fleist Dann mein Seer, uno mid ergreift des judes Sitmer;.

4. Erhalt' aud bei'ns uns= fourlo'gen Epiel in mir Det Erwigfeut (3, fuhl; menn id mid meiner Jugend freu', to gieb Datei, Das id aud flug und másig (e)!

5. 2Benn id fon jtag er: brafien foll, fo na@'s mit mir in Tode nofs; verlás mich niकt in meiner Noth! Durd Did, mein (3ott, wiro mir zum fanften Ediaf Der Iod.

6. Dod foll mein rod nod ferne feyn, Serr, wie Du willf! benn id bin bein; Dann, treuer Gott, berieine min, daß̧ id nur Dir bier lebe, und einft ferbe dit.

53. Das fromme Rino auf rem Sterbebette.

sel. DBer nur Den lieben (Sott. Io lterbe foon; ieood, iक feribe, Damit id ewigleben
Eann; Daḱ i nidot lafterbaft verderbe, ibfieft fió mein \&aufo Der faum begann. Sob werio in mainer fotihlingşeit, als Şimmelgpflanze, Bort gemeiht.

2. Sin ineiner Bruft fårtt eyren (Glauben, ibrelaern, Die igr mict geliebt; Der Joo tant mich niot ganz eud rauben? Da Bott mió eud einft wiedere gieb?. Dalf fen Dir, शater, Dargebradt fir deine Eorgfalts gute शadt!

3. Rnd langem Sdmers und Eurgen Fretiden reißst mi Too aub yiel Befahr; Dod,

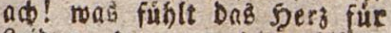
Eeiden, Darunter it gebilbet. war! Dir fen der treu'fte Dane gebradst, getreue s)lutter, gu= te Radt!

4. Berubigt eud, iffr anbers: Meinen, Die ibr mir ourd Das Bifut vermandt: ifr Durft une reid nidt troftos meinen, der Beif if ja in \&ottez Sant: Dec Seib verroel't ju fowin'ree Pramt, wir feb'n uns wieber : gute Pradt!

5. (S) Teich Fråblingeblumetr? wird mein Seben bei'm शhuf blisis in fodon Des Todes Raub: Diéf faran Dir, smeníd! die Lebre geber: Du fey'ft, wie id, nur 2id' und Gtaub. Bei mei. nem (Brabe lene' Den Sinn you Jroiften jum Eiv'gen hin!

6. Tक fferbefoon: lernt afo le ferben! Bsielleidt iff euer Grab nidbt weit. Gind eure Sinder bald'ge Erben, mie id, Der Simmelsherthidefeit; is Denft: fie geb'n unz nur voraits wir treffen fie Dort mieder an! 54. \&ieb am Cirabe. פmel. SAuferftebn, ia auferftehr. 97 iude leget auf daz fille (3) Derfab Der pilger nieber, uno If 4 
feine matten oflieber fótét fanfier SRub.

2. Sarfifer Sdiummer iff fein fobines Sous; er rugt in Er: Denfoouf, befrei't vom Sorme - wie in bem Mutterarme Der Soingling fobluft.

3. N3oir Des Rebens Rínpfen mun befrei's, reift iur tufterb= Jićécit Die midoe sgulle in tiefer Brobernille; einf blugt fie auf!

4. Eole Sedten túbrt im $23 a=$ terland Des guten Vaters 5and aufí nea' hufamtiten; Der renen Liebe Jnmatl glun'n enig oort.

5. Quter! Des für Die lin= fterbiscleit unb Esterblide ge= toeibs, uno eroig's \&eben ourcb Serum uns will geben, fel) bodogefobt!

6. Friede $130 t s e$ sum die fill: de Bruft, mientiloer 23lútben= Duft; bis au Dent sage, mo le: de erdentlage sum Jubel miro! 55. Troftico bei bent Sitabe

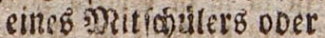
Bseidmifers. Sorige Melodie.

S) eimgegangen an Deb Engelb sotto bift ou ing פater: fand Der Winulsen $b=$ frommen, wobin cuct mir einft fonmen Durdi) Seli Suld.

2. Rofen glcim, baft bu nićt lang gebiuh't; toie man dent Sturm entfieht, bift ou ber erben, Den iunfigen Befämer: Den - von Bort entwinft.

3. Huvollendet giengf du jorat zam Serrn; Dod bleigft ou ifm nicht jern. Ergiebungs: Engei, ibr mandali Sugend = s) Mánger in filarbeit um.

4. Liebend Deiuen Seilano, fangR ou bler fo gern uno roir mit Dir. syit foimmelsualmen. gefranzt, fingt ou uun Plale मren in Engel: Bbor.

5. Dewem Grabe 3f fumen wix, fum \&iebe \$ze Dir; bis frob erfideinet ier Tag, Der uns vereinet: in emigfeit.

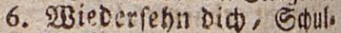
Benoffe (lieber Bruder, liebe (Stmefter), fier, in en'ger Simmelegier, o ne! che Freude! Eitfernt von jedent Letoe find mir aledanu.

56. Uniferblidteit Der Selle. Mtel. Wer nur Den lieten (s)ott. 5) er Lenzerfreuet, mas oales bat; er briót deb gain= ters Feffelii los; Der Bluraen founen flor erbebes ex fribih RUB Der Croe Stodoú; : fremit: fió fádelt et mir gu: unterbs (id), freu diф, MRenín, bift ou!

2. Die Sumne lóft bett Sdbmetteringe $a b$ Datid mit incer Strablen Madt; Da bebt er feine zarte Edowingh

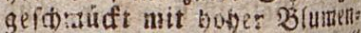
brast, uno minft mir ionell uns nunter freu dia, gRenid, bitt ou!

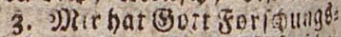
geif gegeben, und wänide! Oie die 2Belt niddt frillt. joil mir! fie bu:gen mir ein qeben! tou fíd De S Sóditien SRath ent: butlt. Sojr', Jefite Ebriftub ruft mir zu: unizerbicis, freu didp פMenfo, bitf Du!

57. 2luferfebung $=$ (5) fant ime Friblinge.

Met. MBe ictoin reuatet ber Morgenftert.

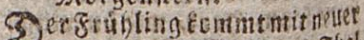
Dradot, uno \%Bald uno thal uno ffur ermact; $e$ a arimet al les gistonne. Die @rop, die ers forbeumar, reiфt@rásøen ung

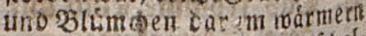
Strabidor Sonne. Eiever fdols len, Gretionenticoer - tonels roieder aub den 2zäldern, Lán wer bupfen auf pen SADern. 
2. Doth, toie fo tur ift frubh= fing E. Suft! Der Sonne Etrab! engt unfre bruft, Die gelben Blátter fallen; Die icobnen Blúmben follen $a b$, teer ftebt Das fe!t, bas frúdote gab, und didote frebel matten Dura bie enfte uber fluren; - Duffre Spuren ftiller Trauer lábrt Des Serbfrminos falter Couuer.

3. Fár ung ein bild det Bterblicfeit! 2Bit armen \$us ger oiefer 3eit, wir blithen, relfen, fallen, mie bort int Ehal Die Blume fáltr. Sind roir benn mebr fúr diefe reselt, ale Diefe slum der alle? Eroe de of: uns; Doch oort oben, Bott ju Ioben auberlefen, toiro uns un= veraoinalich's vefen.

4. Der jerr volljog, maz er verionac estand der Eaa. mo er Durdbracd Der Bråber Dadt unD Brauen. Sebt ibn, ibr vie. ben, auterfteb'n! 2sir nuffen bier Durcs (Bráber geh'n, dą bob'te Sidt zu cosuen. Butt= Iid fiegt er. (Ebritten, finget! - Dpfer bringet, br Erlofien, ibm, Dem serrlidfen, Dem Grósien!

9. Froblodt Dent Serrn! brinat fob uno Dant ibm, Der Des robes nawt bezmang, uno นnE, Den Staub, erhobte! De: bohe Sieger úbermand ; Nadt mar un ihn, uno fie peridomano in belle פgorgentotbe. Seele! máble thn zum fáthret, und $\Re_{e}=$ gierer Deines \&ebenb! 2uf ibn boffit ou nicht vergebens

6. - Tum bóbern Leben fúbrt er Diक, uno niamt gewis Did einft zu fiø, finft beine Sülle nieber. Das Brab umfángt groar Dein Gebein; Docb nidt auf ewig nirb es feyn; verflárt giebt et Dir's mieder. Serrlid wird fid bann auf's neue feine ¿reue offenbaren. 20 olses 5 il wirft du erfabreis.
58. Seliger 3uftanb bes Frommen nad $D$ cm ₹oDe.

anel. Sefus, meitie suverfitot. SDier if nur mein Đrúftng e ftani, aber nidot mein blei= tiend Erbe. Druben ift mein Baterland, Dab erreic ic twon id ferbe. Dodun bring ith oir, o gott, siels uno Daif fút utmen

2. (S)tTEB Stadt Die oroben iff, mo Dir uninotbare mobiet; mo mein s)tittler Sefus (5hrif feinen freunden berrlid lobs: net! foimmel! nie gedent id Dein, obne gottlid frob zu-feon.

3. Er, Dein Iinig, Der oich fier auf Der 2 abih Der sugend leitet; er, mem Szeilano, fat in Dir eine Státte mir bereitet. und an allem deinem seil bat nud meme Seele हुbeil.

4. D mie mobl wird mir vants fenn, mann las eint vids in De. פrabe, frei boh fiummer, 4ngft uno pein, mit vertare tem 2uap febe! Sinfe nue miti Leib jur \&ruft! Bott iftg Der Die Seele ruft.

5. TuDesfarefen formen Dann meinen gelauben nidit er. fduttern. Nur Der Sflan Der Sunde fann yor ber Brabes ?acist ergittern; fromme Sees len fittern nidot, Denn fle find frei vent (Berid).

6. Nabe feis mir oder ferm jenes Sceil, Dab id verlange; ringen rill id, Daßs bom ferrn id) wa's er errbarb, empfange. Sreudig ruft Danti eint mein Beif: Bande der Ratur, zerreift!

7. Dá́s id siefe grreudigfeit einft in meinem Too ertipfinde, mache fiegreich mid im Streis gegen jeben Fiei bet Surtoe! Stárfe mid, o Serr, in lauf? Ttimm mid endit gnádig auf! X s 
59. Sinderfinn. Eigene melobie.

Rafft die Sinder ou mir fous men, labt fie, reekret ihs. nen nimt! Seb!', Die Simo melşrub dee fromsmen frabit ing hettres elrgefitot! 203ie in iarent beiden 15 loe flecienlofer unfotulo egerih, unt dez Eee.

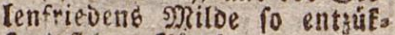
ferto fít verelárt!

2. Shre zarte Soruft emporet nidt ber \&eidenfdaftenSturm; ibres Jergens frieden forres teiner Reue gift'ger भुbrm. Barte fiebe, reine Bute bebt Das yolle Szerzempor, und Der Zugent reinfie blütbe feimt in ibnen bolo bernor.

3. Strifcen fürciten uno verlangen theilt der Sterblide Die Bett. Seitern Sinnes, un. befangen leben fie Der 23 ireficid)= feit; nidbt in Dunfle Gerne fobarend, menn ber soimmel beiter ladt. Sheer EItern Sdus vertraueno, find fie frob bei sang uno Natbt.

4. Mritieid, inniges erbar= melt fúblen fie bei frember Poth; theilen bergliá mit Dem 2lomen, mit bent Solungrigen ibe Srod, ofine lang' erf ab: gumágen: foll id geben, foll id nid)t? eilen fie ibm frob enrgegen, uben fonell Des

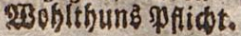

5. EBout ifr jemals múrdis berden, eingugeben in mein Reid, o fo werdet foon auf Eroen unfouldosobllen Sinders gleid! Sourt, o bort Den gros Een Eefrer, mas er ernit und freundido foriot; feine wirk bigen Sierebrer uben froh all Diefe भffidit.

6. Last die Sinder in thit Fommen, lafit fix, webret ihs nen niwt! Yroblt nur pelbet Den $\$$ fad Der Erommen, Der uniouls 2ungefidot! Shic Peime zu entfalten, fie dee Trugesto frúb zu meib'n, inse Reinbeit zit erbalten, mus euф Sergensfreube fevit!

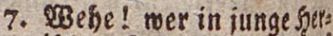
a gift'gen fafterfanen freut! Bitte" find Der Reue Somme" zen, menn er Botteaskito ents

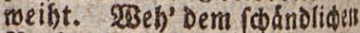
Szerbreduer, Der oiés Sheiligs thum zerftort! Seiner barts ein frenger Fiader, Der Daß (Sørei'n Der unidulo gơrt.

8. Selis, ner ins serg Det Jugend früh Des (S) iten freut, uno Der Bottheit uno Der Cugend ibre garte stuthe meiht! Segen erntet

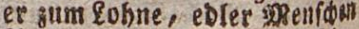
Eieb' und Dane, und Deg fin: melв şirgerlrone, weun fill भbenonieberiant. 

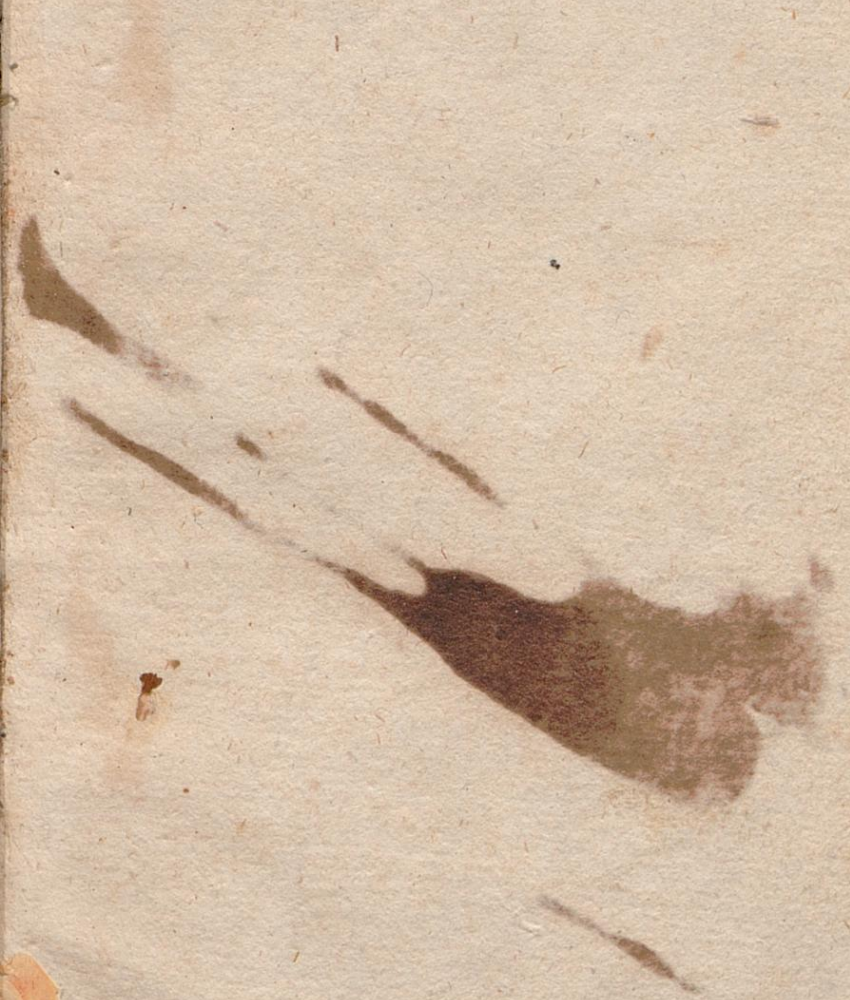

1

(1) UB Universiatsbibliothek Johann Christian Senckenberg

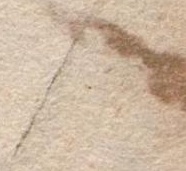

A.

Contans

20

Q 


